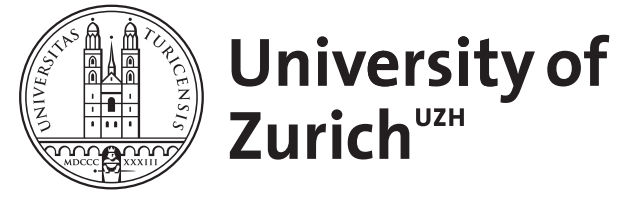

\title{
Ernährung und Gesundheit
}

Faeh, David ; Matzke, Annette

Posted at the Zurich Open Repository and Archive, University of Zurich

ZORA URL: https://doi.org/10.5167/uzh-80800

Book Section

Published Version

Originally published at:

Faeh, David; Matzke, Annette (2012). Ernährung und Gesundheit. In: Bundesamt für Gesundheit; Keller, Ulrich; Battaglia Richi, Evelyne; Beer, Michael; et al. Sechster Schweizerischer Ernährungsbericht. Bern: Bundesamt für Gesundheit (BAG), 227-208. 


\section{Sechster}

\section{Schweizerischer Emährungsbericht}
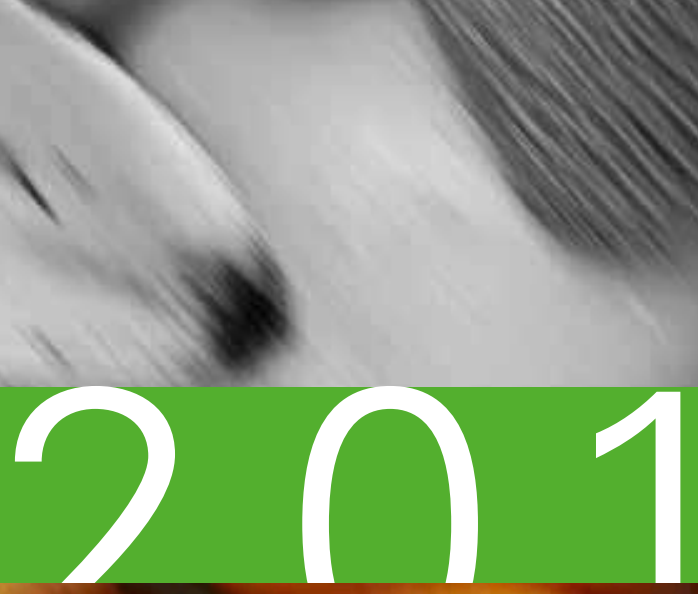



\section{Sechster}

\section{Schweizerischer Ernährungsbericht}




\section{Redaktionskommission}

Ulrich Keller, Evelyne Battaglia Richi, Michael Beer, Roger Darioli, Katharina Meyer, Andrea Renggli, Christine Römer-Lüthi, Nadine Stoffel-Kurt.

\section{Herausgeber}

Bundesamt für Gesundheit (BAG)

\section{Autoren}

Zusammenfassung: Michael Beer

Kapitel 1: Jürg Lüthy, Angelika Hayer, Muriel Jaquet Kapitel 2: Alexandra Schmid, Christine Brombach, Sabine Jacob, Iso Schmid, Robert Sieber, Michael Siegrist

Kapitel 3: David Fäh, Annette Matzke

Kapitel 4: Christian Ryser, Alexia Fournier Fall, Sophie Frei, Andrea May

\section{Projektleitung}

Pascale Mühlemann, Mühlemann Nutrition GmbH www.muehlemann-nutrition.ch

\section{Zitierweise}

Keller U, Battaglia Richi E, Beer M, Darioli R, Meyer K, Renggli A, Römer-Lüthi C, Stoffel-Kurt N.

Sechster Schweizerischer Ernährungsbericht.

Bern: Bundesamt für Gesundheit, 2012.

\section{Gestaltung, Layout und Druck}

Merkur Druck AG

www.merkurdruck.ch

\section{Fotos}

Fotografie Peter Leuenberger www.peter-leuenberger.com

\section{Bezugsquelle}

Bundesamt für Bauten und Logistik BBL, Verkauf Bundespublikationen, $\mathrm{CH}-3003$ Bern www.bundespublikationen.admin.ch BBL-Artikelnummer: 311.106.D ISBN 3-905782-70-7 978-3-905782-70-7

Schweizerische Gesellschaft für Ernährung SGE, www.sge-ssn.ch/shop

Die Publikation ist kostenlos unter www.ernährungsbericht.ch verfügbar.

\section{Hinweise}

Aus produktionstechnischen Gründen wurden die Inhalte des vorliegenden Berichtes in den Jahren 2010 und 2011 verfasst. In diesem Bericht gilt sowohl die männliche als auch die weibliche Schreibweise jeweils auch für das andere Geschlecht. Der Begriff "Schweizer Bevölkerung" umschreibt die in der Schweiz wohnhafte Bevölkerung, womit in der Schweiz wohnhafte Ausländer eingeschlossen sind.

Diese Publikation erscheint auch in französischer und italienischer Sprache.

Gedruckt auf chlorfrei gebleichtem Papier.

(C) Bundesamt für Gesundheit

Publikationszeitpunkt: September 2012

BAG VS 09.12 1'500 D 500 F 200 | 40EXT1202 860295039/1 


\section{Sechster}

\section{Schweizerischer Ernährungsbericht}





\section{Vorwort}


Industrialisierte Länder wie die Schweiz sind zunehmend von nichtübertragbaren Krankheiten, wie Diabetes mellitus Typ 2 und Herz-KreislaufKrankheiten, betroffen. Diese Krankheiten bedeuten für die Betroffenen, aber auch für die Gesundheitssysteme eine enorme Belastung. Vier der bedeutendsten Risikofaktoren für nichtübertragbare Krankheiten hängen mit der Ernährung zusammen: Bluthochdruck, erhöhte Blutcholesterinwerte, Übergewicht oder Adipositas sowie geringer Früchte- und Gemüsekonsum. Ebenso bedeutend sind körperliche Inaktivität sowie übermässiger Alkohol- und Tabakkonsum.

Wie steht es um die Ernährungssituation in der Schweiz? Wie stark ist die Schweizer Bevölkerung von ernährungsabhängigen Krankheiten betroffen? Welche Massnahmen können beitragen, die Situation in der Schweiz zu verbessern? Im vorliegenden Sechsten Schweizerischen Ernährungsbericht beantworten 17 Experten im Auftrag des Bundesamtes für Gesundheit diese Fragen. Beginnend bei den geltenden Ernährungsempfehlungen führt die Lesereise über die Schilderung der Ernährungssituation und das Zusammenspiel von Ernährung und Gesundheit, um als Schlussfolgerung aufzuzeigen, wie die Gesundheit der Schweizer Bevölkerung über die Ernährung erhalten, gefördert und verbessert werden kann.

Das immer vielfältigere und praktisch überall und jederzeit verfügbare Lebensmittelangebot verändert die Ernährungsgewohnheiten der Bevölkerung. Ebenso folgt unser Lebensstil den gesellschaftlichen Veränderungen. Um möglichst wenige negative Auswirkungen auf unsere Gesundheit in Kauf nehmen zu müssen, wollen wir eine Diskussion über anzustrebende Massnahmen führen. Angestrebt wird zu diesem Zwecke eine solide Zusammenarbeit verschiedener Akteure auf nationaler, regionaler und kantonaler Ebene.
Massnahmen zur Verbesserung der ernährungsbezogenen Gesundheitssituation in der Schweiz sollen wissenschaftlich fundiert, d.h. evidenzbasiert, sein. Eine unverzichtbare Voraussetzung für konkrete Empfehlungen sind zuverlässige Daten zum Ernährungsverhalten sowie zur Häufigkeit von ernährungsabhängigen Krankheiten und deren Risikofaktoren. Der Sechste Schweizerische Ernährungsbericht fasst die neuesten Daten zusammen, analysiert und interpretiert diese und bildet somit die Grundlage für eine wirkungsvolle Umsetzung. Der Bericht weist aber auch darauf hin, dass in der Schweiz grosse Datenlücken bestehen. In Zusammenarbeit mit europäischen Institutionen wollen wir diesen Mangel schrittweise, aber prioritär angehen.

Das Bundesamt für Gesundheit als Herausgeberin des Berichtes dankt den Autorinnen und Autoren herzlich für ihren grossen Einsatz, die geleistete Arbeit und die Reduktion des Umfanges; Letztere führt zu einer besseren Lesbarkeit des Berichtes, ohne dass die Aussagekraft darunter leidet. Es hat parallel zur Erstellung des Sechsten Schweizerischen Ernährungsberichtes seine Strategie im Ernährungsbereich überprüft, überarbeitet und konkrete Massnahmen formuliert, um die Gesundheit der Schweizer Bevölkerung zu erhalten oder zu verbessern. Ihnen, liebe Leserinnen und Leser, wünsche ich eine interessante Lektüre und viele Anregungen, wie auch Sie dazu beitragen können, die Ernährungssituation in Ihrer Umgebung zu verbessern.

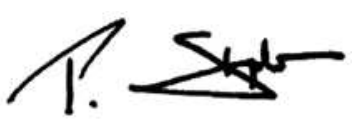

Pascal Strupler

Direktor Bundesamt für Gesundheit 

Vorwort.

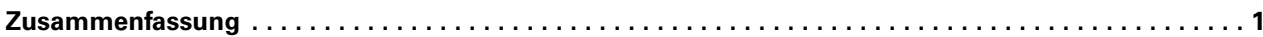

Kapitel 1: Aktuelle Ernährungsempfehlungen $\ldots \ldots \ldots \ldots \ldots \ldots \ldots \ldots \ldots \ldots \ldots \ldots \ldots \ldots \ldots$

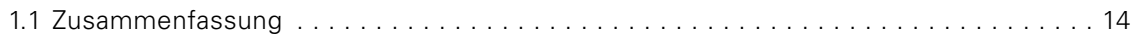

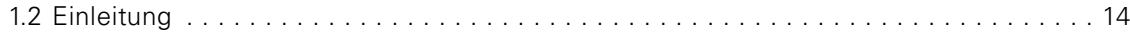

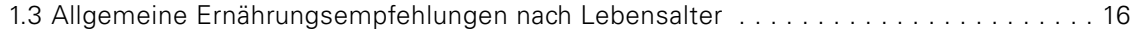

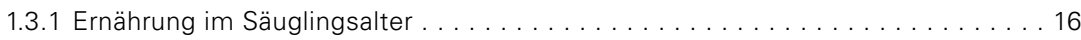

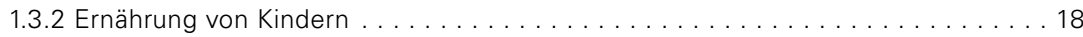

1.3.3 Ernährung von Jugendlichen . . . . . . . . . . . . . . . . 20

1.3.4 Ernährung von Erwachsenen. . . . . . . . . . . . . . . . . . . 21

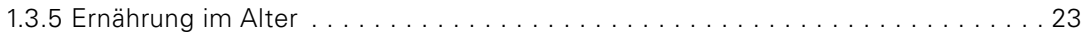

1.4 Ernährungsempfehlungen für spezifische Bevölkerungsgruppen . . . . . . . . . 24

1.4.1 Ernährung in der Schwangerschaft und Stillzeit . . . . . . . . . . . . . 24

1.4 .2 Ernährung von Sportlern . . . . . . . . . . . . . . . . . . . . . 25

1.4 .3 Vegetarische Ernährung. . . . . . . . . . . . . . . . . 26

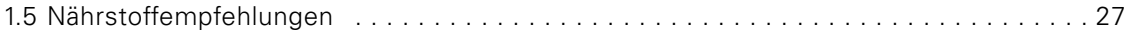

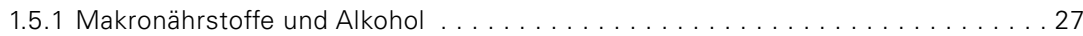

1.5.2 Salz. . . . . . . . . . . . . . . . . . . . . . . . . . . . . . . . . . . 31

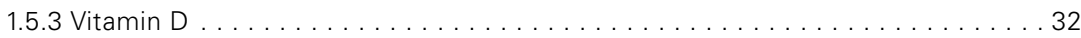

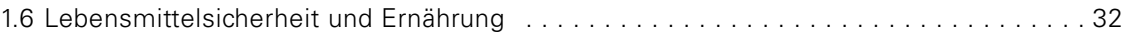

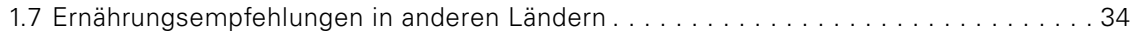

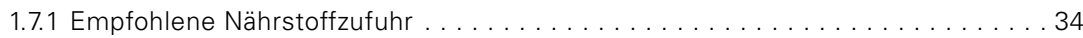

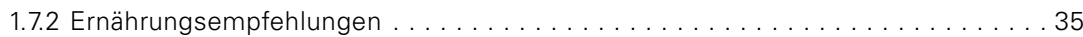

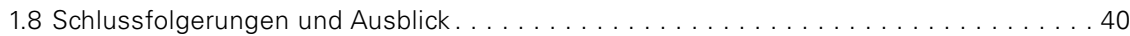

Kapitel 2: Ernährungssituation in der Schweiz. $\ldots \ldots \ldots \ldots \ldots \ldots \ldots \ldots \ldots \ldots \ldots \ldots \ldots \ldots$

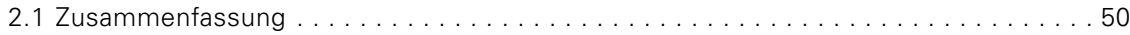

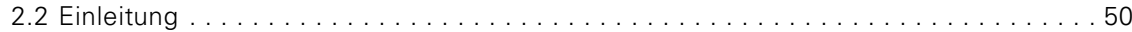

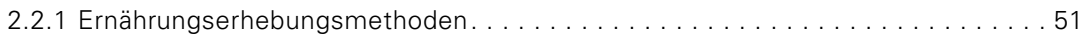

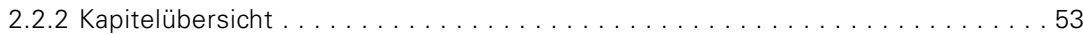

2.3 Lebensmittelverbrauch und -verzehr in der Schweiz. . . . . . . . . . . . . . . . 54

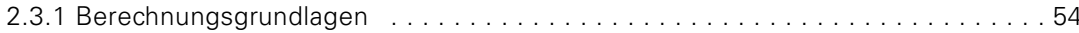

2.3.2 Verbrauchs- und Verzehrsdaten für die einzelnen Lebensmittelgruppen . . . . . . . 54

2.3.3 Ausblick . . . . . . . . . . . . . . . . . . . . . . . . . . . . . . . . . . . 74 
2.4 Energie- und Nährstoffversorgung der Schweizer Bevölkerung . . . . . . . . . 74

2.4 .1 Berechnungsgrundlagen . . . . . . . . . . . . . . . . . . 74

2.4.2 Beitrag einzelner Lebensmittelgruppen an die Energie- und Nährstoffversorgung. . . . 78

2.4.3 Beurteilung der Energie- und Wasserversorgung der Schweizer Bevölkerung . . . . 81

2.4.4 Beurteilung der Versorgung der Schweizer Bevölkerung mit energieliefernden

Nährstoffen und Nahrungsfasern . . . . . . . . . . . . . . . . . . . 82

2.4.5 Beurteilung der Vitaminversorgung der Schweizer Bevölkerung . . . . . . . . . 85

2.4.6 Beurteilung der Mineralstoffversorgung der Schweizer Bevölkerung . . . . . . . . . 90

2.4.7 Energie- und Nährstoffversorgung einzelner Bevölkerungsgruppen . . . . . . . . 100

2.4 .8 Ausblick . . . . . . . . . . . . . . . . . . . . . . . . . . . . . . . . . . . . . 102

2.5 Ernährungssituation in der Schweiz im Vergleich zum europäischen Ausland . . . . . . 102

2.5.1 Allgemeines . . . . . . . . . . . . . . . . . . . . . . . . . . . 102

2.5.2 Schweizer Ernährungssituation und Trends im Vergleich zu Europa . . . . . . . . 103

2.5 .3 Ausblick . . . . . . . . . . . . . . . . . . . . . . . . . . . . . 107

2.6 Ernährungswissen und Ernährungsverhalten der Schweizer Bevölkerung. . . . . . . . 108

2.6.1 Allgemeines . . . . . . . . . . . . . . . . . . . . . . . . . . 108

2.6.2 Ernährungsbewusstsein, Ernährungswissen und Hindernisse für eine ausgewogene Ernährung . . . . . . . . . . . . . . . . . . . 108

2.6.3 Zusammenhang zwischen Ernährungswissen und Verzehrsverhalten . . . . . . 111

2.6.4 Nährwertkennzeichnungssysteme und Labels . . . . . . . . . . . . . . 112

2.6.5 Ernährungsverhalten der Schweizer Bevölkerung . . . . . . . . . . . . . . . . . 114

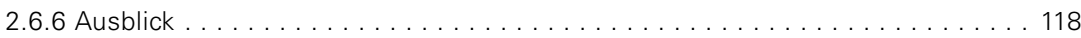

2.7 Schlussfolgerungen und Ausblick. . . . . . . . . . . . . . . . . . 119

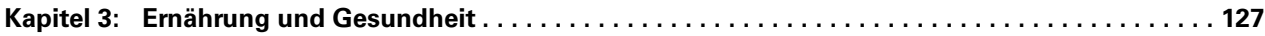

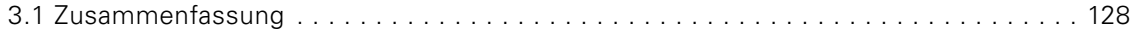

3.2 Einleitung . . . . . . . . . . . . . . . . . . . . . . . . . . . . . . . 129

3.2.1 Ernährung und Gesundheit - Generelle Bedeutung . . . . . . . . . . . . 129

3.2 .2 Datenquellen . . . . . . . . . . . . . . . . . . . . . . 130

3.3 Untergewicht, Übergewicht und Adipositas . . . . . . . . . . . . . . . 133

3.3.1 Häufigkeit. . . . . . . . . . . . . . . . . . . . . . . . . 133

3.3.2 Risiko- und Schutzfaktoren . . . . . . . . . . . . . . . . . . 140

3.3 .3 Bedeutung . . . . . . . . . . . . . . . . . . . . . . . . . 141

3.4 Herz-Kreislauf-Krankheiten . . . . . . . . . . . . . . . . . . . . . 143

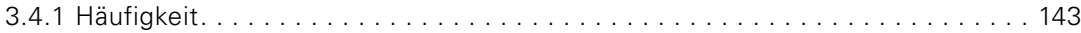

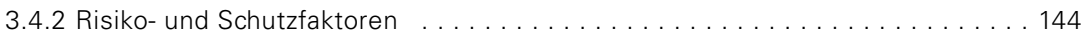

3.4 .3 Bedeutung . . . . . . . . . . . . . . . . . . . . . . . . . . 149 


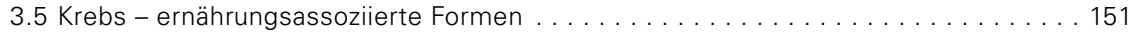

3.5 .1 Häufigkeit. . . . . . . . . . . . . . . . . . . . . . 151

3.5.2 Risiko- und Schutzfaktoren . . . . . . . . . . . . . . . . . . . . . . 151

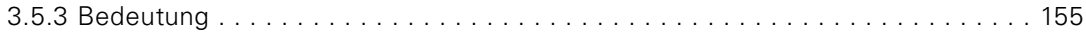

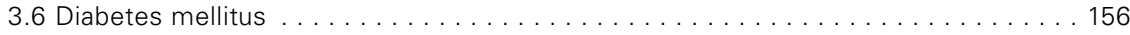

3.6 .1 Häufigkeit. . . . . . . . . . . . . . . . . . . . . . . . . . . . . . . . . . . . . . . . . . . . .

3.6.2 Risiko- und Schutzfaktoren . . . . . . . . . . . . . . . . . . . . . . 156

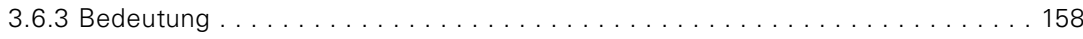

3.7 Osteoporose . . . . . . . . . . . . . . . . . . . . . . . . . . . . . 159

3.7 .1 Häufigkeit . . . . . . . . . . . . . . . . . . . . . . . . . . 159

3.7.2 Risiko- und Schutzfaktoren . . . . . . . . . . . . . . . . . . . . . . . . 159

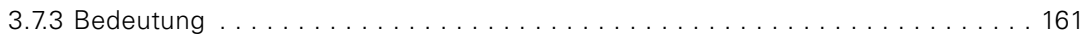

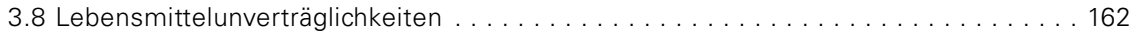

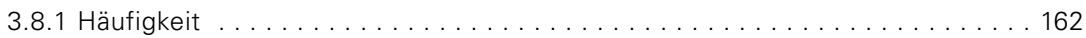

3.8.2 Nicht-immunologische Intoleranzen . . . . . . . . . . . . . . . . 162

3.8 .3 Immunologische Intoleranzen. . . . . . . . . . . . . . . . . 163

3.8 .4 Bedeutung . . . . . . . . . . . . . . . . . . . . . . . . . . 164

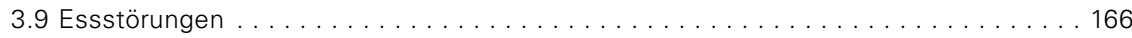

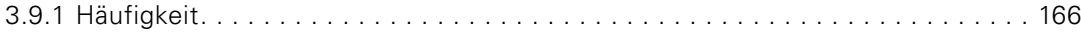

3.9.2 Risiko- und Schutzfaktoren . . . . . . . . . . . . . . . . . . . 169

3.9 .3 Bedeutung . . . . . . . . . . . . . . . . . . . . . 170

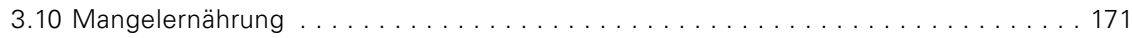

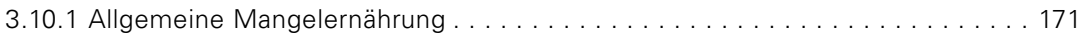

3.10.1.1 Häufigkeit . . . . . . . . . . . . . . . . . . . . . . . . . 171

3.10.1.2 Risiko- und Schutzfaktoren . . . . . . . . . . . . . . . . . . . . . . 172

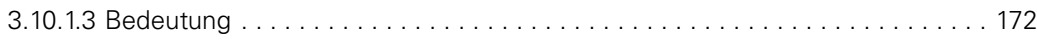

3.10.2 Folsäuremangel . . . . . . . . . . . . . . . . . . . . . . . . . 174

3.10.2.1 Häufigkeit. . . . . . . . . . . . . . . . . . . . . . . . 174

3.10.2.2 Risiko- und Schutzfaktoren. . . . . . . . . . . . . . . . . . . . . . . . . . . 174

3.10.2.3 Bedeutung . . . . . . . . . . . . . . . . . . . . . . . . . 174

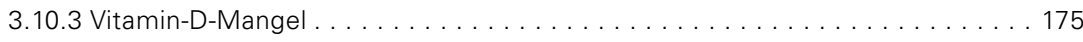

3.10.3.1 Häufigkeit. . . . . . . . . . . . . . . . . . . . . . . . . . 175

3.10.3.2 Risiko- und Schutzfaktoren. . . . . . . . . . . . . . . . . . . . . 175

3.10.3.3 Bedeutung . . . . . . . . . . . . . . . . . . . . . . . . . . 177

3.10 .4 Eisenmangel. . . . . . . . . . . . . . . . . . . . . . . . 179

3.10.4.1 Häufigkeit. . . . . . . . . . . . . . . . . . . . . . . . . . . . . . . 179

3.10.4.2 Risiko- und Schutzfaktoren. . . . . . . . . . . . . . . . . . . . . . . . . 179

3.10.4.3 Bedeutung . . . . . . . . . . . . . . . . . . . . . . . . 179 
3.10 .5 Jodmangel . . . . . . . . . . . . . . . . . . . . . . . 181

3.10.5.1 Häufigkeit. . . . . . . . . . . . . . . . . . . . . . 181

3.10.5.2 Risiko- und Schutzfaktoren. . . . . . . . . . . . . . . . . . . . . . . . 181

3.10.5.3 Bedeutung . . . . . . . . . . . . . . . . . . . . . . . 181

3.10 .6 Selenmangel. . . . . . . . . . . . . . . . . . . . . . . 181

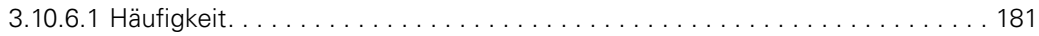

3.10.6.2 Risiko- und Schutzfaktoren. . . . . . . . . . . . . . . . . . . . . . . 182

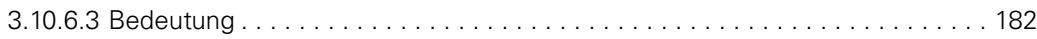

3.11 Zahngesundheit. . . . . . . . . . . . . . . 182

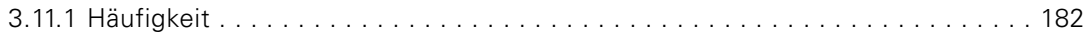

3.11.2 Risiko- und Schutzfaktoren . . . . . . . . . . . . . . . . . . . 184

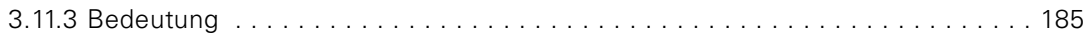

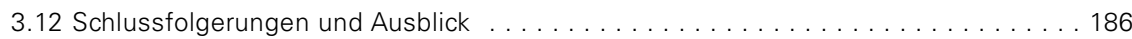

Kapitel 4: Ernährungsmassnahmen zur Förderung der Gesundheit. $\ldots \ldots \ldots \ldots \ldots \ldots \ldots \ldots 209$

4.1 Zusammenfassung . . . . . . . . . . . . . . . . . . . . .

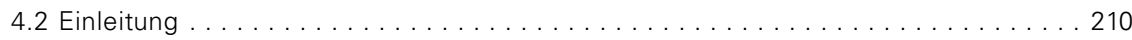

4.3 Politische und gesetzliche Rahmenbedingungen in der Schweiz . . . . . . . . . . 212

4.3 .1 Bundesebene. . . . . . . . . . . . . . . . . . . . . . . . . . 212

4.3 .2 Kantonale Ebene . . . . . . . . . . . . . . . . . . . . . . . . . 213

4.4 Strategische Grundlagen im Ernährungsbereich. . . . . . . . . . . . . . . . . 215

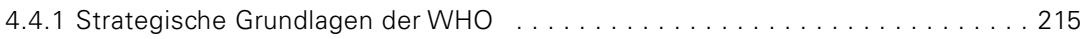

4.4 .2 Strategische Grundlagen in Europa . . . . . . . . . . . . . . . . . . . . 215

4.4.3 Strategische Grundlagen in der Schweiz . . . . . . . . . . . . . . . . 217

4.5 Das Individuum im Spannungsfeld verschiedener Einflüsse . . . . . . . . . . . . . 218

4.5.1 Von der Gesundheitskompetenz zur Ernährungskompetenz . . . . . . . . . . 218

4.5 .2 Lebenswelten . . . . . . . . . . . . . . . . . . . . . . . 218

4.5.3 Von der Bildung über das Wissen zum Verhalten . . . . . . . . . . . . 221

4.6 Laufende Programme und Projekte in der Praxis . . . . . . . . . . . . . . . . . 223

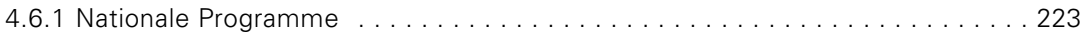

4.6.1.1 Nationales Programm Ernährung und Bewegung . . . . . . . . . . . . 223

4.6.1.2 Strategie 2007-2018 "Gesundes Körpergewicht» . . . . . . . . . . . . . . . . . 225

4.6.1.3 Weitere nationale Programme, Akteure und Netzwerke . . . . . . . . . . . . . 226

4.6.2 Nationale und kantonale Ernährungsprojekte . . . . . . . . . . . . . 227 
4.7 Laufende Aktivitäten auf politischer und gesetzlicher Ebene . . . . . . . . . . . . . . 246

4.7.1 Aktivitäten auf Bundesebene . . . . . . . . . . . . . . . . . . 246

4.7 .2 Aktivitäten auf kantonaler Ebene. . . . . . . . . . . . . . . . . . . . . . . 250

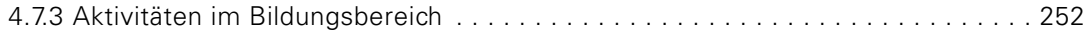

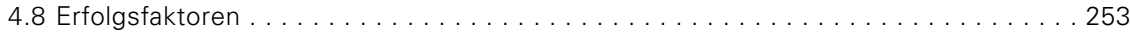

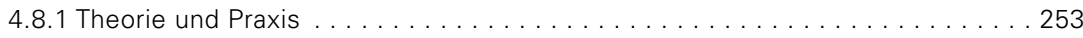

4.8.2 Ernährungsinterventionen in der Schweiz: Erfolgsfaktoren . . . . . . . . . . . . . 254

4.8.3 Ernährungsinterventionen in Europa: Evidenz und Erfolgsfaktoren . . . . . . . . 255

4.9 Handlungsempfehlungen für die Schweiz . . . . . . . . . . . . . . . . . . 257

4.9 .1 Datenlücken schliessen . . . . . . . . . . . . . . . . . . . . . . . . . . . 257

4.9.2 Ernährungskommunikation auf vulnerable Zielgruppen ausrichten. . . . . . . . . 258

4.9.3 Familienergänzende Betreuungseinrichtungen und Schulen stärken . . . . . . . 258

4.9.4 Potenzial föderalistischer Strukturen nutzen . . . . . . . . . . . . . . . . . . . 259

4.9 .5 Politik mobilisieren . . . . . . . . . . . . . . . . . . . . . . . . 259

4.9.6 Privatwirtschaft mobilisieren. . . . . . . . . . . . . . . . . . 260

4.9.7 Dringliche Massnahmen für die Schweiz . . . . . . . . . . . . . . 261

4.10 Schlussfolgerungen und Ausblick. . . . . . . . . . . . . . . . . . . 261

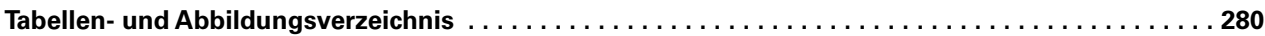

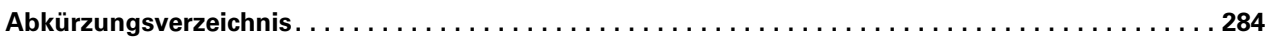

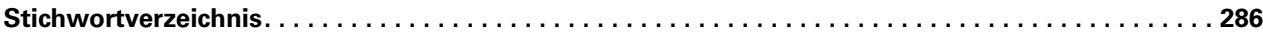

Verzeichnis der Autoren und der Redaktionskommissionsmitglieder $\ldots \ldots \ldots \ldots \ldots \ldots \ldots \ldots \ldots$ 


\section{Zusammenfassung}

Michael Beer 
Der 6. Schweizerische Ernährungsbericht (SEB) fasst die in der Schweiz gültigen Ernährungsempfehlungen zusammen und beschreibt die aktuelle Ernährungssituation in der Schweiz sowie den Einfluss der Ernährung auf bestimmte Risikofaktoren und Krankheitsrisiken. Weiter legt er bestehende und mögliche Massnahmen zur Förderung der Gesundheit durch eine ausgewogene und abwechslungsreiche Ernährung dar. Der Bericht nimmt dabei Bezug auf beobachtete Veränderungen und Trends im Vergleich zum 5. SEB aus dem Jahr 2005 sowie zu früheren Schweizerischen Ernährungsberichten.

\section{Ernährungsempfehlungen und Ernährungssituation in der Schweiz}

\section{Aktuelle Ernährungsempfehlungen}

Die Schweizer Ernährungsempfehlungen basieren auf den Grundlagen der Weltgesundheitsorganisation (WHO) und wurden von verschiedenen Fachgesellschaften und Institutionen gemeinsam an Schweizerische Gegebenheiten angepasst. Sie wurden auf Basis der Referenzwerte für die Nährstoffzufuhr der deutschsprachigen Gesellschaften für Ernährung (DACH-Referenzwerte) sowie weiterer aktueller wissenschaftlicher Erkenntnisse lebensmittelbezogen in Form der Schweizer Lebensmittelpyramide veröffentlicht. Bei deren Entwicklung wurden nationale Ernährungsgewohnheiten sowie Aspekte zur Förderung des Wohlbefindens und der Vorbeugung ernährungsabhängiger Krankheiten berücksichtigt. Die Schweizer Lebensmittelpyramide wurde im
Dezember 2011 in aktualisierter Form von der Schweizerischen Gesellschaft für Ernährung (SGE) und vom Bundesamt für Gesundheit (BAG) gemeinsam veröffentlicht.

Die Ernährungsempfehlungen verfolgen in der Schweiz und in unseren Nachbarländern die gleichen Ziele und basieren grundsätzlich auf den gleichen wissenschaftlichen Grundlagen. Die Art und Weise, wie die Empfehlungen formuliert und graphisch umgesetzt sind, weichen jedoch voneinander $a b$, da sie den jeweiligen nationalen Gegebenheiten angepasst sind.

Die Ernährungsempfehlungen nach Lebensalter und Bevölkerungsgruppe berücksichtigen die spezifischen Bedürfnisse von Säuglingen, Kindern, Jugendlichen, Erwachsenen, älteren Menschen, Schwangeren und Stillenden, SportIern sowie Vegetariern. Neben den Hauptakteuren BAG, Eidgenössische Ernährungskommission (EEK) und SGE waren bei deren Entwicklung noch weitere Fachorganisationen involviert.

Der 6. SEB fasst ferner die nährstoffbezogenen Empfehlungen zusammen und legt neben den Makronährstoffen ein besonderes Augenmerk auf Natrium bzw. Salz und auf Vitamin D.

Die DACH-Referenzwerte aus dem Jahr 2000 empfehlen einen Kohlenhydratanteil von mindestens 50\% der Energiezufuhr, der Rest soll aus Fett (maximal 30\% der Energiezufuhr) und Protein (ca. 15\% der Energiezufuhr) bestehen. Die EEK kommt in ihren Empfehlungen hingegen zum Schluss, dass ein gesundheitlich optimaler Kohlenhydratanteil nicht exakt definiert werden kann und ein Ansatz von mindestens $50 \%$ der Energiezufuhr zu hoch ist. Neuere wissenschaftliche Befunde zeigen, dass auch tiefere Kohlenhydratanteile positive Auswirkungen auf die Gesundheit haben, wenn anstelle der Kohlenhydrate gesundheitlich "günstige» Fette 
konsumiert werden. Diese Erkenntnisse und auch die wissenschaftlichen Diskussionen über den in einer ausgewogenen Ernährung empfohlenen Anteil von Makronährstoffen zeigen, dass Ernährungsempfehlungen periodisch überarbeitet und dem jeweils aktuellen Wissensstand angepasst werden müssen.

Laut den DACH-Referenzwerten wird eine Mindestaufnahme von 550 mg Natrium oder ca. $1.5 \mathrm{~g}$ Salz pro Tag als ausreichend erachtet. Der aktuelle Salzkonsum in der Schweiz liegt mit 8-10 g pro Tag um ein Vielfaches über diesem Wert. Die EEK kommt zum Schluss, dass ein hoher Salzkonsum negative Auswirkungen auf die Gesundheit der Gesamtbevölkerung hat. In der Folge hat das BAG in Abstimmung mit den Empfehlungen der WHO eine Salzstrategie zur Reduktion des Salzkonsums ausgearbeitet, mit dem längerfristigen Ziel, den durchschnittlichen Salzkonsum auf weniger als $5 \mathrm{~g}$ pro Tag zu senken.

Der Vitamin-D-Gehalt der meisten Lebensmittel ist gering; die Versorgung wird hauptsächlich über die endogene Bildung in der Haut unter Einfluss von Sonnenlicht sichergestellt. 25-Hydroxy-Vitamin-D-Konzentrationen im Blutserum von weniger als $50 \mathrm{nmol} / \mathrm{L}$ werden als Mangel bezeichnet. Gemäss aktuellen Empfehlungen sollten gestillte und nichtgestillte Säuglinge im ersten Lebensjahr täglich 300-500 IE $(=7.5-12.5 \mu \mathrm{g})$ Vitamin D erhalten, Kinder bis zum Ende des 3. Lebensjahres $600 \mathrm{IE}(=15 \mu \mathrm{g})$. Eine Erhöhung der täglichen Vitamin-D-Zufuhr auf ca. $20 \mu \mathrm{g}$ bzw. 800 IE bei Erwachsenen ab 60 Jahren wird als sinnvoll erachtet; sie kann allerdings in vielen Fällen nur mit Hilfe von Supplementen erreicht werden.

Beim Herleiten von Ernährungsempfehlungen sind mögliche Risiken durch unerwünschte Stoffe in den jeweiligen Lebensmitteln zu be- achten. So können sich z.B. so genannte persistente Umweltkontaminanten in der Nahrungskette anreichern. Beispiele sind polychlorierte Dioxine und Furane in der Muttermilch oder Schwermetalle in Meerfischen. Solche Stoffe in Lebensmitteln können insbesondere dann zu einem Zielkonflikt bei der Formulierung von Ernährungsempfehlungen führen, wenn zwischen erwünschten und unerwünschten Inhaltsstoffen abgewogen werden muss. Eine sorgfältige Nutzen-Risiko-Abschätzung ist daher eine unerlässliche Voraussetzung, um Gefährdungen auszuschliessen.

\section{Aktuelle Ernährungssituation}

Die im 6. SEB dargelegte Ernährungssituation in der Schweiz zeigt, dass die aktuellen Ernährungsempfehlungen von vielen Personen nur teilweise oder gar nicht umgesetzt werden. Auf der einen Seite werden zu wenig Früchte, zu wenig Gemüse und zu wenig Milch und Milchprodukte konsumiert, und auf der anderen Seite wird zu viel Energie aufgenommen. An die Empfehlungen halten sich nicht alle gleichermassen, da zum einen 30\% der Schweizer Bevölkerung bei ihrer Ernährung laut Eigenaussage auf nichts Bestimmtes achten. Es sind dies hauptsächlich Männer, jüngere Personen und Personen mit einem niedrigen Bildungsniveau. In der Westschweiz scheint das Ernährungsbewusstsein zudem weniger ausgeprägt zu sein als in den beiden anderen Sprachregionen der Schweiz. Zum anderen werden Ernährungsempfehlungen wegen mangelhaftem Ernährungswissen oder unzureichenden Informationen nicht umgesetzt. Für viele Konsumenten ist zum Beispiel die Lebensmittelkennzeichnung (Nährwert- 
kennzeichnung, Front-of-Pack-Kennzeichnungen, Labels) unübersichtlich und teilweise unverständlich, unter anderem weil unterschiedliche Kennzeichnungssysteme parallel verwendet werden. Weiter wird der Ernährung im Zusammenhang mit der Vorbeugung von Krankheiten bzw. der Erhaltung der Gesundheit zu wenig Bedeutung beigemessen. Teils erschweren auch Alltagssituationen, der heutige Lebensstil (z.B. Mobilität) und allgegenwärtige verführerische Verpflegungsangebote die Umsetzung des Ernährungswissens. Schliesslich bestimmen die Verfügbarkeit, der Preis und die Bewerbung von Lebensmitteln zu einem wesentlichen Teil, was auf den Tisch kommt.

Eine Beurteilung der Ernährungssituation in der Schweiz sowie ein Vergleich zu derjenigen in Europa sind nicht vorbehaltlos möglich. Die Schweizer Daten werden aus der Agrarstatistik gewonnen und auf einen Lebensmittelverbrauch pro Kopf der Bevölkerung umgerechnet. Sie erlauben somit keine direkten Rückschlüsse auf den effektiven Lebensmittelverzehr und den Versorgungsgrad einzelner Bevölkerungsgruppen oder einzelner Individuen, da diese Daten nur geschätzt werden. Verlässliche und differenzierte Aussagen bezüglich der Ernährungssituation der Schweizer Bevölkerung und vulnerabler Bevölkerungsgruppen (z.B. Kinder, Schwangere oder ältere Menschen) werden deshalb erst dann möglich sein, wenn repräsentative Schweizer Studien detaillierte Informationen zum tatsächlichen Verzehr liefern. Dazu wäre eine Nationale Ernährungserhebung notwendig - wie sie in den meisten Ländern Europas vorliegt. Erst dann können valide Aussagen zu tatsächlich konsumierten Lebensmittelmengen differenziert nach Alter, Geschlecht, Bildungsniveau und Region gemacht werden. Im- merhin stimmen die aktuellen Verbrauchsdaten mit dem in verschiedenen Studien erhobenen Verzehrsverhalten der Schweizer Bevölkerung weitgehend überein.

Die Gesamtverbrauchsmengen der einzelnen Lebensmittelgruppen in der Schweiz haben sich seit der Veröffentlichung des 5. SEB im Jahr 2005 wenig verändert; hingegen sind teilweise grössere Verschiebungen innerhalb der Lebensmittelgruppen von einem Lebensmittel zum anderen feststellbar. Der Verbrauch von Früchten nahm zwischen 2001/02 (5. SEB) und 2007/08 (6. SEB) weiter ab $(-7 \%$ bezogen auf den Pro-Kopf-Verbrauch), und auch beim Gesamtgemüseverbrauch ist ein Rückgang zu verzeichnen (-6\%). Die Verbrauchsmengen an Nüssen und Samen (-8\%) sowie an alkoholischen Getränken ( $-3 \%$ ) nahmen ebenfalls ab. Der Verbrauch von Fleisch und Fleischprodukten, von Milch und Milchprodukten sowie von Getreide und Kartoffeln blieb seit Veröffentlichung des 5. SEB praktisch unverändert. Eier $(+3 \%)$, Öle und Fette $(+5 \%)$, Zucker und Honig $(+5 \%)$, Fische und Schalentiere $(+8 \%)$ sowie Hülsenfrüchte $(+20 \%$, allerdings auf tiefem Niveau) konnten einen Anstieg im Verbrauch verbuchen. Interessant ist, dass der beobachtete Anstieg bei Ölen und Fetten hauptsächlich auf einen Anstieg beim Verbrauch an pflanzlichen Ölen und Fetten zurückzuführen ist; der Verbrauch an tierischen Ölen und Fetten hat abgenommen. Pflanzliche Öle und Fette zu bevorzugen, entspricht den gängigen Ernährungsempfehlungen.

Der Verbrauch sowie der angenäherte Verzehr an Energie haben sich über die letzten Jahre kaum verändert. Aus dem angenäherten Verzehr lässt sich eine Energieaufnahme von 2'661 kcal pro Person und Tag ableiten (ohne die 
Energie aus alkoholischen Getränken). Diese Aufnahme liegt um ca. 20-30\% über der empfohlenen Zufuhr von 2'010 kcal bzw. 2'245 kcal (je nach körperlicher Aktivität). Allerdings muss bei diesem Vergleich berücksichtigt werden, dass es sich um eine berechnete Energieaufnahme handelt, die von der tatsächlichen individuellen Energieaufnahme abweichen kann. Die wichtigsten Energielieferanten sind unverändert Getreide, Öle und Fette, Zucker und Honig sowie Milch und Milchprodukte. Die Anteile von Proteinen, Fetten und Kohlenhydraten am angenäherten Verzehr an Energie liegen im Bereich der derzeitigen Empfehlungen (DACH-Referenzwerte). Die Empfehlungen werden auf qualitativer Ebene (Fettsäurenanteile, Zuckerkonsum) allerdings nicht eingehalten.

Auf Basis der Daten zum angenäherten Verzehr scheint die Versorgung mit folgenden Mikronährstoffen für die allgemeine Bevölkerung im Durchschnitt ausreichend zu sein: Vitamin A, Carotinoide, Vitamin E, Vitamin B2, Niacin, Vitamin B6, Vitamin B12, Pantothensäure, Vitamin C, Kalium, Natrium und Chlorid, Calcium, Phosphor und Zink. Ungenügend ist hingegen der angenäherte Verzehr an Folsäure und Vitamin D. Nur knapp genügend sind schliesslich die Werte für Eisen, Vitamin B1 und Magnesium. Eine im Durchschnitt ausreichende Versorgung der Schweizer Bevölkerung konnte für Selen im Rahmen spezifischer Studien festgestellt werden. Erste Ergebnisse einer nationalen Studie deuten darauf hin, dass die Jodversorgung eines Teils der Bevölkerung unzureichend sein könnte. Für eine ausreichende Jodzufuhr ist die Salzjodierung unabdingbar. Die ausreichende Zufuhr von Folsäure ist insbesondere für Frauen im gebärfähigen Alter von Bedeutung. Die Versorgung mit Eisen variiert zwi- schen verschiedenen Bevölkerungsgruppen; Frauen weisen im Vergleich zu Männern öfter einen zu geringen Eisenstatus auf. Auch bei anderen Bevölkerungsgruppen ist die Versorgung mit Mikronährstoffen teilweise nicht ausreichend. Dies trifft insbesondere auf ältere Menschen, Kinder und Säuglinge zu. Genaue Angaben zum Ausmass und zur Prävalenz einer allfälligen Mangelernährung lassen sich aufgrund fehlender Daten jedoch nicht machen. Zudem ist in den berechneten Angaben die Zufuhr an Mikronährstoffen über Vitamin- und Mineralstoffsupplemente sowie angereicherte Lebensmittel aufgrund fehlender Daten nicht berücksichtigt.

\section{Auswirkungen auf die Gesundheit}

Die verfügbaren Schweizer Daten zum Einfluss der Ernährung auf Krankheiten und ihre Risikofaktoren sind unzureichend und unsicher. Unter anderem fehlen zuverlässige Daten zur Morbidität von ernährungsabhängigen Krankheiten. Die Schweiz verfügt einzig über relativ zuverlässige und flächendeckende Mortalitätsdaten. Da aber Daten zu den entsprechenden Risikofaktoren fehlen und somit keine gesicherten Aussagen zu allfälligen Zusammenhängen zwischen Risikofaktoren, Morbidität und Mortalität gemacht werden können, lassen sich die Auswirkungen der Ernährung auf die Gesundheit nicht eindeutig ableiten. Es ist deshalb schwierig, auf Basis dieser Ausgangslage erfolgreiche Massnahmen zur Verbesserung der Ernährungssituation in der Schweiz und zur Optimierung der Gesundheit der Schweizer Bevölkerung vorzuschlagen.

Sechster Schweizerischer Ernährungsbericht | 5 


\section{Übergewicht}

In den vergangenen 30 Jahren stieg die Anzahl der übergewichtigen und adipösen Erwachsenen in der Schweiz kontinuierlich an. Männer sind von Übergewicht (Body-Mass-Index = $\left.25.0-29.9 \mathrm{~kg} / \mathrm{m}^{2}\right)$ deutlich stärker betroffen als Frauen (37.6\% vs. 20.8\%); bei Adipositas (BodyMass-Index $\geq 30.0 \mathrm{~kg} / \mathrm{m}^{2}$ ) ist der Geschlechtsunterschied deutlich geringer (8.7\% vs. $7.8 \%$ ). Der Anteil übergewichtiger bzw. adipöser Kinder schwankt je nach Alter und Studie zwischen 5-20\% (Übergewicht) bzw. 0.4-6\% (Adipositas). Die Daten der Schweizerischen Gesundheitsbefragung aus dem Jahr 2007 deuten nun aber darauf hin, dass sich der Anstieg der Prävalenz von Übergewicht und Adipositas bei Erwachsenen in den letzten Jahren abgeflacht haben könnte, insbesondere bei Frauen. Gesicherte national repräsentative Daten über die Situation bei Kindern liegen nicht vor; einige regionale Studien lassen aber auch bei Kindern eine Stabilisierung der Übergewichts- und Adipositasprävalenz vermuten.

Zwischen Stadt und Land gibt es teilweise beträchtliche Unterschiede in der Prävalenz von Übergewicht und Adipositas. So gibt es zum Beispiel im urbanen Kanton Zürich weniger übergewichtige und adipöse Erwachsene als in der restlichen Deutschschweiz. Studien bei Kindern zeigen tendenziell ein umgekehrtes Muster mit einer höheren Prävalenz von Übergewicht und Adipositas in Städten. Unterschiede gibt es auch in Bezug auf Bildungsniveau, Einkommen, Berufsklasse und Migrationshintergrund. Ein niedriges Bildungsniveau ist einer der wichtigsten Risikofaktoren für Übergewicht und Adipositas.

Übergewicht scheint in der Schweiz im Gegensatz zu Adipositas nicht mit einem erhöhten
Sterberisiko verbunden zu sein. Übergewicht und Adipositas verursachen jedoch zahlreiche Folgekrankheiten (u.a. Herz-Kreislauf-Krankheiten, Krebs und Diabetes mellitus) und somit auch erhebliche Gesundheitskosten.

\section{Herz-Kreislauf-Krankheiten}

Herz-Kreislauf-Krankheiten sind nach wie vor die häufigste Todesursache in der Schweiz, erleiden doch jährlich 30'000 Menschen ein akutes koronares Ereignis und 12'500 einen Hirnschlag. Eine unausgewogene Ernährung beeinflusst verschiedene Risikofaktoren für Herz-KreislaufKrankheiten negativ. Inhaltsstoffe wie Salz oder gesättigte Fette können Risikofaktoren wie Bluthochdruck oder ungünstige Blutfettwerte direkt beeinflussen. Eine unausgewogene Ernährung, insbesondere eine übermässige Energieaufnahme, kann aber auch zu Übergewicht und Adipositas führen und das Risiko für Herz-KreislaufKrankheiten über diesen Weg erhöhen. Der Verbrauch von Früchten und Gemüse hat in der Schweiz weiter abgenommen und der Verbrauch von Ölen und Fetten zugenommen; diese Veränderungen können sich negativ auf die Herzgesundheit auswirken. Zu vermerken ist dabei jedoch, dass vor allem der Verbrauch an pflanzlichen Ölen zugenommen hat - was bezüglich Herzgesundheit eher als positiv zu bewerten ist.

Umfassende Informationen über Risikofaktoren für Herz-Kreislauf-Krankheiten fehlen aber, weil bis anhin keine national repräsentativen Messungen - beispielsweise von Blutzucker und -cholesterin - durchgeführt wurden. Auch fehlen zuverlässige und flächendeckende Informationen über die Häufigkeit von HerzKreislauf-Krankheiten in der Schweiz. 


\section{Krebs}

Krebs folgt in der Schweiz als zweithäufigste Todesursache auf Herz-Kreislauf-Krankheiten. Tabakkonsum ist einer der wichtigsten Risikofaktoren für eine Vielzahl von Krebsformen, aber auch die Ernährung kann die Entstehung bestimmter Krebsformen direkt beeinflussen. Alkoholkonsum oder die Einnahme von bakteriellen oder pilzlichen Toxinen oder von Stoffen, welche bei der Lebensmittelproduktion und -zubereitung gebildet werden, können das Risiko bestimmter Krebsformen erhöhen. Auch Übergewicht und Adipositas erhöhen das Risiko für gewisse Krebsformen. So sind Krebsformen der Geschlechtsorgane bei Adipösen häufiger als bei Normalgewichtigen. Allein durch die Vermeidung von Übergewicht und Adipositas würden sich in der Schweiz jährlich schätzungsweise 700 Krebsfälle verhindern lassen.

\section{Diabetes mellitus}

Von Diabetes mellitus sind in der Schweiz zwischen 350'000 und 630'000 Personen betroffen (überwiegend Diabetes mellitus Typ 2). Gesamtschweizerisch wird die Diabetesprävalenz auf ca. $5-6 \%$ bei Männern und $4-5 \%$ bei Frauen geschätzt.

Bei der Entstehung von Diabetes mellitus Typ 2 spielt die Ernährung eine entscheidende Rolle. Die ernährungsabhängigen Risikofaktoren für Diabetes mellitus Typ 2 sind vergleichbar mit jenen für Adipositas und Herz-KreislaufKrankheiten. Neben ungenügender körperlicher Aktivität kann eine energiereiche Ernährung mit einem erhöhten Anteil an raffiniertem Zucker, gesättigten Fetten und Alkohol in grösseren
Mengen das Risiko erhöhen. Fast 90\% der Fälle mit Diabetes mellitus Typ 2 sind auf Übergewicht oder Adipositas zurückzuführen und wären somit mit einer entsprechenden Adipositasprävention vermeidbar.

\section{Osteoporose}

In der Schweiz leben rund 330'000 Menschen mit Osteoporose, betroffen sind vor allem Frauen. Um das 30. Altersjahr herum beginnt ein kontinuierlicher Abbau der Knochenmasse, der in Abhängigkeit von vorhandenen Risikofaktoren mehr oder weniger stark ausgeprägt sein kann. Die Knochenmasse, die Knochendichte und die Knochenfestigkeit sind die für die Knochengesundheit entscheidenden Faktoren. Sie sind neben der genetischen Prädisposition und anderen Einflussgrössen vor allem durch die Nährstoffversorgung (Calcium, Protein, Vitamin D), die körperliche Aktivität und die Muskelmasse beeinflussbar. Eine ungenügende $\mathrm{Cal}$ cium-, Protein- und Vitamin-D-Versorgung korreliert mit einer geringeren Knochenmasse. Die Calcium- und Proteinversorgung der allgemeinen Bevölkerung in der Schweiz ist gemäss Daten zum angenäherten Verzehr ausreichend. Die Versorgung spezifischer Bevölkerungsgruppen mit Vitamin D scheint jedoch nicht immer bedarfsdeckend zu sein. Aufgrund neuerer Erkenntnisse wird älteren Menschen daher eine Supplementierung von Vitamin D empfohlen, da ein Grossteil dieser Bevölkerungsgruppe im Hinblick auf Vitamin D unterversorgt sein könnte. 


\section{Mangelernährung}

Rund 20-30\% der ins Spital eintretenden Personen sind mangelernährt oder weisen ein erhöhtes Risiko für eine Mangelernährung auf. Bei älteren Menschen, die zu Hause gepflegt werden, wird ein noch grösserer Anteil an mangelernährten Personen vermutet. Die möglichen Ursachen sind vielfältig. So können beispielsweise chronische Krankheiten, Medikamente, soziale Isolierung oder Appetitlosigkeit zu einer Mangelernährung führen. Aber auch die einseitige Lebensmittelauswahl birgt die Gefahr einer Mangelernährung in sich, so dass sogar adipöse Menschen mangelernährt sein können.

Neben der allgemeinen Unterversorgung mit Makronährstoffen sind Vitamin D, Folsäure, Eisen, Jod und Selen kritische Nährstoffe, bei denen in gewissen spezifischen Bevölkerungsgruppen oder Teilen der Bevölkerung ein Mangel möglich ist. Während bei der Selenzufuhr vorläufig kein Handlungsbedarf besteht, müssten Teile der Bevölkerung besser mit Jod, Vitamin D (ältere Menschen und Säuglinge) sowie Eisen und Folsäure (letztere beide bei Frauen im gebärfähigen Alter) versorgt werden.

\section{Mögliche Massnahmen}

Das Nationale Programm Ernährung und Bewegung (NPEB) bietet die wichtige Grundlage, um Massnahmen zur Förderung einer ausgewogenen Ernährung und ausreichender Bewegung sowohl auf der Verhaltens- als auch auf der Verhältnisebene in der Schweiz einzuleiten und zu koordinieren. Das NPEB stützt sich auf globale und europäische Strategien. Wirkungsvolle ernährungsbezogene Massnahmen sind dringend notwendig, denn wie in vielen OECD-Staaten nehmen auch in der Schweiz die Gesundheitskosten schneller zu als das Brutto-Inlandprodukt. Ein wesentlicher Kostenfaktor ist die zunehmende Zahl von Menschen mit nichtübertragbaren Krankheiten (Adipositas, Diabetes mellitus, Herz-Kreislauf-Krankheiten, Krebs usw.). Dennoch fliessen in der Schweiz nur gerade 2.3\% aller Ausgaben im Gesundheitssektor in die Gesundheitsförderung und Prävention - der OECD-Durchschnitt liegt bei 3.1\%. Im Hinblick auf den immer grösser werdenden Anteil älterer Menschen ist eine Ausrichtung des Gesundheitssystems auf Grundversorgung und Gesundheitsförderung anzustreben; ansonsten wird eine weitere Zunahme der Gesundheitskosten unausweichlich. Die Kosten für Übergewicht und Adipositas haben sich z.B. in den letzten 5 Jahren mehr als verdoppelt und betragen heute über CHF 5.8 Mia. pro Jahr.

Das Interesse der Schweizer Politiker für den Themenbereich "Gesundheitsförderung und Prävention» nahm im letzten Jahrzehnt stetig zu. So wurden in der Periode von 2000-2010 insgesamt 36 diesbezügliche Vorstösse eingereicht. In der vorhergehenden 10-Jahres-Periode wurden zum gleichen Themengebiet nur 8 Vorstösse gezählt. Diese Entwicklung hat sich auch im Gesetzgebungsprozess niedergeschlagen; so diskutiert doch das Parlament ein Präventionsgesetz und ein revidiertes Lebensmittelgesetz. Gesundheitsförderung und Prävention sollten jedoch auch in anderen Politikbereichen wie Landwirtschaft, Sport, Bildung, Verkehrsund Raumordnung, Umwelt, Soziales, Arbeitsmarkt und Handel sowie Forschung vermehrt berücksichtigt werden.

Das Mass an staatlichen Interventionen, das Recht auf Selbstbestimmung und die Über- 
nahme von Selbstverantwortung im Ernährungs- und Gesundheitsbereich werden in der Öffentlichkeit kontrovers diskutiert. Da die Verbesserung der Ernährungssituation über Veränderungen im individuellen Verhalten (Verhaltensebene) oder über Veränderungen des Umfeldes (Verhältnisebene), mit oder ohne staatliche Intervention, erfolgen kann, sind diese Themen grundlegend für die Prioritätensetzung.

\section{Massnahmen auf Verhaltensebene}

Ein ausreichendes Ernährungswissen bildet die Voraussetzung für ein nachhaltig gesundes Ernährungsverhalten. Die Steigerung des Wissens muss also ein vordringliches Ziel aller Präventionsbemühungen auf der Verhaltensebene sein. Es reicht jedoch nicht aus, das Ernährungsverhalten mittels blosser Wissenssteigerung beeinflussen zu wollen. Insbesondere das soziale Gefälle kann nicht alleine durch Ernährungsaufklärung kompensiert werden. Auch die persönliche Lebenssituation ist relevant und mitentscheidend, ob Empfehlungen umgesetzt werden oder nicht. Massnahmen im Setting Schule, die sich an Kinder richten, versprechen eine nachhaltige Wirkung. Dies nicht zuletzt deshalb, weil die Gewohnheitsbildung und Prägung des Ernährungsverhaltens bereits in den ersten Lebensjahren beginnen. Neben Kindern sind Männer, junge Menschen und sozio-ökonomisch benachteiligte Bevölkerungsgruppen (z.B. solche mit Migrationshintergrund, geringem Einkommen und / oder tiefem Bildungsniveau) Schlüsselzielgruppen. Schliesslich ist zu berücksichtigen, dass das Ernährungsbewusstsein in den verschiedenen Sprachregionen der Schweiz unterschiedlich ausgeprägt ist.
Auf Verhaltensebene läuft in den Kantonen im Rahmen der Kantonalen Aktionsprogramme (KAP) eine grosse Anzahl von Projekten, deren Wirkung laufend evaluiert wird. Angesichts ihrer verhältnismässig kurzen Interventionsdauer war der Nachweis einer nachhaltigen gesundheitsfördernden Verhaltensänderung durch die ergriffenen Massnahmen bisher nicht möglich. Dennoch deuten Erhebungen bei Schulkindern und Erwachsenen in der Schweiz auf eine Stabilisierung der Übergewichts- und Adipositasprävalenz hin. Ob diese Entwicklung auf einzelne Massnahmen zurückzuführen ist, auf die Summe aller Präventionsbemühungen oder auch auf weitere Faktoren und Entwicklungen, kann nicht abschliessend beurteilt werden.

\section{Massnahmen auf Verhältnisebene}

Unser Umfeld ("Verhältnisse») hat einen entscheidenden Einfluss auf unser Verhalten; eine Verbesserung der Verhältnisse kann deshalb nachhaltige Wirkungen erzielen. Verhältnisse können über Anpassungen der gesetzlichen Rahmenbedingungen oder - schneller - über freiwillige Massnahmen der betroffenen Akteure verändert werden.

Unternehmen aus der Privatwirtschaft sollen deshalb weiterhin zu freiwilligen Massnahmen zur Förderung einer ausgewogenen Ernährung motiviert werden, sei dies bei der Anpassung von Portionengrössen oder der Zusammensetzung von Lebensmitteln oder beim Verzicht auf die Bewerbung von Lebensmitteln und nichtalkoholischen Getränken mit einem hohen Gehalt an gesättigten und Transfettsäuren, Zucker oder Salz, die sich an Kinder richten. 
Auf internationaler Ebene sind Lenkungsabgaben auf Lebensmittel zunehmend ein Thema und wurden z.B. in Dänemark umgesetzt. Ob sich solche Massnahmen gesundheitsfördernd auswirken, wird sich erst in einigen Jahren zeigen.

Die Verfügbarkeit, der Preis und die Bewerbung von Lebensmitteln haben einen wesentlichen Einfluss auf das Konsumverhalten. Eine besondere Bedeutung haben diese Einflussfaktoren bei Familien mit Migrationshintergrund und tiefem Bildungsniveau, die sich aufgrund von oftmals schwach ausgebildeten Gesundheits- und Ernährungskompetenzen selten an gesundheitsfördernden Verhaltensstrategien orientieren und solchen Einflüssen deshalb in verstärktem Mass ausgesetzt sind.

\section{Erfolgsfaktoren}

Die Wirkung ernährungsbezogener Massnahmen kann meist erst nach mehreren Jahren nachgewiesen werden. Dementsprechend schwierig ist es, eindeutige Erfolgsfaktoren zu bestimmen. Aus den verschiedenen in der Schweiz und in Europa laufenden Programmen und Projekten können dennoch einzelne kontextbezogene Erfolgsfaktoren abgeleitet werden.

Der Einbezug von Schlüsselpersonen bzw. Schlüsselinstitutionen in die Planung und Umsetzung von Interventionen ist ein wichtiger Erfolgsfaktor. So ist beispielsweise bei Interventionen, die auf Personen mit Migrationshintergrund abzielen, der Einbezug von interkulturellen Vermittlern als Schlüsselpersonen und Ausländervereinen als Schlüsselinstitutionen erfolgversprechend. Bei Interventionen, die auf Kleinkinder abzielen, sind Mütter-Väterberatungsstellen und familienergänzende Betreu- ungseinrichtungen Schlüsselinstitutionen für den Erfolg.

Wichtig für den Erfolg einer Intervention ist auch das Setting, in der sie stattfindet. Die Schulen, insbesondere solche mit Ganztagesstrukturen, scheinen ein idealer Ort für erfolgreiche Interventionen zu sein. Kinder aus allen sozialen Schichten und ihre Bezugspersonen können erreicht werden; auch auf das Lebensmittelangebot in Schulkantinen oder in Automaten kann Einfluss genommen werden. Ein verbessertes Angebot, wie z.B. ein Schulfruchtprogramm mit einer kostenlosen Abgabe der Früchte an alle sowie einem vielfältigen und täglich wechselnden Früchteangebot, hat eine nachhaltige Wirkung und führt zu einer Steigerung des Früchtekonsums. Interventionen im Setting Schule sind vor allem dann wirksam, wenn sie in einem übergeordneten Programm verankert sind und die Familie einbezogen wird.

Generell ist die Einbettung einer Intervention in eine übergeordnete nationale Strategie und die Nutzung bestehender Netzwerke wichtig für den Erfolg. Eine umfassende Kommunikationsstrategie ist zusammen mit der Unterstützung des Staates ebenfalls von entscheidender Bedeutung.

\section{Handlungsempfehlungen}

\section{Datenlage verbessern}

Aufgrund der unzureichenden Schweizer Datenlage im Bereich der Ernährung und Gesundheit ist es schwierig, die Situation in der Schweiz zuverlässig zu analysieren, zu bewerten und Prioritäten zu setzen sowie wirkungsvolle Massnah- 
men zu planen und diese anschliessend zu evaluieren. Es fehlen vor allem qualitativ hochwertige und national repräsentative Daten zum Lebensmittelverzehr und Ernährungsverhalten in der Schweiz, umfassende Daten über die Zusammensetzung (Energie, Makro- und Mikronährstoffe) von in der Schweiz erhältlichen Lebensmitteln sowie Schweizer Daten, die es erlauben, Zusammenhänge zwischen Ernährung und Risikofaktoren bzw. Krankheiten aufzuzeigen. Ohne diese Daten können Massnahmen und das Vorgehen kaum mit jenen in Europa und anderen Ländern verglichen werden.

Die Verbesserung der Datenlage liegt im Interesse und in der Verantwortung vieler Akteure und ist deshalb eine gesamtgesellschaftliche Aufgabe, die entsprechende finanzielle und personelle Ressourcen, die Berücksichtigung internationaler Erkenntnisse und geeignete Forschungs- und Messmethoden voraussetzt.

\section{Ernährungskommunikation auf vulnerable Zielgruppen ausrichten}

Offizielle Ernährungsempfehlungen existieren für alle Altersgruppen bzw. Lebensphasen. Lücken bestehen hingegen im Bereich der Ernährungskommunikation an spezifische Bevölkerungsgruppen mit problematischem Ernährungsbewusstsein und -wissen. Dazu zählen insbesondere Männer, junge Menschen und sozio-ökonomisch benachteiligte Menschen. Diese Zielgruppen sind von besonderer Bedeutung, weil sie gleichzeitig die Bevölkerungsgruppen darstellen, die ernährungsabhängigen Krankheitsrisiken am stärksten ausgesetzt sind. Das Körpergewicht von Kindern steht beispielsweise in einem direkten Zusammenhang zum
Bildungsniveau ihrer Eltern. Diese Bevölkerungsgruppen sind bei der Entwicklung und Ausrichtung künftiger Interventionen und Kommunikationsmassnahmen deshalb besonders zu beachten. Dabei ist nicht nur der Inhalt der vermittelten Ernährungsbotschaften relevant, sondern vor allem auch die Art der Vermittlung. In diesem Zusammenhang sollte die Nährwertkennzeichnung von Lebensmitteln beachtet werden. Die heutigen Kennzeichnungssysteme sind schwierig zu verstehen und werden im Prozess der Kaufentscheidung wenig genutzt. Eine Verbesserung in diesem Bereich ist jedoch eine wichtige Voraussetzung dafür, dass die Selbstverantwortung wahrgenommen werden kann.

\section{Familienergänzende Betreuungs- einrichtungen und Schulen stärken}

Die frühe Bildung und Prägung durch Elternhaus, Schule und Gemeinschaft sind für die Entwicklung von Ernährungskompetenzen und für die Förderung eines gesunden Ernährungsverhaltens wesentlich. Familienergänzende Betreuungseinrichtungen, Kindergärten und Schulen sind von besonderer Bedeutung, denn sie können den Grundstein für eine altersgerechte und nachhaltige Ernährungsbildung legen. Dazu braucht es aber eine Stärkung ihrer Vermittlerfunktion mit finanziellen und personellen Ressourcen.

\section{Potenzial der Gemeinden nutzen}

Die föderalistischen Strukturen in der Schweiz bilden zugleich eine Herausforderung wie auch eine Chance. Eine besondere Rolle kommt den 
rund 2'600 Gemeinden zu, denn sie sind zuständig für budgetrelevante Entscheidungen, welche einen direkten Einfluss auf die Verhältnisebene haben. Viele gesundheitsfördernde Massnahmen, wie z.B. die Verbesserung der Verpflegungsangebote in Kindergärten und Schulen, liegen in ihrem Einflussbereich.

\section{Multisektorale Gesundheitspolitik fördern}

Die multisektorale Gesundheitspolitik verlangt, dass Gesundheitsförderung und Prävention in allen Politikbereichen berücksichtigt werden. Im Hinblick auf eine ausgewogene Ernährung stehen neben der Gesundheitspolitik v.a. die Agrar- und die Bildungspolitik in der Pflicht. Mit zukünftigen Gesetzesrevisionen sollte ein Umfeld geschaffen werden, welches eine ausgewogene Ernährung fördert.

\section{Zusammenarbeit zwischen staatli- chen und privaten Akteuren fördern und Privatwirtschaft mobilisieren}

Der Staat spielt bei der Gesundheitsförderung eine besonders wichtige Rolle, nicht zuletzt aufgrund seiner in der Bundesverfassung verankerten Aufgabe, Massnahmen zum Schutz der Gesundheit zu ergreifen. Allerdings trägt der Staat nicht die alleinige Verantwortung. Eine wirksame Gesundheitsförderung und Prävention ist nur im Verbund mit allen beteiligten Akteuren möglich. Insbesondere die Lebensmittelwirtschaft ist gefordert, ihre Verantwortung aktiv wahrzunehmen. Akteure der Privatwirtschaft können mit freiwilligen Massnahmen zur
Förderung einer ausgewogenen Ernährung beitragen, z.B. durch die Anpassung von Portionengrössen, die Überprüfung der Lebensmittelrezepturen oder die Selbstkontrolle zur Beschränkung der Vermarktung von unausgewogen zusammengesetzten Lebensmitteln, die sich an Kinder richten.

Die Ausser-Haus-Verpflegung, die Schnellverpflegung und die Verwendung von Convenience-Produkten und Fertigmahlzeiten zu Hause haben in der Schweiz in den letzten Jahren an Bedeutung gewonnen und bieten somit grosses Potenzial für eine gesundheitsfördernde Ernährung. Angesprochen sind Lebensmittelindustrie und -handel, die Gastronomie und ihre Lieferanten und alle Entscheidungsträger, die dazu beitragen können, dass die Verpflegung in öffentlichen und privaten Institutionen gesundheitlichen Kriterien genügt.

\section{Erkenntnisse des 6. SEB nutzen}

Der 6. SEB zeigt, dass die verfügbaren Daten in der Schweiz nicht optimal sind, um wirkungsvolle Massnahmen planen und messen zu können und um den Ernährungsstatus der Schweizer Bevölkerung und spezifischer Bevölkerungsgruppen beurteilen zu können. Er liefert aber wichtige Grundlagen, um Massnahmen, die sich als erfolgreich erwiesen haben, auf der Verhältnis- und Verhaltensebene umsetzen zu können. Die Schweizer Ernährungsstrategie 2013-2016 sowie das Nationale Programm Ernährung und Bewegung (NPEB) werden das im 6. SEB generierte Wissen nutzen, um Prioritäten zu setzen und messbare Ziele zu definieren. Dies im Hinblick darauf, die Gesundheit und das Wohlbefinden der Schweizer Bevölkerung weiter zu verbessern. 


\section{Aktuelle \\ Ernährungsempfehlungen}

\section{Jürg Lüthy}

Angelika Hayer

Muriel Jaquet

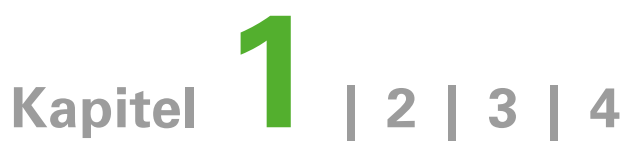




\subsection{Zusammenfassung}

In der Schweiz haben sich verschiedene Fachgesellschaften und Institutionen an der Ausarbeitung von Ernährungsempfehlungen beteiligt. Für die Ernährung im Säuglingsalter sind die Empfehlungen der Ernährungskommission der Schweizerischen Gesellschaft für Pädiatrie sowie die Bestimmungen des Lebensmittelrechtes für die Säuglingsanfangs- und Folgenahrung massgebend. Die Empfehlung der Weltgesundheitsorganisation (WHO) für ausschliessliches Stillen während sechs Monaten (sofern möglich) und anschliessendes Weiterstillen während der Einführung der Beikost bis ins zweite Lebensjahr und länger wurde auch von der Schweiz übernommen, allerdings mit der Einschränkung, dass bei Bedarf eine individuelle Einführung der Beikost frühestens ab dem fünften Lebensmonat möglich und in gewissen Fällen sinnvoll ist.

Für Erwachsene hat die Schweizerische Gesellschaft für Ernährung die Lebensmittelpyramide zur Empfehlung einer bedarfsgerechten und gesundheitsfördernden Ernährung etabliert. Sie bildet eine ausgewogene Mischkost $a b$, welche eine ausreichende Zufuhr sowohl von Energie als auch von lebensnotwendigen Nährstoffen gewährleistet. Die Empfehlungen richten sich an gesunde Erwachsene. Die Grundzüge einer gesunden Kinderernährung sind in der Ernährungsscheibe für Kinder der Schweizerischen Gesellschaft für Ernährung zusammengefasst. Die fünf Hauptbotschaften der Ernährungsscheibe greifen weitverbreitete kritische Ernährungsgewohnheiten auf und appellieren an die Handlungsebene. Bei Jugendlichen kommt es während der Pubertät zu einem Wachstumsschub und zu physiologischen Veränderungen, die mit einem erhöhten Energie- und Nährstoffbedarf einhergehen. Für die Knochenentwicklung, die während der Adoleszenz besonders intensiv verläuft, ist eine ausreichende Calciumversorgung wichtig. Ein erhöhter Bedarf besteht auch bei Phosphor, Eisen und Vitamin B1. Für die Ernährung im Alter gelten grundsätzlich die Empfehlungen der Schweizerischen Gesellschaft für Ernährung für Erwachsene, wobei aber einige Punkte wie genügende Protein-, Calcium- und Flüssigkeitszufuhr von besonderer Bedeutung sind.

Bei den Ernährungsempfehlungen für spezifische Bevölkerungsgruppen wie Schwangere, Sportler oder Vegetarier sind einige besondere Punkte zu berücksichtigen. Eine Herausforderung sind Empfehlungen zu Lebensmitteln, die sowohl erwünschte wie auch unerwünschte Inhaltsstoffe enthalten. Eine teilweise Neubewertung erfuhren die Empfehlungen zur Zufuhr einzelner Nährstoffe. Ein Vergleich mit anderen europäischen Ländern zeigt, dass es auch hier noch Unterschiede gibt. Bei der Umsetzung auf lebensmittelbasierte Ernährungsempfehlungen müssen die nationalen Gegebenheiten im Ernährungsverhalten ebenso berücksichtigt werden wie bestehende ernährungsabhängige Krankheiten und deren Risikofaktoren.

\subsection{Einleitung}

\section{Geschichte der Ernährungsempfehlungen} Geht man die Geschichte der Ernährungsempfehlungen durch, so stellt man fest, dass noch bis in die 50er Jahre des letzten Jahrhunderts die Vermeidung von Unter- und Mangelernährung im Vordergrund stand. Eine eigentliche Pioniertat war in diesem Zusammenhang die in der Schweiz schon 1922 erfolgte Kochsalzjodie- 
rung (1). Während des Zweiten Weltkrieges erhielt das sogenannte "Kriegsernährungsamt» weitgehende Vollmachten auf dem Gebiet der agrarischen Produktion und Versorgung mit in- und ausländischen Nahrungs- und Futtermitteln. Die in der Folge gegründete "Kriegsernährungskommission» stellte einen Ernährungsplan auf, in den die damaligen Ernährungsempfehlungen einflossen, die im Wesentlichen auf einer ausreichenden Versorgung mit der erforderlichen Energiemenge und den notwendigen Nährstoffen basierten. Einige der damals aufgestellten Grundsätze muten erstaunlich modern an, obwohl die wissenschaftlichen Zusammenhänge zwischen Ernährung und Gesundheit noch grosse Lücken aufwiesen (2).

Seit der zweiten Hälfte des letzten Jahrhunderts wurden Ernährungsempfehlungen zunehmend unter dem Gesichtspunkt der Zusammenhänge zwischen Ernährung bzw. Ernährungsfaktoren einerseits und Risiken für ernährungsabhängige Krankheiten andererseits betrachtet. In der Schweiz war es vor allem die schon 1948 gegründete Eidgenössische Ernährungskommission (EEK) sowie die in den 60 er Jahren entstandenen Schweizerische Vereinigung für Ernährung und Schweizerische Gesellschaft für Ernährungsforschung (die sich 2004 zur Schweizerischen Gesellschaft für Ernährung (SGE) zusammenschlossen), die sich darum bemühten, die international erzielten Forschungsresultate auf Schweizer Verhältnisse angepasst umzusetzen. In diesem Kontext können auch die seit 1975 vorerst von der EEK herausgegebenen, später zunehmend vom Bundesamt für Gesundheit (BAG) mitgetragenen Schweizerischen Ernährungsberichte betrachtet werden. Die EEK war es auch, die 1995 erstmals in der Schweiz acht Punkte umfassende allgemeine Ernährungsempfehlungen herausgab, wobei an der Spitze bereits die Empfehlungen standen, Ernährung und Bewegung im Hinblick auf eine ausgewogene Energiebilanz aufeinander abzustimmen und bei der Ernährung nebst der Abwechslung auf einen genügenden Früchte- und Gemüsekonsum zu achten. Später waren es dann die Schweizerische Vereinigung für Ernährung und die EEK, die in Abstimmung mit dem BAG Ernährungsempfehlungen entwickelten.

\section{Kapitelübersicht}

Das Ziel dieses Kapitels ist die Darstellung der in der Schweiz gültigen Ernährungsempfehlungen und ihrer wissenschaftlichen Basis. Unterteilt werden die Ernährungsempfehlungen nach Lebensalter (Kapitel 1.3) und spezifischen Bevölkerungsgruppen (Kapitel 1.4). Eine Grundlage für diese lebensmittelbasierten Empfehlungen sind die in Kapitel 1.5 behandelten Nährstoffempfehlungen. Dabei wurde nicht Vollständigkeit wie bei den Referenzwerten für die Nährstoffzufuhr der deutschsprachigen Gesellschaften für Ernährung (DACH-Referenzwerte) angestrebt (3). Die Kriterien für die Auswahl der diskutierten Nährstoffe sind vielmehr die Bedeutung dieser Nährstoffe für ernährungsabhängige Krankheiten und Mangelernährung.

Die Frage der rechtlich relevanten Sicherheit der Lebensmittel wird in Kapitel 1.6 unter dem Gesichtspunkt diskutiert, inwieweit solche Sicherheitsrisiken mit Ernährungsempfehlungen in Konflikt kommen können. In diesem Zusammenhang stellt sich auch die Frage, wie weit Bemühungen zur Verbesserung der Ernährung im Laufe der Zeit im Lebensmittelrecht Eingang gefunden haben. Das historisch beste Beispiel dafür ist die "Verfügung des Eidgenössischen 
Departements des Innern (EDI) über Zusatz und Anpreisung von Vitaminen bei Lebensmitteln» vom 7. März 1957, die über 40 Jahre Bestand hatte. Ein aktuelles Beispiel ist die bis heute anhaltende Diskussion über eine für den Konsumenten informativere Nährwert- und Frontof-Pack-Kennzeichnung. Im revidierten Lebensmittelgesetz vom 9. Oktober 1992 wurde als wesentliche Neuerung ein Artikel über die «Information der Öffentlichkeit» aufgenommen (4). In diesem Artikel 12 wird die Informationspflicht des Bundes über «besondere Ereignisse» (in der Regel Sicherheitsprobleme mit Lebensmitteln) in dem Sinne erweitert, dass der Bund die Öffentlichkeit auch «über ernährungswissenschaftliche Erkenntnisse von allgemeinem Interesse, welche namentlich für die $\mathrm{Ge}$ sundheitsvorsorge und den Gesundheitsschutz von Bedeutung sind, informieren kann». Dies ist nichts anderes als eine rechtliche Basis für die Erarbeitung von Ernährungsempfehlungen, über welche die Bevölkerung oder spezifische Bevölkerungsgruppen in geeigneter Form informiert werden können.

In den Kapiteln 1.7 und 1.8 wird schliesslich ein Blick auf die Ernährungsempfehlungen anderer Länder geworfen, um u.a. auch die Frage zu beantworten, ob es tatsächlich schweizspezifische Ernährungsempfehlungen braucht oder nur länderspezifische Umsetzungen. Dass es Gemeinsamkeiten im Ernährungsmuster in europäischen Ländern und damit auch wünschenswerte Änderungen im Hinblick auf eine Prävention ernährungsabhängiger Krankheiten gibt, zeigt die Entwicklung des «CINDI Dietary Guide» durch die WHO-Europa (5). CINDI steht für "countrywide integrated noncommunicable diseases intervention». Die darin formulierten zwölf Schritte zur gesunden Ernährung sind sowohl eine Basis für die in der Schweiz geltenden Ernährungsempfehlungen als auch für die Programme der Weltgesundheitsorganisation (WHO) zur Verbesserung der Ernährungssituation in europäischen Ländern. Nährstoffbasierte Ernährungsempfehlungen werden aufgrund von internationalen wissenschaftlichen Erkenntnissen erarbeitet. Deren Umsetzung in lebensmittelbasierte Ernährungsempfehlungen sollte allerdings auf nationale Gegebenheiten im Ernährungsverhalten ebenso Rücksicht nehmen wie auf bestehende ernährungsabhängige gesundheitliche Probleme, die länderspezifisch unterschiedlich sind (6).

\subsection{Allgemeine Ernährungs- empfehlungen nach Lebensalter}

\subsubsection{Ernährung im Säuglingsalter}

Seit 1983 erarbeitet die Ernährungskommission der Schweizerischen Gesellschaft für Pädiatrie (EK SGP) Empfehlungen für die Säuglingsernährung, die periodisch neuen Erkenntnissen angepasst werden, letztmals in den Jahren 2008 (7) und 2009 (8). Berücksichtigt wurden neben neuen wissenschaftlichen Erkenntnissen auch Änderungen des Lebensmittelrechtes (9), wobei auch der international neu ausgehandelte Standard für Säuglingsnahrungen des Codex Alimentarius (10) und das «Statement on dietary fats in infant nutrition» der International Society for the Study of Fatty Acids and Lipids (ISSFAL; 11) einbezogen wurden. Säuglingsanfangs- und Folgenahrung sind die am ausführlichsten regulierten Bereiche des Lebensmittelrechtes. 
Die Säuglingsernährung umfasst zwei Phasen: einerseits die Ernährung ausschliesslich mit Muttermilch oder einer Säuglingsanfangsnahrung in den ersten vier bis sechs Lebensmonaten und andererseits die Ernährung mit Muttermilch oder einer Säuglingsanfangsnahrung bzw. Folgenahrung zusammen mit Beikost ab dem fünften bis siebten Lebensmonat.

Die WHO und die United Nations Children's Fund (UNICEF) empfehlen in ihrer Resolution aus dem Jahr 2001 ausschliessliches Stillen während der ersten sechs Lebensmonate nach der Geburt und anschliessendes Weiterstillen während der Einführung der Beikost bis ins zweite Lebensjahr und länger (12). Diese WHOEmpfehlungen wurden auch von der Schweiz übernommen, allerdings mit der Einschränkung, dass eine individuelle Einführung der Beikost bei Bedarf bereits ab dem fünften Lebensmonat möglich und in gewissen Fällen sinnvoll ist. Eine Einführung der Beikost vor dem fünften Lebensmonat ist nicht zu empfehlen, unter anderem wegen der höheren Belastung der Nieren mit harnpflichtigen Substanzen durch die Beikost und wegen der Gefahr eines möglichen Auftretens von Allergien. Spätestens ab dem siebten Lebensmonat muss die Beikost eingeführt werden, da die Muttermilch den Nährstoffbedarf des Säuglings ab diesem Zeitpunkt nicht mehr decken kann $(7 ; 8)$. Kapitel 1.6 geht auf mögliche toxikologische Risiken ein, die mit durch Rückstände belasteter Muttermilch verbunden sein können.

Die Zusammensetzung der Muttermilchersatzpräparate ist in der Schweiz durch eine Verordnung des EDI über Speziallebensmittel detailliert gesetzlich geregelt, und zwar nicht nur was die Zusammensetzung betrifft, sondern auch bzgl. Gestaltung der Packungen, Nähr- wertkennzeichnung, Etiketten, Beipackzettel sowie Kriterien für Werbeaussagen (9). Sowohl Säuglingsanfangs- als auch Folgenahrungen können Zusätze enthalten, die nicht gesetzlich vorgeschrieben sind. Oft handelt es sich dabei um Substanzen, die auch in der Muttermilch nachgewiesen werden können. Für die Beurteilung solcher Zusätze wird auf die jeweils aktuellen Stellungnahmen von internationalen Expertengremien wie der Ernährungskommission der European Society for Paediatric Gastroenterology, Hepatology and Nutrition (ESPGHAN) verwiesen.

Säuglinge haben einen im Vergleich zu Erwachsenen erhöhten Flüssigkeitsbedarf, abhängig vom Alter, der Aktivität, der zugeführten Energie und der Urinproduktion. Der mittlere Wasserbedarf liegt im ersten Lebensjahr abnehmend zwischen 130 und $120 \mathrm{ml}$ pro kg Körpergewicht und Tag. Voll gestillte Säuglinge brauchen in den ersten sechs Lebensmonaten keine zusätzliche Flüssigkeitszufuhr. Ausnahmen können Perioden mit Fieber, Durchfall und anderes sein, die eine Gabe von Flüssigkeit erforderlich machen (13). Erst mit drei Breien am Tag als Beikost sind aber zusätzliche Flüssigkeitsgaben erforderlich, am besten in Form von Trinkwasser. Vom unkontrollierten Gebrauch der Schoppenflasche wird abgeraten, insbesondere auch wegen der Gefahr des Risikos für Karies und Erosionen durch Milch, gezuckerten Tee oder Fruchtsäfte (7;8; Kapitel 3.11).

Der Energiebedarf ist altersabhängig und liegt im ersten Lebensjahr abnehmend zwischen 95 und $90 \mathrm{kcal}$ pro kg Körpergewicht und Tag (7). Die WHO empfiehlt beim gestillten Säugling vom siebten bis neunten Monat für die Beikost eine Energiezufuhr von ca. $200 \mathrm{kcal}$ pro Tag, vom zehnten bis zwölften Monat von 
ca. $300 \mathrm{kcal}$ pro Tag und im zweiten Lebensjahr von ca. $550 \mathrm{kcal}$ pro Tag.

Während des ersten Lebensjahres wird für alle gestillten und nichtgestillten Säuglinge die tägliche Zufuhr von 300-500 IE Vitamin D empfohlen (= 7.5-12.5 $\mu \mathrm{g}$ ), unabhängig von der individuellen Sonnenexposition (8); für Kinder bis zum Ende des 3. Lebensjahres werden 600 IE $(=15 \mu \mathrm{g})$ empfohlen (65; Kapitel 3.7 und 3.10.3). Eine maximale Zufuhr von täglich 1'000 IE $(=25 \mu \mathrm{g})$ sollte allerdings nicht überschritten werden $(8 ; 65)$.

Spätestens ab dem siebten Lebensmonat braucht der Säugling zusätzliche Nährstoffe, welche durch Beikost gedeckt werden. Für die Beikost eignen sich z.B. Gemüse-KartoffelFleisch-Brei und Getreide-Früchte-Brei. Die Verabreichung von unverdünnter Kuhmilch wird frühestens ab dem zweiten Lebensjahr empfohlen. Joghurt ohne zugesetzten Zucker kann als Zusatz zu Früchte- und Getreidebreien ab dem siebten Monat verabreicht werden. Die Anzahl Mahlzeiten wird nach Einführung der Beikost langsam gesteigert, so dass der Säugling am Ende des ersten Lebensjahres täglich drei bis vier Breimahlzeiten erhält. Über weitere wichtige Punkte wie die Allergieprävention geben die Empfehlungen der EK SGP Auskunft (7;13). Zusätzliche Informationen für die Praxis bietet die Schweizerische Stiftung zur Förderung des Stillens (14).

\subsubsection{Ernährung von Kindern}

Gegen Ende des ersten Lebensjahres sind einige Zähne durchgebrochen und die psychomotorischen Fähigkeiten sowie die Verdauungs- und Stoffwechselfunktionen so weit ausgereift, dass nun der allmähliche Übergang von der
Säuglingsernährung zur Ernährung am Familientisch erfolgen kann. Nach und nach werden die Brei- und Milchmahlzeiten durch drei «normale» Hauptmahlzeiten und zwei Zwischenmahlzeiten ersetzt. Der Übergang richtet sich nach dem Entwicklungsstand des Kindes, z.B. seiner Fähigkeit, zu kauen. Ab dem zweiten Lebensjahr dürfen Kinder auch unverdünnte Kuhmilch und Quark bekommen; spezielle Säuglingsmilch wie beispielsweise Folgemilch oder Juniormilch sind nicht erforderlich. Bei gestillten Kindern empfiehlt die SGP, diese auch über das erste Lebensjahr hinaus weiter zu stillen (8;15-18).

Ab dem Alter von einem Jahr können Kinder somit von allen Lebensmitteln essen. Die Gerichte sollten aber wenig gewürzt, gesalzen und gezuckert werden. Manche Kinder reagieren anfangs noch empfindlich auf stark blähende Lebensmittel wie z.B. Hülsenfrüchte oder Kohlgewächse. Vorsicht ist bei Lebensmitteln geboten, die verschluckt werden könnten. Nüsse sollten deshalb bis zum Ende des zweiten Lebensjahres nur in gemahlener Form gegeben werden.

Grundlage einer gesunden Ernährung für ein- bis zwölfjährige Kinder bildet eine ausgewogene Mischkost. Ausgewogen bedeutet, dass die Lebensmittel abwechslungsreich ausgewählt und in einem bestimmten Mengenverhältnis zueinander verzehrt werden. So wird eine ausreichende Zufuhr von Energie und von lebensnotwendigen Nährstoffen gewährleistet.

Aus den Mengenempfehlungen für ein- bis zwölfjährige Kinder wird ersichtlich, dass Kinder im Vergleich zu Erwachsenen nicht einfach nur kleinere Portionen brauchen (18). Da Kinder sich noch in der Wachstums- und Entwicklungsphase befinden, brauchen sie von manchen Lebensmittelgruppen relativ gesehen mehr oder 
auch weniger als Erwachsene. In manchen Altersstufen haben die Stärkelieferanten (z.B. Getreideprodukte, Kartoffeln) bzw. Milch und Milchprodukte eine etwas stärkere Bedeutung.

Die Grundzüge einer gesunden Kinderernährung sind in der Ernährungsscheibe für Kinder der SGE dargestellt (19; Abbildung 1.1). Diese ist aufgeteilt in fünf Segmente, die jeweils für eine Ernährungsbotschaft stehen.

Die Botschaften greifen kritische Ernährungsgewohnheiten bei Kindern auf, wie z.B. hoher Konsum von Süssgetränken, und appellieren an die Handlungsebene, wie z.B. Wasser trinken (19-22). Zu jeder Hauptbotschaft gibt es zwei praktische Tipps, welche die Hauptbotschaft konkretisieren und die Umsetzung im Alltag erleichtern. Zielgruppe sind insbesondere Kinder im Alter von 5 bis 12 Jahren sowie ihre Eltern, die eine wichtige Vorbildfunktion haben. Grundsätzlich haben die Botschaften aber auch bei Kindern aller anderen Altersgruppen sowie bei Erwachsenen Gültigkeit. Die Ernährungs-

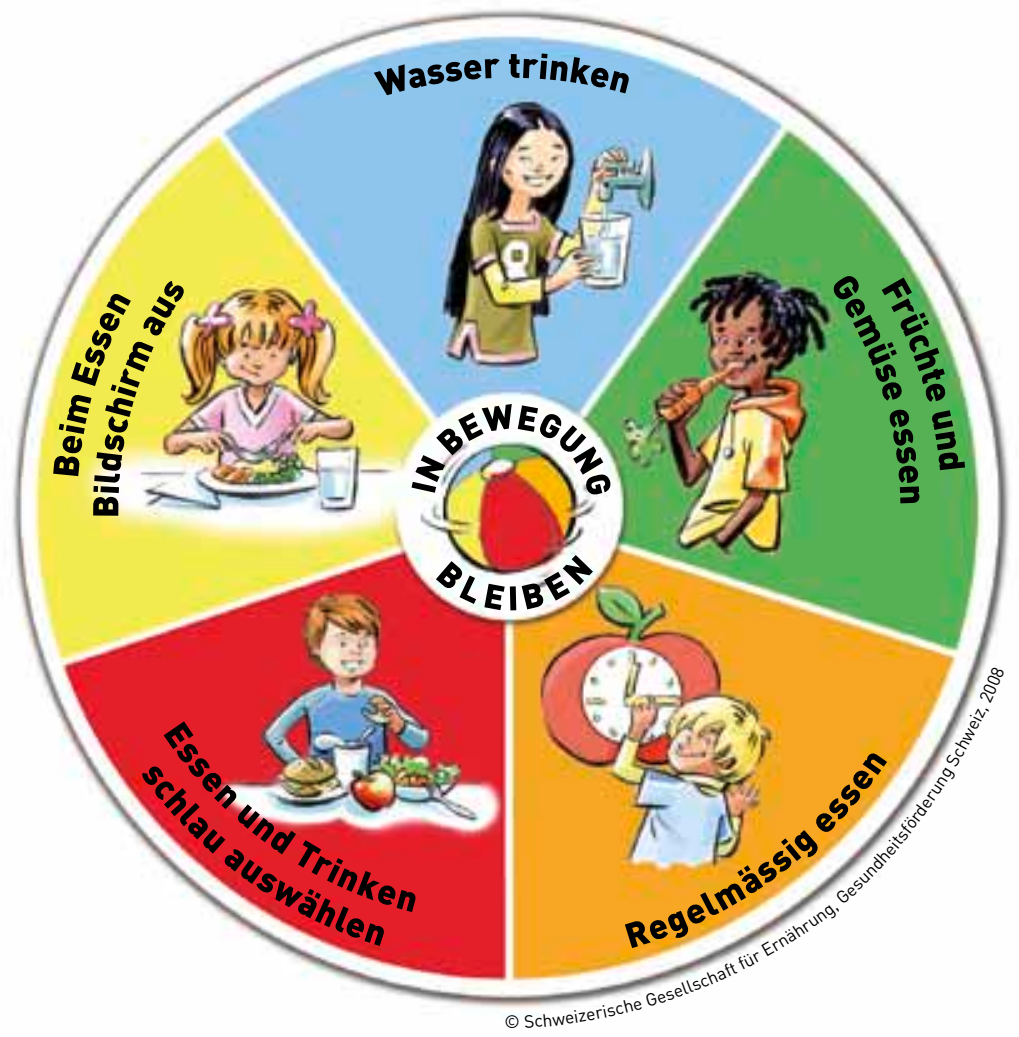

Abbildung 1.1: Ernährungsscheibe für Kinder der SGE (19) 
scheibe für Kinder der SGE zielt darauf $a b$, anschaulich und praxisnah ein gesundes Ess- und Trinkverhalten zu vermitteln. Als didaktisches Hilfsmittel wird sie im Kindergarten und in der Primarschule sowie in ausserschulischen Projekten zur Gesundheitsförderung eingesetzt.

\subsubsection{Ernährung von Jugendlichen}

Während der Pubertät kommt es zu einem Wachstumsschub und zu physiologischen Veränderungen, die mit einem erhöhten Energieund Nährstoffbedarf einhergehen. Der Energiebedarf ist individuell sehr unterschiedlich, da dieser u.a. von Alter, Geschlecht, der körperlichen Aktivität und vom individuellen Entwicklungsstand abhängt. Geht man von Normalgewicht und einer mittleren Aktivität aus, liegt der Richtwert für die durchschnittliche Energiezufuhr der 13- bis 15-Jährigen schätzungsweise bei 2'200 kcal pro Tag (Mädchen) bzw. 2'700 kcal pro Tag (Knaben). Bei den 15- bis 18-Jährigen liegen die entsprechenden Richtwerte bei 2'500 kcal pro Tag (Mädchen) bzw. 3'100 kcal pro Tag (Knaben). Die letztgenannte Altersgruppe hat im Vergleich zu allen anderen - ob jünger oder älter - den höchsten Energiebedarf (3).

Bis ins junge Erwachsenenalter bzw. bis ca. zum 30. Lebensjahr wird Knochenmasse bis zu einem individuellen Maximalwert ( "peak bone mass") aufgebaut. Aus Untersuchungen ist bekannt, dass der Aufbau und die Mineralisation der Knochen nicht gleichmässig verlaufen und dass die Knochenentwicklung während der Adoleszenz sehr intensiv ist (23). Um im Hinblick auf die Osteoporosevorbeugung eine optimale maximale Knochenmasse zu erreichen, ist eine ausreichende Calciumversorgung im Ju- gendalter besonders wichtig. Die Zufuhrempfehlungen von Calcium für 13- bis 18-Jährige liegen bei 1'200 mg pro Tag und damit höher als bei den anderen Altersstufen (3). Für die gesunde Knochenentwicklung spielen ausserdem weitere Faktoren wie z.B. Protein, Vitamin D und Bewegung eine wichtige Rolle (Kapitel 3.7).

Nebst Calcium haben Jugendliche auch von einigen weiteren Nährstoffen einen höheren Bedarf als Kinder oder Erwachsene, so beispielsweise an Phosphor, Eisen und Vitamin B1 (3).

Der höhere Bedarf an Energie und Nährstoffen kann mit einer ausgewogenen und abwechslungsreichen Mischkost gedeckt werden. Gesunde Jugendliche benötigen daher keine speziellen Produkte oder Supplemente. Eine Orientierung zur gesunden Ernährung gibt die Schweizer Lebensmittelpyramide (24; Abbildung 1.2). Für Jugendliche gelten jedoch teilweise andere Mengenempfehlungen als für Erwachsene (25). So sind beispielsweise die angegebenen Mengen für die stärkereichen Lebensmittel wie Brot, Teigwaren, Kartoffeln usw. grösser, um eine ausreichende Energiezufuhr zu gewährleisten.

Jugendliche unterscheiden sich von Erwachsenen aber nicht nur bezüglich ihrer ernährungsphysiologischen Bedürfnisse, sondern auch bezüglich ihres Lebensstils sowie ihrer Ess- und Trinkgewohnheiten $(26 ; 27)$. Was, wo und wie Jugendliche essen, ist eng mit dem Wunsch, sich vom Elternhaus abzugrenzen, und der Entwicklung der eigenen Identität verbunden. Dies trägt dazu bei, dass bestimmte Lebensmittel und Essmuster wie z.B. Fast Food und Snacking gerade bei Jugendlichen sehr beliebt sind (2628). In den Ernährungsempfehlungen für 13-bis 18-Jährige werden daher Themen, die in dieser Altersgruppe eine wichtige Rolle einnehmen, besonders berücksichtigt. Dazu gehören u.a. 
Fast Food, vegetarische Ernährung, Getränkekonsum (inkl. Süssgetränke, Alkohol, Energy Drinks), Mahlzeitenverteilung und Snacking, Körperwahrnehmung und Körpergewicht (25).

\subsubsection{Ernährung von Erwachsenen}

Eine ausgewogene Ernährung ist Teil eines gesundheitsfördernden Lebensstils. Sie deckt den täglichen Bedarf an Energie und Nährstoffen, beeinflusst das geistige und körperliche Wohlbefinden und trägt dazu bei, ernährungsabhängigen Krankheiten vorzubeugen.

Grundsätzlich besteht eine gesunde Ernährung aus reichlich pflanzlichen Lebensmitteln wie Gemüse, Früchten, Hülsenfrüchten, Kartoffeln, Getreideprodukten u.a. Ergänzend hinzu kommen Milchprodukte und in kleineren Mengen Fleisch, Fisch, Eier sowie Nüsse und hochwertige Pflanzenöle. Eine ausreichende Flüssigkeitszufuhr, bevorzugt in Form von ungesüssten Getränken, erhält die körperliche und geistige Konzentrations- und Leistungsfähigkeit. Süssigkeiten, salzige Knabbereien, zucker- und alkoholhaltige Getränke sollten im Rahmen einer ausgewogenen Ernährung massvoll konsumiert werden.

Um die Empfehlungen für eine bedarfsgerechte und gesundheitsfördernde Ernährung zu vermitteln, hat sich in der Schweiz die Lebensmittelpyramide der SGE mit den Empfehlungen zum ausgewogenen und genussvollen Essen und Trinken für Erwachsene etabliert (24; Abbildung 1.2). Sie entstand 1998 in Anlehnung an die US-amerikanische Lebensmittelpyramide und wurde in den Jahren 2005 und 2011 in aktualisierter Form neu veröffentlicht (29). Seit 2011 wird sie unter dem Namen «Schweizer
Lebensmittelpyramide» gemeinsam von SGE und BAG herausgegeben.

Da Empfehlungen zur Nährstoffzufuhr abstrakt und für den Konsumenten wenig hilfreich sind (30), wurden sie auf Lebensmittelebene umgerechnet («nutritiver Ansatz»). Dies bedeutet, dass Lebensmittel entsprechend ihres Gehaltes an "Schlüsselnährstoffen» sowie ihrer traditionellen Verwendung in unserer Esskultur in Lebensmittelgruppen zusammengefasst wurden. Bei der Festlegung der lebensmittelbasierten Empfehlungen ("food based dietary guidelines") wurden ausserdem die Wirkungen von Lebensmitteln auf den Stoffwechsel ( «metabolischer Ansatz») und präventive Aspekte hinsichtlich Übergewicht, Diabetes mellitus Typ 2, Herz-Kreislauf- und Krebserkrankungen berücksichtigt (30-33).

Für die wissenschaftliche Aktualisierung der Schweizer Lebensmittelpyramide von 2011 war eine Gruppe von Experten verschiedener Fachrichtungen verantwortlich. Auf Basis einer umfassenden Literaturrecherche überprüfte sie die bisherigen Empfehlungen auf ihre Gültigkeit und leitete Massnahmen zur Überarbeitung ab. Die Umsetzung der ernährungswissenschaftlichen Erkenntnisse in praxisnahe Empfehlungen erfolgte in Zusammenarbeit mit dipl. Ernährungsberatern FH / HF aus der Praxis, wobei auch die Essgewohnheiten in der Schweiz berücksichtigt wurden. Umfassende Berechnungen gewährleisteten schliesslich, dass mit den lebensmittelbasierten Empfehlungen die Nährstoffempfehlungen erreicht werden.

Sowohl bei der Formulierung der Empfehlungen als auch bei der anschliessenden graphischen Überarbeitung der Schweizer Lebensmittelpyramide wurde Wert auf eine bessere Verständlichkeit und Benutzerfreundlichkeit gelegt. Wichtige 


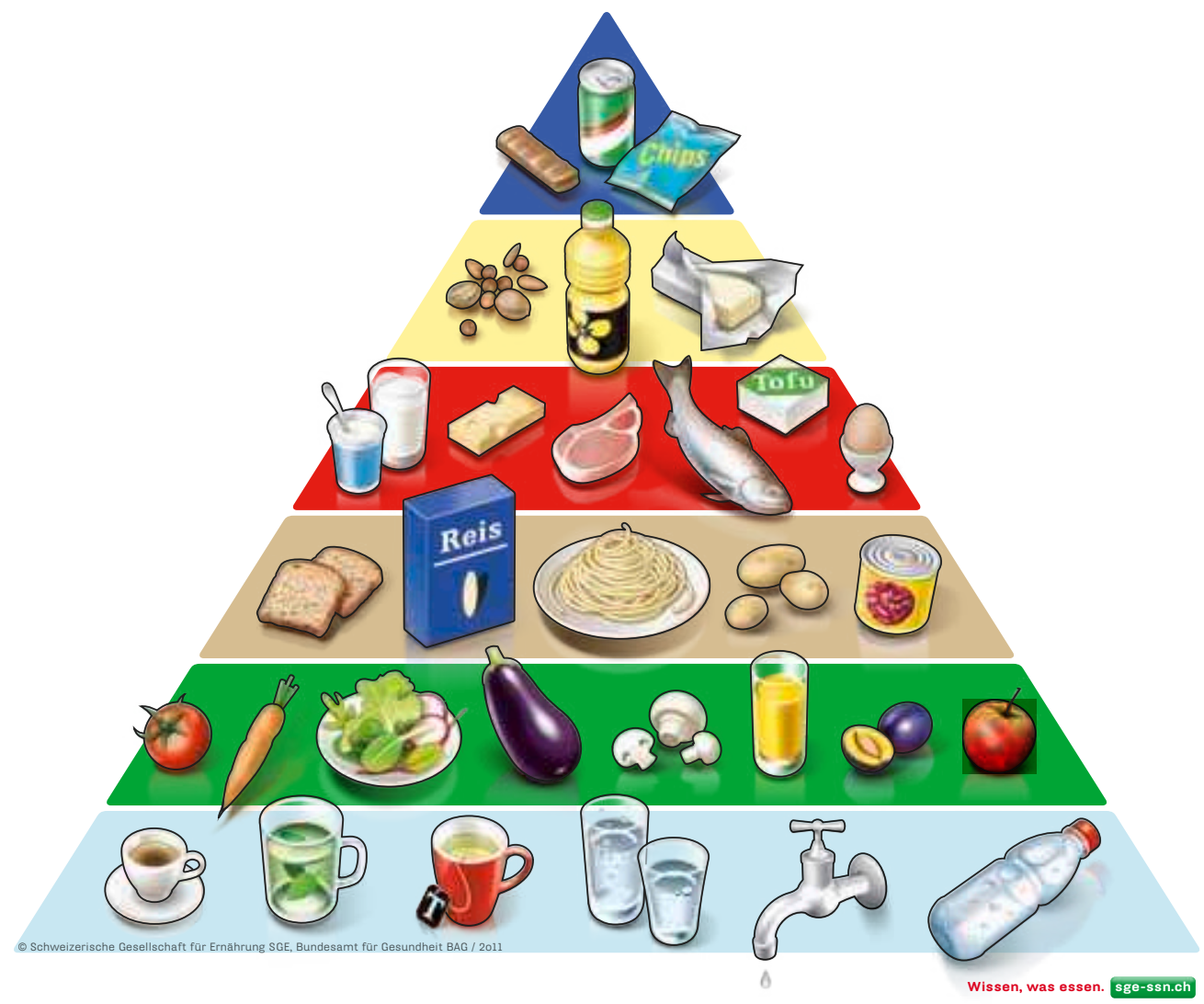

Abbildung 1.2: Schweizer Lebensmittelpyramide für Erwachsene (24)

Hinweise hierfür lieferte die von der SGE durchgeführte Online-Befragung unter Ernährungsfachleuten und Lehrpersonen im 2009 (34).

Die Schweizer Lebensmittelpyramide stellt eine ausgewogene Mischkost dar, welche eine ausreichende Zufuhr von Energie und von lebensnotwendigen Nährstoffen gewährleistet. Der Zielbereich für die Energiezufuhr beträgt 1'800-2'500 kcal und entspricht damit dem Grundumsatz für 19- bis 65-Jährige multipliziert mit einem PAL-Wert ${ }^{1}$ von 1.4 (3). Die Empfehlungen richten sich an gesunde Erwachsene. Für andere Alters- und Bevölkerungsgruppen (z.B. Kinder, Schwangere, Leistungssportler)

${ }^{1}$ Der PAL ("physical activity level») ist als durchschnittlicher täglicher Energiebedarf für körperliche Aktivität als Mehrfaches des Grundumsatzes definiert. Ein PALWert von 1.4 gilt für Personen mit ausschliesslich sitzender Tätigkeit und mit wenig oder keiner anstrengenden Freizeitaktivität. 
sowie bei Krankheit oder einer gewünschten Gewichtsabnahme gelten teilweise abweichende Empfehlungen (Kapitel 1.4).

Die Schweizer Lebensmittelpyramide stellt keinen starren Ernährungsplan dar, sondern lässt Spielraum für die individuelle Gestaltung. Ihr liegen folgende Schlüsselprinzipien zu Grunde:

- Mengenverhältnis: Lebensmittel der unteren Pyramidenebenen sollen in grösseren, solche der oberen Ebenen hingegen in kleineren Mengen gegessen werden. Alle Lebensmittel sind erlaubt.

- Abwechslung: Eine ausgewogene Ernährung ist abwechslungsreich zusammengesetzt und berücksichtigt sowohl verschiedene Lebensmittelgruppen als auch verschiedene Lebensmittel innerhalb der Lebensmittelgruppen.

Einen Überblick über die detaillierten Mengenempfehlungen zu den einzelnen Lebensmittelgruppen gibt Tabelle 1.2. Die Empfehlungen müssen nicht jeden Tag, sondern sollen langfristig eingehalten werden, z.B. über eine Woche. Eine Ausnahme bilden die Empfehlungen zur Flüssigkeitszufuhr, die täglich berücksichtigt werden sollen. Um Nährstoffverluste gering zu halten, ist auf eine schonende Zubereitung der Lebensmittel zu achten.

Mahlzeiten dienen nicht nur der Versorgung mit Energie und Nährstoffen, sondern erfüllen auch emotionale und soziale Bedürfnisse. Sie sind Zeiten des Genusses, der Erholung, des Kontaktes und des Austauschs mit anderen. Die SGE empfiehlt daher, sich für Mahlzeiten Zeit zu nehmen, Nebenbeschäftigungen einzustellen und das Essen in Ruhe zu geniessen.

In der Schweizer Lebensmittelpyramide sind die Empfehlungen zur Ernährung eng mit denen zur Bewegung verknüpft. Entsprechend den Empfehlungen des Bundesamtes für Sport (BASPO) wird täglich mindestens eine halbe Stunde Bewegung in Form von Alltags- und sportlichen Aktivitäten empfohlen wie z.B. Treppensteigen, Spazieren mit dem Hund, Velofahren, Joggen, Schwimmen (35). Neben gesunder Ernährung und ausreichend Bewegung gehören ausserdem der Verzicht aufs Rauchen und der richtige Umgang mit Stresssituationen bzw. bewusstes und gezieltes Entspannen zu einem gesunden Lebensstil.

\subsubsection{Ernährung im Alter}

Die Ernährung spielt auch mit zunehmendem Alter eine wichtige Rolle, weil unsere Gesundheit und unser Wohlbefinden stark davon abhängen, was und wie wir essen. Ausser im Krankheitsfall braucht es keine vollständige Umstellung der gewohnten Ernährungsweise. Sinnvoll könnte es hingegen sein, die persönlichen Ess- und Trinkgewohnheiten frühzeitig den sich ändernden (Alters-) Umständen anzupassen.

Für gesunde ältere Menschen gelten grundsätzlich die Empfehlungen gemäss der Schweizer Lebensmittelpyramide für Erwachsene (Abbildung 1.2). $\mathrm{Zu}$ beachten ist jedoch, dass im Alter der Energiebedarf sinkt, während der Bedarf an Protein, Vitaminen und Mineralstoffen mindestens gleich hoch bleibt wie in jüngeren Jahren. Folglich ist verstärkt auf eine nährstoffdichte Ernährung zu achten, um Mangelernährung vorzubeugen (36; Kapitel 3.10).

Aufgrund von Krankheiten, Medikamenteneinnahme, eingeschränkter Beweglichkeit, Schmerzen, Appetitmangel, psychosozialer sowie weiterer Faktoren kann die Zufuhr von Nähr- 
stoffen bzw. deren Aufnahme im Körper ungenügend sein. Als Folge einer zu geringen oder einer zu einseitigen Nährstoffzufuhr kann Mangelernährung entstehen. In diesem Falle bedarf es einer Abklärung und Betreuung durch eine Fachperson, wie bestehende Mängel behoben und zukünftig vermieden werden können. Eine Schlüsselrolle spielen hierbei die Spitäler. Die Schweiz hat sich im Europarat an der Ausarbeitung und Umsetzung eines Expertenberichtes über diese Thematik beteiligt $(37 ; 38)$. Die empfohlenen Massnahmen beinhalten u.a. Standards für das Risikoscreening sowie die Beurteilung und Überwachung des Ernährungszustandes (Kapitel 3.10).

Ökonomische und soziale Veränderungen sind mögliche Gründe für Vereinsamung und Depressionen im Alter. Eine aktive und gesunde Lebensgestaltung mit regen sozialen Kontakten und Erlebnissen, regelmässige körperliche Aktivität sowie eine ausgewogene und vielseitige Ernährung bieten demgegenüber die beste Grundlage für ein gesundes Leben im Alter. In Gesellschaft machen Bewegung und Essen besonders viel Spass.

\subsection{Ernährungsempfehlungen für spezifische Bevölkerungs- gruppen}

\subsubsection{Ernährung in der Schwanger- schaft und Stillzeit}

Der EEK-Bericht über die Ernährung in Schwangerschaft und Stillzeit vermittelt neben Empfehlungen für eine ausreichende Versorgung an Energie und Nährstoffen auch Empfehlungen zur Vermeidung von Risikoprodukten, zur Mini- mierung der Schadstoffaufnahme und zur Eliminierung bzw. Vermeidung von Krankheitskeimen (39).

Während der Schwangerschaft und Stillzeit ist eine abwechslungsreiche Kost gemäss der Schweizer Lebensmittelpyramide (Abbildung 1.2) unter zusätzlicher Berücksichtigung der speziellen Bedürfnisse, wie sie in den DACH-Referenzwerten festgehalten sind, besonders wichtig: regelmässige über den Tag verteilte Mahlzeiten, viel Früchte und Gemüse, Milch- und Milchprodukte als wichtige Quelle für Protein und Calcium, Fleisch (ausser Wild) für Eisen, Zink und Vitamin B12, pflanzliche Fette als Träger ungesättigter Fettsäuren sowie ein- bis zweimal Fisch pro Woche zur Zufuhr von n-3- und n-6-Fettsäuren (24).

Die Ernährung und dabei insbesondere die empfohlene Energiezufuhr der Schwangeren und Stillenden werden auch durch das Ausgangsgewicht vor der Schwangerschaft bzw. zu Beginn der Stillzeit mitbestimmt. Die Auswahl der Lebensmittel sollte bewusst im Hinblick auf die Deckung des vermehrten Bedarfs an Makronährstoffen, Vitaminen, Mengen- und Spurenelementen und entsprechend individuellen Unverträglichkeiten erfolgen (39).

Eine ausgewogene Ernährung unter Einhaltung der erwünschten mütterlichen Körpergewichtszunahme wirkt sich neben anderen Faktoren auch auf eine optimale Schwangerschaftsdauer und ein optimales Geburtsgewicht des Neugeborenen aus. Damit hat das Kind eine gute Ausgangslage für sein späteres Leben (39).

Frauen im gebärfähigen Alter, die schwanger werden könnten oder möchten, sollten bereits vor der Konzeption und während den ersten zwölf Schwangerschaftswochen zur Verhütung 
eines "offenen Rückens» (Spina bifida) beim Kind zusätzlich Folsäure in einer Dosis von $0.4 \mathrm{mg}$ pro Tag einnehmen, vorzugweise in Form eines Multivitaminsupplementes (40; Kapitel 3.10.3).

Die gleichen Empfehlungen wie während der Schwangerschaft gelten auch für die mütterliche Ernährung während der Stillzeit. Stillen bietet Mutter und Kind grosse Vorteile. Die Vorteile des Stillens und der Muttermilch überwiegen das Risiko der allfällig vorhandenen Schadstoffe in der Muttermilch bei weitem. Kapitel 1.3.1 befasst sich mit den Empfehlungen zur Ernährung im Säuglingsalter.

Empfehlungen zur Vermeidung von Risikoprodukten in Schwangerschaft und Stillzeit umfassen: keine tierischen Rohprodukte wie Rohmilch, rohe Eier oder rohes Fleisch (Tartar), keine Leberprodukte in den ersten 12 Schwangerschaftswochen und keine rohen Schalentiere (z.B. Austern). Während der Schwangerschaft wird weiter vom Konsum von Weich- und Halbhartkäse aus roher und pasteurisierter Milch abgeraten. Der Konsum von entrindeten Teilen von Extra- und Hartkäsen wie z.B. Emmentaler, Greyerzer und Sbrinz ist hingegen auch für schwangere Frauen unbedenklich (41). In der Schwangerschaft sollen ferner keine Genussmittel wie Alkohol oder Suchtmittel sowie höchstens 2-3 Tassen Kaffee oder äquivalente koffeinhaltige Getränke und möglichst keine chininhaltigen Getränke konsumiert werden. Zudem sind auch hygienische Massnahmen zur Eliminierung bzw. Vermeidung von Krankheitskeimen zu beachten.

Auf die Frage der Schadstoffaufnahme während der Schwangerschaft und Stillzeit wird im Kapitel 1.6 eingegangen.

\subsubsection{Ernährung von Sportlern}

Bei Sportlern, die mehr als fünf Stunden Sport pro Woche betreiben, ist von einem Mehrbedarf an Energie und Nährstoffen auszugehen. Das BASPO und das Swiss Forum for Sport Nutrition haben sich dieses Themas angenommen und auf Basis der SGE-Lebensmittelpyramide 2005 eine Lebensmittelpyramide für Sportlerinnen und Sportler mit entsprechenden Empfehlungen ausgearbeitet $(42 ; 43)$.

Die Empfehlungen richten sich somit an gesunde Erwachsene, die an den meisten Tagen pro Woche jeweils ca. eine Stunde oder mehr mit mindestens mittlerer Intensität sportlich aktiv sind und dadurch ein gesamtes Trainingsvolumen von ca. 5 Stunden oder mehr pro Woche erzielen. Eine mittlere Intensität entspricht der "Stop and Go»-Belastung eines durchschnittlichen Eishockey-, Fussball- oder Tennisspiels oder der kontinuierlichen Belastung von ca. $2.5 \mathrm{~km} / \mathrm{h}$ Crawl-Schwimmen, ca. $8 \mathrm{~km} / \mathrm{h}$ Joggen oder ca. 2 Watt/kg Körpergewicht auf dem Veloergometer. Die Lebensmittelpyramide für Sportlerinnen und Sportler gewährleistet eine ausreichende Zufuhr von Energie sowie von lebensnotwendigen Nährstoffen für die jeweiligen Zielpersonen. Die Lebensmittel sollten möglichst abwechslungsreich und vorzugsweise saisongerecht aus den einzelnen Pyramidenebenen gewählt sowie schonend verarbeitet und zubereitet werden. Bei regelmässiger Verwendung von mit Mineralstoffen und / oder Vitaminen angereicherten Lebensmitteln oder bei der Einnahme von Mineralstoff- und / oder Vitaminsupplementen können die maximal tolerierbaren Zufuhrmengen («Tolerable Upper Intake Level», UL) überschritten werden. 
Das Befolgen der Lebensmittelpyramide für Sportlerinnen und Sportler bietet eine gute Grundlage für eine längerfristige, gute sportliche Leistungsfähigkeit. Im Unterschied zur Basispyramide der SGE (Abbildung 1.2), in der die Empfehlungen nicht strikt jeden Tag eingehalten werden müssen, sollten Sportler für eine gute sportliche Leistungsfähigkeit sowie gute Regeneration die Empfehlungen möglichst jeden Tag einhalten. Der Mehrbedarf für den Sport ist für eine tägliche sportliche Aktivität von 1 bis 4 Stunden mit mittlerer Intensität angegeben; für grössere Trainingsumfänge ist der Mehrbedarf entsprechend höher.

Für Sportler erfolgt die Wahl der Portionengrössen in Abhängigkeit ihres Körpergewichtes. Die kleinsten Portionengrössen gelten für ein Körpergewicht von 50 kg, die grössten Portionengrössen für ein Körpergewicht von $85 \mathrm{~kg}$. Für dazwischenliegende Körpergewichte gelten entsprechende Zwischenstufen an Portionengrössen (z.B. mittlere Portionengrössen für $67 \mathrm{~kg}$ Körpergewicht). Weitere Angaben wie der zusätzliche Flüssigkeitsbedarf finden sich in der Lebensmittelpyramide für Sportlerinnen und Sportler (42).

\subsubsection{Vegetarische Ernährung}

\section{Formen der vegetarischen Ernährung}

Es gibt verschiedene Formen der vegetarischen Ernährung. Allgemein gilt, dass sich Vegetarier vorwiegend von pflanzlichen Produkten ernähren unter Verzicht von Fleisch und Fisch. In Bezug auf den Konsum von tierischen Lebensmitteln können verschiedene Gruppen vegetarischer Ernährung unterschieden werden, je nachdem, ob Milch und / oder Eier und daraus hergestellte Produkte verzehrt werden oder nicht. So konsumieren die Ovo-Lacto-Vegetarier zusätzlich Eier und Milch sowie daraus hergestellte Produkte, während Lacto-Vegetarier nur Milch und Milchprodukte und Ovo-Vegetarier nur Eier und daraus hergestellte Produkte in ihre Ernährung mit einbeziehen. Veganer schliesslich meiden alle Lebensmittel tierischen Ursprungs einschliesslich Honig.

Von diesen verschiedenen Formen der vegetarischen Ernährung kann die ovo-lacto-vegetarische Ernährung für gesunde Erwachsene als ausgewogene Ernährungsweise betrachtet werden (44). Ebenso wie bei allen anderen Ernährungsformen gilt aber auch hier, dass die Erhaltung einer guten Gesundheit die Einnahme einer Vielzahl pflanzlicher Lebensmittel, angemessene körperliche Aktivität, kein oder nur mässiger Alkoholkonsum sowie Verzicht auf Rauchen voraussetzt.

\section{Ernährungsphysiologische Aspekte}

Nährstoffe, deren Bedarf bei Nicht-Vegetariern mit der Einnahme von Fleisch und Fisch teilweise gedeckt werden, nehmen Ovo-LactoVegetarier ausschliesslich durch pflanzliche Produkte sowie Milch und Eier ein. Es handelt sich dabei um Selen, Zink, Eisen, n-3-Fettsäuren und die Vitamine D3 und B12 sowie hochwertige Proteine zur Deckung des Bedarfes an essentiellen Aminosäuren. Bei einer ovo-lactovegetarischen Ernährung ist die ausreichende Versorgung der oben erwähnten Nährstoffe in der Regel gewährleistet. In speziellen Lebensphasen mit einem erhöhten Nährstoffbedarf, z.B. bei Krankheit, sollte diesen Nährstoffen besondere Beachtung geschenkt werden. In diesen speziellen Fällen können allenfalls angereicherte Lebensmittel oder auch Supplemen- 
te als sinnvolle Ergänzungen eingesetzt werden (44).

Bei Veganern erhöhen sich durch den Verzicht auf alle tierischen Produkte die Risiken für eine mangelnde Zufuhr verschiedener Nährstoffe. Auf die genügende Zufuhr von Vitamin B12 muss bei veganer Ernährung besonders geachtet werden, da dieses üblicherweise nur über tierische Produkte aufgenommen wird. Zu den bei Ovo-Lacto-Vegetariern in gewissen Lebensphasen kritischen Nährstoffen kommt bei Veganern noch Calcium dazu, das wegen des Verzichts auf Milch und Milchprodukte aus anderen Quellen eingenommen werden muss. Die Erfahrung zeigt, dass es möglich ist, über eine vegane Ernährungsweise genügend Nährstoffe einzunehmen, mit Ausnahme von Vitamin B12, welches mit angereicherten Lebensmitteln oder Supplementen zugeführt werden sollte. Unabdingbar ist dabei jedoch ein entsprechendes Ernährungswissen. Die allgemeine Empfehlung, sich abwechslungsreich zu ernähren, sollten Veganer in besonderem Masse beachten. Da ferner die Risiken für einen Mangel in verschiedenen Lebensphasen (Schwangerschaft, Stillzeit, Wachstum, Alter) sehr gross und kritisch werden können, muss von einer generellen Empfehlung der veganen Ernährung für breite Bevölkerungskreise abgesehen werden. Man kann diese Ernährungsform aber durchaus als eine "Nischenernährungsweise" betrachten, die bei korrekter Anwendung zu einem guten gesundheitlichen Resultat führen kann $(45 ; 46)$.

\section{Nachhaltigkeit}

Der Begriff Nachhaltigkeit, der oft im Zusammenhang mit der vegetarischen Ernährung erwähnt wird, und seine Definition sind in Artikel 73 der Bundesverfassung festgehalten: "Bund und Kantone streben ein auf Dauer ausgewogenes Verhältnis zwischen der Natur und ihrer Erneuerungsfähigkeit einerseits und ihrer Beanspruchung durch den Menschen andererseits an (47).» Bei einer Diskussion über Gesundheitsförderung und nachhaltige Entwicklung können die Nahrungsmittelproduktion und das Ernährungsverhalten nicht ausgeklammert werden. Als nachhaltiges Nahrungsmittelsystem gilt ein "System, in dem sichere und gesunde Nahrungsmittel produziert werden mit positiven sozialen Auswirkungen und geringen Auswirkungen auf die Umwelt» $(48 ; 49)$. Die Produktion von pflanzlichen Nahrungsmitteln belastet die Umwelt hinsichtlich benötigter Landfläche, Energie- und Wasserverbrauch sowie Ausstoss von Treibhausgasen bedeutend weniger, als dies bei der Produktion von Fleisch der Fall ist (50). Eine vegetarische Ernährungsweise kann in diesem Sinne zur Nachhaltigkeit beitragen.

\subsection{Nährstoffempfehlungen}

\subsubsection{Makronährstoffe und Alkohol}

\section{Optimales Verhältnis der drei Makronährstoffe} In welchem Verhältnis die drei Makronährstoffe und Energieträger aufgenommen werden sollen, gab und gibt immer wieder Anlass zu Diskussionen. Die DACH-Referenzwerte empfehlen einen Kohlenhydratanteil von mindestens $50 \%$ der Energiezufuhr, der Rest soll aus Fett (maximal $30 \%$ der Energiezufuhr) und Protein (ca. 15\% der Energiezufuhr) bestehen (3). Die EEK kommt in ihren Empfehlungen zur Kohlenhydratzufuhr allerdings zum Schluss, dass der gesundheitlich 
optimale Kohlenhydratanteil nicht exakt definiert werden kann und der Zielwert der DACH-Referenzwerte zu hoch liegt (51). Neuere wissenschaftliche Befunde zeigen, dass auch tiefere Kohlenhydratanteile positive Auswirkungen auf die Gesundheit haben, wenn anstelle der Kohlenhydrate gesundheitlich "günstige» Fette konsumiert werden (52). Kapitel 2.4 gibt Auskunft darüber, inwiefern die Schweizer Bevölkerung die im vorliegenden Kapitel 1 behandelten Nährstoffempfehlungen einhält.

\section{Empfehlungen zur Fettzufuhr}

In den letzten Jahren wurden neue Erkenntnisse über die physiologische und pathologische Bedeutung der Nahrungsfette und -öle gewonnen, die eine Neubearbeitung der aus dem Jahre 1992 stammenden EEK-Empfehlungen über den Konsum von Nahrungsfetten nach sich zogen (52). Die Empfehlungen für die Fettzufuhr gelten für den gesunden Erwachsenen im Rahmen einer ausgewogenen, abwechslungsreichen Ernährung, wie sie in der Schweizer Lebensmittelpyramide (Abbildung 1.2) dargestellt ist. Sie beziehen sich auf den Tagesbedarf und betreffen die Anteile einzelner Fettsäuregruppen. Nachfolgend sind ausserdem die Empfehlungen der Food and Agriculture Organization (FAO) und WHO für die Zufuhr an Fett und essentiellen Fettsäuren (53) sowie die Empfehlungen der ISSFAL (54) berücksichtigt.

- Gesättigte Fettsäuren (SFA): Der Effekt der SFA auf die Serumlipoproteine sowie ihr Einfluss auf diverse Krankheiten steht derzeit stark im Zentrum der Forschung. Je nach Kettenlänge haben die SFA eine unterschiedliche Wirkung auf das LDL- und HDL-Cholesterin. Aktuelle Studien zeigen, dass eine Reduktion der SFA-Aufnahme bei gleichzeitiger Erhö- hung der PUFA-Aufnahme das Risiko für Herz-Kreislauf-Krankheiten senkt (53). Diese Massnahme steigert möglicherweise auch die Insulinempfindlichkeit und senkt das Diabetesrisiko. Unter Berücksichtigung dieser Gegebenheiten wird eine Beschränkung der SFA-Zufuhr auf maximal 10\% der pro Tag konsumierten Energie zugunsten der PUFA-Aufnahme empfohlen $(53 ; 55)$.

- Transfettsäuren (TFA): Diese entstehen bei der partiellen Fetthärtung, beim Frittieren und bei der Desodorierung von Ölen. Sie haben ungünstige Wirkungen auf die Blutfette (LDL-Cholesterinzunahme, HDL-Cholesterinabnahme) und steigern dadurch das Risiko für atherosklerotische Krankheiten. Die in einzelnen Studien gefundenen neutralen oder gar günstigen gesundheitlichen Wirkungen spezifischer TFA (z.B. konjugierter Linolsäuren), die im Wiederkäuermagen entstehen, sind unter Fachleuten umstritten; deshalb können für diese keine Empfehlungen abgegeben werden. Klar ist jedoch, dass durch industrielle Fetthärtung entstandene TFA in möglichst kleinen Mengen zugeführt werden sollten, d.h. bei Fertigprodukten max. $1 \mathrm{~g}$ TFA pro $100 \mathrm{~g}$ Produkt, bei Ölen und Margarinen max. $1 \%$ des Gesamtfettanteils. Die täglich konsumierte Menge sollte nicht mehr als 1\% des Gesamtenergieanteils betragen.

- Einfach ungesättigte Fettsäuren (MUFA): Diese kommen in relativ grossen Mengen in Oliven- und Rapsöl, aber auch in vielen anderen Ölen und Fetten vor. Sie sind bezüglich Blutfetten und Insulinempfindlichkeit im Vergleich zu SFA neutral. MUFA sollten mit 1015\% der pro Tag konsumierten Energie den grössten Anteil aller Fettsäurengruppen ausmachen (entspricht bei einer Ernährung von 
2'000 kcal oder 8'400 kJ 20-30 g MUFA pro Tag).

- Mehrfach ungesättigte Fettsäuren (PUFA): Sie sind essentiell und können in zwei Untergruppen eingeteilt werden:

- Linolsäure und ihre Abkömmlinge (Gruppe der n-6-Fettsäuren).

Quellen: pflanzliche Öle wie Sonnenblumenöl, Sojaöl, Distelöl, Weizenkeimöl.

- $\alpha$-Linolensäure und ihre Abkömmlinge (Gruppe der n-3-Fettsäuren).

Quellen: Rapsöl, Nüsse, Blattgemüse.

- Ein im Verhältnis zum n-3-Fettsäuren-Konsum zu hoher Konsum von n-6-Fettsäuren kann thrombose- und entzündungsfördernd sein (52). Daher wird empfohlen, das Verhältnis von n-6:n-3 auf 5:1 zu senken. Das aktuelle Verhältnis beträgt ca. 10:1 (51). Die Zufuhr von n-6-Fettsäuren sollte ca. $2.5 \%$ der Tagesenergie oder $6 \mathrm{~g}$ pro 2'000 kcal bzw. 8'400 kJ betragen. Die Zufuhr von n-3-Fettsäuren sollte ca. $0.7 \%$ der Tagesenergie oder $1.7 \mathrm{~g}$ pro 2'000 kcal bzw. 8'400 kJ betragen. Werden hingegen die empfohlenen Zufuhr-

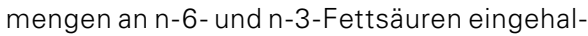
ten, fehlt gemäss FAO / WHO (53) allerdings die wissenschaftliche Evidenz zur Empfehlung eines spezifischen Verhältnisses von n-6:n-3-Fettsäuren.

- Langkettige n-3-Fettsäuren: Diese kommen besonders reichlich in bestimmten Fischen (insbesondere in den fettreichen Sorten) und in Fischöl vor. Sie müssen unter bestimmten Bedingungen oder in bestimmten Lebensphasen (z.B. im Säuglings- und Kleinkindalter) mit der Nahrung eingenommen werden. Es wird empfohlen, pro Woche 1-2 Portionen Fisch (100-240 g pro Woche) zu essen. Wer keinen Fisch isst, sollte den Bedarf an diesen essen- tiellen Fettsäuren mit Kapseln oder Fischöl decken (500 mg Eicosapentaensäure (EPA) und Docosahexaensäure (DHA) pro Tag).

- Nahrungscholesterin: Eine erhöhte Zufuhr von Nahrungscholesterin steigert geringgradig und individuell variabel das LDL-Cholesterin. Aus wissenschaftlicher Sicht kann keine konkrete Beschränkung der Zufuhr von Cholesterin in $\mathrm{mg} / \mathrm{Tag}$ empfohlen werden.

\section{Empfehlungen zur Kohlenhydratzufuhr}

Die aus dem EEK-Bericht über Kohlenhydrate in der Ernährung abgeleiteten Empfehlungen zur Kohlenhydratzufuhr sind nachfolgend in Kürze dargestellt (51), soweit sie nicht schon in früheren Abschnitten dieses Kapitels behandelt worden sind. Auf ein optimales Verhältnis der drei Makronährstoffe wurde bereits am Anfang von Kapitel 1.5.1 eingegangen. Ein zu stark erhöhter Kohlenhydratanteil vermindert die Insulinempfindlichkeit und die HDL-Cholesterinkonzentration und erhöht die Blutfette (Serumglyceride), die Entzündungsproteine im Serum sowie den Blutdruck. Diese Veränderungen sind besonders dann zu beobachten, wenn kohlenhydrathaltige Lebensmittel mit relativ starker Blutzuckerwirkung verzehrt werden, also Lebensmittel mit hohem glykämischem Index (GI). Erfahrungen aus der Sporternährung (Kapitel 1.4.2) legen zudem den Schluss nahe, dass Kohlenhydrate ihr «ungesundes» Potenzial vor allem in Kombination mit körperlicher Inaktivität entfalten. Mengenmässig dürfte der optimale Kohlenhydratanteil deshalb bei 45-55\% der pro Tag konsumierten Energie liegen, wobei wie auch bei Proteinen und Fetten die Empfehlung "Qualität vor Quantität» gilt (Kapitel 3.4.2). 
Die EEK hält in ihrem Bericht fest (51):

- Grundsätzlich gelten die Empfehlungen der SGE für eine ausgewogene und abwechslungsreiche Ernährung. Diese beinhalten eine Bevorzugung der Kohlenhydratquellen Vollkorngetreideprodukte, Früchte und Gemüse sowie Hülsenfrüchte und Kartoffeln. Dadurch wird auch das Ziel der empfohlenen Nahrungsfaserzufuhr von mindestens $30 \mathrm{~g}$ pro Tag erreicht.

- Maximal 10\% der täglichen Energieaufnahme sollte aus zugesetztem Zucker (z.B. Saccharose, Glucose, Fructose) sowie aus Honig, Dicksaft und Fruchtsaft stammen. Für eine erwachsene Person mit vorwiegend sitzender Tätigkeit und einem täglichen Energieverbrauch von ungefähr 2'000 kcal bzw. 8'400 kJ ergibt dies einen maximalen Zuckerkonsum von ca. $50 \mathrm{~g}$ pro Tag.

- Die Beschränkung des Zuckerkonsums limitiert auch die Fructosezufuhr, da Zucker (Saccharose) zur Hälfte aus Fructose besteht. Erhöhte Mengen an Fructose (über $1 \mathrm{~g}$ pro kg Körpergewicht und Tag) haben negative Auswirkungen auf die Blutfette, Insulinempfindlichkeit und den Blutdruck. Es ist wahrscheinlich die Fructosemenge an sich, die dabei relevant ist, unabhängig davon, ob sie als Saccharose gebunden oder frei ist. Bei Risikogruppen (Personen mit Übergewicht, geringer körperlicher Aktivität, Veranlagung zu oder bereits bestehendem Diabetes mellitus, Bluthochdruck, erhöhten Blutfetten) kann sich ein zu hoher Fructosekonsum (z.B. durch gezuckerte Getränke) besonders negativ auf die Gesundheit auswirken.

- Die massvolle Einnahme von Süss- und Zuckeraustauschstoffen im Rahmen einer ausgewogenen Ernährung hat keine negativen
Wirkungen auf die Gesundheit. Süssstoffe sind eine kalorienarme und zahnschonende Alternative zu Saccharose. Ein übermässiger Konsum bestimmter Zuckeraustauschstoffe über 20-30 g pro Tag kann allerdings zu Durchfall führen. Personen mit Phenylketonurie sollten kein Aspartam konsumieren.

\section{Empfehlungen zur Proteinzufuhr}

Bei Erwachsenen wird eine Proteinzufuhr von $0.8 \mathrm{~g}$ pro kg Körpergewicht und Tag empfohlen (3;56). Die $0.8 \mathrm{~g}$ entsprechen der notwendigen täglichen Menge zur Aufrechterhaltung des Stickstoffgleichgewichtes beim gesunden Menschen mit moderater körperlicher Aktivität unter Berücksichtigung individueller Schwankungen und einer verminderten Verdaulichkeit in einer gemischten Kost. Der obere noch tolerierbare Wert (UL) wurde auf $2 \mathrm{~g}$ pro Tag gesetzt, dies aufgrund der Unsicherheit bezüglich gesundheitlicher Effekte beim Verzehr höherer Mengen an Nahrungsproteinen. 10-20\% des Energiebedarfs sollten durch Proteine gedeckt werden. Eine adäquate Energiezufuhr aus Fetten und Kohlenhydraten ist die Voraussetzung für diese Empfehlung. Eine Proteinzufuhr von $0.8 \mathrm{~g}$ pro kg Körpergewicht und Tag wird auch für ältere Menschen empfohlen (57).

Während der Schwangerschaft und Stillzeit ist der Proteinbedarf erhöht: Er liegt während der Schwangerschaft bei $1.1 \mathrm{~g}$ pro kg Körpergewicht und Tag und während der Stillzeit bei $1.3 \mathrm{~g}$ pro kg Körpergewicht und Tag (Kapitel 1.4.1).

\section{Empfehlungen zum Alkoholkonsum}

Alkohol in Bier, Wein und Spirituosen hat verschiedene Wirkungen. Ernährungsphysiologisch relevant sind die hohe Energiedichte, seine ungünstige Wirkung auf die Absorption von 
zahlreichen essentiellen Nährstoffen im Darm und die mögliche Verdrängung lebensnotwendiger Stoffe aus der Nahrung bei Alkoholmissbrauch. Ein übermässiger Alkoholkonsum birgt nicht nur akut, sondern auch langfristig schwerwiegende Krankheitsrisiken (3). Als risikosarmer Alkoholkonsum werden geschlechtsabhängig Maximalwerte von 10-20 g Alkohol pro Tag (entsprechend 1-2 dl Wein) angesehen (58). Diese Mengen dürfen jedoch nicht als Empfehlung zum täglichen Alkoholkonsum missverstanden werden, sondern dienen als Richtwerte für die maximale Zufuhr. Die mit einem moderaten Alkoholkonsum verbundenen, allfällig vorhandenen positiven Aspekte (kardioprotektive Wirkung) werden durch die gesundheitlichen Risiken übertroffen: z.B. Krebs des oberen Verdauungstraktes und der Leber sowie Leberzirrhose und Hirngefässkrankheiten $(21 ; 59$; Kapitel 3.4.2, 3.5.2 und 3.6.2). Ganz auf Alkohol verzichten sollten Kinder und Jugendliche, schwangere und stillende Frauen, Personen, die Medikamente jeder Art zu sich nehmen, Personen, welchen aus medizinischen Gründen vom Alkoholkonsum abgeraten wird, Personen, die unter seelischen Problemen oder Krankheiten leiden, Personen am Steuer sowie Personen mit Untergewicht oder starkem Übergewicht (58).

\subsubsection{Salz}

Die Salzbestandteile Natrium und Chlorid haben beide eine physiologische Bedeutung, und entsprechend ist auch eine bevölkerungsgruppenspezifische minimale Zufuhr über Lebensmittel und Getränke notwendig. In den DACH-Referenzwerten wird eine Mindestaufnahme von 550 mg Natrium oder ca. $1.5 \mathrm{~g}$ Salz pro Tag für
Jugendliche und Erwachsene als ausreichend erachtet (3). Der derzeitige Salzkonsum in der Schweiz liegt allerdings, wie auch in anderen europäischen Ländern, um ein Vielfaches über diesem Wert (Kapitel 2.4.6), so dass bei einer Risikobeurteilung die mit einer derartigen Überversorgung verbundenen Gesundheitsrisiken klar im Vordergrund stehen. In verschiedenen WHO-Studien ist eine derartige Risikobeurteilung bezüglich der Rolle von Salz bei den wichtigsten ernährungsabhängigen Krankheiten vorgenommen worden: Darin wird der derzeitige hohe Salzkonsum mit überzeugender Evidenz als Risikofaktor für Herz-Kreislauf-Krankheiten eingestuft (Kapitel 3.4.2) und aufgrund der Datenlage empfohlen, den durchschnittlichen Salzkonsum auf Bevölkerungsebene auf $5 \mathrm{~g}$ pro Kopf und Tag zu senken (60).

Auch der EEK-Bericht «Salzkonsum und Bluthochdruck" kommt zum Schluss, dass "ein hoher Salzkonsum negative Auswirkungen auf die Gesundheit haben kann, vor allem durch eine Erhöhung des Blutdrucks und insbesondere bei gefährdeten Bevölkerungsgruppen wie älteren Menschen, Übergewichtigen, Hypertonikern und Salzsensitiven" (61). In der Folge hat das BAG - unter Berücksichtigung internationaler Massnahmen - eine Salz-Strategie 2008-2012 zur Reduktion des Salzkonsums $(62 ; 63)$ ausgearbeitet mit dem längerfristigen Ziel, den durchschnittlichen Salzkonsum von derzeit 8-10 g pro Tag in der Schweizer Bevölkerung (64; Kapitel 2.4.6) auf weniger als $5 \mathrm{~g}$ pro Tag zu senken (Kapitel 4.6.1.1). Da 70-80\% der Salzaufnahme über verarbeitete Lebensmittel wie Brot, Käse, Fleischprodukte, Suppen und Fertigmahlzeiten erfolgt, soll dieses Ziel in erster Linie durch eine Senkung des Salzgehaltes in diesen Lebensmitteln erreicht werden. Menüberechnungen auf 
Basis der nationalen Ernährungsempfehlungen haben ergeben, dass der Salzkonsum bei Einhaltung dieser Empfehlungen weniger als $5 \mathrm{~g}$ pro Tag beträgt $(24 ; 62)$.

\subsubsection{Vitamin D}

Der Vitamin-D-Gehalt der meisten Lebensmittel ist gering. Die Vitamin-D-Versorgung wird hauptsächlich über die endogene Bildung in der Haut sichergestellt. Bei gewissen Bevölkerungsgruppen besteht das Risiko einer Unterversorgung mit Vitamin D (Kapitel 3.10.3). Mit Hilfe von Vitamin-D-Supplementen kann ein unzureichender Vitamin-D-Status verbessert werden. Für Säuglinge und Kleinkinder besteht bereits eine entsprechende Empfehlung (Kapitel 1.3.1). 25(OH)D-Serumgehalte von weniger als $50 \mathrm{nmol} / \mathrm{L}$ werden als Mangel bezeichnet (65). Eine Erhöhung der täglichen Vitamin-DZufuhr auf ca. 20 mg bzw. 800 IE bei Erwachsenen ab 60 Jahren, was dem Doppelten der DACH-Empfehlung (3) entspricht, wird aufgrund von Interventionsstudien als sinnvoll erachtet und kann nur mit Hilfe von Supplementen erreicht werden.

\subsection{Lebensmittelsicherheit und Ernährung}

\section{Risikowahrnehmung in Wissenschaft und Öffentlichkeit}

Bereits im Zweiten Schweizerischen Ernährungsbericht (SEB) wurde festgehalten, dass eine unausgewogene Ernährung aus wissenschaftlicher Sicht grössere Risiken für die Gesundheit beinhaltet als mit Lebensmitteln ver- bundene mikrobiologische oder chemische Risiken, obwohl dies in der Öffentlichkeit anders wahrgenommen wird (66). Obwohl im 5. SEB diese Unterschiede sogar quantifiziert wurden, hat sich diese Sichtweise in der Lebensmittelgesetzgebung bisher noch zu wenig durchgesetzt (67). Es ist allerdings geplant, den Zweckartikel im Lebensmittelgesetz zu erweitern, um dem Konsumenten zu ermöglichen, informiert zu wählen $(4 ; 68)$.

Umweltkontaminanten sind Stoffe, die in der Regel ungewollt in die Umwelt gelangen oder nach ihrer bewussten Anwendung unerwünscht lange in der Umwelt verbleiben und sich aufgrund ihrer chemischen und physikalischen Eigenschaften in der Nahrungskette anreichern. Beispiele sind polychlorierte Dioxine und Furane in der Muttermilch, Schwermetalle in Meerfischen und Nitrat in Salat und Gemüse. Solche Stoffe in Lebensmitteln können insbesondere dann zu einem Zielkonflikt bei der Formulierung von Ernährungsempfehlungen führen, wenn zwischen erwünschten und unerwünschten Inhaltsstoffen abgewogen werden muss. Zusatzstoffe dagegen gehören zu den toxikologisch bestuntersuchten Stoffen. Entsprechend gering wird aus wissenschaftlicher Sicht das mit der rechtlich geregelten Verwendung dieser Stoffe verbundene Risiko eingeschätzt.

\section{Risiken durch Rückstände in der Muttermilch versus Vorteile des Stillens}

Die Risiken durch Dioxine, polychlorierte Biphenyle (PCB) und Organochlorpestizide in der Muttermilch sind für den 5. SEB analysiert worden (69). Aufgrund der gesammelten Erkenntnisse empfiehlt das BAG, in Übereinstimmung mit der WHO und der SGP, weiterhin das Stillen der Säuglinge. Die vielen positiven Aspekte der 
Humanmilch als erste Nahrung (wie bessere Immunabwehr, Unterstützung in der psychomotorischen Entwicklung und Förderung der innigen Beziehung zwischen Mutter und Kind) überwiegen die Nachteile der vorhandenen Rückstände (Kapitel 1.3.1).

\section{Risiken durch Quecksilberrückstände in Meerfischen versus Empfehlungen zum Fischkonsum}

Grosse Raubfische wie Hai, Marlin / Speerfisch, Schwertfisch, aber auch frischer Thunfisch sowie ausländischer Hecht können Konzentrationen an Methylquecksilber aufweisen, welche bei häufigem Verzehr zu einer Überschreitung der provisorisch tolerierbaren wöchentlichen Aufnahmemenge von $1.6 \mu \mathrm{g}$ pro kg Körpergewicht Methylquecksilber führen können. Daher wird Schwangeren und Stillenden zur Deckung des Bedarfs an langkettigen PUFA empfohlen, pro Woche 1-2 Portionen möglichst fetthaltige, Methylquecksilber-arme Fische zu essen und auf den Konsum der oben erstgenannten Fischarten zu verzichten. Der Konsum von frischem Thunfisch oder ausländischem Hecht sollte auf eine Portion (130 g) pro Woche beschränkt werden. Thunfisch aus der Konserve darf mit bis zu 4 Portionen à $130 \mathrm{~g}$ pro Woche verzehrt werden, weil dazu eine andere Fischart und / oder kleinere Fische verwendet werden, die geringere Quecksilberkonzentrationen enthalten als frischer Thunfisch (39).

\section{Risiken durch Nitrat in Salaten und Gemüse} versus Empfehlungen zum Gemüsekonsum Eine Übersichtsstudie aus der Schweiz zeigt, dass ca. 70\% des mit der Nahrung aufgenommenen Nitrats aus Salat und Gemüse stammt (70). Aus toxikologischer Sicht ist eine hohe
Nitrataufnahme wegen der in vivo Bildung von kanzerogenen Nitrosaminen unerwünscht. Eine Risikobeurteilung zeigt aber auch hier, dass sich die in Kapitel 1.3 empfohlenen 3 Portionen Gemüse täglich insgesamt trotzdem präventiv günstig auswirken. So ist abgeschätzt worden, dass ungenügender Früchte- und Gemüsekonsum für $31 \%$ der koronaren Herzkrankheiten und zu je 20\% für Magen- und Speiseröhrenkrebs verantwortlich sein könnte 171; Kapitel 3.4 und 3.5). Es liegen experimentelle Hinweise vor, dass der präventive Effekt des Gemüsekonsums auf Herz-Kreislauf-Krankheiten neben anderen Faktoren sogar direkt auf Nitrat zurückzuführen ist (101).

Risiken durch Überschreitung des UL bei Verwendung von Vitaminsupplementen und Functional Food

Zwei Studien zeigen, dass die Nahrung in der Schweiz relativ häufig mit Supplementen bzw. Functional Food ergänzt wird $(72 ; 73)$. Laut der CoLaus-Studie ist dies bei $25.7 \%$ der Studienteilnehmer der Fall (72). Eine Überversorgung bzw. eine Überschreitung des UL kann bei einigen Nährstoffen ein Gesundheitsrisiko darstellen.

Risiken bei Überschreitung des UL von Folsäure versus Vorbeugung von Neuralrohrdefekten

Eine Expertengruppe der EEK hat im Jahre 2002 empfohlen, Getreidemehle in der Schweiz mit $3 \mathrm{mg}$ Folsäure und $10 \mu \mathrm{g}$ Vitamin B12 pro kg Mehl anzureichern (74). Durch diese Massnahme könnte die bisher ungenügende Folsäureversorgung der Bevölkerung um $275 \mu \mathrm{g}$ pro Person und Tag verbessert werden. Mit einer Gesamtsumme von CHF 120'000 pro Jahr könnten mit dieser Massnahme zudem in der Schweiz 30 bis 40 Kinder mit Neuralrohrfehl- 
bildungen bzw. Aborte verhindert werden $(40 ; 74)$. Ein vom BAG in Auftrag gegebenes juristisches Gutachten kam allerdings zum Schluss, dass die rechtlichen Grundlagen für eine derartige Massnahme nicht ausreichen (75). Zudem mehren sich in den letzten Jahren toxikologische Bedenken in Bezug auf diese Massnahme. So kann nicht ausgeschlossen werden, dass eine Überschreitung des UL von $1 \mathrm{mg}$ Folsäure pro Person und Tag das Krebsrisiko erhöht (76; Kapitel 3.5.2 und 3.10.2).

\subsection{Ernährungsempfehlungen in anderen Ländern}

In allen Sprachregionen der Schweiz wird die Bevölkerung über die Medien mit Ernährungsinformationen und Gesundheitsförderungskampagnen konfrontiert, die in den gleichsprachigen Nachbarländern verbreitet werden. So haben auch Ernährungs- und Gesundheitsfachleute der Deutsch- und Westschweiz sowie des Tessins bedingt durch die Sprache und die geografische Nähe einen leichteren Zugang zu Referenzwerten aus Deutschland, Frankreich bzw. Italien. Die Ernährungsempfehlungen basieren auf wissenschaftlichen, grösstenteils internationalen Referenzwerten, doch den Nachbarländern wird ein besonderes Gewicht beigemessen. Daher konzentriert sich der nachfolgende Vergleich der Ernährungsempfehlungen bzw. der Empfehlungen für die Nährstoffzufuhr auf Deutschland, Frankreich und Italien.

\subsubsection{Empfohlene Nährstoffzufuhr}

Das wissenschaftliche Gremium für diätetische Produkte, Ernährung und Allergien der Europäischen Lebensmittelsicherheitsbehörde (EFSA, European Food Safety Authority) hat Referenzwerte für die Zufuhr von Kohlenhydraten (77), Nahrungsfasern (77), Fetten (78) und Wasser (79) festgelegt, die es 2010 veröffentlicht hat. Die wissenschaftlichen Empfehlungen für Proteine, Energiezufuhr, Vitamine und Mineralstoffe werden zurzeit erarbeitet bzw. lagen zum Zeitpunkt der Texterstellung noch nicht vor. Da diese Referenzwerte erst seit kurzem bestehen oder noch erarbeitet werden, hat jedes Land noch seine eigenen Referenzwerte für die empfohlene Nährstoffzufuhr (Tabelle 1.1).

Tabelle 1.1: Referenzwerte für Nährstoffempfehlungen

\begin{tabular}{l|l}
\hline Schweiz & DACH-Referenzwerte, 2008 (3) \\
& $\begin{array}{l}\text { Empfehlungen der Eidgenössischen } \\
\text { Ernährungskommission, 2006-2011 } \\
(51 ; 52 ; 56)\end{array}$ \\
\hline Deutschland & DACH-Referenzwerte, 2008 (3) \\
\hline Frankreich & $\begin{array}{l}\text { Empfohlene Nährstoffzufuhr für die } \\
\text { französische Bevölkerung, 2001 (80) }\end{array}$ \\
& $\begin{array}{l}\text { Aktualisierung der Angaben zu den Fet- } \\
\text { ten durch die AFSSA (Agence française } \\
\text { de sécurité sanitaire des aliments), } \\
\text { 2010 (81) }\end{array}$ \\
\hline Italien & $\begin{array}{l}\text { Empfohlene Mengen der täglichen } \\
\text { Nährstoffzufuhr für die italienische } \\
\text { Bevölkerung, 1996 (82) }\end{array}$ \\
\hline
\end{tabular}




\subsubsection{Ernährungsempfehlungen}

In der Global Strategy on Diet, Physical Activity and Health der WHO vom Mai 2004 (83; Kapitel 4.4.1) und der entsprechenden World Health Assembly-Resolution WHA 57.17 (84; Kapitel 4.4.1), die auch von der Schweiz angenommen und im Nationalen Programm Ernährung und Bewegung (NPEB) (85; Kapitel 4.6.1.1) umgesetzt worden ist, wurde festgehalten, dass an die Bevölkerung gerichtete Ernährungsempfehlungen zumindest die folgenden fünf Punkte enthalten sollten:

- die Beachtung der Energiebilanz und Aufrechterhaltung eines gesunden Körpergewichtes

- die Limitierung des Gesamtfettkonsums, eine Verlagerung des Konsums von Fetten mit hohem SFA-Gehalt hin zu Fetten mit hohen MUFA- / PUFA-Gehalten sowie eine Reduktion bzw. Eliminierung von TFA

- die Erhöhung des Konsums von Früchten und Gemüse, Vollkornprodukten sowie Nüssen

- die Limitierung von freiem Zucker

- die Limitierung von Salz

Diese sind im "Zweiten Europäischen Aktionsplan Nahrung und Ernährung der WHO» mit folgenden Ernährungsempfehlungen genauer festgelegt (86; Kapitel 4.4.2):

- $<10 \%$ der täglichen Energiezufuhr aus SFA

- $<1 \%$ der täglichen Energiezufuhr aus TFA

- $<10 \%$ der täglichen Energiezufuhr aus freien Zuckern

- $\geq 400 \mathrm{~g}$ Früchte und Gemüse pro Tag

- $<5$ g Salz pro Tag

Die Ziele der WHO wurden von den verschiedenen europäischen Ländern weitgehend in ihre
Empfehlungen aufgenommen. Ein Vergleich der Botschaften an die Bevölkerung und der Mengenempfehlungen der Schweiz und der Nachbarländer zeigt, dass alle Länder die Bevölkerung dazu auffordern, sich zu bewegen (Tabelle 1.2). In Deutschland und Italien wird der Bevölkerung zudem empfohlen, für eine ausgeglichene Energiebilanz zu sorgen.

Die Ernährungsempfehlungen stimmen grösstenteils überein. In jedem Land wird die Bevölkerung aufgefordert, reichlich Wasser zu trinken und stärkehaltige Lebensmittel zu konsumieren, bevorzugt Vollkornprodukte. Was die Lebensmittel tierischer Herkunft (Milchprodukte, Fleisch, Fisch, Eier) angeht, sehen die Empfehlungen jedes Landes einen ausreichenden Konsum von Milchprodukten und einen eingeschränkten Konsum von Fleisch und Fisch in der Grössenordnung von ein- bis zweimal pro Woche vor. Überall wird empfohlen, Fett nur in kleinen Mengen sowie Zucker und Salz mit Mass zu konsumieren.

Es wird systematisch dazu aufgefordert, Früchte und Gemüse zu essen, doch bei dieser Kategorie variieren die Mengenangaben von einem Land zum anderen am stärksten. In Frankreich lautet die Empfehlung, mindestens 5 Portionen à 80-100 g Früchte / Gemüse (ohne Unterscheidung) zu konsumieren, was ein Total von mindestens 400-500 g ergibt. Der tiefere Wert entspricht dem von der WHO empfohlenen Minimum. In der Schweiz und in Deutschland wird empfohlen, 3 Portionen Gemüse und 2 Portionen Früchte à insgesamt $600 \mathrm{~g}$ (Schweiz) bzw. mehr als $650 \mathrm{~g}$ (Deutschland) zu konsumieren. Die in Italien empfohlenen Portionen entsprechen den üblichen Portionen und es wird geraten, täglich 3-4 Portionen Früchte und 2 Portionen Gemüse oder Salat à insgesamt 750-900 g zu konsumieren. 
Tabelle 1.2: Ernährungsempfehlungen in der Schweiz, Deutschland, Frankreich und Italien: Botschaften an die Bevölkerung (grün) und Mengenempfehlungen (schwarz)

\begin{tabular}{|c|c|c|c|c|}
\hline & Schweiz & Deutschland & Frankreich & Italien \\
\hline $\begin{array}{l}\text { Massgebende } \\
\text { Herausgeber- } \\
\text { organisation } \\
\text { von Ernäh- } \\
\text { rungsempfeh- } \\
\text { lungen }\end{array}$ & $\begin{array}{l}\text { Schweizerische Gesell- } \\
\text { schaft für Ernährung } \\
\text { (SGE; 87), } \\
\text { Nichtregierungs- } \\
\text { organisation } \\
\text { Eidgenössische } \\
\text { Ernährungskommis- } \\
\text { sion }(88 ; 92)\end{array}$ & $\begin{array}{l}\text { Deutsche Gesellschaft für } \\
\text { Ernährung } \\
\text { (DGE; 89), } \\
\text { Nichtregierungs- } \\
\text { organisation }\end{array}$ & $\begin{array}{l}\text { Programme National } \\
\text { Nutrition Santé } \\
\text { (PNNS; 91), } \\
\text { Regierungs- } \\
\text { organisation }\end{array}$ & $\begin{array}{l}\text { Istituto Nazionale di } \\
\text { Ricerca per gli Ali- } \\
\text { menti e la Nutrizione } \\
\text { (INRAN; 102), } \\
\text { Regierungs- } \\
\text { organisation }\end{array}$ \\
\hline Titel & $\begin{array}{l}\text { Schweizer Lebens- } \\
\text { mittelpyramide }\end{array}$ & 10 Regeln der DGE & $\begin{array}{l}\text { Les } 9 \text { repères nutri- } \\
\text { tionnels journaliers } \\
\text { (Die } 9 \text { Ernährungs- } \\
\text { prinzipien) }\end{array}$ & $\begin{array}{l}\text { Linee guida per una } \\
\text { sana alimentazione } \\
\text { italiana } \\
\text { (Richtlinien für eine } \\
\text { gesunde italienische } \\
\text { Ernährung) }\end{array}$ \\
\hline \multirow[t]{2}{*}{ Getränke } & $\begin{array}{l}\text { Täglich 1-2 Liter } \\
\text { ungesüsste Getränke. } \\
\text { Wasser bevorzugen }\end{array}$ & Reichlich Flüssigkeit & $\begin{array}{l}\text { Wasser nach } \\
\text { Belieben }\end{array}$ & $\begin{array}{l}\text { Trinke jedenTag } \\
\text { reichlich Wasser }\end{array}$ \\
\hline & $\begin{array}{l}\text { Täglich: } \\
\text { 1-2 Liter }\end{array}$ & $\begin{array}{l}\text { Täglich: } \\
\text { mindestens } 1.5 \text { Liter }\end{array}$ & & $\begin{array}{l}\text { Täglich: } \\
\text { 1.5-2 Liter }\end{array}$ \\
\hline \multirow[t]{2}{*}{$\begin{array}{l}\text { Früchte \& } \\
\text { Gemüse }\end{array}$} & $\begin{array}{l}\text { Täglich } 5 \text { Portionen in } \\
\text { verschiedenen Farben }\end{array}$ & Nimm «5 amTag» & $\begin{array}{l}\text { Mindestens fünfmal } \\
\text { proTag }\end{array}$ & $\begin{array}{l}\text { Mehr Getreide, } \\
\text { Hülsenfrüchte, } \\
\text { Gemüse \& Früchte }\end{array}$ \\
\hline & $\begin{array}{l}\text { Täglich: } \\
3 \times 120 \mathrm{~g} \text { Gemüse } \\
+ \\
2 \times 120 \mathrm{~g} \text { Früchte }\end{array}$ & $\begin{array}{l}\text { Täglich: } \\
400 \mathrm{~g} \text { Gemüse oder mehr } \\
+ \\
250 \mathrm{~g} \text { Früchte oder mehr }\end{array}$ & $\begin{array}{l}\text { Täglich: } \\
\text { mindestens } \\
5 \times 80-100 \mathrm{~g}\end{array}$ & $\begin{array}{l}\text { Täglich: } \\
3-4 \times 150 \text { g Früchte } \\
+ \\
2 \times 50 \text { g Salat oder } \\
250 \text { g gekochtes } \\
\text { Gemüse }\end{array}$ \\
\hline $\begin{array}{l}\text { Stärkehaltige } \\
\text { Lebensmittel }\end{array}$ & $\begin{array}{l}\text { Täglich } 3 \text { Portionen. } \\
\text { Bei Getreidepro- } \\
\text { dukten Vollkorn } \\
\text { bevorzugen } \\
\text { Täglich: } \\
\text { 3×75-125 g Brot /Teig } \\
\text { oder 60-100 g Hülsen- } \\
\text { früchte (Rohgewicht) } \\
\text { oder 180-300 g Kar- } \\
\text { toffeln oder } \\
45-75 \text { gTeigwaren / } \\
\text { Reis / usw. (Roh- } \\
\text { gewicht) }\end{array}$ & $\begin{array}{l}\text { Reichlich Getreide- } \\
\text { produkte \& Kartoffeln } \\
\text { Täglich: } \\
200-250 \text { g gekochte Teig- } \\
\text { waren / Kartoffeln oder } \\
\text { 150-180 g gekochter Reis } \\
+ \\
200-300 \text { g Brot (oder } \\
\text { 150-250 g Brot + 50-60 g } \\
\text { Getreideflocken) }\end{array}$ & $\begin{array}{l}\text { Zu jeder Mahlzeit je } \\
\text { nach Appetit }\end{array}$ & $\begin{array}{l}\text { Täglich: } \\
3-6 \times 50 \mathrm{~g} \text { Brot } \\
+ \\
1-2 \times 20 \mathrm{~g} \text { Zwieback } \\
+ \\
1-2 \times 80 \text { gTeigwaren } / \\
\text { Reis } \\
\text { Wöchentlich: } \\
1-2 \times 200 \mathrm{~g} \mathrm{Kar-} \\
\text { toffeln }\end{array}$ \\
\hline
\end{tabular}




\begin{tabular}{|c|c|c|c|c|}
\hline & Schweiz & Deutschland & Frankreich & Italien \\
\hline $\begin{array}{l}\text { Milch- } \\
\text { produkte }\end{array}$ & $\begin{array}{l}\text { Täglich } 3 \text { Portionen } \\
\text { Milch \& Milchprodukte } \\
\text { Täglich: } \\
3 \times 2 \text { dl Milch oder } \\
150-200 \text { g Joghurt / } \\
\text { Quark oder } 30 \text { g Halb- } \\
\text { hart-/ Hartkäse oder } \\
60 \text { gWeichkäse }\end{array}$ & $\begin{array}{l}\text { Täglich Milch \& } \\
\text { Milchprodukte } \\
\text { Täglich: } \\
200-250 \text { g Milch / Joghurt } \\
+ \\
50-60 \text { g Käse }\end{array}$ & $\begin{array}{l}\text { Dreimal proTag } \\
\text { Täglich: } \\
3 \times 125 \mathrm{~g} \text { Joghurt oder } \\
100-120 \mathrm{~g} \text { Frischkäse } \\
\text { oder } 30 \mathrm{~g} \text { Käse oder } \\
1 \text { Glas Milch }\end{array}$ & $\begin{array}{l}\text { Täglich: } \\
3 \times 125 \text { g Milch / } \\
\text { Joghurt } \\
\text { Wöchentlich: } \\
2-3 \times 50 \text { g Käse oder } \\
100 \text { g Frischkäse }\end{array}$ \\
\hline $\begin{array}{l}\text { Fleisch, Fisch, } \\
\text { Eier,Tofu \& } \\
\text { andere pro- } \\
\text { teinhaltige } \\
\text { Lebensmittel }\end{array}$ & $\begin{array}{l}\text { Täglich } 1 \text { Portion } \\
\text { Fleisch, Fisch, Eier, } \\
\text { Tofu ... } \\
\text { Täglich: } \\
1 \times 100-120 \text { g Fleisch / } \\
\text { Fisch /Tofu / Quorn / } \\
\text { Seitan (Rohgewicht) } \\
\text { oder 2-3 Eier oder } \\
30 \text { g Halbhart-/ Hart- } \\
\text { käse oder } 60 \text { gWeich- } \\
\text { käse oder } \\
150-200 \text { g Quark / } \\
\text { Hüttenkäse }\end{array}$ & $\begin{array}{l}\text { Ein- bis zweimal in der } \\
\text { Woche Fisch; Fleisch, } \\
\text { Wurstwaren \& Eier in } \\
\text { Massen } \\
\text { ProWoche: } \\
300-600 \text { g Fleisch } \\
+ \\
80-150 \text { g magerer Fisch } \\
+ \\
70 \text { g fetter Fisch } \\
+ \\
3 \text { Eier }\end{array}$ & $\begin{array}{l}\text { Täglich: } \\
\text { 1-2× } 100 \mathrm{~g} \text { Fleisch / } \\
\text { Fisch* oder } 2 \text { Eier } \\
\text { * mindestens zweimal } \\
\text { wöchentlich Fisch } \\
\text { essen }\end{array}$ & $\begin{array}{l}\text { Täglich: } \\
1-2 \times 50-70 \mathrm{~g} \text { Fleisch } \\
\text { oder } 1 \text { Ei oder } 100 \mathrm{~g} \\
\text { Fisch oder } 30 \mathrm{~g} \\
\text { trockene Hülsen- } \\
\text { früchte oder } \\
80-120 \mathrm{~g} \text { frische } \\
\text { Hülsenfrüchte }\end{array}$ \\
\hline $\begin{array}{l}\text { Öle, Fette \& } \\
\text { Nüsse }\end{array}$ & $\begin{array}{l}\text { Öl \& Nüsse täglich } \\
\text { in kleinen Mengen. } \\
\text { Butter \& Margarine } \\
\text { sparsam } \\
\text { Täglich: } \\
20-30 \text { g Pflanzenöle } \\
+ \\
20-30 \text { g Nüsse } \\
+ \\
\text { Bei Bedarf } 10 \text { g Butter / } \\
\text { Margarine }\end{array}$ & $\begin{array}{l}\text { Wenig Fett \& fettreiche } \\
\text { Lebensmittel } \\
\text { Täglich: } \\
\text { 15-30 g Butter / } \\
\text { Margarine } \\
+ \\
\text { 10-15 g Öl }\end{array}$ & $\begin{array}{l}\text { Konsum } \\
\text { einschränken }\end{array}$ & $\begin{array}{l}\text { Fette: Setze auf } \\
\text { die Qualität \& } \\
\text { beschränke die } \\
\text { Menge } \\
\text { Täglich: } \\
\text { 3-4×10 g }\end{array}$ \\
\hline $\begin{array}{l}\text { Süsses, } \\
\text { Salziges \& } \\
\text { Alkoholisches }\end{array}$ & In kleinen Mengen & Zucker \& Salz in Massen & $\begin{array}{l}\text { Zuckerhaltige } \\
\text { Produkte: Konsum } \\
\text { einschränken }\end{array}$ & $\begin{array}{l}\text { Zucker, Süssigkeiten, } \\
\text { gesüsste Getränke: } \\
\text { in gesundem Mass } \\
\text { Alkoholische } \\
\text { Getränke: wenn ja, } \\
\text { nur in kontrollierten } \\
\text { Mengen }\end{array}$ \\
\hline $\begin{array}{l}\text { Andere } \\
\text { Botschaften } \\
\text { \& Empfeh- } \\
\text { lungen }\end{array}$ & $\begin{array}{l}\text { Speisen zurückhaltend } \\
\text { salzen. Jodiertes \& } \\
\text { fluoridiertes Speisesalz } \\
\text { verwenden } \\
\text { Täglich mindestens } \\
30 \text { Minuten Bewe- } \\
\text { gung \& ausreichend } \\
\text { Entspannung }\end{array}$ & $\begin{array}{l}\text { Schmackhaft \& scho- } \\
\text { nend zubereiten } \\
\text { Sich Zeit nehmen \& } \\
\text { geniessen } \\
\text { Auf das Gewicht achten } \\
\text { \& in Bewegung bleiben } \\
\text { Vielseitig essen }\end{array}$ & $\begin{array}{l}\text { Salz: Konsum ein- } \\
\text { schränken } \\
\text { Bewegung: täglich } \\
\text { mindestens so viel } \\
\text { Bewegung, die } \\
\text { einer halben Stunde } \\
\text { schnellen Gehens } \\
\text { entspricht }\end{array}$ & $\begin{array}{l}\text { Salz? Besser nur } \\
\text { wenig } \\
\text { Variiere deine Mahl- } \\
\text { zeiten häufig } \\
\text { Achte auf das } \\
\text { Gewicht \& bleibe } \\
\text { immer in Bewegung }\end{array}$ \\
\hline
\end{tabular}


Die Einteilung der Lebensmittel in Gruppen wird in allen vier Ländern gleich vorgenommen, ausser bei den Hülsenfrüchten, die in Italien den proteinhaltigen Lebensmitteln und in den drei anderen Ländern den stärkehaltigen Lebensmitteln zugeordnet werden. Die Mengenempfehlungen sind berechnet für eine tägliche Energiezufuhr von 1'800-2'500 kcal in der Schweiz, 1'600-2'400 kcal in Deutschland und 1'7002'600 kcal in Italien. Je nach Ernährungsgewohnheiten des Landes werden sie mit unterschiedlichen Lebensmitteln, Häufigkeiten und Portionen angegeben, insbesondere was die Produkte tierischer Herkunft und die stärkehaltigen Lebensmittel angeht. Doch in Bezug auf die Nährwerte sind sie insgesamt vergleichbar.
In Frankreich werden lediglich für die Produkte tierischer Herkunft und das Gemüse detaillierte Mengenempfehlungen angegeben.

Die Ernährungsempfehlungen in der Schweiz und in den Nachbarländern verfolgen eindeutig sehr ähnliche Ziele. Um die Ernährungsgewohnheiten der jeweiligen Bevölkerung besser wiederzugeben, weichen sie im Wesentlichen in der Art und Weise, wie sie formuliert sind, voneinander ab.

Während die ausgewogene Ernährung in der Schweiz auf Basis von Lebensmittelgruppen in Form einer Lebensmittelpyramide dargestellt wird (Abbildung 1.2), wird die Proportionalität in Deutschland in Form eines Kreises dargestellt. Zudem werden die Konsumempfehlungen für

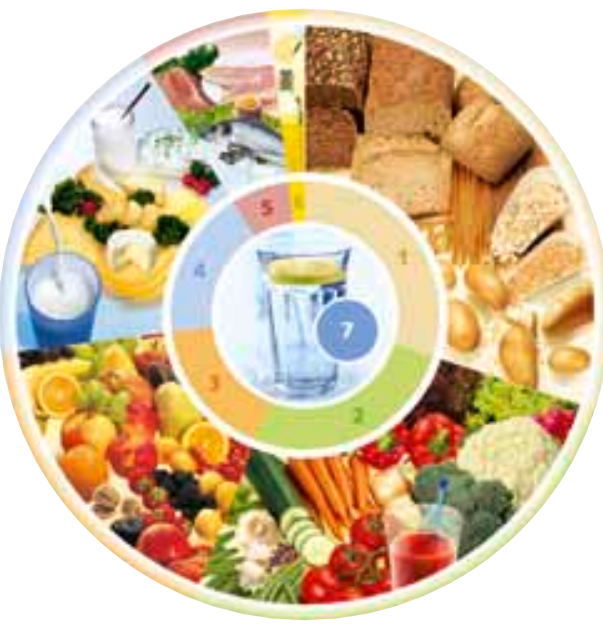

DGE-Ernährungskreis ${ }^{\circledR}$

(C) Deutsche Gesellschaft für Ernährung e.V., Bonn

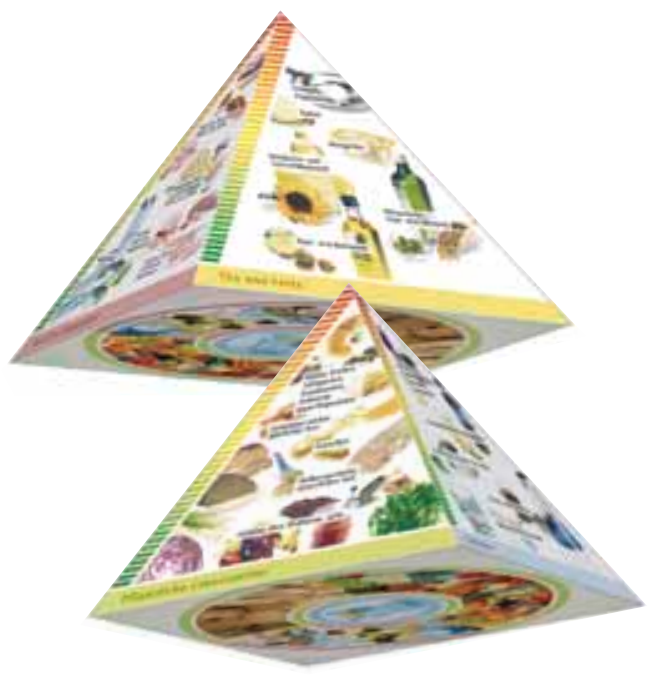

Dreidimensionale Lebensmittelpyramide

(C) Deutsche Gesellschaft für Ernährung e. V., Bonn

Abbildung 1.3: Ernährungskreis und dreidimensionale Lebensmittelpyramide der DGE (89) 


\section{Popolazione adulta (18-65 anni)}

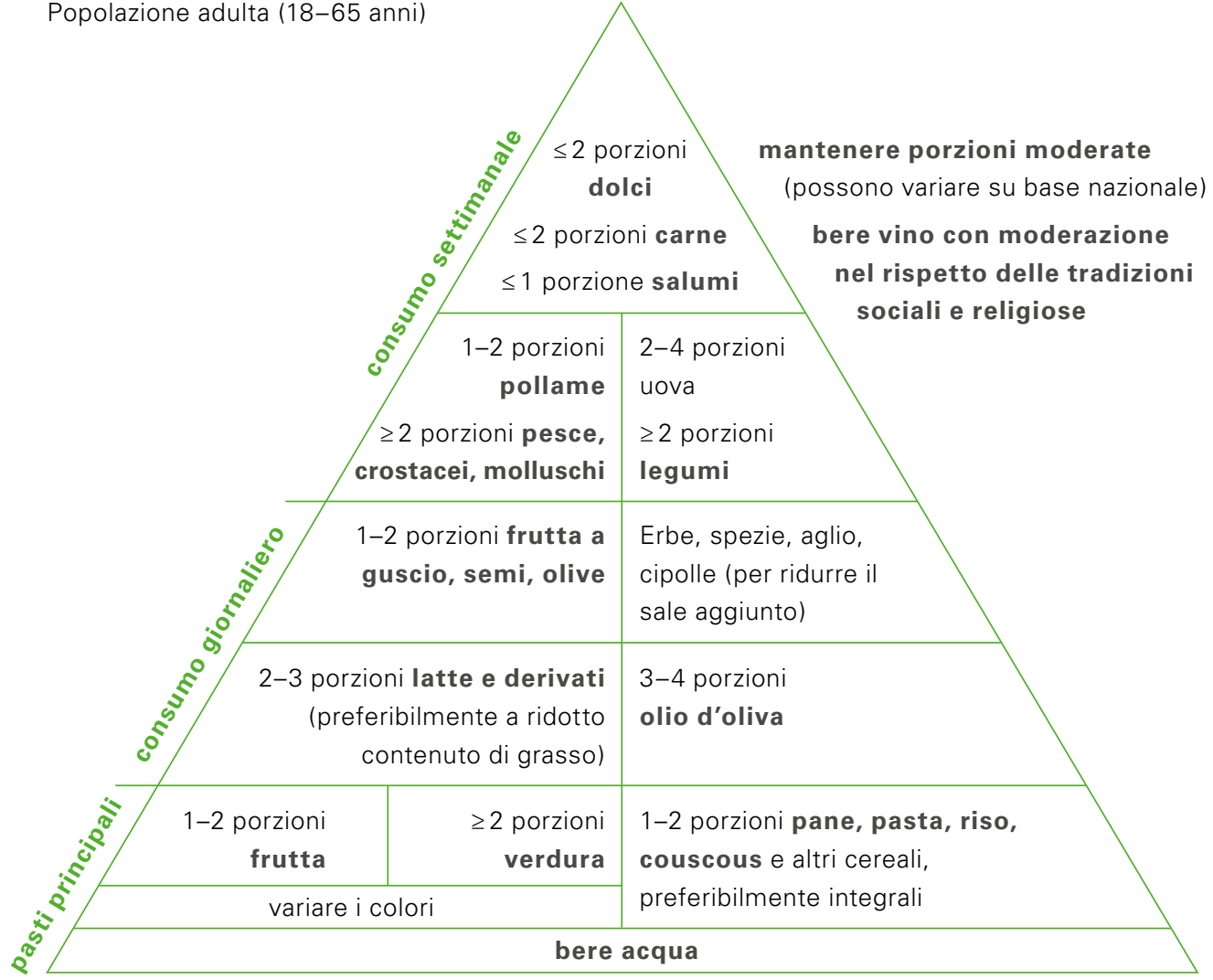

attitivà fisica

convivialità

stagionalità

prodotti locali

Abbildung 1.4: Die neue Lebensmittelpyramide der mediterranen Ernährung (90)

jede Lebensmittelgruppe anhand einer dreidimensionalen Pyramide (Abbildung 1.3) dargestellt. In Italien wurde Ende 2009 im Anschluss an einen internationalen Kongress, der vom Centro Interuniversitario Internazionale di Studi sulle Culture Alimentari Mediterranee (CIISCAM) und dem Istituto Nazionale di Ricerca per gli Alimenti e la Nutrizione (INRAN) or- ganisiert wurde, eine neue Pyramide für die moderne mediterrane Ernährung vorgestellt (Abbildung 1.4; 90). In Frankreich wird die ausgewogene Ernährung nicht graphisch dargestellt, doch es wird das Bild einer Treppe verwendet, um die 9 Ernährungsprinzipien darzustellen (91; Abbildung 1.5).

Sechster Schweizerischer Ernährungsbericht | 39 


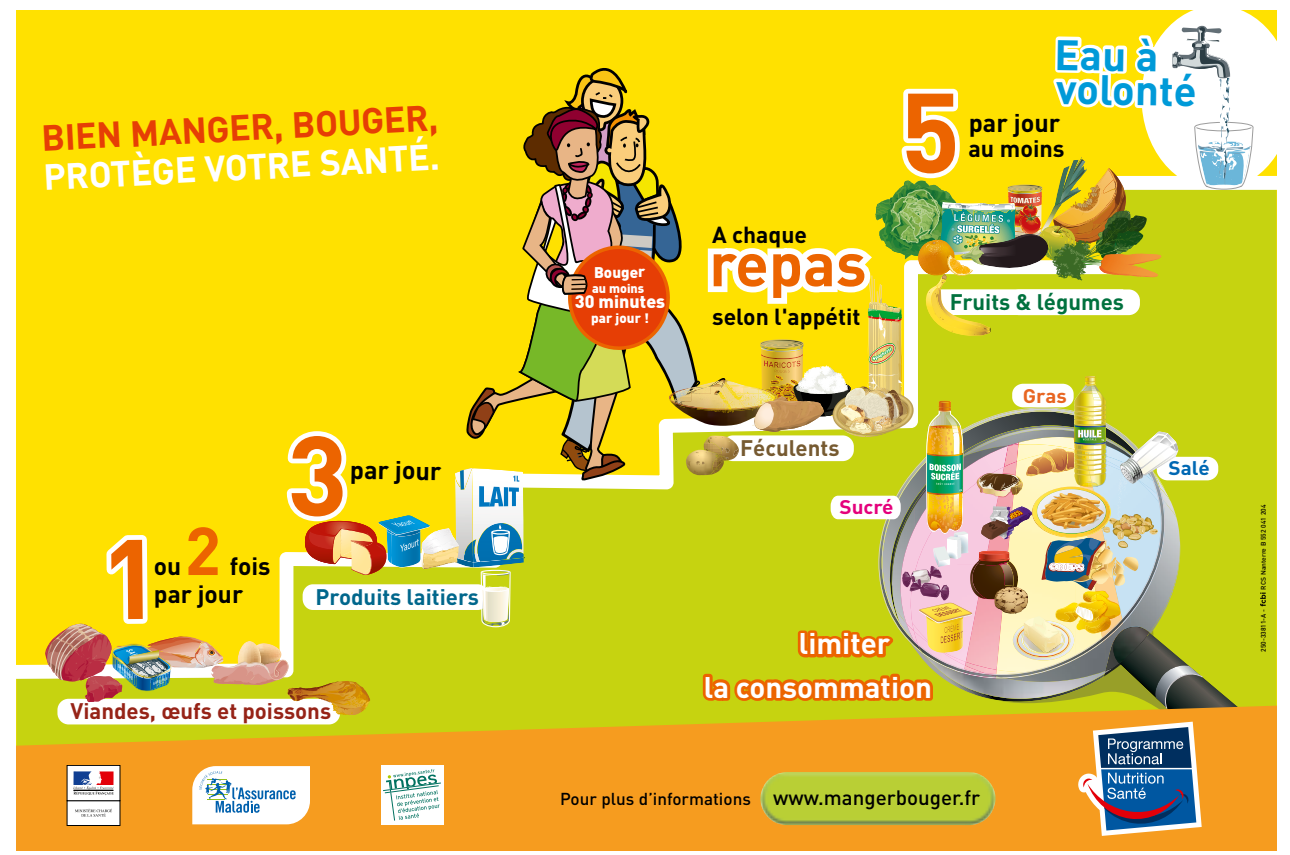

Abbildung 1.5: Plakat der Treppe, welche die 9 Ernährungsprinzipien gemäss PNNS (Programme National Nutrition Santé) für Erwachsene darstellt (91)

\subsection{Schlussfolgerungen und Ausblick}

Eine Grundlage für nährstoffbasierte Ernährungsempfehlungen sind die im Jahre 2000 erstmals publizierten DACH-Referenzwerte (3). Seither hat in der Schweiz vor allem die EEK die wissenschaftliche Literatur über aus verschiedenen Gründen kritische Nährstoffe aufgearbeitet und dem aktuellen Stand entsprechende Empfehlungen publiziert (Kapitel 1.5). Neue Erkenntnisse waren hier namentlich bei den Empfehlungen zur Fettzufuhr zu berücksichtigen. Auf europäischer Ebene bemüht sich die
EFSA um eine Harmonisierung der nährstoffbasierten Ernährungsempfehlungen (77-79). Es ist aber unbestritten, dass deren Umsetzung auf lebensmittelbasierte Ernährungsempfehlungen die länderspezifischen Ernährungsgewohnheiten und das Auftreten ernährungsabhängiger Krankheiten in einer Bevölkerungsgruppe mitberücksichtigen und deshalb vorzugsweise auf nationaler Ebene erfolgen sollte (6). Entsprechend fallen - trotz Gemeinsamkeiten - die Ernährungsempfehlungen und die Botschaften an die Bevölkerung in den verschiedenen Ländern unterschiedlich aus (Tabelle 1.2). 
In den letzten Jahrzehnten sind in den industrialisierten Ländern nichtübertragbare Krankheiten (z.B. Übergewicht und dessen Folgen) als Todesursache im Vergleich zu den übertragbaren Krankheiten (Infektionskrankheiten) immer wichtiger geworden. Früher waren zudem Ernährungsmängel ein häufigeres Problem als Überernährung. Dies hat den Ruf nach einer Ernährungspolitik, die dieser Entwicklung Rechnung trägt, immer stärker werden lassen und ist auch die Basis verschiedener WHO-Programme $(86 ; 93 ; 94)$. Die Grundlage einer Ernährungspolitik ist eine sorgfältige Analyse der Ernährungsgewohnheiten der Bevölkerung und der Entwicklung der ernährungsabhängigen Krankheiten. Auf deren Basis können Ziele und Massnahmen für eine wünschenswerte Entwicklung formuliert werden. In der Schweiz sind diese auf internationaler Ebene erstmals an der Internationalen Ernährungskonferenz der WHO / FAO vom Dezember 1992 in Rom (95) entwickelten Grundsätze durch eine Arbeitsgruppe der EEK im «Aktionsplan Ernährung und Gesundheit: Eine Ernährungspolicy für die Schweiz" vom August 2001 umgesetzt worden $(96 ; 97)$. Die damals aufgestellten Ernährungsempfehlungen bildeten zusammen mit einer Analyse der ernährungsabhängigen Krankheiten in der Schweiz im 4. SEB $(98 ; 99)$ die Grundlage für die Festlegung von acht Zielen für den Zeitraum 2001-2010 (96;97). Ernährungsempfehlungen sind also ein zusätzliches wesentliches Element für die Formulierung einer wünschenswerten Entwicklung auf diesem Gebiet.

Im erwähnten Zeitraum (2001-2010) haben sich die Ernährungsempfehlungen und damit auch die Ziele für eine wünschenswerte Entwicklung verändert. Durch eine verstärkte Verknüpfung der Ernährungs- und Bewegungs- empfehlungen $(24 ; 42)$ ist eine Erreichung des Zieles «Förderung eines gesunden Körpergewichtes» (96) eher in den Bereich des Möglichen gerückt worden. Das im Jahr 2008 vom Bundesrat lancierte Nationale Programm Ernährung und Bewegung (NPEB) hat diese Zielsetzung noch mehr in den Vordergrund gestellt (85; Kapitel 4.6.1.1).

Über die Auswirkungen der Ernährungsempfehlungen auf das Ernährungsverhalten ist zumindest in der Schweiz noch zu wenig bekannt. Verschiedentlich durchgeführte Umfragen zum Ernährungswissen der Schweizer Bevölkerung zeigen Diskrepanzen zu den angenäherten Verzehrsdaten. So etwa entspricht der Früchteund Gemüsekonsum in der Schweiz nicht den Empfehlungen (Kapitel 2.6.5).

Im geplanten Bundesgesetz über Prävention und Gesundheitsförderung (Präventionsgesetz) ist ein Artikel zur Gesundheitsfolgenabschätzung vorgesehen (100; Kapitel 4.7.1). Von grosser Bedeutung ist in diesem Zusammenhang auch, dass die Ernährungsempfehlungen nicht nur eine Grundlage in der Gesundheitspolitik sind, sondern auch in anderen Politikbereichen wie Landwirtschaft, Bildung, Finanzen, Handel und Wirtschaft, Soziales, Konsumentenangelegenheiten, Arbeit sowie Sport und Kultur als Richtschnur gelten. Nur so ist es möglich, der Gesamtbevölkerung das präventive Potenzial einer ausgewogenen Ernährung auch zugänglich zu machen.

Sechster Schweizerischer Ernährungsbericht | 41 


\section{Literaturverzeichnis}

1. Bürgi H. Jodversorgung der Schweizerischen Bevölkerung. In: Keller U, Lüthy J, Amadò R et al., eds. Vierter Schweizerischer Ernährungsbericht. Bern: Bundesamt für Gesundheit, 1998:64-73.

2. Fleisch A. Ernährungsprobleme in Mangelzeiten: Die schweizerische Kriegsernährung 1939-1946. Basel: Benno Schwabe \& Co, 1947.

3. Deutsche Gesellschaft für Ernährung, Österreichische Gesellschaft für Ernährung, Schweizerische Gesellschaft für Ernährungsforschung, Schweizerische Vereinigung für Ernährung. Referenzwerte für die Nährstoffzufuhr. 1. Auflage, 3. vollständig durchgesehener und korrigierter Nachdruck. Neustadt: Neuer Umschau Buchverlag, 2008.

4. Bundesgesetz über Lebensmittel und Gebrauchsgegenstände vom 9. Oktober 1992 (Stand am 1. April 2008); SR 817.0, Art. 12 Information der Öffentlichkeit. Internet: http://www.admin.ch/ch/d/sr/817_0/ a12.html (letzter Zugriff 30. September 2011).

5. World Health Organization Europe. CINDI dietary guide, 2000.

Internet: http://www.euro.who.int/__data/assets/ pdf_file/0010/119926/E70041.pdf (letzter Zugriff 30. September 2011).

6. European Food Safety Authority. Scientific opinion on establishing of food-based dietary guidelines. EFSA Panel on Dietetic Products, Nutrition, and Allergies (NDA). EFSA Journal 2010;8(3):1460.

7. Bähler P, Bänziger $O$, Belli D et al. Empfehlungen für die Säuglingsernährung 2008. Ernährungskommission der Schweizerischen Gesellschaft für Pädiatrie. Schweiz Med Forum 2008;8(20):366-9.

8. Bähler P, Bänziger O, Belli D et al. Empfehlungen für die Säuglingsernährung 2009. Ernährungskommission der Schweizerischen Gesellschaft für Pädiatrie. Paediatrica 2009;20:13-5.
9. Lebensmittel-und Gebrauchsgegenständeverordnung vom 23. November 2005 (Stand am

1. November 2010); SR 817.02.

Internet: http://www.admin.ch/ch/d/sr/ c817_02.html (letzter Zugriff 30. September 2011).

10. Codex Alimentarius. Codex standard for infant formula and formulas for special medical purposes intended for infants. Codex Stan 72-1981.

Internet: http://www.codexalimentarius.net/ download/standards/288/CXS_072e.pdf (letzter Zugriff 30. September 2011).

11. International Society for the Study of Fatty Acids and Lipids. ISSFAL Statement on dietary fats in infant nutrition, May 2008.

Internet: http://www.issfal.org/statements/ pufa-recommendations/statement-2 (letzter Zugriff 30. September 2011).

12. World Health Organization. The optimal duration of exclusive breastfeeding. Report of an expert consultation. Geneva, Switzerland. 28-30 March 2001. Internet: http://www.who.int/nutrition/publications/optimal_duration_of_exc_bfeeding_report_ eng.pdf (letzter Zugriff 30. September 2011).

13. Koletzko B, Brönstrup A, Cremer M, Flothkötter $M$ et al. Säuglingsernährung und Ernährung der stillenden Mutter. Handlungsempfehlungen - Ein Konsensuspapier im Auftrag des bundesweiten Netzwerk Junge Familie. Monatsschr Kinderheilkd 2010;158:679-89.

14. Schweizerische Stiftung zur Förderung des Stillens. Internet: http://www.allaiter.ch/logicio/pmws/ indexDOM.php?client_id=stillen\&page id=aktuell\&lang_iso639=de (letzter Zugriff 30. September 2011).

15. Eugster $\mathrm{G}$. Babyernährung gesund \& richtig. $\mathrm{B}(\mathrm{r}$ )eikost und Fingerfood ab dem 6. Lebensmonat. München: Elsevier GmbH, 2005.

16. Karall D, Meisinger B, Grissenauer G, Scholl-Bürgi $S$. Ernährung des gesunden Säuglings und Kindes. In: Ledochowski M, ed. Klinische Ernährungsmedizin. Wien: Springer-Verlag, 2010:763-72. 
17. Kersting $\mathrm{M}$, Alexy $U$. Empfehlungen für die Ernährung von Säuglingen. 3. überarbeitete Auflage. Dortmund: Forschungsinstitut für Kinderernährung GmbH Dortmund, 2009.

18. Schweizerische Gesellschaft für Ernährung. Ernährung von Kindern, 2011. Internet: http://www.sge-ssn.ch/media/ medialibrary/2012/06/merkblatt_ernaehrung_von_ kindern_2011.pdf (letzter Zugriff 6. Juni 2012).

19. Schweizerische Gesellschaft für Ernährung, Gesundheitsförderung Schweiz. Ernährungsscheibe. Empfehlungen zum gesunden und genussvollen Essen und Trinken für Kinder. Internet: http://www.sge-ssn.ch/ media/medialibrary/2012/03/merkblatt_ ernaehrungsscheibe_fuer_kinder_1.pdf (letzter Zugriff 6. Juni 2012).

20. Vartanian LR, Schwartz MB, Brownell KD. Effects of soft drink consumption on nutrition and health: a systematic review and meta-analysis. Am J Public Health 2007;97:667-75.

21. World Cancer Research Fund, American Institute for Cancer Research. Zusammenfassung: Ernährung, körperliche Aktivität und Krebsprävention: Eine globale Perspektive. Washington, DC:

AICR, 2007.

Internet: http://www.dge.de/pdf/ws/ WCRF-Report-summary-de.pdf (letzter Zugriff 30. September 2011).

22. Suter P. Ernährung, Lifestyle und Adipositas von Kindern und Jugendlichen in der Schweiz. In: Eichholzer M, Camenzind-Frey E, Matzke A et al., eds. Fünfter Schweizerischer Ernährungsbericht. Bern: Bundesamt für Gesundheit, 2005:167-98.

23. Minas K. Skelettentwicklung des Heranwachsenden und ihre Bedeutung für die Spitzenknochenmasse («peak bone mass»). Fortbildung Osteologie 2010;3:5-6.
24. Schweizerische Gesellschaft für Ernährung SGE. Schweizer Lebensmittelpyramide: Empfehlungen zum ausgewogenen und genussvollen Essen und Trinken für Erwachsene, 2011.

Internet: http://www.sge-ssn.ch/media/ medialibrary/pdf/100-ernaehrungsthemen/ 10-gesundes_essen_trinken/ Lebensmittelpyramide/_sge_pyramid_long_D.pdf (letzter Zugriff 6. Juni 2012)

25. Schweizerische Gesellschaft für Ernährung. Ernährung von Jugendlichen, 2011. Internet: http://www.sge-ssn.ch/media/ medialibrary/2012/06/merkblatt_ernaehrung_ von_jugendlichen_2011.pdf (letzter Zugriff 6. Juni 2012).

26. Bartsch S. Jugendesskultur: Bedeutungen des Essens für Jugendliche im Kontext Familie und Peergroup. Köln: Bundeszentrale für gesundheitliche Aufklärung, 2008.

27. Gerhards J, Rössl J. Das Ernährungsverhalten Jugendlicher im Kontext ihrer Lebensstile. Forschung und Praxis der Gesundheitsförderung. Band 30. Köln: Bundeszentrale für gesundheitliche Aufklärung, 2003.

28. Schweizerische Gesellschaft für Ernährung. State-of-the-Art-Bericht: Fast Food und Gesundheit, 2005.

Internet: http://sge-ssn.feinheit.ch/media/ medialibrary/pdf/100-ernaehrungsthemen/ 60-ernaehrungsformen_lebensstil/ State-of-the-Art-Bericht_Fast_Food.pdf (letzter Zugriff 6. Juni 2012).

29. Welsh SO, Davis C, Shaw A. USDA's Food Guide. Background and development. Hyattsville, Maryland: United States Department of Agriculture, 1993.

30. Mozaffarian D, Ludwig DS. Dietary guidelines in the 21st century - a time for food. JAMA 2010; 304:681-2.

31. Boeing $\mathrm{H}$. Lebensmittelbasierte Präventionskonzepte. Ernährungs Umschau 2009;8:458-73. 
32. World Health Organization. Preparation and use of food-based dietary guidelines: report of a joint FAO / WHO consultation. Geneva: World Health Organization, 1998. [WHOTechnical report series; 880] Internet: http://apps.who.int/bookorders/anglais/ detart1.jsp? sesslan $=1 \&$ codlan $=1 \&$ codcol $=$ $10 \& \operatorname{codcch}=880$ (letzter Zugriff 30. September 2011)

33. Stehle P, Oberritter $H$, Büning-Fesel $M$, Heseker $H$. Grafische Umsetzung von Ernährungsrichtlinien traditionelle und neue Ansätze. Ernährungs Umschau 2005;52:128-35.

34. Auswertung der Online-Befragung zur Lebensmittelpyramide 2009. Daten zurVerfügung gestellt von der Schweizerischen Gesellschaft für Ernährung, 2009.

35. Bundesamt für Sport (BASPO). Gesundheitswirksame Bewegung für Erwachsene, 2002. Internet: http://www.hepa.ch/internet/hepa/de/ home/dokumentation/grundlagendokumente. parsys.74026. downloadList.77540.DownloadFile. tmp/bewegungsempferw20074hepa.pdf (letzter Zugriff 30. September 2011).

36. Schweizerische Gesellschaft für Ernährung SGE. Schweizer Lebensmittelpyramide: Empfehlungen zum ausgewogenen und genussvollen Essen und Trinken für Erwachsene. Ergänzende Empfehlungen für ältere Erwachsene, 2011. Internet: http://www.sge-ssn.ch/media/medialibrary/pdf/100-ernaehrungsthemen/10-gesundes_ essen_trinken/Lebensmittelpyramide/_sge_pyramid_basic_D.pdf (letzter Zugriff 6. Juni 2012).

37. Council of Europe. Food and nutritional care in hospitals: how to prevent undernutrition. Report and recommendations of the Committee of Experts on Nutrition, Food Safety and Consumer Protection. Council of Europe Publishing. Strasbourg, November 2002.
38. Expertengruppe des Europarates und Eidgenössische Ernährungskommission. Mangelernährung im Spital. Stellungnahme einer Expertengruppe des Europarates und Empfehlungen der Eidgenössischen Ernährungskommission. Bern: Bundesamt für Gesundheit, 2005.

Internet: http://www.bag.admin.ch/themen/ ernaehrung_bewegung/05207/05226/index.html? lang=de\&download=NHzLpZeg7t, Inp6IONTU042 I2Z6In1acy4Zn4Z2qZpnO2Yuq2Z6gpJCFe4R6g2ym162epYbg2c_JjKbNoKSn6A-- (letzter Zugriff 30. September 2011).

39. Eidgenössische Ernährungskommission. Ernährung in Schwangerschaft und Stillzeit. Gefahren für Mutter und Kind? Expertenbericht der EEK für eine optimale Ernährung von Mutter und Kind. Bern: Bundesamt für Gesundheit, 2006. Internet: http://www.bag.admin.ch/themen/ ernaehrung_bewegung/05207/05217/index.html? lang=de\&download=NHzLpZeg7t, Inp6IONTU042 I2Z6In1acy4Zn4Z2qZpnO2Yuq2Z6gpJCGeHt3f2ym162epYbg2c_JjKbNoKSn6A-- (letzter Zugriff 30. September 2011).

40. Eichholzer M, Camenzind-Frey E, Amberg J et al. Folsäure ist unentbehrlich für die normale embryonale Entwicklung des Kindes. Ist die Bevölkerung mit diesem Vitamin genügend versorgt? Bern: Bundesamt für Gesundheit, 2008. Internet: http://www.bag.admin.ch/shop/00012/ 00272/index.html? lang =de\&download $=\mathrm{NHz}-$ LpZeg7t,Inp6I0NTU042I2Z6In1acy4Zn4Z2qZpn O2Yuq2Z6gpJCHeHt5gWym162epYbg2c_JjKbNoKSn6A-- (letzter Zugriff 30. September 2011).

41. Eidgenössische Ernährungskommission. (Extra-) Hartkäse aus Rohmilch in der Schwangerschaft. Addendum zum Bericht der EEK. Bern: Bundesamt für Gesundheit, 2010.

Internet: http://www.bag.admin.ch/themen/ ernaehrung_bewegung/05207/05217/index.html? lang=de\&download=NHzLpZeg7t, Inp6IONTU042 | 2Z6In1acy4Zn4Z2qZpnO2Yuq2Z6gpJCIdX95g2ym 162epYbg2c_JjKbNoKSn6A-- (letzter Zugriff 30. September 2011). 
42. Swiss Forum for Sport Nutrition. Lebensmittelpyramide für Sportlerinnen und Sportler, 2008. Internet: http://www.sfsn.ethz.ch/sportnutr/ pyramide (letzter Zugriff 30. September 2011).

43. Mettler S, Mannhart Ch, Colombani P. Development and validation of a food pyramid for swiss athletes. Int J Sport Nutr Exerc Metabol 2009;19:504-18.

44. Eidgenössische Ernährungskommission. Gesundheitliche Vor- und Nachteile einer vegetarischen Ernährung. Expertenbericht der EEK. Bern: Bundesamt für Gesundheit, 2006. Internet: http://www.bag.admin.ch/themen/ ernaehrung_bewegung/05207/05219/index.html? lang=de\&download=NHzLpZeg7t, Inp6IONTU042। 2Z6In1acy4Zn4Z2qZpnO2Yuq2Z6gpJCGd396gWym162epYbg2c_JjKbNoKSn6A-- (letzter Zugriff 30. September 2011).

45. Fraser GE. Vegetarian diets: what do we know of their effects on common chronic diseases? Am J Clin Nutr 2008;89(suppl):1-6.

46. Craig WJ. Health effects of vegan diets. Am J Clin Nutr 2009;89(suppl):1627-33.

47. Bundesverfassung der Schweizerischen Eidgenossenschaft vom 18. April 1999 (Stand am 1. Januar 2011); SR 101, Art. 73 Nachhaltigkeit. Internet: http://www.admin.ch/ch/d/sr/101/a73.html (letzter Zugriff 30. September 2011).

48. Ambler-Edwards S, Bailey K, Kiff A et al. Food Futures: Rethinking UK Strategy. London: Royal Institute of International Affairs (Chatham House), 2009.

49. Kickbusch I. The Food System: a prism of present and future challenges for health promotion and sustainable development. Bern: Health Promotion Switzerland, 2010.

Internet: http://graduateinstitute.ch/webdav/site/ globalhealth/shared/1894/White\%20Paper\%20\%20The\%20Food\%20System.pdf (letzter Zugriff 30. September 2011).

50. Pimental D, Pimental M. Sustainability of meatbased and plant-based diets and the environment. Am J Clin Nutr 2003;78(suppl):660-3.
51. Eidgenössische Ernährungskommission. Kohlenhydrate in der Ernährung. Stellungnahme und Empfehlungen der EEK. Zürich: Bundesamt für Gesundheit, 2009.

Internet: http://www.bag.admin.ch/themen/ ernaehrung_bewegung/05207/07326/index.html? lang=de\&download=NHzLpZeg7t, Inp6IONTU042 I2Z6In1acy4Zn4Z2qZpnO2Yuq2Z6gpJCHfIR5fWym162epYbg2c_JjKbNoKSn6A-- (letzter Zugriff 30. September 2011).

52. Eidgenössische Ernährungskommission. Fette in der Ernährung. Empfehlungen der EEK. Bern: Bundesamt für Gesundheit, 2006.

Internet: http://www.bag.admin.ch/themen/ ernaehrung_bewegung/05207/05211/index.html?

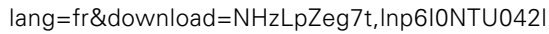
2Z6In1ae2IZn4Z2qZpnO2Yuq2Z6gpJCFfYR_gWym162epYbg2c_JjKbNoKSn6A-- (letzter Zugriff 30. September 2011).

53. World Health Organization. Interim summary of conclusion and dietary recommendations on total fat $\&$ fatty acids. From the joint FAO /WHO expert consultation on fats and fatty acids in human nutrition, 10-14 November 2008, Geneva. Internet: http://www.who.int/nutrition/topics/ FFA_summary_rec_conclusion.pdf (letzter Zugriff 30. September 2011).

54. International Society for the Study of Fatty Acids and Lipids.

Internet: www.issfal.org (letzter Zugriff 30. September 2011).

55. Calder PC, Dangour AD, Diekman C et al. Essential fats for future health. Proceedings of the 9th Unilever Nutrition Symposium, 26-27 May 2010. Eur J Clin Nutr 2010;64:1-13.

56. Federal Commission for Nutrition. Proteins in human nutrition. Expert report of the FCN. Bern: Federal Office of Public Health, 2011. Internet: http://www.bag.admin.ch/themen/ ernaehrung_bewegung/05207/11924/index.html? lang=de (letzter Zugriff 30. September 2011). 
57. World Health Organization. Protein and amino acid requirement in human nutrition: report of a joint WHO / FAO / UNU expert consultation. Geneva: World Health Organization, 2007. [WHOTechnical report series; 935] Internet: http://whqlibdoc.who.int/trs/WHO_TRS_ 935_eng.pdf (letzter Zugriff 30. September 2011).

58. Schweizerische Gesellschaft für Ernährung. Alkohol, 2007.

Internet: http://sge-ssn.feinheit.ch/media/ medialibrary/pdf/100-ernaehrungsthemen/40lebensmittel/7-suessigkeiten_knabbereien/Merkblatt_Alkohol.pdf (letzter Zugriff 6. Juni 2012).

59. Schütze $M$, Boeing $H$, PischonT et al. Alcohol attributable burden of incidence of cancer in eight European countries based on results from prospective cohort study. Brit Med J 2011;342:d1584.

60. World Health Organization. Reducing salt intake in populations. Report of a WHO Forum andTechnical Meeting. Paris: World Health Organization, 2006.

61. Bachmann M, Keller U. Salzkonsum und Bluthochdruck. Schweiz Med Forum 2008;8(50):968-72.

62. Bundesamt für Gesundheit. Salz Strategie 20082012. Strategiepapier zur Reduktion des Kochsalzkonsums. Bern: Bundesamt für Gesundheit, 2009 Internet: http://www.bag.admin.ch/themen/ ernaehrung_bewegung/05207/05216/index.html? lang=de\&download=NHzLpZeg7t,Inp6IONTU042। 2Z6In1acy4Zn4Z2qZpnO2Yuq2Z6gpJCHfYJ7f2ym 162epYbg2c_JjKbNoKSn6A-- (letzter Zugriff 30. September 2011).

63. Lüthy J, Beer M. Eine Salz-Strategie für die Schweiz. Cardiovasc 2009;5:17-9.

64. Chappuis A, Bochud M, Glatz N, Vuistiner P, Paccaud F, Burnier M. Swiss survey on salt intake: main results. Bern: Federal office of public health, 2011.

Internet: http://www.bag.admin.ch/themen/ ernaehrung_bewegung/05207/05216/12335/ index.html? lang=de\&download=NHzLpZeg7t, Inp 6IONTU042I2Z6In1acy4Zn4Z2qZpnO2Yuq2Z6gpJ CKdoN3fWym162epYbg2c_JjKbNoKSn6A-- (letzter Zugriff 5. Dezember 2011).
65. Federal Commission for Nutrition. Vitamin D deficiency: Evidence, safety, and recommendations for the Swiss population. Expert report of the FCN. Zurich: Federal Office of Public Health, 2012. Internet: http://www.bag.admin.ch/themen/ ernaehrung_bewegung/05207/13246/index. html?lang=de (letzter Zugriff 30. Juni 2012).

66. Frey U. Synopsis. In: Aebi H, Blumenthal A, Bohren-Hoerni M et al., eds. Zweiter Schweizerischer Ernährungsbericht. Bern: H. Huber, 1984:424-46.

67. Lüthy J, Beer M. Einleitung zu Kapitel 3. In: Eichholzer M, Camenzind-Frey E, Matzke A et al., eds. Fünfter Schweizerischer Ernährungsbericht. Bern: Bundesamt für Gesundheit, 2005:343-6.

68. Bundesamt für Gesundheit. Bericht über die Ergebnisse des Vernehmlassungsverfahrens zur Totalrevision des Bundesgesetzes über Lebensmittel und Gebrauchsgegenstände (Lebensmittelgesetz, LMG, SR 817.0). Bern: Bundesamt für Gesundheit, 2010.

Internet: http://www.bag.admin.ch/themen/ lebensmittel/04865/05022/07826/index.html? lang=de\&download=NHzLpZeg7t, Inp6I0NTU042 I2Z6In1 acy4Zn4Z2qZpnO2Yuq2Z6gpJCJeIN6fWym162epYbg2c_JjKbNoKSn6A-- (letzter Zugriff 30. September 2011).

69. Kuchen A. Rückstände von Umweltkontaminanten in Lebensmitteln. In: Eichholzer M, CamenzindFrey E, Matzke A et al., eds. Fünfter Schweizerischer Ernährungsbericht. Bern: Bundesamt für Gesundheit, 2005:389-402.

70. Tremp E. Nitrat, Nitrit, N-Nitrosoverbindungen. In: Stähelin HB, Lüthy J, Casabianca A et al., eds. Dritter Schweizerischer Ernährungsbericht. Bern: Bundesamt für Gesundheit, 1991:181-91.

71. Lock K, Pomerleau J, Causer L, Altman DR, Mc Kee M. The global burden of disease attributable to low consumption of fruits and vegetables: Implications for the global strategy on diet. Bulletin of the World Health Organization 2005;83(2):100-8.

72. Marques-Vidal P, Pecoud A, Hayoz D et al. Prevalence and characteristics of vitamin or dietary supplement users in Lausanne, Switzerland: the CoLaus study. Eur J Clin Nutr 2009;63:273-81. 
73. Beer-Borst S, Costanza M C, Morabia A. Die Bedeutung von "Functional Food» in der Ernährung der erwachsenen Genfer Bevölkerung - eine Bestandesaufnahme. In: Eichholzer M, Camenzind-Frey E, Matzke A et al., eds. Fünfter Schweizerischer Ernährungsbericht. Bern: Bundesamt für Gesundheit, 2005:751-66.

74. Eidgenössische Ernährungskommission. Folsäure: Expertenbericht der EEK zur Prophylaxe von Neuralrohrdefekten. Bern: Bundesamt für Gesundheit, 2002.

Internet: http://www.bag.admin.ch/themen/ ernaehrung_bewegung/05207/05212/index.html? lang=de\&download=NHzLpZeg7t, Inp6IONTU042 | 2Z6In1acy4Zn4Z2qZpnO2Yuq2Z6gpJCFeX1_fWym162epYbg2c_JjKbNoKSn6A-- (letzter Zugriff 30. September 2011).

75. Zenger A, Ischi L. Rechtsgutachten über die Verfassungsmässigkeit einer "obligaten» Anreicherung von Getreidemehl mit Folsäure zur Verhütung von Spina bifida und zu weiteren gesundheitsbezogenen Zwecken. Bern: Universität Bern, 2004. Internet: http://www.bag.admin.ch/themen/ ernaehrung_bewegung/05207/05212/index.html? lang=de\&download=NHzLpZeg7t, Inp6IONTU04 2I2Z6In1acy4Zn4Z2qZpnO2Yuq2Z6gpJCGdH9_ gWym162epYbg2c_JjKbNoKSn6A-- (letzter Zugriff 30. September 2011).

76. European Food Safety Authority. Folic Acid: An update on scientific developments, meeting summary report, 21-22 January 2009, Uppsala, Sweden.

Internet: http://www.efsa.europa.eu/en/home/ publication/efsafolicacid.pdf (letzter Zugriff 30. September 2011).

77. European Food Safety Authority. Scientific opinion on dietary reference values for carbohydrates and dietary fibre. EFSA Panel on Dietetic Products, Nutrition, and Allergies (NDA). EFSA Journal 2010;8(3):1462.

Internet: http://www.efsa.europa.eu/fr/efsajournal/doc/1462.pdf (letzter Zugriff 30. September 2011).
78. European Food Safety Authority. Scientific opinion on dietary reference values for fats, including saturated fatty acids, polyunsaturated fatty acids, monounsaturated fatty acids, trans fatty acids, and cholesterol. EFSA Panel on Dietetic Products, Nutrition, and Allergies (NDA). EFSA Journal 2010; 8(3):1461.

Internet: http://www.efsa.europa.eu/fr/efsajournal/ doc/1461.pdf (letzter Zugriff 30. September 2011).

79. European Food Safety Authority. Scientific opinion on dietary reference values for water. EFSA Panel on Dietetic Products, Nutrition, and Allergies (NDA). EFSA Journal 2010;8(3):1459.

Internet: http://www.efsa.europa.eu/fr/efsajournal/ doc/1459.pdf (letzter Zugriff 30. September 2011).

80. Ambroise Martin. Apports nutritionnels conseillés pour la population française. $3^{\mathrm{ème}}$ édition. Paris: Technique \& Documentation, 2001.

81. Agence Française de sécurité sanitaire des aliments. Opinion of the French Food Safety Agency on the update of French population reference intakes (ANCs) for fatty acids. AFSSA - Request no. 2006-SA-0359. March 2010. Internet: http://www.afssa.fr/Documents/ NUT2006sa0359EN.pdf (letzter Zugriff 30. September 2011).

82. Società Italiana di Nutrizione Umana. Internet: http://www.sinu.it/pubblicazioni.asp (letzter Zugriff 30. September 2011).

83. Organisation mondiale de la Santé. Stratégie mondiale pour l'alimentation, I'exercice physique et la santé, 2004.

Internet: http://www.who.int/dietphysicalactivity/ strategy/eb11344/strategy_french_web.pdf (letzter Zugriff 30. September 2011).

84. World Health Organization. Fifty-seventh World Health Assembly. WHA 57.17 Global strategy on diet, physical activity and health. 2004. Internet: http://apps.who.int/gb/ebwha/pdf_files/ WHA57/A57_R17-en.pdf (letzter Zugriff 30. September 2011). 
85. Bundesamt für Gesundheit. Nationales Programm Ernährung und Bewegung (NPEB) 2008-2012.

Bern: Bundesamt für Gesundheit, 2008. Internet: http://www.bag.admin.ch/themen/ernaehrung_bewegung/13227/index.html?lang=de (letzter Zugriff 6. Juni 2012).

86. Weltgesundheitsorganisation Europa.

Europäischer Aktionsplan Nahrung und Ernährung derWHO 2007-2012.

Internet: http://www.euro.who.int/_data/assets/ pdf_file/0019/74404/E91153G.pdf

(letzter Zugriff 6. Juni 2012).

87. Schweizerische Gesellschaft für Ernährung. Internet: http://www.sge-ssn.ch (letzter Zugriff 30. September 2011).

88. Eidgenössische Ernährungskommission. Internet: http://www.bag.admin.ch/themen/ ernaehrung_bewegung/05194/06310/index.html? lang=de (letzter Zugriff 30. September 2011).

89. Deutsche Gesellschaft für Ernährung. Internet: http://www.dge.de (letzter Zugriff 30. September 2011).

90. Centro Interuniversitario Internazionale di Studi sulle Culture Alimentari Mediterranee. Internet: http://www.ciiscam.org (letzter Zugriff 30. September 2011).

91. Programme National Nutrition Santé. Internet: http://www.mangerbouger.fr (letzter Zugriff 30. September 2011).

92. Bundesamt für Gesundheit. Empfehlungen für eine ausgewogene Ernährung. Bern: Bundesamt für Gesundheit, 2007.

93. World Health Organization Europe. The first action plan for food and nutrition policy. Copenhagen:World Health Organization, Regional Office for Europe, 2001. Internet: http://www.euro.who.int/_data/assets/ pdf_file/0013/120244/E72199.pdf (letzter Zugriff 30. September 2011).

94. World Health Organization Europe. European charter on counteracting obesity. Copenhagen:World Health Organization, Regional Office for Europe, 2006. Internet: http://www.euro.who.int/_data/assets/ pdf_file/0009/87462/E89567.pdf (letzter Zugriff 30. September 2011).
95. Food and Agriculture Organization of the United Nations / World Health Organization. International Conference on Nutrition. Final Report on the Conference. Rome: December 1992.

Internet: http://whqlibdoc.who.int/hq/1992/ a34812.pdf (letzter Zugriff 30. September 2011).

96. Bundesamt für Gesundheit. Aktionsplan Ernährung und Gesundheit: Eine Ernährungspolicy für die Schweiz. Bern: Bundesamt für Gesundheit, 2001. Internet: http://www.bag.admin.ch/themen/ ernaehrung_bewegung/05207/05215/index.html? lang=de\&download=NHzLpZeg7t, Inp6IONTU042| 2Z6In1acy4Zn4Z2qZpnO2Yuq2Z6gpJCFeXx7fWym162epYbg2c_JjKbNoKSn6A-- (letzter Zugriff 30. September 2011).

97. Lüthy J. Aktionsplan Ernährung und Gesundheit: Eine Ernährungspolicy für die Schweiz. In: Eichholzer M, Camenzind-Frey E, Matzke A et al., eds. Fünfter Schweizerischer Ernährungsbericht. Bern: Bundesamt für Gesundheit, 2005:863-7.

98. Lüthy J, Eichholzer M. Ernährung und ernährungsabhängige Krankheiten in der Schweiz. Mitt Gebiete Lebensm Hyg 1998;81:548-80.

99. Keller U, Lüthy J, Amadò R, Battaglia-Richi E et al., eds. Vierter Schweizerischer Ernährungsbericht. Bern: Bundesamt für Gesundheit, 1998.

100. Bundesamt für Gesundheit. Botschaft zum Bundesgesetz über Prävention und Gesundheitsförderung (Präventionsgesetz, PrävG) vom 30. September 2009.

Internet: http://www.bag.admin.ch/themen/ gesundheitspolitik/07492/index.html?lang=de\& download=NHzLpZeg7t, Inp6I0NTU042I2Z6In 1a cy4Zn4Z2qZpnO2Yuq2Z6gpJCHfYN5gWym162 epYbg2c_JjKbNoKSn6A-- (letzter Zugriff 30. September 2011).

101. Webb AJ, Patel N, Loukogeorgakis S et al. Acute blood pressure lowering, vasoprotective and antiplatelet properties of dietary nitrate via bioconversion to nitrite. Hypertension 2008:51(3):784-90.

102. Istituto Nazionale di Ricerca per gli Alimenti e la Nutrizione. Internet: http://www.inran.it (letzter Zugriff 30. September 2011). 


\section{Ernährungssituation in der Schweiz}

Alexandra Schmid

Christine Brombach

Sabine Jacob

Iso Schmid

Robert Sieber

Michael Siegrist 


\subsection{Zusammenfassung}

Wie in den früheren Schweizerischen Ernährungsberichten wurden auch im aktuellen die auf der Agrarstatistik basierenden Verbrauchsdaten berechnet, womit sich nun die Entwicklung des Verbrauchs über rund 30 Jahre hinweg abbilden lässt. Die rückläufigen Trends im Verbrauch von Fleisch und Fleischprodukten sowie auch von Milch und Milchprodukten setzten sich nicht weiter fort; hingegen nahm der Verbrauch von Früchten und auch der Konsum von alkoholischen Getränken weiter ab. Der Gemüseverbrauch weist zum ersten Mal keinen Anstieg auf. Zucker, Öle und Fette, Hülsenfrüchte sowie Fische und Schalentiere konnten eine geringfügige Zunahme im Verbrauch verbuchen; Getreide- und Kartoffelverbrauch blieben konstant.

Analog zum Lebensmittelverbrauch veränderte sich auch der daraus berechnete Verbrauch und angenäherte Verzehr an Energie, energieliefernden Nährstoffen, Nahrungsfasern und Mikronährstoffen nicht wesentlich. Der Energieverbrauch liegt auf dem gleichen Niveau wie in den früheren Schweizerischen Ernährungsberichten. Bei den energieliefernden Nährstoffen weisen nur die mehrfach ungesättigten Fettsäuren einen leicht ansteigenden Trend auf, ansonsten sind die Verbrauchsmengen vergleichbar mit dem 4. und 5. Schweizerischen Ernährungsbericht. Die Anteile von Protein, Fett und Kohlenhydraten am angenäherten Gesamtverzehr an Energie liegen im Bereich der Empfehlungen. Nicht eingehalten werden jedoch die Empfehlungen bezüglich Anteile der einzelnen Fettsäuregruppen. Unter den Mikronährstoffen ist der angenäherte Verzehr von Vitamin D und Folsäure weiterhin unzureichend; die anderen
Mikronährstoffe erreichen oder übertreffen die gewichtete empfohlene Zufuhr in unterschiedlichem Ausmass. Dies schliesst jedoch nicht aus, dass gewisse Bevölkerungsgruppen bzw. Teile der Bevölkerung bei einzelnen Nährstoffen (Eisen, Jod) eine Unterversorgung aufweisen können.

Die Verbrauchsdaten stimmen mit dem in verschiedenen Studien erhobenen Verzehrsverhalten der Schweizer Bevölkerung weitgehend überein. Die von der Schweizerischen Gesellschaft für Ernährung empfohlene Anzahl Portionen an Gemüse, Früchten sowie Milch und Milchprodukten wird von der Mehrheit nicht erreicht. Das Ernährungswissen hat einen gewissen, wenn auch nicht sehr ausgeprägten Einfluss auf das Verzehrsverhalten. Frauen, besser Ausgebildete und ältere Personen verfügen über ein grösseres Ernährungswissen, und es sind auch hauptsächlich diese Gruppen, welchen die Bedeutung der Ernährung für die Gesundheit bewusst ist. An Wichtigkeit gewonnen haben die Ausser-Haus-Verpflegung sowie die Verwendung von Convenience-Produkten zu Hause.

Generell ist die Datenlage zur Ernährungssituation in der Schweiz unbefriedigend, und ein quantitativer Vergleich der Schweizer mit den europäischen Daten ist aufgrund der unterschiedlichen Erhebungsmethoden schwierig.

\subsection{Einleitung}

Das Essen ist zentraler Bestandteil des menschlichen Alltags. Die Schweizer Bevölkerung lebt grundsätzlich im Überfluss, was das Lebensmittelangebot und die dadurch mögliche Versorgung mit Energie und Nährstoffen angeht. Theoretisch wäre durch diese Fülle eine gesundheitsfördernde, angemessene Ernährung 
uneingeschränkt möglich. Allerdings kann sich Überfluss auch negativ auswirken, was sich in Form von Übergewicht und Adipositas manifestiert (Kapitel 3.3), und trotz des Überflusses finden sich auch Bevölkerungsgruppen, die unausgewogen oder mangelhaft ernährt sind (Kapitel 3.10). Die Untersuchung und Darstellung der Ernährungssituation ist deshalb auch in einer Überflussgesellschaft unerlässlich. Das Wissen über die Ernährungssituation eines Landes ist Ausgangspunkt und Grundlage für Forschung, Prävention, Monitoring und Evaluation im Bereich Ernährung.

Die Ernährungssituation, d.h. die Ernährungsgewohnheiten sowie die Energie- und Nährstoffversorgung der gesamten Bevölkerung eines Landes zu bestimmen, ist ein schwieriges Unterfangen. Jeder Mensch konsumiert seine eigene, täglich wechselnde, individuelle $\mathrm{Mi}$ schung und Menge an pflanzlichen und tierischen Lebensmitteln wie auch an Getränken. Beeinflusst wird die Nahrungszufuhr durch geschmackliche Vorlieben, kulturelle Traditionen, soziales Umfeld, Bildungsniveau, finanzielle Verhältnisse, Medien, Werbung, Angebot, Gesundheitszustand und vieles mehr. Da länderspezifische Einflüsse vorhanden sind, lässt sich aus Angaben anderer Länder nicht auf die Situation in der Schweiz zurückschliessen, obwohl sich gewisse Trends grenzübergreifend manifestieren können oder zeitverschoben in verschiedenen Ländern auftreten. Ziel ist deshalb, über möglichst aktuelle, vergleichbare, differenzierte und valide Schweizer Daten zur Ernährungssituation der Bevölkerung zu verfügen. Die diesbezügliche Datenlage ist in der Schweiz noch immer unzureichend, wenn auch Anstrengungen im Gange sind, dies zu ändern. So stellt das Monitoring-System Ernährung und Bewe- gung (MOSEB) über definierte Indikatoren seit 2009 fortlaufend repräsentative Daten im Bereich Ernährung und Bewegung zusammen (1). Auch die Resultate der Nationalen Ernährungserhebung, die zum Ziel hat, repräsentative Daten zur Beurteilung der Lebensmittelsicherheit und Ernährungssituation in der Schweiz zu sammeln, werden in der Zukunft ein zuverlässiges Bild über den Verzehr verschiedener Lebensmittel durch die Schweizer Bevölkerung ermöglichen (2). Geplant ist, in dieser Studie den 24-h-Lebensmittelverzehr und das Ernährungsverhalten der erwachsenen Schweizer Bevölkerung zu erheben.

\subsubsection{Ernährungserhebungs- methoden}

Zur Beurteilung der Ernährungssituation einer Bevölkerungsgruppe ist die Verfügbarkeit von qualitativ hochwertigen Daten zum Lebensmittelverzehr und Ernährungsverhalten unabdingbar. Diese Daten werden durch Ernährungserhebungen gesammelt. Je nach Fragestellung stehen verschiedene Methoden zur Verfügung, die alle sowohl Vor- als auch Nachteile aufweisen. Um Daten von verschiedenen Studien interpretieren und vergleichen zu können, muss zuerst beachtet werden, wie die Daten erhoben wurden, und ob sie einander direkt gegenübergestellt werden können oder zuerst noch aufgearbeitet werden müssen. Meist werden Studien so geplant und durchgeführt, dass man den Ernährungszustand einer bestimmten Bevölkerungsgruppe anhand der aus der erhobenen Lebensmittelzufuhr berechneten Einnahme von spezifischen Makro- und Mikronährstoffen sowie der Energieaufnahme beurteilen kann. 
Ernährungsdaten können sowohl direkt als auch indirekt erhoben werden (Abbildung 2.1). Indirekte Methoden basieren auf statistischen Angaben wie Agrar-, Verbrauchs- oder Einkommensstatistiken, die auf den Pro-Kopf-Verbrauch umgerechnet werden. Dabei handelt es sich nicht um Daten des tatsächlichen Verzehrs, sondern um den Verbrauch (Kapitel 2.3.1). Demgegenüber erfassen die direkten Erhebungsmethoden den Lebensmittelverzehr einzelner Personen. Dabei werden entweder der aktuelle oder der zurückliegende Lebensmittelkonsum von Individuen erfasst (prospektive oder retrospektive Erhebung). Retrospektive Erhebungsmethoden haben den Vorteil, dass das Ernährungsverhalten durch die Untersuchung nicht beeinflusst wird. Ihr Nachteil liegt jedoch darin, dass der Befragte sich gut an den Lebensmittel- konsum erinnern sollte, was je nach abgefragtem Zeitraum oder Alter der Befragten schwierig sein kann. Bei den prospektiven Methoden, bei denen der aktuelle Lebensmittelverzehr erfasst wird, besteht der Nachteil darin, dass sich das Ernährungsverhalten des Studienteilnehmers durch die Aufzeichnung verändern kann. Zudem sind solche Methoden mit viel mehr Aufwand verbunden. Da die prospektiven Methoden die genaueren sind (v.a. Wiegeprotokoll), wird für Erhebungen oft eine Kombination verschiedener Methoden eingesetzt, um die Nachteile der einzelnen Methoden zu minimieren (3).

Bei denjenigen Methoden, die nach geschätzten Portionengrössen verlangen (z.B. Schätzprotokoll), besteht die Herausforderung darin, Standardportionen zu definieren. Da es

\section{Ernährungserhebungsmethoden}

\section{Indirekte Methoden}

- Agrarstatistiken

- Verbrauchsstatistiken

- Einkommensstatistiken

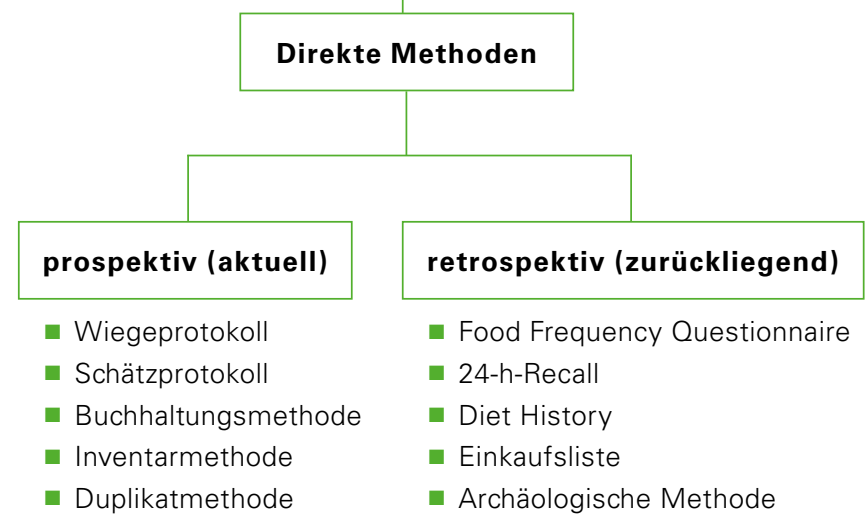

Abbildung 2.1: Verschiedene Methoden zur Erhebung von Ernährungsdaten (3) 
bis heute keine einheitliche und vollständige Datenbank zu definierten verzehrsüblichen Portionengrössen gibt, werden in vielen Studien diverse Quellen über Portionenangaben benutzt und mit eigenen Angaben ergänzt. In der Schweiz oft verwendete Quellen für Standardportionengrössen sind diejenigen der Schweizerischen Gesellschaft für Ernährung (SGE; 4), das Programm DISHES des Robert Koch-Institutes (5) oder die MONICA-Mengenliste (6).

\subsubsection{Kapitelübersicht}

Wie in den früheren Schweizerischen Ernährungsberichten (SEB) stützt sich auch der vorliegende SEB hauptsächlich auf Verbrauchsdaten, die der Agrarstatistik entnommen wurden. Das heisst, es wird nicht der Verzehr einzelner Individuen oder Bevölkerungsgruppen wiedergegeben, sondern die auf dem nationalen Markt zur Verfügung stehenden Lebensmittelmengen. Der Lebensmittelverbrauch pro Kopf wird in Kapitel 2.3 dargestellt. Verfeinert werden die Angaben durch Abschätzung und Abzug der Zubereitungsverluste, nicht verzehrbarer Lebensmittelanteile und nicht mehr verwertbarer Produkte, woraus der "angenäherte Verzehr» resultiert (7). Der effektive Verzehr kann daraus nicht abgeleitet werden, denn es fehlen Hinweise auf die weiteren im Haushalt entstehenden Verluste, wie zum Beispiel verdorbene Lebensmittel, weggeworfene Reste oder an Haustiere verfütterte Lebensmittel. Auch der individuelle Konsum kann aus den Bevölkerungsdaten nicht hergeleitet werden.

Basierend auf den Verbrauchsdaten wurde die durchschnittliche Versorgung mit Energie und Nährstoffen berechnet, wobei wiederum zwischen Verbrauch und angenähertem Verzehr unterschieden wird. Die Resultate werden in Kapitel 2.4 dargestellt und diskutiert. Wo immer möglich werden diesen Daten zusätzliche Schweizer Zahlen aus anderen Quellen als Ergänzung und für die Diskussion zur Seite gestellt.

Die für die Schweiz vorgestellten Daten werden im Kapitel 2.5 mit jenen anderer europäischer Länder verglichen, wobei sowohl länderübergreifende Tendenzen wie auch Spezifisches der Schweiz zur Sprache kommen. Unterschiedliche Erhebungsmethoden in den verschiedenen Ländern schränken die Vergleichbarkeit der Daten jedoch ein.

Die Abschätzung der Versorgung der Schweizer Bevölkerung mit Energie und Nährstoffen beruht auf dem Verbrauch von Lebensmitteln. Dieser wiederum hängt unter anderem ab vom Wissen der Schweizer Bevölkerung bezüglich Ernährung, von ihrer Einstellung gegenüber der Ernährung und von ihrem konkreten Verzehrsverhalten. Verschiedene Faktoren - zum Beispiel soziodemographische oder ökonomische - wie auch Kenntnisse bezüglich Ernährungsempfehlungen und Nährwertkennzeichnung beeinflussen das Ernährungsverhalten. Dieser Thematik widmet sich Kapitel 2.6.

Sechster Schweizerischer Ernährungsbericht | 53 


\subsection{Lebensmittelverbrauch und -verzehr in der Schweiz}

\subsubsection{Berechnungsgrundlagen}

Das Vorgehen zur Berechnung der Verbrauchszahlen ist das gleiche wie in den vorangehenden SEB (8-11). Der Verbrauch berechnet sich mittels unten aufgeführter Formel. Rohstoffmengen, die für die Verwendung als Tierfutter oder für nicht-alimentäre Zwecke produziert werden, gelangen nicht in diese Gleichung.

Verbrauch $=$ Produktion \pm Vorratsveränderung

$$
\text { - Export + Import }
$$

Für den vorliegenden SEB wurden die Verbrauchsdaten der Jahre 2007 und 2008 gemittelt. Die Mengenangaben beruhen auf der vom Schweizerischen Bauernverband in Brugg zur Verfügung gestellten Agrarstatistik (12). Die Mengen werden auf den Durchschnittsverbraucher bezogen. Zur Kalkulation der Pro-KopfMengen wurde die geschätzte mittlere ortsanwesende Bevölkerung der Schweiz (2007: 7.72 Mio.; 2008: 7.82 Mio.) verwendet. Eine Ausnahme stellen die Pro-Kopf-Mengen an alkoholischen Getränken dar, die auf der Bevölkerungszahl der über 15 Jahre alten Personen (2007: 6.26 Mio.; 2008: 6.44 Mio.) beruhen.

Der angenäherte Verzehr pro Person und Tag wurde ausgehend von den Verbrauchsmengen mit Hilfe lebensmittelspezifischer "Abfallfaktoren» berechnet, die den Nährwert-Tabellen von Souci, Fachmann und Kraut entnommen wurden (13). Die Abzüge variieren stark je nach Lebensmittelgruppe und erstrecken sich von $1 \%$
(Feigen) bis 58\% (Haselnüsse). Vor allem $\mathrm{Ge}$ müse und Früchte (Rüstabfälle, Steine, Hartschalen), Fleisch, Fische und Weichtiere (Knochen, Sehnen, Schalen), Hartkäse (Rinde) sowie Fette und Öle (Fette zum Braten und Frittieren) sind Verlusten unterworfen.

Obwohl mit den Angaben zu Verbrauch und angenähertem Verzehr nur sehr allgemeine Aussagen über die Versorgung der Schweizer Bevölkerung gemacht werden können, lassen sich durch deren Fortführung über die Jahre hinweg wertvolle Längsvergleiche anstellen und Veränderungen im Verbrauch von Lebensmitteln aufzeigen.

\subsubsection{Verbrauchs- und Verzehrs- daten für die einzelnen Lebensmittelgruppen}

Die Tabellen 2.1-2.10 weisen den Lebensmittelverbrauch pro Kopf und Jahr basierend auf den Angaben der Agrarstatistik aus (12). Neben den aktuellen Zahlen werden in den Tabellen auch Angaben aus früheren SEB wiedergegeben (8-11). Lebensmittelverbrauch und angenäherter Verzehr pro Person und Tag finden sich in Tabelle 2.12. Abbildung 2.2 stellt den aktuellen Verbrauch als prozentuale Veränderung gegenüber 2001/02 dar. Das regelmässige Erscheinen der SEB alle 6-7 Jahre und die damit einhergehende regelmässige Veröffentlichung der Verbrauchszahlen ermöglicht nun einen Vergleich über annähernd 30 Jahre hinweg. In Abbildung 2.3 sind die Verbrauchsmengen der verschiedenen Lebensmittelgruppen pro Kopf und Jahr über die gesamte erfasste Zeitdauer dargestellt. Im Folgenden wird detaillierter auf die einzelnen Lebensmittelgruppen eingegangen. 


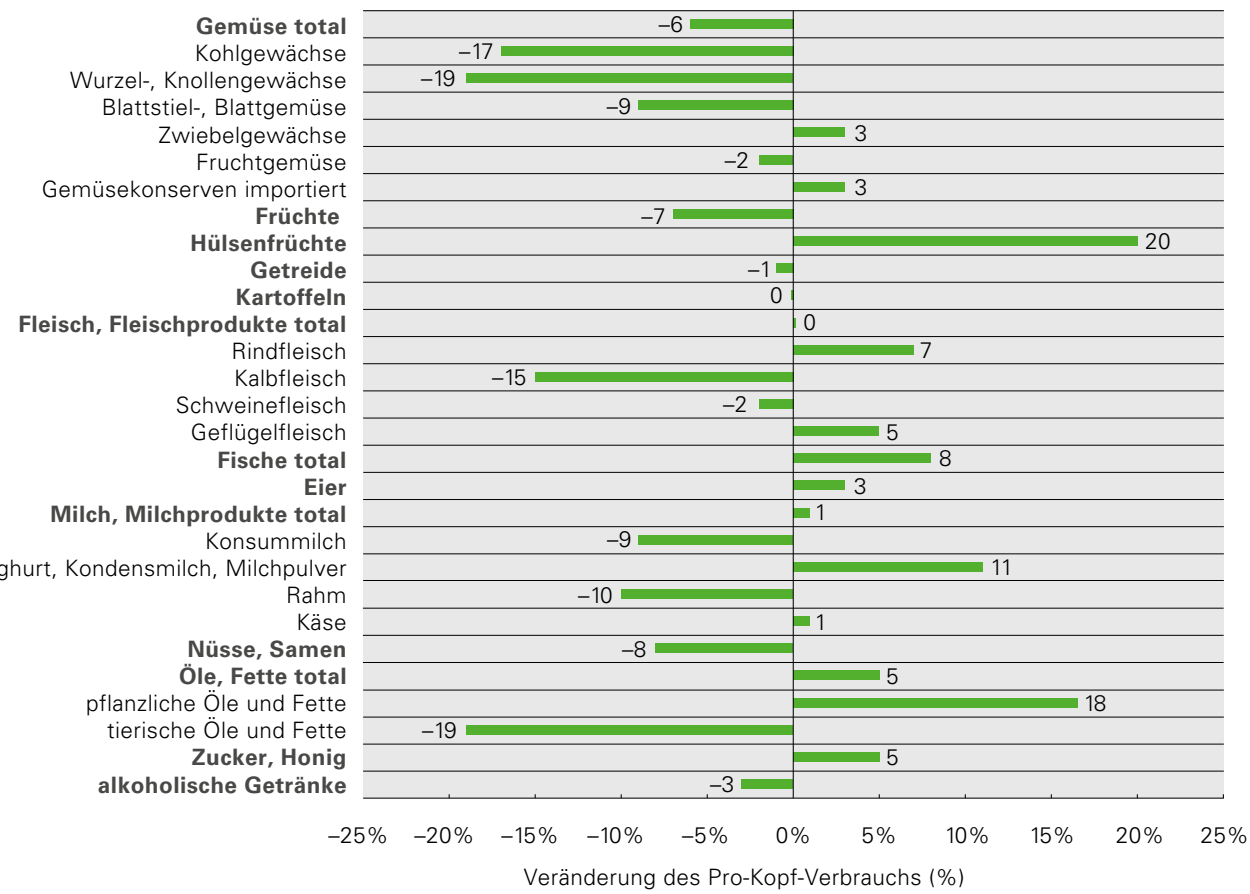

Gemüse total: ohne Kartoffeln und Hülsenfrüchte

Milch, Milchprodukte: ohne Butter

Nichtalkoholische Getränke: nicht aufgeführt, da die in 2001/02 verwendete Datengrundlage nicht mit derjenigen in 2007/08 vergleichbar ist

Den Gruppen zugehörende Lebensmittel sieheTabellen 2.1-2.10.

Abbildung 2.2: Prozentuale Veränderungen im Pro-Kopf-Verbrauch einzelner Lebensmittelgruppen 2007/08 im Vergleich zu 2001/02

\section{Gemüse}

Beim Gemüseverbrauch mussten rückwirkend für 1994/95 und 2001/02 die Mengen an importierten Gemüsekonserven nach oben korrigiert werden, weshalb nun für diese Erfassungsperioden von höheren Gesamtverbrauchszahlen ausgegangen werden muss, als sie im 4. und 5. SEB $(8 ; 10)$ publiziert worden sind. Der Gesamtgemü- severbrauch hat damit in 2007/08 zum ersten Mal nicht weiter zugenommen, sondern ist im Vergleich zum 5. SEB sogar um durchschnittlich $6 \%$ gesunken (Abbildungen 2.2 und 2.3 sowie Tabelle 2.1). Dies, obwohl die Ernährungsempfehlungen einen höheren Gemüsekonsum anregen (Kapitel 1.3) und die Jahre 2007 und 2008 in der Schweiz durchschnittliche Erntejahre wa- 


\section{$>$ Ernährungssituation in der Schweiz}

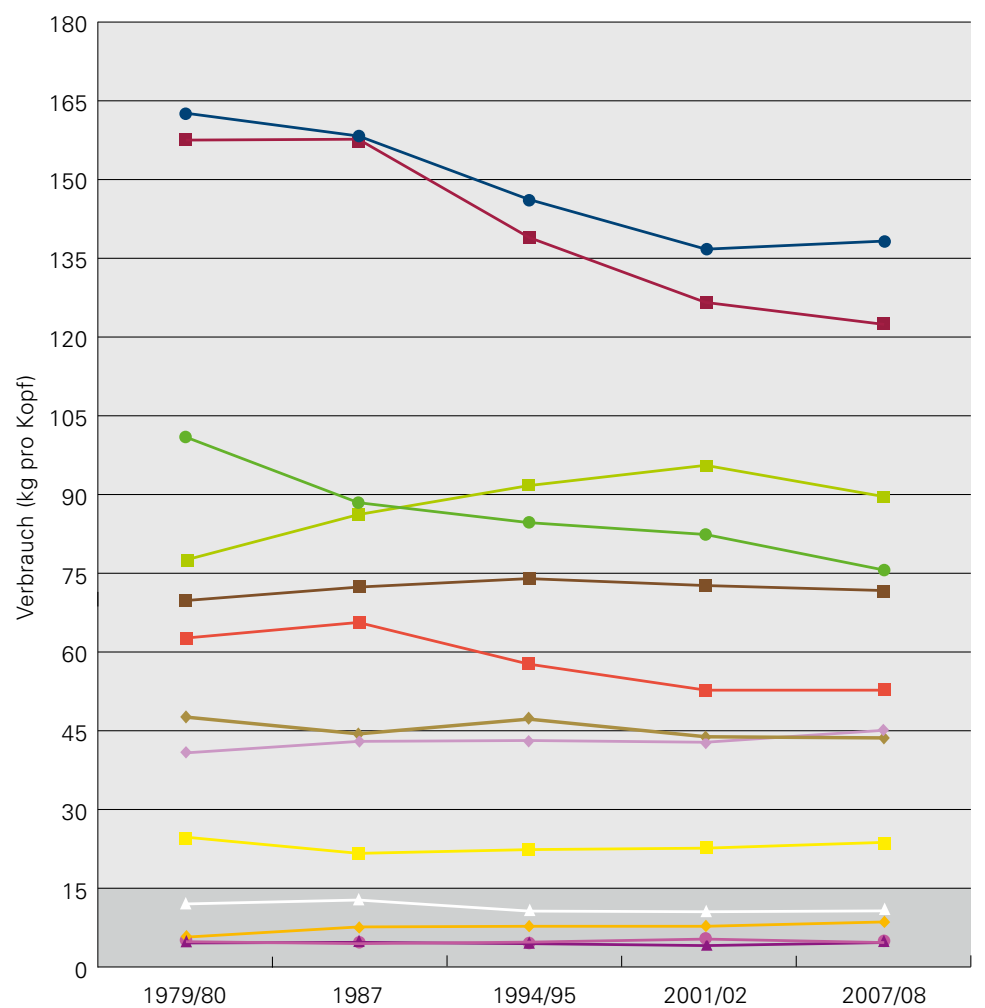

- Getreide

$\rightarrow$ Kartoffeln

$\leadsto$ Zucker

- - Gemüse

$\neg$ Hülsenfrüchte

- - Nüsse, Samen

$\rightarrow$ Früchte

$\rightarrow-$ Fleisch, Fleischprodukte

$\rightarrow$ Fisch

$\rightarrow$ Eier

$\rightarrow$ Milch, Milchprodukte

- Öle, Fette

$\rightarrow$ - alkoholische Getränke

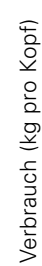

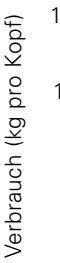

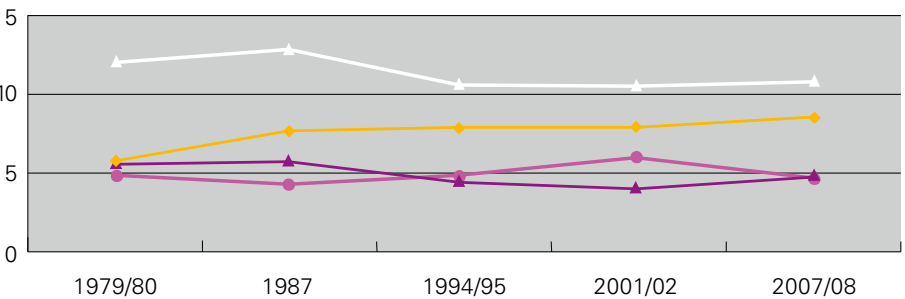

$\neg$ Hülsenfrüchte

$\rightarrow$ Nüsse, Samen

$\rightarrow-$ Fisch

$\leftrightarrow$ Eier

Gemüse: ohne Kartoffeln und Hülsenfrüchte

Milch, Milchprodukte: ohne Butter

Nichtalkoholische Getränke: nicht aufgeführt, da die in den verschiedenen Jahren verwendeten Datengrundlagen nicht vergleichbar sind

Den Gruppen zugehörende Lebensmittel siehe Tabellen 2.1-2.10.

Abbildung 2.3: Verbrauchsmengen der verschiedenen Lebensmittelgruppen von 1979/80 bis 2007/08 (in kg pro Kopf und Jahr) 
ren $(14 ; 15)$. Den grössten Rückgang weisen Weisskohl, Spitzkabis, Randen, Karotten, Sellerie, Treibzichorien, Kopf- und Eichblattsalat, Endivien, Tomaten und Küchenkräuter auf (Tabelle 2.1). Im Verbrauch zugenommen haben hingegen Broccoli, Spinat, Peperoni, Zucchetti, Eisbergsalat und Zwiebeln.

Durchschnittlich liegt der angenäherte Verzehr an Gemüse pro Person und Tag bei $209 \mathrm{~g}$ (Tabelle 2.12). Insgesamt entspräche dies durchschnittlich 1.7 Gemüseportionen, wenn man die Empfehlungen der SGE (1 Portion = $120 \mathrm{~g}$ ) als Grundlage nimmt (4). In Realität werden die Mengen aber tiefer liegen, da im Haushalt auftretende Verluste, z.B. durch Verderb, bei dieser Berechnungsweise nicht berücksichtigt sind. Auch laut der Schweizerischen Gesundheitsbefragung 2007 (SGB07) nehmen 87\% der Befragten weniger als dreimal täglich Gemüse zu sich (16; Kapitel 2.6.5). Bei den Männern liegt dieser Prozentsatz höher als bei den Frauen (91\% vs. $83 \%)$. Ergebnisse der Genfer Studie Bus Santé weisen auf eine signifikante Zunahme bei der durchschnittlichen Häufigkeit des Gemüseverzehrs zwischen 1993 und 2007 hin (17), sowohl bei den Männern (von 1.15- auf 1.35-mal pro Tag) wie auch bei den Frauen (von 1.30- auf 1.57-mal pro Tag). Angaben zur Häufigkeit des Gemüseverzehrs lassen jedoch keine Aussagen über die tatsächlichen Verzehrsmengen zu, was den Widerspruch zwischen den Resultaten der verschiedenen Datensätze erklären könnte.

\section{Früchte}

Der schon im 5. SEB erwähnte rückläufige Trend beim Verbrauch von Früchten setzt sich weiter fort und verstärkt sich noch (Tabelle 2.2 sowie Abbildungen 2.2 und 2.3). Vor allem die traditionellen Früchtesorten werden weniger nachge- fragt, denn hauptsächlich Äpfel, Birnen, Kirschen und Trauben sind vom Rückgang betroffen. Ein entgegengesetzter Trend zeigt sich hingegen bei vielen exotischen Früchten; so weisen Bananen, Ananas und auch Mango eine Verbrauchszunahme auf, die insgesamt jedoch geringer ausfällt als die Verbrauchsabnahmen der oben erwähnten Früchte.

Der angenäherte Verzehr von Früchten liegt bei durchschnittlich $172 \mathrm{~g}$ pro Person und Tag (Tabelle 2.12) und damit um $8 \mathrm{~g}$ unter dem im 5. SEB ausgewiesenen Wert von $180 \mathrm{~g}$ (18). Von der SGE werden $120 \mathrm{~g}$ als eine Tagesportion definiert (4), womit $172 \mathrm{~g} 1.4$ Portionen entsprechen. Zusammen mit dem angenäherten Verzehr an Gemüse erreicht die Schweizer Bevölkerung damit etwas über drei Portionen Gemüse und Früchte pro Tag. Dies liegt unter den empfohlenen fünf Portionen am Tag (Kapitel 1.3.4). Der angenäherte Verzehr an Fruchtsäften kann die Bilanz mit durchschnittlich $33 \mathrm{~g}$ pro Person und Tag (Tabelle 2.12) nur leicht verbessern. Dass die Empfehlungen in diesem Bereich nicht durchwegs erfüllt werden, zeigen auch die SGBO7 und die Genfer Studie Bus Santé (16;17). Beide Untersuchungen weisen ausserdem auf geschlechtsspezifische Unterschiede hin. Laut der SGB07 halten 38\% der Frauen, aber nur 21\% der Männer die Empfehlung «5 am Tag» ein (16; Kapitel 2.6.5).

\section{Getreide, Kartoffeln und Hülsenfrüchte}

Der im 5. SEB festgestellte leichte Rückgang des Gesamtgetreideverbrauchs setzt sich weiter fort. Nach dem Höchstwert in 1994/95 liegt er nun unter dem Niveau von 1987 (Tabelle 2.3). Weichweizen (Inlandproduktion und Import) macht rund $67 \%$ des Getreideverbrauchs aus, gegenüber 2001/02 hat sein Verbrauch um 7\% 
Tabelle 2.1: Durchschnittlicher Verbrauch an Gemüse pro Kopf und Jahr in kgab

\begin{tabular}{|c|c|c|c|c|c|}
\hline & $1979 / 80$ & 1987 & $1994 / 95$ & $2001 / 02$ & $2007 / 08$ \\
\hline Total Gemüse & 77.6 & 86.1 & $91.9^{\mathrm{c}}$ & $95.6^{\mathrm{C}}$ & 89.7 \\
\hline Kohlgewächse & 7.4 & 9.8 & 10.4 & 10.1 & 8.4 \\
\hline Blumenkohl & 2.1 & 2.7 & 2.7 & 2.1 & 2.0 \\
\hline Broccoli & 0.3 & 0.3 & 0.9 & 1.2 & 1.4 \\
\hline Chinakohl & - & - & - & $0.9^{d}$ & 0.9 \\
\hline Kohlrabi, Spitzkabis & 0.5 & 0.4 & 0.7 & 1.4 & - \\
\hline Kohlrabi & - & - & - & $0.7^{d}$ & 0.6 \\
\hline Spitzkabis & - & - & - & $0.70^{d}$ & 0.05 \\
\hline Rosenkohl & 0.4 & 1.0 & 0.4 & 0.2 & 0.3 \\
\hline Rot-, Weisskohl & 2.7 & 3.7 & 3.6 & 3.1 & - \\
\hline Rotkohl & - & - & - & $0.7^{d}$ & 0.6 \\
\hline Weisskohl & - & - & - & $2.2^{d}$ & 1.8 \\
\hline Wirsing & 1.4 & 1.0 & 1.0 & 1.0 & 0.8 \\
\hline Andere Kohlgewächse & - & 0.70 & 1.10 & 1.10 & 0.12 \\
\hline Wurzel- und Knollengewächse (ohne Kartoffeln) & 16.2 & 16.7 & 16.7 & 15.4 & 12.4 \\
\hline Fenchel & 1.9 & 1.2 & 1.6 & 1.6 & 1.5 \\
\hline Karotten & 8.4 & 9.3 & 8.7 & 9.2 & 7.8 \\
\hline Radieschen & 0.5 & 0.3 & 0.3 & 0.3 & 0.3 \\
\hline Randen & 2.1 & 1.9 & 1.7 & 1.4 & 0.9 \\
\hline Rettiche & 0.6 & 0.4 & 0.4 & 0.3 & 0.3 \\
\hline Schwarzwurzeln & 0.20 & 0.50 & 0.10 & 0.01 & 0.01 \\
\hline Sellerie & 1.8 & 1.5 & 1.4 & 1.7 & 1.3 \\
\hline Weisse Rüben & 0.1 & 0.8 & 0.7 & 0.4 & 0.3 \\
\hline AndereWurzel- und Knollengewächse & 0.60 & 0.80 & 1.80 & 0.50 & 0.02 \\
\hline Blattstiel- und Blattgemüse, Salate & 16.4 & 15.8 & 20.0 & 21.7 & 19.8 \\
\hline Artischocken & 0.30 & 0.20 & 0.20 & 0.20 & 0.15 \\
\hline Cicorino & - & - & - & 0.9 & 0.8 \\
\hline Endivien & 2.0 & 2.2 & 2.6 & 2.7 & 2.2 \\
\hline Eisbergsalat & - & - & - & 3.3 & 3.5 \\
\hline Kopfsalat, Eichblattsalat & 5.7 & 3.6 & 3.0 & 2.8 & 2.2 \\
\hline Krachsalat & - & - & - & 0.9 & 0.9 \\
\hline Küchenkräuter & 0.5 & 0.4 & 0.5 & 0.7 & 0.4 \\
\hline Lattich & 1.1 & 0.8 & 0.8 & 0.6 & 0.6 \\
\hline
\end{tabular}




\begin{tabular}{|c|c|c|c|c|c|}
\hline & $1979 / 80$ & 1987 & $1994 / 95$ & $2001 / 02$ & $2007 / 08$ \\
\hline Lollo & - & - & - & 1.4 & 1.2 \\
\hline Mangold & 0.6 & 0.4 & 0.4 & 0.4 & 0.3 \\
\hline Nüsslisalat & 0.3 & 0.3 & 0.6 & 0.5 & 0.5 \\
\hline Rhabarber & 0.60 & 0.30 & 0.30 & 0.20 & 0.14 \\
\hline Spargeln & 0.5 & 0.9 & 1.2 & 1.4 & 1.2 \\
\hline Spinat & 2.1 & 2.0 & 2.8 & 2.4 & 2.7 \\
\hline Treibzichorien & 2.1 & 1.8 & 2.0 & 2.0 & 1.3 \\
\hline Zuckerhut & 0.5 & 0.3 & 0.6 & 0.9 & 0.9 \\
\hline Andere Salate & 0.6 & 3.0 & 5.5 & 1.1 & 0.9 \\
\hline Zwiebelgewächse & 5.5 & 6.3 & 6.6 & 6.3 & 6.5 \\
\hline Lauch & 1.8 & 1.7 & 1.8 & 1.8 & 1.7 \\
\hline Knoblauch, Zwiebeln & 3.7 & 4.6 & 4.8 & 4.5 & - \\
\hline Knoblauch & - & - & - & $0.3^{d}$ & 0.4 \\
\hline Zwiebeln & - & - & - & $4.2^{\mathrm{d}}$ & 4.4 \\
\hline Fruchtgemüse & 15.5 & 15.9 & 18.2 & 20.1 & 19.6 \\
\hline Auberginen & 0.3 & 0.3 & 0.5 & 0.7 & 0.7 \\
\hline Gurken und Cornichons & 3.5 & 3.7 & 4.0 & 3.1 & 3.0 \\
\hline Kürbisse & - & - & - & $0.20^{\mathrm{d}}$ & 0.16 \\
\hline Peperoni & 1.3 & 1.6 & 2.2 & 2.5 & 2.8 \\
\hline Tomaten & 8.6 & 8.3 & 9.1 & 10.2 & 9.4 \\
\hline Zucchetti & 1.3 & 1.2 & 1.7 & 1.3 & 2.2 \\
\hline Andere Frischgemüse & - & 0.4 & 0.2 & 2.4 & 1.4 \\
\hline Gemüsekonserven importiert & 16.6 & 20.6 & $18.6^{\mathrm{c}}$ & $21.0^{c}$ & 21.7 \\
\hline Pilze & - & 1.0 & 1.4 & 1.3 & 1.3 \\
\hline
\end{tabular}

- = keine Daten vorhanden

a Die Verbrauchsdaten von 1979/80 stammen aus dem 2. SEB (9), diejenigen von 1987 aus dem 3. SEB (11), von 1994/95 aus dem 4. SEB (10) und von 2001/02 aus dem 5. SEB (8). Die Daten für 2007/08 beziehen sich auf die Statistiken und Erhebungen des Schweizerischen Bauernverbandes, Brugg (Agrarstatistik).

b Kartoffeln und Hülsenfrüchte finden sich neu in Tabelle 2.3.

c Nachträgliche Korrektur: Für 1994/95 und 2001/02 musste die Menge an importierten Gemüsekonserven rückwirkend nach oben korrigiert werden.

d Sind 2007/08 Daten für einzelne Lebensmittel verfügbar, die früher in Gruppen mit anderen Lebensmitteln zusammengefasst waren, so werden jeweils auch die Zahlen für 2001/02 angegeben. 
abgenommen. Roggen und Mischel ${ }^{1}$ sind hingegen nach der leicht rückläufigen Tendenz im 5. SEB wieder auf den Wert von 1994/95 angestiegen, was an einem höheren Einsatz von Mischel in der Lebensmittelproduktion liegt. Der erhöhte Mischelverbrauch wiegt die Abnahme beim Weichweizen jedoch nicht auf. Neben Mischel weist auch Dinkel eine substanzielle Verbrauchssteigerung auf. Bei der Gerste zeigt sich ein leichter Aufwärtstrend, der jedoch frühere Verbrauchszahlen nicht erreicht. Der Mais erfährt nach dem Anstieg zwischen dem 4. und 5. SEB (d.h. zwischen 1994/95 und 2001/02) wieder einen leichten Rückgang. Der von $1979 / 80$ bis 2001/02 kontinuierlich ansteigende Reisverbrauch ist auf dem Niveau von 2001/02 verblieben (Tabelle 2.3).

2007/08 liegt der angenäherte Verzehr von Getreide bei durchschnittlich $197 \mathrm{~g}$ pro Person und Tag (Tabelle 2.12). Darin enthalten sind die Getreidemengen, die über Brot- und Backwaren

\footnotetext{
1 Mischel ist eine Mischung aus 10-50\% Roggen und 50-90\% Weizen.
}

sowie andere verarbeitete Lebensmittel (z.B. Teigwaren) eingenommen werden, wie auch wenig verarbeitetes Getreide (z.B. in Form von Frühstücksflocken). Laut der Brotkonsumstatistik des Bundesamtes für Landwirtschaft macht der Verbrauch an Brot- und Backwaren (ohne brotähnliche Gebäcke) im gleichen Zeitraum durchschnittlich $133 \mathrm{~g}$ pro Person und Tag aus. In den Jahren 1992 bis 2009 bewegte er sich zwischen $125 \mathrm{~g}$ und $142 \mathrm{~g}$ pro Person und Tag $(19 ; 20)$.

Der Verbrauch an Kartoffeln hat sich seit dem 5. SEB nicht verändert (Abbildung 2.2). Er liegt weiterhin unter den Mengen, die von $1979 / 80$ bis 1994/95 ausgewiesen wurden (Tabelle 2.3). Der angenäherte Verzehr beläuft sich auf $96 \mathrm{~g}$ pro Person und Tag (Tabelle 2.12).

Bei den Hülsenfrüchten ging der Verbrauch in den drei vorangehenden SEB zurück, hat nun jedoch wieder angezogen. Er ist im Vergleich zum 5. SEB um 20\% gestiegen (Abbildung 2.2), zurückzuführen hauptsächlich auf einen Mehrverbrauch von getrockneten Hülsenfrüchten, was jedoch aufgrund der insgesamt geringen Menge kein substanzielles Wachstum darstellt.

Tabelle 2.2: Durchschnittlicher Verbrauch an Früchten pro Kopf und Jahr in kgab

\begin{tabular}{|c|c|c|c|c|c|}
\hline & $1979 / 80$ & 1987 & $1994 / 95$ & $2001 / 02$ & $2007 / 08$ \\
\hline Total Früchte & 101.0 & 88.5 & 84.8 & $82.5^{c}$ & 76.6 \\
\hline Ananas & - & - & - & 1.4 & 2.2 \\
\hline Äpfel & 29.0 & 20.2 & 18.5 & 18.0 & 15.8 \\
\hline Aprikosen & 2.4 & 3.9 & 2.5 & 1.7 & 1.6 \\
\hline Avocados & - & - & - & 0.5 & 0.6 \\
\hline Bananen & 8.8 & 8.9 & 9.5 & 9.0 & 9.3 \\
\hline Birnen & 6.8 & 4.1 & 4.2 & 4.5 & 3.3 \\
\hline
\end{tabular}




\begin{tabular}{|c|c|c|c|c|c|}
\hline & $1979 / 80$ & 1987 & $1994 / 95$ & $2001 / 02$ & $2007 / 08$ \\
\hline Datteln & - & - & - & 0.2 & 0.3 \\
\hline Erdbeeren & 2.7 & 3.2 & 3.5 & 3.1 & 3.0 \\
\hline Feigen & - & - & - & 0.4 & 0.4 \\
\hline Grapefruits & 2.4 & 1.6 & 1.5 & 1.2 & 1.0 \\
\hline Kirschen & 5.4 & 2.1 & 1.6 & 1.6 & 0.7 \\
\hline Kiwis & - & - & - & 1.4 & 1.4 \\
\hline Mangos, Guaven & - & - & - & 0.5 & 0.8 \\
\hline Melonen & 1.9 & 2.6 & 4.6 & 3.1 & 3.0 \\
\hline Oliven & - & - & - & 0.5 & 0.7 \\
\hline Orangen, Mandarinen & 14.9 & 15.7 & 14.8 & $12.9^{\mathrm{c}}$ & - \\
\hline Orangen & - & - & - & $8.1^{d}$ & 7.8 \\
\hline Mandarinen, Clementinen & - & - & - & $4.8^{d}$ & 4.9 \\
\hline Papayas & - & - & - & 0.1 & 0.2 \\
\hline Pfirsiche und Nektarinen & 4.6 & 5.3 & 4.4 & 4.0 & - \\
\hline Pfirsiche & - & - & - & $1.5^{d}$ & 1.2 \\
\hline Nektarinen & - & - & - & $2.5^{d}$ & 2.3 \\
\hline Pflaumen und Zwetschgen & 6.7 & 2.2 & 1.0 & 1.1 & 1.2 \\
\hline Tafeltrauben & 5.5 & 6.0 & 5.5 & 5.2 & 4.5 \\
\hline Wassermelonen & - & - & - & 1.6 & 1.7 \\
\hline Zitronen, Limetten & 2.5 & 3.2 & 2.6 & 2.3 & 2.1 \\
\hline Andere Beeren & 0.7 & 0.5 & 3.2 & 1.9 & 2.2 \\
\hline Andere Früchte & 1.5 & 3.2 & 3.1 & 1.2 & 1.7 \\
\hline Früchte getrocknet & - & - & - & $1.0^{d}$ & 1.0 \\
\hline Pulpen und Konserven importiert & 5.2 & 5.8 & 4.3 & 2.6 & - \\
\hline Fruchtkonserven & - & - & - & - & 1.8 \\
\hline
\end{tabular}

- = keine Daten vorhanden

a Die Verbrauchsdaten von 1979/80 stammen aus dem 2. SEB (9), diejenigen von 1987 aus dem 3 . SEB (11), von 1994/95 aus dem 4. SEB (10) und von 2001/02 aus dem 5. SEB (8). Die Daten für 2007/08 beziehen sich auf die Statistiken und Erhebungen des Schweizerischen Bauernverbandes, Brugg (Agrarstatistik).

b Fruchtsäfte: sieheTabelle 2.9 (nichtalkoholische Getränke).

c Nachträgliche Korrektur: Für 2001/02 musste die Menge an Orangen und Mandarinen rückwirkend nach unten korrigiert werden.

d Sind 2007/08 Daten für einzelne Lebensmittel verfügbar, die früher in Gruppen mit anderen Lebensmitteln zusammengefasst waren, so werden jeweils auch die Zahlen für 2001/02 angegeben. 
Tabelle 2.3: Durchschnittlicher Verbrauch an Getreide, Kartoffeln und Hülsenfrüchten pro Kopf und Jahr in $\mathrm{kg}^{\mathrm{a}}$

\begin{tabular}{|c|c|c|c|c|c|}
\hline & $1979 / 80$ & 1987 & $1994 / 95$ & $2001 / 02$ & $2007 / 08$ \\
\hline Total Getreide (in Mehl berechnet) & 69.9 & 72.2 & 74.0 & 72.7 & 71.8 \\
\hline Dinkel & - & - & - & 0.4 & 2.0 \\
\hline Gerste & 1.8 & 1.3 & 0.7 & 0.8 & 1.0 \\
\hline Hafer & 1.1 & 1.0 & 1.0 & 0.8 & 0.7 \\
\hline Mais & 1.5 & 1.7 & 1.5 & 2.1 & 1.8 \\
\hline Reis & 3.6 & 4.3 & 4.8 & 5.2 & 5.2 \\
\hline Roggen und Mischel' & 3.1 & 2.5 & 2.3 & 1.2 & - \\
\hline Roggen & - & - & - & $1.0^{b}$ & 1.1 \\
\hline Mischele & - & - & - & $0.3^{b}$ & 1.0 \\
\hline Weizen und Hartweizengriess & 58.8 & 61.4 & 63.7 & 62.6 & - \\
\hline Hartweizen & - & - & - & $11.0^{\mathrm{b}}$ & 11.0 \\
\hline Weichweizen & - & - & - & $51.9^{b}$ & 48.1 \\
\hline Kartoffeln und Kartoffelprodukte & 47.6 & 44.3 & 47.1 & 43.8 & 43.7 \\
\hline Hülsenfrüchte & 5.5 & 5.7 & 4.4 & 4.0 & 4.8 \\
\hline Bohnen & 2.8 & 2.8 & 2.2 & 1.9 & 2.0 \\
\hline Erbsen, Kefen & 1.6 & 1.6 & 1.0 & 0.7 & 0.8 \\
\hline Trockene Hülsenfrüchte & 1.1 & 1.3 & 1.2 & 1.1 & 1.8 \\
\hline Andere Hülsenfrüchte & - & - & - & $0.30^{\mathrm{b}}$ & 0.14 \\
\hline
\end{tabular}

- = keine Daten vorhanden

a Die Verbrauchsdaten von 1979/80 stammen aus dem 2. SEB (9), diejenigen von 1987 aus dem 3. SEB (11), von 1994/95 aus dem 4. SEB (10) und von 2001/02 aus dem 5. SEB (8). Die Daten für 2007/08 beziehen sich auf die Statistiken und Erhebungen des Schweizerischen Bauernverbandes, Brugg (Agrarstatistik).

b Sind 2007/08 Daten für einzelne Lebensmittel verfügbar, die früher in Gruppen mit anderen Lebensmitteln zusammengefasst waren, so werden jeweils auch die Zahlen für 2001/02 angegeben.

c Mischel ist eine Mischung aus 10-50\% Roggen und 50-90\% Weizen.

\section{Milch und Milchprodukte}

Der Gesamtverbrauch an Milch und Milchprodukten ist nach einer kontinuierlichen Abnahme zwischen 1979/80 und 2001/02 in 2007/08 erstmals stabil geblieben (Abbildung 2.3). Wie schon in den früheren SEB ging der Verbrauch an Konsummilch jedoch weiter zurück (Abbildung 2.2), was hauptsächlich durch einen Nach- fragerückgang bei der Vollmilch begründet ist. Hingegen hat sich der Verbrauch der im vorliegenden SEB erstmals separat ausgewiesenen Milchgetränke (z.B. Energy milk, Caffè latte, Ovo-Drink) seit 2001/02 verdoppelt (Tabelle 2.4). Der zwischen 1987/88 und 2001/02 konstant gebliebene Joghurtverbrauch hat seither um $11 \%$ zugenommen, und der im 5 . SEB kons- 
tatierte substanzielle Rückgang bei Magermilchpulver, einhergehend mit einer leichten Erhöhung des Verbrauchs an Vollmilchpulver, hat angehalten. Der Verbrauch von Rahm hat sich weiter reduziert und liegt nun auf dem Niveau von 1979/80, wobei der grösste Verbrauchsrückgang beim Kaffeerahm zu verzeichnen ist. Insgesamt ist der Käseverbrauch im Vergleich zu 2001/02 annähernd konstant geblieben, wobei der Trend auch weiterhin in Richtung Zunahme geht (Tabelle 2.4). Zu Käse liegt im vorliegenden SEB eine wesentlich detailliertere Sortenaufschlüsselung vor als in früheren SEB.

Der angenäherte Verzehr von Milch und Milchprodukten liegt bei $378 \mathrm{~g}$ pro Person und Tag (Tabelle 2.12). Laut der SGB07 konsumieren $65 \%$ der Schweizer Bevölkerung täglich Milch oder Milchprodukte (Kapitel 2.6.5). Neunzig Prozent der Befragten liegen dabei unter den von der SGE empfohlenen drei Portionen pro Tag. Gar keine Milch und Milchprodukte konsumieren 5\% der Befragten (16). Der Anteil der Personen, die nie Milch oder Milchprodukte konsumieren, ist damit seit der SGB02, wo er $1 \%$ betrug, deutlich angestiegen (21). Über mögliche Gründe für diese Veränderung liegen keine Angaben vor.

\section{Fleisch, Fisch und Eier}

Zwischen 1987 und 2001/02 hat der Fleischverbrauch gesamthaft um $20 \%$ abgenommen (Abbildung 2.3). Die Jahre 2007/08 zeigen nun eine Stagnation des Verbrauchs auf dem Niveau von 2001/02. Die verschiedenen $S G B$ bestätigen diesen Befund: Von 1992 bis 2002 nahm der Anteil der Personen, die täglich mehr als einmal Fleisch und/oder Wurstwaren konsumierten, von $24.8 \%$ auf $19.5 \%$ ab und blieb dann bis 2007 unverändert (16). Zu erkennen sind jedoch gewisse mengenmässige Verschiebungen bei den einzelnen Fleischsorten. So scheint sich der Rindfleischverbrauch von der BSE-Krise in den 1990 er Jahren langsam zu erholen, denn er hat zwischen 2001/02 und 2007/08 um 7\% zugenommen (Abbildung 2.2). Auch der Geflügelverbrauch ist nach einem leichten Rückgang 2001/02 wieder auf die Werte von 1994/95 angestiegen, und beim Verbrauch von Organteilen (Innereien) hat sich der Abwärtstrend in eine leichte Zunahme gewandelt (Tabelle 2.5). Beim Schweinefleisch setzt sich hingegen der seit 1987 zu beobachtende Trend zur Verbrauchsabnahme weiter fort (-2\% verglichen mit 2001/02). Beim Kalbfleisch fällt der Rückgang im Verbrauch noch wesentlich deutlicher aus $1-15 \%$ verglichen mit 2001/02; Abbildung 2.2 und Tabelle 2.5).

Der angenäherte Verzehr von Fleisch und Fleischprodukten liegt bei $130 \mathrm{~g}$ pro Person und Tag (Tabelle 2.12). Die SGE definiert 100-120 g Fleisch (Frischgewicht) als eine Portion, wobei Fleisch nicht täglich, sondern abwechslungsweise mit anderen Proteinquellen verzehrt werden soll (4).

In Bezug auf den Fischverbrauch zeigt sich eine Zunahme von $8 \%$ im Vergleich zum 5. SEB. Damit setzt sich der seit 1979/80 feststellbare Trend fort (Abbildung 2.3 und Tabelle 2.5).

Die Ernährungsempfehlungen lauten auf ein bis zwei Portionen Fisch pro Woche (Kapitel 1.5.1). Der angenäherte Verzehr von Fisch und Schalentieren liegt aktuell bei $119 \mathrm{~g}$ pro Person und Woche (Tabelle 2.12), was laut der SGE einer Portion entspricht (4).

Der Eierverbrauch ist seit 1994/95 mehr oder weniger konstant geblieben und hat sich auch 2007/08 nicht verändert (Tabelle 2.5).

Sechster Schweizerischer Ernährungsbericht | 63 
Tabelle 2.4: Durchschnittlicher Verbrauch an Milch und Milchprodukten pro Kopf und Jahr in kga

\begin{tabular}{|c|c|c|c|c|c|}
\hline & $1979 / 80$ & $1987 / 88$ & $1994 / 95$ & $2001 / 02$ & $2007 / 08$ \\
\hline Total Milch und Milchprodukte (ohne Butter) & 162.9 & 158.1 & 146.1 & 136.9 & 138.1 \\
\hline Total Konsummilch & 122.4 & 110.7 & 96.8 & 83.9 & 76.7 \\
\hline Magermilch & 4.3 & 5.7 & 3.0 & 2.3 & 2.1 \\
\hline Teilentrahmte Milch & 29.0 & 25.9 & 24.1 & 26.2 & 25.4 \\
\hline Vollmilch & 89.1 & 79.1 & 69.7 & 55.4 & 49.2 \\
\hline Milchgetränkeb & - & - & - & $3.4^{c}$ & 9.3 \\
\hline Total Joghurt, Kondensmilch und Milchpulver & 17.9 & 22.9 & 22.8 & 20.6 & 22.8 \\
\hline Joghurt & 13.4 & 16.7 & 16.9 & 16.5 & 18.3 \\
\hline Kondensmilch & 0.8 & 0.7 & 0.3 & 0.4 & 0.8 \\
\hline Magermilchpulver & 2.3 & 3.4 & 4.2 & 1.4 & 1.2 \\
\hline Vollmilchpulver & 1.4 & 2.1 & 1.4 & 2.3 & 2.6 \\
\hline Total Rahm (nicht in Vollrahm umgerechnet) & 8.8 & 10.1 & 9.6 & 9.3 & 8.4 \\
\hline Halbrahm & 0.4 & 1.3 & 1.7 & 2.0 & 1.8 \\
\hline Kaffeerahm & 5.1 & 5.3 & 4.6 & 3.6 & 3.3 \\
\hline Vollrahm & 3.3 & 3.5 & 3.3 & 3.7 & 3.4 \\
\hline Total Käse & 13.8 & 14.4 & 16.9 & 20.7 & 21.0 \\
\hline $\begin{array}{l}\text { Frischkäse (Mascarpone, Mozzarella, Quark, } \\
\text { Rohzieger, Ricotta) }\end{array}$ & 0.6 & - & 1.9 & 6.0 & - \\
\hline Mascarpone & - & - & - & $0.2^{c}$ & 0.2 \\
\hline Mozzarella & - & - & - & $2.2^{\mathrm{c}}$ & 2.4 \\
\hline Quark & - & - & - & $1.4^{c}$ & 1.3 \\
\hline Andere Frischkäse & - & - & - & $2.2^{c}$ & 2.7 \\
\hline Emmentaler & 1.5 & 1.6 & 1.1 & 0.7 & 0.8 \\
\hline Gruyère & 2.5 & 2.2 & 2.0 & 1.9 & 1.9 \\
\hline Parmigiano, Grana Padano & - & - & - & 0.8 & 0.6 \\
\hline Sbrinz & 0.4 & 0.4 & 0.3 & 0.2 & 0.2 \\
\hline Appenzeller & 0.5 & 0.5 & 0.4 & 0.4 & 0.4 \\
\hline Schweizer Raclette & - & - & - & $1.6^{c}$ & 1.3 \\
\hline Freiburger Vacherin & - & - & - & $0.3^{c}$ & 0.3 \\
\hline Tête de Moine & - & - & - & $0.1^{c}$ & 0.1 \\
\hline Tilsiter & 1.2 & 1.0 & 0.8 & 0.7 & 0.4 \\
\hline Andere Hart- und Halbhartkäse & 1.8 & 4.4 & 4.9 & 6.4 & - \\
\hline Andere Halbhartkäse & - & - & - & $2.8^{\mathrm{c}}$ & 3.4 \\
\hline
\end{tabular}




\begin{tabular}{|c|c|c|c|c|c|}
\hline & $1979 / 80$ & $1987 / 88$ & $1994 / 95$ & $2001 / 02$ & $2007 / 08$ \\
\hline Andere Hartkäse & - & - & - & - & 1.3 \\
\hline Weichkäse (Danablu, Gorgonzola, Roquefort, Brie) & 3.9 & 2.8 & 3.7 & 1.9 & 2.0 \\
\hline Fertigfondue & 0.4 & 0.4 & 0.5 & 0.5 & 0.5 \\
\hline Magerkäse & - & - & 0.1 & - & - \\
\hline Schabziger & - & - & - & $0.3^{c}$ & 0.2 \\
\hline Schmelzkäse & 1.0 & 1.1 & 1.2 & 1.2 & 1.1 \\
\hline
\end{tabular}

- = keine Daten vorhanden

a Die Verbrauchsdaten von 1979/80 stammen aus dem 2. SEB (9), diejenigen von 1987/88 aus dem 3 . SEB (11), von 1994/95 aus dem 4. SEB (10) und von 2001/02 aus dem 5. SEB (8). Die Daten für 2007/08 beziehen sich auf die Statistiken und Erhebungen des Schweizerischen Bauernverbandes, Brugg (Agrarstatistik).

b z.B. Energy milk, Caffè latte, Ovo-Drink

c Sind 2007/08 Daten für einzelne Lebensmittel verfügbar, die früher in Gruppen mit anderen Lebensmitteln zusammengefasst waren, so werden jeweils auch die Zahlen für 2001/02 angegeben.

\section{Nüsse und Samen}

Bei den Nüssen und Samen zeigt sich ein leichter Verbrauchsrückgang verglichen mit den Zahlen im 5. SEB, der vorwiegend auf einer Abnahme der Baumnussmenge beruht (Tabelle 2.6). Der Gesamtverbrauch bewegt sich jedoch immer noch innerhalb der bisher festgestellten Bandbreite.

\section{Öle und Fette}

Der Gesamtverbrauch an Ölen und Fetten hat im Vergleich zum 4. und 5. SEB leicht zugenommen (Tabelle 2.7). Ein Anstieg zeigt sich hauptsächlich beim Verbrauch an pflanzlichen Ölen und Fetten (ausgenommen Erdnussöl), wohingegen der Verbrauch an tierischen Ölen und Fetten (vor allem Schweineschmalz und andere tierische Öle und Fette) weiter rückläufig ist (Abbildung 2.2). Die seit 1987 nachweisbare Abkehr von tierischen Fetten und der Mehrverbrauch an pflanzlichen Ölen und Fetten entspricht der gängigen Empfehlung, welche den vermehrten Konsum mehrfach ungesättigter Fettsäuren (PUFA) auf Kosten der gesättigten empfiehlt (22). Spitzenreiter unter den Ölen ist noch immer das Sonnenblumenöl, obschon seit längerem aufgrund der Fettsäurenzusammensetzung hauptsächlich zum Konsum von Olivenund Rapsöl geraten wird (23;24; Kapitel 1.5.1). Diese beiden Ölsorten verzeichnen denn auch seit 2001/02 eine Verbrauchszunahme von knapp $25 \%$. Sonnenblumenöl wird als Zutat in vielen Fertigprodukten und verbrauchsfertigen Salatsaucen verwendet.

Insgesamt beläuft sich der angenäherte Verzehr an Ölen und Fetten auf $49 \mathrm{~g}$ pro Person und Tag (Tabelle 2.12), was leicht über den Empfehlungen der SGE liegt (4).

Sechster Schweizerischer Ernährungsbericht | 65 
Tabelle 2.5: Durchschnittlicher Verbrauch an Fleisch, Fisch und Eiern pro Kopf und Jahr in kga

\begin{tabular}{|c|c|c|c|c|c|}
\hline & $1979 / 80$ & 1987 & $1994 / 95$ & $2001 / 02$ & $2007 / 08$ \\
\hline Total Fleisch (ohne Fisch, Eier) & 62.8 & 65.7 & 57.7 & 52.7 & 52.8 \\
\hline Geflügel & 7.3 & 9.8 & 10.2 & 9.7 & 10.2 \\
\hline Kalb & 4.2 & 4.0 & 3.3 & 3.3 & 2.8 \\
\hline Kaninchen & 0.6 & 0.7 & 0.7 & 0.5 & 0.3 \\
\hline Pferd & 0.6 & 0.6 & 0.6 & 0.7 & 0.7 \\
\hline Rind & 15.0 & 15.9 & 12.8 & 9.8 & 10.5 \\
\hline Schaf & 1.1 & 1.1 & 1.3 & 1.5 & 1.3 \\
\hline Schwein & 29.7 & 30.0 & 25.2 & 24.6 & 24.0 \\
\hline Wild & 0.7 & 0.7 & 0.8 & 0.7 & 0.6 \\
\hline Ziegen & 0.1 & 0.1 & 0.1 & 0.1 & 0.1 \\
\hline Organteile (Innereien) & 3.4 & 2.7 & 2.6 & 1.8 & 2.2 \\
\hline Fleischkonserven & 0.1 & 0.1 & 0.1 & - & - \\
\hline Total Fische & 5.8 & 7.7 & 7.9 & 7.9 & 8.5 \\
\hline Fische, frisch & 2.8 & 3.7 & 4.1 & 3.8 & - \\
\hline Meerwasserfische (frisch oder gefroren) & - & - & - & $2.3^{b}$ & 2.3 \\
\hline Meerwasserfische (Konserven) & - & - & - & $2.5^{b}$ & 2.6 \\
\hline Süsswasserfische (frisch oder gefroren, Konserven) & - & - & - & $1.5^{\mathrm{b}}$ & 1.7 \\
\hline Krebs- undWeichtiere & - & - & - & $1.6^{b}$ & 1.9 \\
\hline Krebse, Fischkonserven & 3.0 & 4.0 & 3.8 & 4.1 & - \\
\hline Eier & 12.0 & 12.9 & 10.6 & 10.5 & 10.8 \\
\hline
\end{tabular}

- = keine Daten vorhanden

a Die Verbrauchsdaten von 1979/80 stammen aus dem 2. SEB (9), diejenigen von 1987 aus dem 3. SEB (11), von 1994/95 aus dem 4. SEB (10) und von 2001/02 aus dem 5. SEB (8). Die Daten für 2007/08 beziehen sich auf die Statistiken und Erhebungen des Schweizerischen Bauernverbandes, Brugg (Agrarstatistik).

b Sind 2007/08 Daten für einzelne Lebensmittel verfügbar, die früher in Gruppen mit anderen Lebensmitteln zusammengefasst waren, so werden jeweils auch die Zahlen für 2001/02 angegeben. 
Tabelle 2.6: Durchschnittlicher Verbrauch an Nüssen und Samen pro Kopf und Jahr in kga

\begin{tabular}{|c|c|c|c|c|c|}
\hline & $1979 / 80$ & 1987 & $1994 / 95$ & $2001 / 02$ & $2007 / 08$ \\
\hline Total Nüsse und Samen & 4.9 & 4.4 & 4.9 & 5.1 & 4.7 \\
\hline Acajounüsse & - & - & - & 0.09 & 0.12 \\
\hline Erdnüsse & 0.8 & 1.2 & 0.9 & 0.5 & 0.4 \\
\hline Haselnüsse, Baumnüsse & 3.2 & 2.3 & 2.4 & 2.5 & - \\
\hline Haselnüsse & - & - & - & $1.5^{b}$ & 1.5 \\
\hline Baumnüsse & - & - & - & $0.8^{b}$ & 0.4 \\
\hline Kastanien & - & - & 0.5 & 0.4 & 0.4 \\
\hline Kokosnüsse & - & - & - & 0.2 & 0.2 \\
\hline Leinsamen & - & - & - & 0.12 & 0.18 \\
\hline Mandeln & 0.9 & 0.9 & 1.1 & 1.1 & 1.3 \\
\hline Pistazien & - & - & - & 0.02 & 0.03 \\
\hline Sonnenblumenkerne & - & - & - & 0.20 & 0.26 \\
\hline
\end{tabular}

- = keine Daten vorhanden

a Die Verbrauchsdaten von 1979/80 stammen aus dem 2. SEB (9), diejenigen von 1987 aus dem 3. SEB (11), von 1994/95 aus dem 4. SEB (10) und von 2001/02 aus dem 5. SEB (8). Die Daten für 2007/08 beziehen sich auf die Statistiken und Erhebungen des Schweizerischen Bauernverbandes, Brugg (Agrarstatistik).

b Sind 2007/08 Daten für einzelne Lebensmittel verfügbar, die früher in Gruppen mit anderen Lebensmitteln zusammengefasst waren, so werden jeweils auch die Zahlen für 2001/02 angegeben.

Tabelle 2.7: Durchschnittlicher Verbrauch an Ölen und Fetten pro Kopf und Jahr in kga

\begin{tabular}{l|c|c|c|c|c} 
& $\mathbf{1 9 7 9 / 8 0}$ & $\mathbf{1 9 8 7}$ & $\mathbf{1 9 9 4 / 9 5}$ & $\mathbf{2 0 0 1 / 0 2}$ & $\mathbf{2 0 0 7 / 0 8}$ \\
\hline Total Öle und Fette & $\mathbf{2 4 . 9}$ & $\mathbf{2 1 . 7}$ & $\mathbf{2 2 . 2}$ & $\mathbf{2 2 . 6}$ & $\mathbf{2 3 . 8}$ \\
\hline Total pflanzliche Öle und Fette & 15.0 & 12.3 & 13.6 & 15.3 & 18.0 \\
\hline Erdnussöl & - & - & - & 1.1 & 0.5 \\
\hline Olivenöl & - & - & - & 1.3 & 1.6 \\
\hline Rapsöl & - & - & - & 2.8 & 3.5 \\
\hline Sonnenblumenöl & - & - & - & 6.0 & 6.9 \\
\hline Margarine und andere pflanzliche Öle & - & - & - & 4.1 & 5.7 \\
\hline Total tierische Öle und Fette & 9.9 & 9.4 & 8.6 & 7.3 & 5.9 \\
\hline Butter & 7.5 & 6.8 & 6.2 & 5.9 & 5.6 \\
\hline Schweineschmalz & 1.2 & 1.0 & 0.9 & 0.6 & 0.1 \\
\hline $\begin{array}{l}\text { Andere tierische Öle und Fette (Fischöle, Geflügelfett, } \\
\text { Rinderfett) }\end{array}$ & 1.2 & 1.6 & 1.5 & 0.8 & 0.3 \\
\hline
\end{tabular}

- = keine Daten vorhanden

a Die Verbrauchsdaten von 1979/80 stammen aus dem 2. SEB (9), diejenigen von 1987 aus dem 3. SEB (11), von 1994/95 aus dem 4. SEB (10) und von 2001/02 aus dem 5. SEB (8). Die Daten für 2007/08 beziehen sich auf die Statistiken und Erhebungen des Schweizerischen Bauernverbandes, Brugg (Agrarstatistik). 


\section{Zucker und Honig}

Im vorliegenden SEB wird zusätzlich zum Zuckerverbrauch neu auch der Verbrauch von importiertem Sirup erfasst (Tabelle 2.8), bei dem es sich hauptsächlich um Glucose- (ca. 85\%) und Fructosesirup (ca. 10\%) handelt. Ausserdem musste der Zuckerverbrauch von 2001/02 rückwirkend nach unten korrigiert werden (siehe Fussnote e in Tabelle 2.8). Wird der Sirup dem Zucker hinzugerechnet, blieb der Verbrauch zwischen 1987 und 2001/02 konstant und nahm in den letzten Jahren etwas zu (Abbildung 2.3). Der Honigverbrauch hält das bisherige Niveau.

Angaben des Verbands Schweizerischer Schokoladefabrikanten (Chocosuisse) bezüglich Schokoladenverbrauch weisen für die Schweiz einen Pro-Kopf-Verbrauch von $12.0 \mathrm{~kg}$ Schokolade im Jahr 2010 aus (25). Darin inbegriffen sind auch Einkäufe von Feriengästen und Grenzgängern.

\section{Nichtalkoholische Getränke}

Bei den nichtalkoholischen Getränken zeigt sich eine Zunahme des Kaffeeverbrauchs, wohingegen sich der Tee- und Kräuterteeverbrauch nicht verändert hat (Tabelle 2.9). Der Kakao ist nach dem Rückgang im 2001/02 wieder auf das Niveau von 1994/95 zurückgekehrt. Bei den Fruchtsäften wird neu auch der Grapefruitsaft separat ausgewiesen, der damit nicht mehr in den "anderen Fruchtsäften» enthalten ist. Insgesamt ist der Fruchtsaftverbrauch auf das bisher tiefste Niveau seit Veröffentlichung des ersten SEB gesunken. Der Mineralwasserverbrauch liegt im Vergleich zum 5. SEB höher.

Zahlen des Marktforschungsinstituts AC Nielsen (Marktzahlen des Detailhandels aus der ganzen Schweiz; 26) weisen einen Pro-Kopf-Verbrauch von 136 L nichtalkoholischer Getränke aus, davon sind 46\% Mineralwasser und 54\% Süssgetränke (wozu auch Frucht- und Gemüsesäfte gezählt werden). Rund die Hälfte der Süss-

Tabelle 2.8: Durchschnittlicher Verbrauch an Zucker und Honig pro Kopf und Jahr in kga

\begin{tabular}{|c|c|c|c|c|c|}
\hline & $1979 / 80$ & 1987 & $1994 / 95$ & $2001 / 02$ & $2007 / 08$ \\
\hline Honig & 1.0 & 1.3 & 1.4 & 1.4 & 1.3 \\
\hline Sirup für Getränkebc & - & - & - & $2.2^{d}$ & 2.6 \\
\hline Zucker (Saccharose) ${ }^{c}$ & 40.9 & 43.0 & 43.1 & $40.7^{e}$ & 42.4 \\
\hline
\end{tabular}

- = keine Daten vorhanden

a Die Verbrauchsdaten von 1979/80 stammen aus dem 2. SEB (9), diejenigen von 1987 aus dem 3. SEB (11), von 1994/95 aus dem 4. SEB (10) und von 2001/02 aus dem 5. SEB (8). Die Daten für 2007/08 beziehen sich auf die Statistiken und Erhebungen des Schweizerischen Bauernverbandes, Brugg (Agrarstatistik).

b hauptsächlich Glucosesirup (ca. 85\%) und Fructosesirup (ca. 10\%)

c EinTeil des Sirup- und Zuckerverbrauchs wird für die Herstellung von Süssgetränken verwendet.

d Sind 2007/08 Daten für einzelne Lebensmittel verfügbar, die früher in Gruppen mit anderen Lebensmitteln zusammengefasst waren, so werden jeweils auch die Zahlen für 2001/02 angegeben.

e Korrigierter Wert: Nachträglich wurde festgestellt, dass von der im 5. SEB angegebenen Zuckermenge 7.0 kg in Form von Veredelungsprodukten wieder exportiert wurden. 
Tabelle 2.9: Durchschnittlicher Verbrauch an nichtalkoholischen Getränken pro Kopf und Jahr in kga

\begin{tabular}{|c|c|c|c|c|c|}
\hline & $1979 / 80$ & 1987 & $1994 / 95$ & $2001 / 02$ & $2007 / 08$ \\
\hline$K_{\text {affee }}^{b}$ & 7.4 & 7.5 & 8.3 & 8.0 & 10.2 \\
\hline Kakaob & 6.0 & 5.0 & 5.5 & 4.5 & 5.5 \\
\hline Tee und Kräutertee ${ }^{b}$ & 0.3 & 0.3 & 0.3 & 0.3 & 0.3 \\
\hline Mineralwasser ${ }^{c}$ & - & - & - & 106.9 & 116.5 \\
\hline Total Fruchtsäfte & 17.8 & 17.8 & 13.2 & 15.2 & 12.0 \\
\hline Grapefruitsaft & - & - & - & - & 0.4 \\
\hline Orangensaft & - & - & - & 6.0 & 5.5 \\
\hline Traubensaft & - & - & - & 2.5 & 1.9 \\
\hline Andere Fruchtsäfte & 17.8 & 17.8 & 13.2 & 6.7 & 4.2 \\
\hline
\end{tabular}

- = keine Daten vorhanden

a Die Verbrauchsdaten von 1979/80 stammen aus dem 2. SEB (9), diejenigen von 1987 aus dem 3. SEB (11), von 1994/95 aus dem 4. SEB (10) und von 2001/02 aus dem 5. SEB (8). Die Daten für 2007/08 beziehen sich auf die Statistiken und Erhebungen des Schweizerischen Bauernverbandes, Brugg (Agrarstatistik).

b Angaben in Trockensubstanz

c ohne Süssgetränke (Süssgetränke sind in Tabelle 2.8 miterfasst, siehe Fussnote c)

Tabelle 2.10: Durchschnittlicher Verbrauch an alkoholischen Getränken pro Kopf ${ }^{a}$ und Jahr in Liter ${ }^{b}$

\begin{tabular}{l|c|c|c|c|c} 
& $1979 / 80$ & 1987 & $1994 / 95$ & $2001 / 02$ & $2007 / 08$ \\
\hline Total akoholische Getränke & 157.7 & 157.9 & 139.0 & 126.6 & 122.3 \\
\hline Bier & 86.6 & 85.6 & 77.2 & 67.8 & 69.0 \\
\hline Obstwein & 6.2 & 5.9 & 3.9 & 2.9 & 2.0 \\
\hline Spirituosen 40Vol.-\% & 6.6 & 6.2 & 4.6 & 4.7 & 4.8 \\
\hline Wein & 58.3 & 60.2 & 53.3 & 51.2 & 46.6 \\
\hline
\end{tabular}

a nur Personen über 15 Jahre

b Die Verbrauchsdaten von 1979/80 stammen aus dem 2. SEB (9), diejenigen von 1987 aus dem 3. SEB (11), von 1994/95 aus dem 4. SEB (10) und von 2001/02 aus dem 5. SEB (8). Die Daten für 2007/08 beziehen sich auf die Statistiken und Erhebungen des Schweizerischen Bauernverbandes, Brugg (Agrarstatistik). 
getränke machen die Süsswasser (alle süssen Getränke ausser Frucht- und Gemüsesäfte, Sport- und Energydrinks, Eistee) aus. Die realen Verbrauchsmengen werden hier jedoch, wie das auch beim Mineralwasser der Fall ist, höher liegen, da der Ausser-Haus-Konsum sowie der Verkauf bei kleineren Detaillisten und an Automaten nicht erfasst werden. Die Zahlen sollten deshalb mit Vorsicht aufgenommen werden.

Über den Verbrauch von Leitungswasser als Getränk können keine Angaben gemacht werden.

\section{Alkoholische Getränke}

Die Pro-Kopf-Verbrauchszahlen für alkoholische Getränke werden basierend auf der Bevölkerungszahl der Personen im Alter von über 15 Jahren berechnet. Insgesamt hat sich der Verbrauch alkoholischer Getränke seit dem 5. SEB um 3\% verringert, womit sich der seit 1987 feststellbare rückläufige Trend fortsetzt (Abbildungen 2.2 und 2.3). Der aktuelle Rückgang ist hauptsächlich auf einen geringeren Wein- und Obstweinverbrauch zurückzuführen (Tabelle 2.10). Auch laut der Eidgenössischen Alkoholverwaltung ist der Verbrauch an alkoholischen Getränken seit Jahrzehnten rückläufig (27). Nur bei Bier und Spirituosen zeigte sich zwischen 2005 und 2008 eine leichte Zunahme, wohingegen 2009 ein Rückgang zu vermelden war. Der Pro-Kopf-Verbrauch reinen Alkohols in der Wohnbevölkerung der Schweiz (d.h. über alle Einwohner gerechnet, nicht nur diejenigen älter als 15 Jahre) lag 2007 bei 8.8 L, 2008 bei $8.7 \mathrm{~L}, 2009$ bei $8.6 \mathrm{~L}$ und 2010 bei $8.5 \mathrm{~L}$; im Jahr 2000 hatte er noch durchschnittlich $9.2 \mathrm{~L}$ pro Jahr betragen (Abbildung 2.4).

Der tägliche Durchschnittskonsum in der Gesamtbevölkerung ab 15 Jahren ist laut Zahlen der SGB zwischen 1997 und 2002 von $10.5 \mathrm{~g}$ auf $9.8 \mathrm{~g}$ reinen Alkohols gesunken (28). Berücksichtigt man nur die Konsumierenden, so hat sich hingegen deren Durchschnittskonsum kaum verändert (1997: 13.0 g, 2002: 12.8 g reiner Alkohol).

Der Alkoholkonsum variiert individuell sehr stark. Die fortlaufenden Daten der SGB zeigen bei den Frauen seit 1997 eine leichte Abnahme der Abstinenten und seltenen Konsumenten von Alkohol bei gleichzeitiger Zunahme der Gelegenheitstrinker (ein- bis zweimal wöchentlich), während der Anteil Frauen mit täglichem Alkoholkonsum relativ konstant geblieben ist (Abbildung 2.5). Auch bei den Männern hat der gelegentliche Alkoholkonsum zugenommen; im Gegensatz zu den Frauen ist bei den Männern jedoch die Zahl der täglichen Konsumenten rückläufig und die seltenen Konsumenten und Abstinenten blieben zahlenmässig relativ konstant (29). Die Hälfte des konsumierten Alkohols wird von $12.5 \%$ der erwachsenen Bevölkerung getrunken (30). Generell konsumieren Männer häufiger Alkohol als Frauen (31); es finden sich bei den Männern auch zweimal mehr Konsumenten mit risikoreichem Trinkverhalten (> $60 \mathrm{~g}$ reiner Alkohol/Tag bei Männern, > $40 \mathrm{~g} / \mathrm{Tag}$ bei Frauen) als bei den Frauen ( $2 \%$ vs. 1\% im Jahr 2007). In der Schweiz leben schätzungsweise rund 250'000 alkoholabhängige Personen (32) . Die Mehrheit der Schweizer Bevölkerung ab 15 Jahren (95.5\% im Jahr 2007) konsumiert Alkohol jedoch auf eine risikoarme Art und Weise oder lebt abstinent, wobei der Anteil dieser beiden Gruppen zwischen 1997 und 2007 angestiegen ist (31).

In der Westschweiz und im Tessin trinken deutlich mehr Personen täglich Alkohol als in der Deutschschweiz. Auch der Durchschnittskonsum an Alkohol liegt in diesen beiden Lan- 


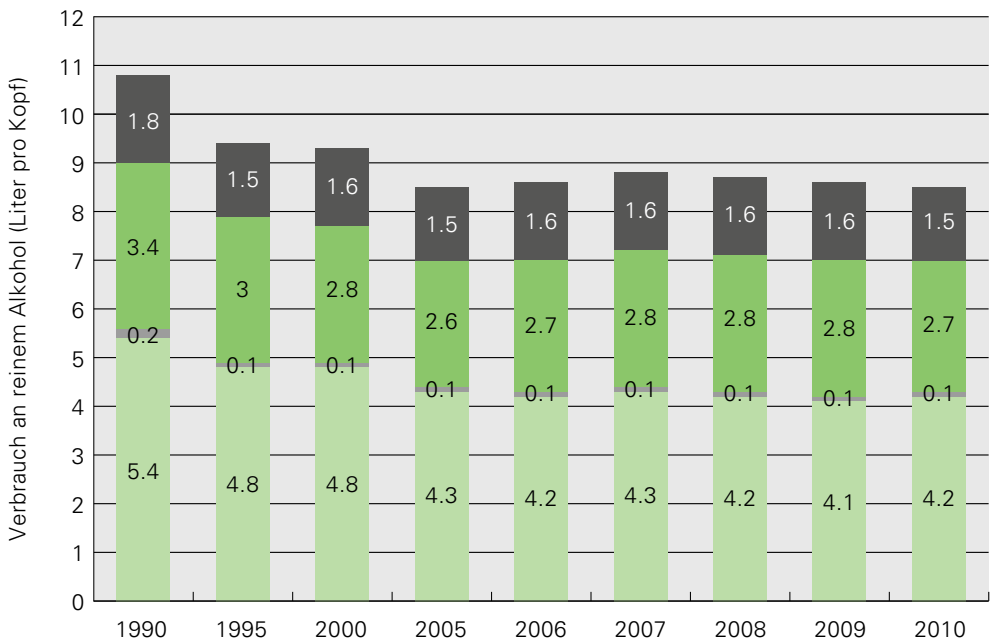

Spirituosen (40 Vol.-\%)

Bier (4.8 Vol.-\%)

- Obstwein (4.5 Vol.-\%)

Wein (11 Vol.-\%)

Abbildung 2.4: Jährlicher Pro-Kopf-Verbrauch alkoholischer Getränke in der Schweiz zwischen 1990 und 2010 (Liter reiner Alkohol; 27)

desteilen höher als in der Deutschschweiz (hauptsächlich in Bezug auf Wein). Die Deutschschweiz weist im regionalen Vergleich den grössten Anteil Gelegenheitstrinker (ein- bis zweimal pro Woche) auf, wohingegen der höchste Anteil an Abstinenten im Tessin zu finden ist (29).

Laut Erhebungen in der CoLaus-Studie trinken $84.0 \%$ der befragten Männer und $63.1 \%$ der Frauen Alkohol. 53.4\% der Männer bzw. $56.3 \%$ der Frauen kommen auf 1-13 Einheiten pro Woche, $26.8 \%$ bzw. $6.5 \%$ auf $14-34$ Einheiten pro Woche und $3.8 \%$ bzw. $0.3 \%$ auf mehr als 34 Einheiten, wobei eine Einheit als ein Glas Wein, eine Flasche Bier oder eine kleine Menge eines hochprozentigen alkoholischen Getränks (10-12 g Ethanol/Einheit) definiert ist (33). In der gleichen Studienpopulation gaben $73 \%$ an, in der letzten Woche Alkohol getrunken zu haben (34). Bei 55\% lag das Alkoholkonsumrisiko tief, bei $16 \%$ in einem mittleren bis hohen und bei $2 \%$ in einem sehr hohen Bereich. Laut der Genfer Studie Bus Santé bejahten in den Jahren 1993 bis 2007 durchschnittlich 84-94\% der befragten Männer, in den letzten vier Wochen Alkohol getrunken zu haben; bei den Frauen belief sich der Anteil auf 72-83\%, wobei der Anteil bei beiden Geschlechtern abnimmt (17). Auch die konsumierte Menge an Alkohol geht zurück, dies hauptsächlich bei den Männern; bei den Frauen liegt sie auf tieferem Niveau und die Abnahme ist geringer.

Bei jüngeren Schweizern (im Alter zwischen 15 und 24 Jahren) liegt die Abstinenzrate mit 29.3\% höher als im späteren Leben, erst im Alter steigt sie wieder an (31). Eine Erhebung im 


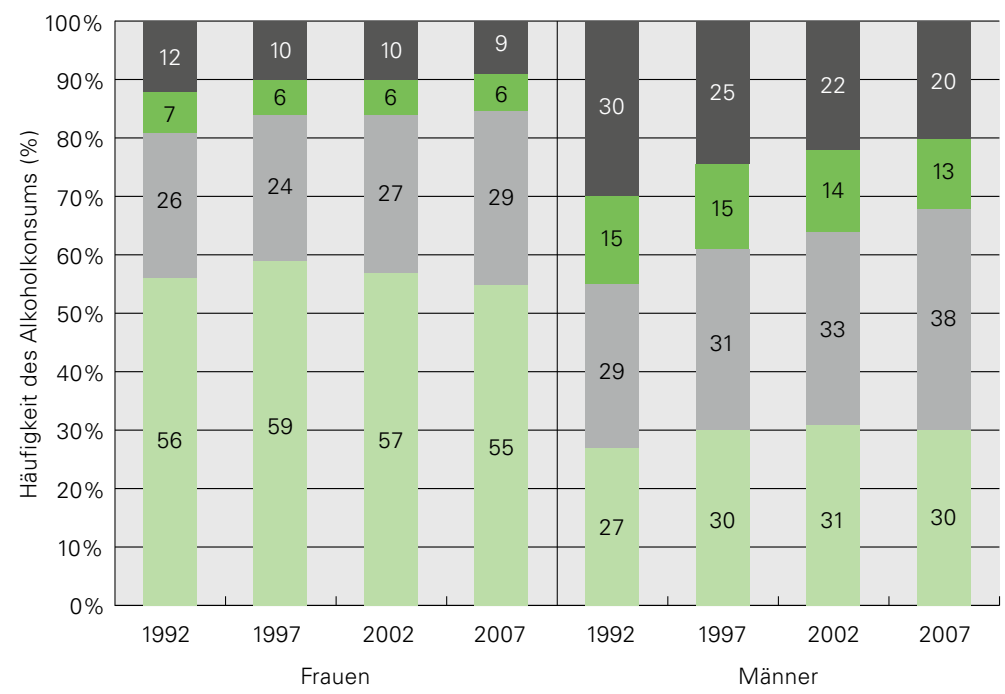

ein- oder mehrmals täglich

- mehrmals pro Woche

ein- bis zweimal wöchentlich

weniger als einmal pro Woche oder abstinent

Abbildung 2.5: Häufigkeit des Alkoholkonsums (in \%) nach Geschlecht (Gesamtbevölkerung ab 15 Jahren) zwischen 1992 und 2007 (29)

Lausanner Militärrekrutierungszentrum im Jahr 2007 bei 3'536 Männern im Alter von 19 Jahren ergab, dass nur $7.2 \%$ der Befragten in den letzten 12 Monaten keinen Alkohol konsumiert hatten (35). Der grösste Teil (75.5\%) dieser 19-jährigen Westschweizer wies ein episodisch-risikoreiches Konsumverhalten (Rauschtrinken) auf mit einer hohen Alkoholzufuhr an 1 bis 3 Tagen pro Woche (hauptsächlich am Wochenende). Ein episodisch zu hoher Alkoholkonsum nimmt mit zunehmendem Alter ab, wohingegen der regelmässig-risikoreiche Konsum zunimmt (28). Die Schülerbefragung Health Behaviour in School-aged Children (HBSC) 2006 weist darauf hin, dass in der Schweiz rund ein Viertel der 15-jährigen Knaben und knapp $18 \%$ der gleichaltrigen Mädchen mindestens einmal wöchentlich Alkohol trinken (36). Die neusten Zahlen aus dem Jahr 2010 zeigen geringfügig höhere Werte bei den Knaben (27\%) und tiefere (13\%) bei den Mädchen (37). Bei den 11-jährigen Knaben ist der wöchentliche Alkoholkonsum rückläufig, bei den Mädchen desselben Alters auf tiefem Niveau stabil; kein ausgeprägter Trend zeigt sich bei den 13-Jährigen (Abbildung 2.6). Die bevorzugten Getränke bei den 15-Jährigen sind Bier und Alcopops. Nach einem substanziellen Anstieg im Jahr 2002 lag der Alcopopkonsum 2006 wieder deutlich tiefer $19.6 \%$ vs. $17.2 \%$ der Mädchen; $9.6 \%$ vs. $17.3 \%$ der Knaben), was in Zusammenhang stehen könnte mit der breiten Medienarbeit im Jahr 2003 sowie dem 2004 eingeführten Sondersteuersatz auf süssen Mischgeträn- 


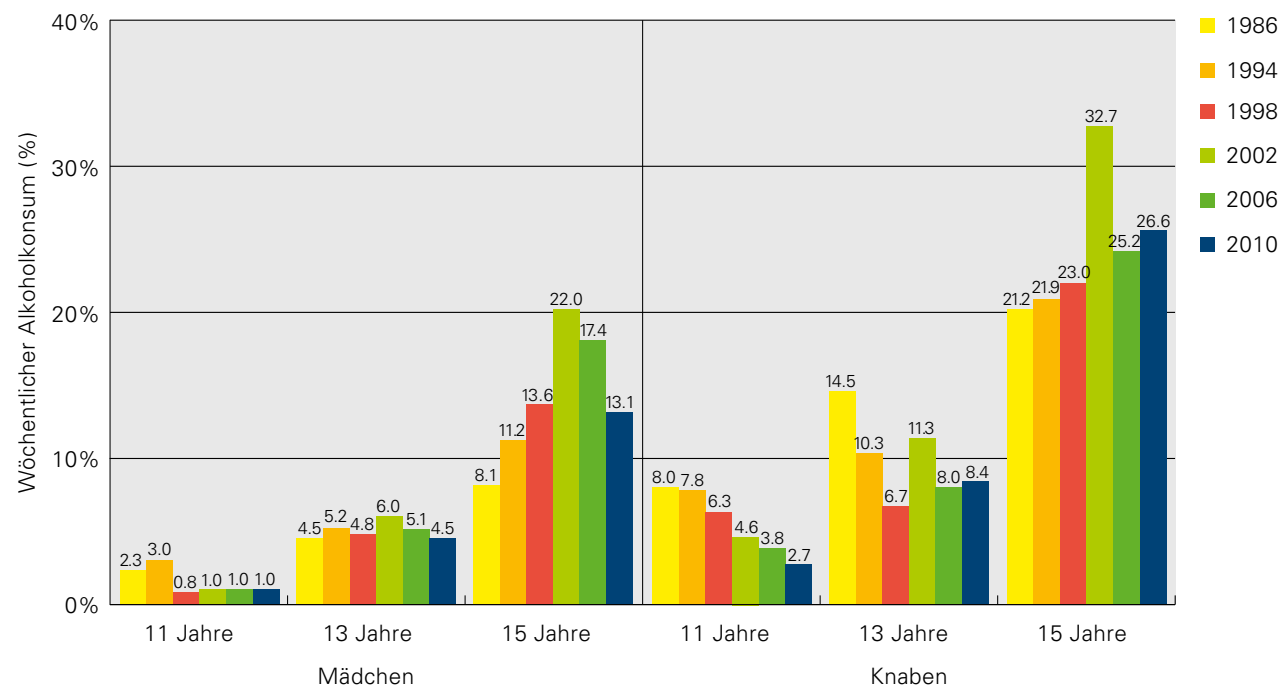

Abbildung 2.6: Prozentuale Anteile der 11- bis 15-jährigen Knaben und Mädchen mit wöchentlichem Alkoholkonsum (mindestens einmal pro Woche) zwischen 1986 und 2010 (37)

ken (37;38). Der Trend ist auch weiterhin rückläufig: Im Jahr 2010 konsumierten noch 9.1\% der Knaben und 6.1\% der Mädchen Alcopops (37). Bei den Knaben stieg der wöchentliche Bierkonsum zwischen 2006 und 2010 von $19.1 \%$ auf $20.6 \%$ an, bei den Mädchen ging der Anteil von $9.1 \%$ auf $6.6 \%$ zurück. Im Jahr 2010 zeigte sich im Vergleich zum Jahr 2006 eine starke Zunahme beim Konsum von Likör und Spirituosen: Knapp doppelt so viele 15-jährige Knaben und Mädchen konsumierten mindestens wöchentlich Likör und Spirituosen $19.8 \%$ vs. $5.0 \%$ der Knaben und $5.2 \%$ vs. $2.3 \%$ der Mädchen). Die Stärke der Zunahme könnte jedoch methodologisch bedingt leicht überschätzt sein (37).
Befragte Personen tendieren dazu, den Alkoholkonsum zu unterschätzen oder auch aufgrund sozialer Erwünschtheit bewusst zu tief anzugeben (28). Hinzu kommt, dass hoch risikoreich Konsumierende teilweise nicht befragt werden können (z.B. Randständige). Dies sollte bei der Beurteilung der obigen Angaben berücksichtigt werden. Zu den gesundheitlichen Folgen des Alkoholkonsums siehe Kapitel 3.4.2 und 3.5.2. 


\subsubsection{Ausblick}

Die Gesamtverbrauchsmengen der einzelnen Lebensmittelgruppen weisen gegenüber dem 5. SEB keine grossen Veränderungen auf. Die rückläufigen Trends im Verbrauch von Fleisch und Fleischprodukten sowie von Milch und Milchprodukten setzten sich nicht weiter fort; hingegen nahm der Verbrauch von Früchten und auch von alkoholischen Getränken weiter ab. Der Gesamtgemüseverbrauch hat nach einer steten Zunahme zum ersten Mal einen geringen Rückgang verzeichnet. Öle und Fette sowie Fische und Schalentiere konnten einen leichten Anstieg im Verbrauch verbuchen. Auch wenn die Mengenveränderungen der einzelnen Lebensmittelgruppen im Vergleich zum 5. SEB insgesamt gering sind, so zeigen sich innerhalb der einzelnen Lebensmittelgruppen teilweise grössere Verschiebungen von einem Lebensmittel zum anderen.

Verbrauchsdaten zeichnen sich durch Aktualität und Kontinuität und damit durch eine gute Vergleichbarkeit aus. Es handelt sich bei ihnen aber nicht um individuelle Verzehrsdaten, was ihre Aussagekraft einschränkt. Ausserdem sind die Daten wenig differenziert; sie geben keinerlei Hinweise auf die Lebensmittelversorgung einzelner Bevölkerungsgruppen oder Regionen und auch nicht bezüglich saisonaler Schwankungen. Es kann deshalb nur ein relativ grobes Bild der Ernährungssituation in der Schweiz gezeichnet werden. In der Schweiz fehlen weiterhin repräsentative, nationale Daten zu den verzehrten Lebensmittelmengen sowohl im Haushalt wie auch ausser Haus, differenziert nach Alter, Geschlecht, Bildungsniveau, Region u.a.

\subsection{Energie- und Nährstoff- versorgung der Schweizer Bevölkerung}

\subsubsection{Berechnungsgrundlagen}

Die Berechnungen des Verbrauchs und des angenäherten Verzehrs an Energie, energieliefernden Nährstoffen, Nahrungsfasern und Mikronährstoffen dienen dazu, die durchschnittliche Versorgung der Schweizer Bevölkerung mit den Zufuhrempfehlungen zu vergleichen. Die Daten lassen dank der über Jahre hinweg gleichbleibenden Methodik Schlüsse auf Veränderungen in der Nährstoffversorgung zu; es lassen sich jedoch keine Aussagen über den Versorgungsgrad einzelner Bevölkerungsgruppen machen. Die Verwendung unterschiedlicher Nährwertdatenbanken und -angaben zur Berechnung der Energie, Makro- und Mikronährstoffe kann in den einzelnen SEB zu leicht voneinander abweichenden Zahlen führen, was einschränkend berücksichtigt werden muss (39).

Berechnung des Verbrauchs und angenäherten Verzehrs an Energie, energieliefernden Nährstoffen, Nahrungsfasern und Mikronährstoffen Die durchschnittlichen Verbrauchsmengen an Lebensmitteln in den Jahren 2007/08 (Kapitel 2.3) dienen als Grundlage für die Berechnungen des Verbrauchs an Energie, energieliefernden Nährstoffen, Nahrungsfasern und Mikronährstoffen. Der angenäherte Verzehr wurde auf der Basis der Verbrauchsmengen unter Abzug lebensmittelspezifischer Abfälle und Verluste kalkuliert, wie in Kapitel 2.3.1 dargelegt. Wie schon beim 5. SEB wurden die Schweizer Nährwertdaten für die Berechnungen herange- 
zogen (18;40). In der Schweizer Nährwertdatenbank fehlende Angaben zu Mikronährstoffen wurden aus deutschen Nährwerttabellen und -datenbanken ergänzt $(13 ; 41)$. Für Fleischprodukte wurden aktuelle Analysendaten der Forschungsanstalt Agroscope Liebefeld-Posieux ALP verwendet (42-44). Neben der Energieversorgung wurde die Versorgung mit 31 Nährstoffen und Nährstoffkomponenten berechnet. Für Transfettsäuren (TFA), Vitamin K, Biotin, Kupfer, Mangan, Fluor und Selen konnte der Verbrauch mangels Daten nicht bestimmt werden.

Für die Berechnungen wurden die verbrauchten Lebensmittel den ihnen entsprechenden Lebensmitteln in der Schweizer Nährwertdatenbank zugeordnet. Waren die Lebensmittelbezeichnungen nicht eindeutig oder nur Angaben zu Lebensmittelgruppen vorhanden, wurden die Werte für das entsprechend am häufigsten verwendete Lebensmittel oder ein Mittelwert der häufigsten Sorten, Arten oder Verarbeitungsformen verwendet. Diese Zuordnungen sind in Tabelle 2.11 aufgeführt.

Der Verbrauch an Energie, energieliefernden Nährstoffen, Nahrungsfasern und Mikronährstoffen von Kaffee-, Tee- und Kräuterteepulver wurde als Getränk berechnet und in der Lebensmittelgruppe "nichtalkoholische Getränke» erfasst. Das Mineralwasser wurde aus den Nährstoffberechnungen ausgeschlossen, da die individuellen Wasser sehr unterschiedliche Mineralstoffmengen bereitstellen und keine Angaben bezüglich Verbrauchsmengen der einzelnen Wasser zur Verfügung stehen. Die Umrechnung des Verbrauchs an Rind-, Kalb- und Schweinefleisch auf einzelne Fleischstücke erfolgte auf Basis des von der GfK Switzerland AG, Hergiswil, für Privathaushaltungen ermittelten Fleischkonsums für die Jahre 2007 und 2008 (45).
Alle Berechnungen wurden mit MS Excel ${ }^{\circledR}$ durchgeführt. Die angegebenen Werte beziehen sich auf den essbaren Anteil des unverarbeiteten Lebensmittels. Nicht mit einbezogen sind allfällige Nährstoffverluste durch Lagerung bzw. Zubereitung der Lebensmittel im Haushalt. Dies muss bei der Interpretation der Daten berücksichtigt werden. Ausserdem können sich durch die Verwendung der neuen Analysendaten bei den Fleischprodukten im Vergleich zu den Berechnungen in früheren SEB trotz gleichbleibendem Lebensmittelverbrauch Veränderungen in den Energie- und Nährstoffwerten ergeben.

In den berechneten Angaben ist die Zufuhr an Mikronährstoffen über Vitamin- und Mineralstoffsupplemente sowie angereicherte Lebensmittel nicht berücksichtigt. Die einzige Ausnahme bilden vitaminisierte Margarinen und Milchgetränke. Die Lausanner CoLaus-Studie ergab, dass $25.7 \%$ der befragten Studienteilnehmer im Alter von 35-75 Jahren die Nahrung in irgendeiner Form mit Nährstoffen ergänzen (46). Das ist doppelt so viel, wie im Jahr 1997 in der $S G B(47)$ ermittelt wurde $(13.1 \%)$. Von $19.8 \%$ der Befragten werden Vitamin- oder Mineralstoffsupplemente eingenommen, von 10.0\% Nahrungsergänzungen wie z.B. n-3-und n-6-Fettsäuren, Tier- bzw. Pflanzenextrakte (z.B. Ginseng, Gelée Royale, Haifischknorpel) oder Mikroorganismen. Calcium wird von $6.6 \%$ supplementiert und Eisen von 1.8\%. Bei den Frauen liegen die Anteile jeweils höher als bei den Männern (46).

Sechster Schweizerischer Ernährungsbericht | 75 
Tabelle 2.11: Zuordnung von Lebensmitteln in der Schweizer Nährwertdatenbank zu den verbrauchten Lebensmitteln

\begin{tabular}{|c|c|}
\hline Verbrauchte Lebensmittel & $\begin{array}{l}\text { Zugeordnete Lebensmittel aus der Schweizer } \\
\text { Nährwertdatenbank }\end{array}$ \\
\hline \multicolumn{2}{|l|}{ Gemüse } \\
\hline Andere Fruchtgemüse & $\begin{array}{l}\text { Auberginen, Gurken, Peperoni grüne/rote, Tomaten, } \\
\text { Zucchetti }\end{array}$ \\
\hline Andere Kohlgewächse & Grünkohl \\
\hline Andere Wurzel- und Knollengewächse & $\begin{array}{l}\text { Fenchel, Karotten, Knollensellerie, Radieschen, Randen, } \\
\text { Rettich, Schwarzwurzeln, Weisse Rüben }\end{array}$ \\
\hline Gemüsekonserven & $\begin{array}{l}\text { Erbsen, Erbsen und Karotten, Maiskölbchen, Pelati, Sauer- } \\
\text { kraut, Silberzwiebeln, Zuckermais }\end{array}$ \\
\hline Wildpilze & Eierschwämme \\
\hline Zuchtpilze & Champignons \\
\hline \multicolumn{2}{|l|}{ Früchte } \\
\hline Andere Beeren & $\begin{array}{l}\text { Brombeeren, Heidelbeeren, Himbeeren, Johannisbeeren } \\
\text { rote/schwarze, Stachelbeeren }\end{array}$ \\
\hline Andere Früchte & Kaki, Mirabelle, Passionsfrucht, Quitte \\
\hline Früchte getrocknet & Apfel, Aprikose, Banane, Birne, Dattel, Pflaume, Rosine \\
\hline Fruchtkonserven & Ananas, Apfelmus, Aprikose, Birne, Fruchtsalat, Pfirsich \\
\hline \multicolumn{2}{|l|}{ Getreide, Kartoffeln, Hülsenfrüchte } \\
\hline Dinkel & Weizenmehl/VollkornmehlTyp 1900 \\
\hline Gerste & Rollgerste (Gerstengraupen) \\
\hline Hafer & Haferflocken \\
\hline Reis & je $50 \%$ parboiled und weisser Reis \\
\hline Trockene Hülsenfrüchte & Linsen \\
\hline \multicolumn{2}{|l|}{ Nüsse, Samen } \\
\hline Kokosnüsse & Kokosflocken, Kokosraspel \\
\hline \multicolumn{2}{|l|}{ Milch, Milchprodukte } \\
\hline Milchgetränke & Energy milk, Caffè latte, Schokoladengetränke, Ovo-Drink \\
\hline Anderer Frischkäse & Blanc battu, Hüttenkäse \\
\hline \multicolumn{2}{|l|}{ Fleisch, Fisch, Eier } \\
\hline Schaf & Filet, Gigot, Koteletten \\
\hline Geflügel & Brust mit/ohne Haut, Poulet ganz, Schenkel mit Haut \\
\hline Wild & Hirsch, Reh \\
\hline Organteile & Kalb: Niere, Herz; Rind: Leber, Zunge; Schwein: Leber \\
\hline Süsswasserfische, frisch, gefroren oder Konserven & Egli, Forelle \\
\hline
\end{tabular}


Verbrauchte Lebensmittel

Meerfische, frisch oder gefroren

Meerfische, Konserven

Krebs- undWeichtiere

Öle, Fette

Andere Fette und Öle

Nichtalkoholische Getränke

Andere Fruchtsäfte

Tee und Kräutertee

Alkoholische Getränke

Wein

Berechnung der gewichteten empfohlenen Zufuhr

Um die ermittelten Daten zum Verbrauch und angenäherten Verzehr mit der täglichen empfohlenen Zufuhr vergleichen zu können, wurde die empfohlene Zufuhr gewichtet. Mit Hilfe der Referenzwerte für die Nährstoffzufuhr der deutschsprachigen Gesellschaften für Ernährung (DACH-Referenzwerte; 48) und den in der laufenden Bevölkerungsstatistik (49) gemachten Angaben zur Anzahl in der Schweiz lebenden männlichen (3.79 Mio.) und weiblichen (3.91 Mio.) Personen jeglichen Alters im Jahr 2008 wurde für jeden Nährstoff die empfohlene Zufuhr eines hypothetischen Durchschnittsschweizers berechnet. Bei der Berechnung der täglichen gewichteten Energiezufuhr wurden die in den DACH-Referenzwerten (48) angegebenen Richtwerte für die durchschnittliche Energiezufuhr bei Personen mit einem BodyMass-Index (BMI) im Normbereich und mit ei-
Zugeordnete Lebensmittel aus der Schweizer Nährwertdatenbank

Flunder, Heilbutt, Lachs, Scholle, Seelachs, Seezunge

Lachs geräuchert, Sardelle im Öl, Sardine im ÖI, Thon im Öl, Thon im Wasser

Garnele, Scampi, Steingarnele

Fischöle, Geflügelfett, Rinderfett

Ananas, Aprikose, Birne, Süsskirsche

Kaffee, trinkfertig

Tee, trinkfertig ner körperlichen Aktivität von 1.4 und 1.6 PAL (physical activtiy level) verwendet, was einer mehrheitlich sitzenden Tätigkeit entspricht. Die DACH-Referenzwerte decken den Nährstoffbedarf von rund $98 \%$ der Bevölkerung ab. Eine Ausnahme stellt die Energie dar, bei der es sich nur um den durchschnittlichen Bedarf (d.h. Bedarf von $50 \%$ der Bevölkerung) handelt (48). 


\subsubsection{Beitrag einzelner Lebens- mittelgruppen an die Energie- und Nährstoffversorgung}

Der durchschnittliche Verbrauch und angenäherte Verzehr an Energie, energieliefernden Nährstoffen, Nahrungsfasern, Mikronährstoffen sowie Wasser pro Person und Tag, aufgeschlüsselt nach den einzelnen Lebensmittelgruppen, ist in den Tabellen 2.12-2.16 dargestellt. Die einzelnen Lebensmittelgruppen tragen in unterschiedlichem Masse zur Versorgung mit Energie und Nährstoffen bei. Nachfolgend werden nur diejenigen Beiträge erwähnt, bei denen eine Lebensmittelgruppe mehr als $15 \%$ an den Gesamtverbrauch eines Nährstoffs bzw. an den Gesamtenergieverbrauch liefert, so wie es bereits im 5. SEB gehandhabt wurde (39). Aber auch Beiträge in geringerer Höhe sind wertvoll und tragen mit zur Bedarfsdeckung bei. Ein umfassendes Bild über die prozentualen Beiträge der einzelnen Lebensmittelgruppen an die Energie- und Nährstoffversorgung geben die Abbildungen 2.7 bis 2.9.

Getreide ist der wichtigste Lieferant für Energie und Nahrungsfasern; der Grund für Ersteres liegt im hohen Anteil an Protein und Kohlenhydraten. Ausserdem trägt Getreide zur Versorgung mit Vitamin B1 sowie mit Phosphor, Eisen und Zink bei.

Auch Kartoffeln sind für die Versorgung mit Vitamin B1 wichtig; zusätzlich liefern sie wertvolle Mengen an Vitamin B6.

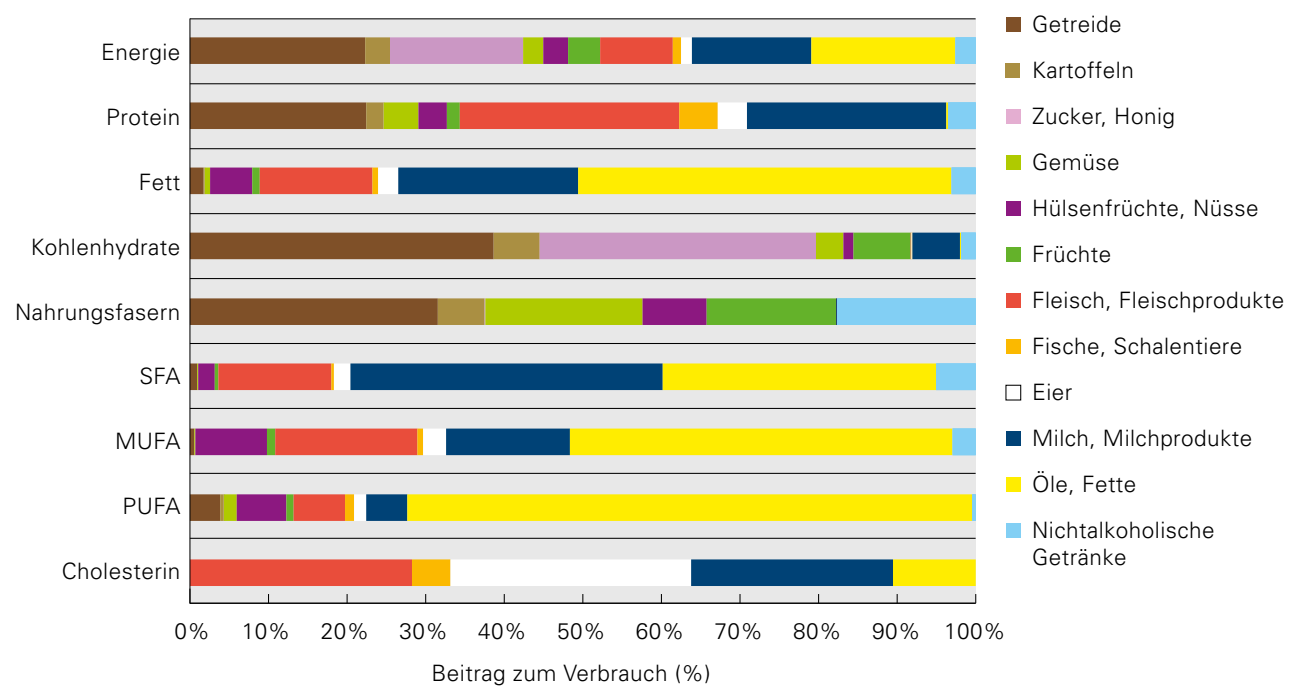

Abbildung 2.7: Prozentualer Beitrag der einzelnen Lebensmittelgruppen zum Verbrauch von Energie, energieliefernden Nährstoffen, Nahrungsfasern und Cholesterin 
Zucker und Honig sind neben Getreide die grössten Quellen für Kohlenhydrate, was zu einem substanziellen Beitrag zur Energiezufuhr führt.

Gemüse ist neben Getreide ein weiterer wichtiger Lieferant für Nahrungsfasern. Ferner spielt es eine grosse Rolle als Vitaminquelle (Carotinoide, Vitamin B1, Vitamin B6, Folsäure und Vitamin C) und trägt auch zur Versorgung mit Kalium, Calcium und Eisen bei. Allerdings ist die Bioverfügbarkeit von Calcium und Eisen aus pflanzlichen Lebensmitteln geringer als aus Lebensmitteln tierischer Herkunft (50-52).
Auch Früchte tragen zur Versorgung mit Nahrungsfasern bei und sind eine gute Quelle für Carotinoide, Folsäure und Vitamin C.

Der Beitrag von Hülsenfrüchten und Nüssen liegt bei allen Nährstoffen unter 15\%, was hauptsächlich auf die niedrigen Verbrauchsmengen zurückzuführen ist.

Fleisch und Fleischprodukte sind die wichtigsten Lieferanten für Protein, Vitamin A (hauptsächlich aus Leber), Vitamin B1, Vitamin B12, Niacin, Natrium und Chlorid. Sie liefern ausserdem grössere Mengen an einfach ungesät-

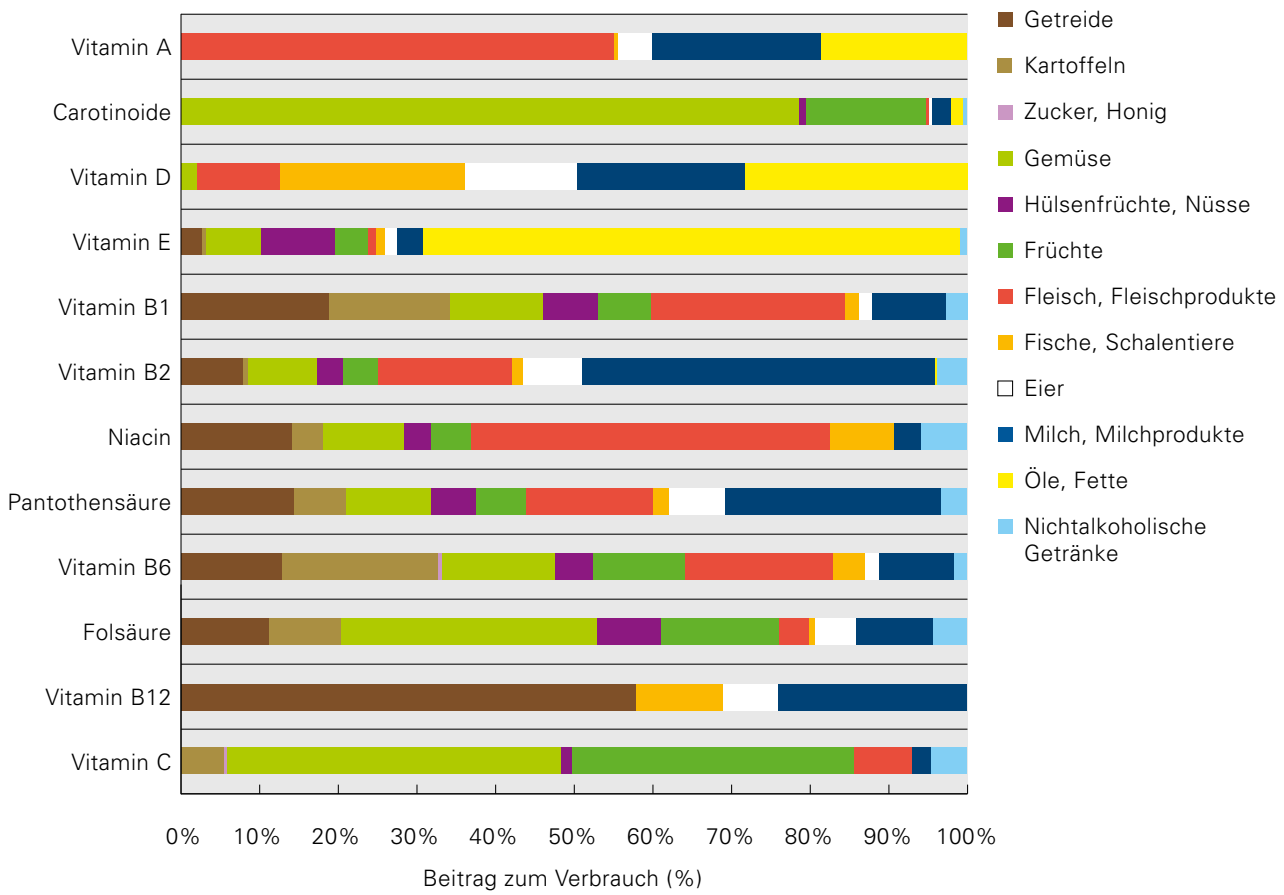

Abbildung 2.8: Prozentualer Beitrag der einzelnen Lebensmittelgruppen zum Verbrauch von Vitaminen 
tigten Fettsäuren (MUFA), Cholesterin, Vitamin B2, Vitamin B6, Pantothensäure, Phosphor, Eisen und Zink.

Fische und Schalentiere liefern Vitamin D. Eier tragen hauptsächlich zur Cholesterinversorgung bei. Bei den übrigen Nährstoffen liegt der Beitrag von Fisch und Eiern unter 15\%, was durch die geringen Mengen, die verzehrt werden, bedingt ist.

Milch und Milchprodukte tragen am meisten zur Versorgung mit gesättigten Fettsäuren (SFA), Vitamin B2, Pantothensäure, Kalium, Calcium, Phosphor, Zink und Jod bei. Sie sind auch bedeutende Lieferanten für Energie, Protein, Fett, MUFA, Cholesterin, die Vitamine A, D und B12 sowie die Mineralstoffe Natrium, Magnesium und Chlorid.
Öle und Fette sind erwartungsgemäss die Hauptquellen für Fett, MUFA und PUFA sowie die Vitamine D und E. Ausserdem leisten sie einen substanziellen Beitrag zur Versorgung mit SFA und Vitamin A, die beide je etwa zur Hälfte aus Butter und aus Margarine und anderen pflanzlichen Ölen stammen.

Nichtalkoholische Getränke sind Lieferanten für Nahrungsfasern, Magnesium und Eisen, was hauptsächlich auf den Verbrauch an Kakao zurückzuführen ist. Da Süssgetränke nicht gesondert eingeschlossen und berechnet werden konnten, ist ihr Beitrag an Energie und Kohlenhydraten im Gesamttotal zwar enthalten (der Zuckergehalt der Süssgetränke ist Teil des Zuckerverbrauchs), kann jedoch nicht separat ausgewiesen werden.

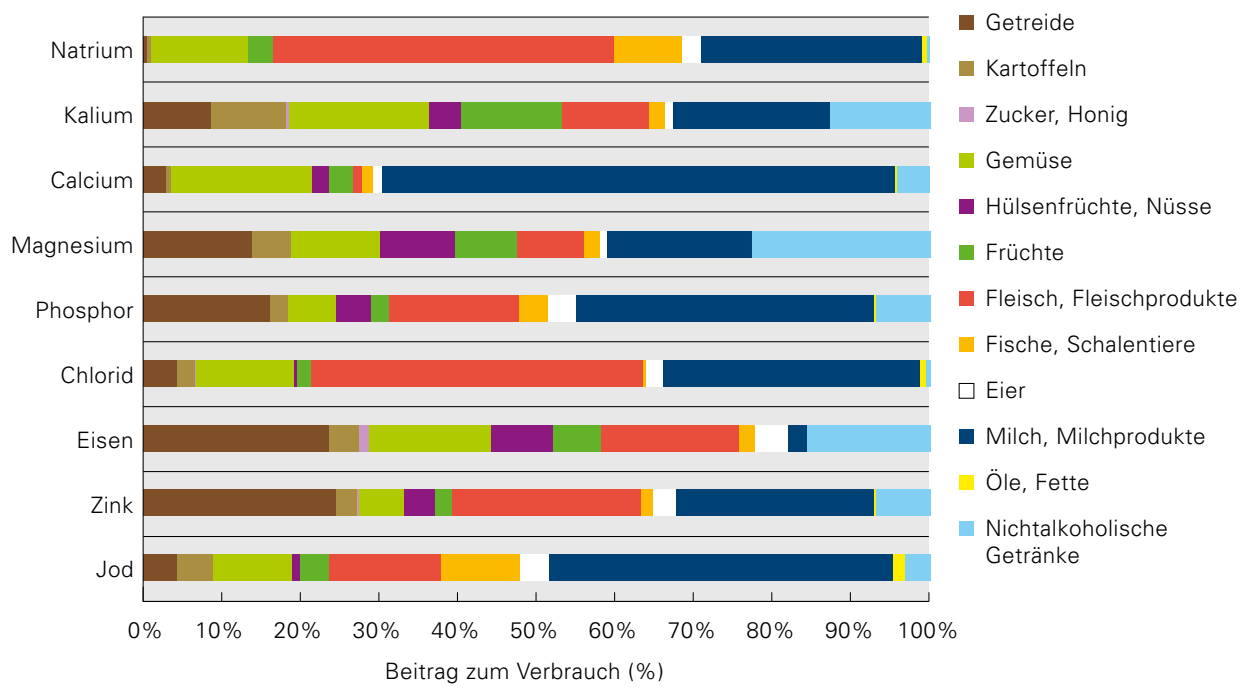

Abbildung 2.9: Prozentualer Beitrag der einzelnen Lebensmittelgruppen zum Verbrauch von Mineralstoffen 


\subsubsection{Beurteilung der Energie- und Wasserversorgung der Schweizer Bevölkerung}

\section{Energie}

Der Energieverbrauch entspricht den Werten in den vorangehenden SEB. Dasselbe trifft auf den angenäherten Verzehr an Energie zu (Abbildung 2.10). Wie schon dort diskutiert $(39 ; 53)$, wird die auf Basis des Lebensmittelverbrauchs berechnete Energiezufuhr überbewertet, da Verluste wie nicht verzehrte und verdorbene Lebensmittel unberücksichtigt bleiben. Die dem Durchschnittsverbraucher zurechenbare Energiemenge aus den verbrauchten Lebensmitteln (Tabellen 2.17 und 2.18) beläuft sich auf 2'923 kcal (12'230 kJ), der angenäherte Verzehr ergibt noch einen Wert von 2'661 kcal (11'135 kJ). In beiden Werten ist die Energie aus alkoholischen Getränken nicht berücksichtigt. Im Vergleich mit der gewichteten empfohlenen Zufuhr von 2'010 kcal bzw. 2'245 kcal (8'415 kJ bzw. 9'395 kJ) je nach körperlicher Aktivität (PAL $=1.4$ bzw. 1.6) liegt der Verbrauch des Durchschnittsschweizers rund 700-900 kcal (2'800-3'800 kJ) bzw. $30-45 \%$ höher. Auch ausgehend vom angenäherten Verzehr liegen die Zahlen noch 18-32\% über den Empfehlungen. Würde dieser Energieverbrauch den Tatsachen entsprechen, kann davon ausgegangen werden, dass grosse Teile der Bevölkerung übergewichtig oder adipös wären. Dies ist jedoch nicht der Fall, auch wenn der Anteil übergewichtiger und adipöser Personen in der Schweiz in den letzten 15 Jahren auf 38.9\% der erwachsenen Bevölkerung angestiegen ist (16; Kapitel 3.3). Die wichtigsten Energielieferanten sind unverändert Getreide, Öle und Fette, Zucker und Honig sowie Milch und Milchprodukte (in absteigender Reihenfolge; Abbildung 2.7).
Wasser

Die Wasserzufuhr lässt sich mit Hilfe der vorhandenen Verbrauchsdaten nicht genau bestimmen. Aus dem Verbrauch von alkoholischen und nichtalkoholischen Getränken resultiert eine Zufuhr von $719 \mathrm{ml}$ Wasser pro Person und Tag (Tabelle 2.12). Da jedoch der Verbrauch an Mineralwasser, Süssgetränken und Leitungswasser darin nicht enthalten ist, ist dieser Wert nicht aussagekräftig.

Aus dem Verbrauch an Lebensmitteln (ohne Getränke) resultieren $961 \mathrm{ml}$ Wasser pro Person und Tag (angenäherter Verzehr: 855 ml; Tabelle 2.12). Dies reicht aus, um die DACH-Richtwerte zur Wasserzufuhr durch feste Nahrung abzudecken, die je nach Alter der Person zwischen $350 \mathrm{ml}$ und $920 \mathrm{ml} /$ Tag betragen (48). Nur die Empfehlungen für Stillende (1'000 ml/Tag) liegen über dem berechneten Durchschnittswert. Der Verbrauch ist vergleichbar mit demjenigen im 4. und 5. SEB: Dort machte er ohne Getränke 1'005 ml bzw. $952 \mathrm{ml}$ aus (18;54). Unter den Lebensmitteln sind Milch und Milchprodukte, Gemüse sowie Früchte die Hauptquellen für Wasser (Beitrag von $\geq 15 \%$ an den Gesamtverbrauch).

Sechster Schweizerischer Ernährungsbericht | 81 


\subsubsection{Beurteilung der Versorgung der Schweizer Bevölkerung mit energieliefernden Nähr- stoffen und Nahrungsfasern}

\section{Protein}

Der Proteinverbrauch liegt, wie schon in den beiden vorangehenden SEB, knapp unter $100 \mathrm{~g}$ pro Kopf und Tag, womit der Verbrauch doppelt so hoch ist wie die gewichtete Empfehlung, die auf dem empfohlenen Minimalbedarf basiert
(Tabelle 2.17). Bezogen auf die gesamte Energiemenge (ohne und mit alkoholischen Getränken) trägt Protein 13.3 Energie-\% bzw. 12.6 Energie-\% bei (auf Basis des Verbrauchs). Legt man den angenäherten Verzehr zugrunde, liegt der Anteil an der Gesamtenergie bei $13.6 \%$ bzw. $12.7 \%$. Dies entspricht den Anteilen in den früheren SEB (Abbildung 2.10). Die Hauptquellen für Protein sind Fleisch und Fleischprodukte, Milch und Milchprodukte sowie Getreide (Abbildung 2.7).

Tabelle 2.12: Durchschnittlicher Verbrauch (Vb) und angenäherter Verzehr (aV) an Energie, energieliefernden Nährstoffen und Wasser pro Person und Tag (Durchschnitt 2007/08)

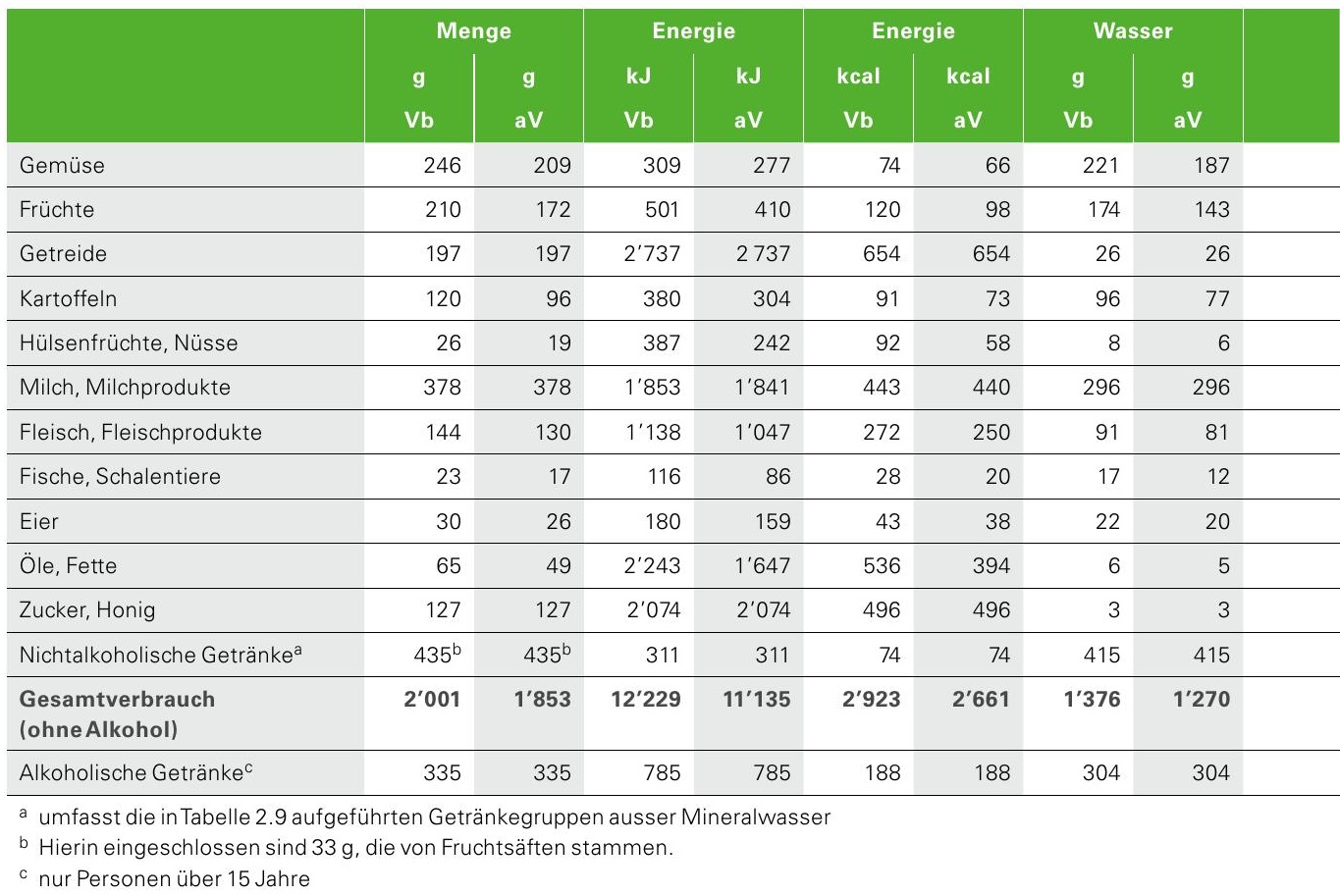


Fett, Fettsäuren und Cholesterin

Der Verbrauch an Fett hat sich im Vergleich zu den Werten im 4. und 5. SEB nicht verändert (Tabelle 2.17). Der Durchschnittsschweizer nimmt über Fett $38.5 \%$ bzw. 36.2\% der Gesamtenergie auf (Verbrauch; ohne und mit alkoholischen Getränken) oder 35.1 Energie-\% bzw. 32.8 Energie-\%, wenn der angenäherte Verzehr als Berechnungsbasis verwendet wird. Der Fettanteil an der Gesamtenergie unterscheidet sich damit nicht wesentlich vom 3. und

\begin{tabular}{|c|c|c|c|c|c|}
\hline \multicolumn{2}{|c|}{ Protein } & \multicolumn{2}{|c|}{ Fett } & \multicolumn{2}{|c|}{ Kohlenhydrate } \\
\hline $\mathbf{g}$ & $\mathbf{g}$ & g & $\mathbf{g}$ & $\mathbf{g}$ & g \\
\hline Vb & aV & $\mathbf{V b}$ & aV & $\mathbf{V b}$ & $\mathbf{a V}$ \\
\hline 4.3 & 3.7 & 0.9 & 0.8 & 12 & 11 \\
\hline 1.6 & 1.3 & 1.2 & 1.0 & 26 & 21 \\
\hline 21.9 & 21.9 & 2.3 & 2.3 & 136 & 136 \\
\hline 2.2 & 1.7 & 0.1 & 0.1 & 20 & 16 \\
\hline 3.5 & 2.6 & 6.7 & 3.6 & 5 & 4 \\
\hline 24.6 & 24.4 & 28.6 & 28.4 & 22 & 22 \\
\hline 27.1 & 24.4 & 17.9 & 16.7 & 1 & 0 \\
\hline 4.7 & 3.4 & 1.0 & 0.7 & 0 & 0 \\
\hline 3.7 & 3.2 & 3.1 & 2.7 & 0 & 0 \\
\hline 0.1 & 0.1 & 59.5 & 43.7 & 0 & 0 \\
\hline 0.0 & 0.0 & 0.0 & 0.0 & 124 & 124 \\
\hline 3.4 & 3.4 & 3.8 & 3.8 & 7 & 7 \\
\hline 97.1 & 90.2 & 125.1 & 103.8 & 352 & 341 \\
\hline 1.0 & 1.0 & 0.0 & 0.0 & 5 & 5 \\
\hline
\end{tabular}

5. SEB (Abbildung 2.10). Die pauschale Empfehlung zu einem geringeren Fettanteil in der Nahrung ist in den letzten Jahren ergänzt worden mit Empfehlungen hinsichtlich Verbesserungen der Fettqualität (24; Kapitel 1.5.1 und 1.7.2). Die Verbrauchsmengen an SFA, MUFA und PUFA haben sich jedoch im Vergleich zum 5. SEB nicht wesentlich verändert. Positiv zu vermerken ist der leichte Anstieg bei den PUFA. Die Beiträge der einzelnen Fettsäuregruppen an die Gesamtenergie (Verbrauch ohne alkoholische Getränke) liegen bei $13.3 \%$ (SFA), $13.0 \%$ (MUFA) und $7.9 \%$ (PUFA) bzw. bei $12.5 \%$, $12.2 \%$ und $7.4 \%$ unter Berücksichtigung der alkoholischen Getränke in der Gesamtenergie. Die Hauptquellen für Fett, SFA und MUFA sind Öle und Fette sowie Milch und Milchprodukte. Bei den MUFA kommen noch Fleisch und Fleischprodukte als Hauptquellen hinzu. Die PUFA stammen überwiegend aus Ölen und Fetten (Abbildung 2.7 und Tabelle 2.13).

Mangels Angaben in der Nährwertdatenbank konnte der Verbrauch an TFA nicht berechnet werden. Diese werden in Zusammenhang gebracht mit negativen gesundheitlichen Effekten v.a. in Bezug auf Herz-Kreislauf-Krankheiten (55). Da in der Schweiz durch eine Verordnung des Eidgenössischen Departements des Innern (EDI) von März 2008 der TFA-Gehalt in Speisefetten und -ölen auf $2 \mathrm{~g}$ pro $100 \mathrm{~g}$ beschränkt wurde (56), sollten die zuvor gefundenen, teilweise beachtlichen Mengen an TFA in Lebensmitteln mit teilgehärteten Fetten (57) der Vergangenheit angehören. Der quantitativ geringe Konsum von TFA über tierische Lebensmittel stellt kein gesundheitliches Problem dar (58).

Der kontinuierliche Rückgang des Cholesterinverbrauchs, der in den früheren SEB festgestellt wurde, hat sich nicht fortgesetzt; die Wer- 


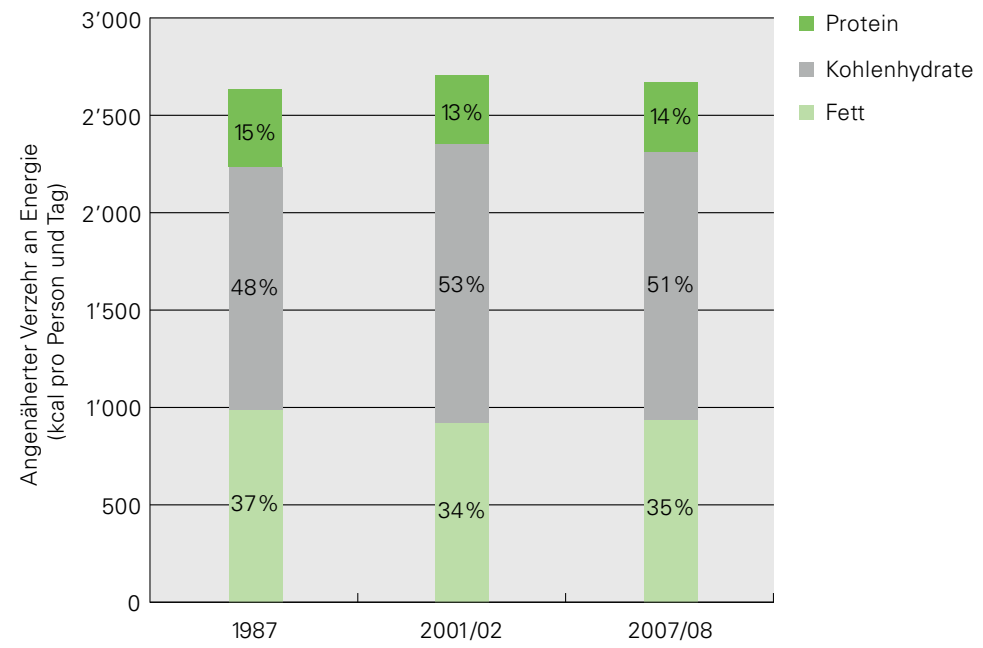

Abbildung 2.10: Angenäherter Verzehr an Energie (in kcal) und prozentualer Anteil der energieliefernden Nährstoffe pro Person und Tag in den Jahren 1987, 2001/02 und 2007/08 (ohne Berücksichtigung von Alkohol)

te sind mit $367 \mathrm{mg}$ (Verbrauch) und $336 \mathrm{mg}$ (angenäherter Verzehr) auf gleicher Höhe wie diejenigen des 5. SEB (Tabellen 2.17 und 2.18). Das könnte daran liegen, dass sich die diesbezüglichen Ernährungsempfehlungen in den letzten Jahren verändert haben (Kapitel 1.5.1). Die Beschränkungen der Cholesterinzufuhr sind weggefallen, da laut neueren Erkenntnissen der Einfluss des Nahrungscholesterins auf den Serumcholesterinspiegel als gering eingestuft und die Cholesterinzufuhr mit der Nahrung als wenig relevant für die Entwicklung von Herz-KreislaufKrankheiten angesehen wird (24). Naturgemäss tragen nur Lebensmittel tierischer Herkunft zum Cholesterinverbrauch bei; die Hauptquellen sind Eier, Fleisch und Fleischprodukte sowie Milch und Milchprodukte (Abbildung 2.7).

\section{Kohlenhydrate}

Der im 5. SEB ausgewiesene Kohlenhydratverbrauch (und angenäherte Verzehr) musste nachträglich nach unten korrigiert werden (siehe Fussnote e in Tabelle 2.8). Der damals erwähnte Anstieg fällt damit geringer aus als angenommen und hat sich in den folgenden Jahren auch nicht fortgesetzt, wie die aktuellen Zahlen zeigen. Diese liegen gleichauf mit den Zahlen aus dem 4. und 3. SEB (Tabelle 2.17). Der angenäherte Verzehr hat sich hingegen gegenüber dem 3. SEB leicht erhöht und im Vergleich zum 5. SEB geringfügig verringert (Tabelle 2.18 und Abbildung 2.10). Der Kohlenhydratverbrauch macht $48.2 \%$ des Gesamtenergieverbrauchs ohne alkoholische Getränke aus (angenäherter Verzehr 51.3 Energie-\%). Lange Zeit wurde ein 
relativ hoher Kohlenhydratanteil (50-75 Energie-\%) in der Nahrung empfohlen. Neue Erkenntnisse weisen jedoch auf ungünstige Wirkungen einer stark kohlenhydratlastigen Ernährungsweise hin (Kapitel 3.3.2, 3.4.2, 3.5.2 und 3.6.2), vor allem wenn die kohlenhydrathaltigen Lebensmittel einen hohen glykämischen Index aufweisen. Die Eidgenössische Ernährungskommission (EEK) kommt deshalb zum Schluss, dass der optimale Kohlenhydratanteil zwischen $45 \%$ und 55\% der Gesamtenergie liegt (59). Der berechnete Kohlenhydratverbrauch des Durchschnittsschweizers befindet sich innerhalb dieser Bandbreite. Aussagen zur Kohlenhydratqualität lassen sich auf Basis der Verbrauchsdaten nicht machen. Die Hauptquellen für Kohlenhydrate sind Getreide und Zucker (Abbildung 2.7).

Der Verbrauch an Saccharose trägt 17\% zum Gesamtenergieverbrauch bei, womit der Beitrag gleich hoch ist wie im 5. SEB und deutlich über den Empfehlungen der Weltgesundheitsorganisation (WHO; < 10\%) liegt (60; Kapitel 1.7.2).

\section{Alkohol}

Der tägliche Verbrauch und angenäherte Verzehr an Alkohol liegt bei $23.2 \mathrm{~g}$ pro Person (Tabellen 2.17 und 2.18), berechnet für den Bevölkerungsanteil über 15 Jahren. Diese Alkoholmenge stammt ausschliesslich aus alkoholischen Getränken, andere Lebensmittelgruppen liefern keine relevanten Mengen (Tabelle 2.13). Weitere Angaben zum Alkoholkonsum finden sich im Abschnitt «Alkoholische Getränke» in Kapitel 2.3.2.

\section{Nahrungsfasern}

Der tägliche Verbrauch an Nahrungsfasern liegt mit $28 \mathrm{~g}$ pro Person tendenziell etwas über den Werten im 4. und 5. SEB, ebenso der angenä- herte Verzehr (Tabellen 2.17 und 2.18). Der DACH-Richtwert von mindestens $30 \mathrm{~g}$ pro Tag wird damit nach wie vor knapp nicht erreicht (48). Die wichtigsten Lieferanten für Nahrungsfasern sind Getreide, Gemüse, Früchte und nichtalkoholische Getränke (hauptsächlich Kakao; Abbildung 2.7).

\subsubsection{Beurteilung der Vitamin- versorgung der Schweizer Bevölkerung}

\section{Vitamin A und Carotinoide}

Die verbrauchte Menge an Vitamin A und Carotinoiden ist konstant geblieben. Umgerechnet auf Retinoläquivalente (RE) resultiert ein Gesamtverbrauch bzw. angenäherter Verzehr von $1.8 \mathrm{mg}$ bzw. $1.6 \mathrm{mg}$, was weiterhin deutlich über der gewichteten Empfehlung von $0.9 \mathrm{mg}$ liegt (Tabellen 2.19 und 2.20). Vitamin A wird vor allem über Fleisch und Fleischprodukte (hauptsächlich Leber), Milch und Milchprodukte sowie Öle und Fette aufgenommen. Die Carotinoide stammen hingegen vorwiegend aus $\mathrm{Ge}$ müse und Früchten (Abbildung 2.8).

\section{Vitamin D}

Beim Vitamin D kann zwar ein leichter Anstieg im Verbrauch und angenäherten Verzehr gegenüber den früheren SEB festgestellt werden; die Zufuhr ist aber insgesamt noch immer zu gering (Tabellen 2.19 und 2.20). Da Vitamin D auch bei Sonnenbestrahlung in der Haut gebildet wird, wurde die Unterversorgung bisher nur bei einzelnen Risikogruppen wie zum Beispiel älteren Frauen (61) als problematisch angesehen. In den letzten Jahren hat sich jedoch die Erkenntnis durchgesetzt, dass in unseren Breitengraden im 
Tabelle 2.13: Durchschnittlicher Verbrauch (Vb) und angenäherter Verzehr (aV) an Fettsäuren, Cholesterin, Alkohol und Nahrungsfasern pro Person und Tag (Durchschnitt 2007/08)

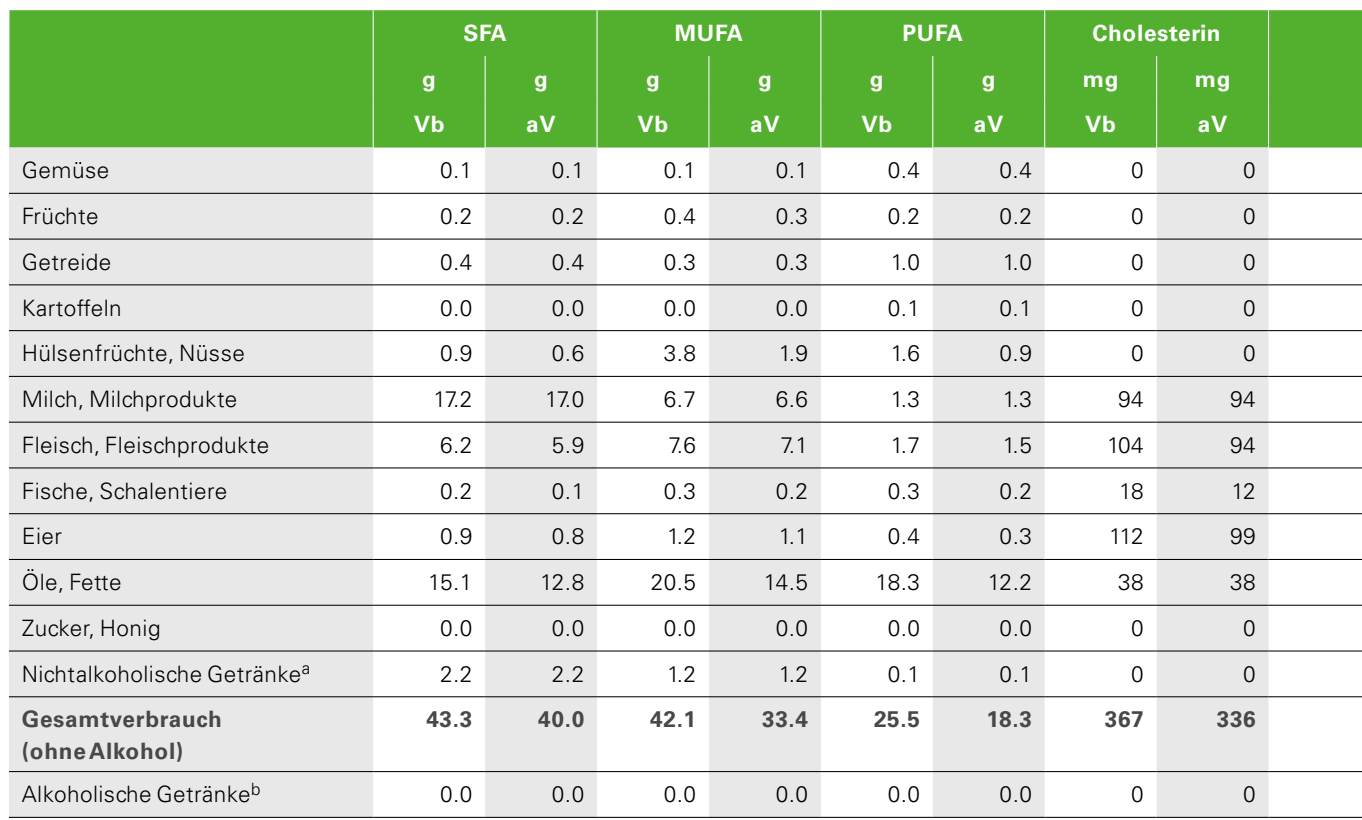

a umfasst die in Tabelle 2.9 aufgeführten Getränkegruppen ausser Mineralwasser

b nur Personen über 15 Jahre

Winter die Eigenproduktion an Vitamin D generell als ungenügend einzustufen ist (62). In den meisten europäischen Ländern ist folglich die Vitamin-D-Unterversorgung in der Bevölkerung hoch bis sehr hoch (63), die Schweiz stellt dabei keine Ausnahme dar (Kapitel 3.10.3). Durch neue wissenschaftliche Erkenntnisse über Vitamin D, die ihm verschiedenste, über die Vorbeugung und Behandlung von Knochenerkrankungen hinausgehende Wirkungen attestieren (64), gewinnt die Thematik zunehmend an Bedeutung. Zweifellos muss der Zufuhr von Vitamin D künftig ein grösserer Stellenwert beigemessen werden, als dies bisher der Fall war, was sich auch in den Diskussionen um eine Erhöhung der Zufuhrempfehlungen bzw. einer Empfehlung zur Supplementierung zeigt (65; Kapitel 1.5.3 und 3.10.3). Bei Säuglingen wird eine Vitamin-DSupplementierung während des ersten Lebensjahres seit vielen Jahren empfohlen (66; Kapitel 1.3.1). Diese wird im Durchschnitt jedoch nur bei $64 \%$ der Babys umgesetzt (im Sommer bei $62 \%$, im Winter bei 72\%; 67). Die wichtigsten Vitamin-D-Quellen in unserer Nahrung sind Öle und Fette, Fische und Schalentiere sowie Milch und Milchprodukte (Abbildung 2.8). 


\begin{tabular}{|c|c|c|c|}
\hline \multicolumn{2}{|c|}{ Alkohol } & \multicolumn{2}{|c|}{ Nahrungsfasern } \\
\hline $\mathbf{g}$ & g & g & g \\
\hline Vb & aV & Vb & aV \\
\hline 0.0 & 0.0 & 5.6 & 4.8 \\
\hline 0.0 & 0.0 & 4.6 & 3.9 \\
\hline 0.0 & 0.0 & 8.8 & 8.8 \\
\hline 0.0 & 0.0 & 1.7 & 1.3 \\
\hline 0.0 & 0.0 & 2.3 & 1.7 \\
\hline 0.0 & 0.0 & 0.0 & 0.0 \\
\hline 0.0 & 0.0 & 0.0 & 0.0 \\
\hline 0.0 & 0.0 & 0.0 & 0.0 \\
\hline 0.0 & 0.0 & 0.0 & 0.0 \\
\hline 0.0 & 0.0 & 0.0 & 0.0 \\
\hline 0.0 & 0.0 & 0.1 & 0.1 \\
\hline 0.0 & 0.0 & 4.9 & 4.9 \\
\hline 0.0 & 0.0 & 28.0 & 25.5 \\
\hline 23.2 & 23.2 & 0.4 & 0.4 \\
\hline
\end{tabular}

\section{Vitamin B1 und Vitamin B2}

Verglichen mit den Werten im 5. SEB sind Verbrauch und angenäherter Verzehr an Vitamin B1 (Thiamin) und Vitamin B2 (Riboflavin) konstant geblieben (Tabellen 2.19 und 2.20). Die Versorgung ist ausreichend, wenn auch nur knapp. Die Hauptmenge an Vitamin B1 stammt aus Fleisch und Fleischprodukten, des Weiteren tragen auch Getreide, Kartoffeln und Gemüse grössere Mengen bei. Vitamin B2 wird hauptsächlich über Milch und Milchprodukte zugeführt, deutlich geringere Mengen liefern Fleisch und Fleischprodukte (Abbildung 2.8).

\section{Vitamin B6}

Auch beim Vitamin B6 (Pyridoxin) zeigen sich keine Veränderungen in Verbrauch und angenähertem Verzehr im Vergleich zum 5. SEB (Tabellen 2.19 und 2.20). Die verfügbare Menge deckt die gewichtete empfohlene Zufuhr deutlich ab. Vitamin B6 wird hauptsächlich über Kartoffeln, Fleisch und Fleischprodukte sowie Gemüse eingenommen (Abbildung 2.8).

\section{Niacin}

Der Niacinverbrauch ist im Vergleich zu den An-

\section{Vitamin $E$}

Verbrauch und angenäherter Verzehr an Vitamin $E$ sind im Vergleich zu den früheren SEB wiederum etwas angestiegen (Tabellen 2.19 und 2.20), was vermutlich am zunehmenden Verbrauch an pflanzlichen Ölen und Fetten (inkl. Margarine) liegt, denn diese stellen die Hauptquellen für Vitamin $E$ dar (Abbildung 2.8). Die gewichtete empfohlene Zufuhr wird wie auch in den vorangehenden SEB übertroffen. gaben im 5. SEB leicht angestiegen, erreicht jedoch nicht das Niveau im 4. SEB (Tabelle 2.19). Durch den Anstieg kommt der angenäherte Verzehr etwas über die gewichtete empfohlene Zufuhr zu liegen (Tabelle 2.20). Fleisch und Fleischprodukte tragen am meisten zur Niacinversorgung bei, gefolgt von Getreide. Die anderen Lebensmittelgruppen decken jeweils weniger als $15 \%$ des Verbrauchs ab (Abbildung 2.8). 
Tabelle 2.14: Durchschnittlicher Verbrauch (Vb) und angenäherter Verzehr (aV) an fettlöslichen Vitaminen pro Person und Tag (Durchschnitt 2007/08)

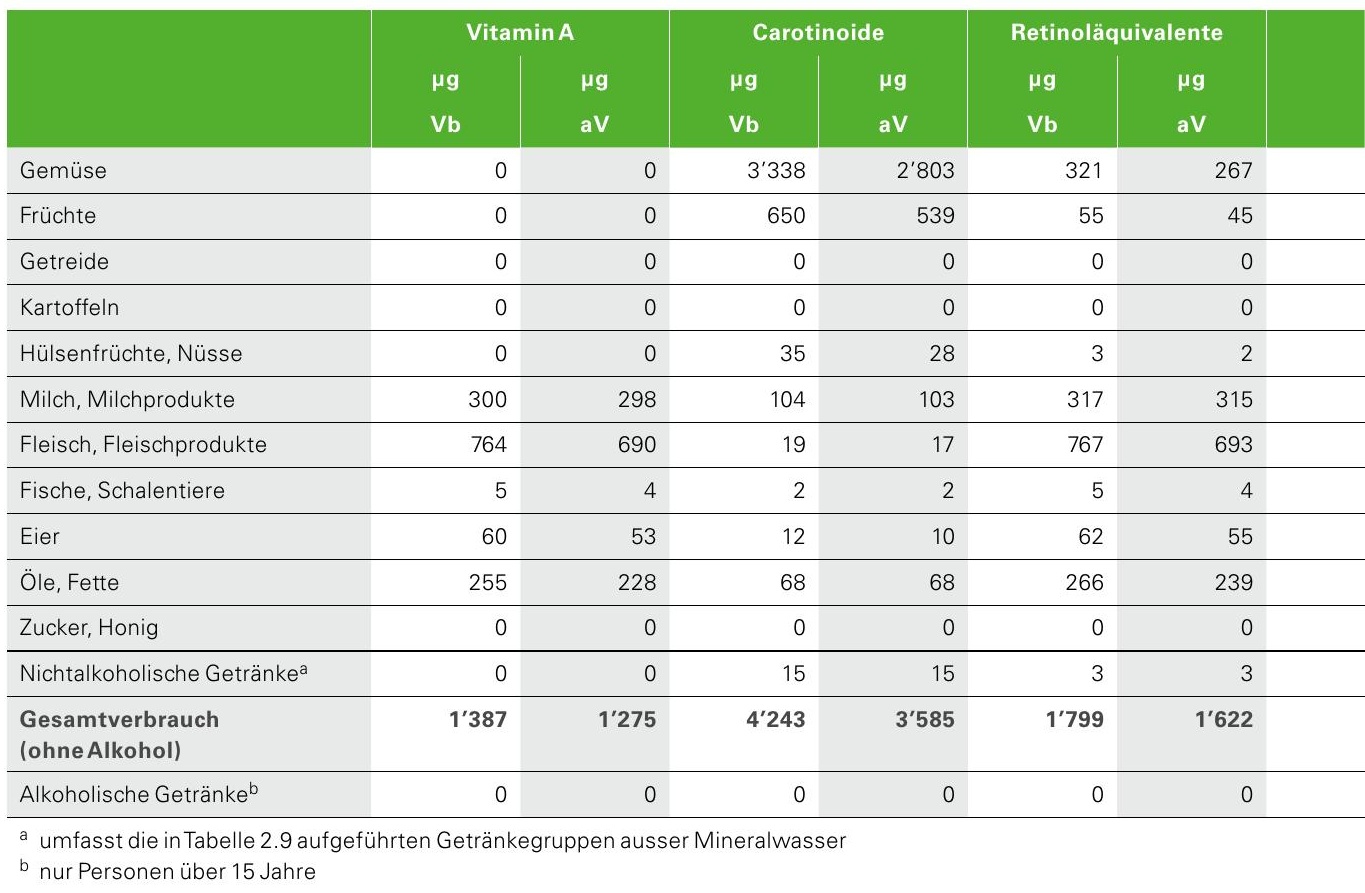

\section{Folsäure}

Die Folsäureversorgung ist weiterhin ungenügend. Die Differenz zwischen Verbrauch bzw. angenähertem Verzehr einerseits und gewichteter empfohlener Zufuhr andererseits hat sich jedoch weiter verringert (Tabellen 2.19 und 2.20). Vermutlich fällt die tatsächliche Folsäurezufuhr über Lebensmittel noch tiefer aus als im angenäherten Verzehr ausgewiesen, da Folsäure hauptsächlich über Gemüse und Früchte aufgenommen wird (Abbildung 2.8). Beides sind Lebensmittelgruppen, die grösseren Zuberei- tungsverlusten und Verlusten durch Verderbnis (z.B. während der Lagerung) ausgesetzt sind. Nicht berücksichtigt ist die Folsäurezufuhr durch die Einnahme von Folsäuresupplementen, die Frauen im gebärfähigen Alter empfohlen wird (68; Kapitel 1.4.1), oder mit Folsäure angereicherte Lebensmittel. Die Bedeutung einer unzureichenden Folsäureversorgung wird in Kapitel 3.10.2 aufgezeigt. 


\begin{tabular}{|c|c|c|c|}
\hline \multicolumn{2}{|c|}{ Vitamin D } & \multicolumn{2}{|c|}{ Vitamin-E-Äquivalente } \\
\hline$\mu g$ & $\mu \mathrm{g}$ & mg & mg \\
\hline Vb & aV & Vb & aV \\
\hline 0.1 & 0.1 & 1.7 & 1.5 \\
\hline 0.0 & 0.0 & 1.0 & 0.9 \\
\hline 0.0 & 0.0 & 0.7 & 0.7 \\
\hline 0.0 & 0.0 & 0.1 & 0.1 \\
\hline 0.0 & 0.0 & 2.3 & 1.1 \\
\hline 0.7 & 0.7 & 0.8 & 0.8 \\
\hline 0.4 & 0.4 & 0.3 & 0.2 \\
\hline 0.8 & 0.6 & 0.3 & 0.2 \\
\hline 0.5 & 0.4 & 0.4 & 0.3 \\
\hline 1.0 & 0.8 & 16.8 & 11.2 \\
\hline 0.0 & 0.0 & 0.0 & 0.0 \\
\hline 0.0 & 0.0 & 0.2 & 0.2 \\
\hline 3.5 & 3.1 & 24.5 & 17.2 \\
\hline 0.0 & 0.0 & 0.0 & 0.0 \\
\hline
\end{tabular}

Fleisch, Fleischprodukten, Fisch und Schalentieren beliefe sich der Verbrauch an Vitamin B12 nur auf $2.1 \mu \mathrm{g}$ (Tabelle 2.15) und würde die gewichtete empfohlene Zufuhr von $2.8 \mu \mathrm{g}$ nicht mehr abdecken. Veganer und in einem geringeren Umfang auch Ovo-Lacto-Vegetarier stellen deshalb eine Risikogruppe für eine Vitamin-B12Unterversorgung dar (69).

\section{Pantothensäure}

Die gleiche Entwicklung wie Vitamin B12 - wenn auch in geringerem Umfang - weist auch Pantothensäure auf: ein Rückgang des Verbrauchs zwischen dem 4. und 5. SEB, der sich nicht weiter fortsetzt (Tabelle 2.19). Der angenäherte Verzehr verzeichnet sogar eine leichte Zunahme (Tabelle 2.20). Sowohl Verbrauch wie auch angenäherter Verzehr liegen etwas über der gewichteten empfohlenen Zufuhr. Die Veränderungen im Pantothensäureverbrauch hängen möglicherweise mit dem Verbrauch an Fleisch und Fleischprodukten zusammen, denn diese tragen neben Milch und Milchprodukten am meisten zur Pantothensäurezufuhr bei (Abbildung 2.8).

\section{Vitamin C}

Beim Vitamin $\mathrm{C}$ finden sich die gleichen Ver-

\section{Vitamin B12}

Nach dem starken Rückgang des Verbrauchs an Vitamin B12 (Cobalamin) zwischen dem 4. und 5. SEB ist dieser nun konstant geblieben (Tabelle 2.19). Das Gleiche gilt für den angenäherten Verzehr (Tabelle 2.20), womit dieser die gewichtete Empfehlung weiterhin deutlich übertrifft. Vitamin B12 ist fast ausschliesslich in Lebensmitteln tierischer Herkunft enthalten. Hauptquellen sind deshalb Fleisch und Fleischprodukte, gefolgt von Milch und Milchprodukten (Abbildung 2.8). Ohne die Zufuhr aus 
Tabelle 2.15: Durchschnittlicher Verbrauch (Vb) und angenäherter Verzehr (aV) an wasserlöslichen Vitaminen pro Person und Tag (Durchschnitt 2007/08)

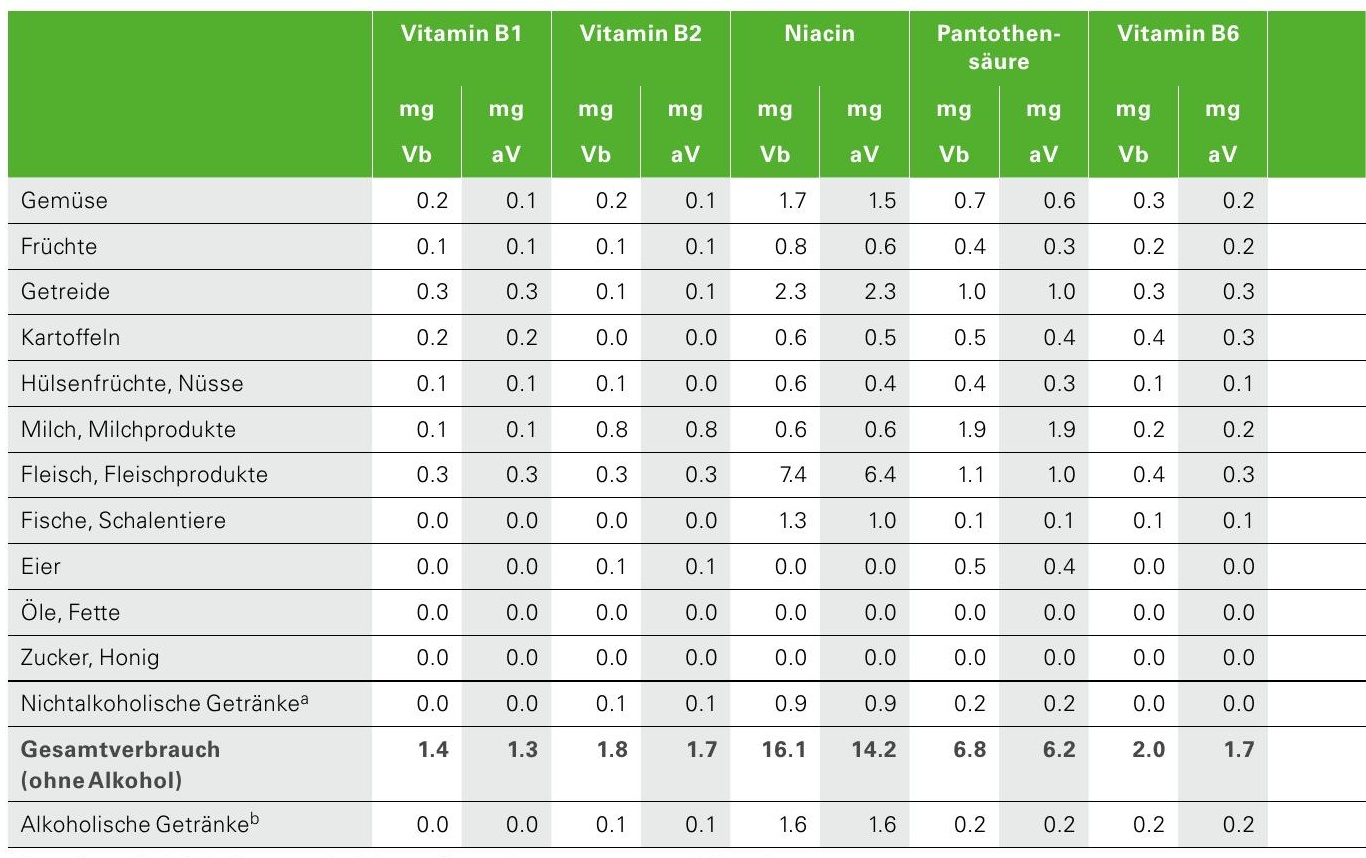

a umfasst die in Tabelle 2.9 aufgeführten Getränkegruppen ausser Mineralwasser

b nur Personen über 15 Jahre

\subsubsection{Beurteilung der Mineral- stoffversorgung der Schweizer Bevölkerung}

\section{Kalium}

Die im 5. SEB beschriebene Abnahme in Verbrauch und angenähertem Verzehr von Kalium setzt sich nicht weiter fort (Tabellen 2.21 und 2.22). Die Mengen liegen noch immer deutlich über der gewichteten empfohlenen Zufuhr, bei der es sich um einen Schätzwert für eine mini- male Zufuhr handelt. Die Hauptquellen für Kalium sind Milch und Milchprodukte sowie Gemüse (Abbildung 2.9).

\section{Natrium und Chlorid}

Wie für Kalium sind auch für Natrium und Chlorid nur Schätzwerte für eine minimale Zufuhr vorhanden, die von den Verbrauchsmengen und vom angenäherten Verzehr im vorliegenden SEB um das Dreifache übertroffen werden (Tabellen 2.21 und 2.22). Gegenüber dem 5. SEB haben sich die 


\begin{tabular}{|c|c|c|c|c|c|}
\hline \multicolumn{2}{|c|}{ Folsäure } & \multicolumn{2}{|c|}{ Vitamin B12 } & \multicolumn{2}{|c|}{ Vitamin C } \\
\hline$\mu \mathbf{g}$ & $\mu \mathrm{g}$ & $\mu \mathrm{g}$ & $\mu \mathrm{g}$ & $\mathrm{mg}$ & $\mathrm{mg}$ \\
\hline Vb & aV & Vb & aV & Vb & aV \\
\hline 112 & 91 & 0.0 & 0.0 & 55 & 44 \\
\hline 52 & 42 & 0.0 & 0.0 & 47 & 37 \\
\hline 39 & 39 & 0.0 & 0.0 & 0 & 0 \\
\hline 31 & 25 & 0.0 & 0.0 & 7 & 6 \\
\hline 28 & 21 & 0.0 & 0.0 & 2 & 1 \\
\hline 34 & 34 & 1.6 & 1.6 & 3 & 3 \\
\hline 13 & 11 & 3.8 & 3.5 & 9 & 9 \\
\hline 3 & 2 & 0.7 & 0.6 & 0 & 0 \\
\hline 18 & 16 & 0.5 & 0.4 & 0 & 0 \\
\hline 0 & 0 & 0.0 & 0.0 & 0 & 0 \\
\hline 0 & 0 & 0.0 & 0.0 & 0 & 0 \\
\hline 15 & 15 & 0.0 & 0.0 & 6 & 6 \\
\hline 344 & 295 & 6.6 & 6.0 & 130 & 106 \\
\hline 10 & 10 & 0.2 & 0.2 & 0 & 0 \\
\hline
\end{tabular}

Aus den Natrium-Verbrauchsdaten lässt sich eine Salzzufuhr von $4.1 \mathrm{~g}$ Salz pro Tag und Person berechnen. Hinzu kommen jedoch noch die bei der Verarbeitung von Lebensmitteln eingesetzten Mengen (erfasst sind nur die Mengen in Milchund Fleischprodukten) sowie das im Haushalt bei der Zubereitung und dem Verzehr hinzugefügte Salz. In der Genfer Studie Bus Santé wurde über 12 Jahre hinweg (1993-2004) der Salzkonsum von Erwachsenen im Alter von 35-74 Jahren ermittelt (71). Die durchschnittliche Salzzufuhr lag bei $10.6 \mathrm{~g}$ (Männer) bzw. $8.1 \mathrm{~g}$ (Frauen) und hat sich im Lauf der Jahre nicht verändert. Fünf Lebensmittelgruppen machten $47 \%$ bzw. 48\% (Männer bzw. Frauen) der Gesamtaufnahme aus: Brot $117 \%$ der Gesamtzufuhr bei beiden $\mathrm{Ge}$ schlechtern), Käse (11\% bzw. 10\%), Fleisch, Fleischprodukte und Eier ( $8 \%$ bzw. 7\%), Suppen ( $6 \%$ bzw. $9 \%$ ) sowie Fertigprodukte ( $5 \%$ bei beiden Geschlechtern). Hauptquelle für Salz war mit $41 \%$ bzw. 35\% der Gesamtmenge das durch Zusalzen beim Kochen und Essen konsumierte Salz, wobei dies vermutlich leicht überschätzt ist, da nicht erfasste Lebensmittel noch einen Teil davon ausmachen dürften. Die Resultate einer aktuellen nationalen Studie bestätigen die Daten aus Genf zur durchschnittlichen Salzzufuhr (109).

Verbrauchsmengen nicht verändert. Die Hauptquellen für Natrium und Chlorid sind Fleisch und Fleischprodukte sowie Milch und Milchprodukte (Abbildung 2.9). Das hängt unter anderem damit zusammen, dass beide Lebensmittelgruppen auch verarbeitete Produkte umfassen. Die Salzstrategie des Bundesamtes für Gesundheit (BAG) zielt langfristig darauf $a b$, in der Bevölkerung einen täglichen Salzkonsum von weniger als $5 \mathrm{~g}$ pro Person zu erreichen (70), was den Empfehlungen der WHO (60) entspricht (Kapitel 1.5.2 und 4.6.1.1).

\section{Calcium}

Der Verbrauch an Calcium ist über die Jahre hinweg kontinuierlich leicht angestiegen und liegt nun um 24\% höher als im 2. SEB. Im Vergleich zum 5. SEB zeigen sich jedoch keine wesentlichen Unterschiede (Tabelle 2.21). Sowohl Verbrauch wie auch angenäherter Verzehr (Tabelle 2.22) decken die gewichtete empfohlene Zufuhr ab. Milch und Milchprodukte liefern $65 \%$ der Gesamtmenge an Calcium. Gemüse ist die zweitwichtigste Quelle mit 18\% (Abbil- 
dung 2.9), wobei die Bioverfügbarkeit von Calcium aus Gemüse jedoch meist geringer ist (51). In diesen Angaben nicht enthalten ist die Calciumzufuhr aus Mineralwasser und Leitungswasser, die je nach Trinkgewohnheiten und Calciumgehalt des konsumierten Wassers unterschiedlich hoch sein kann.

\section{Phosphor}

Der Verbrauch und der angenäherte Verzehr an Phosphor haben sich seit dem 5. SEB nicht verändert und liegen jeweils etwa doppelt so hoch wie die gewichtete empfohlene Zufuhr (Tabellen 2.21 und 2.22). Die Hauptmenge an Phosphor wird über Milch und Milchprodukte eingenommen, gefolgt von Fleisch und Fleischprodukten sowie Getreide (Abbildung 2.9).

\section{Magnesium}

Die Schweizer Bevölkerung ist ausreichend mit Magnesium versorgt, wenn auch der angenäherte Verzehr nur knapp über der gewichteten empfohlenen Zufuhr liegt (Tabellen 2.21 und 2.22). Hauptquellen für Magnesium sind die nichtalko-

Tabelle 2.16: Durchschnittlicher Verbrauch (Vb) und angenäherter Verzehr (aV) an Mineralstoffen pro Person und Tag (Durchschnitt 2007/08)

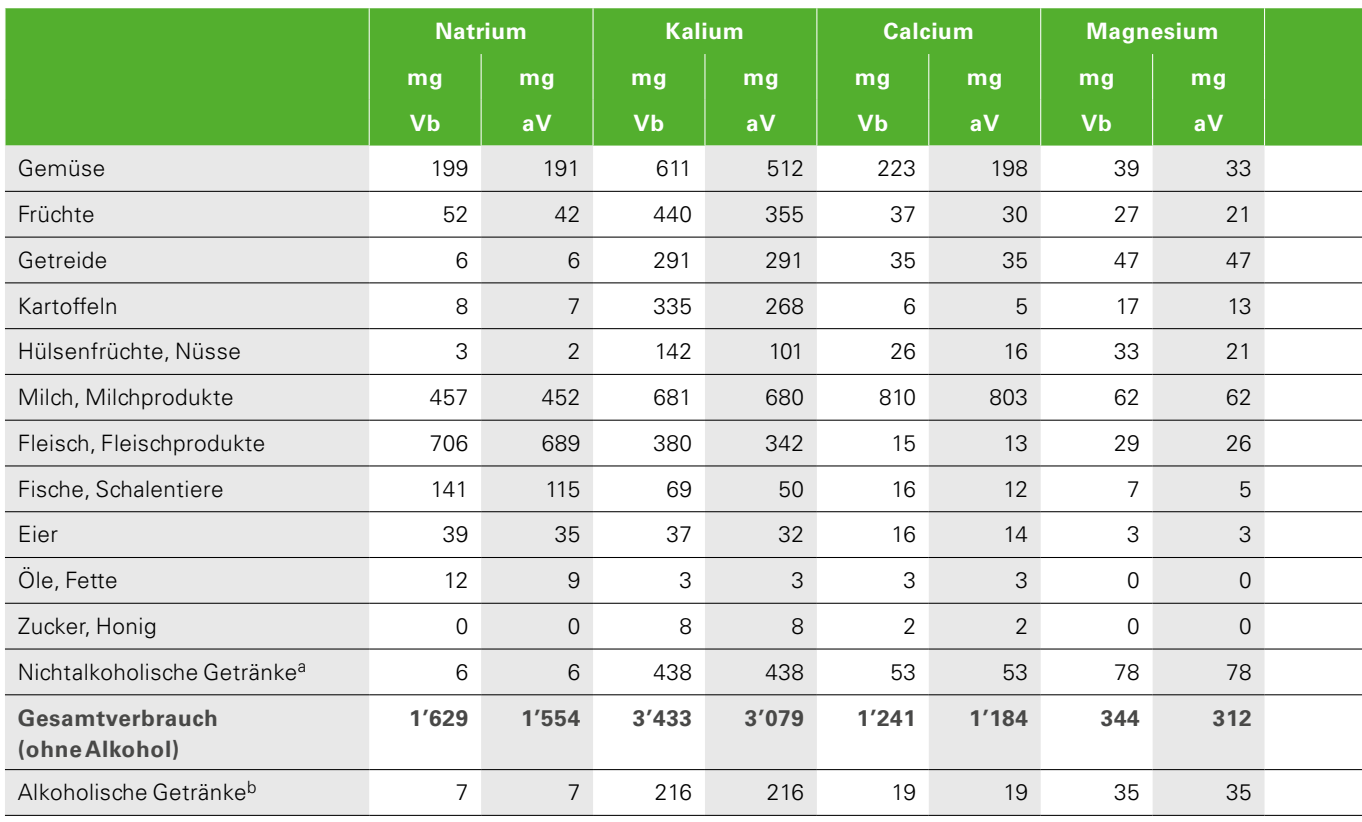

\footnotetext{
a umfasst die in Tabelle 2.9 aufgeführten Getränkegruppen ausser Mineralwasser
}

b nur Personen über 15 Jahre 
holischen Getränke Kakao und Kaffee sowie Milch und Milchprodukte (Abbildung 2.9).

\section{Eisen}

Der Eisenverbrauch hatte zwischen dem 4. und 5. SEB deutlich abgenommen und ist nun wieder leicht angestiegen (Tabelle 2.21). Damit liegt der angenäherte Verzehr gleichauf mit der gewichteten empfohlenen Zufuhr (Tabelle 2.22). Verschiedene Studien weisen jedoch darauf hin, dass die Versorgung nicht bei allen Bevölkerungsgruppen ausreichend ist. Eine Un- tersuchung bei 672 Frauen aus dem Zürcher Unterland im Alter von 18-42 Jahren wies bei $22.7 \%$ einen Eisenmangel und bei $2.2 \%$ eine Eisenmangelanämie nach (72). Die gleichzeitige Erfassung der Nahrungsaufnahme bei 176 der Frauen mittels 24-h-Recall ergab, dass die durchschnittliche Eisenaufnahme nur $60 \%$ der für diese Bevölkerungsgruppe empfohlenen 15 mg entsprach und es sich dabei fast nur um das schlechter absorbierbare Nicht-Hämeisen handelte (73). Vergleichbare Prozentzahlen fanden sich auch in einer Zürcher Studie bei Mara-

\begin{tabular}{|c|c|c|c|c|c|c|c|c|c|}
\hline \multicolumn{2}{|c|}{ Phosphor } & \multicolumn{2}{|c|}{ Chlorid } & \multicolumn{2}{|c|}{ Eisen } & \multicolumn{2}{|c|}{ Zink } & \multicolumn{2}{|c|}{ Jod } \\
\hline mg & mg & mg & $\mathrm{mg}$ & mg & mg & mg & mg & $\mu \mathrm{g}$ & $\mu \mathrm{g}$ \\
\hline Vb & aV & Vb & aV & Vb & aV & Vb & aV & Vb & aV \\
\hline 98 & 85 & 310 & 292 & 2.0 & 1.7 & 0.8 & 0.7 & 8 & 7 \\
\hline 38 & 31 & 44 & 32 & 0.8 & 0.7 & 0.3 & 0.2 & 3 & 2 \\
\hline 256 & 256 & 103 & 103 & 3.0 & 3.0 & 3.2 & 3.2 & 3 & 3 \\
\hline 37 & 30 & 54 & 43 & 0.5 & 0.4 & 0.4 & 0.3 & 4 & 3 \\
\hline 68 & 46 & 10 & 8 & 1.0 & 0.8 & 0.5 & 0.4 & 1 & 1 \\
\hline 606 & 601 & 807 & 799 & 0.3 & 0.3 & 3.3 & 3.3 & 34 & 34 \\
\hline 264 & 238 & $1^{\prime} 038$ & $1^{\prime} 024$ & 2.2 & 2.0 & 3.2 & 3.0 & 11 & 11 \\
\hline 61 & 44 & 11 & 8 & 0.3 & 0.2 & 0.2 & 0.1 & 8 & 5 \\
\hline 56 & 49 & 53 & 47 & 0.5 & 0.5 & 0.4 & 0.3 & 3 & 2 \\
\hline 3 & 3 & 17 & 14 & 0.0 & 0.0 & 0.0 & 0.0 & 1 & 1 \\
\hline 1 & 1 & 3 & 3 & 0.2 & 0.2 & 0.0 & 0.0 & 0 & 0 \\
\hline 111 & 111 & 13 & 13 & 2.0 & 2.0 & 0.9 & 0.9 & 3 & 3 \\
\hline 1'598 & 1'495 & $2^{\prime} 464$ & 2'386 & 12.7 & 11.6 & 13.2 & 12.5 & 78 & 72 \\
\hline 41 & 41 & 42 & 42 & 0.9 & 0.9 & 0.2 & 0.2 & 13 & 13 \\
\hline
\end{tabular}


Tabelle 2.17: Täglicher Verbrauch an Energie, energieliefernden Nährstoffen, Nahrungsfasern und Cholesterin im Vergleich zu den Angaben im 5., 4., 3. und 2. Schweizerischen

Ernährungsbericht und zur gewichteten empfohlenen Zufuhr

\begin{tabular}{|c|c|c|c|c|c|c|c|}
\hline Parameter & $\begin{array}{l}\text { Ein- } \\
\text { heit }\end{array}$ & $\begin{array}{c}\text { 6. SEB } \\
2007 / 08\end{array}$ & $\begin{array}{c}\text { 5. SEB } \\
2001 / 02\end{array}$ & $\begin{array}{c}\text { 4. SEB } \\
1994 / 95\end{array}$ & $\begin{array}{c}\text { 3. SEB } \\
1987\end{array}$ & $\begin{array}{c}\text { 2. SEB } \\
1979 / 80\end{array}$ & $\begin{array}{l}\text { empfohle- } \\
\text { ne Zufuhr } \\
\text { gewichtet }^{\mathrm{c}}\end{array}$ \\
\hline \multirow[t]{2}{*}{ Energie } & kcal & $2^{\prime} 923+188^{a}$ & $2^{\prime} 960^{b}+196^{a}$ & $2^{\prime} 962+239^{a}$ & $3^{\prime} 084+283^{a}$ & $3^{\prime} 083+344^{a}$ & $2^{\prime} 010 / 2^{\prime} 245^{d}$ \\
\hline & kJ & $12^{\prime} 230+785^{a}$ & $12^{\prime} 385^{b}+818^{a}$ & $12^{\prime} 395+1^{\prime} 000^{a}$ & $12^{\prime} 900+1^{\prime} 180^{a}$ & $12^{\prime} 900+1^{\prime} 440^{a}$ & $8^{\prime} 415 / 9^{\prime} 395^{d}$ \\
\hline Protein & $g$ & $97.1+1.0^{a}$ & $95.0+1.0^{a}$ & $98.8+1.2^{a}$ & 104.7 & 98.8 & $48.0^{e}$ \\
\hline Fett & $g$ & 125.1 & 122.0 & 125.2 & 132.2 & 142.9 & $69-75 / 69-77^{d}$ \\
\hline Kohlenhydrate & $g$ & $352+6^{a}$ & $369^{b}+6^{a}$ & $356+11^{a}$ & 351 & 373 & - \\
\hline Nahrungsfasern & $g$ & $28.0+0.4^{a}$ & 25.7 & 25.0 & 21.8 & 22.4 & - \\
\hline SFA & $g$ & 43.3 & 43.8 & 50.2 & - & - & - \\
\hline MUFA & $g$ & 42.1 & 41.0 & 45.2 & - & - & - \\
\hline PUFA & $g$ & 25.5 & 23.8 & 21.8 & - & - & - \\
\hline Cholesterin & $\mathrm{mg}$ & 367 & 363 & 390 & 480 & 534 & - \\
\hline Alkohol & $g$ & $0.0+23.2^{\mathrm{a}}$ & - & - & - & - & - \\
\hline
\end{tabular}

- = keine bzw. nicht ausreichend Daten vorhanden

a Zusätzliche Menge aus alkoholischen Getränken für Personen über 15 Jahren. Lesebeispiel für 2007/08: Der durchschnittliche Energieverbrauch ohne Alkohol ist 2'923 kcal; mit Alkohol liegt der Energieverbrauch um 188 kcal höher.

b korrigiert gegenüber den im 5. SEB publizierten Werten (sieheTabelle 2.8, Fussnote e)

c Berechnung der gewichteten empfohlenen Zufuhr basierend auf den DACH-Referenzwerten, siehe Kapitel 2.4.1.

d Energiebedarf für die gesamte Bevölkerung mit PAL-Wert 1.4 (ausschliesslich sitzende Tätigkeit mit wenig oder keiner anstrengenden Freizeitaktivität) und 1.6 (sitzende Tätigkeit, zeitweilig auch zusätzlicher Energieaufwand für gehende und stehende Tätigkeiten) berechnet (Anzahl Personen gemäss Bevölkerungsstatistik).

e Minimum

thonläuferinnen und -läufern: 12 der untersuchten 43 Frauen (28\%) wiesen einen Eisenmangel auf (74). Bei den Männern lag der Prozentsatz bei $1.6 \%$ (2 von 127). Aber auch das Gegenteil ist möglich: So fand sich in der gleichen Studiengruppe bei $15 \%$ der Männer und $4.7 \%$ der Frauen eine Eisenüberladung, was wohl auf den weitverbreiteten Konsum von Eisensupplementen oder sogar Eiseninfusionen bei Sportlern zurückzuführen ist. In der allgemeinen Schweizer Bevölkerung ist die Eisensupplementierung hingegen nicht üblich (durchschnittlich 1.8\% der
Bevölkerung), wie die CoLaus-Studie aufzeigt (46). Im Jahr 2004 wurden in sechs Schweizer Armeerekrutierungszentren 7'714 Rekruten untersucht. Bei 7.2\% wurde ein latenter Eisenmangel festgestellt (75). Für weitere Informationen zum Thema sei auf Kapitel 3.10.4 verwiesen.

Fleisch und Fleischprodukte, Gemüse und nichtalkoholische Getränke (hauptsächlich Kakao) sind die Hauptquellen für Eisen (Abbildung 2.9), wobei das Hämeisen aus Fleisch eine höhere Bioverfügbarkeit aufweist als das NichtHämeisen aus pflanzlichen Lebensmitteln (52). 
Tabelle 2.18: Täglicher angenäherter Verzehr an Energie, energieliefernden Nährstoffen, Nahrungsfasern und Cholesterin im Vergleich zu den Angaben im 5. und 3. Schweizerischen Ernährungsbericht und zur gewichteten empfohlenen Zufuhra

\begin{tabular}{|c|c|c|c|c|c|}
\hline Parameter & $\begin{array}{l}\text { Ein- } \\
\text { heit }\end{array}$ & $\begin{array}{c}\text { 6. SEB } \\
2007 / 08\end{array}$ & $\begin{array}{l}\text { 5. SEB } \\
2001 / 02\end{array}$ & $\begin{array}{c}\text { 3. SEB } \\
1985-1987\end{array}$ & $\begin{array}{l}\text { empfohlene } \\
\text { Zufuhr } \\
\text { gewichtet }^{\text {d }}\end{array}$ \\
\hline \multirow[t]{2}{*}{ Energie } & kcal & $2^{\prime} 661+188^{b}$ & $2^{\prime} 703^{c}+196^{b}$ & & $2^{\prime} 010 / 2^{\prime} 245^{e}$ \\
\hline & $\mathrm{kJ}$ & $11^{\prime} 135+785^{b}$ & $11^{\prime} 310^{c}+818^{b}$ & $11^{\prime} 060+1^{\prime} 270^{b}$ & $8^{\prime} 415 / 9^{\prime} 395^{e}$ \\
\hline Protein & $g$ & $90.2+1.0^{b}$ & $87.7+1.0^{b}$ & $97.8+1.2^{b}$ & $48.0^{f}$ \\
\hline Fett & $g$ & 103.8 & 101.4 & 108.9 & $69-75 / 69-77^{e}$ \\
\hline Kohlenhydrate & $g$ & $341+5^{b}$ & $358^{c}+6^{b}$ & $313+25^{b}$ & - \\
\hline Nahrungsfasern & $g$ & $25.5+0.4^{b}$ & $23.3+0.4^{b}$ & $23.4+1.0^{b}$ & - \\
\hline SFA & g & 40.0 & 40.3 & 45.5 & - \\
\hline MUFA & $g$ & 33.4 & 32.5 & 34.4 & - \\
\hline PUFA & $g$ & 18.3 & 17.2 & 16.2 & - \\
\hline Cholesterin & $\mathrm{mg}$ & 336 & 331 & 450 & - \\
\hline Alkohol & $\mathrm{g}$ & $0.0+23.2^{b}$ & - & - & - \\
\hline $\begin{array}{l}\text { - = keine bzw. ni } \\
\text { a keine Daten zu } \\
\text { b zusätzliche Me } \\
{ }^{c} \text { korrigiert geger } \\
\text { d Berechnung de } \\
\text { e Energiebedarf } \\
\text { anstrengenden } \\
\text { stehendeTätig } \\
\text { f Minimum }\end{array}$ & $\begin{array}{l}\text { end D } \\
\text { erten V } \\
\text { oholisc } \\
\text { n } 5 \text {. SE } \\
\text { en em } \\
\text { amte E } \\
\text { ivität) } \\
\text { chnet }\end{array}$ & $\begin{array}{l}\text { vorhanden } \\
\text { hr im } 4 \text {. und } 2.5 \\
\text { Getränken für F } \\
\text { blizierten Wert } \\
\text { enen Zufuhr ba } \\
\text { kerung mit PA } \\
6 \text { (sitzende Tät } \\
\text { hl Personen ge }\end{array}$ & $\begin{array}{l}\text { d auf den DACH } \\
1.4 \text { (ausschlies } \\
\text { zeitweilig auch } \\
\text { Bevölkerungsst }\end{array}$ & $\begin{array}{l}\text { renzwerten, sieh } \\
\text { sitzende Tätigkei } \\
\text { zlicher Energieau } \\
\text { k). }\end{array}$ & $\begin{array}{l}\text { pitel 2.4.1. } \\
\text { wenig oder keiner } \\
\text { nd für gehende und }\end{array}$ \\
\hline
\end{tabular}

\section{Zink}

Auch beim Zink hat sich der Verbrauch nach dem Rückgang zwischen dem 4. und 5. SEB wieder geringfügig verbessert (Tabelle 2.21). Im Gegensatz zum Eisen liegt der angenäherte Verzehr aber deutlich über der gewichteten empfohlenen Zufuhr (Tabelle 2.22). Zink wird hauptsächlich über Milch und Milchprodukte, Fleisch und Fleischprodukte sowie Getreide zugeführt (Abbildung 2.9).
Jod

Bei den Angaben zur Jodzufuhr in den TabelIen 2.21 und 2.22 handelt es sich nur um die aus Lebensmitteln berechneten Mengen. Verbrauch und angenäherter Verzehr liegen im gleichen Bereich wie die Werte des 4. und 5. SEB und decken nur rund die Hälfte der empfohlenen gewichteten Zufuhr ab. Das Jod stammt dabei überwiegend aus Milch und Milchprodukten (Abbildung 2.9). In den Zahlen nur teilweise enthalten ist der Jodverbrauch durch den Einsatz von jodiertem Salz in der Lebensmittelproduk- 
Tabelle 2.19: Täglicher Verbrauch an Vitaminen im Vergleich zu den Angaben im 5. und 4. Schweizerischen Ernährungsbericht und zur gewichteten empfohlenen Zufuhr

\begin{tabular}{|c|c|c|c|c|c|}
\hline Vitamin & Einheit & $\begin{array}{c}\text { 6. SEB } \\
2007 / 08\end{array}$ & $\begin{array}{c}\text { 5. SEB } \\
2001 / 02\end{array}$ & $\begin{array}{c}\text { 4. SEB } \\
1994 / 95\end{array}$ & $\begin{array}{c}\text { empfohlene } \\
\text { Zufuhr } \\
\text { gewichtet }^{c}\end{array}$ \\
\hline A & $\mathrm{mg}$ & 1.4 & 1.3 & 1.3 & 0.9 \\
\hline Carotinoide & $\mathrm{mg}$ & 4.2 & 4.3 & 5.2 & - \\
\hline A + Carotinoide & $m g R E^{b}$ & 1.8 & 1.7 & 2.3 & - \\
\hline D & $\mu \mathrm{g}$ & 3.5 & 2.9 & - & 5.9 \\
\hline$E$ & mgTEb & 24.5 & 21.8 & 15.6 & 12.2 \\
\hline B1 (Thiamin) & $\mathrm{mg}$ & 1.4 & 1.4 & 1.7 & 1.1 \\
\hline B2 (Riboflavin) & $\mathrm{mg}$ & $1.8+0.1^{a}$ & $1.9+0.1^{a}$ & $2.1+0.1^{a}$ & 1.2 \\
\hline Niacin & $\mathrm{mg}$ & $16.1+1.6^{a}$ & $15.3+1.7^{a}$ & $17.4+1.5^{a}$ & 13.8 \\
\hline B6 (Pyridoxin) & $\mathrm{mg}$ & $2.0+0.2^{a}$ & $2.0+0.2^{a}$ & $2.3+0.1^{a}$ & 1.3 \\
\hline Folsäure gesamt & $\mu \mathrm{g}$ & $344+10^{a}$ & $334+10^{a}$ & $306+12^{a}$ & 388 \\
\hline B12 (Cobalamin) & $\mu \mathrm{g}$ & $6.6+0.2^{a}$ & $6.7+0.2^{a}$ & $9.5+0.6^{a}$ & 2.8 \\
\hline Pantothensäure & $\mathrm{mg}$ & $6.8+0.2^{a}$ & $6.4+0.2^{a}$ & $7.2+0.3^{a}$ & 5.8 \\
\hline C & $\mathrm{mg}$ & 130 & 130 & 143 & 97 \\
\hline
\end{tabular}

- = keine bzw. nicht ausreichend Daten vorhanden

a Zusätzliche Menge aus alkoholischen Getränken für Personen über 15 Jahren. Lesebeispiel für 2007/08: Der durchschnittliche Vitamin-B2-Verbrauch ohne alkoholische Getränke ist 1.8 mg; mit dem Beitrag von alkoholischen Getränken liegt er um $0.1 \mathrm{mg}$ höher.

b $\mathrm{RE}=$ Retinoläquivalente; $\mathrm{TE}=$ Tocopheroläquivalente

c Berechnung der gewichteten empfohlenen Zufuhr basierend auf den DACH-Referenzwerten, siehe Kapitel 2.4.1.

tion und -verarbeitung. Auch nicht mit einbezogen sind die über die Verwendung von jodiertem Salz im Haushalt aufgenommenen Mengen. Nimmt man die in der Genfer Studie Bus Santé (71) ermittelten Daten bezüglich der Salzverwendung bei der Zubereitung von Lebensmitteln und am Tisch (2.8 g bzw. $4.3 \mathrm{~g}$ Salz bei Frauen bzw. Männern) als Grundlage, so kann bei einer Jodkonzentration von 20 mg Jodid pro kg Salz bei Frauen und Männern eine zusätzliche Zufuhr von $56 \mu \mathrm{g}$ bzw. $86 \mu \mathrm{g}$ Jod pro Person und Tag angenommen werden. Zusammen ergäbe dies einen Verbrauch von $128 \mu \mathrm{g}$ bzw. $158 \mu \mathrm{g}$ Jod pro Person und Tag, wozu dann noch die in verarbeiteten Lebensmitteln (durch Einsatz von jodiertem Salz) enthaltenen Mengen kommen würden. Diese Menge liegt in der Grössenordnung der gewichteten empfohlenen Zufuhr. Eine repräsentative Untersuchung der Eidgenössischen Technischen Hochschule Zürich (ETH Zürich) im Jahr 2004 hat festgestellt, dass der durchschnittliche Jodgehalt im Urin von Kindern $(N=610)$ und Schwangeren $(N=511)$ bei $141 \mu \mathrm{g} / \mathrm{L}$ bzw. $249 \mu \mathrm{g} / \mathrm{L}$ liegt, was auf eine ausreichende 
Tabelle 2.20: Täglicher angenäherter Verzehr an Vitaminen im Vergleich zu den Angaben im 5. und 3. Schweizerischen Ernährungsbericht und zur gewichteten empfohlenen Zufuhra

\begin{tabular}{|c|c|c|c|c|c|}
\hline Vitamin & Einheit & $\begin{array}{c}\text { 6. SEB } \\
2007 / 08\end{array}$ & $\begin{array}{l}\text { 5. SEB } \\
2001 / 02\end{array}$ & $\begin{array}{c}\text { 3. SEB } \\
1985-1987\end{array}$ & $\begin{array}{l}\text { empfohlene } \\
\text { Zufuhr } \\
\text { gewichtet }^{\text {d }}\end{array}$ \\
\hline A & $\mathrm{mg}$ & 1.3 & 1.6 & - & 0.9 \\
\hline Carotinoide & $\mathrm{mg}$ & 3.6 & 3.5 & - & - \\
\hline A + Carotinoide & $m g R E^{c}$ & 1.6 & 1.6 & 2.3 & - \\
\hline D & $\mu \mathrm{g}$ & 3.1 & 2.5 & - & 5.9 \\
\hline$E$ & $m g T E^{\mathrm{C}}$ & 17.2 & 15.3 & 15.8 & 12.2 \\
\hline B1 (Thiamin) & $\mathrm{mg}$ & 1.3 & 1.3 & 2.3 & 1.1 \\
\hline B2 (Riboflavin) & $\mathrm{mg}$ & $1.7+0.1^{b}$ & $1.8+0.1^{b}$ & $2.1+0.1^{b}$ & 1.2 \\
\hline Niacin & $\mathrm{mg}$ & $14.2+1.6^{b}$ & $13.4+1.6^{b}$ & $24.0+1.6^{b}$ & 13.8 \\
\hline B6 (Pyridoxin) & $\mathrm{mg}$ & $1.7+0.2^{b}$ & $1.7+0.2^{b}$ & $2.3+0.1^{b}$ & 1.3 \\
\hline Folsäure gesamt & $\mu \mathrm{g}$ & $295+10^{b}$ & $284+10^{b}$ & $262+12^{b}$ & 388 \\
\hline B12 (Cobalamin) & $\mu \mathrm{g}$ & $6.0+0.2^{b}$ & $6.1+0.2^{b}$ & 11.4 & 2.8 \\
\hline Pantothensäure & $\mathrm{mg}$ & $6.2+0.2^{b}$ & $5.8+0.2^{b}$ & $6.5+0.3^{b}$ & 5.8 \\
\hline C & $\mathrm{mg}$ & 106 & 108 & $119+2^{b}$ & 97 \\
\hline
\end{tabular}

- = keine bzw. nicht ausreichend Daten vorhanden

a keine Daten zum angenäherten Verzehr im 4. und 2. SEB

b zusätzliche Menge aus alkoholischen Getränken für Personen über 15 Jahren

c $R E=$ Retinoläquivalente; $T E=$ Tocopheroläquivalente

d Berechnung der gewichteten empfohlenen Zufuhr basierend auf den DACH-Referenzwerten, siehe Kapitel 2.4.1.

Jodzufuhr in der Bevölkerung schliessen lässt (76). Eine Wiederholung der Studie im Jahr 2009 ergab durchschnittliche Jodkonzentrationen im Urin von $120 \mu \mathrm{g} / \mathrm{L}$ (Schulkinder, $\mathrm{N}=916$ ) und $162 \mu \mathrm{g} / \mathrm{L}$ (schwangere Frauen, $\mathrm{N}=648$ ), was auf eine weiterhin genügende Jodversorgung der Schweizer Bevölkerung hinweist (77). Erste Ergebnisse aus einer aktuellen nationalen Studie deuten jedoch darauf hin, dass die Jodversorgung eines Teils der Schweizer Bevölkerung nicht der Empfehlung für eine ausreichende Jodzufuhr von $150 \mu \mathrm{g}$ pro Tag entspricht $(48 ; 110)$.
Jodiertes Salz wird von 92\% der Haushalte verwendet; trotzdem weist der Vergleich der Zahlen von 2004 und 2009 auf einen Rückgang in der Jodzufuhr hin. Da sich die Verbrauchszahlen nicht verändert haben, hängt dies vermutlich mit dem Ersatz von jodiertem durch unjodiertes Salz in der Lebensmittelproduktion und / oder einem verringerten Salzeinsatz im Haushalt zusammen. Die Ermittlung des Jodstatus von Neugeborenen ( $N=875)$ ergab eine nur marginal genügende Versorgung, wobei vor allem ausschliesslich gestillte Neugeborene ohne 
Tabelle 2.21: Täglicher Verbrauch an Mineralstoffen im Vergleich zu den Angaben im 5., 4. und 2. Schweizerischen Ernährungsbericht und zur gewichteten empfohlenen Zufuhra

\begin{tabular}{|c|c|c|c|c|c|c|}
\hline Mineralstoff & $\begin{array}{l}\text { Ein- } \\
\text { heit }\end{array}$ & $\begin{array}{c}\text { 6. SEB } \\
2007 / 08\end{array}$ & $\begin{array}{c}\text { 5. SEB } \\
2001 / 02\end{array}$ & $\begin{array}{c}\text { 4. SEB } \\
1994 / 95\end{array}$ & $\begin{array}{c}\text { 2. SEB } \\
1979 / 80\end{array}$ & $\begin{array}{l}\text { empfohle- } \\
\text { ne Zufuhr } \\
\text { gewichtet }^{\text {e }}\end{array}$ \\
\hline Kalium & $\mathrm{mg}$ & $3^{\prime} 433+216^{b}$ & $3^{\prime} 287+230^{b}$ & $3^{\prime} 776+228^{b}$ & 3'700 & $1^{\prime} 915^{c}$ \\
\hline Natrium & $\mathrm{mg}$ & $1^{\prime} 629+7^{b}$ & $1^{\prime} 598+8^{b}$ & $960+40^{b}$ & $4^{\prime} 700$ & $530^{c}$ \\
\hline Chlorid & $\mathrm{mg}$ & $2^{\prime} 464+42^{b}$ & $2^{\prime} 253+42^{b}$ & $1^{\prime} 756+80^{b}$ & 6'900 & $800^{c}$ \\
\hline Calcium & $\mathrm{mg}$ & $1^{\prime} 241+19^{b}$ & $1^{\prime} 146+20^{b}$ & $1^{\prime} 096+32^{b}$ & 1'000 & 987 \\
\hline Phosphor & $\mathrm{mg}$ & $1^{\prime} 598+41^{b}$ & $1^{\prime} 510+43^{b}$ & $1^{\prime} 700+60^{b}$ & $1^{\prime} 600$ & 748 \\
\hline Magnesium & $\mathrm{mg}$ & $344+35^{b}$ & $344+36^{b}$ & $380+30^{b}$ & 370 & 306 \\
\hline Eisen & $\mathrm{mg}$ & $12.9+0.7^{b}$ & $11.9+1.0^{b}$ & $15.0+0.8^{b}$ & 14.7 & 11.5 \\
\hline Zink & $\mathrm{mg}$ & $13.2+0.2^{b}$ & $12.5+0.2^{b}$ & $15.1+0.4^{b}$ & 11.2 & 8.1 \\
\hline Jod & $\mu \mathrm{g}$ & $78^{d}+13^{b}$ & $92^{d}+15^{b}$ & $83^{d}+19^{b}$ & 160 & 144 \\
\hline
\end{tabular}

a keine Angaben zum Mineralstoffverbrauch im 3. SEB

b Zusätzliche Menge aus alkoholischen Getränken für Personen über 15 Jahren. Lesebeispiel für 2007/08: Der durchschnittliche Kaliumverbrauch ohne alkoholische Getränke ist 3'433 mg; mit dem Beitrag von alkoholischen Getränken liegt er um 216 mg höher.

c berechnet aus den Schätzwerten für eine minimale Zufuhr

d Es handelt sich hier nur um die aus den Lebensmitteln berechneten Mengen.

e Berechnung der gewichteten empfohlenen Zufuhr basierend auf den DACH-Referenzwerten, siehe Kapitel 2.4.1.

jodhaltige Beikost oder Muttermilchersatz ein erhöhtes Risiko einer Jodunterversorgung aufweisen (77; Kapitel 3.10.5).

\section{Selen}

Wie schon in den vorangehenden SEB wurde die Selenzufuhr basierend auf den Verbrauchsdaten nicht berechnet, da in den Nährwertdatenbanken nicht genügend Angaben zu den Selengehalten der Lebensmittel verfügbar sind. Berechnungen des BAG, die auf Analysen der Selenkonzentration in Lebensmitteln in Kombination mit Lebensmittelverbrauchsdaten des Schweizerischen Bauernverbandes aus dem Jahr 2006 basieren, ergeben eine durchschnitt- liche Selenaufnahme von $50 \mu \mathrm{g}$ pro Person und Tag (78). Eine in den Jahren 2005 und 2006 in der ganzen Schweiz durchgeführte Untersuchung von Blutproben gesunder, erwachsener Blutspender ( $N=1^{\prime} 847$ ) beiderlei Geschlechts weist auf eine ausreichende Selenversorgung der Schweizer Bevölkerung hin (79). Die durchschnittliche Selenkonzentration im Blut (inkl. Standardabweichung) lag bei $98.4 \pm 12.9 \mu \mathrm{g} / \mathrm{L}$ (Kapitel 3.10.6), wobei die Männer einen höheren Durchschnittswert aufwiesen als die Frauen (99.9 $\mu \mathrm{g} / \mathrm{L}$ vs. $95.6 \mu \mathrm{g} / \mathrm{L})$. Geht man von diesen Blutwerten aus, so müsste die tägliche Seleneinnahme mit der Nahrung bei $66 \mu$ Selen pro Person liegen (78). Eine Duplikatsstudie 
Tabelle 2.22: Täglicher angenäherter Verzehr an Mineralstoffen im Vergleich zu den Angaben im 5. und 3. Schweizerischen Ernährungsbericht und zur gewichteten empfohlenen Zufuhra

\begin{tabular}{l|c|c|c|c|c} 
Mineralstoff & Einheit & $\begin{array}{c}\text { 6. SEB } \\
\mathbf{2 0 0 7 / 0 8}\end{array}$ & $\begin{array}{c}\text { 5. SEB } \\
\mathbf{2 0 0 1 / 0 2}\end{array}$ & $\begin{array}{c}\text { 3. SEB } \\
1985-1987\end{array}$ & $\begin{array}{c}\text { empfohlene } \\
\text { Zufuhr } \\
\text { gewichtet }\end{array}$ \\
\hline Kalium & $\mathrm{mg}$ & $3^{\prime} 079+216^{\mathrm{b}}$ & $2^{\prime} 932+230^{\mathrm{b}}$ & $3^{\prime} 863+250^{\mathrm{b}}$ & $1^{\prime} 915^{\mathrm{c}}$ \\
\hline Natrium & $\mathrm{mg}$ & $1^{\prime} 554+7^{\mathrm{b}}$ & $1^{\prime} 515+8^{\mathrm{b}}$ & $3^{\prime} 768+18^{\mathrm{b}}$ & $530^{\mathrm{c}}$ \\
\hline Chlorid & $\mathrm{mg}$ & $2^{\prime} 386+42^{\mathrm{b}}$ & $2^{\prime} 167+42^{\mathrm{b}}$ & $5^{\prime} 846+97^{\mathrm{b}}$ & $800^{\mathrm{c}}$ \\
\hline Calcium & $\mathrm{mg}$ & $1^{\prime} 184+19^{\mathrm{b}}$ & $1^{\prime} 098+20^{\mathrm{b}}$ & $981+28^{\mathrm{b}}$ & 987 \\
\hline Phosphor & $\mathrm{mg}$ & $1^{\prime} 495+111^{\mathrm{b}}$ & $1^{\prime} 405+43^{\mathrm{b}}$ & $1^{\prime} 605+71^{\mathrm{b}}$ & 748 \\
\hline Magnesium & $\mathrm{mg}$ & $312+35^{\mathrm{b}}$ & $312+36^{\mathrm{b}}$ & $365+33^{\mathrm{b}}$ & 306 \\
\hline Eisen & $\mathrm{mg}$ & $11.6+0.9^{\mathrm{b}}$ & $10.8+1.0^{\mathrm{b}}$ & $16.2+1.4^{\mathrm{b}}$ & 11.5 \\
\hline Zink & $\mathrm{mg}$ & $12.5+0.2^{\mathrm{b}}$ & $11.8+0.2^{\mathrm{b}}$ & $14.5+0.4^{\mathrm{b}}$ & 8.1 \\
\hline Jod & $\mu \mathrm{gg}$ & $72+13^{\mathrm{b}}$ & $86^{\mathrm{d}}+15^{\mathrm{b}}$ & $105+20^{\mathrm{b}}$ & 144 \\
\hline
\end{tabular}

a keine Daten zum angenäherten Verzehr im 4. und 2. SEB

b zusätzliche Menge aus alkoholischen Getränken für Personen über 15 Jahren

${ }^{c}$ berechnet aus den Schätzwerten für eine minimale Zufuhr

d korrigiert gegenüber dem im 5. SEB publizierten Wert

e Berechnung der gewichteten empfohlenen Zufuhr basierend auf den DACH-Referenzwerten, siehe Kapitel 2.4.1.

$(N=72)$, durchgeführt von der gleichen Forschergruppe, ergab eine Seleneinnahme von $62 \pm 27 \mu \mathrm{g}$ (Durchschnitt und Standardabweichung) pro Tag (78). Laut den DACH-Referenzwerten wird für Erwachsene eine Zufuhr von 30-70 $\mathrm{gg}$ pro Tag als notwendig erachtet (48). Da die Schweiz selenarme Böden besitzt, gelangt das Selen vor allem durch aus den USA importierten, selenreichen Weizen sowie über tierische Produkte (Selenanreicherung des Futters) in unsere Nahrung. Oben genannte Untersuchungen bestätigen dies: Der ausreichende Selenstatus wird zurzeit hauptsächlich durch den Konsum von Schweizer Teigwaren, hergestellt mit nordamerikanischem Durum-Weizen, sowie über den Fleischverzehr sichergestellt. Daneben stellen auch Fisch, Eier, Weizenmehl und Milch wichtige Selenquellen dar (78).

Sechster Schweizerischer Ernährungsbericht | 99 


\subsubsection{Energie- und Nährstoff- versorgung einzelner Bevölkerungsgruppen}

Basierend auf den Verbrauchszahlen können keine Angaben zur Versorgung einzelner Bevölkerungsgruppen mit Energie, energieliefernden Nährstoffen, Nahrungsfasern und Mikronährstoffen gemacht werden. Vereinzelt existieren jedoch Studien, die bestimmte Bevölkerungsgruppen untersucht haben und Aussagen zu deren Nährstoffversorgung zulassen.

\section{Ältere Menschen}

Eine Studie aus Lausanne hat die Nährstoffversorgung von Frauen im Alter von 75-87 Jahren untersucht (61). Die Auswertung der Food Frequency Questionnaires (FFQ) von 401 Teilnehmerinnen der Kohortenstudie SEMOF (Swiss Evaluation of the Methods of Measurement of Osteoporotic Fracture Risk) zeigt, dass bei einigen Nährstoffen die durchschnittliche Zufuhr im Vergleich zu den DACH-Referenzwerten zu gering ausfällt (Tabelle 2.23). Beinahe alle Frauen (99.8\%) wiesen eine massiv ungenügende $\mathrm{Vi-}$ tamin-D-Zufuhr auf; bei über 50\% der Frauen war der tägliche Bedarf an Energie, Protein, Fett, Kohlenhydraten, Calcium, Magnesium, Vitamin B6, Vitamin C und Vitamin E nicht gedeckt. Da es sich bei den Studienteilnehmerinnen um sich selbständig versorgende Frauen handelte mit einem besseren Gesundheitszu-

Tabelle 2.23: Mittels Food Frequency Questionnaire erhobene durchschnittliche Nährstoffeinnahme im Jahr 2003 (und Standardabweichung) bei 401 Frauen im Alter von 75-87 Jahren (61)

\begin{tabular}{l|c|c|c}
\hline Nährstoff & Einheit & Zufuhr & $\begin{array}{c}\text { DACH- Referenzwert } \\
\text { (für Frauen ab 65 Jahren; 48) }\end{array}$ \\
\hline Energie & $\mathrm{kcal}$ & $1^{\prime} 544.0(447.7)$ & $2^{\prime} 069$ \\
\hline Protein & $\mathrm{g}$ & $65.2(19.9)$ & 63 \\
\hline Fett & $\mathrm{g}$ & $63.9(21.3)$ & 69 \\
\hline Kohlenhydrate & $\mathrm{g}$ & $163.8(54.4)$ & $>259$ \\
\hline Calcium & $\mathrm{mg}$ & $983.1(388.7)$ & $1^{\prime} 000$ \\
\hline Phosphor & $\mathrm{mg}$ & $1^{\prime} 163.7(391.5)$ & 700 \\
\hline Magnesium & $\mathrm{mg}$ & $287.7(93.1)$ & 300 \\
\hline Kalium & $\mathrm{mg}$ & $22^{\prime} 761.4(874.6)$ & 10 \\
\hline Eisen & $\mathrm{mg}$ & $11.6(3.7)$ & 1.2 \\
\hline Vitamin B6 & $\mathrm{mg}$ & $1.25(0.44)$ & 100 \\
\hline Vitamin C & $\mathrm{mg}$ & $93.1(45.2)$ & 10 \\
\hline Vitamin D & $\mu \mathrm{gg}$ & $2.49(1.45)$ & 11 \\
\hline Vitamin E & $\mathrm{mg}$ & $9.39(3.88)$ & \\
\hline & & & \\
\hline
\end{tabular}


stand als in dieser Altersgruppe sonst üblich, ist für den Durchschnitt der Bevölkerung in diesem Alter von einer noch ungenügenderen Versorgung auszugehen.

\section{Kinder}

Auch für Kinder aus der Deutschschweiz ( $N=156)$ im Alter von 6-14 Jahren liegen spezifische Daten vor (80). Ihr Lebensmittelverzehr wurde mittels 24-h-Recall sowie eintägigen Verzehrsprotokollen erhoben. In Tabelle 2.24 wird die Nährstoffzufuhr der normalgewichtigen Kinder (aufgeteilt nach Geschlecht) den DACH-Referenzwerten gegenübergestellt. Die Werte von übergewichtigen Kindern waren vergleichbar mit denjenigen der normalgewichti- gen Kinder, nur die Proteinzufuhr (in Gramm und in Prozent der Gesamtenergie) lag bei den übergewichtigen Kindern signifikant höher (80; Daten nicht aufgeführt in Tabelle 2.24).

\section{Säuglinge}

Wenig ist bekannt bezüglich der Ernährung von Säuglingen und Kleinkindern. Rund 90\% der Mütter, welche an der SGBO7 teilnahmen, gaben an, ihr jüngstes Kind gestillt zu haben, über $50 \%$ davon länger als 3 Monate. Der Anteil nicht stillender Mütter hat sich seit 2002 nicht verändert (81).

Tabelle 2.24: Durchschnittliche Nährstoffeinnahme (Median und Interquartile) von normalgewichtigen Kindern in der Deutschschweiz im Alter von 6-14 Jahren (80)

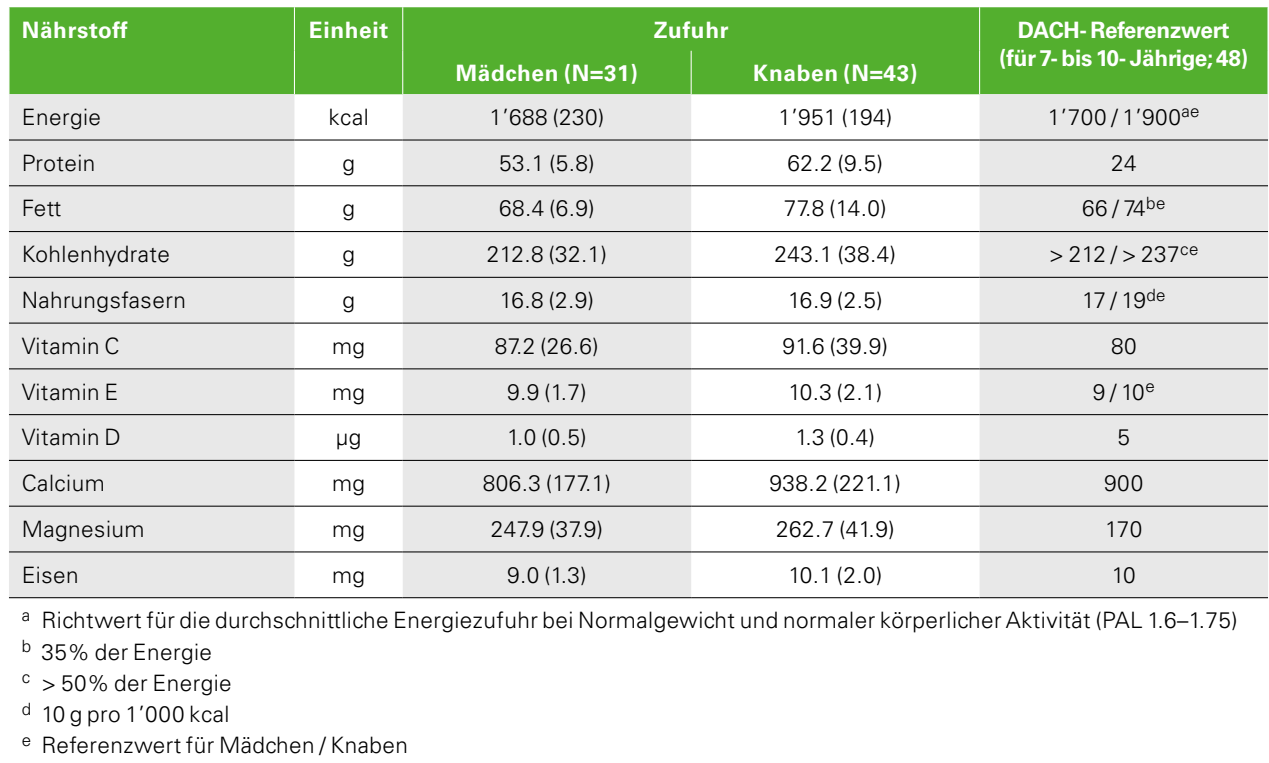




\subsubsection{Ausblick}

Analog zum Lebensmittelverbrauch haben sich auch bei dem daraus berechneten täglichen Verbrauch und angenäherten Verzehr an Energie, energieliefernden Nährstoffen, Nahrungsfasern und Mikronährstoffen keine grossen Veränderungen ergeben seit dem 5. SEB. Wie schon in den früheren SEB diskutiert, überschätzt die berechnete Energiezufuhr die reale Energieaufnahme. Daraus lässt sich ableiten, dass der berechnete Verbrauch auch bei den Nährstoffen über den tatsächlichen Zufuhrmengen liegt.

Unter den energieliefernden Nährstoffen ist bei den PUFA ein leicht positiver Trend auszumachen, ansonsten sind die Zahlen vergleichbar mit dem 4. und 5. SEB. Bezüglich Anteilen an der Energiezufuhr liegt der angenäherte Verzehr von Protein, Fett und Kohlenhydraten innerhalb der empfohlenen Bandbreiten, wenn auch beim Fett an der oberen Grenze. Der berechnete angenäherte Verzehr an Mikronährstoffen weist auf eine weiterhin vorherrschende Unterversorgung mit Vitamin D und Folsäure hin. Hinzuweisen ist auch auf Vitamin B1, Magnesium und Eisen, für welche der angenäherte Verzehr nur sehr knapp über der gewichteten empfohlenen Zufuhr liegt. Die anderen Mikronährstoffe übertreffen die gewichtete empfohlene Zufuhr in unterschiedlichem Masse, wobei für eine ausreichende Jodzufuhr der Einsatz von jodiertem Salz unabdingbar ist.

Die Ernährungssituation der Bevölkerung kann nur dann realistisch beurteilt werden, wenn die tatsächlich zugeführten Mengen an Makro- und Mikronährstoffen bekannt sind. Repräsentative und differenzierte Daten zur Nährstoffversorgung sind unverzichtbar für die Erarbeitung von sinnvollen und konkreten Mass- nahmen zur Aufrechterhaltung und Verbesserung der Ernährungssituation. In der Schweiz ist die diesbezügliche Datenlage als ungenügend einzustufen (Kapitel 4.9.1). Die hier vorgestellten Zahlen geben nur ein grobes Bild der Situation. Auf die Einschränkungen bezüglich Verbrauchsdaten wurde schon in Kapitel 2.3.3 eingegangen. Sie gelten analog auch für die daraus berechneten Zahlen zur Nährstoffversorgung. Einige Studien mit dem Ziel, den Nährstoffstatus der Schweizer Bevölkerung zu erheben, sind angelaufen (u.a. zu Vitamin D und Jod). Zum Zeitpunkt der Texterstellung lagen jedoch noch keine Endergebnisse dazu vor.

\subsection{Ernährungssituation in der Schweiz im Vergleich zum europäischen Ausland}

\subsubsection{Allgemeines}

Wie in den vorangehenden Kapiteln beschrieben, liegen bis heute zu wenig Daten zur präzisen Beschreibung der Ernährungssituation der Schweizer Bevölkerung vor. Die bis anhin veröffentlichten Daten basieren auf Agrarstatistiken, mit deren Hilfe indirekt der Lebensmittelverbrauch in kg pro Kopf und Jahr und der angenäherte Verzehr pro Person und Tag berechnet wurden. Der Nachteil dieser Daten besteht darin, dass sie den tatsächlichen Lebensmittelverzehr überschätzen und keine Verzehrsmuster bestimmter Bevölkerungsgruppen aufzeigen können (Kapitel 2.3).

Die meisten Länder Europas führen nationale Verzehrsstudien durch. Die European Food Safety Authority (EFSA) hat die aktuellsten Verzehrs- 
daten aus den vorhandenen nationalen Verzehrserhebungen in einer Datenbank (Comprehensive Food Consumption Database) zusammengeführt (82). Die unterschiedlichen Erhebungsmethoden erschweren bzw. verunmöglichen jedoch einen direkten Ländervergleich. Aus diesem Grund wurden von der EFSA Richtlinien herausgegeben, anhand derer zukünftige Daten einheitlich erhoben werden sollen. Neben zahlreichen anderen Studien waren EU-Programme wie die European Food Consumption Survey Method (EFCOSUM) und die European Food Consumption Validation (EFCOVAL) an der Umsetzung und Weiterentwicklung dieser Richtlinien mitbeteiligt (83;84). So hatte EFCOVAL zum Ziel, die von der EFSA empfohlene Methode der wiederholten, nicht-konsekutiven 24-h-Recalls weiterzuentwickeln und zu standardisieren, um damit für den internationalen Vergleich zuverlässige Daten zu erzielen (84). Darauf basierend ist eine paneuropäische Erhebung über den Lebensmittelverzehr mit dem Namen "Wie ernährt sich Europa» geplant (85).

Um die Ernährungssituation in der Schweiz mit derjenigen anderer Länder in Europa zu vergleichen, wurde der Europäische Ernährungs- und Gesundheitsbericht 2009 (ENHR II) herangezogen (86). In diesem wurden die vorhandenen Informationen zur Ernährungs- und Gesundheitssituation in 24 Ländern der Europäischen Union (EU) und in Norwegen zusammengefasst. Angaben über Trends im Verbrauch von Lebensmitteln im ENHR II basieren auf Bilanzen der Food and Agriculture Organization (FAO). Die Daten zum Lebensmittelverzehr wurden von der EFSA aus unterschiedlichen Ernährungserhebungsstudien in 16 Ländern zusammengestellt. Zweiundzwanzig Länder lieferten für den ENHR II Daten aus einzelnen Verzehrs- studien zur Einnahme von Vitaminen und Mineralstoffen. Die Daten dieser Länder wurden nach unterschiedlichen Alterskategorien gegliedert (Kinder verschiedener Altersgruppen, Jugendliche, Erwachsene, ältere Menschen); zwecks Wahrung der Übersichtlichkeit werden nachfolgend nur die Daten der Erwachsenen den Schweizer Daten gegenübergestellt.

\subsubsection{Schweizer Ernährungs- situation und Trends im Vergleich zu Europa}

\section{Lebensmittelverbrauch und-verzehr}

In der Schweiz blieben die jährlichen Verbrauchsmengen pro Kopf für die meisten Lebensmittelgruppen seit 2001/02 stabil (Kapitel 2.3.2 und Abbildung 2.3). Auffällig sind der ansteigende Verbrauch an Fisch (8\% mehr als im 5. SEB), der erneute Rückgang im Verbrauch von Früchten (7\% weniger als im 5. SEB) und die erstmals auftretende Abnahme im Gemüseverbrauch (6\% weniger als im 5. SEB). Im Vergleich dazu gibt es innerhalb der Europäischen Union verschiedene Entwicklungen (86;87). Laut den Bilanzen der FAO über die letzten 40 Jahre nimmt europaweit der Verbrauch an Früchten, Gemüse, pflanzlichen Ölen, rotem Fleisch, Schweinefleisch, Geflügel, Fisch, Milch und Milchprodukten zu, während der Verbrauch an Getreide, Kartoffeln, Hülsenfrüchten, tierischen Fetten und Eiern tendenziell stagniert oder sogar sinkt (86).

Tabelle 2.25 stellt für verschiedene Lebensmittelkategorien die Daten des schweizerischen Pro-Kopf-Verbrauchs dem durchschnittlichen Verzehr in Europa gegenüber. Die Zahlen sind jedoch beschränkt vergleichbar, da die schweizerischen Daten indirekt (gesamter Verbrauch) 
Tabelle 2.25: Verbrauch von Lebensmitteln durch die Schweizer Bevölkerung im Vergleich zum Verzehr von Lebensmitteln durch die europäische Bevölkerung (Angaben in kg pro Kopf und Jahr)

\begin{tabular}{|c|c|c|c|c|}
\hline & $\begin{array}{l}\text { Schweiz } \\
2007 / 08^{a}\end{array}$ & $\begin{array}{l}\text { Durchschnitt } \\
\text { der EU-Länder } \\
\text { (N=16) }\end{array}$ & Minimum (Land) & Maximum (Land) \\
\hline Gemüse & 89.7 & 80.3 & 43.1 (Schweden) & 107.7 (Polen) \\
\hline Früchte & 76.6 & 60.6 & 34.5 (Grossbritannien) & 102.9 (Polen) \\
\hline Getreide & 71.8 & 101.1 & 55.8 (Finnland) & 120.5 (Österreich) \\
\hline Milch, Milchprodukte & 138.1 & 97.1 & 62.4 (Österreich) & 190.5 (Norwegen) \\
\hline Fleisch, Fleischprodukte & 52.8 & 63.5 & 39.8 (Norwegen) & 94.5 (Polen) \\
\hline Fisch & 8.5 & 10.2 & 3.3 (Ungarn) & 23.0 (Norwegen) \\
\hline Eier & 10.8 & 7.3 & 1.8 (Österreich) & 11.3 (Polen) \\
\hline Fette, Öle & 23.8 & 12.4 & 4.4 (Österreich) & 21.9 (Polen) \\
\hline Alkoholische Getränke & $122.3^{\mathrm{c}}$ & 71.2 & 24.8 (Polen) & 150.8 (Tschechien) \\
\hline
\end{tabular}

a Daten basieren auf Agrarstatistiken (siehe Kapitel 2.3).

b Daten basieren auf verschiedenen Ernährungserhebungsstudien von EU-Ländern in der Altersgruppe 15-64 Jahre (86) .

c nur Personen über 15 Jahre

Tabelle 2.26: Täglicher Verbrauch (Vb) und angenäherter Verzehr (aV) an Energie und energieliefernden Nährstoffen durch die Schweizer Bevölkerung im Vergleich zu den Angaben im Europäischen Ernährungs- und Gesundheitsbericht 2009 (ENHR II)

\begin{tabular}{|c|c|c|c|c|c|}
\hline & \multirow[t]{2}{*}{ Einheit } & \multicolumn{2}{|c|}{ Schweiz 2007/08a } & \multicolumn{2}{|c|}{ ENHR II $2009^{b c}$} \\
\hline & & $\mathbf{V b}$ & aV & Männer & Frauen \\
\hline \multirow[t]{2}{*}{ Energie } & kcal & $2^{\prime} 923$ & $2^{\prime} 661$ & $2^{\prime} 030-3^{\prime} 320$ & 1'500-2'730 \\
\hline & kJ & $12^{\prime} 230$ & $11^{\prime} 135$ & $8^{\prime} 500-13^{\prime} 900$ & $6^{\prime} 300-11^{\prime} 400$ \\
\hline Protein & Energie-\% ${ }^{d}$ & 13.3 & 13.5 & $13.5-18.5$ & 13.1-19.3 \\
\hline Fett & Energie-\% ${ }^{d}$ & 38.5 & 35.1 & $28.4-45.0$ & $29.9-47.2$ \\
\hline Kohlenhydrate & Energie-\% ${ }^{d}$ & 48.2 & 51.3 & $36.8-51.0$ & $37.7-51.8$ \\
\hline
\end{tabular}

a Daten basieren auf Agrarstatistiken (siehe Kapitel 2.4).

b Daten basieren auf verschiedenen Ernährungserhebungsstudien von EU-Ländern in der Altersgruppe 18-64 Jahre (86).

c Angaben als Spannweite (Minimum-Maximum) der Durchschnitte verschiedener EU-Länder

d Energie-\%: in Prozent der Gesamtenergie 
Tabelle 2.27: Durchschnittlicher Verbrauch (Vb) und angenäherter Verzehr (aV) an Vitaminen durch die Schweizer Bevölkerung im Vergleich zu den Angaben im Europäischen Ernährungsund Gesundheitsbericht 2009 (ENHR II)

\begin{tabular}{|c|c|c|c|c|c|c|}
\hline & \multicolumn{2}{|c|}{$\begin{array}{l}\text { Schweiz } \\
2007 / 08^{a}\end{array}$} & \multicolumn{2}{|c|}{$\begin{array}{l}\text { ENHR II 2009b } \\
\text { (min.-max.) }\end{array}$} & \multicolumn{2}{|c|}{ DACH-Referenzwerte } \\
\hline & $\mathbf{V b}$ & aV & Männer & Frauen & Männerc & Frauenc \\
\hline Vitamin A [mg] & 1.4 & 1.3 & $0.5-2.2$ & $0.5-2.0$ & 1.0 & 0.8 \\
\hline Carotinoide [mg] & 4.2 & 3.6 & $1.4-5.3$ & $1.4-5.6$ & $-d$ & $-d$ \\
\hline Vitamin D [ $\mu \mathrm{g}]$ & 3.5 & 3.1 & $1.6-10.9$ & $1.2-10.1$ & 5.0 & 5.0 \\
\hline Vitamin E [mg] & 24.5 & 17.2 & $3.3-17.4$ & $4.2-16.1$ & 15 & 12 \\
\hline Vitamin B1 [mg] & 1.4 & 1.3 & $1.1-2.3$ & $0.9-2.1$ & 1.3 & 1.0 \\
\hline Vitamin B2 [mg] & 1.8 & 1.7 & $1.4-2.4$ & $1.2-2.8$ & 1.5 & 1.2 \\
\hline Niacin [mg] & 16.1 & 14.2 & $9.2-41.3$ & $6.4-30.6$ & 17 & 13 \\
\hline Vitamin B6 [mg] & 2.0 & 1.7 & $1.6-3.5$ & $1.3-2.1$ & 1.5 & 1.2 \\
\hline Folsäure [ $\mu \mathrm{g}]$ & 344 & 295 & $203-494$ & $131-392$ & 400 & 400 \\
\hline Vitamin B12 [ [ $\mathrm{g}]$ & 6.6 & 6.0 & $1.9-9.3$ & $1.0-8.8$ & 3.0 & 3.0 \\
\hline Vitamin C [mg] & 130 & 106 & $64-153$ & $62-153$ & 100 & 100 \\
\hline
\end{tabular}

a Daten basieren auf Agrarstatistiken (siehe Kapitel 2.4).

b Daten basieren auf verschiedenen Ernährungserhebungsstudien von EU-Ländern in der Altersgruppe 18-64 Jahre (86) .

c DACH-Referenzwerte für einen gesunden Erwachsenen (19-bis 50-Jährige; 48)

d nicht vorhanden

und die EU-Daten direkt (individueller Verzehr) erhoben wurden. Zudem handelt es sich bei den Lebensmittelkategorien um Gruppierungen, die je nach nationalem Kontext unterschiedliche Lebensmittel umfassen können. Des Weiteren fallen grosse Streuungen zwischen den EULändern auf: In fünf der neun dargestellten Lebensmittelkategorien weist Polen den höchsten Verzehr auf. Dies kann an den unterschiedlichen Ernährungserhebungsmethoden und Lebensmittelkategorien liegen, aber auch darin begründet sein, dass die Erhebungen in Polen während der Hochsaison von Früchten, Gemüse und Kar- toffeln durchgeführt wurden und Polen eine sehr hohe Selbstversorgungsrate aufweist (86).

\section{Energiezufuhr}

Der Energieverbrauch pro Person und Tag blieb in den letzten Jahren in der Schweiz konstant bei ca. 3'000 kcal bzw. ca. 12'500 kJ (Kapitel 2.4.3). Hingegen kann eine Abnahme von Alkohol als Energiequelle beobachtet werden. Im ENHR II werden als tägliche Energiezufuhr eines Erwachsenen je nach Land zwischen 2'030 kcal und 3'320 kcal (8'500-13'900 kJ) für Männer und zwischen 1'500 kcal und 2'730 kcal (6'300-11'400 kJ) für 
Tabelle 2.28: Durchschnittlicher Verbrauch (Vb) und angenäherter Verzehr (aV) an Mineralstoffen durch die Schweizer Bevölkerung im Vergleich zu den Angaben im Europäischen Ernährungsund Gesundheitsbericht 2009 (ENHR II)

\begin{tabular}{|c|c|c|c|c|c|c|}
\hline & \multicolumn{2}{|c|}{$\begin{array}{l}\text { Schweiz } \\
2007 / 08^{a}\end{array}$} & \multicolumn{2}{|c|}{$\begin{array}{l}\text { ENHR II 200gb } \\
\text { (min.-max.) }\end{array}$} & \multicolumn{2}{|c|}{ DACH Referenzwerte } \\
\hline & $\mathbf{V b}$ & aV & Männer & Frauen & Männerc & Frauen ${ }^{c}$ \\
\hline Natrium [g] & 1.63 & 1.55 & $2.6-7.3$ & $1.7-5.6$ & 0.55 & 0.55 \\
\hline Kalium [g] & 3.4 & 3.1 & $2.7-4.4$ & $2.3-3.6$ & 2 & 2 \\
\hline Calcium [mg] & $1^{\prime} 241$ & $1^{\prime} 184$ & 687-1'171 & $508-1^{\prime} 047$ & $1^{\prime} 000$ & $1^{\prime} 000$ \\
\hline Magnesium [mg] & 344 & 312 & $256-465$ & $192-372$ & 350 & 300 \\
\hline Phosphor [mg] & $1^{\prime} 598$ & $1^{\prime} 495$ & $1^{\prime} 264-1 ' 778$ & $1^{\prime} 017-1^{\prime} 422$ & 700 & 700 \\
\hline Eisen [mg] & 12.7 & 11.6 & $10.6-26.9$ & $8.2-22.2$ & 10 & 15 \\
\hline Zink [mg] & 13.2 & 12.5 & $8.6-14.6$ & $6.7-10.7$ & 10 & 7 \\
\hline $\operatorname{Jod}[\mu \mathrm{g}]$ & 78 & 72 & $67-264$ & $48-200$ & 200 & 150 \\
\hline
\end{tabular}

a Daten basieren auf Agrarstatistiken (siehe Kapitel 2.4).

b Daten basieren auf verschiedenen Ernährungserhebungsstudien von EU-Ländern in der Altersgruppe 18-64 Jahre (86).

c DACH-Referenzwerte gelten für einen gesunden Erwachsenen (19- bis 50-Jährige). Für Natrium und Kalium: Schätzwerte für eine minimale Zufuhr (48).

Frauen angegeben (86; Tabelle 2.26). Ein direkter Vergleich dieser Daten mit den Schweizer Daten ist aufgrund der unterschiedlichen Datengrundlagen kaum möglich (Durchschnittswerte vs. individuelle Befragungen, Gesamtbevölkerung vs. Bevölkerungsgruppen).

\section{Vitamin- und Mineralstoffufuhr}

In der Schweiz scheint die Vitaminversorgung der Bevölkerung tendenziell besser zu sein als in den meisten Ländern der EU (Tabelle 2.27), wobei jedoch wiederum die verschiedenen Datenerhebungsmethoden berücksichtigt werden müssen. Eine Unterversorgung an Vitaminen findet sich in der Schweiz v.a. bei Vitamin D und Folsäure (Kapitel 2.4.5). Die Vitamin-D-Einnahme liegt auch in den meisten europäischen Län- dern unter den DACH-Referenzwerten von $5 \mu \mathrm{g} / \mathrm{Tag}$. Nur finnische, litauische, norwegische und polnische Männer sowie finnische und norwegische Frauen nehmen täglich genug Vitamin D zu sich. Die tägliche Einnahme von Folsäure liegt zwischen $203 \mu \mathrm{g}$ und $494 \mu \mathrm{g}$ bei europäischen Männern und zwischen $131 \mu \mathrm{g}$ und $392 \mu \mathrm{g}$ bei europäischen Frauen. Einzig die Männer in Estland erreichen die empfohlene Zufuhr von $400 \mu \mathrm{g}$ pro Tag (86).

In der Schweiz werden die Empfehlungen zur Mineralstoffzufuhr in unterschiedlichem Masse erreicht (Kapitel 2.4.6). In den EU-Ländern variiert die Natriumeinnahme zwischen $2.6 \mathrm{~g}$ und $7.3 \mathrm{~g}$ pro Tag bei Männern und zwischen $1.7 \mathrm{~g}$ und $5.6 \mathrm{~g}$ pro Tag bei Frauen (Tabelle 2.28). Somit befinden sich alle teilnehmenden 
Länder weit über dem minimalen Schätzwert von $0.55 \mathrm{~g} / \mathrm{Tag}$. Auch in der Schweiz übertrifft der angenäherte Verzehr von Natrium die Empfehlungen um das Dreifache. Das Bild der Eisenzufuhr sieht in der EU ähnlich aus wie in der Schweiz: Einzelne Bevölkerungsgruppen weisen ein erhöhtes Risiko für eine Unterversorgung auf. So sind junge Frauen und Schwangere häufiger von Eisenmangel betroffen als andere Bevölkerungsgruppen (Kapitel 2.4.6 und 3.10.4). Die Empfehlung von $10 \mathrm{mg} / \mathrm{Tag}$ für Männer wird in allen EU-Ländern erreicht. Bei den Frauen erreichen nur tschechische, portugiesische und rumänische Frauen die Empfehlungen von $15 \mathrm{mg} / \mathrm{Tag}$ (86). Die Jodzufuhr scheint gemäss den Verbrauchszahlen in der Schweiz auf den ersten Blick ungenügend $z u$ sein, wobei beachtet werden muss, dass in den Angaben der Einsatz von jodiertem Salz in der Lebensmittelproduktion und im Haushalt nicht einberechnet wurde. In den meisten EU-Ländern ist die Jodzufuhr genügend. Ausser deutschen und litauischen Männern und Frauen sowie portugiesischen Frauen erreichen alle die empfohlenen Mengen.

\subsubsection{Ausblick}

Grundsätzlich sind der Lebensmittelverbrauch sowie der angenäherte Verzehr an Energie, energieliefernden Nährstoffen und Mikronährstoffen in der Schweiz vergleichbar mit anderen Ländern Europas. Der hohe Anteil an übergewichtigen und adipösen Kindern und Erwachsenen weist darauf hin, dass das Ernährungsverhalten in ganz Europa einschliesslich der Schweiz noch verbessert werden könnte (16; Kapitel 3.3).
Durch die unterschiedliche Art der Datengewinnung (Verbrauch vs. Verzehr) ist ein quantitativer Vergleich der Schweizer mit den europäischen Daten nur bedingt möglich. Selbst innerhalb der europäischen Daten ist die Vergleichbarkeit eingeschränkt, da je nach Studie verschiedene Ernährungserhebungsmethoden angewendet wurden. Zudem werden unterschiedliche Nährwertdatenbanken für die Datenauswertung herangezogen. Daher sollte es ein Ziel sein, dass künftig in ganz Europa und in der Schweiz Daten nach einheitlichen Methoden erhoben und nach übereinstimmenden Kriterien ausgewertet werden.

Die vom BAG geplante Nationale Ernährungserhebung, welche repräsentative Aussagen über den Lebensmittelverzehr der Schweizer Bevölkerung basierend auf 24-h-Recalls erlauben wird, ist ein Schritt in diese Richtung (2). Erstmals werden aktuelle und vergleichbare Daten zur Ernährungssituation in der Schweiz zur Verfügung stehen, die mit denjenigen der verschiedenen EU-Länder direkt verglichen werden können. Ein weiterer Schritt in die richtige Richtung ist auch das Projekt EuroFIR (European Food Information Resource) im Bereich Nährwertdatenbanken, bei dem die Schweizer Nährwertdatenbank Partner ist (88). Die Schweiz anerkennt generell den Bedarf an genormten Lebensmittelverzehrsdaten und unterstützt deshalb die Planung und Durchführung einer paneuropäischen Ernährungserhebung durch die EFSA (85).

Sechster Schweizerischer Ernährungsbericht | 107 


\subsection{Ernährungswissen und Ernährungsverhalten der Schweizer Bevölkerung}

\subsubsection{Allgemeines}

Um sich ausgewogen ernähren zu können, müssen Konsumenten über relevantes Wissen verfügen. Sie benötigen implizites oder explizites Wissen über die geltenden Empfehlungen für eine ausgewogene und bedarfsgerechte Ernährung. Wissen alleine genügt allerdings nicht, eine ausgewogene Ernährung muss auch als wichtig erachtet und umgesetzt werden können. Auch die Einstellung zur Ernährung ist deshalb ein relevanter Einflussfaktor auf das Ernährungsverhalten.

Ernährungsverhalten wird als umfassender Begriff für die Beschaffung, Zubereitung und Verwendung der Nahrung benutzt. Mit dem Begriff Verzehrsverhalten ist der Teil des Ernährungsverhaltens gemeint, der sich auf den Lebensmittelkonsum bezieht. Das Verzehrsverhalten kann unter anderem mit einem FFO oder mit einem 24-h-Recall untersucht werden (Kapitel 2.2.1). In der bereits erwähnten Nationalen Ernährungserhebung werden Daten zum Lebensmittelverzehr mittels 24-h-Recalls erfasst und zusätzlich weitere Fragen zum Ernährungsverhalten gestellt werden. Die in diesem Kapitel dargelegte Beschreibung des Ernährungsverhaltens der Schweizer Bevölkerung basiert allerdings noch ausschliesslich auf FFO-Studien. Als Hauptdatenquelle wird die $S G B$ herangezogen, die mit einem kurzen FFQ und weiteren Fragen zum Ernährungsverhalten arbeitet $(16 ; 89)$. Diese Befragung wird in Abständen von fünf Jahren in allen drei Sprachregionen durchgeführt, die aktuellste stammt aus dem
Jahr 2007 ( $\left.N=18^{\prime} 760\right)$. Daneben wird in diesem Kapitel auf weitere in der Schweiz durchgeführte Studien zurückgegriffen. Berücksichtigt werden dabei ausschliesslich quantitative Studien, weil qualitative Studien keine Aussagen über das Ernährungswissen oder -verhalten der Gesamtbevölkerung erlauben.

\subsubsection{Ernährungsbewusstsein, Ernährungswissen und Hindernisse für eine ausgewogene Ernährung}

\section{Ernährungsbewusstsein}

In der $S G B$ wird das Ernährungsbewusstsein mit der Frage "Achten Sie auf bestimmte Sachen bei Ihrer Ernährung?» erhoben $(16 ; 21)$. Im Jahr 2007 gaben 30\% der Befragten an, auf nichts Bestimmtes zu achten. Frauen sind dabei ernährungsbewusster als Männer, und ältere Personen sind ernährungsbewusster als jüngere Personen. Auch das Bildungsniveau hat einen Einfluss: Besser Ausgebildete achten häufiger auf ihre Ernährung als schlechter Ausgebildete. Auffällig sind die Unterschiede zwischen den Sprachregionen (Abbildung 2.11). In der Westschweiz ist das Ernährungsbewusstsein weniger ausgeprägt als in den beiden anderen Sprachregionen. Insgesamt hat sich das Ernährungsbewusstsein der Schweizer Bevölkerung zwischen 1992 und 2007 nur wenig verändert.

In anderen Befragungen fanden sich vergleichbare Anteile an wenig ernährungsbewussten Personen in der Bevölkerung wie in der SGB07. So sind in einer von Coop lancierten Online-Umfrage 28\% der Befragten ( $N=514)$ der Meinung, dass Ernährung keinen oder nur einen geringen Einfluss auf das Krankheitsrisiko 
hat (90). In derselben Umfrage geben 33\% der Befragten an, der Genuss von Alkohol habe keinen oder nur einen kleinen Einfluss auf das Krankheitsrisiko.

Eine allzu positive Einschätzung des eigenen Verhaltens kann sich negativ auf das Ernährungsverhalten auswirken. Laut einer telefonischen Befragung in allen drei Sprachregionen ( $N=1^{\prime} 008$ ) sind $80 \%$ der Personen mit normalem Körpergewicht der Meinung, dass sie sich alles in allem gesund ernähren. Auch bei den Personen mit Untergewicht (81\%), Übergewicht (68\%) oder Adipositas (64\%) ist eine Mehrheit überzeugt, sich gesund zu ernähren (91). Einem Teil der Bevölkerung könnte es damit an einem Problembewusstsein fehlen, welches für eine Verhaltensänderung in Richtung ausgewogenere Ernährung notwendig wäre.
Mit dem Essen werden verschiedene Bedürfnisse gestillt. In einer Studie der Universität St. Gallen wurden 1'081 Personen gefragt, welche Ernährungsbedürfnisse für sie am wichtigsten sind (92). Das Bedürfnis "genussvoll essen» wurde dabei von den Befragten am häufigsten genannt, dicht gefolgt von "gesund essen». Die Verzehrssituation hat einen starken Einfluss darauf, welches Bedürfnis dominiert: Unter der Woche steht bei den Verzehrssituationen Frühstück, Mittagessen und Abendessen bei vielen Befragten der Aspekt "gesund essen» im Vordergrund (über $40 \%$ der Befragten). Am Wochenende tritt hingegen das Bedürfnis "genussvoll essen" in den Vordergrund. Einschränkend erwähnt werden muss, dass bei dieser Studie die Studienteilnehmer vor Supermärkten und Einkaufszentren befragt wurden.

Achten Sie auf bestimmte Sachen bei Ihrer Ernährung?

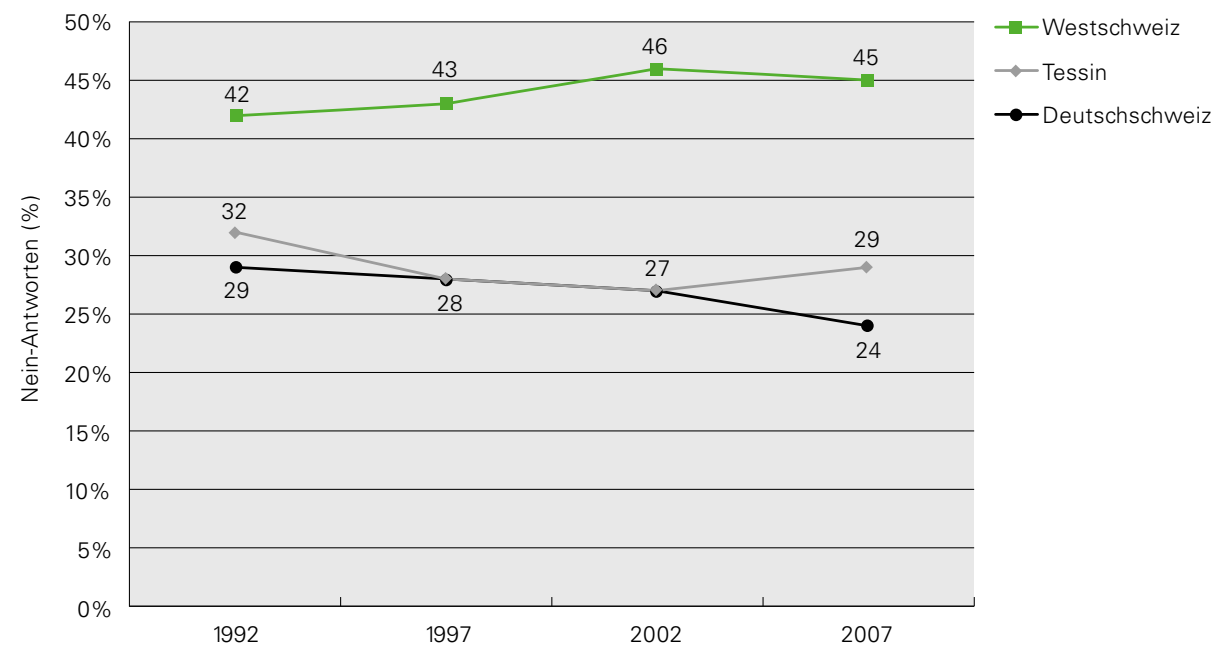

Abbildung 2.11: Anteil Personen, die nicht auf bestimmte Sachen in ihrer Ernährung achten, aufgeschlüsselt nach Sprachregion und Erhebungsjahr (16;21) 
Früchte kann man vollständig durch Vitamin- und Mineralstofftabletten ersetzen.

Wenn man stark fetthaltige Dinge gegessen hat, kann man dies danach durch den Verzehr von Äpfeln wieder rückgängig machen.

Eine Ernährung mit einem hohen Anteil an Früchten und Gemüse ist genauso einseitig wie eine Ernährung mit einem hohen Fettanteil.

Ein gesundes Menü sollte etwa zur Hälfte aus Fleisch, zu einem Viertel aus Gemüse und zu einem Viertel aus Beilagen bestehen.

Fett ist immer schlecht für die Gesundheit, man sollte es daher möglichst vermeiden.

Ausgewogene Ernährung bedeutet, von allen Lebensmitteln etwa gleich viel zu essen.

Um sich gesund zu ernähren, sollte man vor allem weniger Fett essen. Ob man zusätzlich mehr Früchte und Gemüse isst, spielt keine Rolle.

Brauner Zucker ist viel gesünder als weisser Zucker.

Für eine gesunde Ernährung sollten Milchprodukte etwa in gleicher Menge wie Früchte und Gemüse verzehrt werden.

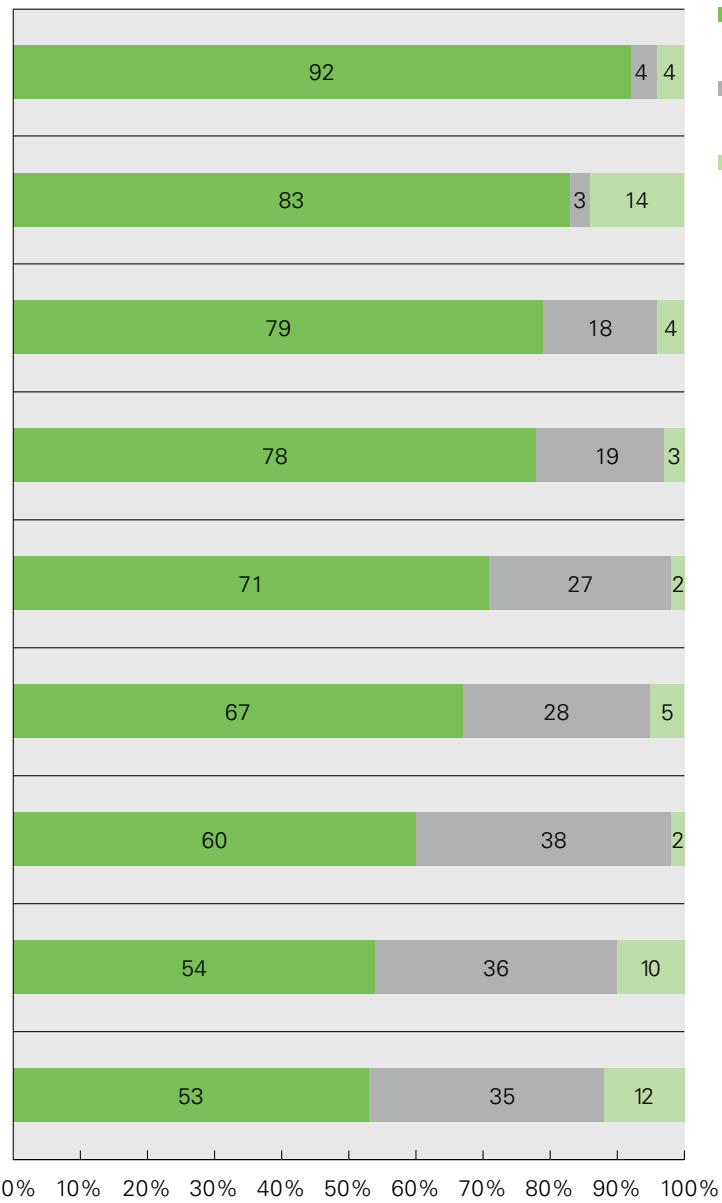

Richtige Antworten

- Falsche Antworten

Weiss nicht 
Auch wenn dabei darauf geachtet wurde, dass die Befragten die Schweizer Bevölkerung möglichst gut abbilden, ist das Auswahlverfahren dafür nicht optimal.

\section{Ernährungswissen}

Ernährungsbewusstsein ist eine notwendige, aber keine hinreichende Voraussetzung für ein gesundheitsförderndes Ernährungsverhalten. Die Konsumenten müssen heutzutage bei der riesigen Auswahl an Produkten grundlegendes Wissen über Lebensmittel haben, um sich ausgewogen ernähren zu können (Kapitel 4.5.1 und 4.5.3). Das vorhandene Ernährungswissen wurde in der Deutschschweiz mit einer schriftlichen Befragung bei 1'053 zufällig aus dem Telefonbuch ausgewählten Personen untersucht $(93 ; 94)$. In Abbildung 2.12 wird der Anteil richtiger und falscher Beurteilungen von vorgegebenen Aussagen dargestellt. Die Ergebnisse zeigen, dass verschiedene Ernährungsmythen weit verbreitet sind: Fett wird generell als ungesund und schlecht beurteilt und der Anteil an Milchprodukten für eine gesunde Ernährung wird überschätzt. Die Aussage «Brauner Zucker ist viel gesünder als weisser Zucker» beurteilten nur $54 \%$ der Befragten richtigerweise als nicht korrekt. Diese drei Aussagen weisen von allen untersuchten Äusserungen den kleinsten Anteil an richtigen Beurteilungen auf. Frauen verfügen über ein signifikant grösseres Ernährungswissen als Männer. Besser Ausgebildete und ältere Personen haben ein signifikant grösseres Ernährungswissen als schlechter Ausgebildete und jüngere Personen. Da diese Studie nur in der Deutschschweiz durchgeführt wurde, bleibt offen, ob bezüglich Ernährungswissen ähnliche Unterschiede zwischen den Sprachregionen bestehen wie bezüglich Ernährungsbewusstsein.
Assoziationen zu gesunder Ernährung wurden auch in zwei telefonischen Befragungen untersucht (91;95). Die Daten wurden 2006 $\left(\mathrm{N}=1^{\prime} 441\right)$ und $2009\left(\mathrm{~N}=1^{\prime} 008\right)$ in allen drei Sprachregionen bei Personen über 16 Jahren erhoben. Die Befragten mussten unter anderem die offene Frage "Was muss man beachten, wenn man sich gesund ernähren will?» beantworten. Mehr als jeder zweite Befragte nannte "Gemüse und Früchte essen» (2006: 50\%; 2009: 63\%). "Ausgewogenes Essen / Lebensmittelpyramide" war die am zweithäufigsten genannte Antwort (2006: 39\%; 2009: 49\%) und an dritter Stelle folgte "Nicht zu viel Fett» (2006: 35\%; 2009: 38\%).

Hindernisse für eine ausgewogene Ernährung Die Universität St. Gallen ging in ihrer Konsumentenbefragung ( $\left.N=1^{\prime} 081\right)$ auch der Frage nach, welche Faktoren die Konsumenten daran hindern, sich ausgewogen zu ernähren (92). Als grösstes Hindernis für eine bessere Ernährungsweise wurde von den Befragten mangelnde Disziplin genannt (36\%). Fehlende Zeit (27\%) und fehlendes Gastro-Angebot (16\%) waren die am zweit- und dritthäufigsten genannten Hinderungsgründe für eine ausgewogene Ernährung.

\subsubsection{Zusammenhang zwischen Ernährungswissen und Verzehrsverhalten}

Zwischen dem Verzehrsverhalten, gemessen mit einem FFQ, und dem Ernährungswissen können gewisse Zusammenhänge beobachtet werden $(93 ; 94)$. Eine positive Korrelation von $r=0.29$ findet sich zwischen Gemüsekonsum 
und Ernährungswissen (94). Das Ernährungswissen weist lediglich einen schwach positiven Zusammenhang mit dem Konsum von Früchten, Wasser, Getreideflocken, Salat, Fisch und Vollkornbrot ( $r=0.10$ bis 0.20 ) sowie einen schwach negativen Zusammenhang mit dem Konsum von Würsten, Süssgetränken, Eierteigwaren, Chips und Pommes frites ( $r=-0.10$ bis -0.20 ) auf (94). Die Grösse des in der Schweiz beobachteten Zusammenhangs zwischen Ernährungswissen und Verzehrsverhalten entspricht den Beobachtungen aus anderen Ländern (96). Aus den Korrelationen in den verschiedenen Studien lassen sich jedoch nur beschränkt Aussagen über Kausalitäten ableiten.

\subsubsection{Nährwertkennzeichnungs- systeme und Labels}

In der Pilotstudie der Nationalen Ernährungserhebung ( $N=1$ '500, Quotenstichprobe für Deutschschweiz, Westschweiz und Tessin) wurde den Teilnehmern die Frage "Beachten Sie beim Lebensmitteleinkauf die Nährwertangaben?" gestellt. Die Mehrheit der Befragten beantwortete diese Frage mit "Nein» (68\%) und nur 27\% mit «Ja». Vier Prozent gaben an, keine Lebensmittel einzukaufen (97).

In einer Studie der ETH Zürich wurden 1'162 Deutschschweizer schriftlich befragt, wie häufig sie die Nährwertkennzeichnung benut-

Wie häufig benutzen Sie Nährwerttabellen, wenn Sie ein Lebensmittel auswählen, das Sie noch nie gekauft haben?

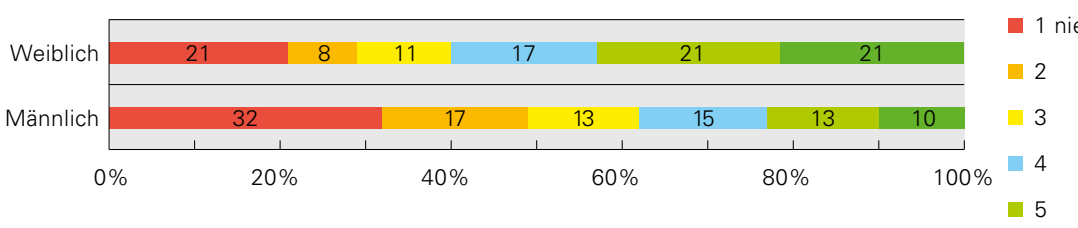

Wie häufig benutzen Sie Nährwerttabellen, wenn Sie sich zwischen zwei oder mehr Lebensmitteln entscheiden müssen?

6 sehr häufig

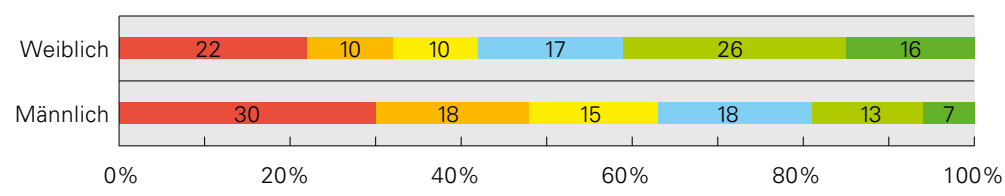

Die Befragten ( $N=1^{\prime}$ '133) erhielten folgende Anweisung: "Auf der Verpackung von Lebensmitteln sind jeweils Nährwerttabellen angegeben (z.B. Angabe der Energie/Kalorien pro $100 \mathrm{~g}$, Anteil Zucker pro $100 \mathrm{~g}$, Anteil Fett pro $100 \mathrm{~g}$ usw.). Bitte geben Sie an, wie häufig und wofür Sie solche Nährwerttabellen verwenden.» Der in der Befragung verwendete Begriff «Nährwerttabellen» entspricht dabei der Nährwertkennzeichnung. 
zen, wenn sie ein Lebensmittel auswählen, das sie noch nie gekauft haben, oder wenn sie sich zwischen zwei Lebensmitteln entscheiden müssen (98). Ein Drittel der Befragten achtet in diesen beiden Kaufsituationen häufig auf die Nährwertkennzeichnung und ein Drittel beachtet sie sehr selten oder nie. Frauen konsultieren die Nährwertinformationen deutlich häufiger als Männer (Abbildung 2.13). Ernährungsbewusstsein, Ernährungswissen, Gesundheitsbewusstsein und Kompetenz im Umgang mit Zahlen sind weitere Faktoren, die positiv mit der Nutzung der Nährwertkennzeichnung zusammenhängen. Personen, die das Essen in erster Linie mit Genuss in Verbindung bringen, verwenden die Nährwertkennzeichnung hingegen weniger häufig (98).

Den meisten Konsumenten fällt es schwer, aufgrund der Nährwertkennzeichnung das Produkt auszuwählen, welches am besten ihren Bedürfnissen entspricht (99). Front-of-PackKennzeichnungen (z.B. Ampel, Guideline Daily Amount [GDA] oder Choice Label) ${ }^{2}$ können deshalb ein wichtiges Hilfsmittel für die Konsumenten sein. Eine repräsentative Online-Studie der SGE zeigt, dass die Konsumenten Ampel und GDA als deutlich hilfreicher einstufen als das

\footnotetext{
2 Die Ampelkennzeichnung informiert, wie viel Fett, gesättigte Fettsäuren, Zucker und Salz pro $100 \mathrm{~g}$ bzw. $100 \mathrm{ml}$ in einem Produkt enthalten sind. Mit Farben wird den Konsumenten signalisiert, ob der entsprechende Nährstoffgehalt bezogen auf die Ernährungsempfehlungen hoch (rot), mittel (orange) oder tief (grün) ist. Beim Kennzeichnungssystem mittels GDA wird angegeben, wie viel Kalorien, Fett, gesättigte Fettsäuren, Zucker und Salz eine Portion des Lebensmittels enthält. Zusätzlich wird in Prozent angegeben, wie viel vom durchschnittlichen Richtwert für dieTageszufuhr einer erwachsenen Frau durch eine Portion des Lebensmittels gedeckt wird. Mit dem Choice Label werden empfehlenswerte Produkte innerhalb definierter Lebensmittelkategorien gekennzeichnet (100).
}

Choice Label (100). In einem Experiment mussten die Studienteilnehmer jeweils zwei Produkte miteinander vergleichen und das ihrer Meinung nach gesündere Produkt auswählen. Bei der Ampel war die Wahl signifikant häufiger richtig (71\%) als beim Choice Label (67\%) oder GDA (66\%). Beim Choice Label war jedoch die Entscheidungszeit signifikant kürzer als bei den anderen beiden Kennzeichnungssystemen. Beachtet werden muss dabei, dass von den befragten Personen $81 \%$ GDA kannten, aber nur $33 \%$ die Ampel (die in der Schweiz nicht verwendet wird) und $14 \%$ das Choice Label.

Befragungen zur Nährwertkennzeichnung oder Experimente, in denen die Probanden mit Front-of-Pack-Kennzeichnungen konfrontiert werden, dürften die Nutzungshäufigkeit dieser Hilfsmittel allerdings überschätzen. Eine Alternative stellen Eye-Tracker Studien dar, bei denen gemessen wird, welche Informationen die Konsumenten bei der Auswahl eines Produktes wirklich beachten. In einer Studie mit dieser Datenerhebungsmethode achteten die Versuchspersonen signifikant länger auf die Nährwertkennzeichnung, wenn sie aus fünf Getreideflocken ein Produkt für den Kindergarten auswählen mussten, als wenn das Produkt für die Mensa bestimmt war (101). Eine weitere Erkenntnis aus dieser Studie ist, dass die Verpackung eines Produktes das Verhalten ebenfalls beeinflusst. Bei einfach gestalteten Verpackungsdesigns schenkten die Studienteilnehmer der Nährwert- und Front-of-Pack-Kennzeichnung mehr Aufmerksamkeit als bei Verpackungen, die mit Informationen überfüllt waren. Gesundheitsmotivation der Konsumenten und Packungsdesign nehmen also bedeutenden Einfluss darauf, ob Nährwertkennzeichnungen auch tatsächlich beachtet werden (101). 


\subsubsection{Ernährungsverhalten der Schweizer Bevölkerung}

Eine Mehrheit der Schweizer Bevölkerung kocht gemäss der Online-Umfrage von Coop regelmässig zu Hause warme Mahlzeiten: 64\% mindestens einmal täglich und $85 \%$ mehrmals pro Woche oder häufiger (102). Im Ernährungs-Panel der ETH Zürich ( $N=6$ '193, Zufallsstichprobe aus dem Telefonbuch für die Deutsch- und Westschweiz) wurden die Studienteilnehmer gefragt, wie häufig sie die Mahlzeit vor dem Fernseher einnehmen (103). Sechs Prozent der Befragten schauen täglich und weitere $6 \%$ schauen vier- bis sechsmal pro Woche fern, währenddem sie gleichzeitig eine Hauptmahlzeit essen. Die Häufigkeit dieser Verhaltensweise birgt Risiken, weil Essen bei gleichzeitigem Fernsehen mit einer erhöhten Energieaufnahme verbunden sein kann (104).

Das Abendessen ist die am stärksten verbreitete Hauptmahlzeit. Im Ernährungs-Panel der ETH Zürich gaben $83 \%$ der Befragten an, täglich ein Abendessen zu konsumieren (103). Das Mittagessen wird von $75 \%$ und das Frühstück von 69\% der Befragten täglich eingenommen. Abbildung 2.14 zeigt die Konsumhäufigkeit von Haupt- und Zwischenmahlzeiten aufgeschlüsselt nach Geschlecht. Die Daten dokumentieren, dass Frauen über den Tag hinweg öfter etwas essen als Männer.

Die Ausser-Haus-Verpflegung hat in den letzten Jahren an Bedeutung gewonnen (92; Kapitel 4.5.2). Die Verpflegungsorte «warme Theke im Supermarkt», "Fachgeschäfte» und "über die Gasse / unterwegs" weisen für die letzten Jahre die grössten relativen Zuwachsraten aus. Gemäss dem Ernährungs-Panel der ETH Zürich essen 3\% der Befragten täglich und 14\% mehr- mals pro Woche in einem Restaurant, in einer Kantine oder Mensa oder in einem Bistro (103). Dabei nehmen jüngere Personen ihre Mahlzeiten deutlich häufiger ausser Haus ein als ältere Personen (Abbildung 2.15).

\section{Konsum verschiedener Lebensmittelgruppen}

In den $S G B$ wird jeweils nach dem Konsum verschiedener Lebensmittelgruppen gefragt. Die Ergebnisse aus dem Jahr 2007 werden nachstehend zusammengefasst. In der SGBO7 gaben $87 \%$ der Befragten an, weniger als die drei empfohlenen Portionen Gemüse oder Gemüsesäfte pro Tag zu konsumieren, wobei in der Westschweiz mehr Personen die empfohlene Menge verzehrten als in den restlichen Landesteilen (16; Abbildung 2.16). Drei Viertel der befragten Männer und Frauen gaben weiter an, pro Tag maximal zwei Portionen Früchte oder Fruchtsäfte zu konsumieren. Frauen verzehren insgesamt häufiger Früchte oder Fruchtsäfte und auch Gemüse oder Gemüsesäfte als Männer (Abbildung 2.16). Diese Daten lassen darauf schliessen, dass lediglich $30 \%$ der Schweizer Bevölkerung mindestens fünf Portionen Gemüse und Früchte pro Tag zu sich nehmen. Bei den Frauen ist der Anteil höher (38\%) als bei den Männern (21\%). Die Zahlen fallen tiefer aus, wenn als Kriterium drei Portionen Gemüse und zwei Portionen Früchte genommen werden, wie dies von der SGE empfohlen wird (16).

Eine Mehrheit der Schweizer Bevölkerung (65\%) konsumiert täglich Milch und Milchprodukte, lediglich 10\% nehmen jedoch die empfohlenen drei Portionen pro Tag zu sich. Fisch wird von 37\% der Bevölkerung selten oder nie gegessen, $58 \%$ konsumieren ihn an ein bis zwei Tagen pro Woche. Hierbei zeigen sich deutliche Unterschiede zwischen den Sprachregionen: In 
Wie häufig essen Sie im Durchschnitt...

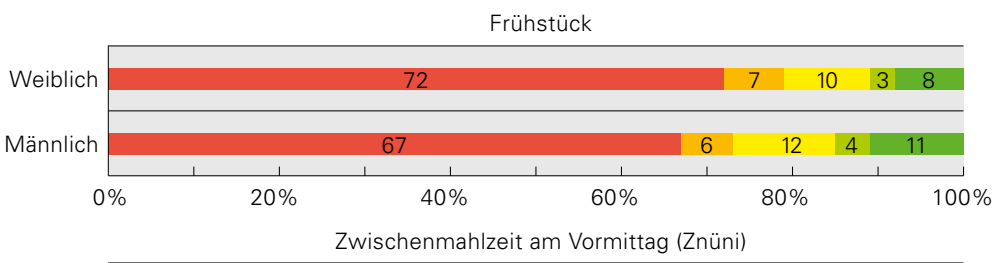

Täglich

4-bis 6-mal pro Woche

1- bis 3-mal pro Woche

1- bis 3-mal pro Monat

Seltener oder nie

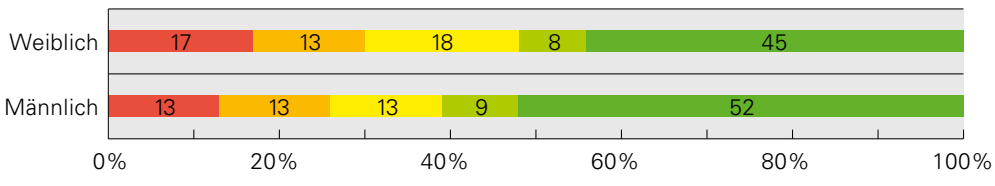

Mittagessen

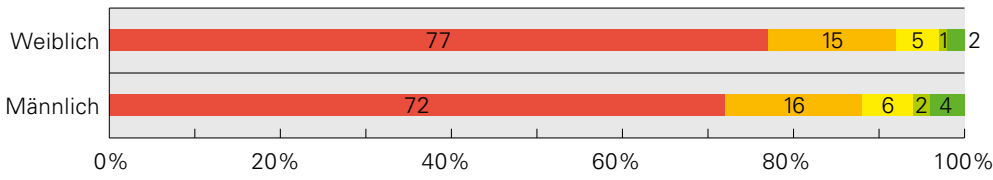

Zwischenmahlzeit am Nachmittag (Zvieri)

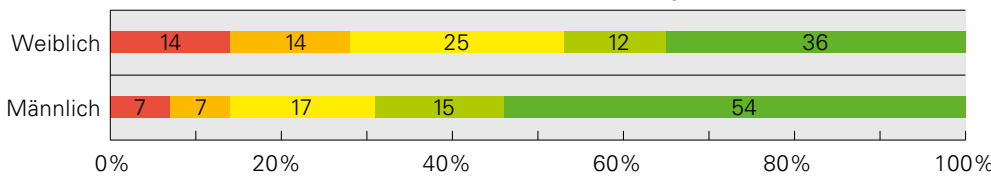

Abendessen

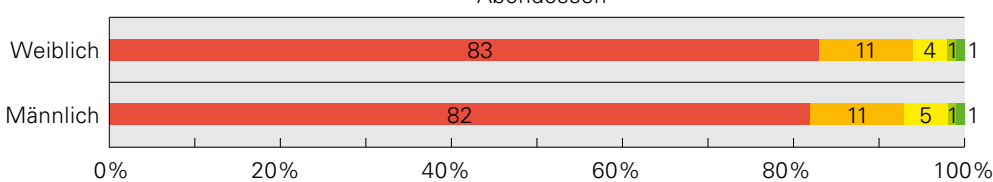

Zwischenmahlzeit nach dem Abendessen und während der Nacht

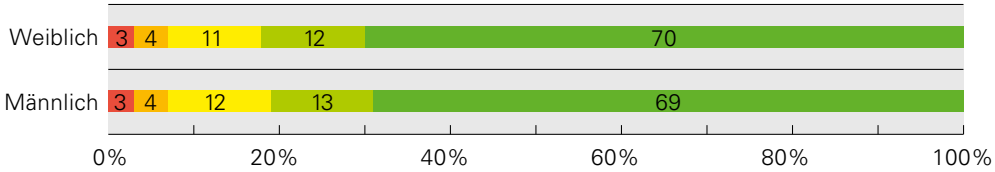

Weitere Zwischenmahlzeit (z.B. Naschen, süsse oder salzige Snacks)

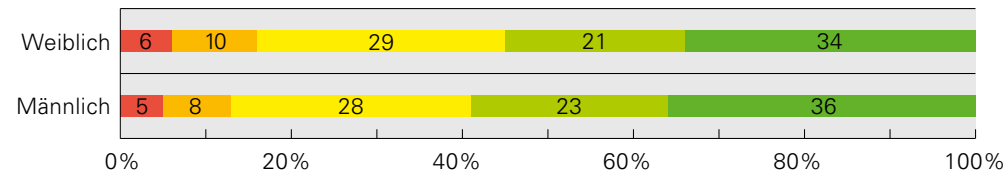


der Deutschschweiz gibt es mehr Personen, die selten oder nie Fisch essen, als in den anderen beiden Landesteilen (Abbildung 2.16). Beim Konsum von Fleisch und Wurstwaren geben $20 \%$ an, diese mindestens einmal pro Tag zu verzehren. Die Unterschiede zwischen den Geschlechtern und den Sprachregionen sind auffällig. Männer essen deutlich mehr Fleisch und Wurstwaren als Frauen. Im Tessin ist der Anteil der Personen, die mindestens einmal pro Tag Fleisch und Wurstwaren zu sich nehmen, kleiner als in den anderen Sprachregionen (16; Abbildung 2.16).

Im Ernährungs-Panel der ETH Zürich mussten die Befragten Aussagen zum Konsum von Schokolade, Guetzli, Keksen und süssen Backwaren machen (103; Abbildung 2.17). Zehn Prozent der Befragten essen mehrmals täglich
Schokolade; $6 \%$ essen täglich Guetzli und Kekse. Süsse Backwaren werden nur von $2 \%$ mehrmals täglich konsumiert.

In der SGB wird auch der Flüssigkeitskonsum erhoben. Drei von vier Personen geben an, täglich mindestens $1.5 \mathrm{~L}$ nichtalkoholische Getränke zu konsumieren; lediglich 4\% trinken weniger als $1 \mathrm{~L}$ pro Tag (16). Beim Konsum alkoholischer Getränke geben 20\% der Männer und 9\% der Frauen an, "mindestens einmal pro Tag» Alkohol zu trinken. 13\% der Männer und 6\% der Frauen nehmen mehrmals pro Woche alkoholische Getränke zu sich (105; Kapitel 2.3.2 und Abbildung 2.5).

Eine schriftliche Befragung der ETH Zürich in der Deutschschweiz ( $N=918$, Zufallsstichprobe aus dem Telefonbuch) zeigt, dass der Konsum von Convenience-Produkten häufig ist (106). Auch Fertigmahlzeiten werden regelmässig

Wie häufig essen Sie im Durchschnitt in einem Restaurant, Kantine / Mensa, Bistro usw. (nicht Fast-Food-Restaurant)?

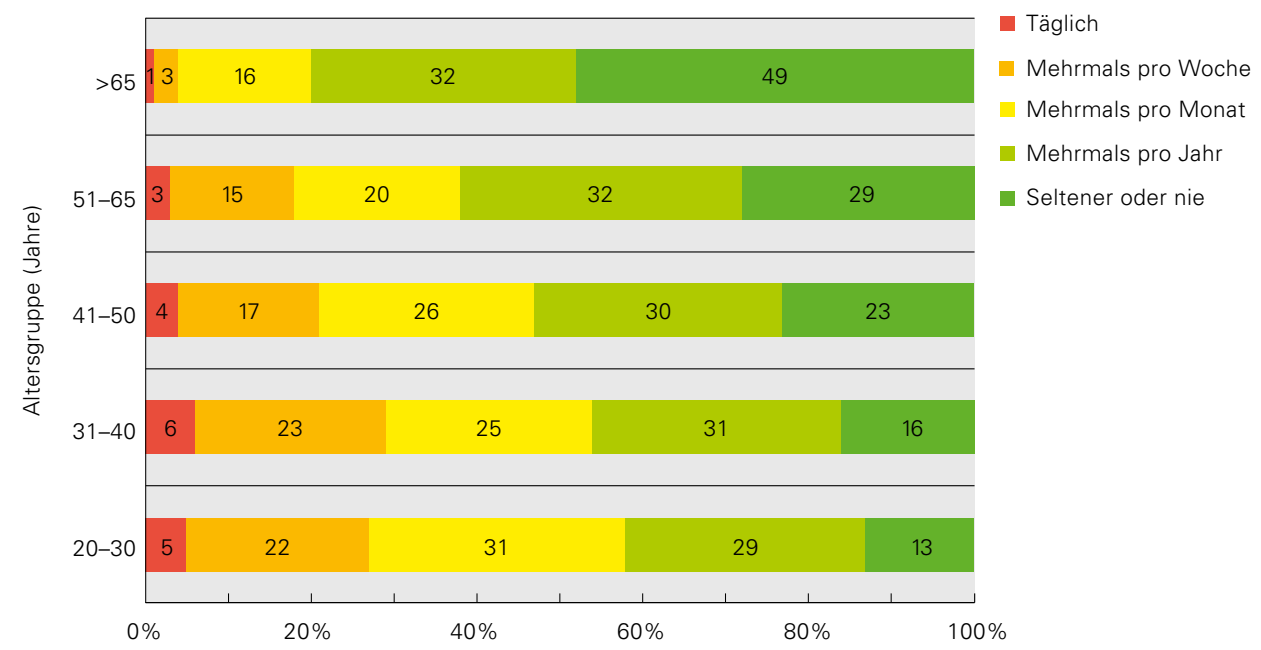

Abbildung 2.15: Verpflegung in Restaurant, Kantine / Mensa und Bistro (103) 
Ich esse oder trinke weniger als dreimal täglich Gemüse oder Gemüsesäfte (ohne Kartoffeln und Mais)

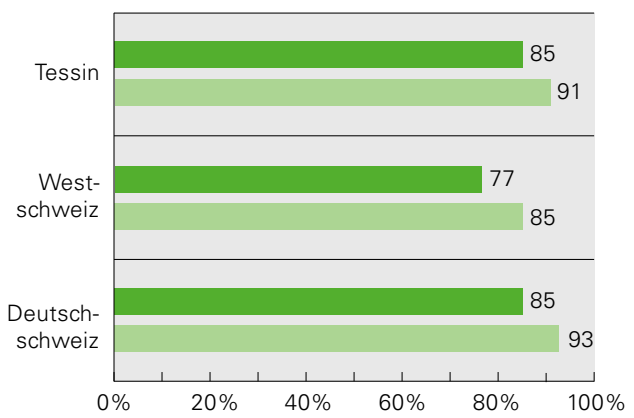

Ich esse oder trinke weniger als dreimal täglich Früchte oder Fruchtsäfte

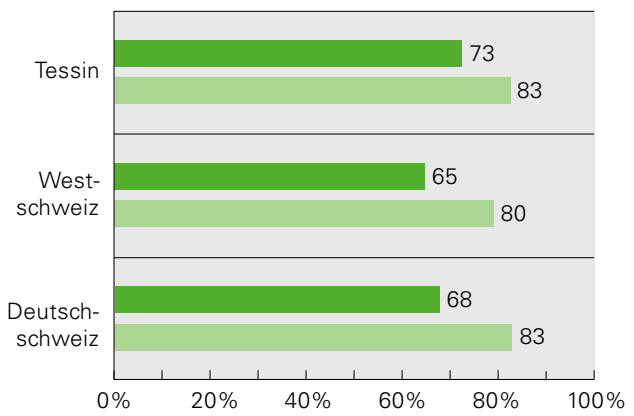

Weiblich Männlich
Ich esse selten oder nie Fisch

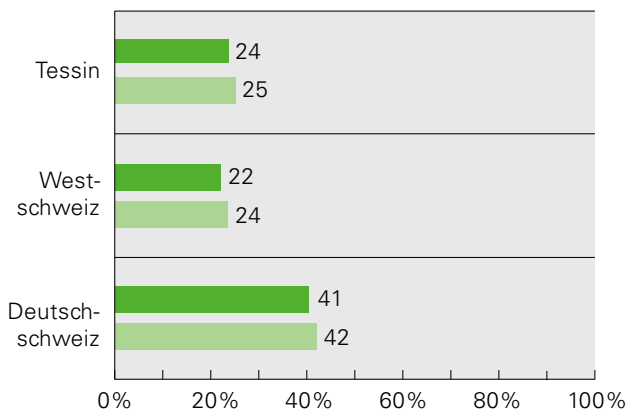

Ich esse ein- oder mehrmals täglich Fleischoder Wurstwaren

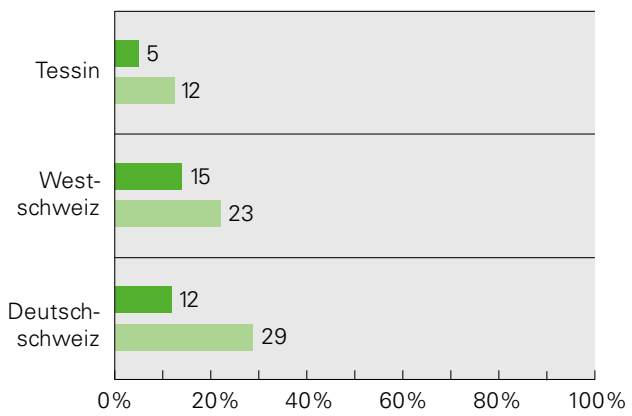

Abbildung 2.16: Konsum von Gemüse und Gemüsesäften, Früchten und Fruchtsäften, Fisch sowie Fleisch und Wurstwaren in den drei Sprachregionen (16)

konsumiert, obschon damit die Begriffe «viele Zusatzstoffe, Geschmacksverstärker, Zucker, Salz und Fett» assoziiert werden (107). Zudem werden in Fertigmahlzeiten kaum Vitamine und andere Nährstoffe erwartet. Männer und übergewichtige Personen beurteilen Fertigmahlzeiten insgesamt positiver als Frauen und normalgewichtige Personen. Geringe Kochfähigkeiten und Übergewicht hängen mit einem grösseren Konsum von Fertigmahlzeiten zusammen. Jüngere Personen konsumieren häufiger Fertigmahlzeiten als ältere Personen und Männer häufiger als Frauen (107). 
Wie häufig essen Sie im Durchschnitt...

Guetzli, Kekse

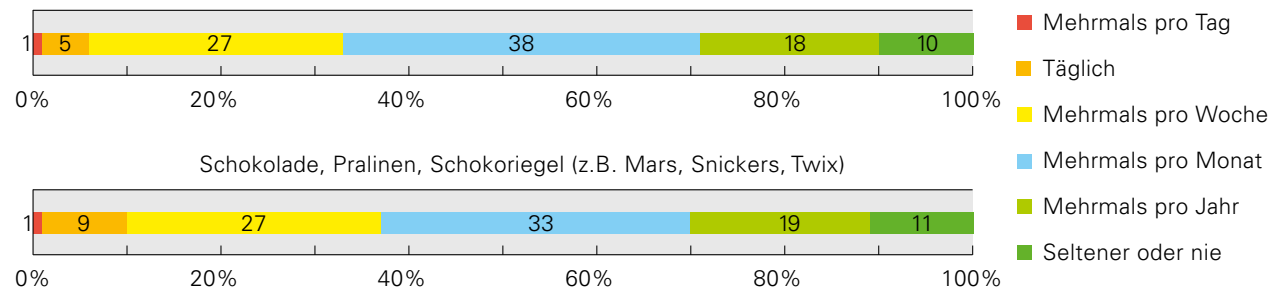

Süsse Backwaren (z.B. Berliner, Kuchen, Nussgipfel)

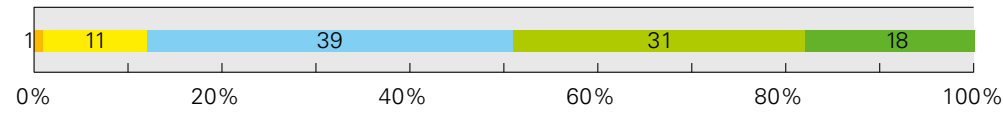

Abbildung 2.17: Konsumhäufigkeit von Guetzli, Keksen, süssen Backwaren sowie Schokolade, Pralinen und Schokoriegeln (103)

\subsubsection{Ausblick}

Rund 30\% der Schweizer Bevölkerung achtet auf nichts in der Ernährung. Es sind hauptsächlich Männer, jüngere Personen und Personen mit einem niedrigen Bildungsniveau, bei denen sich ein geringes Ernährungsbewusstsein findet. Dieselben soziodemografischen Indikatoren stehen auch in Zusammenhang mit einem geringen Ernährungswissen. Da das Ernährungswissen das Verzehrsverhalten nachweislich, wenn auch nur schwach, positiv beeinflusst, sollte die Wissensvermittlung über eine ausgewogene Ernährung nicht vernachlässigt werden. Weiter ist es auch wichtig, die Bevölkerung für die gesundheitlichen Vorteile einer ausgewogenen Ernährung zu sensibilisieren (Kapitel 4.5 und 4.9).

Kennzeichnungssysteme (Nährwertkennzeichnung, Front-of-Pack-Kennzeichnungen, La- bels) sollen den Konsumenten die Wahl erleichtern. Aufgrund der wissenschaftlichen Datenlage lassen sich keine eindeutigen Aussagen darüber machen, welches Kennzeichnungssystem am verständlichsten und wirkungsvollsten ist. Für den Konsumenten ist die heutige Situation in der Schweiz eher unübersichtlich, unter anderem weil unterschiedliche Kennzeichnungssysteme parallel verwendet werden. Selbst bei Produkten des gleichen Herstellers aus der gleichen Kategorie gibt es zum Teil grosse Unterschiede in den Angaben. Sich für eine Standardisierung der Kennzeichnung zu entscheiden, wäre deshalb wichtiger als die Entscheidung für ein bestimmtes Kennzeichnungssystem (Kapitel 4.9.2).

Ausser-Haus-Verpflegung und ConvenienceProdukte spielen eine wichtige Rolle in der Ernährung eines Grossteils der Bevölkerung. Die von der Industrie gewählten Rezepturen nehmen damit zunehmend Einfluss auf die Nähr- 
stoffversorgung der Schweizer Bevölkerung, denn in vielen Konsumsituationen können gesundheitsrelevante Entscheidungen nicht mehr vom Konsumenten getroffen werden. Richtlinien für die Gastronomie und Industrie sind deshalb ebenso wichtig wie Informationen für die Bevölkerung (Kapitel 4.9.6 und 4.9.7).

Die Ergebnisse der SGB07zeigen, dass eine Mehrheit der Bevölkerung sich nicht nach den aktuellen Ernährungsempfehlungen richtet. Nimmt man die SGE-Empfehlungen bezüglich Anzahl Portionen als Grundlage, so werden zu wenig Früchte, zu wenig Gemüse und zu wenig Milch und Milchprodukte konsumiert. Männer, jüngere Personen und Personen mit niedrigem Bildungsniveau berücksichtigen die Ernährungsempfehlungen vergleichsweise am seltensten. Gleichzeitig wissen fast alle Konsumenten, dass Gemüse und Früchte wichtig für eine ausgewogene Ernährung sind.

Über das Ernährungsverhalten der Schweizer Bevölkerung liegen bisher nur wenige Informationen vor. Die Datenlage wird durch die Nationale Ernährungserhebung verbessert werden. Der dabei geplante 24-h-Recall wird ein Bild darüber liefern, was im Durchschnitt gegessen wird. Auf Grund der grossen Variabilität in den Daten braucht es zusätzlich noch einen regelmässig erhobenen $\mathrm{FFQ}$, der detailliertere Informationen als die SGB liefert. Ohne zusätzliche Daten wird es schwierig, Massnahmen im Bereich Ernährung zu planen und zu evaluieren. Zusätzlich sind auch Lebensmittelverzehrsdaten notwendig, um die Nährstoffaufnahme oder die Exposition von synthetischen Chemikalien über Lebensmittel zu modellieren (108). Es ist zu hoffen, dass diese Datenlücken in Zukunft geschlossen werden können (Kapitel 4.9.1).

\subsection{Schlussfolgerungen und Ausblick}

Die vorliegenden Daten zum Verbrauch und angenäherten Verzehr von Lebensmitteln und Inhaltsstoffen zeichnen das Bild einer guten Ernährungssituation in der Schweiz. Mit Ausnahme von Vitamin D und Folsäure scheint der Bedarf an Makro- und Mikronährstoffen gedeckt zu werden und die Energiezufuhr ist mehr als ausreichend. Dies, obschon die Ernährung häufig nicht der Zusammensetzung einer ausgewogenen Mischkost gemäss der Schweizer Lebensmittelpyramide zu entsprechen scheint und auch kein genereller Trend in diese Richtung auszumachen ist. Auf Letzteres weisen die vorhandenen Daten über das Ernährungsverhalten in der Schweiz hin.

Eine Beurteilung der Ernährungssituation in der Schweiz ist nicht vorbehaltlos möglich. Die Daten zur Nährstoffversorgung werden aus der Agrarstatistik gewonnen und auf einen Lebensmittelverbrauch pro Kopf der Bevölkerung umgerechnet. Sie erlauben keine Rückschlüsse auf den Versorgungsgrad einzelner Bevölkerungsgruppen und schon gar nicht einzelner Individuen. Gerade bei der Versorgung mit Vitamin D, Eisen oder Jod zeigt sich, dass es in der Schweiz spezifische Bevölkerungsgruppen gibt, bei denen das Risiko einer Unterversorgung mit einzelnen Nährstoffen hoch ist oder gar weite Teile der Bevölkerung vom Risiko einer Unterversorgung betroffen sein könnten. Es muss auch berücksichtigt werden, dass der in Kapitel 2.2 angesprochene Überfluss an vorhandener Nahrung und Nährstoffen nicht von allen Menschen gleich gut genutzt wird oder werden kann, wobei die Gründe dafür mannigfaltig sind. Das Ernährungswissen und das Ernährungsbewusstsein stellen dabei nur zwei Aspekte unter vielen 
dar. Exakte, differenzierte und valide Angaben bezüglich der Ernährungssituation der Schweizer Bevölkerung werden erst dann möglich sein, wenn repräsentative Schweizer Studien detaillierte Informationen zum wirklichen Verzehr liefern. Die Ergebnisse der Nationalen Ernährungserhebung und anderer momentan laufender Untersuchungen werden bestehende Lücken füllen, was einen grossen und dringend notwendigen Schritt vorwärts bedeutet. Um ein Monitoring der Ernährungssituation aufzubauen, müssen diese Untersuchungen jedoch regelmässig und wiederholt durchgeführt werden.

Die vorgestellten Verbrauchsdaten dürfen aber nicht einfach als undifferenziert und deshalb nicht aussagekräftig abgetan werden. Sie stellen eine umfassende Datengrundlage dar, aus der viele Informationen gewonnen werden können. Die Resultate verschiedener Schweizer Einzelstudien weisen in die gleiche Richtung wie die Zahlen der Verbrauchsberechnungen, was darauf hinweist, dass die den Berechnungen zugrunde gelegten Annahmen realistisch sind. Durch die lückenlose Verfügbarkeit der Verbrauchszahlen und deren einheitlicher Berechnung über viele Jahre hinweg werden Veränderungen dokumentierbar. Konstanz, Veränderungen oder Trends im Verbrauch von einzelnen Lebensmitteln oder Lebensmittelgruppen können sichtbar gemacht werden.

\section{Danksagung}

Wir danken Frau Dr. Stephanie Good für die Hilfe beim Verfassen des Unterkapitels 2.5 und auch allen Personen, die wir während der Kapitelerstellung kontaktiert haben, für ihre Auskünfte und Unterstützung.

\section{Literaturverzeichnis}

1. Bundesamt für Gesundheit. Kurzbeschrieb des Monitoring-Systems Ernährung und Bewegung (MOSEB).

Internet: http://www.bag.admin.ch/themen/ ernaehrung_bewegung/05190/index.html? lang=de (letzter Zugriff 30. September 2011).

2. Bundesamt für Gesundheit. Nationale Ernährungserhebung.

Internet: http://www.bag.admin.ch/themen/ ernaehrung_bewegung/05190/05297/index.html? lang=de (letzter Zugriff 30. September 2011).

3. Strassburg A. Ernährungserhebungen: Methoden und Instrumente. Ernährungs Umschau 2010; 57:422-30.

4. Schweizerische Gesellschaft für Ernährung SGE. Schweizer Lebensmittelpyramide: Empfehlungen zum ausgewogenen und genussvollen Essen und Trinken für Erwachsene, 2011.

Internet: http://www.sge-ssn.ch/media/medialibrary/pdf/100-ernaehrungsthemen/10-gesundes_ essen_trinken/Lebensmittelpyramide/_sge_pyramid_long_D.pdf (letzter Zugriff 6. Juni 2012).

5. Robert Koch-Institut. DISHES (Dietary Interview Software for Health Examination Studies) - Ein Ernährungserhebungsprogramm.

6. aid Infodienst Ernährung. Monica-Mengenliste. Internet: http://www.aid.de/shop/pdf/654_2009_ monica.pdf (letzter Zugriff 30. September 2011).

7. Sieber R. Einleitung zu Kapitel 1. In: Stähelin HB, Lüthy J, Casabianca A et al., eds. Dritter Schweizerischer Ernährungsbericht. Bern: Bundesamt für Gesundheitswesen, 1991:18-9.

8. Gremaud G, Schmid I, Sieber R. Estimation de I'utilisation des denrées alimentaires en Suisse pour les années 2001/2002. In: Eichholzer M, Camenzind-Frey E, Matzke A et al., eds. Fünfter Schweizerischer Ernährungsbericht. Bern: Bundesamt für Gesundheit, 2005:7-23. 
9. Sieber R, Grüter R. Lebensmittelverbrauch in der Schweiz während den Jahren 1973/74-1974/75 und 1979-1980. In: Aebi H, Blumenthal A, BohrenHoerni M et al., eds. Zweiter Schweizerischer Ernährungsbericht. Bern: Verlag H. Huber, 1984: 18-29.

10. Grüter R, Schmid I, Sieber R. Verbrauch an Lebensmitteln in der Schweiz in den Jahren 1994/95. In: Keller U, Lüthy J, Amadò A et al., eds. Vierter Schweizerischer Ernährungsbericht. Bern: Bundesamt für Gesundheit, 1998:4-16.

11. Erard M, Sieber R. Verbrauch und angenäherter Verzehr von Lebensmitteln in der Schweiz. In: Stähelin HB, Lüthy J, Casabianca A et al., eds. Dritter Schweizerischer Ernährungsbericht. Bern: Bundesamt für Gesundheitswesen, 1991:31-41.

12. Schweizerischer Bauernverband. Nahrungsmittelverbrauch pro Kopf.

Internet: http://www.sbv-usp.ch/fileadmin/user_ upload/bauernverband/Statistik/Ernaehrungsbilanz/se_0602.pdf (letzter Zugriff 30. September 2011).

13. Souci SW, Fachmann W, Kraut H. Die Zusammensetzung der Lebensmittel. Nährwert-Tabellen. 6. Auflage. Stuttgart: medpharm Scientific Publisher, 2000.

14. Schweizerischer Bauernverband. Vielfältiges Unternehmertum in der Landwirtschaft - Situationsbericht 2007. Internet: http://www.sbv-usp.ch/fileadmin/user_ upload/bauernverband/Taetigkeit/Situationsberichte/080104_SBV_Situationsbericht.pdf (letzter Zugriff 30. September 2011).

15. Schweizerischer Bauernverband. Mythos teure Landwirtschaft - Situationsbericht 2008. Internet: http://www.sbv-usp.ch/fileadmin/ user_upload/bauernverband/Taetigkeit/Situationsberichte/090105_SBV_Situationsbericht.pdf (letzter Zugriff 30. September 2011).

16. Eichholzer M, Bovey F, Jordan P, Probst-Hensch N, Stoffel-Kurt N. Daten zum Übergewicht und zu Ernährungsgewohnheiten aus der Schweizerischen Gesundheitsbefragung 2007. PRAXIS 2010;99:17-25.
17. Gaspoz JM, Beer-Borst S, Costanza MC, Morabia A. Suivi des facteurs de risque cardiovasculaire dans la population genevoise de 1993 à 2007. Rapport destiné à l'Unité de Direction Santé publique de l'Office fédéral de la santé publique.

Genève, 2009.

Internet: http://www.bag.admin.ch/themen/ ernaehrung_bewegung/05192/05943/index.html? lang=de (letzter Zugriff 30. September 2011).

18. Jacob S. Berechnung des Verbrauchs und angenäherten Verzehrs an Nahrungsenergie und Nährstoffen. In: Eichholzer M, Camenzind-Frey E, Matzke A et al., eds. Fünfter Schweizerischer Ernährungsbericht. Bern: Bundesamt für Gesundheit, 2005:25-35.

19. Bundesamt für Landwirtschaft. Marktbeobachtung Brot und Getreide. Brotkonsumstatistik April 2010. Internet: http://www.blw.admin.ch/dokumentation/00844/01044/01084/index.html?lang=de (letzter Zugriff 30. September 2011).

20. Daten zur Verfügung gestellt von U. Vock, Bundesamt für Landwirtschaft. Juni 2010.

21. Tschannen A, Calmonte R. Ernährungsgewohnheiten in der Schweiz. Stand und Entwicklungen auf der Grundlage der Daten der Schweizerischen Gesundheitsbefragungen 1992, 1997 und 2002. Neuchâtel: Bundesamt für Statistik, 2005. Internet: http://www.bfs.admin.ch/bfs/portal/de/ index/themen/14/22/publ.html?publicationID= 2028 (letzter Zugriff 30. September 2011).

22. Mozaffarian D, Micha R, Wallace S. Effects on coronary heart disease of increasing polyunsaturated fat in place of saturated fat: A systematic review and meta-analysis of randomized controlled trials. PLoS Medicine 2010;7:e1000252.

23. Schweizerische Gesellschaft für Ernährung. Merkblatt 3.1: Fette in der Ernährung.

24. Eidgenössische Ernährungskommission. Fette in der Ernährung. Empfehlungen der EEK. Bern: Bundesamt für Gesundheit, 2006. Internet: http://www.bag.admin.ch/themen/ernaehrung_bewegung/05207/05211/index.html? lang=de (letzter Zugriff 30. September 2011). 
25. Verband Schweizerischer Schokoladefabrikanten. Schweizer Schokoladeindustrie im Jahr 2010. Internet: http://www.chocosuisse.ch/web/chocosuisse/de/documentation/press_release.html (letzter Zugriff 30. September 2011).

26. AC Nielsen. Handelspanel-Daten 2008/2009. The Nielsen Company, Root. 2009.

27. Eidgenössische Alkoholverwaltung. Alkohol in Zahlen 2011.

Internet: http://www.eav.admin.ch/dokumentation/ 00445/00582/index.html?lang=de (letzter Zugriff 30. September 2011).

28. Annaheim B, Gmel G. Alkoholkonsum in der Schweiz. Lausanne: Schweizerische Fachstelle für Alkoholund andere Drogenprobleme, 2004. Internet: http://www.sucht-info.ch/infos-und-fakten/ alkohol/konsum/ (letzter Zugriff 30. September 2011).

29. Sucht Info Schweiz. Alkoholkonsum - Konsumhäufigkeit nach Geschlecht und Sprachregion. Internet: http://www.suchtschweiz.ch/ infos-und-fakten/alkohol/konsum/ konsumhaeufigkeit (letzter Zugriff 20. Februar 2012).

30. Sucht Info Schweiz. Alkoholkonsum in der Schweiz. Internet: http://www.suchtschweiz.ch/ infos-und-fakten/alkohol/konsum (letzter Zugriff 20. Februar 2012).

31. Sucht Info Schweiz. Alkoholkonsum - Risikokonsum.

Internet: http://www.suchtschweiz.ch/ infos-und-fakten/alkohol/konsum/risikokonsum (letzter Zugriff 20. Februar 2012).

32. Kuendig, H. Estimation du nombre de personnes alcoolo-dépendantes dans la population helvétique (Rapport de recherche No 56). Lausanne:

Addiction Info Suisse, 2010. Internet: http://www.suchtschweiz.ch/fileadmin/ user_upload/estimation_alcoolodependants_ RR56.pdf (letzter Zugriff 20. Februar 2012).

33. Marques-Vidal P, Bochud M, Paccaud F et al. No interaction between alcohol consumption and HDL-related genes on HDL cholesterol levels. Atherosclerosis 2010;211:551-7.
34. Clerc O, Nanchen D, Cornuz J et al. Alcohol drinking, the metabolic syndrome and diabetes in a population with high mean alcohol consumption. Diabetic Medicine 2010;27:1241-9.

35. Gmel G, Gaume J, Faouzi M, Kulling J-P, Daeppen J-B. Who drinks most of the total alcohol in young men - risky single occasion drinking as normative behaviour. Alcohol \& Alcoholism 2008;43:692-7.

36. Delgrande Jordan M, Annaheim B. Habitudes alimentaires, activité physique et statut pondéral chez les élèves de 11 à 15 ans en Suisse. Situation en 2006 et évolution récente - Résultats de l'Enquête internationale Health Behaviour in School-aged Children (HBSC). Lausanne: Schweizerische Fachstelle für Alkohol- und andere Drogenprobleme, 2009.

Internet: http://www.sfa-ispa.ch/DocUpload/ rr_no45_HBSC_2006.pdf (letzter Zugriff 30. September 2011).

37. Windlin B, Kuntsche E, Delgrande Jordan M. Konsum psychoaktiver Substanzen Jugendlicher in der Schweiz - Zeitliche Entwicklungen und aktueller Stand. Resultate der internationalen Studie "Health Behaviour in School-aged Children" (HBSC) (Forschungsbericht Nr. 58). Lausanne: Sucht Info Schweiz, 2011. Internet: http://www.addictionsuisse.ch/fileadmin/user_upload/DocUpload/RR_58.pdf (letzter Zugriff 20. Februar 2012).

38. Schweizerische Fachstelle für Alkohol- und andere Drogenprobleme. Trends im Tabak-, Alkoholund Cannabisgebrauch von 1986 bis 2006 bei Jugendlichen in der Schweiz. Bern, Bundesamt für Gesundheit, 2008.

Internet: http://www.bag.admin.ch/pdf_link.php? \&download=Trends_ACT (letzter Zugriff 30. September 2011).

39. Camenzind-Frey E, Sutter-Leuzinger A, Schmid A, Sieber R. Beurteilung des Verbrauchs und angenäherten Verzehrs an Nahrungsenergie und Nährstoffen. In: Eichholzer M, Camenzind-Frey E, Matzke A et al., eds. Fünfter Schweizerischer Ernährungsbericht. Bern: Bundesamt für Gesundheit, 2005:51-70. 
40. Bundesamt für Gesundheit, EidgenössischeTechnische Hochschule Zürich. Schweizer Nährwertdatenbank (V 3.01).

Internet: http://www.swissfir.ethz.ch/datenbank (letzter Zugriff 30. September 2011).

41. Bundesinstitut für gesundheitlichen Verbraucherschutz und Veterinärmedizin (BgVV). Der Bundeslebensmittelschlüssel (BLS ii.3). Konzeption, Aufbau und Dokumentation der Datenbank blsdat. BgVV-Heft 08/1999.

42. Schmid A, Ampuero S, Badertscher R et al. Zusammensetzung diverser Schweizer Brühwürste.

ALP science 531, 2009.

Internet: http://www.agroscope.admin.ch/data/ publikationen/as531.pdf (letzter Zugriff

30. September 2011).

43. Schmid A, Badertscher R, Collomb M et al. Zusammensetzung verschiedener Rohwürste Schweizer Herkunft. Mitt Lebensm Umweltchem 2012;100 (im Druck).

44. Schmid A, Badertscher R, Collomb M et al. Die Zusammensetzung diverser Schweizer Rohpökelwaren. Fleischwirtschaft 2011;91:84-8.

45. Fleischkonsum in Privathaushalten. Daten zur Verfügung gestellt von R. Kennel und M. Schneider, Proviande. April 2010.

46. Marques-Vidal P, Pecoud A, Hayoz D et al. Prevalence and characteristics of vitamin or dietary supplement users in Lausanne, Switzerland: the CoLaus study. Eur J Clin Nutr 2009;63:273-81.

47. Eichholzer M, Bisig B, Gutzwiller F, Lüthy J. Aktuelle Ernährungsprobleme in der Schweiz. Resultate der Schweizerischen Gesundheitsbefragung 1997. Mitt Lebensm Hyg 2000;91:251-73.

48. Deutsche Gesellschaft für Ernährung, Österreichische Gesellschaft für Ernährung, Schweizerische Gesellschaft für Ernährungsforschung, Schweizerische Vereinigung für Ernährung. Referenzwerte für die Nährstoffzufuhr. 1. Auflage, 3. vollständig durchgesehener und korrigierter Nachdruck. Neustadt: Neuer Umschau Buchverlag, 2008.
49. Bundesamt für Statistik. Statistik des jährlichen Bevölkerungsstandes (ESPOP) 2008. Neuchâtel: Bundesamt für Statistik BFS, 2009. Internet: http://www.bfs.admin.ch/bfs/portal/de/ index/news/publikationen.Document.123463.pdf (letzter Zugriff 30. September 2011).

50. Hunt JR. Bioavailability of iron, zinc, and other trace minerals from vegetarian diets. Am J Clin Nutr 2003;78:633S-639S.

51. Guéguen L, Pointillart A. The bioavailability of dietary calcium. J Am Coll Nutr 2000;19:119S-136S.

52. Hurrell RF. Bioavailability of iron. Eur J Clin Nutr 1997;51:S4-S8.

53. Sutter-Leuzinger A, Sieber R. Beurteilung des Verbrauchs an Nahrungsenergie, Energieträgern, Nahrungsfasern, Vitaminen, Mineralstoffen und Spurenelementen. In: Keller U, Lüthy J, Amadò A et al., eds. Vierter Schweizerischer Ernährungsbericht. Bern: Bundesamt für Gesundheit 1998:28-50.

54. Schlotke F, Sieber R. Berechnung des Verbrauchs an Nahrungsenergie, Energieträgern, Nahrungsfasern, Vitaminen, Mineralstoffen und Spurenelementen. In: Keller U, Lüthy J, Amadò A et al., eds. Vierter Schweizerischer Ernährungsbericht. Bern: Bundesamt für Gesundheit 1998:18-27.

55. Mozaffarian D, Aro A, WillettWC. Health effects of trans-fatty acids: experimental and observational evidence. Eur J Clin Nutr 2009;63:S5-S21.

56. Eidgenössisches Departement des Innern. Verordnung des EDI über Speiseöl, Speisefett und daraus hergestellte Erzeugnisse. Stand 22. März 2009. Internet: http://www.admin.ch/ch/d/sr/8/ 817.022.105.de.pdf (letzter Zugriff 30. September 2011).

57. Richter EK, Shawish KA, Scheeder MRL, Colombani PC. Trans fatty acid content of selected Swiss foods: The TransSwissPilot study. J Food Compost Anal 2009;22:479-84.

58. Uauy R, Aro A, Clarke R et al. WHO Scientific Update on trans fatty acids: summary and conclusions. Eur J Clin Nutr 2009;63:S68-S75. 
59. Eidgenössische Ernährungskommission. Kohlenhydrate in der Ernährung. Stellungnahme und Empfehlungen der EEK. Zürich: Bundesamt für Gesundheit, 2009.

Internet: http://www.bag.admin.ch/themen/ ernaehrung_bewegung/05207/07326/index. html? lang=de (letzter Zugriff 30. September 2011).

60. World Health Organization. Diet, nutrition and the prevention of chronic diseases: report of a joint WHO / FAO expert consultation. Geneva:World Health Organization, 2003. [WHOTechnical report series; 916].

Internet: http://whqlibdoc.who.int/trs/WHO_ TRS_916.pdf (letzter Zugriff 30. September 2011).

61. Wynn-Dumartheray E, Krieg MA, Cornuz J, Whittamore DR, Lanham-New SA, Burckhardt P. Energy and nutrient intake of Swiss women aged 75-87 years. J Hum Nutr Diet 2006;19:431-5.

62. Holick MF. Environmental factors that influence the cutaneous production of vitamin D. Am J Clin Nutr 1995;61:S638-S645.

63. Mithal A, Wahl D, Bonjour JP et al. Global vitamin D status and determinants of hypovitaminosis D. Osteoporos Int 2009;20:1807-20.

64. Holick MF, Chen TC. Vitamin D deficiency: a worldwide problem with health consequences. Am J Clin Nutr 2008;87:1080S-1086S.

65. Bischoff-Ferrari H. Vitamin D: What is an adequate vitamin $D$ level and how much supplementation is necessary? Best Pract Res Clin Rheumatol 2009; 23:789-95.

66. Spalinger J, Schubiger G, Baerlocher K. Ernährung gesunder Neugeborener in den ersten Lebenstagen. Paediatrica 2003;14:24-5.

67. Dratva J, Merten S, Ackermann-Liebrich U. Vitamin D supplementation in Swiss infants. Swiss MedWkI 2006;136:473-81.
68. Eidgenössische Ernährungskommission. Folsäure. Expertenbericht der EEK zur Prophylaxe von Neuralrohrdefekten. Bern: Bundesamt für Gesundheit, 2002.

Internet: http://www.bag.admin.ch/themen/ ernaehrung_bewegung/05207/05212/index.html? lang=de (letzter Zugriff 30. September 2011).

69. Eidgenössische Ernährungskommission. Gesundheitliche Vor- und Nachteile einer vegetarischen Ernährung. Expertenbericht der EEK. Bern: Bundesamt für Gesundheit, 2006. Internet: http://www.bag.admin.ch/themen/ ernaehrung_bewegung/05207/05219/index.html? lang=de (letzter Zugriff 30. September 2011).

70. Bundesamt für Gesundheit. Salz Strategie 20082012.

Internet: http://www.bag.admin.ch/themen/ ernaehrung_bewegung/05207/05216/index.html? lang=de (letzter Zugriff 30. September 2011).

71. Beer-Borst $S$, Costanza MC, Pechère-Bertschi A, Morabia A. Twelve-year trends and correlates of dietary salt intakes for the general adult population of Geneva, Switzerland. Eur J Clin Nutr 2009;63:155-64.

72. Andersson M, Egli IM, Zimmermann MB. Eisenmangel. Schweizerische Zeitschrift für Ernährungsmedizin 2010;8:13-9.

73. Andersson M, Zimmermann MB, Tajeri Foman J, Theis W, Hurrell RF. Prevalence and etiology of functional iron deficiency in young women with a low anemia prevalence. In: Andersson M. Impact evaluation of food fortification with iron in school children and women of reproductive age. Diss ETH 19032. Zürich: ETH Zürich 2010:189-211.

74. Mettler S, Zimmermann MB. Iron excess in recreational marathon runners. Eur J Clin Nutr 2010;64:490-4.

75. Schleiffenbaum BE, Schaer DJ, Burki D et al. Unexpected high prevalence of metabolic disorders and chronic disease among young male draftees the Swiss Army XXI experience. Swiss MedWkI 2006;136:175-84. 
76. Zimmermann MB, Aeberli I, TorresaniT, Burgi H. Increasing the iodine concentration in the Swiss iodized salt program markedly improved iodine status in pregnant women and children: a 5-y prospective national study. Am J Clin Nutr 2005;82:388-92.

77. Andersson M, Aeberli I, Wust $\mathrm{N}$ et al. The Swiss iodized salt program provides adequate iodine for school children and pregnant women, but weaning infants not receiving iodine-containing complementary foods as well as their mothers are iodine deficient. J Clin Endocrinol Metab 2010; 95:5217-24.

78. Jenny-Burri J, Haldimann M, DudlerV. Selenium intake in Switzerland in relation to selected food groups. Food Addit Contam 2010;27:1516-31.

79. Burri J, Haldimann M, DudlerV. Selenium status of the Swiss population: Assessment and change over a decade. JTrace Elem Med Biol 2008; 22:112-9.

80. Aeberli I, Kaspar M, Zimmermann MB. Dietary intake and physical activity of normal weight and overweight 6- to 14-year-old Swiss children. Swiss MedWkI 2007;137:424-30.

81. Stamm H, Wiegand D, Lamprecht M. MonitoringSystem Ernährung und Bewegung. Indikatorensammlung zum NPEB 2008-2012. Indikator 2.7: Stillen. Bern: Bundesamt für Gesundheit, 2010. Internet: http://www.bag.admin.ch/themen/ ernaehrung_bewegung/05190/07835/07864/ index.html?lang=de (letzter Zugriff 30. September 2011).

82. European Food Safety Authority. The EFSA Comprehensive European Food Consumption Database. Internet: http://www.efsa.europa.eu/en/datex/ datexfooddb.htm (letzter Zugriff 30. September 2011).

83. European Food Consumption Survey Method (EFCOSUM). Internet: http://www.public-health.tu-dresden. de/dotnetnuke3/eu/Projects/PastProjects/ EFCOSUM/tabid/338/Default.aspx (letzter Zugriff 30. September 2011).
84. European Food Consumption Validation (EFCOVAL). Internet: http://www.efcoval.eu/ (letzter Zugriff 30. September 2011).

85. European Food Safety Authority. Erklärung des Beirats der EFSA zur paneuropäischen Erhebung über den Lebensmittelverzehr. Internet: http://www.efsa.europa.eu/en/eumenu/ docs/eumenudeclarationde.pdf (letzter Zugriff 30. September 2011).

86. European Nutrition and Health Report 2009. Elmadfa I (ed). Forum of nutrition, Vol. 62. Internet: http://www.univie.ac.at/enhr/index.htm (letzter Zugriff 30. September 2011).

87. Ernährungsbericht 2008. Bonn: Deutsche Gesellschaft für Ernährung e.V., 2008.

88. EuroFIR.

Internet: http://www.eurofir.org/home (letzter Zugriff 30. September 2011).

89. Bundesamt für Statistik. Erhebungen, QuellenSchweizerische Gesundheitsbefragung (SGB). Internet: http://www.bfs.admin.ch/bfs/portal/de/ index/infothek/erhebungen__quellen/blank/blank/ ess/04.html (letzter Zugriff 30. September 2011).

90. Coop. Ess-Trends im Fokus: Essen und Gesundheit. Basel: Coop, 2009.

Internet: http://www.coop.ch/pb/site/common/ get/documents/system/elements/gesundessen/ pdf/fokus/Studienbericht_III_d.pdf (letzter Zugriff 30. September 2011).

91. Frisch AL, Schulz PJ. Ernährung, Bewegung und Körpergewicht: Wissen, Einstellung undWahrnehmung der Schweizer Bevölkerung. Lugano: Institute of Communication and Health, Università della Svizzera italiana, 2010. Internet: http://www.gesundheitsfoerderung.ch/ pdf_doc_xls/d/gesundes_koerpergewicht/ grundlagen_wissen/Monitoring/Bevoelkerungsbefragung_Bericht.pdf (letzter Zugriff 30. September 2011).

92. Rudolph T, Glas A. Food Consumption 2008: Essund Verzehrverhalten in der Schweiz. St. Gallen: Thexis, 2008. 
93. Dickson-Spillmann M, Siegrist M, Keller C. Development and validation of a short, consumer-oriented nutrition knowledge questionnaire. Appetite 2011;56:617-20.

94. Dickson-Spillmann M, Siegrist M. Consumers' knowledge about healthy diets and its correlation with dietary behaviour. J Hum Nutr Diet 2011; 24:54-60.

95. Schulz PJ, Hartung U, Faustinelli C, Keller S. Ernährung, Körperbewegung und Körpergewicht: Einstellungen und Wahrnehmungen der Schweizer Bevölkerung. Lugano: Institute of Communication and Health, Università della Svizzera italiana, 2007.

96. Wardle J, Parmenter K, Waller J. Nutrition knowledge and food intake. Appetite 2000;34:269-75.

97. Schaub A, Palladino C. Schlussbericht NANUSS Pilot: Ernährungsverhalten. Zürich: gfs-zürich, 2010.

Internet: http://www.bag.admin.ch/themen/ ernaehrung_bewegung/05192/05943/index.html? lang=de (letzter Zugriff 30. September 2011).

98. Hess R, Visschers VHM, Siegrist M. The role of health-related, motivational and socio-demographic aspects in predicting food label use: A comprehensive study. Public Health Nutr 2012; 15: 407-14.

99. Visschers VHM, Siegrist M. Applying the evaluability principle to nutrition table information. How reference information changes people's perception of food products. Appetite 2009;52:505-12.

100. Infanger E, Ryser C, Beer M. Wirksamkeit von drei verschiedenen Front-of-Pack-Kennzeichnungen auf Lebensmitteln zur Unterstützung des Konsumenten bei der gesunden Wahl. Bern: Schweizerische Gesellschaft für Ernährung, 2010. Internet: http://www.sge-ssn.ch/media/medialibrary/pdf/500-fuer_experten/70-labelling/Studienbericht_FINAL.pdf (letzter Zugriff 6. Juni 2012).

101. Visschers VHM, Hess R, Siegrist M. Health motivation and product design determine consumers' visual attention to nutrition information on food products. Public Health Nutr 2010;13:1099-106.
102. Coop. Ess-Trends im Fokus: So kocht die Schweiz. Basel: Coop, 2010.

Internet: http://www.coop.ch/pb/site/common/ get/documents/system/elements/gesundessen/ pdf/fokus/Studienbericht_IV_d.pdf (letzter Zugriff 30. September 2011).

103. Das Ernährungs-Panel Schweiz. Daten zurVerfügung gestellt von M. Siegrist und K. van der Horst, ETH Zürich. August 2010.

104. Higgs S, Woodward M. Television watching during lunch increases afternoon snack intake of young women. Appetite 2009;52:39-43.

105. Bundesamt für Statistik. Schweizerische Gesundheitsbefragung 2007: Erste Ergebnisse. Neuchâtel: Bundesamt für Statistik BFS, 2008. Internet: http://www.bfs.admin.ch/bfs/portal/de/ index/themen/14/22/publ.html?publication ID= 3338 (letzter Zugriff 30. September 2011).

106. BrunnerT, van der Horst K, Siegrist M. Convenience food products: Drivers for consumption. Appetite 2010;55:498-506.

107. van der Horst K, BrunnerT, Siegrist M. Ready-meal consumption: Associations with weight status and cooking skills. Public Health Nutr 2011;14:239-45.

108. Dickson-Spillmann M, Siegrist M, Keller C, Wormuth M. Phthalate exposure through food and consumers' risk perception of chemicals in food. Risk Analysis 2009;29:1170-81.

109. Chappuis A, Bochud M, Glatz N, Vuistiner P, Paccaud $F$, Burnier $M$. Swiss survey on salt intake: main results. Bern: Federal Office of Public Health, 2011. Internet: http://www.bag.admin.ch/themen/ ernaehrung_bewegung/05207/05216/12335/ index.html? lang=de\&download=NHzLpZeg7t, Inp 6IONTU042I2Z6In1acy4Zn4Z2qZpnO2Yuq2Z6gpJ CKdoN3fWym162epYbg2c_JjKbNoKSn6A-(letzter Zugriff 5. Dezember 2011).

110. Bundesamt für Gesundheit. Jodversorgung der Schweizer Bevölkerung. Ergebnisse aus einer nationalen Multizenterstudie. Bisher unveröffentlichte Daten, 2011. 


\section{Ernährung und Gesundheit}

\section{David Fäh}

Annette Matzke

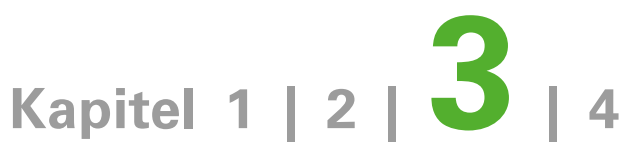




\subsection{Zusammenfassung}

Verfügbare Schweizer Daten zur Beurteilung des Einflusses der Ernährung auf Risikofaktoren und Krankheitsrisiken sind unzureichend und unsicher. Die ausgeprägte Abnahme der HerzKreislauf-Mortalität über die letzten Jahre kann nicht mit Veränderungen der Ernährungsgewohnheiten erklärt werden, sondern eher mit verbesserter Früherkennung und Behandlung. Risikofaktoren, die mit Ernährung zusammenhängen, wie hoher Blutdruck, Fettstoffwechselstörungen und Diabetes mellitus Typ 2, scheinen zugenommen zu haben. Neben der Mortalität von Herz-Kreislauf-Krankheiten hat auch diejenige von Krebs abgenommen, allerdings weniger stark. Hält dieser Trend an, wird Krebs im Verhältnis an Bedeutung gewinnen. Die Inzidenz von Magenkrebs ging zurück, während diejenige von anderen ernährungsassoziierten Krebsformen gleich blieb oder zunahm. Übergewicht und Adipositas, der konstant hohe Salzund Zuckerkonsum und der abnehmende Früchtekonsum in der Schweiz sprechen dagegen, dass die Ernährung zu einer Abnahme chronischer Krankheiten führen wird. Eher dafür sprechen hingegen der in den letzten Jahrzehnten gestiegene Konsum von Nahrungsfasern, Fisch und Gemüse, der gesunkene Konsum von Alkohol sowie von Fleisch und Fleischprodukten und der Trend weg von tierischem hin zu pflanzlichem Fett. Ausserdem blieben Gesamtkalorienkonsum und körperliche Aktivität konstant (Datenbasis: verschiedene Erhebungsmethoden).

Viele Erkrankungen liessen sich durch eine Ernährung vermeiden, die Übergewicht vorbeugt. Erfreulicherweise gibt es erstmals Hinweise darauf, dass die Prävalenz von Übergewicht und Adipositas nicht gleich stark zunimmt wie in der Vergangenheit - bei Kindern und Erwachsenen. Allerdings schwankt die Adipositasprävalenz in Abhängigkeit von Bildungsniveau und Nationalität. Adipositas ist mit Essstörungen assoziiert. Mit ca. 3\% ist die Prävalenz klinisch manifester Essstörungen zwar relativ niedrig, allerdings sind problematisches Essverhalten und gestörte Körperwahrnehmung weit verbreitet.

An allgemeiner Mangelernährung leidet ca. ein Fünftel aller ins Spital eintretenden Menschen. Mangelernährung ist auch in Heimen und in spitalexterner Pflege häufig und bleibt dort oft unerkannt. Einzelne Bevölkerungsgruppen zeigen eine ungenügende Versorgung mit gewissen Nährstoffen. So ist die einer Osteoporose vorbeugende Calcium-, Protein- und Vitamin-D-Versorgung insbesondere bei vielen älteren Menschen unzureichend. Auch bei vollgestillten Säuglingen und im Winter könnte die Vitamin-D-Versorgung besser sein. Eisenmangel weisen besonders Frauen im gebärfähigen Alter auf. Der Selenstatus scheint hingegen beim Grossteil der Bevölkerung ausreichend zu sein. Unklar bleibt, inwieweit die Folsäurezufuhr durch Supplemente oder angereicherte Lebensmittel die Häufigkeit von Neuralrohrdefekten vermindert. Problematisch dürfte schliesslich die Jodversorgung eines Teils der Bevölkerung sein. Bezüglich Lebensmittelunverträglichkeiten muss aufgrund der Zunahme kreuzreagierender Pollenallergien mit häufigeren Lebensmittelallergien gerechnet werden.

Karies kommt wesentlich seltener vor als in den 1980er Jahren. 


\subsection{Einleitung}

\subsubsection{Ernährung und Gesundheit - Generelle Bedeutung}

\section{Bedeutung der Ernährung}

Essen bedeutet Leben - allerdings essen wir nicht nur, um unseren Hunger zu stillen. Essen bedeutet auch Lust, Genuss und Lebensqualität. Was und wie ein Mensch isst, hängt stark von seiner Erziehung und seinen Wertvorstellungen ab, aber auch von seinem sozialen, kulturellen und ökonomischen Umfeld. Der Mensch braucht Nahrung und Flüssigkeit, um den Aufbau, die Entwicklung und den Betrieb seines Körpers zu gewährleisten. Isst er über längere Zeit zu viel, zu wenig oder das Falsche, kann er krank werden. Dabei wirkt die Ernährung nicht isoliert, sondern im Zusammenspiel mit anderen Risiko- und Schutzfaktoren wie körperlicher Aktivität oder Tabakkonsum.

\section{Evidenz über den Zusammenhang zwischen Ernährung und Gesundheitszustand}

Der Zusammenhang zwischen Ernährung und Gesundheitszustand ist schwer zu bestimmen, weil er von vielen Faktoren wie den Genen und der Entwicklung im Mutterleib beeinflusst wird. Auch dauert es lange, bis sicht- bzw. spürbare Symptome einer Störung als Folge einer Fehlernährung auftreten. So können Trends bei den Risikofaktoren für Übergewicht und Adipositas der letzten 10-15 Jahre beispielsweise nur mit Einschränkung für die Erklärung der heutigen Prävalenz in der Bevölkerung herangezogen werden. Die dafür relevanten Altersgruppen $(50+)$ haben den Grossteil ihres überschüssigen Gewichts wahrscheinlich in den 1970er und
1980 er Jahren zugelegt, also im Alter zwischen 20 und 50 Jahren, und für diese Zeit ist die Datenlage ungenügend. Beim gleichzeitigen Erfassen von Krankheiten und deren Risikofaktoren kann es also sein, dass fehlerhafte Rückschlüsse über die Ursächlichkeit eines Zusammenhangs gezogen werden.

Zwischen der Ernährung und anderen Lebensstilfaktoren gibt es auch viele Wechselwirkungen, die schwer messbar sind. Das Beispiel Krebs zeigt, dass die lange Latenzzeit bis zur klinischen Manifestation und die komplexen Zusammenhänge zwischen Umwelt-, Verhaltensund genetischen Faktoren die Abschätzung der ursächlichen Beteiligung der Ernährung an der Entstehung oder Vorbeugung von Krebserkrankungen besonders stark erschweren (Kapitel 3.5). Oft ist auch unklar, ob die unmittelbaren oder die langfristigen Folgen einer Fehlernährung für ein erhöhtes Erkrankungsrisiko verantwortlich sind. Zum Beispiel ist nicht eindeutig, inwiefern die mit Adipositas einhergehenden Risiken auf den Überschuss an Fettgewebe oder auf die damit zusammenhängende Ernährung zurückzuführen sind. Es ist schwierig zu unterscheiden, ob das Gesundheitsrisiko von Adipositas auf die insgesamt zu hohe Energiezufuhr oder auf eine ungünstige Zusammensetzung der Nahrung zurückzuführen ist.

Nicht nur Menge und Qualität, sondern auch die Lagerung der Nahrung kann das Krankheitsrisiko beeinflussen. Bei manchen potenziellen Risikofaktoren - beispielsweise beim Salzkonsum - bestehen auch Unterschiede zwischen individuellem und Bevölkerungsrisiko (Kapitel 3.4.2).

Oft ist die Datenlage ungenügend. Ernährungsstudien haben zudem Schwächen und / oder werden teilweise nicht veröffentlicht, beispiels- 
weise weil das Resultat zu wenig Interesse weckt oder einem breiten Konsens widerspricht. Um die Relevanz einer Studie besser beurteilen zu können, muss das Studiendesign berücksichtigt werden. Interventionsstudien haben häufig eine stärkere Beweiskraft für die Kausalität eines Zusammenhangs als epidemiologische Beobachtungsstudien. So liegen für die Schweiz zur möglichen Ursache von Krebs vor allem Fall-Kontroll-Studien vor, die nur beschränkt dazu geeignet sind, einen ursächlichen Zusammenhang nachzuweisen. Klinische Interventionsstudien sind aber selten national repräsentativ, weshalb die Resultate nicht für die gesamte Bevölkerung geltend gemacht werden können. Aus ethischen Gründen liegen ausserdem häufig Tierstudien vor, deren Resultate nur begrenzt auf den Menschen übertragbar sind. Schliesslich gestaltet sich auch die Erfassung der Ernährung problematisch (Kapitel 2.2.1). Befragungen eignen sich dazu nur bedingt, weil Menschen dazu tendieren, unbewusst zu essen, zu unterschätzen, wie viel sie essen, und schliesslich zu vergessen, was sie gegessen haben. Für den Zusammenhang zwischen Ernährung und Erkrankung wurden unterschiedliche Stufen der Evidenz beschrieben, von überzeugend bis ungenügend $(1 ; 2)$.

\section{Ziel, Struktur und Inhalt dieses Kapitels}

Das Ziel dieses Kapitels ist es, eine Grundlage zu schaffen, die es erlauben soll, die Entwicklung ernährungsabhängiger Krankheiten und deren Zusammenhänge zu Ernährungsfaktoren zu verfolgen. Zu diesem Zweck wurden möglichst alle in der Schweiz verfügbaren Datenquellen gesammelt und relevante Resultate daraus dargestellt. Dieses Kapitel ist nach Krankheiten gegliedert mit dem Fokus auf de- ren Häufigkeit und auf Risiko- und Schutzfaktoren, die mit der Ernährung zusammenhängen. Klinische Aspekte, beispielsweise die Ernährung bei der Prävention von Komplikationen oder bei der Therapie bestimmter Krankheiten, werden höchstens am Rande erwähnt. Die Auswahl der Krankheiten erfolgte nach ihrer Bedeutung für die Schweiz und danach, ob sie entweder ursächlich mit der Ernährung zusammenhängen oder sich auf den Ernährungszustand auswirken (z.B. Essstörungen, Mangelernährung, Lebensmittelunverträglichkeiten).

Im Zentrum des Interesses liegen Daten aus der Schweiz, wenn möglich solche, die von bevölkerungsrepräsentativen Studien stammen. Fehlten diese, wurden fallspezifisch Daten aus anderen europäischen Ländern oder von weniger repräsentativen Gruppen aus der Schweiz beigezogen.

\subsubsection{Datenquellen}

Daten zum Lebensmittelverbrauch und-verzehr sowie zum Ess- und Trinkverhalten

Was und wie viel in der Schweiz konsumiert wird, kann bisher nur aufgrund von nationalen Verbrauchsdaten geschätzt werden, weil es keine für die gesamte Schweiz repräsentativen individuellen Verzehrsdaten gibt. Die verfügbaren Verbrauchsdaten erlauben es, Veränderungen über die Zeit abzuschätzen (Kapitel 2.3). Ernährungsdaten wurden in der Vergangenheit auch mit Gesundheitsstudien gesammelt. Diese sind aber weit weniger detailliert und stammen überwiegend aus der Westschweiz und dem Tessin (3). Die beiden aktuellsten grösseren Erhebungen wurden in der Stadt Lausanne und im Kanton Genf durchgeführt $(4 ; 5)$. In eini- 
gen Belangen decken sich die Resultate der Gesundheitsstudien mit den Verbrauchsdaten (6). Die Schweizerische Gesundheitsbefragung $(S G B)$, die seit 1992 alle fünf Jahre durchgeführt wird (7), sowie die Studie Health Behaviour in School-aged Children (HBSC), die seit 1986 alle vier Jahre stattfindet (8), liefern einige, allerdings oberflächliche Informationen über das Ess- und Trinkverhalten.

\section{Erfassung von Risikofaktoren für ernährungsab- hängige Krankheiten}

Die Erfassung von Risikofaktoren wie Übergewicht, ungünstigen Blutfettwerten oder hohem Blutdruck ist wichtig, weil diese einen Zwischenschritt zwischen Ernährung und einer Krankheit darstellen. Bei vielen Risikofaktoren braucht es Messungen, um die Prävalenz vollumfänglich erfassen zu können. Studien wie die $S G B$, die Personen nach ihrer eigenen Einschätzung fragen, unterschätzen die Prävalenz, weil sie Betroffene nicht erfassen, die nichts von einem allfälligen erhöhten Blutdruck, erhöhten Cholesterinwerten oder der Existenz eines Diabetes mellitus wissen. Auch die Adipositasprävalenz wird durch Befragungen unterschätzt (Kapitel 3.3.1). Messungen fanden in der Schweiz bisher meist nur im Rahmen von zeitlich und räumlich begrenzten Studien statt $(3-5 ; 10)$. Abgesehen von zwei national wenig repräsentativen Studien $(11 ; 12)$ fand die erste grössere, landesweite Befragung 1992/93 in Form der ersten SGB statt (7). Auch das Schweizer HaushaltPanel (SHP) erfasst seit 1999 Gesundheitsdaten (13). Neben diesen generellen Gesundheitsstudien gibt es Untersuchungen, die sich mit spezifischen Aspekten der Ernährung befassen. So wird der Alkoholkonsum im Rahmen von Studien erhoben, die von Sucht Info Schweiz (ehemals
Schweizerische Fachstelle für Alkohol- und andere Drogenprobleme) in Auftrag gegeben oder begleitet werden $(8 ; 14)$.

\section{Morbidität ernährungsabhängiger Krankheiten}

Die Schweiz besitzt keine national repräsentativen Zahlen zur Morbidität. Die SGB liefert zwar Informationen darüber, allerdings nur über Selbstangaben (7;15; Tabelle 3.1). Für gewisse spezifische Krankheiten (z.B. Herzinfarkt) sind Zahlen verfügbar, allerdings nicht flächendeckend (16). In vielen Kantonen wird die Inzidenz von Krebserkrankungen erhoben (d.h. die Anzahl Neuerkrankungen pro Jahr; Kapitel 3.5) und in manchen Spitälern Mangelernährung (Kapitel 3.10). 2010 wurden erstmals Essstörungen mit einer national repräsentativen Studie erfasst (17). Gewisse Hinweise auf die Morbidität können Daten zu Begleitdiagnosen bei Sterbefällen, zu stationären Behandlungsfällen in Spitälern und die Verordnungshäufigkeit von Medikamenten in Arztpraxen geben (18-22).

Generell wäre es wichtig, die Inzidenz von Krankheiten zu kennen. Angaben zur Prävalenz (d.h. Angaben über die Anzahl Erkrankter in der Bevölkerung) sind ungenügend, weil sie keine Auskunft darüber geben, ob eine Zunahme auf ein vermehrtes Auftreten einer Erkrankung oder auf bessere Überlebenschancen der Erkrankten zurückzuführen ist (Kapitel 3.6.1).

\section{Mortalität ernährungsabhängiger Krankheiten}

Die Schweiz verfügt über relativ zuverlässige und flächendeckende Mortalitätsdaten (19;23; 24). Dank der über Jahre vergleichbaren Erfassung sind die Analyse von Zeit-Trends und internationale Vergleiche möglich. Die Verknüpfung von Mortalitätsdaten mit Daten aus den Volkszählungen 1990 und 2000 (Swiss National Co- 
hort, SNC) bietet erweiterte Möglichkeiten (25). Mortalitätsraten sind in den Tabellen 3.4 und 3.6 zusammengefasst. Die Tabellen werden in den entsprechenden Kapiteln näher erläutert (Kapitel 3.4.1 und 3.5.1).

\section{Alle Datenquellen haben Einschränkungen}

Daten und Studien sind grundsätzlich immer nur beschränkt aussagekräftig. Beispielsweise ist selbst die $S G B$ nur bedingt repräsentativ für die
Gesamtbevölkerung. Ein Grund ist die begrenzte Teilnehmerquote von $60-70 \%$ im telefonischen und von rund $50 \%$ im schriftlichen Teil $(7 ; 26)$. Aus anderen Studien ist zudem bekannt, dass tendenziell Gesündere an solchen Befragungen teilnehmen und Verweigerer einen weniger gesunden Lebensstil pflegen. Eine ungewollte Selektion könnte schliesslich bereits bei der Stichprobenziehung entstehen, weil nur Personen mit eingetragenem Festnetzan-

Tabelle 3.1: Prävalenz von erfragten Erkrankungen und Störungen bei Personen ab 15 Jahren in Privathaushalten in der Schweiz, nach Geschlecht, 1992-2007 (15)

\begin{tabular}{|c|c|c|c|c|c|c|c|c|}
\hline & \multicolumn{4}{|c|}{ Prävalenz bei Männern (in \%) } & \multicolumn{4}{|c|}{ Prävalenz bei Frauen (in \%) } \\
\hline & $1992 / 93$ & 1997 & 2002 & 2007 & $1992 / 93$ & 1997 & 2002 & 2007 \\
\hline Untergewicht ${ }^{a}$ & 1.3 & 1.4 & 1.6 & 1.0 & 7.8 & 6.7 & 6.2 & 5.9 \\
\hline Übergewicht ${ }^{a}$ & 33.1 & 35.4 & 37.4 & 37.6 & 17.1 & 21.1 & 21.7 & 20.8 \\
\hline Adipositas (Klassen I-III)a & 6.1 & 6.8 & 8.0 & 8.7 & 4.7 & 7.0 & 7.5 & 7.8 \\
\hline Adipositas (Klassen II+III) & 1.0 & 1.0 & 1.0 & 1.7 & 1.1 & 1.4 & 1.8 & 1.8 \\
\hline Gewichtsabnahme erwünscht ${ }^{b}$ & - & 20.0 & 15.4 & 15.7 & - & 51.1 & 46.0 & 45.8 \\
\hline Essen, ohne aufhören zu können b & - & 26.4 & - & 25.1 & - & 28.9 & - & 27.1 \\
\hline Sich selber zum Erbrechen bringen ${ }^{b}$ & - & 1.9 & - & 1.1 & - & 3.1 & - & 2.1 \\
\hline Bluthochdruck (Arztdiagnose) & - & 19.1 & 20.5 & 23.2 & - & 21.1 & 21.2 & 20.5 \\
\hline Bluthochdruck (Behandlung, täglich) & 7.6 & 8.8 & 10.3 & 13.5 & 8.5 & 11.4 & 12.1 & 13.7 \\
\hline Zu hoher Cholesterinwert (Arztdiagnose) & - & 12.8 & 16.5 & 18.1 & - & 10.9 & 13.0 & 13.9 \\
\hline Zu hoher Cholesterinwert (Behandlung, täglich) & - & - & 5.0 & 7.4 & - & - & 3.4 & 4.9 \\
\hline Diabetes mellitus (Arztdiagnose) & - & 3.3 & 4.0 & 4.9 & - & 3.3 & 3.6 & 4.2 \\
\hline Diabetes mellitus (Behandlung, täglich) & - & - & - & 2.9 & - & - & - & 2.0 \\
\hline Herzinfarkt (Behandlung, aktuell und gewesen) ${ }^{c}$ & 2.1 & 2.7 & 3.2 & 3.1 & 0.9 & 1.3 & 1.2 & 1.2 \\
\hline Hirnschlag (Behandlung, aktuell und gewesen) ${ }^{c}$ & 0.5 & 0.8 & 1.2 & 1.3 & 0.4 & 0.6 & 0.7 & 0.8 \\
\hline Osteoporose (Behandlung, aktuell und gewesen)c & - & - & - & 0.6 & - & - & - & 4.9 \\
\hline
\end{tabular}

Die Daten stammen aus den Schweizerischen Gesundheitsbefragungen 1992/93, 1997, 2002 und 2007 und sind bevölkerungsgewichtet.

\footnotetext{
a Definitionen der BMI-Klassen (Untergewicht bis Adipositas) sind derTabelle 3.2 zu entnehmen.

b nur 15-bis 24-Jährige

c schriftlicherTeil der Befragung (Fragebogen)

- = keine Angabe
} 
schluss dafür berücksichtigt werden. Junge sowie gut gebildete Personen haben vermehrt keinen Festnetzanschluss mehr, sondern telefonieren über Internet, Kabel oder mobil und sind damit für die $S G B$ unerreichbar (27).

Die Erfassung von Inzidenz und Prävalenz von Krebserkrankungen könnte verbessert werden, wenn alle Kantone über ein Register verfügen würden. Entsprechende Anstrengungen sind im Gange (28). Auch die Daten der Spitäler bieten Verbesserungspotenzial (29). Zurzeit erfassen sie nur stationäre Behandlungen; und Längsschnittanalysen sind fehleranfällig, weil sich die Datenerhebung und -verarbeitung (z.B. Anzahl möglicher Diagnosen, ICD-Klassifikation oder Kodierrichtlinien) über die Zeit unterscheiden $(20 ; 30)$. Auch bei Diagnosedaten sind Längsschnittanalysen zurzeit schwer interpretierbar (18). Mortalitätsdaten haben ebenfalls Einschränkungen, z.B. beim Vergleich mit anderen Ländern: So gibt es kulturelle Unterschiede in der Art und Weise, wie der Arzt den Tod bescheinigt (31). Zudem ist bei älteren Verstorbenen die Bescheinigung häufig ungenau.

Trotz dieser Einschränkungen können die bestehenden Daten genutzt werden, um Erkenntnisse über ernährungsabhängige Krankheiten zu gewinnen, und ihr Potenzial ist nicht ausgeschöpft. Beispielsweise können unterschiedliche Datensätze auf anonyme Art miteinander verknüpft werden, wodurch ein Mehrwert entsteht. So kann eine Verknüpfung von Daten der SNC mit Daten historischer Studien helfen, mit Ernährung verbundene Sterberisiken abzuschätzen (9). Dieses Vorgehen kann allerdings nur teilweise eine national repräsentative Kohorte ersetzen, bei der die gleichen Studienteilnehmer in regelmässigen Abständen befragt und deren Gesundheitsparameter gemessen würden, wie z.B. bei der European Prospective Investigation into Cancer and Nutrition (EPIC; 32;33). Weitere Lücken könnten mit einer fundierteren Verzehrserhebung und einer konsequenteren Erfassung von Krankheiten und Risikofaktoren geschlossen werden.

\subsection{Untergewicht, Übergewicht und Adipositas}

\subsubsection{Häufigkeit}

\section{Zunehmendes öffentliches Interesse}

In den vergangenen zwei Jahrzehnten hat das Wissen der Bevölkerung über Ursachen und Folgen von Unter- und Übergewicht und über die Bedeutung der Ernährung zugenommen (34). Im Zuge des demografischen Wandels und steigender Gesundheitskosten wurden viele für die Problematik sensibilisiert (35). Eine Rolle spielt auch die zunehmende und flächendeckende Erfassung von Untergewicht, Übergewicht und Adipositas bei Kindern und Erwachsenen. Möglicherweise hat auch der stärker werdende Kontrast zwischen Realität und der von den Medien präsentierten Idealvorstellung eines schlanken Körpers den Fokus stärker auf Personen gerichtet, die diesem vermeintlichen Ideal nicht entsprechen. In den folgenden Unterkapiteln wird die Situation bei Kindern und Jugendlichen jeweils am Ende des Absatzes besprochen.

\section{Definition}

Untergewicht, Übergewicht und Adipositas werden weltweit meistens mit den Grenzwerten des Body-Mass-Index (BMI) definiert (36; 
Tabelle 3.2). Der BMI hat den Nachteil, dass er weder die Zusammensetzung der Körpermasse noch die Verteilung der Fettmasse widerspiegelt. Das kann zu einer Fehleinschätzung des individuellen Gesundheitsrisikos führen. So haben Männer mit relativ grosser Muskelmasse und Frauen mit Hüft- und Oberschenkel-betonter Fettverteilung bei gleichem BMI ein niedrigeres Erkrankungs- und Sterberisiko als Männer mit bauchbetonter (viszeraler) Fettverteilung (37). Neben der BMI-Klassifikation wurden für die Definition von Adipositas auch der Körperfettanteil, der Bauchumfang oder dessen Verhältnis zum Hüftumfang vorgeschlagen $(37 ; 38)$. Obwohl diese Definitionen oft besser mit gewissen Risikofaktoren (Kapitel 3.3.2) und Erkrankungs- und Sterberisiken korrelieren (37-39), haben sie sich gegenüber dem BMI nicht durchgesetzt. Das liegt am grösseren Aufwand für die Messungen, an deren höheren Fehleranfälligkeit und daran, dass sich die Daten nicht oder nur schlecht erfragen lassen (40).

Neben der Messgrösse BMI gibt auch dessen Klassifikation Anlass zur Kritik. Die Beurtei-

Tabelle 3.2: Definition von BMI-Klassen bei Erwachsenen nach WHO (36)

\begin{tabular}{|c|c|c|}
\hline Definition & $\begin{array}{c}\text { BMI } \\
\left(\mathrm{kg} / \mathrm{m}^{2}\right)\end{array}$ & $\begin{array}{l}\text { Erkrankungs- / } \\
\text { Sterberisiko }\end{array}$ \\
\hline Untergewicht & $<18.5$ & $\begin{array}{l}\text { nicht erhöht bis } \\
\text { mässig erhöht }\end{array}$ \\
\hline Normalgewicht & $18.5-24.9$ & Referenz \\
\hline Übergewicht & $25.0-29.9$ & $\begin{array}{l}\text { nicht erhöht bis } \\
\text { mässig erhöht }\end{array}$ \\
\hline Adipositas (Klasse I) & $30.0-34.9$ & erhöht \\
\hline Adipositas (Klasse II) & $35.0-39.9$ & deutlich erhöht \\
\hline Adipositas (Klasse III) & $\geq 40.0$ & sehr stark erhöht \\
\hline
\end{tabular}

lung von $\mathrm{BMI} \geq 25 \mathrm{~kg} / \mathrm{m}^{2}$ macht in Ländern wie den USA nur noch wenig Sinn, weil Übergewicht und Adipositas sehr häufig sind und Normalgewichtige inzwischen eine Minderheit darstellen. Vorsicht ist auch angebracht bei der Interpretation der BMI-Klassifizierung hinsichtlich eines damit verbundenen Erkrankungs- und Sterberisikos (Tabelle 3.2), weil sich dieses je nachdem, ob Grösse und Gewicht erfragt oder gemessen wurden, auch zwischen Bevölkerungen, Altersklassen und über die Zeit verändern kann (41).

Für Kinder und Jugendliche (< 18 Jahre) gelten andere Definitionen der BMI-Klassen. Da es anders als bei Erwachsenen nicht nur eine BMIEinteilung gibt, sollte bei Vergleichen auch berücksichtigt werden, welche Definition angewandt wurde $(42 ; 43)$.

\section{Datenquellen}

Die ersten repräsentativen Erhebungen des BMI wurden in der Schweiz in den 1970er Jahren durchgeführt (10). In den 1980er Jahren wurden Grösse und Gewicht im Rahmen der Vorläuferstudie zur $S G B$ erfragt $(12 ; 44)$. Die $S G B$ lieferte erstmals eine national repräsentative Prävalenz von Untergewicht, Übergewicht und Adipositas (7). Gewicht und Grösse wurden auch in der MONICA-Studie (MONItoring of trends and determinants in CArdiovascular disease, 1984-1993) und im Rahmen von Gesundheitsstudien in Lausanne (2003) und im Kanton Genf (seit 1993) gemessen (3-5). Die Aussagekraft dieser Studien ist aufgrund der Erfassungsmethode (siehe unten) oder Einschränkungen bei der Stichprobe (Kapitel 3.2.2) allerdings begrenzt. So werden bei der $S G B$ methodisch bedingt junge, urbane und besser gebildete Personen, die tendenziell seltener übergewichtig sind, wahrscheinlich häufiger 
ausgeschlossen als andere Bevölkerungsgruppen. Dies könnte eher zu einer Überschätzung als zu einer Unterschätzung der effektiven Prävalenz von Übergewicht und Adipositas führen. Die beschränkte Teilnahme aus der Bruttostichprobe (Kapitel 3.2.2) führt hingegen wahrscheinlich zu einer Unterschätzung dieser Prävalenz, weil Verweigerer häufiger übergewichtig oder adipös sind als Teilnahmewillige (45).

Angaben über den BMI liefern auch Daten von Stellungspflichtigen, allerdings wurden bei den letzten Aushebungen nicht mehr alle Männer des entsprechenden Jahrgangs erfasst $(46 ; 47)$.

Neben den hier erwähnten Datenquellen gibt es noch weitere, die Informationen zum Körpergewicht und damit verwandten Themen liefern (48). Deren Resultate sind im Vergleich allerdings weniger aussagekräftig.

Über die Prävalenz von Übergewicht und Adipositas bei Kindern geben Schulbefragungen oder schulärztliche Untersuchungen Auskunft (49-52). Diese Daten schliessen aber nicht alle Kinder der entsprechenden Altersklassen ein, und auch die Erhebungsmethoden sind uneinheitlich, was Quervergleiche erschwert. Die Prävalenz von Übergewicht und Adipositas nach Quelle und Geschlecht ist in Tabelle 3.3 dargestellt.

\section{Unterschiedliche Prävalenz: Befragung versus Messung}

Befragungen wie die $S G B$ unterschätzen den effektiven (gemessenen) BMI, weil die meisten Menschen ihr Körpergewicht unterschätzen und ihre Körpergrösse überschätzen (53). Diese Fehleinschätzung kann entstehen, weil Menschen mit dem Alter an Grösse verlieren und meistens an Gewicht zunehmen (53). In der Schweiz hat diese Abweichung einen deutlichen Einfluss auf die Adipositasprävalenz: Bei Studien mit Messun- gen ist sie durchwegs höher als die der SGB (45). Aus diesem Unterschied ergibt sich, dass die Adipositasprävalenz in der Schweiz zwischen 13 und $16 \%$ liegen könnte - anstelle der ca. $8 \%$, wenn die Teilnehmer der SGBO7 gemessen und gewogen statt befragt würden. Die auf Studien mit gemessenen und erfragten Daten basierende Adipositasprävalenz lässt sich angleichen, wenn für die Definition von Adipositas Grenzwerte für den BMI von $28.2 \mathrm{~kg} / \mathrm{m}^{2}$ bei Frauen und $28.6 \mathrm{~kg} / \mathrm{m}^{2}$ bei Männern statt $30 \mathrm{~kg} / \mathrm{m}^{2}$ herangezogen werden (54). Bei der Prävalenz von Übergewicht sind die Diskrepanzen zwischen Erfassung von Grösse und Gewicht mittels Befragung und Messung wahrscheinlich deutlich geringer (45). Auch scheint die Fehleinschätzung von Grösse und Gewicht über die Zeit konstant zu bleiben, weshalb anzunehmen ist, dass Zeit-Trends mit Befragungen korrekt abgebildet werden $(45 ; 54)$. Offen bleibt, inwiefern sich die Fehleinschätzung innerhalb der Schweiz, beispielsweise zwischen den Sprachregionen, unterscheidet $(55 ; 56)$. Wahrscheinlich führt auch die Befragung von Kindern und Jugendlichen zu einer Fehleinschätzung der Prävalenz von Übergewicht und Adipositas. Resultate aus Studien mit Messungen und Befragungen sollten deshalb nicht ohne Vorbehalt miteinander verglichen werden (49-51).

\section{Prävalenz und Zeit-Trends}

In den vergangenen drei Jahrzehnten stieg die Prävalenz von Übergewicht und Adipositas bei Erwachsenen kontinuierlich, während Untergewicht seltener wurde (7;57; Tabellen 3.1 und 3.5). Allerdings deuten die SGB darauf hin, dass sich dieser Anstieg seit 1997 abgeflacht haben könnte; diese Annahme drängt sich besonders bei Frauen auf (26). Im Kanton Zürich nahm die Prävalenz von $\mathrm{BMI} \geq 25 \mathrm{~kg} / \mathrm{m}^{2}$ bei den Frauen seit 
Tabelle 3.3: Prävalenz von Übergewicht und Adipositas bei Erwachsenen, Kindern und Jugendlichen in der Schweiz: Neuere Datenquellen (Auswahl, seit 2003)

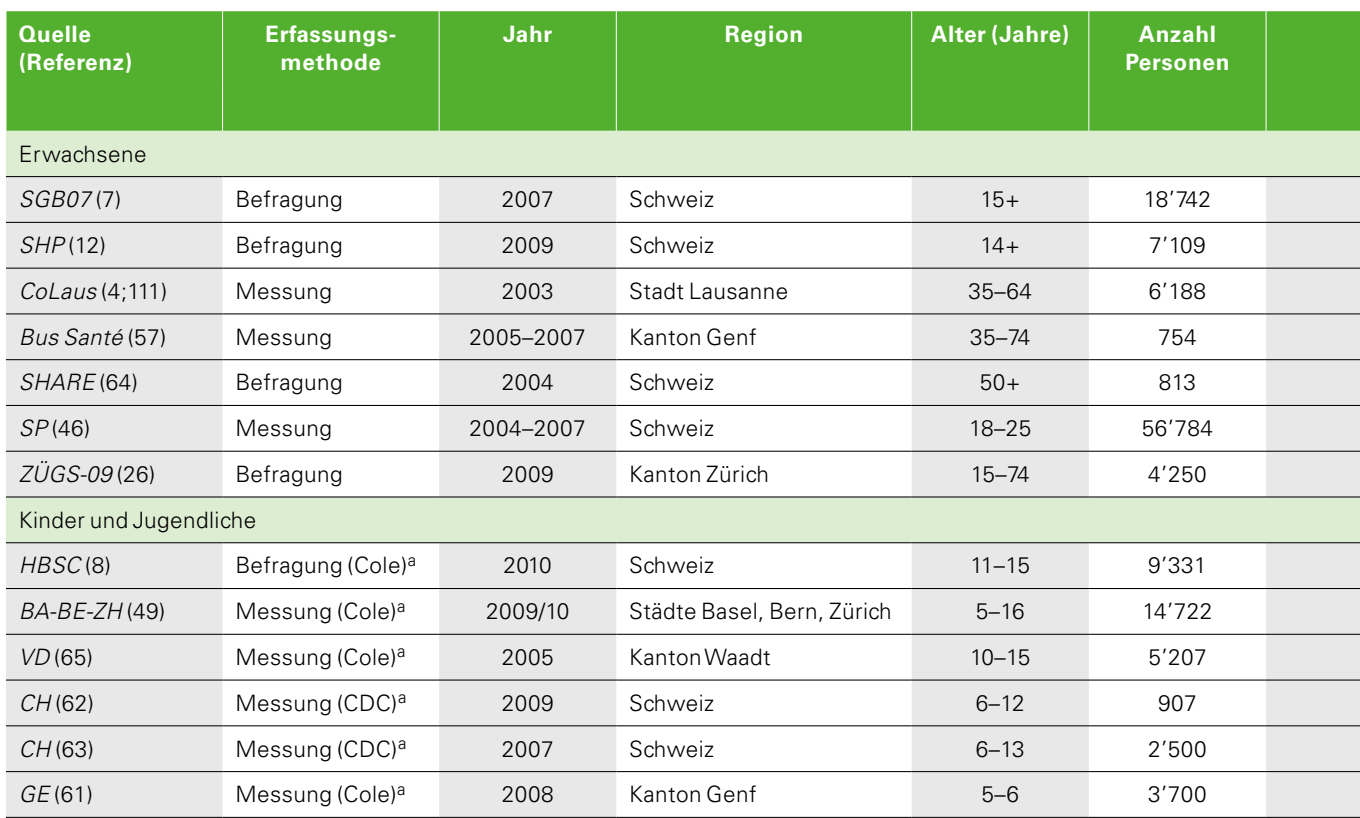

a Cole: Definition von Übergewicht / Adipositas nach Cole et al. (42); CDC: Definition von Übergewicht / Adipositas nach

Centers for Disease Control and Prevention (43)

- = keine Angabe

SGB07: Schweizerische Gesundheitsbefragung 2007

SHP: $\quad$ Schweizer Haushalt-Panel

CoLaus: Cohorte Lausannoise

SHARE: The Study of Health, Ageing and Retirement in Europe

SP: $\quad$ Studie mit Stellungspflichtigen

ZÜGS-09: Zürcher Übergewichtsstudie 2009

1997 ab, während sie bei den Männern stagnierte (26; Abbildung 3.1). Messungen der Studie Bus Santé zeigen hingegen einen kontinuierlichen Anstieg des mittleren BMI (Tabelle 3.5).

Die Häufigkeit von untergewichtigen Männern blieb relativ konstant; diejenige von untergewichtigen Frauen nahm leicht, aber kontinuierlich ab (57; Tabelle 3.1). Studien mit
HBSC: Health Behaviour in School-aged Children

BA-BE-ZH: Monitoring der schulärztlichen Dienste der Städte Basel, Bern und Zürich

VD: $\quad$ Studie aus dem Kanton Waadt

$\mathrm{CH}$ : $\quad$ Studie mit national repräsentativer Stichprobe

GE: $\quad$ Studie aus dem Kanton Genf

Messungen ergeben eine niedrigere Prävalenz für Untergewicht als Befragungen, z.B. CoLausStudie (2003): $0.7 \%$ bei Männern und $2.6 \%$ bei Frauen (58). Allerdings unterschied sich bei dieser Studie das Einschlussalter (35-75 Jahre) von jenem bei der $S G B$ ( $\geq 15$ Jahre).

National repräsentative Studien mit Kindern, die in einem vergleichbaren Zeitraum stattfan- 
Aufgrund zweier kleinerer Studien aus der Stadt Zürich aus den Jahren 1960/65 und 1980/90 kann angenommen werden, dass dieser Anstieg über einen grösseren Zeitraum hin\begin{tabular}{l|l|l|l} 
Männlich & Weiblich & Männlich & Weiblich
\end{tabular}

\begin{tabular}{|c|c|c|c|}
\hline 37.6 & 20.8 & 8.7 & 7.8 \\
\hline 36.0 & 21.0 & 9.5 & 8.2 \\
\hline 45.7 & 28.4 & 17.1 & 14.5 \\
\hline 48.9 & 22.9 & 12.9 & 14.1 \\
\hline
\end{tabular}

37.9

16.8

33.8

11.3

6.5

1.6

0.6

19.3

15.0

12.5

11.5

12.4

$12.4 \quad 1.8$

4.9

$\begin{array}{ll}8 & 1.7\end{array}$

$6.2 \quad 4.2$

5.3

3.3

9.8

10.0

2.8 den wie die Studien mit Erwachsenen, liegen keine vor. Die vorhandenen Studien ergeben teilweise widersprüchliche Resultate. Gemäss Messungen bei Schülern der 4. und 8. Klasse aus der Stadt Zürich wurden Übergewicht und Adipositas innerhalb des Beobachtungszeitraumes häufiger (52; Abbildung 3.2). weg stattfand $(59 ; 60)$. Auch die HBSC-Studie weist bei 13-jährigen Knaben auf eine höhere Übergewichtsprävalenz im 2006 verglichen mit 2002 hin (51), zwischen 2006 und 2010 stagnierte die Prävalenz hingegen (8). Auch Erhebungen aus den Städten Basel, Bern und Zürich (Schuljahre 2005/06 bis 2009/10) sowie Untersuchungen bei 5 - bis 6-Jährigen aus dem Kanton Genf (2003-2008) deuten auf eine Stabilisierung der Übergewichts- und Adipositasprävalenz hin, wobei Adipositas tendenziell rückläufig ist $(49 ; 61)$. Zwei national repräsentative Studien bei 6 - bis 13-Jährigen zeigen eine Stabilisierung (2007-2009; 62) oder eine Abnahme (2002-2007; 63) der Übergewichts- und Adipositasprävalenz. Laut HBSC-Studie nehmen Übergewicht und Adipositas tendenziell auch bei 11- und 13-jährigen Mädchen leicht ab oder bleiben stabil (51).

Zu Untergewicht bei Kindern wurden nur wenige Resultate publiziert. Eine national repräsentative Studie von 2007 weist eine Prävalenz von $3.5 \%$ (Knaben) und 2.6\% (Mädchen) aus; diese Resultate unterscheiden sich nur wenig von solchen früherer Studien (63). Eine Analyse von Perzentilverteilungen aus Messungen der schulärztlichen Dienste weist auf ein geringes Vorkommen von Untergewicht bei Kindern hin (50).

\section{Regionale Unterschiede}

Gemäss SGB07 sind Unterschiede in der Prävalenz von Übergewicht und Adipositas zwischen den Sprachregionen vernachlässigbar. Hingegen scheint es Stadt-Land-Unterschiede zu geben: Im urbanen Kanton Zürich gibt es weniger 


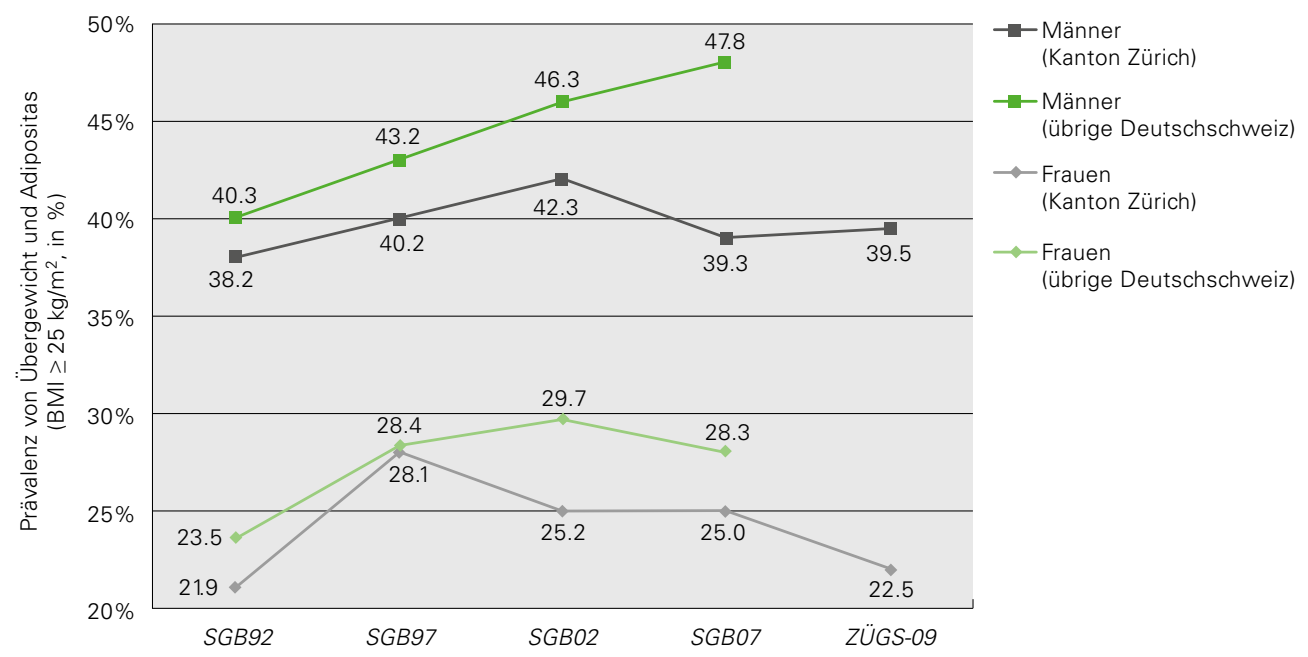

Die Daten sind bevölkerungsgewichtet und altersstandardisiert ( $N=41^{\prime} 628$, Alter: 15-74 Jahre).

SGB92, SGB97, SGB02, SGB07: Schweizerische Gesundheitsbefragung 1992, 1997, 2002, 2007 ZÜGS-09: Zürcher Übergewichtsstudie 2009

Abbildung 3.1: Prävalenz von Übergewicht und Adipositas kombiniert (BMl $\left.\geq 25 \mathrm{~kg} / \mathrm{m}^{2}\right) \mathrm{im}$ Kanton Zürich und in der übrigen Deutschschweiz, nach Geschlecht, 1992-2009 (7;26)

übergewichtige und adipöse Erwachsene als in der restlichen Deutschschweiz (26; Abbildung 3.1). Besonders niedrig ist die Prävalenz in der Stadt Zürich (26). Wahrscheinlich gibt es auch bei älteren Menschen solche Stadt-LandUnterschiede (64). In der Stadt Bern ist der Anteil an übergewichtigen und adipösen Kindern geringer als in den Städten Basel und Zürich, in denen die Zahlen ähnlich sind (49).

Bezüglich Stadt-Land-Unterschieden bei Kindern gibt es widersprüchliche Resultate. Die Prävalenz von Übergewicht und Adipositas ist in den ländlichen Kantonen Graubünden, Wallis und Jura niedriger als in Basel-Stadt und in der
Stadt Zürich (50). In den Kantonen Waadt und Genf ist die Prävalenz in städtischen Gebieten höher als in der Agglomeration $(50 ; 65)$. Laut zwei national repräsentativen Studien ist bei 6- bis 13-jährigen Kindern die Prävalenz von Übergewicht und Adipositas in grösseren Gemeinden (über 100'000 Einwohner) höher als in kleineren und im Süden der Schweiz höher als in den übrigen Regionen $(62 ; 63)$. Eine andere Studie fand eine höhere Adipositasprävalenz bei Kindern in der Westschweiz als in der Deutschschweiz (66). 


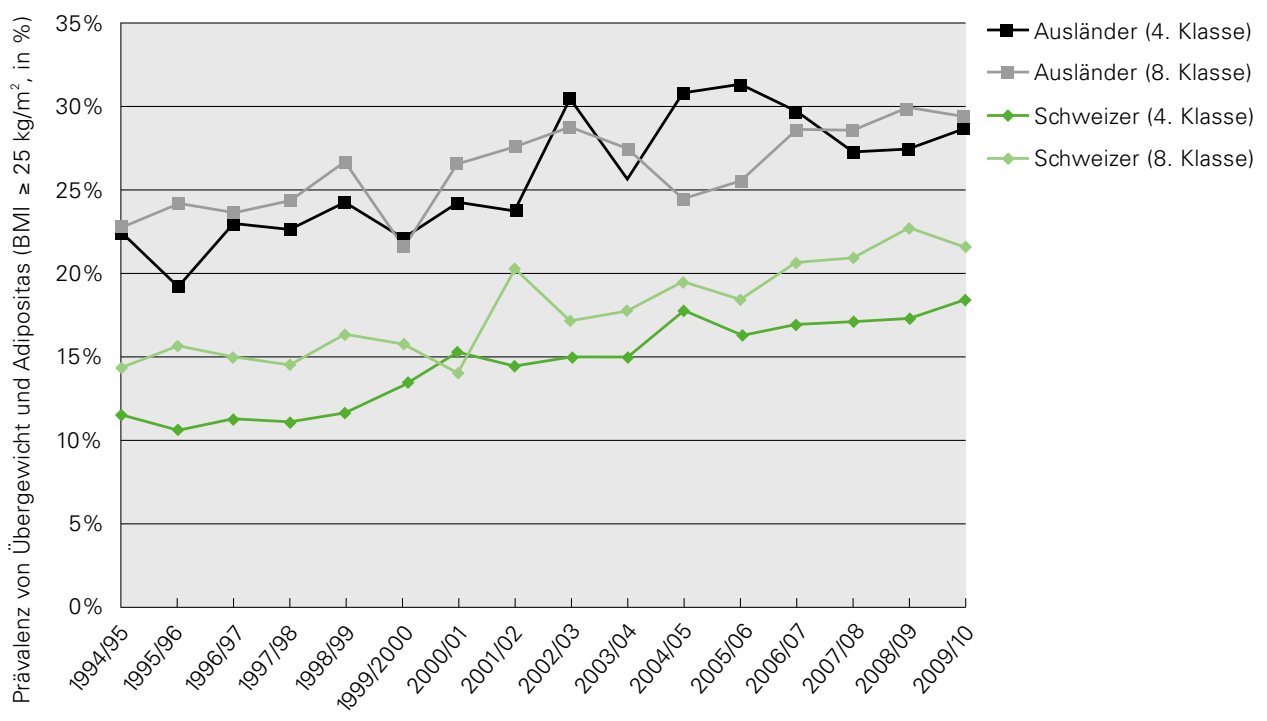

Die Daten stammen aus schulärztlichen Untersuchungen (Messungen).

Die Fallzahlen pro Jahr und Klasse betragen jeweils zwischen ca. 1'400 und ca. 2'400 Personen.

Abbildung 3.2: Prävalenz von Übergewicht und Adipositas kombiniert (BMI $\left.\geq 25 \mathrm{~kg} / \mathrm{m}^{2}\right)$ bei Schülern der 4. und 8. Klasse nach Nationalität (Schweizer oder Ausländer) in der Stadt Zürich, Schuljahre 1994/95 bis 2009/10 (52)

\section{Unterschiede nach Geschlecht}

Die Prävalenz von Übergewicht bei Männern unterscheidet sich deutlich von derjenigen bei Frauen (Tabelle 3.3). Hingegen ist die Prävalenz von Adipositas vergleichbar.

Bei übergewichtigen Kindern und Jugendlichen sind die Unterschiede zwischen Mädchen und Knaben gering. Tendenziell sind Knaben der Oberstufe etwas häufiger betroffen (50). Adipositas ist generell etwas häufiger bei Knaben als bei Mädchen (Tabelle 3.3).

\section{Internationaler Vergleich}

Im europäischen Vergleich hat die Schweiz zusammen mit Rumänien die niedrigste Adipositasprävalenz bei Erwachsenen (67-69). Sie ist niedriger als die von Italien (9.9\%), Frankreich $(11.2 \%)$ und Deutschland $(13.6 \%)$ und deutlich niedriger als die von Grossbritannien (24.5\%). Das gute Abschneiden der Schweiz liegt aber auch am Grenzwert von Adipositas (BMI $\geq 30 \mathrm{~kg} / \mathrm{m}^{2}$ ). Würde Übergewicht plus Adipositas (BMI $\geq 25 \mathrm{~kg} / \mathrm{m}^{2}$ ) oder der mittlere BMI als Vergleichskriterium herangezogen werden, läge die Schweiz näher am Mittelfeld, da es viele Menschen mit einem BMI knapp unter $30 \mathrm{~kg} / \mathrm{m}^{2}$ gibt (68). 
Aufgrund erheblicher methodischer Unterschiede sind internationale Vergleiche von Studien mit Kindern schwierig. Wahrscheinlich liegt die Schweiz hier zwischen dem unteren Drittel und dem Durchschnitt mit einer Prävalenz von Übergewicht und Adipositas, die vergleichbar ist mit jener von Frankreich und den Niederlanden, aber niedriger ist als jene von Grossbritannien und den meisten südeuropäischen Ländern $(67 ; 68 ; 70)$. Bei der Zunahme von Adipositas zwischen 2001/02 und 2005/06 liegt die Schweiz knapp unter dem Durchschnitt (68).

\subsubsection{Risiko- und Schutzfaktoren}

Übergewicht und Adipositas sind das Resultat einer positiven Energiebilanz und damit von Faktoren, die diese beeinflussen können. Das können gewisse Lebensmittel und Verhaltensweisen sein, aber auch kulturelle und soziale Rahmenbedingungen. Unabhängig davon sind in der Schweiz männliches Geschlecht und das Alter Risikofaktoren. Mögliche beeinflussbare Risiko- und Schutzfaktoren werden im Folgenden diskutiert.

\section{Energieeinnahme und Energieverbrauch}

Eine zunehmende Gesamtenergieeinnahme pro Kopf scheint in der Schweiz kein bedeutender Risikofaktor zu sein. Laut Verbrauchsdaten (Kapitel 2.4.3) und der Studie Bus Santé (71) ist der Energieverbrauch über die letzten drei Jahrzehnte nicht gestiegen. Eine mögliche Erklärung für die Zunahme von Übergewicht und Adipositas könnte die Abnahme des Energieverbrauchs sein. Hier gibt es widersprüchliche Ergebnisse. In der Studie Bus Santé blieb der Energieverbrauch seit 1997 konstant (71). An- dere Studien deuten auf ein relativ stabiles $\mathrm{Ni}$ veau von körperlicher Aktivität in der Schweizer Bevölkerung hin (72). Laut SGB hat seit 1997 die Anzahl derjenigen, die in der Freizeit ins Schwitzen geraten, Sport treiben, die tägliche Wegstrecke zu Fuss und mit dem Velo zurücklegen, leicht zugenommen (7). In der SGBO7 sind hingegen sowohl Übergewichtige als auch Adipöse häufiger teilinaktiv oder inaktiv als Normalgewichtige (73). Entsprechend dem Mikrozensus zum Verkehrsverhalten haben die zu Fuss zurückgelegten Kilometer seit 1984 markant und kontinuierlich zugenommen (74).

Die HBSC-Studie zeigt, dass sich das Bewegungsverhalten von Kindern und Jugendlichen zwischen 1994 und 2006 nicht grundlegend verändert hat (75). Drei Studien können aber unabhängig voneinander einen starken Zusammenhang zwischen Fernseh- und Videospielkonsum und Adipositas bei Jugendlichen nachweisen $(65 ; 76 ; 77)$.

\section{Lebensmittel und Nährstoffzusammensetzung}

Betrachtet man den Lebensmittelverbrauch der vergangenen 30 Jahre, dann sind der zunehmende Verbrauch an Gemüse und Fisch sowie der abnehmende Verbrauch an Fleisch wünschenswerte Entwicklungen, nicht dagegen der zunehmende Verbrauch an Zucker und der abnehmende Verbrauch an Früchten. Der Verbrauch an Fetten und Ölen hat seit 1987 zugenommen, liegt 2007/08 aber auf ähnlichem Niveau wie 1979/80 (Abbildung 2.3). Positiv zu bewerten ist der Alkoholkonsum, der in den letzten Jahrzehnten tendenziell abgenommen hat. Laut SGBO7 achten Adipöse im Vergleich zu Normalgewichtigen weniger häufig auf die Ernährung, trinken seltener Alkohol, konsumieren aber mehr Fleisch und Wurstwaren (73). 
Zwischen 1994 und 2006 hat der Früchtekonsum bei Kindern zugenommen und der Konsum von Fast Food abgenommen (75). Bei städtischen Schulkindern (6-14 Jahre) ist erhöhter Fleischkonsum mit Adipositas assoziiert (76). Bei Schulkindern aus dem Kanton Waadt werden folgende - teilweise unerwartete - Zusammenhänge mit Übergewicht gefunden: ein positiver mit Früchtekonsum, keiner mit Gemüsekonsum und ein inverser Zusammenhang mit dem Konsum von Snacks und Süssgetränken. Diese Studie hatte aber verschiedene Einschränkungen, und die Resultate sollten entsprechend vorsichtig interpretiert werden (65). Bei Kindern mit Migrationshintergrund besteht eine Ernährungsweise, die Adipositas begünstigt $(66 ; 78-80)$.

\section{Essverhalten}

Bei Kindern und Erwachsenen sind Übergewicht und Adipositas mit ungünstigem Essverhalten und / oder Essstörungen (Kapitel 3.9) assoziiert. Die SGB weisen aber nicht darauf hin, dass eine grundlegende Verschlechterung des Essverhaltens stattgefunden hat (74; Kapitel 2.6). Auch Fasten und die fehlende Einnahme regelmässiger Hauptmahlzeiten haben gemäss $S G B$ eher ab- als zugenommen. Das Essverhalten von Kindern wird wahrscheinlich durch Werbung mitbeeinflusst. Problematisch ist, dass die an Kinder gerichtete Werbung in der Schweiz überwiegend energiedichte Lebensmittel bewirbt (81).

\section{Andere Risiko- und Schutzfaktoren}

Ein niedriges Bildungsniveau ist einer der wichtigsten Risikofaktoren für Übergewicht und vor allem für Adipositas (82-85). Das gilt besonders für Frauen. Bei Frauen mit Grundschulab- schluss ist Adipositas dreimal häufiger als bei Akademikerinnen $(83 ; 85)$. Etwas geringere Unterschiede gibt es nach Einkommen und Berufsklasse. Diese ungleiche Verteilung von Adipositas nach Bildungsniveau, Einkommen und Berufsklasse ist seit 1992 unverändert (85).

Diese drei Faktoren sind mitverantwortlich dafür, dass manche Kinder und Erwachsene mit Migrationshintergrund ein erhöhtes Risiko für Übergewicht und Adipositas haben $(7 ; 49 ; 52$; $65 ; 66 ; 78-80 ; 86-88)$. Betroffen sind vor allem Migranten aus dem Balkan und aus Süd- und Osteuropa (87;88). Tatsächlich sind Migranten aus diesen Regionen häufig schlechter gebildet als Schweizer (80).

Analysen von Personen in der gleichen Jahrgangsgruppe lassen vermuten, dass Menschen, die zwischen 1960 und 1979 geboren wurden, ein höheres Adipositasrisiko haben als solche, die früher zur Welt kamen, unabhängig vom Alter (Kohorteneffekt; 89). Untergewicht kommt vermehrt bei jungen Frauen und älteren Menschen vor, bei Letzteren häufig in Folge von Mangelernährung (Kapitel 3.10.1).

\subsubsection{Bedeutung}

Wie in Abbildung 3.3 ersichtlich, scheint Übergewicht in der Schweiz nicht mit einem erhöhten Sterberisiko verbunden zu sein - weder für die Todesursache Herz-Kreislauf-Krankheit noch für Krebs oder andere Ursachen (41). Ab einem BMI von $30 \mathrm{~kg} / \mathrm{m}^{2}$ steigt das Sterberisiko hingegen kontinuierlich an. Verglichen mit Nichtrauchern mit einem BMI zwischen 20 und $22.4 \mathrm{~kg} / \mathrm{m}^{2}$ haben adipöse Nichtraucher ein etwa zweifach und adipöse Raucher ein 3- bis 4.5-fach höheres Sterberisiko (41). 


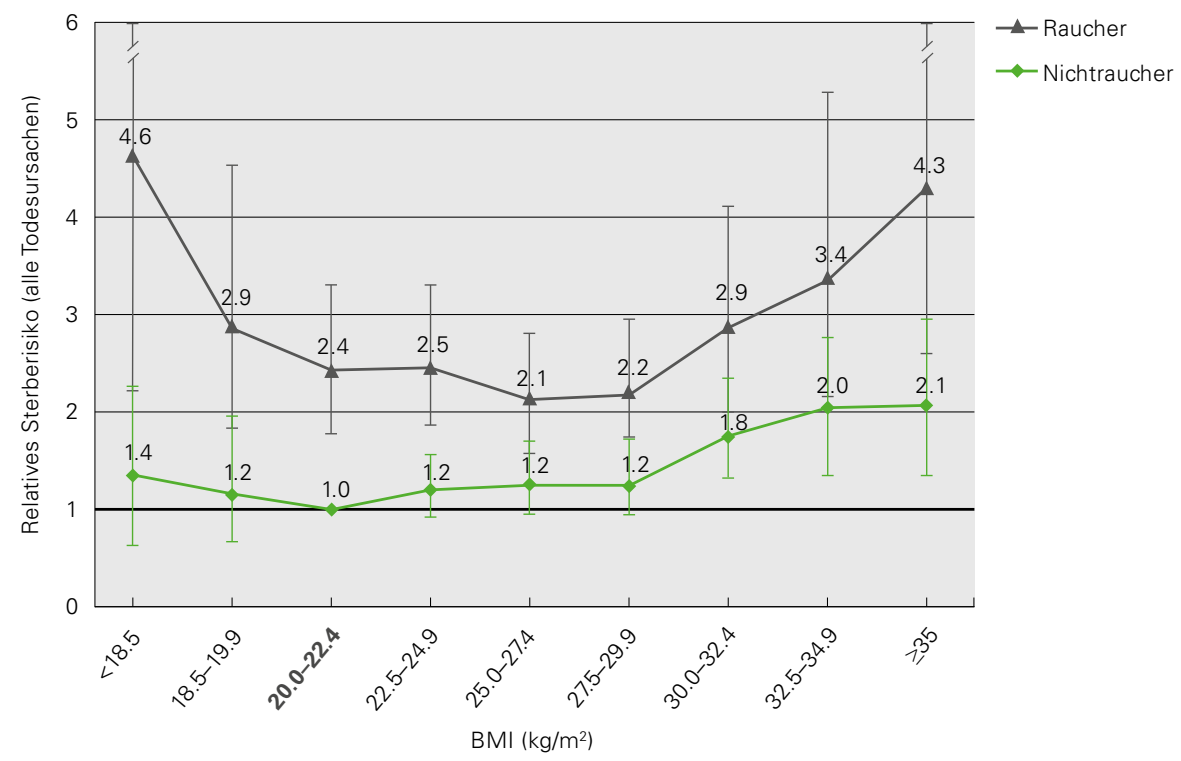

Die Daten stammen aus der MONICA-Studie (Schweizer Daten, Männer und Frauen zusammen, 1983-1992).

Es sind die relativen Risiken und die dazugehörigen $95 \% \mathrm{Cl}$ angegeben. Die Referenzklasse mit einem relativen Risiko von 1 sind Nichtraucher mit BMI 20.0-22.4 (fett gedruckt); diese Klasse hat im Vergleich die niedrigste Mortalität (alleTodesursachen).

Abbildung 3.3: Relatives Sterberisiko nach BMI-Klasse bei MONICA-Teilnehmern nach Raucherstatus (41)

Übergewicht und Adipositas verursachen verschiedene ernährungsabhängige Krankheiten (Kapitel 3.4.2, 3.5.2 und 3.6.2) und erhebliche Gesundheitskosten (35; Kapitel 4.2). Im Jahr 2006 waren es gesamtschweizerisch geschätzte CHF 5.8 Mia. Wichtig für die Abschätzung zukünftiger Kosten ist die Entwicklung der Prävalenz von Übergewicht und Adipositas. Hier gibt es widersprüchliche Indizien. Zahlen aus dem Kanton Zürich deuten bei Frauen auf eine Trendumkehr hin, und auch bei Kindern gibt es zumindest Hinweise auf eine Stagnation $(8 ; 26 ; 49 ; 51 ; 61-63)$. Projektionen der zukünftigen Entwicklung gehen in die gleiche Richtung. Demnach dürfte die Prävalenz von Übergewicht und Adipositas bei Frauen seit 2010 nicht weiter ansteigen, bei Männern wird ein Plateau 2022 erreicht sein (90). Analysen von Geburtskohorten lassen aber auch einen weiteren Anstieg bei beiden Geschlechtern denkbar erscheinen (89). Darauf deutet die Schätzung hin, dass Übergewicht bei Kindern bis 2022 weiter zunehmen 
wird (59). Es ist also vorstellbar, dass die Abnahme oder Abflachung der Prävalenz zeitlich begrenzt ist und den seit mindestens 30 Jahren vorherrschenden Trend zur Zunahme nur kurz unterbricht. Mehr darüber wird man im Rahmen der SGB12 erfahren.

Wie bei anderen Ländern mit hohem Ausländeranteil ist es denkbar, dass auch in der Schweiz Migrationsströme die Prävalenz von Übergewicht und Adipositas beeinflussen. So könnte die nationale Prävalenz sinken, wenn statt wenig qualifizierter Personen vermehrt hochgebildete Arbeitskräfte in die Schweiz migrieren. Ein hohes Bildungsniveau ist grundsätzlich eine der wichtigsten Voraussetzungen für ein gesundes Körpergewicht (82). Wichtig ist aber auch eine Umwelt, die den Menschen weniger dazu verlockt, überschüssige Energie aufzunehmen, und inm vor allem mehr Energie abverlangt (91).

Lebensstilinterventionen zur Verringerung der Adipositasprävalenz könnten nicht nur wirksam, sondern auch kosteneffizient sein $(92 ; 93)$. Beispielsweise kann gezielte Bewegungsförderung in der Schule die körperliche Aktivität steigern und die Adipositasprävalenz senken (94). Es ist ungleich schwieriger, die Zahl Adipöser zu senken, als zu verhindern, dass Menschen adipös werden. Adipositasprävention muss sich also an alle richten - auch an Normalgewichtige. Welche Empfehlungen und Massnahmen dazu sinnvoll und nötig sind, wird in den Kapiteln 4.8.2 und 4.9 erörtert.

Die Prävalenz von Untergewicht hat seit 1992 abgenommen (Tabelle 3.1). Untergewicht kann mit einem erhöhten Sterberisiko einhergehen. Meist ist aber nicht das Untergewicht selbst dafür verantwortlich, sondern Mangelernährung oder eine zugrunde liegende (oft unerkannte) Krankheit (Kapitel 3.9 und 3.10). In der
Schweiz haben gesunde untergewichtige Nichtraucher im Vergleich zu normalgewichtigen Nichtrauchern wahrscheinlich kein höheres $\mathrm{Ge}$ samtsterberisiko (41; Abbildung 3.3).

\subsection{Herz-Kreislauf-Krankheiten}

\subsubsection{Häufigkeit}

Am häufigsten betreffen Herz-Kreislauf-Krankheiten die Gefässe des Herzens und des Gehirns. Landesweit erleiden jährlich 30'000 Menschen ein akutes koronares Ereignis und 12'500 einen Hirnschlag (82). Genauere, national repräsentative Zahlen zur Morbidität sind in der Schweiz nicht verfügbar. Einige Informationen dazu liefern die $S G B$, die Analyse von Spital- und Todesursachenstatistiken sowie die Verordnungshäufigkeit von Medikamenten in Arztpraxen (18;22;95; Tabelle 3.1).

Herz-Kreislauf-Krankheiten sind aber nach wie vor die häufigste Todesursache in der Schweiz. Diese Erkrankungen sind hierzulande deshalb so häufig, weil die Menschen immer älter werden. Wird dies durch Altersstandardisierung berücksichtigt, hat die Herz-KreislaufMortalität in den vergangenen 30 Jahren abgenommen (Tabelle 3.4). Dies war vor allem bei Männern ausgeprägt und ist besonders auf die Abnahme der Hirngefässkrankheiten (z.B. Hirnschlag) zurückzuführen (96-100). Über die Gründe dafür kann nur spekuliert werden: Wahrscheinlich trugen Fortschritte bei der Früherkennung und Behandlung von Risikofaktoren und bei Diagnose und Therapie der Krankheiten dazu bei. Bezüglich Herz-Kreislauf-Mortalität steht die Schweiz international gut da (96-98;100). 
Tabelle 3.4: Altersstandardisierte Mortalitätsraten ernährungsabhängiger Krankheiten pro 100'000 Männer oder Frauen in der Schweiz, 1980-2008 (23)

\begin{tabular}{|c|c|c|c|c|c|c|c|c|}
\hline & \multicolumn{4}{|c|}{$\begin{array}{l}\text { Mortalitätsrate bei Männern } \\
\left.\text { (pro } 100^{\prime} 000\right)\end{array}$} & \multicolumn{4}{|c|}{$\begin{array}{l}\text { Mortalitätsrate bei Frauen } \\
\left.\text { (pro } 100^{\prime} 000\right)\end{array}$} \\
\hline & 1980 & 1990 & 2000 & 2008 & 1980 & 1990 & 2000 & 2008 \\
\hline Alle Ursachen & $1^{\prime} 070$ & 944 & 750 & 596 & 634 & 535 & 457 & 386 \\
\hline Herz-Kreislauf-Krankheiten & 485 & 377 & 264 & 191 & 308 & 225 & 167 & 126 \\
\hline Koronare Herzkrankheit & 199 & 176 & 129 & 89 & 79 & 77 & 65 & 43 \\
\hline Hirnschlag & 97 & 65 & 42 & 29 & 81 & 49 & 34 & 26 \\
\hline Diabetes mellitus & 17 & 15 & 15 & 13 & 17 & 13 & 12 & 10 \\
\hline Alkoholische Leberzirrhose & 23 & 15 & 8 & 8 & 6 & 6 & 3 & 3 \\
\hline
\end{tabular}

Mortalität: Anzahı Sterbefälle

Die Mortalität von Herz-Kreislauf-Krankheiten ist bei Männern immer noch höher als bei Frauen, der Unterschied hat sich aber verringert. Aufgrund koronarer Herzkrankheiten werden immer noch mehr als doppelt so viele Männer wie Frauen ins Spital eingewiesen $(22 ; 101)$. Unterschiede gibt es auch zwischen den Landesteilen: Tessiner und Westschweizer haben trotz ähnlicher Herz-Kreislauf-Risiken eine niedrigere Herz-Kreislauf-Mortalität als Deutschschweizer (31). Auch der sozio-ökonomische Status, z.B. gemessen an der Bildung, spielt eine Rolle (102). Entsprechende Unterschiede sind bei Frauen besonders ausgeprägt $(83 ; 85 ; 103)$.

\subsubsection{Risiko- und Schutzfaktoren}

Eine ungesunde Ernährung führt meist durch eine Verschlechterung von Risikofaktoren zu Herz-Kreislauf-Krankheiten. Zu diesen so genannten intermediären Risikofaktoren gehören
Adipositas, Diabetes mellitus Typ 2, Bluthochdruck und Fettstoffwechselstörungen. Die erste Messung von Herz-Kreislauf-Risikofaktoren in der Schweiz erfolgte im Jahr 1977 in den Städten Aarau, Solothurn, Nyon, Vevey und Lugano (10). Bei den darauf folgenden Studien fehlt eine Beteiligung der Deutschschweiz: $M O$ NICA (1984-1993, Kantone Fribourg, Waadt, Tessin; 3), CoLaus-Studie (2003/04, Stadt Lausanne; 4) und Studie Bus Santé (seit 1993, Kanton Genf; 5;57). Umfassende Informationen über Herz-Kreislauf-Risikofaktoren fehlen hingegen, weil bis anhin keine national repräsentativen Messungen - beispielsweise von Blutdruck, Blutzucker und -cholesterin - durchgeführt wurden (Kapitel 3.2.2). Seit 2011 gibt es erstmals national repräsentative Zahlen über die Prävalenz von Bluthochdruck aufgrund von Messungen. Die Resultate lagen zum Zeitpunkt der Texterstellung noch nicht vor.

Die verfügbaren Schweizer Datenquellen zu den Risikofaktoren von Herz-Kreislauf-Krankheiten würden eher eine Zu- als eine Abnahme der 
Herz-Kreislauf-Mortalität vermuten lassen. Beispielsweise die Zunahme von Übergewicht und Adipositas (Kapitel 3.3.1 sowie Tabellen 3.1 und 3.5) sowie von Diabetes mellitus (Kapitel 3.6.1). Zahlen der Studie Bus Santé lassen vermuten, dass die Prävalenz von Bluthochdruck aufgrund zunehmender Behandlung abgenommen hat, während die Prävalenz von hohem Gesamtcholesterin trotz vermehrter Behandlung zwischen 1993 und 2007 zugenommen hat (57;104; Tabelle 3.5). Resultate der SGB gehen in eine ähnliche Richtung (95; Tabelle 3.1). Bei Stellungspflichtigen wurden bei $1.4 \%$ Cholesterinwerte über $6.2 \mathrm{mmol} / \mathrm{L}$ und bei $9.1 \%$ Werte über $5.2 \mathrm{mmol} / \mathrm{L}$ gefunden (105). Eine Messung bei über 5'000 Schulkindern im Kanton Waadt ergab, dass $2.2 \%$ Bluthochdruck hatten (106).

\section{Übergewicht und Adipositas}

Übergewicht und Adipositas können das Risiko für Herz-Kreislauf-Krankheiten erhöhen, entweder direkt oder über andere Herz-Kreislauf-Risikofaktoren (37;53;106-109). So haben 37\% der untersuchten Schulkinder mit Übergewicht und Adipositas auch Bluthochdruck (106) und 35\% eine Fettstoffwechselstörung (107). Auch bei über 50-Jährigen sind Fettstoffwechselstörungen wesentlich häufiger bei Übergewichtigen und Adipösen als bei Normalgewichtigen (110).

Im Vergleich zu Normalgewichtigen haben adipöse Männer und Frauen ein etwa doppelt so hohes Risiko, an einer Herz-Kreislauf-Krankheit zu sterben. Das liegt teilweise daran, dass Adipöse einen höheren Blutdruck oder schlechtere Blutfettwerte haben als Normalgewichtige. Aber selbst wenn diese intermediären Risikofaktoren berücksichtigt werden, haben Adipöse immer noch ein um 50\% erhöhtes Herz-Kreislauf-Sterberisiko (41). Auch vom Lebensstil Adi- pöser kann ein Risiko ausgehen, indem sie sich weniger bewegen und etwas weniger gesund ernähren als Normalgewichtige (41; Kapitel 3.3.2). Je nachdem, ob Messungen oder Selbstangaben zur Bestimmung der Adipositasprävalenz in der Schweiz herangezogen werden, gehen 9-14\% aller Herz-Kreislauf-Todesfälle auf das Konto der Adipositas. Übergewichtige scheinen gegenüber Normalgewichtigen kein erhöhtes Herz-Kreislauf-Sterberisiko zu haben (41). Je nach Studie oder beobachteter Population kann sich aber ein unterschiedlicher Zusammenhang zwischen BMI und Sterberisiko ergeben (41).

\section{Metabolisches Syndrom}

Das gleichzeitige Auftreten von Adipositas (v.a. stammbetonte) mit Zucker- und Fettstoffwechselstörungen, Bluthochdruck, schlechten Leber- und Insulinwerten und Hyperurikämie wird als «metabolisches Syndrom» bezeichnet (104; 107;111-113). Die Prävalenz des metabolischen Syndroms liegt bei Erwachsenen (20-79 Jahre) in der Schweiz schätzungsweise bei $15 \%$ : bei $18 \%$ bei Männern und bei $10 \%$ bei Frauen (114). In der CoLaus-Studie (35-75 Jahre) ist die Prävalenz höher, nämlich zwischen 20 und $25 \%$ (115).

Je nachdem, welche Definitionen für Adipositas (Kapitel 3.3.1) und das metabolische Syndrom herangezogen werden, korrelieren die beiden Krankheiten unterschiedlich stark miteinander $(39 ; 114)$. Allerdings muss Adipositas nicht zwingend mit Stoffwechselstörungen verbunden sein: Körperliche Aktivität mindert und familiäres Risiko für Diabetes mellitus erhöht das Risiko (39). Umgekehrt sind Elemente des metabolischen Syndroms bei Normalgewichtigen selten $(113 ; 115)$. Einige Elemente des me- 
Tabelle 3.5: Prävalenz von Risikofaktoren für Herz-Kreislauf-Krankheiten nach Geschlecht in zwei Studienpopulationen, 1993-2007 (57;111)

\begin{tabular}{|c|c|c|c|c|c|c|c|c|c|c|}
\hline & \multicolumn{5}{|c|}{ Prävalenz bei Männern (in \%) } & \multicolumn{5}{|c|}{ Prävalenz bei Frauen (in \%) } \\
\hline & $\begin{array}{l}1993- \\
1995\end{array}$ & $\begin{array}{l}1996- \\
1998\end{array}$ & $\begin{array}{l}1999- \\
2001\end{array}$ & $\begin{array}{l}2002- \\
2004\end{array}$ & $\begin{array}{l}2005- \\
2007\end{array}$ & $\begin{array}{l}1993- \\
1995\end{array}$ & $\begin{array}{l}1996- \\
1998\end{array}$ & $\begin{array}{l}1999- \\
2001\end{array}$ & $\begin{array}{l}2002- \\
2004\end{array}$ & $\begin{array}{l}2005- \\
2007\end{array}$ \\
\hline $\begin{array}{l}\text { Bus Santé (1993-2007), } \\
\text { Kanton Genf, 35-74 Jahre, } \\
N(\max )=\end{array}$ & 1'202 & $1^{\prime} 784$ & $1 ' 848$ & 1'801 & 378 & $1^{\prime} 140$ & 1'815 & $1^{\prime} 804$ & 1'799 & 376 \\
\hline Mittlerer BMI (kg/m²) & 25.6 & 26.1 & 26.1 & 26.2 & 26.4 & 23.9 & 24.2 & 24.2 & 24.3 & 24.7 \\
\hline Adipositas & 11.5 & 14.0 & 13.2 & 14.5 & 12.9 & 8.7 & 9.5 & 10.5 & 10.8 & 14.1 \\
\hline Übergewicht & 39.8 & 44.6 & 44.7 & 45.1 & 48.9 & 21.7 & 24.5 & 24.2 & 24.3 & 22.9 \\
\hline Hoher Cholesterinwert ${ }^{a}$ & 23.1 & 29.3 & 29.9 & 30.5 & 30.7 & 18.2 & 22.1 & 23.4 & 26.0 & 28.9 \\
\hline $\begin{array}{l}\text { Behandlung hoher } \\
\text { Cholesterinwert }\end{array}$ & 6.0 & 8.1 & 10.8 & 12.5 & 12.2 & 3.0 & 4.2 & 5.5 & 6.5 & 8.2 \\
\hline Hoher Blutdruck ${ }^{b}$ & 48.8 & 49.0 & 41.9 & 41.7 & 36.6 & 36.2 & 32.5 & 27.4 & 25.4 & 22.8 \\
\hline $\begin{array}{l}\text { Behandlung hoher } \\
\text { Blutdruck }\end{array}$ & 12.1 & 12.3 & 12.9 & 15.9 & 17.5 & 9.3 & 11.6 & 11.4 & 10.9 & 13.1 \\
\hline $\begin{array}{l}\text { CoLaus (2003), Stadt } \\
\text { Lausanne, 35-75 Jahre, N = }\end{array}$ & - & - & - & 2'937 & - & - & - & - & $3^{\prime} 251$ & - \\
\hline Mittlerer BMI (kg/m²) & - & - & - & 26.6 & - & - & - & - & 25.1 & - \\
\hline Adipositas & - & - & - & 17.1 & - & - & - & - & 14.5 & - \\
\hline Übergewicht & - & - & - & 45.7 & - & - & - & - & 28.4 & - \\
\hline Fettstoffwechselstörung ${ }^{c}$ & - & - & - & 42.7 & - & - & - & - & 26.6 & - \\
\hline $\begin{array}{l}\text { Behandlung Fettstoff- } \\
\text { wechselstörung }\end{array}$ & - & - & - & 15.3 & - & - & - & - & 11.0 & - \\
\hline Hoher Blutdruck ${ }^{b}$ & - & - & - & 43.0 & - & - & - & - & 30.9 & - \\
\hline $\begin{array}{l}\text { Behandlung hoher } \\
\text { Blutdruck }\end{array}$ & - & - & - & 47.1 & - & - & - & - & 53.8 & - \\
\hline
\end{tabular}

Ausser der Bluthochdruckbehandlung bei Frauen waren alle Zu- oder Abnahmen zwischen 1993 und 2007 statistisch signifikant $(p<0.05)$.

a Gesamtcholesterin $\geq 6.5 \mathrm{mmol} / \mathrm{L}(57)$

b Blutdruck $\geq 140 / 90 \mathrm{mmHg}$ und / oder antihypertensive Therapie $(57 ; 111)$

c Triglyceride $\geq 2.2 \mathrm{mmol} / \mathrm{L}$ und / oder HDL-Cholesterin $<1.0 \mathrm{mmol} / \mathrm{L}$ und / oder LDL-Cholesterin $\geq 4.1 \mathrm{mmol} / \mathrm{L}$ oder $\geq 2.6 \mathrm{mmol} / \mathrm{L}$ bei berichtetem Status nach Myokardinfarkt, Hirnschlag, koronarer Herzkrankheit oder Diabetes mellitus (111)

- = keine Angabe 
tabolischen Syndroms wurden auch bei Kindern und Stellungspflichtigen gefunden $(105 ; 112)$.

\section{Salz}

Selbst wenn der Blutdruck bei den meisten Menschen nach Salzeinnahme nahezu konstant bleibt, würde eine Reduktion der Salzzufuhr den Blutdruck in der Bevölkerung senken (116-120; Kapitel 1.5.2). Ein hoher Blutdruck erhöht das Risiko für Herz-Kreislauf-Krankheiten und ist der häufigste Grund für eine ärztliche Behandlung und oft die Ursache einer Spitaleinweisung $(41 ; 57 ; 101 ; 121)$. Der geschätzte Salzkonsum in der Schweiz ist etwa doppelt so hoch wie die Empfehlung (Kapitel 2.4.6).

\section{Fett}

Menge und Qualität des konsumierten Fetts können das Risiko für Herz-Kreislauf-Krankheiten beeinträchtigen $(112 ; 122 ; 123$; Kapitel 1.5.1). Die Gesamtfetteinnahme und die Einnahme an gesättigten Fetten ist bei übergewichtigen Jugendlichen mit hohem Blutdruck und Insulinresistenz verbunden (112). Auch Entzündungsreaktionen, die bei der Entstehung von Herz-Kreislauf-Krankheiten eine Rolle spielen, kommen bei hohem Fettkonsum gehäuft vor (123). Transfettsäuren pflanzlichen Ursprungs aus verarbeiteten Lebensmitteln können das Risiko für Herz-Kreislauf-Krankheiten erhöhen $(122 ; 124)$. Ob gesättigte Fette als Ersatz für Kohlenhydrate oder Nahrungscholesterin die Herz-Kreislauf-Morbidität und -Mortalität bei Gesunden tatsächlich erhöhen, ist jedoch umstritten $(122 ; 125 ; 126)$. Hingegen gibt es überzeugende Evidenz, dass der Ersatz von gesättigten durch ungesättigte Fettsäuren das Risiko für Herz-Kreislauf-Krankheiten vermindert $(122 ; 125 ; 126)$. Möglicherweise beeinflussen auch Unterschiede in der Fettsäurezusammensetzung von Milchprodukten die Herz-KreislaufMortalität (108;127-129).

Es ist vorstellbar, dass der insgesamt abnehmende Fettkonsum und die vermehrte Verwendung von pflanzlichen statt tierischen Fetten (Kapitel 2.3.2) zu einer Senkung des Risikos für Herz-Kreislauf-Krankheiten beiträgt (125). Aufgrund des Verbrauchs an pflanzlichen Ölen ist allerdings anzunehmen, dass die Einnahme an ungesättigten Fettsäuren nicht den Empfehlungen entspricht (122;126; Kapitel 1.5.1). Der Verbrauch an n-6-Fettsäuren ist im Verhältnis zu demjenigen an n-3-Fettsäuren zu hoch. Allerdings wird die Bedeutung dieses Verhältnisses zurzeit diskutiert (Kapitel 1.5.1). Möglicherweise trägt die Zunahme des Fischkonsums in der Schweiz zu einer Senkung des Risikos für HerzKreislauf-Krankheiten bei, zumal der überwiegende Anteil aus Meerwasserfisch besteht, der reich an mehrfach ungesättigten Fettsäuren ist (122). Der potenziell risikosenkende Einfluss von Nüssen kommt in der Schweiz nicht zum Tragen, weil der Verbrauch auf tiefem Niveau stagniert (122; Kapitel 2.3.2).

\section{Kohlenhydrate}

Im Gegensatz zu nahrungsfaserreichen Stärkelieferanten erhöhen Mono- und Disaccharide wie Saccharose, Glucose oder Fructose wahrscheinlich das Risiko für Herz-Kreislauf-Krankheiten (Kapitel 1.5.1). Vor allem Fructose verschlechtert bei hohem Konsum die Blutfett- und Harnsäurewerte sowie die Insulinempfindlichkeit und kann damit das Risiko für Herz-Kreislauf-Krankheiten erhöhen (130). Der Einsatz von Fructose als Süssungsmittel ist in der Schweiz zwar verhältnismässig gering, die Einfuhr stieg jedoch zwischen 2007 und 2008 an (Kapi- 
tel 2.3.2). Besonders häufig kommt Fructose in Form von Saccharose in Süssgetränken und Fruchtsäften vor (130). Vor diesem Hintergrund ist der unverändert hohe Verbrauch dieser Getränke als ungünstig zu beurteilen. Unerfreulich ist ebenso, dass bei konstant hohem Kohlenhydratverbrauch der Anteil an komplexen Kohlenhydraten aus Stärkeprodukten abgenommen und derjenige an einfachen Kohlenhydraten zugenommen hat (Kapitel 2.3.2 und 2.4.4).

\section{Alkohol}

Alkohol wird in der Schweiz relativ häufig konsumiert (Kapitel 2.3.2) und hat daher vermutlich einen wesentlichen Einfluss auf die Herz-Kreislauf-Mortalität (68). Darauf deuten Vergleiche zwischen der Deutsch- und Westschweiz hin. Die Herz-Kreislauf-Mortalität ist in der Westschweiz generell geringer. Da bei den sonstigen Herz-Kreislauf-Risikofaktoren kaum Unterschiede bestehen, könnte dies auf den regelmässigen Alkoholkonsum in der Westschweiz zurückgeführt werden. Dazu passt auch, dass in der Westschweiz andere Sterbeursachen, die mit Alkohol zusammenhängen, häufiger sind als in der Deutschschweiz: z.B. Krebs des oberen Verdauungstraktes und der Leber, Leberzirrhose und Strassenunfälle (31).

Regelmässiger, aber massvoller Alkoholkonsum kann Herz-Kreislauf-Krankheiten und dabei insbesondere koronaren Herzkrankheiten vorbeugen, wahrscheinlich vor allem dann, wenn er im Rahmen eines auch sonst gesunden Lebensstils eingenommen wird $(131 ; 132$; Kapitel 1.5.1). Der Effekt scheint unabhängig von der Art des alkoholischen Getränks (z.B. Bier oder Wein) zu sein (131-133) und ist u.a. auf eine Erhöhung des HDL-Cholesterins zurückzuführen $(133 ; 134)$. Das niedrigste Risiko für
Herz-Kreislauf-Krankheiten haben Personen mit einem wöchentlichen Konsum von 7-13 Standardgetränken $(115 ; 133)$. Ein Standardgetränk entspricht einem Glas Bier (2.5 dl), Wein (1 dl) oder Spirituose $(2.5 \mathrm{cl})$.

Das Sterbe- und Erkrankungsrisiko für Hirnschlag steigt im Gegensatz zum Herzinfarktrisiko mit zunehmendem Alkoholkonsum an (135). Wesentlicher Grund dafür könnte die Erhöhung des Blutdrucks mit zunehmendem Alkoholkonsum sein (133). Möglicherweise trug der sinkende Alkoholkonsum (Abbildung 2.4) und der zunehmende Anteil an Abstinenten zur Abnahme der Hirnschlag-Mortalität bei. Ein U-förmiger Zusammenhang mit dem niedrigsten Risiko bei moderatem Konsum besteht auch zwischen Alkoholkonsum und dem metabolischen Syndrom (115).

\section{Andere Risiko- und Schutzfaktoren}

Abgesehen von den bereits besprochenen Nährstoffen wurde bei spezifischen Lebensmittelgruppen bzw. Ernährungsweisen ein vorbeugender Effekt auf Herz und Kreislauf nachgewiesen. Dazu gehört die Einnahme von Früchten und Gemüse, von anderen Lebensmitteln mit hohem Anteil an Nahrungsfasern, von Kaffee und Tee sowie von Schokolade $(136 ; 137)$. Die Schweizer Bevölkerung profitiert nur teilweise davon: Der Gemüsekonsum hat in der Schweiz in den letzten Jahrzehnten in der Tendenz zwar zugenommen, der Früchtekonsum hingegen sank. Zugenommen hat weiter der Kaffee-, nicht aber der Teeverbrauch (Kapitel 2.3.2). Allenfalls birgt auch der vergleichsweise hohe Schokoladenkonsum ein HerzKreislauf-schützendes Potenzial (137). Aus Herz-Kreislauf-Sicht erfreulich ist der deutliche Anstieg des Nahrungsfaserverbrauchs um knapp einen Drittel seit 1979. 
Einer der wichtigsten Risikofaktoren für Herz-Kreislauf-Krankheiten ist der Tabakkonsum. Der Raucheranteil blieb seit 1992 bei den Frauen unverändert bzw. sank bei den Männern geringfügig $(7 ; 57)$. Insgesamt raucht aber immer noch rund ein Drittel der Schweizer Bevölkerung $(7 ; 57 ; 68 ; 102 ; 104 ; 111 ; 138)$. Ein ebenfalls weitverbreiteter Risikofaktor ist körperliche Inaktivität. Auch eine niedrige Bildung ist mit einem erhöhten Risiko für Herz-Kreislauf-Krankheiten verbunden, unter anderem, weil diese mit Adipositas (Kapitel 3.3.2) und Tabakkonsum assoziiert ist $(83 ; 85 ; 103 ; 139)$. Die Einnahme von Calcium-Supplementen könnte das Risiko für Herz-Kreislauf-Krankheiten ebenfalls erhöhen (140). In der CoLaus-Studie nahmen im Jahr 2003 16.8\% kombinierte Vitamin- und Mineralstoffsupplemente ein und weitere $6.6 \%$ Calcium-Supplemente (141). Neben der Einnahme von Supplementen ist in der Schweiz auch die Einnahme nährstoffangereicherter Lebensmittel verbreitet (141; Kapitel 1.6 und 2.4.1). Es ist nicht auszuschliessen, dass es in Teilen der Bevölkerung zu einer Überversorgung mit einzelnen Nährstoffen kommt, die mit einer Erhöhung des Risikos für Herz-Kreislauf-Krankheiten einhergehen könnte (142). Die wissenschaftliche Beweislage ist aber noch ungenügend und ein kausaler Zusammenhang nicht gesichert.

\subsubsection{Bedeutung}

Da Herz-Kreislauf-Krankheiten und damit zusammenhängende Todesfälle meistens erst in höherem Alter auftreten, ist deren Anteil an potenziell verlorenen Lebensjahren (zwischen 1 und 70 Jahren) mit $17.6 \%$ bei den Männern und $10.1 \%$ bei den Frauen relativ gering (143). Herz-
Kreislauf-Krankheiten als Todesursache haben im Verhältnis zu Krebs an Bedeutung verloren und werden das in Zukunft vermutlich weiter tun $(23 ; 24)$. Da die Bevölkerung aber weiter altert, wird diese Krankheit wichtig bleiben: Im 2008 war eine Herz-Kreislauf-Krankheit bei rund $10 \%$ der Hospitalisierten (2002: 10.5\%) der Einweisungsgrund, bei ca. 12\% (2002: $13 \%$ ) der Grund für den Besuch einer Arztpraxis und bei ca. $5 \%$ der Personen unter 65 Jahren (2002: 7\%) der Grund für Invalidität $(30 ; 100$; $101 ; 144 ; 145)$. Die Abnahme der altersstandardisierten Herz-Kreislauf-Mortalität ist wahrscheinlich zu einem bedeutenden Teil auf Fortschritte bei der Früherkennung und Behandlung zurückzuführen $(57 ; 67)$. Das präventive Potenzial einer möglichst frühen Erfassung der wichtigsten Risikofaktoren ist aber noch nicht ausgeschöpft (135;146; Kapitel 3.6.3). Welche Bedeutung Herz-Kreislauf-Krankheiten gegenüber Krebs tatsächlich haben, kann nur mit Hilfe der Inzidenz beurteilt werden. Vergleichbare Angaben für Herz-Kreislauf-Krankheiten fehlen aber. Die möglicherweise hohe Prävalenz mancher Herz-Kreislauf-Krankheiten könnte darauf zurückzuführen sein, dass immer mehr Menschen einen Herzinfarkt oder Hirnschlag überleben und nicht, dass effektiv mehr Menschen einen Herzinfarkt oder Hirnschlag erleiden (22).

Die hauptsächlichen vermeidbaren Risikofaktoren für Herz-Kreislauf-Krankheiten sind neben Tabakkonsum, körperlicher Inaktivität und Adipositas auch Bluthochdruck, Diabetes mellitus und Fettstoffwechselstörungen. Bezüglich dieser intermediären Risikofaktoren ist die Datenlage in der Schweiz ungenügend und muss verbessert werden (Kapitel 4.9.1). Die Ernährung kann diese Risikofaktoren direkt, d.h. über den unmittelbaren Effekt der eingenom- 
menen Nährstoffe und Energie, aber auch indirekt über Übergewicht und Adipositas beeinflussen. Die Prävention von Übergewicht und Adipositas ist für eine weitere Senkung des Risikos für Herz-Kreislauf-Krankheiten deshalb zentral (Kapitel 3.3.3).

Präventionspotenzial bietet auch die Senkung der Salzzufuhr. Dabei sollte die Rolle von Salz als Vehikel für die Versorgung der Schweizer Bevölkerung mit Jod berücksichtigt werden (116;147;148; Kapitel 3.10.5.3). Wahrscheinlich haben Adipositas und körperliche Inaktivität in der Schweizer Bevölkerung einen weitaus grösseren Einfluss auf den Blutdruck als die Salzzufuhr (41). Massnahmen zur Verringerung der Salzzufuhr sind aber einfacher umzusetzen und versprechen mehr Erfolg als solche, die darauf abzielen, die Adipositasprävalenz zu reduzieren oder die körperliche Aktivität zu erhöhen. Mit der Salzstrategie verstärkt der Bund seine Anstrengungen, die Salzzufuhr in der Bevölkerung zu senken (117; Kapitel 1.5.2, 2.4.6 und 4.6.1.1).

Wahrscheinlich ist nur ein geringer Teil der Fettstoffwechselstörungen nicht durch Lebensstilveränderungen modifizierbar (146). Mit einer Abnahme des Verbrauchs an tierischen zugunsten von pflanzlichen Fetten geht der Trend in die richtige Richtung. In Anbetracht des wahrscheinlich immer noch nicht den Empfehlungen entsprechenden Musters der Fettsäureeinnahme ist der vermehrte Einsatz von einfach ungesättigten Fettsäuren aus Oliven- und Rapsöl begrüssenswert (Kapitel 2.3.2). Seit dem 1. April 2008 darf der Gehalt an Transfettsäuren in pflanzlichen Fetten und Ölen nur noch maximal $2 \%$ bezogen auf den Gesamtfettgehalt betragen. Dieser Grenzwert führte zu Rezepturanpassungen industriell hergestellter Produkte $(122 ; 149)$. Weil die aufgenommene Menge wahrscheinlich weiter abnehmen wird, ist anzunehmen, dass von Transfettsäuren kein bedeutendes Risiko mehr ausgehen wird.

Im Gegensatz zur Entwicklung beim Fettverbrauch (Kapitel 2.4.4) ist beim Kohlenhydratverbrauch keine vergleichbare potenzielle vorbeugende Veränderung in der Schweiz zu beobachten. Vor allem die Zufuhr von Monound Disacchariden müsste gesenkt werden. Der Ersatz von Fructose durch Glucose in Lebensmitteln ist aber nicht sinnvoll $(130 ; 150)$. Wünschenswert wäre eine Verlagerung weg von zugesetzten Mono- und Disacchariden hin zu nahrungsfaserreichen Kohlenhydratquellen, wie Vollkornprodukten und Hülsenfrüchten (Kapitel 1.5.1). Da einfache Zucker zu einem grossen Teil in flüssiger Form konsumiert werden (Kapitel 2.3.2), würde sich eine schrittweise Reduktion des Gehalts seitens der Getränkehersteller anbieten, was sich wahrscheinlich auch positiv auf die Adipositasprävalenz auswirken würde (130).

Eine zusätzliche Senkung von Hirngefässkrankheiten wäre zu erwarten, wenn weniger Menschen Alkohol in grossen Mengen konsumieren würden. Gegen moderaten Alkoholkonsum ist vor allem aus Sicht der Herzgesundheit nichts einzuwenden. Alkoholkonsum sollte trotzdem nicht empfohlen werden, da dieser andere Risiken erhöht (Kapitel 3.5.2). Aus HerzKreislauf-Optik müsste der Verbrauch von Nahrungsfasern und von Früchten und vor allem Gemüse in der Bevölkerung zunehmen. Massnahmen sind in Kapitel 4.9 dargestellt. 


\subsection{Krebs - ernährungsassoziierte Formen}

\subsubsection{Häufigkeit}

Jährlich erkranken in der Schweiz über 19'000 Männer und über 16'000 Frauen an Krebs und 8'500 Männer und 7'000 Frauen sterben daran $(151 ; 152)$. Krebs ist nach Herz-Kreislauf-Krankheiten die zweithäufigste Todesursache. Die Inzidenz der meisten Krebsformen blieb in den letzten Jahrzehnten unverändert, während die Mortalität bei fast allen Formen sank (Tabelle 3.6). Eine Ausnahme bildet Magenkrebs, dessen Inzidenz zwischen 1983 und 2007 auch deutlich sank (23;152-154). Zunehmend häufiger treten Leberkrebs und bestimmte Krebsformen der Speiseröhre auf (154-156).

Bei den ernährungsassoziierten Krebserkrankungen (Tabelle 3.6) liegt die Schweiz im europäischen Mittelfeld. Internationale Vergleiche deuten darauf hin, dass Magenkrebs seltener und Prostata- und Brustkrebs häufiger vorkommen als in den meisten anderen Ländern $(68 ; 152)$. Möglicherweise sind Risiko- und Schutzfaktoren in der Ernährung für die internationalen Unterschiede mitverantwortlich $(31 ; 157)$. Auch innerhalb der Schweiz gibt es regionale Unterschiede: Im Tessin ist die Inzidenz von Magenkrebs höher und diejenige von Prostatakrebs niedriger als in der Deutschschweiz. Zudem haben das Tessin und die Westschweiz eine etwas höhere Brustkrebsinzidenz als die Deutschschweiz (152). Die Mortalität von alkoholassoziierten Krebsformen (Tabelle 3.6) ist in der Westschweiz höher als in der Deutschschweiz $(31 ; 152)$.

Bei den meisten Krebsformen sind Mortalitätsunterschiede nach Bildungsniveau deutlich geringer als bei den Herz-Kreislauf-Krankheiten $(82 ; 83)$. Hat das Bildungsniveau einen Einfluss auf die Inzidenz oder Mortalität bestimmter Krebsformen, dann beruht dies meist auf Risikofaktoren, die vom Bildungsniveau abhängig sind $(83 ; 102)$. Der soziale Status, der von der Bildung geprägt wird, spielt aber auch bei der Voruntersuchung (Screening), der Diagnose und der Behandlung von Krebs eine Rolle $(158 ; 159)$.

\subsubsection{Risiko- und Schutzfaktoren}

Tabakkonsum ist einer der wichtigsten Risikofaktoren für praktisch alle Krebsformen (160162). Zudem kann körperliche Inaktivität das Risiko erhöhen (1;82; Kapitel 3.3.2). Die Ernährung kann die Krebsentstehung direkt beeinflussen. Bei einigen Ernährungsfaktoren ist die ursächliche Beteiligung an der Krebsentstehung noch unklar, bei anderen ist die Evidenz überzeugend (Kapitel 3.2.1 und Tabelle 3.6). Alkohol z.B. spielt eine wichtige Rolle, weil dieser in der Schweiz häufig konsumiert wird (Kapitel 2.3.2). Zudem können Toxine, die durch Bakterien oder Pilze (z.B. Aflatoxin) gebildet werden oder die bei der Produktion und Zubereitung von Lebensmitteln entstehen, das Krebsrisiko erhöhen. Eine Ernährung mit einem hohen Anteil an pflanzlichen Produkten und wenig rotem Fleisch gilt hingegen als protektiv. Indirekt kann die Ernährung das Krebsrisiko auch über die Körperfettmasse beeinflussen (Tabelle 3.6). In den folgenden Abschnitten werden diese Aspekte im Detail aufgegriffen. 
Tabelle 3.6: Häufigste Krebsformen in der Schweiz (Inzidenz und Mortalität pro 100'000 Männern oder Frauen), 5-Jahres-Perioden zwischen 1983-2007 (151;152)

\begin{tabular}{|c|c|c|c|c|c|c|c|}
\hline \multirow[t]{2}{*}{ Lokalisation } & \multirow{2}{*}{$\begin{array}{c}\text { Mögliche } \\
\text { Risikofaktoren } \\
\text { (Zusammenhang } \\
\text { Ernährung }{ }^{\text {a) }}\end{array}$} & \multirow{2}{*}{$\begin{array}{c}\text { Mögliche } \\
\text { Schutzfaktoren } \\
\text { (Zusammenhang } \\
\text { Ernährung }{ }^{\text {a) }}\end{array}$} & \multicolumn{5}{|c|}{ Inzidenz und Mortalität bei Männern (pro 100'000) } \\
\hline & & & $\begin{array}{l}1983- \\
1987\end{array}$ & $\begin{array}{l}1988- \\
1992\end{array}$ & $\begin{array}{l}1993- \\
1997\end{array}$ & $\begin{array}{l}1998- \\
2002\end{array}$ & $\begin{array}{l}2003- \\
2007\end{array}$ \\
\hline \multirow{2}{*}{$\begin{array}{l}\text { Mundhöhle und } \\
\text { Rachen, Speise- } \\
\text { röhre, Kehlkopf }\end{array}$} & Alkohol $(+++)$ & $\begin{array}{l}\text { Früchte und } \\
\text { Gemüse }(++)\end{array}$ & 33.6 & 35.2 & 34.3 & 32.7 & 32.5 \\
\hline & & & 21.0 & 19.6 & 17.0 & 16.6 & 15.1 \\
\hline \multirow{2}{*}{$\begin{array}{l}\text { Leber, Gallenblase } \\
\text { und extrahepa- } \\
\text { tische Gallenwege }\end{array}$} & $\begin{array}{l}\text { Aflatoxin }(+++) \\
\text { Alkohol }(++)\end{array}$ & - & & 12.9 & 13.3 & 13.7 & 14.0 \\
\hline & & & 10.4 & 10.5 & 10.7 & 10.6 & 10.4 \\
\hline \multirow[t]{2}{*}{ Nieren } & Körperfett $(+++)$ & - & 12.1 & 12.5 & 11.9 & 11.8 & 11.6 \\
\hline & & & 6.6 & 6.5 & 5.5 & 4.7 & 4.3 \\
\hline \multirow[t]{2}{*}{ Magen } & $\begin{array}{l}\text { Salz und Salzhal- } \\
\text { tiges }(++)\end{array}$ & $\begin{array}{l}\text { Früchte und } \\
\text { Gemüse (++) }\end{array}$ & 20.7 & 18.2 & 15.9 & 13.7 & 10.7 \\
\hline & & & 17.4 & 14.2 & 11.1 & 8.8 & 6.9 \\
\hline \multirow[t]{2}{*}{ Dickdarm } & $\begin{array}{l}\text { Fleisch und } \\
\text { Fleischprodukte, }\end{array}$ & $\begin{array}{l}\text { Nahrungsfasern, } \\
\text { Calcium (++) }\end{array}$ & 51.4 & 49.9 & 51.6 & 52.7 & 50.2 \\
\hline & $\begin{array}{l}\text { Alkohol, Körperfett } \\
(+++)\end{array}$ & & 27.5 & 26.3 & 23.7 & 21.2 & 19.1 \\
\hline \multirow[t]{2}{*}{ Prostata } & $\begin{array}{l}\text { Calciumreiche } \\
\text { Lebensmittel }(++)\end{array}$ & $\begin{array}{l}\text { Lykopen, Selen } \\
(++)\end{array}$ & 78.5 & 91.1 & 106.5 & 128.0 & 133.7 \\
\hline & & & 30.9 & 32.1 & 32.5 & 30.4 & 26.5 \\
\hline \multirow[t]{2}{*}{ Brust } & $\begin{array}{l}\text { Alkohol, Körper- } \\
\text { fett }^{\mathrm{b}}(+++)\end{array}$ & - & - & - & - & - & - \\
\hline & & & - & - & - & - & - \\
\hline \multirow[t]{2}{*}{$\begin{array}{l}\text { Gebärmutter- } \\
\text { körper }\end{array}$} & Körperfett ${ }^{\mathrm{C}}(+++)$ & - & - & - & - & - & - \\
\hline & & & - & - & - & - & - \\
\hline
\end{tabular}

Daten zur Inzidenz (Anzahl Neuerkrankungen) sind in normaler Schrift, und Daten zur Mortalität (Anzahl Sterbefälle) sind kursiv geschrieben.

\footnotetext{
a Zusammenhänge entsprechend $(1 ; 33)$

b Nur in der Postmenopause

c Gilt für Krebs der Gebärmutterschleimhaut

$(+++)=$ überzeugender Zusammenhang

$(++)=$ wahrscheinlicher Zusammenhang

- $\quad=$ keine Angabe
} 


\begin{tabular}{|c|c|c|c|c|}
\hline \multicolumn{5}{|c|}{ Inzidenz und Mortalität bei Frauen (pro $100^{\prime} 000$ ) } \\
\hline $\begin{array}{l}1983- \\
1987\end{array}$ & $\begin{array}{l}1988- \\
1992\end{array}$ & $\begin{array}{l}1993- \\
1997\end{array}$ & $\begin{array}{l}1998- \\
2002\end{array}$ & $\begin{array}{l}2003- \\
2007\end{array}$ \\
\hline 6.6 & 7.6 & 8.8 & 9.4 & 9.3 \\
\hline 3.3 & 3.5 & 3.4 & 3.7 & 3.6 \\
\hline 6.4 & 6.1 & 5.7 & 5.9 & 5.8 \\
\hline 6.0 & 5.5 & 5.3 & 4.6 & 4.8 \\
\hline 5.3 & 5.4 & 5.7 & 4.8 & 4.9 \\
\hline 3.1 & 2.8 & 2.5 & 2.1 & 1.9 \\
\hline 9.5 & 8.2 & 7.5 & 5.9 & 5.6 \\
\hline 8.3 & 6.5 & 5.0 & 3.9 & 3.3 \\
\hline 33.2 & 31.8 & 32.1 & 32.2 & 31.5 \\
\hline 17.5 & 15.6 & 13.9 & 12.4 & 11.1 \\
\hline- & - & - & - & - \\
\hline - & - & - & - & - \\
\hline 89.9 & 95.6 & 101.6 & 114.4 & 110.5 \\
\hline 34.2 & 33.0 & 30.7 & 25.8 & 23.9 \\
\hline 21.1 & 18.9 & 18.7 & 18.7 & 17.6 \\
\hline 5.4 & 4.9 & 4.0 & 3.5 & 3.2 \\
\hline
\end{tabular}

\section{Übergewicht und Adipositas}

Ein Übermass an Körperfett ist mit einem erhöhten Krebsrisiko verbunden (156;163; Tabelle 3.6). So sind z.B. Krebsformen der Geschlechtsorgane bei Adipösen häufiger als bei Normalgewichtigen (156). In der Schweiz ist das Krebssterberisiko (alle Krebsformen) bei Adipösen um etwa $30 \%$ höher als bei Normalgewichtigen, wohingegen Übergewichtige (BMI $25-29.9 \mathrm{~kg} / \mathrm{m}^{2}$ ) kein erhöhtes Krebsrisiko haben (41). Hingegen wurde kein statistisch signifikanter Zusammenhang zwischen BMI und Krebsmortalität bei einer Studie an Männern aus Basel-Stadt festgestellt (164).

Unklar ist, zu welchem Anteil der Überschuss an Fettgewebe einerseits oder die mit Adipositas zusammenhängende Ernährung (Energieeinnahme, Nährstoffzusammensetzung) andererseits für das erhöhte Risiko verantwortlich ist. Tatsächlich ist die Energieeinnahme (ohne Alkohol) unabhängig vom BMI positiv mit dem Auftreten von Kehlkopfkrebs assoziiert (165). Der glykämische Index und die glykämische Last der Nahrung sind ebenfalls unabhängig vom Körpergewicht mit Krebs der Gebärmutterschleimhaut assoziiert (166). Ein besonders hohes Risiko für diese Krebsform haben auch adipöse Frauen mit Diabetes mellitus Typ 2 (167). Ein Grund dafür könnte die Insulinresistenz bei Adipösen sein (156).

\section{Alkohol}

Das Krebsrisiko kann schon ab dem Konsum von einem Standardgetränk pro Tag ansteigen, hauptsächlich betroffene Organe sind in Tabelle 3.6 erwähnt (160-162;168). Unterschiede bei der Krebsmortalität und beim Alkoholkonsum zwischen Deutsch- und Westschweiz weisen darauf hin, dass vor allem der obere Verdau- 
ungstrakt (Mund, Rachen, Speiseröhre) und die Leber betroffen sind $(31 ; 152)$. Kehlkopfkrebs ist mehr als viermal häufiger bei Personen mit hohem Alkoholkonsum (fünf oder mehr Standardgetränke pro Tag) als bei Abstinenten, unabhängig vom Tabakkonsum (162). Zusammen mit anderen Ernährungsfaktoren macht der Konsum von Alkohol rund $30 \%$ der Kehlkopfkrebsfälle aus (161). Ein besonders hohes Krebsrisiko birgt Alkohol in Verbindung mit Tabakkonsum (160162). Die konsumierte Menge des alkoholischen Getränkes hat einen grösseren Effekt auf das Krebsrisiko als die Art des Getränkes (169). So hat das Polyphenol Resveratrol aus Wein wahrscheinlich keinen protektiven Effekt auf das Krebsrisiko: Rotweinkonsum ist mit einem ähnlichen Risiko verbunden wie das Trinken von Bier oder Spirituosen (169).

Leberkrebs ist in der Schweiz vergleichsweise häufig (170). Die in der Schweiz beobachtete Zunahme von Leberkrebs bei Männern seit den 1970er Jahren wurde auch in den Nachbarländern nachgewiesen. Möglicherweise sind hauptsächlich Hepatitis-Viren für diesen Anstieg verantwortlich und weniger der veränderte Alkoholkonsum, da die alkoholische Leberzirrhose als Todesursache in der Schweiz seit den 1970er Jahren abnimmt (Tabellen 3.4 und 3.6).

\section{Andere Risiko- und Schutzfaktoren}

Häufiger Konsum von verarbeitetem Fleisch ist mit einem 2.5- bis 5-fach höheren Erkrankungsrisiko an Krebs von Mund, Rachen, Speiseröhre, Kehlkopf und Dickdarm verbunden (171). Zudem ist Kehlkopfkrebs mit der Einnahme von tierischen Produkten und Cholesterin assoziiert (165). Die abnehmende Magenkrebsmortalität (Tabelle 3.6) spricht aber dafür, dass bestimmte potenziell krebserregende Stoffe aus Fleisch und Fleischprodukten wie Nitritpökelsalz als Risikofaktoren an Bedeutung verloren haben $(23 ; 153 ; 154)$. Tatsächlich sinkt der Verbrauch von Fleisch- und Fleischprodukten in der Schweiz seit drei Jahrzehnten kontinuierlich (Kapitel 2.3.2).

Auch Acrylamid ist potenziell kanzerogen. Es kann entstehen, wenn kohlenhydratreiche Lebensmittel erhitzt werden $(172 ; 173)$. Gemäss einer Fall-Kontroll-Studie ist Essen von Frittiertem mit einem erhöhten Risiko für Mund-, Rachen- und Speiseröhrenkrebs assoziiert (174). Laut anderen Studien geht hingegen weder von gebratenen oder frittierten Kartoffeln noch von anderen frittierten Lebensmitteln ein erhöhtes Krebsrisiko aus $(175 ; 176)$. Schliesslich konnte auch kein Zusammenhang festgestellt werden zwischen der Einnahme von Acrylamid und dem Risiko für irgendeine der untersuchten Krebsformen (173). Der Acrylamidgehalt von in der Schweiz erhältlichen Lebensmitteln sank in den letzten Jahren kontinuierlich. Trotzdem wurden 2006 im Rahmen eines Tests in einigen Produkten immer noch zu hohe Acrylamidwerte gefunden (177).

Zurzeit kann nicht ausgeschlossen werden, dass die langfristige Einnahme von bestimmten Vitaminsupplementen (z.B. Folsäure) das Krebsrisiko erhöht $(178 ; 179)$. Dies könnte von Bedeutung sein, da gemäss der CoLaus-Studie die Supplementeinnahme wahrscheinlich weit verbreitet ist (141; Kapitel 3.4.1).

Hingegen senkt eine Ernährung mit hohem Anteil an Mikronährstoffen das Krebsrisiko, unabhängig vom Tabak- und Alkoholkonsum (178; 180-182). Der Konsum von Früchten und Gemüse ist möglicherweise mit einem niedrigeren Krebsrisiko verbunden $(32 ; 161 ; 180 ; 183 ; 184)$. Dabei könnten nicht nur Menge und Häufigkeit, 
sondern auch die Vielfalt eine Rolle spielen (183). Für die in Früchten und Gemüse enthaltenen Vitamine und sekundären Pflanzeninhaltsstoffe wurde ein unabhängiger schützender Einfluss nachgewiesen (160;180-182). Im Kanton Waadt ist die Aufnahme von Resveratrol aus Traubensaft mit einem geringeren Brustkrebsrisiko verbunden (181). Der Konsum von koffeinhaltigem Kaffee, aber auch von Zwiebeln und Knoblauch ist mit einem niedrigeren Krebsrisiko assoziiert. Das Potenzial, Krebs vorzubeugen, haben auch n-3-Fettsäuren (184-186). Aufgrund der zahlreichen Einschränkungen der herangezogenen Studien müssen die Resultate aber mit Zurückhaltung beurteilt werden (Kapitel 3.2.1).

\subsubsection{Bedeutung}

Menschen, die an Krebs sterben, sind im Durchschnitt jünger als solche, die an einer HerzKreislauf-Krankheit sterben (152). Deshalb ist mit $27.7 \%$ bei den Männern und $43.5 \%$ bei den Frauen der Anteil an potenziell verlorenen Lebensjahren (zwischen 1 und 70 Jahren) bei Krebs höher als bei Herz-Kreislauf-Krankheiten (143). Wegen ihrer kulturellen Vielfalt bietet die Schweiz ein gutes Umfeld, um den Zusammenhang zwischen Lebensstil und Krebsentstehung zu untersuchen. Mit der Bildung eines nationalen Krebsregisters und der Schaffung der dazu notwendigen rechtlichen Grundlagen wird ein wichtiger Grundstein dafür gelegt (28).

Obwohl einzelne Krebsformen seltener auftreten, ist Krebs nach wie vor häufiger Grund für Spitaleinweisung, Invalidität, Arbeitsausfall und vorzeitigen Tod $(101 ; 121)$. Im 2008 war eine Krebserkrankung bei rund $7.2 \%$ der Hospitali- sierten (2002: 7.3\%) der Einweisungsgrund und bei ca. 3.5\% der Personen unter 65 Jahre (2002: $3.5 \%)$ der Grund für Invalidität $(30 ; 100 ; 101 ; 144$; 145). Krebserkrankungen stellen nicht nur für Betroffene, sondern auch für die Gesellschaft eine grosse Belastung dar, zumal sie im Vergleich zu Herz-Kreislauf-Krankheiten nicht überwiegend ältere Menschen betreffen. Die Entwicklung der letzten Jahre lässt annehmen, dass es nicht mehr lange dauern wird, bis die altersstandardisierte Mortalität von Krebs höher ist als diejenige von Herz-Kreislauf-Krankheiten. Krebs als Ursache für Krankheit und Tod wird wahrscheinlich weiter an Bedeutung gewinnen. Dies liegt auch daran, dass sich viele Krebsformen schlechter früherkennen, vorbeugen und behandeln lassen als Herz-Kreislauf-Krankheiten.

Weil viele Krebsformen, bei denen ein $\mathrm{Zu}$ sammenhang zu Ernährungsfaktoren vermutet wird, zum Zeitpunkt der Diagnose nur noch schlechte Heilungschancen haben, spielt Prävention eine wichtige Rolle (163). Neben dem Vermeiden von Tabakkonsum gehört auch eine ausgewogene Ernährung und körperliche Aktivität dazu. Allein durch die Vermeidung von Übergewicht und Adipositas würden sich in der Schweiz jährlich 700 Krebsfälle verhindern lassen $(41 ; 156)$. Der Trend zur Stabilisierung von Übergewicht und Adipositas stimmt zuversichtlich, aber es besteht weiteres Präventionspotenzial (Kapitel 3.3.3). Auch durch massvollen Umgang mit Alkohol und eine ausgewogene mehrheitlich pflanzliche Ernährung liesse sich die Häufigkeit mancher Krebsformen senken. Einige Trends bewegen sich in diese Richtung (Kapitel 2.3.2). Allerdings wird risikoreicher Alkoholkonsum von Verbrauchsdaten oder Gesundheitsstudien nur ungenügend erfasst, was das Abschätzen von Trends erschwert. Wie in 
Kapitel 2.3.2 dargestellt, dürfte diese Art von Konsum in der Schweiz aber nach wie vor häufig sein (14;187-189). Schliesslich ist vorstellbar, dass Fortschritte bei der Produktion, Konservierung, Lagerung und Zubereitung von Lebensmitteln zu einer Senkung des Krebsrisikos beigetragen haben und noch beitragen werden.

\subsection{Diabetes mellitus}

\subsubsection{Häufigkeit}

Je nach Quelle waren 2010 in der Schweiz zwischen 350'000 und 630'000 Personen von Diabetes mellitus betroffen (93;190-192), davon entfallen schätzungsweise $10^{\prime} 000$ bis 30'000 auf Diabetes mellitus Typ 1 (192). Bei dessen Entstehung spielt die Ernährung eine untergeordnete Rolle. Aufgrund unterschiedlicher Datenquellen ist gesamtschweizerisch eine Diabetesprävalenz von ca. 5-6\% bei Männern und 4-5\% bei Frauen realistisch $(21 ; 93 ; 190-192$; Tabelle 3.1). Die Prävalenz von Diabetes mellitus nahm besonders bei älteren Menschen und bei Männern zu (21;57;102;104;111; Abbildung 3.4). Wahrscheinlich ist dieser Anstieg teilweise auf Verbesserungen bei der Früherkennung und Behandlung und auf die höhere Überlebenswahrscheinlichkeit zurückzuführen (193).

In der Stadt Lausanne betrug die Diabetesprävalenz 2003 bei 35- bis 75-jährigen Männern 9.5\% und $4.0 \%$ bei gleichaltrigen Frauen; bei rund einem Drittel der Betroffenen wurde der Diabetes mellitus erstmals im Rahmen der Studie diagnostiziert (111). Im Kanton Genf gaben 2007 rund 4\% der Männer und 2\% der Frauen (35-74 Jahre) an, wegen Diabetes mellitus in
Behandlung zu sein; aufgrund einer Nüchternblutzuckermessung hatten 2004-2009 7.5\% der Männer und 2.9\% der Frauen Diabetes mellitus (57). Wahrscheinlich kommen zu den Menschen mit Diabetes mellitus nochmals gleich viele Personen mit einem erhöhten Blutzuckerspiegel unterhalb des Schwellenwertes für die Diagnose Diabetes mellitus hinzu $(4 ; 46 ; 107 ; 111$; 194). Bei Rekruten wurden bei $0.4 \%$ anormal hohe Werte gefunden (105).

\subsubsection{Risiko- und Schutzfaktoren}

Die beeinflussbaren Risikofaktoren für Diabetes mellitus Typ 2 sind vergleichbar mit denen für Adipositas und Herz-Kreislauf-Krankheiten (Kapitel 3.3.2 und 3.4.2). Adipositas ist einer der wichtigsten Risikofaktoren für Diabetes mellitus Typ 2 (37;46;107;113;195;196). Neben Bewegungsmangel kann auch eine energiereiche Ernährung mit einem hohen Anteil an raffiniertem Zucker, gesättigten Fetten und Alkohol in grösseren Mengen das Risiko erhöhen (115;122; 130). Ausserdem spielt auch die Vererbung eine Rolle $(113 ; 195)$ : 42.1\% der Typ-2-Diabetiker haben mindestens ein Familienmitglied mit Diabetes mellitus, während es bei den Nicht-Diabetikern nur $21.1 \%$ sind (195).

\section{Übergewicht und Adipositas}

Diabetes mellitus Typ 2 ist bei Normalgewichtigen selten $(113 ; 196)$. Obwohl die Gene eine wichtige Rolle bei der Entstehung spielen, ist Übergewicht meist die Voraussetzung dafür, dass eine Veranlagung manifest wird $(195 ; 196)$. Problematisch ist insbesondere eine stammbetonte Fettansammlung, welche auch ein wichtiges Element des metabolischen Syndroms ist 

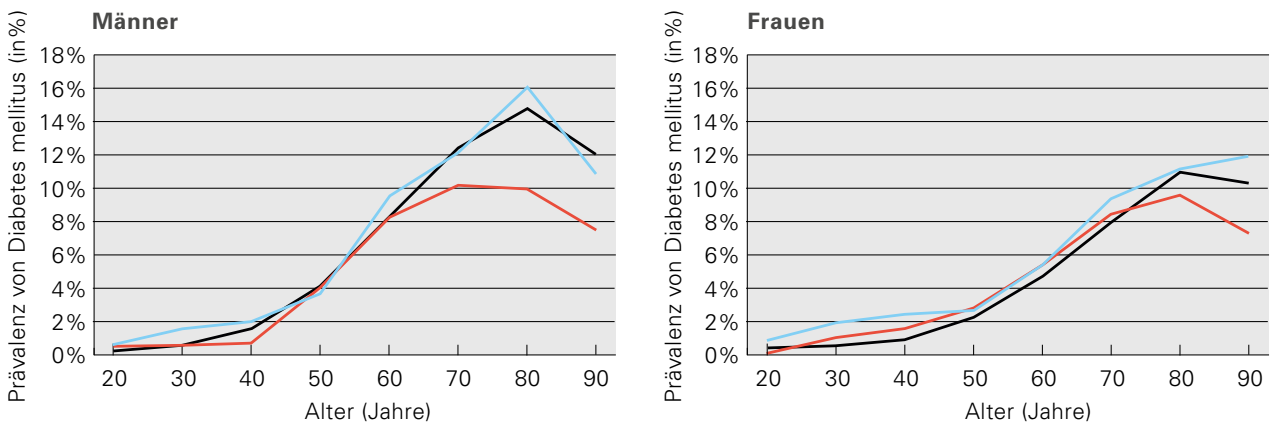

Medizinische Statistik der Krankenhäuser, Nebendiagnose, 2008

Todesursachenstatistik, Begleitursache, 2007/08

Schweizerische Gesundheitsbefragung, jemals diagnostiziert, 2007
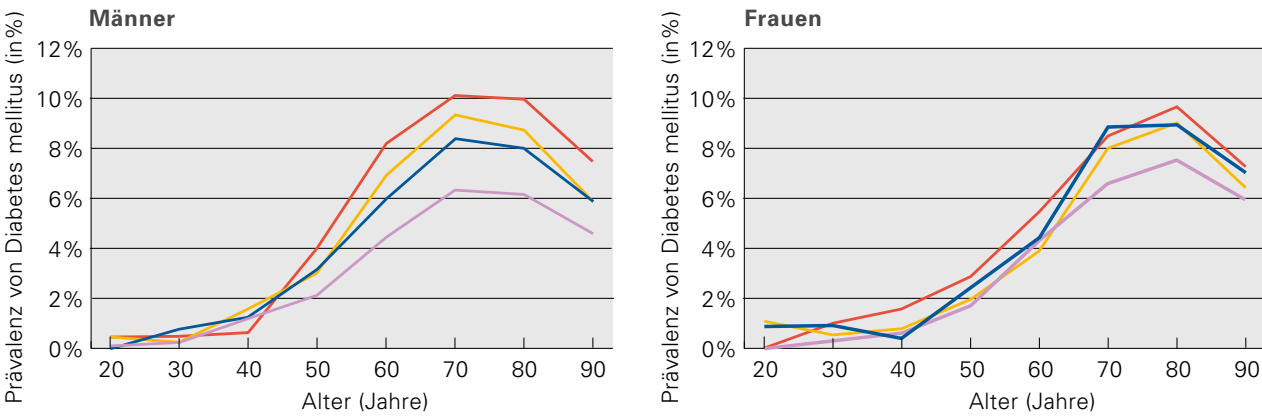

Todesursachenstatistik, Begleitursache, Jahr:

$1995 / 96$

$1999 / 2000$

2003/04

$2007 / 08$

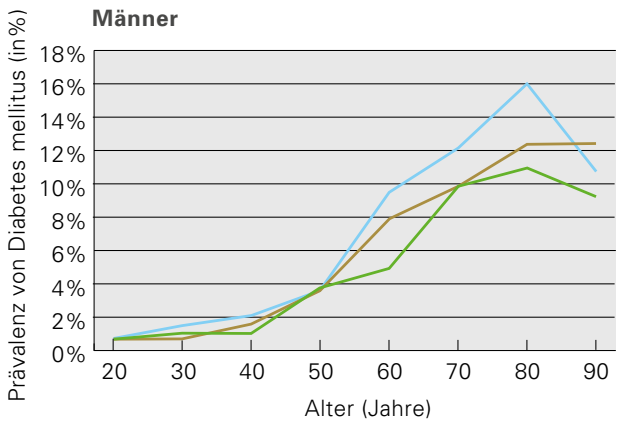

Schweizerische Gesundheitsbefragung, Jahr:

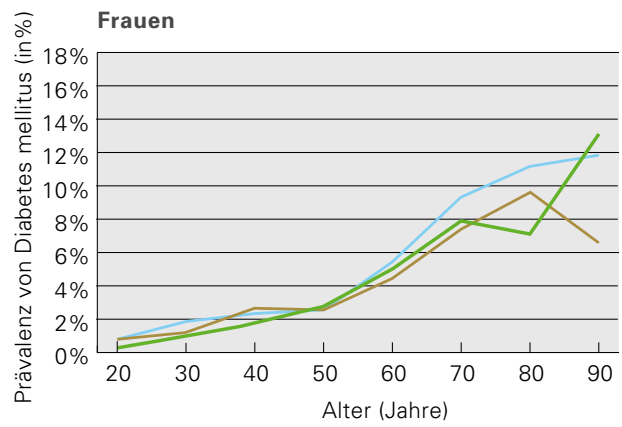
$2002-2007$

Abbildung 3.4: Prävalenz von Diabetes mellitus in der Schweiz aufgrund unterschiedlicher Quellen, nach Alter, Geschlecht und Jahr, Daten aus den Jahren 1995-2008 (21) 
(Kapitel 3.4.2). Im Vergleich zu Nicht-Diabetikern haben Typ-2-Diabetiker einen höheren mittleren BMI $\left(30.4 \mathrm{~kg} / \mathrm{m}^{2}\right.$ vs. $\left.25.5 \mathrm{~kg} / \mathrm{m}^{2}\right)$ und werden häufiger (22.8\% vs. $7.6 \%$ ) wegen einer Fettstoffwechselstörung behandelt (195). Die Studie Bus Santé weist über die Jahre 1993-2007 auf einen Trend hin zu einer Zunahme von Übergewichtigen und Adipösen sowie von Personen mit Diabetesbehandlung $(57 ; 104)$. Nachhaltiger Gewichtsverlust bei Diabetikern, wie er z.B. mit der bariatrischen Chirurgie möglich ist, führt häufig zu einer Normalisierung der Blutzuckerwerte (197).

In der Schweiz ist Diabetes mellitus Typ 2 bei Kindern sehr selten. Allerdings wurde bei übergewichtigen und adipösen Kindern in 6.5\% der Fälle ein Frühstadium einer Zuckerstoffwechselstörung gefunden, die ein Risikofaktor für Diabetes mellitus Typ 2 ist (107).

\section{Andere Risiko- und Schutzfaktoren}

Die Zusammensetzung der zugeführten Nährstoffe kann unabhängig davon, ob sie zu Übergewicht führt, das Diabetesrisiko beeinflussen (Kapitel 1.5.1). Übermässiger Konsum von Fett ist ein Risikofaktor für Störungen des Zuckerstoffwechsels bei Erwachsenen und Kindern $(112 ; 122 ; 123)$. Bei Kindern ist die Einnahme von Gesamtfett und Fett mit hohem Anteil an gesättigten Fettsäuren unabhängig vom BMI positiv mit dem Nüchterninsulinspiegel assoziiert (112). Hohe Insulinwerte im Blut spiegeln eine Insulinresistenz wider, welche das Risiko für Diabetes mellitus Typ 2 erhöht.

Art und Anteil von Kohlenhydraten an der Gesamtenergieaufnahme können das Diabetesrisiko ebenfalls beeinflussen. Im Fokus steht insbesondere Fructose (Kapitel 3.4.2), da eine fructosereiche Ernährung zu einer Insulinresis- tenz führen kann, die wiederum ein Risikofaktor für Zuckerstoffwechselstörungen ist (130). Diese Eigenschaft von Fructose tritt bei Nachkommen von Typ-2-Diabetikern häufiger in Erscheinung als bei Personen ohne familiäres Risiko (198). Neben Fructose stehen kohlenhydrathaltige Lebensmittel mit hohem glykämischem Index und hoher glykämischer Last im Verdacht, bei der Entstehung von Diabetes mellitus mitbeteiligt zu sein (130).

Der Zusammenhang zwischen der Menge an konsumiertem Alkohol und dem Diabetesrisiko ist U-förmig: Moderater Alkoholkonsum (bis zu 20 Standardgetränken pro Woche) schützt, während grössere Alkoholmengen das Risiko erhöhen können $(115 ; 199)$.

\subsubsection{Bedeutung}

Im 2008 waren Hormon- und Stoffwechselkrankheiten bei ca. 1.4\% (2002: 1.2\%) der Hospitalisierten der Einweisungsgrund und bei ca. 6.7\% (2002: 6\%) der Grund für den Besuch einer Arztpraxis $(30 ; 100 ; 144 ; 145)$. Im 2005 waren rund $10 \%$ der im Basler Universitätsspital hospitalisierten medizinischen Patienten Diabetiker (200).

Entsprechend hoch sind die Kosten von jährlich rund CHF 6'000.- pro Diabetiker $135 ; 194$; 201-203). Obwohl das Sterberisiko von Diabetikern in den vergangenen 20 Jahren abnahm, sterben Diabetiker immer noch früher als NichtDiabetiker, wobei weibliche Diabetiker stärker betroffen zu sein scheinen als männliche. Diabetes mellitus ist auch ein Risikofaktor für zahlreiche Krankheiten, besonders für Gefässkrankheiten (193; Kapitel 3.4). Die Inzidenz und Prävalenz von Diabetes mellitus werden voraus- 
sichtlich weiter steigen: Die Prävalenz von Diabetes mellitus soll in der Schweiz im Jahr 2030 bei den 20- bis 79-Jährigen schätzungsweise $10 \%$ betragen (194).

Fast $90 \%$ der Fälle mit Diabetes mellitus Typ 2 sind auf Übergewicht oder Adipositas zurückzuführen und sind somit mit einer entsprechenden Adipositasprävention vermeidbar (196; Kapitel 3.3.3). Neben regelmässiger körperlicher Aktivität wirkt auch eine ausgewogene Ernährung zusätzlich präventiv (Kapitel 3.3.3 und 3.4.3). Da Diabetes mellitus häufig unerkannt bleibt, aber bereits früh gesundheitliche Schäden verursachen kann, ist Früherkennung im Rahmen von Screenings wichtig, besonders bei Risikogruppen wie Schwangeren, Adipösen und Personen mit familiärem Risiko $(111 ; 113$; $146 ; 193 ; 195 ; 204)$. Die Zunahme von Diabetes mellitus in den vergangenen Jahren war bei älteren Männern wahrscheinlich stärker als bei gleichaltrigen Frauen (21). Dementsprechend müssen auch bei dieser Gruppe verstärkt Anstrengungen zur Früherkennung und Prävention unternommen werden.

\subsection{Osteoporose}

\subsubsection{Häufigkeit}

Gemäss Schätzungen lebten im Jahr 2000 in der Schweiz 1 bis 1.2 Mio. Menschen (> 50 Jahre alt) mit Osteopenie und 315'700 bis 348'400 Menschen mit Osteoporose (205). Laut SGB07 waren im Jahr 2007 1.4\% der Befragten ( $N=13^{\prime} 328$ ) wegen Osteoporose in Behandlung, und 1.3\% hatten in der Vergangenheit bereits eine Therapie zur Behandlung von Osteoporose erhalten (15).
Betroffen sind in erster Linie Frauen. Sie erreichen generell eine niedrigere maximale Knochenmasse ("peak bone mass») als Männer (206) und erleiden nach der Menopause zusätzlich einen Abnahmeschub (207). Im Allgemeinen beginnt um das 30. Altersjahr herum ein kontinuierlicher Knochenmasseabbau, und zwar umso stärker, je mehr Risikofaktoren vorhanden und je stärker ausgeprägt sie sind (208). Je mehr sich die individuelle Knochenmasse der maximal erreichbaren Knochenmasse annähert, desto geringer ist das Osteoporoserisiko. So haben Frauen mit einer 10\% höheren Knochenmasse als der Durchschnitt nach den Wechseljahren ein um $50 \%$ niedrigeres Risiko für osteoporotisch bedingte Frakturen (209).

\subsubsection{Risiko- und Schutzfaktoren}

Für die Knochengesundheit sind Merkmale wie Knochenmasse, Knochendichte und Knochenfestigkeit massgebend. Diese resultieren aus einem Zusammenspiel von mehreren Faktoren wie Vererbung, Geschlecht, Nährstoffversorgung (Calcium, Protein, Vitamin D), körperliche Aktivität, Muskelmasse, aber auch Medikamentenkonsum und Krankheiten. Zusätzlich beeinflussen auch hormonelle Veränderungen in der Pubertät die Effekte der Nährstoffe $(210 ; 211)$; einzelne Studien versuchten, dem Verständnis dieser komplexen Zusammenhänge näherzukommen $(212 ; 213)$.

\section{Calcium / Protein / Vitamin D}

Die ungenügende Calcium-, Protein- und Vitamin-D-Versorgung korreliert mit einem geringeren Knochenaufbau bzw. stärkeren Knochenabbau; möglicherweise sind noch weitere Mineral- 
stoffe und Vitamine beteiligt $(208 ; 210 ; 211 ; 214)$. Eine hohe Zufuhr gesättigter Fettsäuren könnte sich nachteilig auswirken (215).

Der geschätzte angenäherte Verzehr von Calcium ist in der Schweiz ausreichend und derjenige von Protein mehr als ausreichend; die Hauptquelle für Calcium sind Milch und deren Produkte, die ausserdem einen Viertel der Proteinzufuhr ausmachen (Kapitel 2). Da Lebensmittel nur wenig zur Vitamin-D-Versorgung beitragen und die Sonnenexposition für den Vitamin-D-Status einer Person entscheidend ist, sind Blutwerte in Bezug auf die Vitamin-DVersorgung aussagekräftiger als Verzehrsdaten. Die Vitamin-D-Versorgung der Schweizer Bevölkerung ist im Sommer ausreichend, im Winter dagegen häufig suboptimal; dann ist ein Knochenmasseabbau sehr wahrscheinlich (Kapitel 3.10.3).

Einzelne Studien weisen allerdings auf Versorgungslücken mit gewissen Nährstoffen bei bestimmten Bevölkerungsgruppen hin. So könnten Jugendliche unzureichend mit Calcium $(76 ; 212 ; 213)$ und Vitamin $D(213)$ versorgt sein. Verbesserungsbedarf in der Vitamin-D-Versorgung besteht vermutlich auch bei vollgestillten Säuglingen (216; Kapitel 3.10.3). Was schon in früheren Ernährungsberichten erwähnt wurde $(208 ; 217 ; 218)$, bestätigen neuere Studien: Ältere, in Heimen wohnhafte Menschen weisen häufig niedrige Serum-Vitamin-D-Spiegel sowie eine unzureichende Calcium- und Proteinzufuhr auf (219-221).

Umgekehrt zeigt die CoLaus-Studie, dass Menschen sich durch Einnahme von Supplementen vor einem Versorgungsmangel schützen wollen: 246 der 6'186 Studienteilnehmer haben Osteoporose, 44 von ihnen supplementieren mit Calcium, 91 mit Calcium plus Vita- min D $(141 ; 222)$. Das Risiko, erneut eine Fraktur zu erleiden, könnten hohe Vitamin-D-Gaben (> $17.5 \mu \mathrm{g}$ ) bei älteren Menschen mit einer bereits vorliegenden osteoporotischen Fraktur reduzieren $(223 ; 224)$.

\section{Körperliche Aktivität}

Verschiedene Studien belegen den positiven Einfluss von gewichtstragender körperlicher Aktivität und Kraftübungen wie Sprüngen, Sportspielen mit Richtungswechseln oder Krafttraining auf die Knochenbildung und Knochenfestigkeit $(211 ; 225 ; 226)$ sowie auch auf den Erhalt der Knochenmasse bei Frauen nach der Menopause (227-229) und bei Männern über 50 Jahren (230). Von gewichtsneutralen Ausdauersportarten wie Radfahren und Schwimmen profitiert der Knochen kaum (226). Besonders im Kindesalter und in der Adoleszenz hilft Bewegung, eine möglichst hohe Knochenmasse zu erreichen (211). Aber erst eine ausreichende Calcium- und Proteinzufuhr ermöglicht den positiven Effekt der körperlichen Aktivität auf die Knochensubstanz $(210 ; 212)$.

Ein Blick auf die Resultate des Sportobservatoriums zeigt eine regelmässig körperlich aktive Schweizer Bevölkerung, deren Anteil sich seit 2000 vergrösserte (231). Erfreulicherweise treibt die Mehrheit der Kinder und Jugendlichen neben dem obligatorischen Schulsport zusätzlich Sport (232; Kapitel 3.3.2).

\section{Andere Risiko- und Schutzfaktoren}

Störungen wie z.B. ein Mangel an Sexualhormonen, eine Überfunktion der Schilddrüse oder Cortisonüberschuss, aber auch Tabakkonsum und übermässiger Alkoholkonsum sowie viszerales Fett gehen mit einem erhöhten Osteoporoserisiko einher (233-237). 
Die Rolle von Vitamin K ist unklar (235). Die Bedeutung einer latenten, durch die Ernährung verursachten Acidose für die Knochengesundheit wird kontrovers diskutiert (238-242). Hoher Salzkonsum könnte einen negativen Einfluss auf die Knochenmasse haben (243).

\subsubsection{Bedeutung}

\section{Gesundheitliche und finanzielle Folgen}

Die Folgen von Osteoporose sind so genannte osteoporotische Frakturen, wie z.B. Wirbeleinbrüche oder Hüft-, Oberschenkelhals- und Unterarmfrakturen infolge eines Sturzes aus eigener Höhe. Daraus folgt eine erhöhte Morbidität sowie Einschränkung der Lebensqualität und Funktionalität im Alltag (Hilfsbedürftigkeit, Pflegebedürftigkeit). Im Jahr 2000 gab es in der Schweiz 35'560 im Spital behandelte Knochenbrüche (244). Ambulant behandelte Knochenbrüche (z.B. des Unterarms) wurden dabei nicht erfasst. Die jährliche Inzidenz für Wirbelfrakturen beträgt bei postmenopausalen Frauen über 50 Jahre 486 pro 100'000, bei Männern über 50 Jahre 243 pro 100'000; für Hüftfrakturen beträgt sie 621 pro 100'000 bzw. 232 pro 100'000; die Inzidenz nimmt mit dem Alter stark zu. Das Risiko, ab einem Alter von 50 Jahren eine osteoporotische Fraktur zu erleiden, wird bei Frauen auf $51.3 \%$ und bei Männern auf 20.2\% geschätzt $(245 ; 246)$.

Die WHO schätzte 2004, dass in Amerika und Europa im Jahr 2002 durch osteoporotische Frakturen 2.8 Mio. Lebensjahre aufgrund von Behinderung und / oder frühzeitigem Tod verloren gingen (zum Vergleich: Diabetes mellitus 5.8 Mio., Bluthochdruck 2 Mio., Prostatakrebs 1 Mio.), 1.2 Mio. alleine wegen einer Hüftfraktur (247).
Angesichts der Bevölkerungsentwicklung werden für das Jahr 2020 25-26\% mehr Osteoporose-Fälle erwartet als für das Jahr 2000 geschätzt wurde; die Anzahl Spitalbehandlungen aufgrund von osteoporotischen Hüft-, Wirbelkörper- und distaler Unterarmfrakturen wird voraussichtlich auf rund 46'000 im Jahr 2020 ansteigen. Die mit Osteoporose verbundenen stationären Kosten (Spital, Rehabilitationseinrichtung, Alters- und Pflegeheim) werden von ca. CHF 765 Mio. im Jahr 2000 auf CHF 1'011 Mio. im Jahr 2020 ansteigen (244).

\section{Vorbeugung und Früherkennung}

Das schon im 5. Schweizerischen Ernährungsbericht (5. SEB) gezogene Fazit gilt weiterhin: Eine lebenslange ausreichende alimentäre Zufuhr an Calcium und Protein (z.B. als Milchprodukt), eine genügende Sonnenexposition für die lebenswichtige subcutane Vitamin-D-Bildung sowie geeignete körperliche Aktivität ganz besonders im Kindes- und Adoleszentenalter sind für eine möglichst hohe Knochenmasse und gute Knochenstruktur und damit zur Vorbeugung von Knochenbrüchen im Alter unerlässlich (233). Im fortgeschrittenen Alter dienen dieselben Empfehlungen der Verlangsamung des altersbedingten Knochenabbaus (227;230; 248-251). Bereits die für Säuglinge empfohlene Vitamin-D-Supplementierung beugt Osteoporose vor, weil sie zu einer höheren Knochenmasse führt (250). Aufgrund neuerer Erkenntnisse wird auch eine Supplementierung von Vitamin D bei älteren Menschen empfohlen und selbst für die gesamte Bevölkerung in Erwägung gezogen (Kapitel 3.10.3).

Auf der Beliebtheitsskala der sportlich aktiven Schweizer stehen allerdings auch gewichtsneutrale, für die Knochengesundheit 
eher unwichtige Sportarten wie Radfahren und Schwimmen (231;232), welche mit Krafttraining ergänzt werden sollten.

Es gibt keine Studien, die zeigen, dass eine systematische Früherfassung (Screening) von Osteoporose zur Verhütung von Frakturen sinnvoll ist. Prinzipiell sollten Patienten mit einer Fraktur ohne adäquatem Trauma (Sturz aus eigener Höhe) sowie jene mit langdauernder Cortisontherapie auf Osteoporose untersucht werden. Das Frakturrisiko sollte bei Risikopersonen entsprechend den Empfehlungen der Schweizerischen Vereinigung gegen Osteoporose erfasst und osteoporotisch bedingte Frakturen entsprechend behandelt werden $(233 ; 252)$.

\subsection{Lebensmittelunverträglich- keiten}

\subsubsection{Häufigkeit}

Lebensmittelunverträglichkeiten können in nicht-immunologische und immunologische Intoleranzen aufgeteilt werden. Für detaillierte Beschreibungen der Krankheiten, deren (mögliche) Ursachen, Diagnostik, Therapie und Konsequenzen für die Ernährungsberatung wird auf entsprechende Übersichtsarbeiten verwiesen (Fructoseunverträglichkeit: 253; Lactoseintoleranz: 254;255; Zöliakie: 256; Lebensmittelallergie: 257).

Die Prävalenz von Lebensmittelunverträglichkeiten in der Schweiz kann nur geschätzt werden (Tabelle 3.7).

\subsubsection{Nicht-immunologische Intoleranzen}

\section{Fructoseunverträglichkeit}

Eine Fructoseunverträglichkeit kann aufgrund eines genetischen Defektes des Enzyms Aldolase B (hereditäre Fructoseintoleranz) oder einer gestörten enteralen Absorption (Fructosemalabsorption) vorliegen.

Schätzungsweise weist in Zentraleuropa eine von 26'100 Personen eine hereditäre Fructoseintoleranz auf (258). Sie kommt viel seltener vor als die Fructosemalabsorption, die je nach Ursache reversibel ist (253). Davon ausgehend, dass $80 \%$ der lactoseinteroleranten Personen auch Fructose nicht vertragen (259), könnte eine Fructosemalabsorption bei 12-16\% der Schweizer Bevölkerung angenommen werden (Tabelle 3.7).

\section{Lactoseintoleranz}

Die Häufigkeit von Lactoseintoleranz nimmt von Nordeuropa (<10\%) über Deutschland (15-20\%) nach Süd- und Osteuropa (bis 70\% in Süditalien und Türkei) zu $(254 ; 260)$. Es kann davon ausgegangen werden, dass in der Schweiz ähnlich wie in Deutschland 15-20\% der Bevölkerung Lactose nicht vertragen (Tabelle 3.7). Da Lactoseintoleranz in gewissen Ethnien weiter verbreitet ist $(253 ; 261 ; 262)$, ist ein häufigeres Vorkommen in gewissen Gruppen der Migrationsbevölkerung in der Schweiz denkbar.

Die Prävalenz eines primären kongenitalen Lactasemangels in Nordeuropa wird auf $2 \%$ geschätzt (255; Tabelle 3.7). 
Tabelle 3.7: Häufigkeit von Lebensmittelunverträglichkeiten in der Schweiz

\begin{tabular}{|c|c|c|}
\hline & Geschätzte Häufigkeit (in \%) & Referenz \\
\hline \multicolumn{3}{|c|}{ Nicht-Immunologische Intoleranzen } \\
\hline Fructosemalabsorption & $12-16 \%$ & $254 ; 259$ \\
\hline Fructoseintoleranz, hereditär & $1: 26^{\prime} 100$ & 258 \\
\hline Lactoseintoleranz & $15-20 \%$ & $254 ; 260$ \\
\hline Primärer Lactasemangel & $2 \%$ & 255 \\
\hline \multicolumn{3}{|l|}{ Immunologische Intoleranzen } \\
\hline Zöliakie & $1 \%$ & 256 \\
\hline \multirow[t]{5}{*}{ Lebensmittelallergien } & 2-3\% Erwachsene & 272 \\
\hline & 2-4\% Erwachsene & $257 ; 273 ; 274$ \\
\hline & 3.1\% Kinder (0-17 Jahre) & 271 \\
\hline & 2-6\% Kleinkinder & $257 ; 273 ; 274$ \\
\hline & 1-5\% Gesamtbevölkerung & 268 \\
\hline
\end{tabular}

Geschätzte Häufigkeiten, basierend auf Studien europäischer Länder und der Schweiz aus dem Zeitraum 1990-2010.

\subsubsection{Immunologische Intoleranzen}

\section{Zöliakie}

In der Schweiz leidet schätzungsweise 1\% der Bevölkerung an Zöliakie (256; Tabelle 3.7). Über die Verteilung zwischen den Geschlechtern oder die Prävalenz bei der Migrationsbevölkerung sind keine Angaben vorhanden. Da in der Türkei und in Kroatien Zöliakie häufiger als in der Schweiz vorkommt $(263 ; 264)$, kann eine höhere Prävalenz bei in der Schweiz wohnhaften Menschen mit türkischer oder kroatischer Herkunft angenommen werden.

\section{Lebensmittelallergien}

Die Liste der am häufigsten eine Allergie auslösenden Lebensmittel hat sich seit dem 5. SEB nicht verändert: Kuhmilch, Hühnerei, Erdnuss, Baumnuss, Fisch, Krebs und Krebstiere sowie Haselnuss, Sellerie, Apfel, Karotte, Mandel,
Pfirsich, Soja, Tomate und Erbse $(257 ; 265)$. Eine Fleischallergie ist in der Schweiz eher selten (266).

Das EU-Projekt EuroPrevall (267) hat den Kenntnisstand über die Prävalenz von Lebensmittelallergien, allergene Lebensmittel und betroffene Altersgruppen in der Europäischen Union einschliesslich der Schweiz verbessert, eine Datensammlung für Wissenschaftler sowie eine informative Website für Interessierte geschaffen (268). Gemäss diesen Analysen liegt die Prävalenz von klinisch belegten Lebensmittelallergien in Europa bei 1-5\%. Kinder reagieren hauptsächlich auf Kuhmilch und Eier allergisch, was mit dem Eintritt ins Schulalter häufig verschwindet; anschliessend sind sie meist auf Erdnüsse, Baumnüsse, Fisch und Früchte allergisch. Bei Erwachsenen wird überwiegend eine Allergie auf Früchte, Gemüse, Baumnüsse, Erdnüsse und meistens als Kreuz- 
Tabelle 3.8: Auswahl von häufigen kreuzreagierenden Pollen- und Lebensmittelallergien (269)

\begin{tabular}{l|l}
$\begin{array}{l}\text { Pollen / andere } \\
\text { Allergenquellen }\end{array}$ & Kreuzreagierende Lebensmittel \\
\hline Birke & $\begin{array}{l}\text { Apfel, Aprikose, Birne, Erdnuss, } \\
\text { Haselnuss, Karotte, Kartoffel, } \\
\text { Kirsche, Kiwi, Mandel, Nektarine, } \\
\text { Pfirsich, Pflaume, Sellerie, Soja }\end{array}$ \\
\hline Gräser & $\begin{array}{l}\text { Erdnuss, Kartoffel, Krautstiele, Melo- } \\
\text { ne, Orange, Tomate }\end{array}$ \\
\hline Beifuss & Banane, Gurke, Melonen, Zucchetti \\
\hline Platane & $\begin{array}{l}\text { Apfel, Erdnuss, grüne Bohnen, } \\
\text { Haselnuss, Kichererbse, Kiwi, Lat- } \\
\text { tich, Mais, Melonen, Pfirsich }\end{array}$ \\
\hline
\end{tabular}

a (270)

reaktion mit einer Pollenallergie oder Latexallergie festgestellt (Tabelle 3.8). Beispielsweise leiden $8 \%$ der europäischen Bevölkerung an einer Birkenpollenallergie, und $80 \%$ von ihnen entwickeln zusätzlich eine Lebensmittelallergie $(265 ; 269 ; 270)$.

Zur Inzidenz von Lebensmittelallergien liegen keine aktuellen Schweizer Daten vor; hingegen kann für die Schätzung der Prävalenz zusätzlich zu den Ergebnissen aus EuroPrevall auf verschiedene weitere Quellen zurückgegriffen werden (Tabelle 3.7).

Eine 2003 in der Schweiz durchgeführte repräsentative telefonische Befragung von Eltern nach einer Lebensmittelallergie ihrer Kinder (0-17 Jahre) ermittelte eine Prävalenz von 3.1\% (271). Folgende allergene Lebensmittel wurden identifiziert: Milch 34.8\%, Früchte 26.1\%, Eier $21.7 \%$, Fisch $17.4 \%$, Gemüse, Nüsse, Meeresfrüchte und Weizen jeweils $13.0 \%$, Fleisch und Hülsenfrüchte jeweils 8.7\%. Diese Studie, an der neben der Schweiz noch neun andere euro- päische Länder mitmachten, brachte nicht nur sehr unterschiedliche Prävalenzen (1.7-11.7\%) im Ländervergleich zu Tage, sondern zeigte auch, dass in den verschiedenen Ländern unterschiedlich viele Kinder auf ein bestimmtes Lebensmittel allergisch reagieren, z.B. auf Eier $7.1 \%$ in Österreich und $27.9 \%$ in Slowenien.

Auf Basis klinischer Untersuchungen (double-blind, placebo-controlled, food challenge) in ausgewählten europäischen Ländern liegt die geschätzte Häufigkeit von Lebensmittelallergien bei durchschnittlich 2-3\% (272). Im gleichen Rahmen liegen Daten anderer Schweizer Studien $(257 ; 271 ; 273 ; 274)$ : 2-6\% aller Kleinkinder, $3.1 \%$ der übrigen Kinder und ca. $2-4 \%$ der Erwachsenen sind von Lebensmittelallergien betroffen (Tabelle 3.7).

\subsubsection{Bedeutung}

Prävalenzentwicklung von Lebensmittelunverträglichkeiten

Neben der fehlenden Datenerfassung verhindert die heutzutage gegenüber früher verbesserte und häufigere Diagnosestellung eine objektive Beurteilung der Prävalenzentwicklung von Lebensmittelunverträglichkeiten. Da in der Schweiz mehr Menschen südeuropäischer, afrikanischer und asiatischer Herkunft leben als vor rund 30 Jahren und in diesen Ländern Lactoseintoleranz häufiger vorkommt, müsste sich dies entsprechend auf die Prävalenz von Lactoseintoleranz in der Schweiz auswirken.

Die Konsumgewohnheiten haben sich seit dem 5. SEB nicht wesentlich geändert (Kapitel 2.3.2); eine Änderung der Häufigkeit von Lebensmittelallergien kann darauf beruhend kaum erwartet werden. Allerdings hat die Häu- 
figkeit von Pollenallergien in der Schweiz zugenommen, weshalb mit mehr pollenassoziierten Lebensmittelallergien (Kreuzallergien) gerechnet werden muss (265).

\section{Prävention}

Früher wurde zur Prävention von Allergien die Vermeidungsstrategie verfolgt; heute wird eine Exposition mit Allergenen im Sinne einer potenziellen Toleranzinduktion diskutiert $(275 ; 276)$.

Zur Vermeidung von Zöliakie kann nur sehr wenig getan werden. Stillen und frühestens im 5. bzw. spätestens im 7. Lebensmonat während der Stillphase kleine Glutenmengen in der Beikost einführen, scheint Zöliakie vorzubeugen. Um ein Fortschreiten der Krankheit bzw. Rückfälle zu vermeiden, ist eine glutenfreie Ernährung unumgänglich (256). Die Lebensmittelkennzeichnung hilft bei der Wahl (277).

\section{Früherkennung}

Eine Lebensmittelunverträglichkeit sollte so früh wie möglich erkannt werden, um Folgeerkrankungen wie Nährstoffmangel, Wachstumsverzögerung bei Kindern und irreversible Darmschleimhautveränderungen zu vermeiden. In der Schweiz gibt es keine routinemässigen Früherkennungsmassnahmen für Lebensmittelunverträglichkeiten. Es bestehen jedoch Bemühungen verschiedener Fachgesellschaften und Organisationen, Kinder- und Schulärzte für die Symptome zu sensibilisieren und so betroffene Kinder möglichst früh zu erfassen und zu therapieren.

\section{Folgeerkrankungen und Begleiterscheinungen} Bei einigen Lebensmittelunverträglichkeiten besteht die Gefahr einer unzureichenden Versorgung mit einzelnen Nährstoffen. Diese Gefahr kann nicht in Zahlen gefasst, muss aber bedacht werden:

- Um den Folgen einer hereditären Fructoseintoleranz auszuweichen, müssen Betroffene jegliche Fructosequellen meiden; sie erkranken bei einer Exposition schwer oder können sogar versterben. Diejenigen mit einer Fructosemalabsorption meiden dagegen "nur» fructosereiche Früchte- und Gemüsesorten. Es stellt sich somit die Frage, ob z.B. eine ausreichende Versorgung mit Folsäure gewährleistet oder eine entsprechende Supplementierung notwendig ist. Bei Personen mit Fructosemalabsorption wird Folsäure- und Zinkmangel beobachtet (253).

- Wenn nicht auf lactosefreie Produkte und Lactasepräparate zurückgegriffen wird, birgt Lactoseintoleranz durch den Verzicht auf Milch und Milchprodukte das Risiko einer unzureichenden Calciumversorgung und eines erhöhten Osteoporoserisikos (254).

- Personen mit Zöliakie müssen glutenhaltige Getreideprodukte meiden und können deshalb eine unzureichende Einnahme an Nahrungsfasern aufweisen und einen Mangel an B-Vitaminen entwickeln (256).

- Auch Lebensmittelallergiker müssen darauf achten, dass sie keinen Nährstoffmangel entwickeln - je nachdem, wie viele verschiedene und welche Lebensmittel sie in welchem Ausmass meiden müssen. 
Von der Therapie einer Lebensmittelunverträglichkeit in Eigenregie muss dringend abgeraten werden. Eine eindeutige Diagnose sowie individuelle medizinische und ernährungstherapeutische Betreuung sind wichtig und nötig.

\section{Soziale und finanzielle Aspekte}

Um die Folgen einer Lebensmittelunverträglichkeit zu vermeiden, umgehen Betroffene den Konsum entsprechender Lebensmittel, was bei hereditärer Fructoseintoleranz, Zöliakie und einzelnen Lebensmittelallergien lebenswichtig ist. Zöliakiebetroffene dürfen eine breite Palette von Lebensmitteln nicht verzehren; Essen auswärts wird kompliziert. Entsprechende glutenfreie Produkte wie Teigwaren, Mehle und Brot sind in der Regel um ein Mehrfaches teurer als herkömmliche Produkte (256) und dadurch für Personen mit geringem Einkommen kaum oder nicht erschwinglich. Ebenso kann eine Lebensmittelallergie zu höheren Lebenshaltungskosten führen (278). Neben körperlichen und ernährungsbezogenen Einschränkungen können somit auch finanzielle Engpässe das Leben der Betroffenen erschweren.

\subsection{Essstörungen}

\subsubsection{Häufigkeit}

Die Hauptformen von Essstörungen sind Anorexia nervosa (AN, ICD-10: F50.0) und Bulimia nervosa (BN, ICD-10: F50.2). Daneben existieren Übergangsformen und Formen von schwer fassbaren problematischen Ernährungsverhaltensweisen $($ EDNOS = Eating Disorder Not Otherwise Specified, ICD-10: F50.1 / F50.3 /
F50.9). Zu diesen gehört die Binge-Eating-Störung (BED), welche zu einer eigenständigen Diagnosekategorie werden soll (276). Zusätzlich existiert die medizinisch nicht klassifizierte Orthorexia nervosa (ON) - die Sucht, richtig zu essen (279). Das Night-Eating-Syndrom, bei dem mindestens ein Viertel des Energiebedarfs am Abend bzw. in der Nacht konsumiert wird, wird auch in der Schweiz diskutiert. Ob dieses Syndrom eine Form abnormen Essverhaltens oder eher eine psychische Störung darstellt, ist Gegenstand der Forschung (280).

\section{Gestörtes Essverhalten (subklinische} Essstörung)

Vier aktuelle Befragungen versuchten Symptome für ein gestörtes Essverhalten bei Jugendlichen und Erwachsenen zu erfassen $(15 ; 17 ; 281$; 282). Die bereits weiter zurückliegende Studie Swiss Multicenter Adolescent Survey on Health (SMASH) wird hier in die Darstellung einbezogen, weil es die einzige grössere Studie mit Schweizer Jugendlichen ist (283). Die Resultate der Studien basieren auf unterschiedlichen, teilweise nicht validierten Methoden, was nur einen eingeschränkten Vergleich erlaubt; ausserdem werden bei der SGB und der SMASH-Studie die Kriterien nicht näher spezifiziert. Die Studien zeigen, dass willentliches Erbrechen zwar selten ist, aber Kontrollverlust und Essanfälle bzw. "viel essen» und "nicht aufhören können zu essen" recht häufig erwähnt werden (Tabelle 3.9). Über ähnliche Resultate bei Jugendlichen berichtete schon der 4. SEB (284).

Fragen zum Umgang mit dem Körpergewicht lassen das Vorhandensein von Essstörungen oder zumindest eine verzerrte Körperwahrnehmung vermuten. Seit der ersten $S G B$ ist der Anteil der Personen, die mit dem eigenen Kör- 
Tabelle 3.9: Häufigkeit von gestörtem Essverhalten in der Schweiz, Daten aus den Jahren 2002-2010

\begin{tabular}{|c|c|c|c|c|c|c|c|c|}
\hline \multirow[t]{2}{*}{$\begin{array}{l}\text { Studie } \\
\text { (Referenz) } \\
\text { Erhebungs- } \\
\text { zeitraum }\end{array}$} & \multirow[t]{2}{*}{$\begin{array}{l}\text { Anzahl } \\
\text { Personen }\end{array}$} & \multirow[t]{2}{*}{$\begin{array}{l}\text { Alter } \\
\text { (Jahre) }\end{array}$} & \multicolumn{2}{|c|}{$\begin{array}{c}\text { Enorm viel essen / } \\
\text { Essanfall in den letzten } \\
\text { 28 Tagen } \\
\text { (Häufigkeit in \%) }\end{array}$} & \multicolumn{2}{|c|}{$\begin{array}{l}\text { Nicht aufhören } \\
\text { können zu essen in } \\
\text { den letzten } 28 \text { Tagen } \\
\text { (Häufigkeit in \%) }\end{array}$} & \multicolumn{2}{|c|}{$\begin{array}{l}\text { Willentliches } \\
\text { Erbrechen in den } \\
\text { letzten } 28 \text { Tagen } \\
\text { (Häufigkeit in \%) }\end{array}$} \\
\hline & & & mind. $1 x^{b}$ & mind. $8 x^{c}$ & mind. $1 x^{b}$ & $\operatorname{mind} .8 x^{c}$ & mind. $1 x^{b}$ & $\operatorname{mind} .8 x^{c}$ \\
\hline $\begin{array}{l}\text { "Basel» } \\
\text { Befragung } \\
2009 / 10(281)\end{array}$ & $\begin{array}{l}\text { 1'514 (1'121 } \\
\text { Frauen und } \\
393 \text { Männer) }\end{array}$ & 21.7 & $58.7 \%$ & $14.6 \%$ & $29.9 \%$ & $5.2 \%$ & $4.3 \%$ & $0.9 \%$ \\
\hline $\begin{array}{l}\text { "Zürich» } \\
\text { Befragung }^{a} \\
2010(282)\end{array}$ & $\begin{array}{l}285 \text { prämeno- } \\
\text { pausale } \\
\text { Frauen }\end{array}$ & 45.9 & $31.9 \%$ & $8.5 \%$ & $21.4 \%$ & $6.5 \%$ & $2.1 \%$ & $1.1 \%$ \\
\hline $\begin{array}{l}\text { «Zürich» } \\
\text { Befragung } \\
2010(282)\end{array}$ & $\begin{array}{l}255 \text { post- } \\
\text { menopausale } \\
\text { Frauen }\end{array}$ & 55.9 & $30.2 \%$ & $9.9 \%$ & $25.5 \%$ & $7.9 \%$ & $2 \%$ & $1.2 \%$ \\
\hline \multirow[t]{2}{*}{$\begin{array}{l}\text { Studie } \\
\text { (Referenz) } \\
\text { Erhebungs- } \\
\text { zeitraum }\end{array}$} & \multirow[t]{2}{*}{$\begin{array}{l}\text { Anzahl } \\
\text { Personen }\end{array}$} & \multirow[t]{2}{*}{$\begin{array}{l}\text { Alter } \\
\text { (Jahre) }\end{array}$} & \multicolumn{3}{|c|}{$\begin{array}{c}\text { Viel und nicht aufhören können } \\
\text { zu essen } \\
\text { (Häufigkeit in \%) }\end{array}$} & \multicolumn{3}{|c|}{$\begin{array}{l}\text { Willentliches Erbrechend } \\
\text { (Häufigkeit in \%) }\end{array}$} \\
\hline & & & täglich & $\begin{array}{l}\text { ein-bis } \\
\text { mehrmals } \\
\text { proWoche }\end{array}$ & selten & täglich & $\begin{array}{l}\text { ein-bis } \\
\text { mehrmals } \\
\text { proWoche }\end{array}$ & selten \\
\hline $\begin{array}{l}\text { SGBO7 } \\
\text { Befragung }^{a} \\
2007(15)\end{array}$ & $\begin{array}{l}\text { 4'924 Frauen } \\
\text { 4'354 Männer }\end{array}$ & $15-49$ & $\begin{array}{c}0.9 \% \\
\text { Frauen } \\
1.1 \% \\
\text { Männer }\end{array}$ & $\begin{array}{l}5.1 \% \\
\text { Frauen } \\
4.5 \% \\
\text { Männer }\end{array}$ & $\begin{array}{l}16.5 \% \\
\text { Frauen } \\
15.2 \% \\
\text { Männer }\end{array}$ & 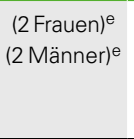 & $\begin{array}{c}0.3 \% \\
\text { (14 Frauen) } \\
0.1 \% \\
\text { (4 Männer) }\end{array}$ & $\begin{array}{c}1 \% \\
\text { Frauen } \\
1 \% \\
\text { Männer }\end{array}$ \\
\hline $\begin{array}{l}\text { SMASH } \\
\text { Befragung }^{\text {a }} \\
2002(283)\end{array}$ & $7^{\prime} 428$ & $16-20$ & - & $\begin{array}{l}16 \% \\
\text { Frauen } \\
9 \% \\
\text { Männer }\end{array}$ & - & - & $\begin{array}{l}2.7 \% \\
\text { Frauen } \\
0.7 \% \\
\text { Männer }\end{array}$ & - \\
\hline $\begin{array}{l}\text { Studie } \\
\text { (Referenz) }\end{array}$ & $\begin{array}{l}\text { Anzahl } \\
\text { Personen }\end{array}$ & $\begin{array}{l}\text { Alter } \\
\text { (Jahre) }\end{array}$ & \multicolumn{6}{|c|}{$\begin{array}{l}\text { Wiederkehrende Episoden von Essanfällenf, Essanfälle mind. 2x pro } \\
\text { Woche über mehrere Monate oder länger }\end{array}$} \\
\hline $\begin{array}{l}\text { Erhebungs- } \\
\text { zeitraum }\end{array}$ & & & \multicolumn{3}{|c|}{ Lebenszeitprävalenz $^{\mathrm{g}}$} & \multicolumn{3}{|c|}{ 12-Monate-Prävalenz ${ }^{h}$} \\
\hline $\begin{array}{l}\text { "Schweiz» } \\
\text { Befragunga } \\
2010(17)\end{array}$ & $\begin{array}{l}\text { 5'615 Frauen } \\
\text { 4'423 Männer }\end{array}$ & $15-60$ & \multicolumn{3}{|c|}{$\begin{array}{l}\text { 5.3\% Frauen } \\
\text { 2.9\% Männer }\end{array}$} & \multicolumn{3}{|c|}{$\begin{array}{l}\text { 1.56\% Frauen } \\
1.03 \% \text { Männer }\end{array}$} \\
\hline
\end{tabular}

a Für die genaue Befragungsmethodik und den exakten Wortlaut der Fragen wird auf die entsprechenden Originalpublikationen verwiesen.

b 1 x pro Monat bedeutet auffälliges Essverhalten

c $8 \times$ pro Monat bedeutet klinische Relevanz

d SGB07: in den letzten 3 Monaten, SMASH 2002: seit einigen Monaten

e Zahlen in Klammern zeigen bei 10-29 Antworten eine eingeschränkte statistische Zuverlässigkeit und bei $<10$ Antworten keine statistische Zuverlässigkeit; daher Angabe der absoluten Antworten. $f$ Essanfälle sind charakterisiert durch 1. Aufnahme einer ausserordentlich grossen Nahrungsmenge in sehr kurzer Zeitspanne und 2. Kontrollverlust während der Essanfälle

g Häufigkeit bezogen auf das ganze Leben

h Häufigkeit bezogen auf die letzten 12 Monate

- = keine Angabe 
pergewicht unzufrieden sind, nahezu konstant geblieben. So sind 33\% der an der SGB07 befragten 15- bis 49-jährigen Personen mit ihrem Körpergewicht unzufrieden $137 \%$ der Frauen, $29 \%$ der Männer) und rund $52 \%$ wollen es verändern (55\% der Frauen, 48\% der Männer): $45 \%$ wollen abnehmen und $7 \%$ zunehmen (Tabelle 3.1). Bei den Männern sind prozentual deutlich mehr mit hohem Bildungsniveau nicht mit ihrem Körpergewicht zufrieden und möchten abnehmen als solche mit tiefem Bildungsniveau; beim Wunsch zuzunehmen verhält es sich genau umgekehrt. Bei den Frauen hat das Bildungsniveau keinen Einfluss auf die Zufriedenheit mit dem Körpergewicht bzw. dem Wunsch, das Gewicht zu reduzieren; es sind hingegen mehr Ausländerinnen (40.1\%) als Schweizerinnen $(36.1 \%)$ unzufrieden mit ihrem Körpergewicht. Weiter geben $4.2 \%$ der Frauen und $2.1 \%$ der Männer an, ständig daran zu denken, dass sie abnehmen wollen. Auffällig ist, dass $29.8 \%$ der normalgewichtigen und $6.1 \%$ der untergewichtigen Frauen sowie $13.3 \%$ der normalgewichtigen und $1.5 \%$ der untergewichtigen Männer abnehmen wollen (15). Betrachtet man nur die 15- bis 24-Jährigen, so ist der Anteil derjenigen, die trotz Unter- oder Normalgewicht abnehmen wollen, seit 2002 konstant geblieben (74). Ein analoger Vergleich für die Gruppe der 25- bis 49-Jährigen ist aufgrund fehlender Daten nicht möglich.

Die SMASH-Studie 2002 kommt zu ähnlichen Ergebnissen: $60 \%$ der Mädchen und 40\% der Knaben (16-20 Jahre) sind mit ihrem Körpergewicht unzufrieden; 70\% dieser Mädchen (gegenüber 55\% im Jahr 1993; 285) und 50\% dieser Knaben (keine Daten für 1993) äusserten den Wunsch abzunehmen, 20\% der 16- bis 20-jährigen Mädchen denken sogar ständig daran (283).
Ca. 58\% der im Rahmen der HBSC-Studie 2010 befragten 11- bis 15-Jährigen schätzen ihr Körpergewicht als "ungefähr richtig» ein; dieser Anteil ist gegenüber 2006 (56\%) und 1994 (48\%) weiter gestiegen $(74 ; 286)$.

Eine Befragung von 709 Schweizer Athletinnen in neun Sportarten zu ihrer Körpergewichtsentwicklung lässt bei $9.0 \%$ mit Untergewicht und insbesondere bei $5.5 \%$ mit einem beträchtlichen Gewichtsverlust in den letzten zwei Jahren ein gestörtes Essverhalten vermuten; der Gewichtsverlust fand selbst während des normalen Wachstums in der Jugend statt (287).

Die Fragen in der SGB07 zu Fastengewohnheiten oder dazu, regelmässig zu essen oder Mahlzeiten auszulassen, können im Hinblick auf eine (sub-)klinische Essstörung nicht weiter interpretiert werden, weil der Kontext ungefragt blieb.

\section{Klinisch manifeste Essstörungen}

Erstmals liegen repräsentative Daten zu klinisch manifesten Formen von Essstörungen vor, welche die bisherigen Annahmen zur Prävalenz übertreffen (17;288). Von 5'615 befragten Frauen und 4'424 befragten Männern zwischen 15 und 60 Jahren weisen im Laufe ihres Lebens $1.2 \%$ der Frauen eine $A N, 2.4 \%$ eine $B N$ und $2.4 \%$ eine BED auf; bei den Männern belaufen sich die Prävalenzen auf $0.2 \% \mathrm{AN}, 0.9 \% \mathrm{BN}$, $0.7 \%$ BED (17). Die 12-Monate-Prävalenz ist entsprechend tiefer (17): Sie liegt bei Frauen bei $0.07 \%$ (AN), $0.56 \%$ (BN), $0.87 \%$ (BED) und bei Männern bei $0.03 \%$ (AN), $0.45 \%(B N)$, $0.28 \%$ (BED).

Eine internetbasierte Befragung an einem kleineren Kollektiv von 1'514 erwachsenen Schweizern aus dem Raum Basel (393 Männer, 1'121 Frauen) zwischen 18 und 26 Jahren fand 
ähnliche Häufigkeiten: $0.3 \% \mathrm{AN}, 1.3 \% \mathrm{BN}$ und $3.4 \%$ BED, wobei kein Mann mit AN und ca. halb so viele Männer wie Frauen mit BN bzw. BED dabei waren (280).

Die Inzidenz von klinisch manifester AN im Kanton Zürich beträgt seit den 1970er Jahren ca. 1.2 pro 100'000 Personen bzw. 19.7 pro 100'000 Frauen zwischen 12 und 25 Jahren (289).

BED kommt häufiger bei Adipösen als bei Normalgewichtigen vor $(288 ; 290)$; die Häufigkeit nimmt mit steigendem BMI zu (291). Gemäss ausländischen Studien weist ca. ein Drittel der Adipösen in Gewichtsabnahmeprogrammen eine BED auf $(290 ; 292)$.

Anzeichen für ON kommen relativ häufig vor: starke Anzeichen bei $27.9 \%$ und einige Anzeichen bei $41.2 \%$ der Personen ohne sonstige Essstörungen sowie bei ca. der Hälfte der Personen mit einer Lebenszeitdiagnose BN und BED (17).

\subsubsection{Risiko- und Schutzfaktoren}

Die Krankheitsbilder von Essstörungen, ihre somatischen Folgen und möglichen Ursachen wurden andernorts ausführlich beschrieben (288; 293-297). Auch neuroendokrine Ursachen müssen in Betracht gezogen werden (298). Über die Schutzfaktoren ist erst relativ wenig bekannt. Da Essstörungen in erster Linie psychisch begründet sind, liegen die meisten Schweizer Daten zu möglichen Risiko- und Schutzfaktoren für Essstörungen im psychischen Bereich vor.

\section{Psychische Merkmale}

Störungen der Affekte, des Selbstwerts und des Verhaltens kommen bei Personen mit Essstörungen gehäuft vor, wobei nicht immer klar abzugrenzen ist, welches Störungsbild zuerst vorlag $(17 ; 299)$.

Die repräsentative Schweizer Studie zu Essstörungen zeigte, dass Personen mit einer Lebenszeitdiagnose einer Essstörung vier- bis sechsmal häufiger ernsthaft psychisch erkrankten als Personen ohne eine Essstörung (17). Von 277 sich im Universitätsspital Zürich in Therapie befindlichen Frauen mit Essstörungen waren nur 44 frei von psychischen Abnormitäten (300). Eine detaillierte Analyse der Daten von 3'890 Knaben der SMASH-Studie 2002 zeigte bei $20.5 \%$ eine Kombination von Störungen im Essverhalten mit einer Reihe von Auffälligkeiten wie problematisches Gesundheitsverhalten (z.B. Diäterfahrung, häufige Episoden von Trunkenheit), soziale Schwierigkeiten (z.B. Hänseleien) oder traumatisierende Lebensereignisse (z.B. Depression, sexueller Missbrauch). Unter den Knaben mit traumatisierenden Lebensereignissen fanden sich ausserdem diejenigen mit dem höchsten BMI (> 90. Perzentile; 301).

\section{Sport}

Übermässiges Training und restriktives Essverhalten bei Leistungssportlern sind Hinweise auf eine Essstörung (302). Besonders Sportler der kompositorischen Sportarten (z.B. rhythmische Gymnastik, Eiskunstlauf, Ballett), Sportarten mit Gewichtslimiten, Ausdauersportarten sowie Sportarten, bei denen das Gewicht eine leistungsbestimmende Grösse ist, sind gefährdet, ein gestörtes Essverhalten zu entwickeln (287;303). Einerseits sind Personen mit dafür passenden körperlichen Voraussetzungen erfolgreicher, andererseits fördern Training und sportspezifische Ernährung die leistungsbestimmende Körperzusammensetzung und Kondition. In beiden Fällen kommt es zu einer engen 
Beziehung zwischen Selbstwert, sportlichem Erfolg und Körperform bzw. -gewicht. Allerdings kann Sport auch schützend wirken (304;305).

\section{Lebensmittelunverträglichkeiten und}

\section{Stoffwechselerkrankungen}

Das Meiden von bestimmten Lebensmitteln aufgrund einer Unverträglichkeit (z.B. bei Zöliakie) sowie das Beachten eines genauen Essplans (z.B. bei Diabetes mellitus Typ 1) erfordert eine kontrollierte Lebensmittelauswahl und kann daher ein rigides Essverhalten und andere zwanghafte Verhaltensweisen fördern, welche möglicherweise in eine Essstörung münden $(293 ; 295 ; 306)$. So zeigt eine Literaturübersicht, dass Frauen mit Diabetes mellitus Typ 1 im Vergleich zu gesunden Frauen ein zwei- bis dreifach erhöhtes Risiko für eine Essstörung (BN) aufweisen (295).

\section{Weitere Risikofaktoren}

Reduktionsdiäten und andere Massnahmen zur Gewichtsreduktion (fasten, wenig essen, Nikotinkonsum, Abführmittel, usw.) können das Risiko einer Essstörung erhöhen (284;301).

Ungewollte sexuelle Erlebnisse gehen mit einem erhöhten Risiko, eine Essstörung zu entwickeln, einher. So berichten $24.5 \%$ von 277 Frauen mit Essstörungen in einer Zürcher Kohortenstudie sowie 3.5\% von 798 Knaben mit gestörtem Essverhalten und $2.5 \%$ von 1'223 Knaben ohne gestörtem Essverhalten, aber mit Auffälligkeiten wie z.B. Angst vor einer Gewichtszunahme oder Lieben eines leeren Magens (unter $1 \%$ in der Kontrollgruppe), von einem sexuellen Missbrauch $(301 ; 307)$.

\subsubsection{Bedeutung}

Die Fragen, welches Körperbild in der westlichen Gesellschaft vorherrscht, welche Rolle dieses in der Identitätsfindung eines Menschen spielt, und wie die Medien damit umgehen, werden diskutiert (308). Der Anteil klinisch manifester Essstörungen in der Bevölkerung scheint zwar nicht alarmierend zu sein. Wenn allerdings ca. ein Viertel der bei der SGBO7 befragten 15- bis 49-Jährigen abnehmen wollen, obwohl sie normal- oder gar untergewichtig sind, sowie BN und BED in jeder Altersgruppe auftreten kann (17), sollte das nachdenklich stimmen und zum Handeln auffordern. Ebenso die Beobachtung, dass Adipositas und Essstörungen nicht selten gemeinsam angetroffen werden (17), zumal Adipositas in der Schweiz eine grosse Bedeutung hat (Kapitel 3.3). Hinzu kommen ca. 10-16\% von geschätzten 30'000 Typ-1-Diabetikern (192) mit einem erhöhten Risiko für Essstörungen. Schliesslich werden ein Viertel (AN, BN) bis knapp die Hälfte (BED) aller Betroffenen nicht von einer Fachperson behandelt (17).

\section{Langzeitverlauf / Prognose}

Die Prognosen für Frauen mit AN sind recht gut bekannt, diejenigen für Frauen mit BN noch nicht ausreichend $(296 ; 297)$. Von den an AN erkrankten Patientinnen sterben ca. $5 \%$ vorzeitig, von den überlebenden werden ca. $47 \%$ symptomfrei, ca. $35 \%$ befinden sich auf dem Wege der Besserung, und ca. 21\% leiden chronisch (309). Ca. 45\% der an BN erkrankten Frauen genesen vollständig oder fast vollständig und $27 \%$ partiell, $23 \%$ entwickeln eine chronische Bulimie, und $0.3 \%$ sterben vorzeitig (296). Liegen psychische Störungen vor, ist die 
Prognose schlechter (310). Hinzu kommt das "Wandern» einer essgestörten Person durch die diagnostischen Kategorien (310), was mit einer Verlagerung der medizinischen Begleiterscheinungen einhergeht.

Als prognostisch günstige Faktoren für $\mathrm{BN}$ können soziale Unterstützung und langfristig wirkende Bewältigungsstrategien («adaptive coping») angesehen werden (296).

Da sich die Prävalenz von AN in den vergangenen 30 Jahren in der Schweiz kaum verändert hat (289), kann von einer gewissen stabilen Häufigkeit ausgegangen werden. Auch in Deutschland geht man davon aus, dass die Häufigkeit von AN und BN stabil bleiben wird (311). Anzunehmen ist allerdings, dass zukünftig subklinische Formen der BED häufiger diagnostiziert werden, weil die Sensibilisierung für die entsprechenden Symptome zugenommen hat und es nicht wenige Adipöse gibt. BED bergen ihrerseits ein Risiko für Adipositas und Stoffwechselstörungen (312).

Da das gestörte mütterliche Verhältnis zu Essen, Körper und Körpergewicht an Töchter weitergegeben wird $(313 ; 314)$, beeinflusst der Therapieerfolg auch die Inzidenz von Essstörungen.

AN und BN beeinträchtigen auf unterschiedliche Weise die Zahngesundheit (315) und ziehen nicht unerhebliche zahnmedizinische Kosten nach sich.

\subsection{Mangelernährung}

Dieses Kapitel befasst sich mit der allgemeinen Mangelernährung sowie mit der kritischen Versorgung spezifischer Nährstoffe wie Folsäure, Vitamin D, Eisen, Jod und Selen. Die Versorgung mit Folsäure (Kapitel 2.4.5) scheint gemäss angenähertem Verzehr unzureichend zu sein, diejenige mit Jod und Selen wird schon länger verfolgt. Letztere stellt zwar im Moment kein Problem dar (Kapitel 2.4.6), aber sie hängt von wirtschaftlichen und politischen Entscheidungen ab und könnte in Zukunft eine neue Bedeutung erlangen. Beim Jod weist schliesslich eine aktuelle Studie auf eine Unterversorgung eines Teils der Bevölkerung hin (397; Kapitel 2.4.6). Obwohl der geschätzte angenäherte Verzehr von Eisen genügend ist (Kapitel 2.4.6), weisen doch Studien auf häufigen Mangel hin. Beim Vitamin D gibt es neue Fakten und Empfehlungen (316). Andere Nährstoffe wie Magnesium, Zink und Vitamin B12 sind wohl klinisch relevant, aber sie scheinen gemäss geschätztem angenähertem Verzehr in ausreichender Menge zugeführt zu werden (Kapitel 2.4.5 und 2.4.6); Studien mit detaillierten Hinweisen zur Versorgung mit diesen Nährstoffen liegen keine vor.

\subsubsection{Allgemeine Mangelernährung}

\subsubsection{Häufigkeit}

Im Durchschnitt sind ca. ein Fünftel bis ein Viertel der ins Spital eintretenden Personen mangelernährt bzw. weisen ein Risiko für Mangelernährung auf (317-320). Bei der Pflege zu Hause scheint der Anteil wesentlich grösser zu sein (321-323; Tabelle 3.10). Die geschätzte Häufigkeit hat sich seit ca. 10 Jahren nicht verändert (324). 


\subsubsection{Risiko- und Schutzfaktoren}

Eine ungenügende Versorgung mit nahezu allen Nährstoffen hat multifaktorielle Ursachen und betrifft verschiedene medizinische Bereiche. So können beispielsweise chronische Krankheiten, Medikamente, organische Einschränkungen (Störungen der Absorption von Nährstoffen), den Alltagsablauf beeinträchtigende muskuloskeletale Probleme, soziale Isolierung oder Appetitlosigkeit zu einer Mangelernährung führen (324-328). Gravierend wirkt sich ein durch die Schwere der Krankheit bedingter Körperproteinverlust aus, der wesentlich durch die alimentäre Protein- und Energiezufuhr beeinflusst wird. Wird diese sichergestellt, kann der Körperproteinverlust vermindert werden (265).
Aber auch die falsche Lebensmittelauswahl birgt die Gefahr einer Mangelernährung in sich, so dass sogar adipöse Menschen qualitativ mangelernährt sein können (325;329).

Vor Mangelernährung schützende Faktoren lassen sich von den Risiken ableiten und liegen im sozialen, medizinischen, pflegerischen und technischen Bereich $(325 ; 330)$. Eine Steigerung der Kauleistung durch eine Zahnprothese verbessert den Ernährungszustand nicht zwangsläufig (331).

\subsubsection{Bedeutung}

Persönliche und gesellschaftliche Dimension Mangelernährung verschlechtert die Lebensqualität durch erhöhte Krankheitsanfälligkeit

Tabelle 3.10: Häufigkeit von Mangelernährung in der Schweiz, Daten aus den Jahren 2003-2010

\begin{tabular}{|c|c|c|c|c|}
\hline $\begin{array}{l}\text { Anzahl } \\
\text { Personen }\end{array}$ & $\begin{array}{l}\text { Studieng } \\
\text { Durchschnitts- } \\
\text { alter }\end{array}$ & $\begin{array}{l}\text { uppe } \\
\text { Beschreibung der } \\
\text { Studiengruppe }\end{array}$ & Ort der Datenerhebung & $\begin{array}{l}\text { Mangelernährt } \\
\text { oder Risiko für } \\
\text { Mangelernährung } \\
\text { (Häufigkeit in \%) }\end{array}$ \\
\hline $32^{\prime} 837$ & $\begin{array}{l}\text { < } 45 \text { Jahre: } 8 \% \text { d } \\
\text { 45-64 Jahre: } 11 \% \text { d } \\
\text { 65-84 Jahre: } 22 \% \text { d } \\
\text { > } 85 \text { Jahre: } 28 \%{ }^{d}\end{array}$ & Spitaleintritt & $\begin{array}{l}7 \text { Deutschschweizer } \\
\text { Spitäler }\end{array}$ & $\begin{array}{l}20 \% \text { mit Risiko für } \\
\text { Mangelernährung oder } \\
\text { stark mangelernährt }\end{array}$ \\
\hline 430 & 63 Jahre & Spitaleintritt & Kantonsspital Graubünden & $\begin{array}{l}20 \% \text { mit Risiko für } \\
\text { Mangelernährung } \\
10 \% \text { mangelernährt }\end{array}$ \\
\hline 115 & 84.6 Jahre & Heimbewohner & $\begin{array}{l}\text { Alters- und Pflegeheime } \\
\text { im Raum St. Gallen }\end{array}$ & $16.9 \%$ mangelernährt \\
\hline 78 & 86 Jahre & $\begin{array}{l}\text { In Pflegeheimen wohnende } \\
\text { Frauen ohne lebensbe- } \\
\text { drohliche Krankheit }\end{array}$ & $\begin{array}{l}11 \text { Pflegeheime im Raum } \\
\text { Lausanne }\end{array}$ & $\begin{array}{l}58 \% \text { mit Risiko für } \\
\text { Mangelernährung } \\
15 \% \text { mangelernährt }\end{array}$ \\
\hline
\end{tabular}

\footnotetext{
a NRS 2000: Nutritional Risk Screening 2000

b MNA: Mini Nutritional Assessment

c MUST: Malnutrition Universal Screening Tool

d Anteil derTeilnehmer in den Altersgruppen

- = keine Angabe
} 
und Mortalität $(251 ; 324)$. Auch ist Mangelernährung mit Sarkopenie und Gebrechlichkeit (Frailty) assoziiert (332). Die Daten einer Longitudinalstudie von Lausanne werden mehr Hinweise über Manifestationen und Determinanten von Gebrechlichkeit liefern; sie lagen zum Zeitpunkt der Texterstellung aber noch nicht vor (333). In einer europäischen Stichprobe (inkl. Schweiz) mit 16'584 50- bis 65-Jährigen waren $4.1 \%$ (95\% Cl 3.4-4.7) gebrechlich und 37.4\% (95\% Cl 35.8-39.1) befanden sich im Vorstadium der Gebrechlichkeit (329). In der Gruppe der über 65-Jährigen waren es 17.0\% (95\% Cl 15.3-18.7) bzw. 42.3\% (95\% Cl 40.5-44.1). $\mathrm{Ob}$ und inwieweit die untersuchten Personen mangelernährt waren, wurde nicht untersucht.

\begin{tabular}{|c|c|c|}
\hline $\begin{array}{c}\text { Erhebungs- } \\
\text { methode }\end{array}$ & Erhebungsjahr & Referenz \\
\hline NRS 2000a & $2003-2006$ & 317 \\
\hline MNA $^{b}$ & 2004 & 318 \\
\hline MUST $^{c}$ & & 321 \\
\hline NRS 2000 & & 322 \\
\hline MNA & 2010 & \\
\hline b & & \\
\hline
\end{tabular}

Mangelernährung zieht hohe Kosten nach sich: Im Jahr 2004 führte sie bei 118'000236'000 hospitalisierten Personen zu Mehrkosten von ca. CHF 526 Mio. (334).

Da hauptsächlich ältere Menschen betroffen sind und diese Altersgruppe in den nächsten Jahren anwachsen wird, gewinnt Mangelernährung an Bedeutung. Man geht von einer Verdoppelung des gesamten Pflegebedarfs in den nächsten 20 Jahren aus (335). Das Problem wird daher Spitäler, Heime und die Pflege zuhause («Spitex») besonders betreffen (322;336).

Eine weitere Risikogruppe sind Personen, die wegen einer massiven Adipositas operativ behandelt wurden (Magenband, Magenbypass oder andere Verfahren); sie weisen ein erhöhtes Risiko für eine Mangelernährung auf (326). Diese Operationen bedingen deshalb eine konsequente Nachsorge.

\section{Politische Dimension}

Bereits im 5. SEB wurde auf die Existenz und die Gefahren von Mangelernährung aufmerksam gemacht - welche Änderungen Spitäler und andere betroffene Institutionen seitdem vollzogen haben, ist allerdings kaum bekannt. In den Spitälern, in denen im Rahmen von Studien der Ernährungszustand von Patienten erfasst wurde, wurden die Spitalangestellten nachweislich für Mangelernährung sensibilisiert (317;337): Der Anteil der als mangelernährt eingestuften Personen, die eine entsprechende Ernährungstherapie erhielten, stieg von $63 \%$ im ersten, über $72 \%$ im zweiten auf $78 \%$ im dritten Jahr der Erhebung (317). Diese erfreuliche Entwicklung darf aber nicht darüber hinwegtäuschen, dass immer noch viele mangelernährte Personen unerkannt bleiben und somit keine adäquate Ernährungstherapie erhalten. Trotz 
bestehender anerkannter Instrumente zur Erfassung und zum Monitoring im Spital sowie zur Ernährungstherapie $(317 ; 324 ; 336 ; 338 ; 339)$ fehlt der politische Wille und die finanzielle Unterstützung zur Schaffung notwendiger Rahmenbedingungen für die Umsetzung im Spital.

\subsubsection{Folsäuremangel}

\subsubsection{Häufigkeit}

Auf Basis des niedrigen geschätzten Verzehrs von $295 \mu \mathrm{g} / \mathrm{Tag}$ (Tabelle 2.20; empfohlen sind $400 \mu \mathrm{g} / \mathrm{Tag}$ ) und der Resultate der SGB07 kann von einer unzureichenden Folsäureversorgung ausgegangen werden: Nahezu 4 von 5 der in der SGB07 Befragten verzehren weniger Früchte und Gemüse (Hauptquellen von Folsäure) als empfohlen (340). Die Situation hat sich gegenüber dem 5. SEB bzw. der SGBO2 nicht verbessert. Welche Rolle mit Folsäure ergänzte Lebensmittel in der Folsäureversorgung spielen, ist unbekannt. Neuere Daten über die Folsäuresupplementierung vor und zu Beginn der Schwangerschaft zur Prävention von Neuralrohrdefekten wurden seit dem 5. SEB nicht publiziert. Damals wurde ein Verbesserungspotenzial vor allem bei Frauen mit Migrationshintergrund (Südosteuropa) festgestellt (341).

\subsubsection{Risiko- und Schutzfaktoren}

Gemüse, Früchte und Getreide sind die wichtigsten Lieferanten von Folsäure über die Nahrung (Kapitel 2.4.5). Entsprechend ist ein geringer Verzehr dieser Lebensmittel ein Risiko für eine unzureichende Folsäureaufnahme. Weitere Risikofaktoren sind die Einnahme bestimmter Medikamente und Nikotinkonsum (342).

\subsubsection{Bedeutung}

Neuralrohrdefekte (Spina bifida, Anenzephalie) kommen bei Säuglingen von Müttern mit einer unzureichenden Folsäureversorgung gehäuft vor. Es gibt Hinweise, dass eine Supplementierung mit Folsäure zusammen mit anderen Vitaminen während der Schwangerschaft neben Neuralrohrdefekten auch das Risiko für weitere Fehlbildungen wie z.B. Gaumen-Lippen-Spalten oder am Herzen vermindert (342). Aus diesem Grunde wird Frauen, die schwanger werden wollen oder könnten, die tägliche Einnahme eines Folsäuresupplementes à $400 \mu \mathrm{g}$ empfohlen (Kapitel 1.4.1). In allen pädiatrischen Ausbildungszentren und 4 Ultraschallzentren wurde in den Jahren 2001-2007 in 167 Fällen eine Diagnose für Neuralrohrdefekt gestellt (343). Der Kanton Waadt erfasst seit 1992 Neuralrohrdefekte und ermittelte im gleichen Zeitraum 58 Neuralrohrdefekte (344). In beiden Erhebungen schwankt die Inzidenz, und kein Trend ist ableitbar. Leider liegen nicht genügend Angaben über die Einnahme von Folsäuresupplementen vor. Aber obige Studien lassen vermuten, dass sich die Folsäurezufuhr über angereicherte Lebensmittel und Supplemente nicht auf die Häufigkeit von Neuralrohrdefekten in der Schweiz ausgewirkt hat. Ganz im Gegensatz zu USA und Kanada, die seit der obligatorischen Anreicherung von Mehl mit Folsäure weniger Neuralrohrdefekte aufweisen (345).

Die Anreicherung eines Grundnahrungsmittels wie z.B. Mehl mit Folsäure, bei der die Gesamtbevölkerung betroffen ist, sollte allerdings kritisch betrachtet werden: Es kann nicht ausgeschlossen, aber auch nicht bestätigt werden, dass zusätzlich verabreichte Folsäure das Wachstum von Krebszellen, die sich bereits in einem späten Entwicklungsstadium befinden, 
im Menschen fördert (346; Kapitel 1.6 und 3.5.2). Der Nutzen der perikonzeptionellen Einnahme von Folsäuresupplementen für Frauen, die schwanger werden wollen oder könnten, bleibt grundsätzlich aber unbestritten.

\subsubsection{Vitamin-D-Mangel}

\subsubsection{Häufigkeit}

Neben den Resultaten weniger Studien (219;325; $347 ; 348 ; 396)$ an einzelnen Bevölkerungsgruppen gibt eine aktuelle Studie (221) Auskunft über die 25-Hydroxy-Vitamin-D-Konzentration [25(OH)D2 und 25(OH)D3] im Serum ${ }^{1}$ gesunder erwachsener Schweizer (Tabelle 3.11): Der Jahresdurchschnitt liegt bei $65.1 \mathrm{nmol} / \mathrm{L}$, wobei in den Winter- und ersten Frühjahrsmonaten der Anteil Personen mit weniger als $25 \mathrm{nmol} / \mathrm{L}$ $25(\mathrm{OH}) \mathrm{D}$ bei maximal $13 \%$ liegt (Abbildung 3.5). Der Anteil des pflanzlichen 25(OH)D beträgt dabei nur 2.8\% des durchschnittlichen Vitamin-DSpiegels (221). Wird nur der Durchschnitt Oktober bis Juni angeschaut (53.9 nmol/L), dann entspricht dieser ungefähr demjenigen, der im Rahmen der MONICA-Studie festgestellt worden war: durchschnittlich $50 \mathrm{nmol} / \mathrm{L}$ (349). Eine $25(\mathrm{OH})$ D-Konzentration von > $50 \mathrm{nmol} / \mathrm{L}$ wird als optimal erachtet (316; Kapitel 3.10.3.3).

Über den Vitamin-D-Status bei Schweizer Kindern und Jugendlichen liegen keine repräsentativen Daten vor. Eventuell sind gestillte Säuglinge nicht ausreichend mit Vitamin D versorgt: Bei einer 2003 durchgeführten Erhebung der Vitamin-D3-Gabe an Säuglinge in der Schweiz wurde festgestellt, dass nur $64 \%$ von

\footnotetext{
$120 \mathrm{nmol} / \mathrm{L} 25(\mathrm{OH}) \mathrm{D}=8 \mathrm{ng} / \mathrm{ml} 25(\mathrm{OH}) \mathrm{D} ; 10 \mu \mathrm{g}$ Vitamin $\mathrm{D}=$ 400 IE (Internationale Einheiten) Vitamin D
}

2'861 Säuglingen die empfohlenen Vitamin-DTropfen bekamen - und zwar in der Deutschschweiz weniger Säuglinge als in den übrigen Schweizer Regionen. Mütter aus Ländern des Balkans befolgten die Vitamin-D-Empfehlungen häufiger als andere Mütter, ältere Mütter eher als jüngere und ausschliesslich stillende Frauen weniger als teilweise stillende Frauen (216). Mit den empfohlenen $10 \mu \mathrm{g}$ Vitamin D3 pro Tag im ersten Lebensjahr wird Rachitis vorgebeugt. Eine kleine Waadtländer Studie fand bei $15 \%$ von 92 Knaben und 17\% von 104 Mädchen (11-16 Jahre) einen 25(OH)D3-Gehalt von $<30 \mathrm{nmol} / \mathrm{L}$ (213).

\subsubsection{Risiko- und Schutzfaktoren}

Zur Vitamin-D-Versorgung trägt der Lebensmittelverzehr nur wenig bei (Kapitel 2.4.5). Die Hauptquelle für Vitamin $D$ ist die körpereigene, von der UV-Strahlung abhängige Vitamin-DSynthese. Bei einem erheblichen Vitamin-DMangel treten Rachitis und Osteomalazie auf (350; Kapitel 1.5.3).

Die Effektivität der Sonneneinstrahlung zur Vitamin-D-Synthese in der Haut hängt vom Hauttyp, Alter, der Jahreszeit, der geografischen Lage des Wohnortes und der Verwendung von Sonnenschutzmitteln ab. Je dunkler die Haut, umso weniger Vitamin D bildet sie. Ab dem 35. Breitengrad Nord reicht die Sonneneinstrahlung im Winter nicht für eine subcutane Vitamin-D-Synthese (350) - die Schweiz liegt auf dem 47. Breitengrad. Die gemessenen 25(OH)D-Konzentrationen im Serum gesunder Erwachsener unterliegen entsprechend grossen jahreszeitlichen Schwankungen (Abbildung 3.5). Da Vitamin D nur eine Halbwertszeit von ca. 6-8 Wochen hat (351), reicht die im Sommer gebildete Vitamin-D-Menge nicht aus, 
Tabelle 3.11: Vitamin-D-Status verschiedener Bevölkerungsgruppen der Schweiz, Daten aus den Jahren 2004-2010

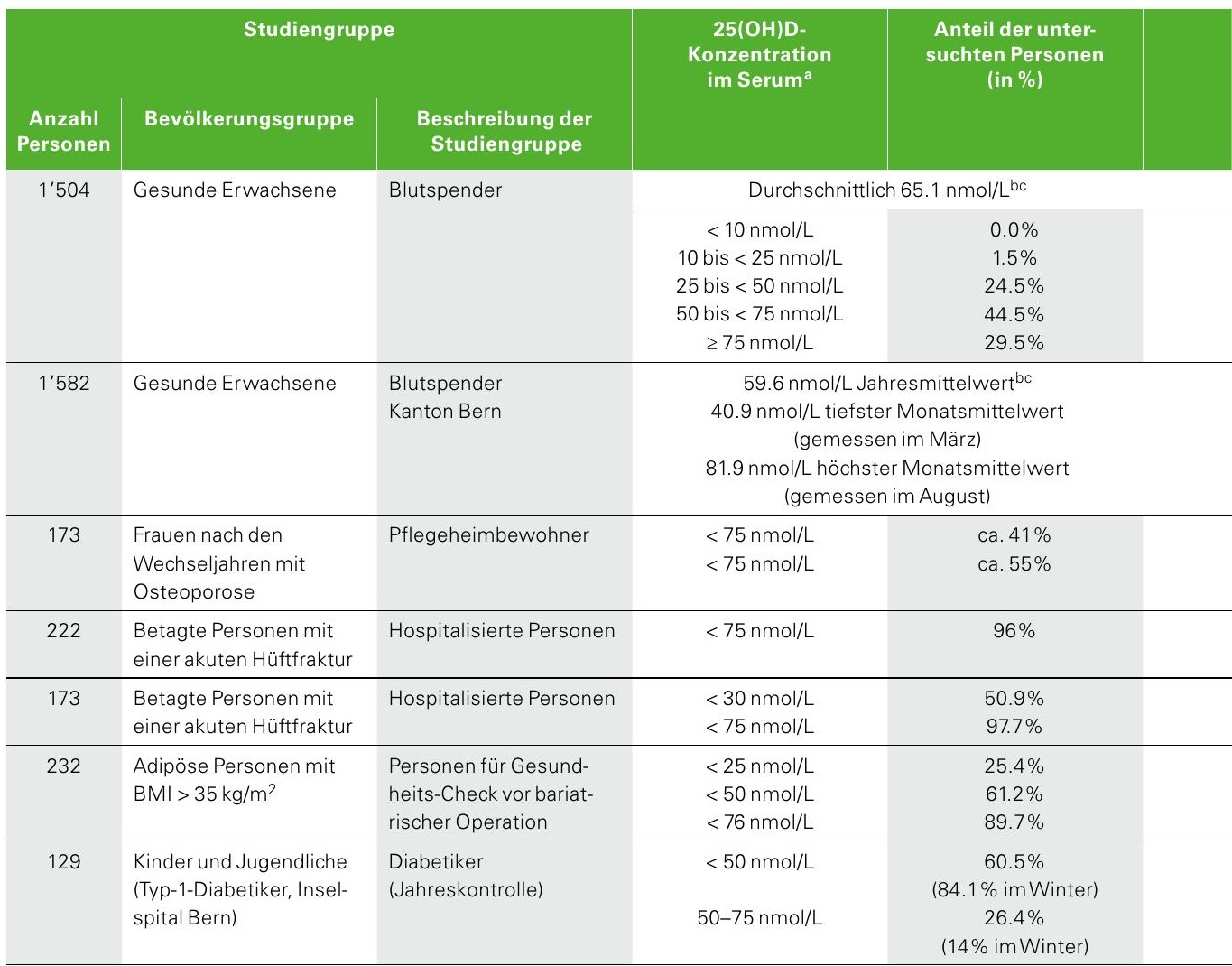

a Konzentrationsbereiche von den einzelnen Studienleitern festgelegt; 25(OH)D: 25-Hydroxy-Vitamin-D

b Vitamin D2 + Vitamin D3

c Mittelwert der Monatsmittelwerte

- = keine Angabe

um bis zum kommenden Frühjahr einen ausreichenden Vitamin-D-Status zu gewährleisten.

Personen, die keine Vitamin-D-haltigen Lebensmittel verzehren und die sich vor allem nicht genügend der Sonne aussetzen, riskieren ein Vitamin-D-Defizit. Unabhängig davon be- steht ein Risiko für Vitamin-D-Mangel bei älteren Menschen (352;353) und Säuglingen (Kapitel 1.3.1). Die Einnahme von Vitamin D als Supplement kann helfen, einem Vitamin-DMangel in speziellen Lebenssituationen vorzubeugen. 


\section{$>$ Ernährung und Gesundheit}

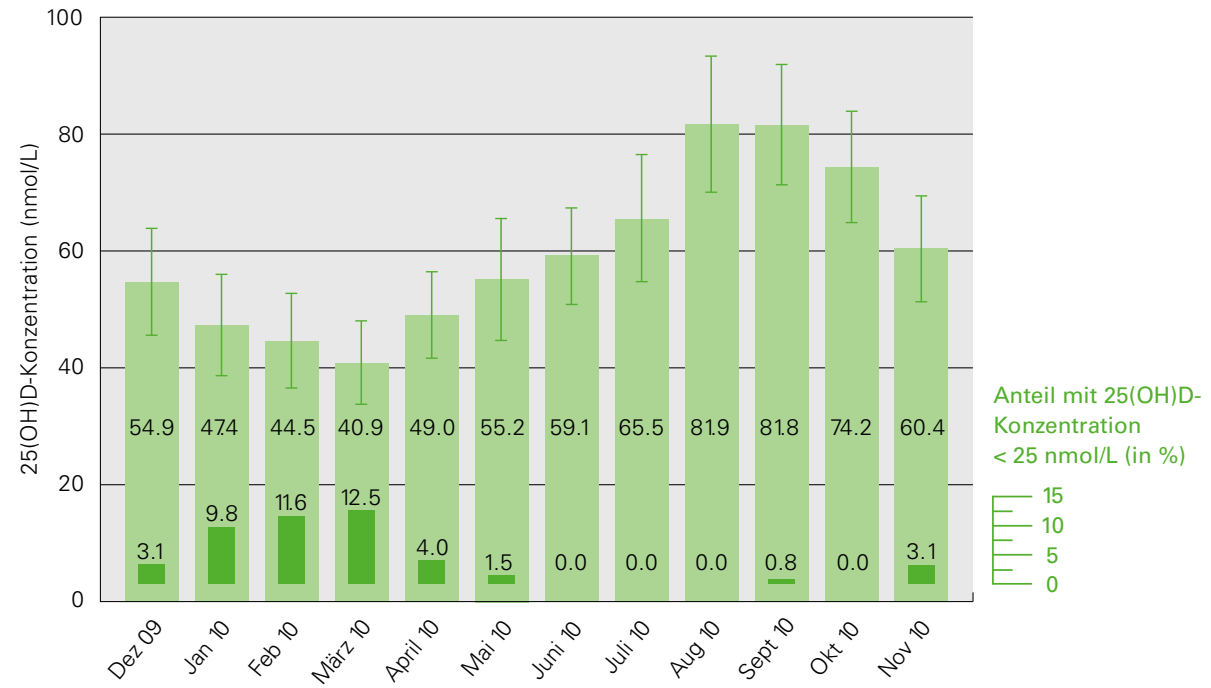

Jeden Monat wurden 130 Personen erfasst, über den gesamten Zeitraum waren es 1'582 Personen.

Es sind die Mittelwerte und die Standardabweichungen angegeben.

25(OH)D: 25-Hydroxy-Vitamin-D

Abbildung 3.5: 25-Hydroxy-Vitamin-D-Konzentrationen im Serum von gesunden, erwachsenen Blutspendern, Dezember 2009 bis November 2010 (221)

über den Stellenwert der alimentären, supplementären und subcutanen Herkunft von Vitamin D, noch über die Auswirkungen auf die Gesundheit, noch über die Notwendigkeit einer bevölkerungsweiten Supplementierung zu (Kapitel 1.5.3).

Neben der belegten Wirkung von Vitamin D auf die Knochengesundheit wird aktuell die Wirkung bei anderen Krankheiten wie Immunschwäche, Krebs und Asthma diskutiert (316; 355). Neue wissenschaftliche Erkenntnisse sind in diesen Bereichen zu erwarten. 


\subsubsection{Eisenmangel}

\subsubsection{Häufigkeit}

Der tägliche Eisenverzehr wird auf $11.6 \mathrm{mg}$ pro Person geschätzt (Tabelle 2.22), was zwar der gewichteten Zufuhr entspricht, aber vermutlich für Frauen ungenügend ist, um einen wünschenswerten Hämoglobingehalt von mindestens $12-13 \mathrm{~g} / \mathrm{dl}$ und eine Serumferritinkonzentration von mindestens $15 \mu \mathrm{g} / \mathrm{L}$ zu erzielen (356). Verschiedene, teils auch ältere Studien lassen einen nicht seltenen Eisenmangel bei Frauen vermuten (105;357-362; Tabelle 3.12).

\subsubsection{Risiko- und Schutzfaktoren}

Als Risikofaktoren für einen ungenügenden $\mathrm{Ei}$ senstatus können u.a. genannt werden: eisenarme Ernährung, starke Blutverluste (Menstruation, häufige Blutspenden, Operationen), häufige Schwangerschaften, Hämodialyse oder Resorptionsstörungen (357;361-366). Auch Übergewicht oder Adipositas könnten ein Risikofaktor sein: Trotz gleich hoher Eisenzufuhr und -verfügbarkeit zeigen übergewichtige 6-bis 12-jährige Schweizer Kinder ( $N=85)$ häufiger Eisenmangel als normalgewichtige $(\mathrm{N}=33)$, wobei die Ursache noch unklar ist (367).

Die alimentäre Eisenaufnahme spielt bei der Vorbeugung eines ungenügenden Eisenstatus eine zentrale Rolle. Da die Nahrung aber auch Verbindungen enthält, welche die Eisenaufnahme im Darm fördern (z.B. Vitamin C) oder hemmen (z.B. Phytinsäure, Polyphenole), sowie die Verfügbarkeit von Eisen aus Fleisch höher ist als jene aus pflanzlichen Lebensmitteln, bestimmt die Auswahl und Zusammenstellung von Lebensmitteln den Eisenstatus ebenfalls (368). Diese Zusammenhänge sind bei einer Therapie eines ungenügenden Eisenstatus zu bedenken.
Welche Rolle die Ernährung für den Eisenstatus der Schweizer Bevölkerung spielt, kann mangels Daten zurzeit nicht abgeschätzt werden.

\subsubsection{Bedeutung}

Wenn bei mehr als 5\% der Bevölkerung ein niedriger Hämoglobinwert festgestellt wird, stellt dies laut der Weltgesundheitsorganisation (World Health Organization, WHO), United Nations Children's Fund (UNICEF) und United Nations University (UNU) ein Problem der öffentlichen Gesundheit dar (369). Legt man die Resultate der nur Teile der Schweizer Bevölkerung umfassenden Studien (Tabelle 3.12) sowie die ernsthaften Folgen von Eisenmangel zugrunde $(357 ; 365 ; 370)$, sollte der Eisenversorgung in der Schweiz mehr Beachtung geschenkt werden.

Sicher ist, dass Frauen vor der Menopause ein erhöhtes Risiko für einen ungenügenden Eisenstatus haben. Das Risiko "häufige Schwangerschaften» traf 2009 auf 24'765 Frauen mit drei Kindern, 7'552 mit vier, 1'716 mit fünf und 518 mit mehr als fünf Kindern zu (371). Zur gynäkologischen Kontrolle gehört die Messung des Hämoglobingehaltes, so dass bei allen Frauen, die diese Kontrolle regelmässig machen, eine Anämie - spätestens dann - erkannt wird.

Weitere Risikogruppen für Eisenmangel und Anämie sind Ausdauersportler, insbesondere Spitzenläufer (372), sowie Adipöse. 
Tabelle 3.12: Prävalenz von Eisenmangel, Anämie und Eisenmangelanämie in der Schweizer Bevölkerung: Resultate einzelner Studien (Daten aus den Jahren 1996-2009)

\begin{tabular}{|c|c|c|c|c|}
\hline $\begin{array}{l}\text { Anzahl } \\
\text { Personen }\end{array}$ & Studiengruppe & $\begin{array}{l}\text { Anteil in der Studiengruppe (in \%) bzw. } \\
\text { Anzahl Personen (absolute Zahlen) }\end{array}$ & $\begin{array}{l}\text { Erhebungs- } \\
\text { zeitraum }\end{array}$ & Referenz \\
\hline 672 & $\begin{array}{l}\text { 18- bis 42-jährige Frauen } \\
\text { (Region Zürich) }\end{array}$ & $\begin{array}{l}22.7 \% \text { Eisenmangel (Serumferritin }<15 \mu \mathrm{g} / \mathrm{L} \text { ) } \\
2.8 \% \text { Anämie } \\
2.2 \% \text { Eisenmangelanämie }\end{array}$ & 2009 & 357 \\
\hline 7'714 & $\begin{array}{l}\text { 18- bis } 20 \text {-jährige Schweizer } \\
\text { Rekruten }\end{array}$ & $\begin{array}{l}\text { 7.2\% Eisenmangel (Serumferritin }<30 \mu \mathrm{g} / \mathrm{L} \text { ) } \\
0.1 \% \text { Anämie }\end{array}$ & 2004 & 105 \\
\hline 127 & $\begin{array}{l}\text { Durchschnittlich 43-jährige } \\
\text { männliche Marathonläufer } \\
\text { aus der gesamten Schweiz } \\
\text { (Zürich-Marathon) }\end{array}$ & $\begin{array}{l}1 \text { Eisenmangel (Serumferritin }<15 \mu \mathrm{g} / \mathrm{L} \text { ) } \\
1 \text { Eisenmangelanämie }\end{array}$ & 2006 & 359 \\
\hline 43 & $\begin{array}{l}\text { Durchschnittlich 39-jährige } \\
\text { weibliche Marathonläufe- } \\
\text { rinnen aus der gesamten } \\
\text { Schweiz } \\
\text { (Zürich-Marathon) }\end{array}$ & $\begin{array}{l}6 \text { Eisenmangel (Serumferritin }<15 \mu \mathrm{g} / \mathrm{L} \text { ) } \\
6 \text { Eisenmangelanämie }\end{array}$ & 2006 & 359 \\
\hline 381 & $\begin{array}{l}\text { 16- bis } 42 \text {-jährige Schwangere } \\
\text { im 2. und 3. Trimenon } \\
\text { ( } 8 \text { Geburtskliniken, } 15 \text { Privat- } \\
\text { praxen) }\end{array}$ & $\begin{array}{l}19 \% \text { Eisenmangel (Serumferritin }<12 \mu \mathrm{g} / \mathrm{L} \text { ) } \\
6 \% \text { Anämie } \\
3 \% \text { Eisenmangelanämie }\end{array}$ & 1999 & 360 \\
\hline 699 & $\begin{array}{l}\text { 18- bis } 50 \text {-jährige weibliche } \\
\text { Blutspenderinnen } \\
\text { (Region Lausanne) }\end{array}$ & $18 \%$ Eisenmangel (Serumferritin $<16 \mu \mathrm{g} / \mathrm{L}$ ) & 2007 & 361 \\
\hline $23^{\prime} 557$ & $\begin{array}{l}\text { 10'893 durchschnittlich } \\
\text { 47-jährige Männer } \\
\text { 12'664 durchschnittlich } \\
\text { 47-jährige Frauen }\end{array}$ & $\begin{array}{l}6.5 \% \text { Eisenmangel (Serumferritin }<10 \mu \mathrm{g} / \mathrm{L} \text { ) } \\
0.2 \% \text { Eisenmangelanämie } \\
16 \% \text { Eisenmangel (Serumferritin }<10 \mu \mathrm{g} / \mathrm{L} \text { ) } \\
1.5 \% \text { Eisenmangelanämie }\end{array}$ & 1996-2009 & 362 \\
\hline 854 & $\begin{array}{l}\text { Patienten, Median } 59.8 \text { Jahre } \\
\text { (allgemeine Hausarztpraxis } \\
\text { Luzern) }\end{array}$ & $\begin{array}{l}6 \% \text { Anämie; diese Personen litten an Krank- } \\
\text { heiten, die eine Anämie förderten }\end{array}$ & 2007 & 358 \\
\hline
\end{tabular}

Eisenmangel (iron deficiency ID) kann mit oder ohne Anämie auftreten und ist gekennzeichnet durch einen tiefen Ferritingehalt im Serum (<15 $\mu \mathrm{g} / \mathrm{L})$; die Grenzwerte sind alters- und geschlechtsabhängig. Zusatzinformationen liefert auch der Gehalt an Transferrinrezeptoren.

Anämie ist definiert durch tiefe, alters- und geschlechtsabhängige Hämoglobinwerte $(<12 \mathrm{~g} / \mathrm{dl}$ bei Frauen, $<11 \mathrm{~g} / \mathrm{dl}$ bei Männern).

Eisenmangelanämie (iron deficiency anaemia IDA) ist eine Kombination von alters- und geschlechtsabhängigen niedrigen Mengen an Hämoglobin und Serumferritin (356). 


\subsubsection{Jodmangel}

\subsubsection{Häufigkeit}

Die Jodversorgung ist vermutlich nicht in der ganzen Bevölkerung ausreichend (Kapitel 2.4.6). Gemäss einer nationalen, repräsentativen Studie in der Schweiz ist der Jodstatus (gemessen als Jod im Spot-Urin) bei Schulkindern, bei Schwangeren und bei Säuglingen, die ausschliesslich oder ergänzend zu Muttermilch Säuglingsmilch erhielten, ausreichend (Mediane: $120 \mu \mathrm{g} / \mathrm{L}, 162 \mu \mathrm{g} / \mathrm{L}$ bzw. $109 \mu \mathrm{g} / \mathrm{L})$. Dagegen muss derjenige von Stillenden, Neugeborenen und hauptsächlich gestillten Säuglingen (Mediane: $67 \mu \mathrm{g} / \mathrm{L}, 91 \mu \mathrm{g} / \mathrm{L}$ bzw. $82 \mu \mathrm{g} / \mathrm{L}$ ) als tief bewertet werden (373). Der Zielwert für Kinder $<2$ Jahren beträgt $\geq 100 \mu \mathrm{g} / \mathrm{L}$, für Schulkinder 100-199 $\mu \mathrm{g} / \mathrm{L}$, für Schwangere 150$249 \mu \mathrm{g} / \mathrm{L}$ und für Stillende $\geq 100 \mu \mathrm{g} / \mathrm{L}(374 ; 375)$. Eine neue Studie weist darauf hin, dass Teile der Bevölkerung vom Risiko einer Unterversorgung mit Jod betroffen sein könnten (397; Kapitel 2.4.6).

\subsubsection{Risiko- und Schutzfaktoren}

Da die Schweiz ein Jodmangelgebiet ist, wird mit Hilfe einer landesweiten Salzjodierung für eine ausreichende Jodzufuhr der Bevölkerung gesorgt (Kapitel 1.2). So ist das jodierte Salz die Hauptjodquelle in der Schweizer Ernährung (Kapitel 2.4.6). Wird vorwiegend jodfreies Salz verwendet und keine alternative Jodquelle konsumiert, besteht das Risiko einer ungenügenden Jodversorgung. Bei den staatlichen Bemühungen, den Salzkonsum zu senken, um das Risiko eines Bluthochdrucks zu verringern, ist gleichzeitig die ausreichende Jodzufuhr durch geeignete Massnahmen sicherzustellen.
Eine ausreichende Jodversorgung der schwangeren bzw. stillenden Frau sorgt auch für einen angemessenen Jodstatus bei ihrem ungeborenen Kind bzw. Säugling.

\subsubsection{Bedeutung}

Die Folgen von Jodmangel (376) sind nur noch selten in der Schweiz. Notwendig ist jedoch die Verwendung von jodiertem Salz. Bei Schwangeren wird häufig eine Schilddrüsenunterfunktion zusammen mit einem Eisenmangel festgestellt (377;378). Diese Beobachtung rechtfertigt die Überprüfung des Eisenstatus bei einer Schilddrüsenunterfunktion.

Die Jodierung des Salzes ist eine einfache und wirksame Massnahme zur Sicherstellung einer ausreichenden Jodzufuhr. Diese Massnahme sollte beibehalten und im Falle eines reduzierten Salzkonsums angepasst werden. Mit Hilfe des Jodmonitorings des Bundesamtes für Gesundheit (BAG) kann eine Verschlechterung der Jodversorgung frühzeitig erkannt und Risikogruppen gezielt angesprochen werden.

\subsubsection{Selenmangel}

\subsubsection{Häufigkeit}

Bei erwachsenen Blutspendern wurden 2006 höhere Selenspiegel als 1993 festgestellt $(379 ; 380)$. Während 1993 die Serumwerte durchschnittlich 92.7 $\pm 14.7 \mu \mathrm{g}$ Selen pro Liter (bei Männern 96.0 $013.3 \mu \mathrm{g} / \mathrm{L}$ und bei Frauen $87.9 \pm 14.4 \mu \mathrm{g} / \mathrm{L})$ betrugen, waren es 13 Jahre

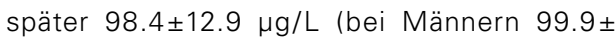
$12.5 \mu \mathrm{g} / \mathrm{L}$, bei Frauen $95.6 \pm 13.3 \mu \mathrm{g} / \mathrm{L}) .1 .5 \%$ der Teilnehmenden hatten 2006 Selenkonzentrationen von mehr als $132.8 \mu \mathrm{g} / \mathrm{L} ; 1993$ waren es nur $0.3 \%$. Statistische Auswertungen lassen 
darauf schliessen, dass Selenkonzentrationen über $132.8 \mu \mathrm{g} / \mathrm{L}$ nur durch die zusätzliche Einnahme von Selensupplementen erreicht werden können.

\subsubsection{Risiko- und Schutzfaktoren}

Der Selengehalt in Lebensmitteln hängt von dessen Vorkommen im Boden ab. Die Schweiz hat eher selenarme Böden, weshalb die Selenversorgung in der Schweiz von Lebensmitteln aus importiertem, selenreichem, nordamerikanischem Getreide sowie von tierischen Lebensmitteln abhängt. Die Tiere erhalten aufgrund ihres eigenen Selenbedarfs selenangereichertes Futter. Eine Studie bestätigt, dass der ausreichende Selenstatus in der Schweiz hauptsächlich durch den Konsum von Teigwaren auf Basis von nordamerikanischem Durum-Weizen sowie von Fleisch sichergestellt wird (381). Weitere wichtige Selenlieferanten sind Eier, Fisch, Milch und Milchprodukte sowie Fleischersatzprodukte auf Soja- und Weizenbasis (Kapitel 2.4.6). Dabei spielt nicht nur die Selenkonzentration dieser Lebensmittel eine wichtige Rolle, sondern auch die tägliche Verzehrsmenge. Im Vergleich zu Fleisch oder anderen tierischen Produkten ist die Selenkonzentration der Milch relativ niedrig; durch die hohe Verzehrsmenge wird sie trotzdem zu einer nicht vernachlässigbaren Selenquelle.

\subsubsection{Bedeutung}

Da Selen an vielen enzymatischen Prozessen beteiligt ist, wirkt sich Selenmangel vielfältig aus (382). Selenmangel ist in der Schweiz wahrscheinlich selten (379). Die Zielwerte für Selen werden in drei Kategorien eingeteilt: Selenkonzentrationen zwischen 50 bis $<60 \mu \mathrm{g} / \mathrm{L}$ gelten als niedrig, solche zwischen 60 bis $100 \mu \mathrm{g} / \mathrm{L}$ als mittel und solche zwischen $>100$ bis $120 \mu \mathrm{g} / \mathrm{L}$ als hoch (383). Darauf basierend kann die Situation in der Schweiz als ausreichend bezeichnet werden. Welche Selenkonzentrationen optimal sind, beruht auf Schätzungen und hängt vom betrachteten Kriterium ab: Zurzeit gilt eine Selenplasmakonzentration von 80-95 $\mu \mathrm{g} / \mathrm{L}$ als ausreichend, um eine maximale Aktivität der Glutathionperoxidase und des Selenoprotein-P zu erreichen (384); dagegen werden $120 \mu \mathrm{g} / \mathrm{L}$ als optimal für die Prävention von Tumoren erachtet (385).

Die Selenversorgung wird nur gewährleistet, wenn die Konsumenten Produkte aus selenreichem Durum-Weizen und tierische Produkte essen (381). Da Tiere selber auf eine genügende Selenzufuhr angewiesen sind und ihr Futter daher mit Selen ergänzt wird, werden Lebensmittel tierischen Ursprungs stets eine Selenquelle für den Menschen sein. Eine Anreicherung pflanzlicher Lebensmittel via Selendüngung ist in der Schweiz zurzeit verboten.

\subsection{Zahngesundheit}

\subsubsection{Häufigkeit}

Lebensmittel und Ernährungsgewohnheiten können zu Schädigungen der Zahnhartsubstanz (Schmelz und Dentin) führen, d.h. zu Karies und Erosionen.

\section{Karies}

Weit verbreitet und deshalb von volksgesundheitlicher Bedeutung war und ist Karies. Darunter versteht man einen in die Tiefe fortschreitenden Demineralisationsprozess der Zahnhart- 
Tabelle 3.13: Kariesprävalenz in der Schweiz nach Bevölkerungsgruppe, Daten aus den Jahren 1999-2006 (386)

\begin{tabular}{|c|c|c|}
\hline Angaben zur Stichprobe & $\begin{array}{l}\text { Kariesprävalenz } \\
\text { (in \%) }\end{array}$ & $\begin{array}{l}\text { Durchschnittlicher Kariesbefall } \\
\text { dmft / DMFTa }\end{array}$ \\
\hline 2-Jährige, Stadt Zürich, 2003 (N=771) & $13 \%$ & - \\
\hline 5-Jährige, Winterthur, 2001 (N=291) & $45 \%$ & $2.4 \mathrm{dmft}$ \\
\hline \multicolumn{3}{|l|}{ 7-Jährige, Kanton Zürich, 2005} \\
\hline Schweizer u.a.A. ${ }^{b}(N=196)$ & $32 \%$ & $1.1 \mathrm{dmft}$ \\
\hline Ex-Jugoslawen $(\mathrm{N}=13)$ & $92 \%$ & $7.4 \mathrm{dmft}$ \\
\hline \multicolumn{3}{|l|}{ Stadt Zürich, 2006} \\
\hline Schweizer u.a.A. ${ }^{b}(N=145)$ & $43 \%$ & $1.7 \mathrm{dmft}$ \\
\hline Ex-Jugoslawen, Albaner ( $\mathrm{N}=26)$ & $88 \%$ & $6.6 \mathrm{dmft}$ \\
\hline \multicolumn{3}{|l|}{ 12-Jährige, Kanton Zürich, 2005} \\
\hline Schweizer u.a.A. ${ }^{b}(N=265)$ & $38 \%$ & $0.8 \mathrm{DMFT}$ \\
\hline Ex-Jugoslawen $(\mathrm{N}=26)$ & $77 \%$ & 2.2 DMFT \\
\hline \multicolumn{3}{|l|}{ Stadt Zürich, 2006} \\
\hline Schweizer $(\mathrm{N}=161)$ & $26 \%$ & $0.5 \mathrm{DMFT}$ \\
\hline u.a. $A^{c}(N=46)$ & $46 \%$ & 1.1 DMFT \\
\hline Ex-Jugoslawen $(\mathrm{N}=22)$ & $82 \%$ & 2.5 DMFT \\
\hline 20-Jährige, Rekruten, Thun, 2006 (N=606) & $77 \%$ & 3.1 DMFT \\
\hline \multicolumn{3}{|l|}{ Erwachsene, Kanton Zürich, 1999 ( $N=304)$} \\
\hline 20-bis 29-Jährige & - & 6.9 DMFT (alle mit $\geq 20$ Zähnend) \\
\hline 30- bis 39-Jährige & - & 11.2 DMFT (alle mit $\geq 20$ Zähnen) \\
\hline 40- bis 49-Jährige & - & 17.8 DMFT (99\% mit $\geq 20$ Zähnen) \\
\hline 50- bis 59-Jährige & - & 20.2 DMFT (89\% mit z 20 Zähnen) \\
\hline 60- bis 69-Jährige & - & 22.0 DMFT (67\% mit $\geq 20$ Zähnen) \\
\hline 70- bis 79-Jährige & - & 22.9 DMFT (44\% mit $\geq 20$ Zähnen) \\
\hline \multicolumn{3}{|c|}{$\begin{array}{l}\text { DMFT: Summe der kariösen (D), extrahierten (M) und gefüllten (F) bleibenden Zähne (T); } \\
\text { dmft: Summe der kariösen, extrahierten und gefüllten Zähne im Milchgebiss }\end{array}$} \\
\hline \multicolumn{3}{|c|}{$\begin{array}{l}\text { b u.a.A.: und andere Ausländer, nicht aus Ex-Jugoslawien und Albanien; Unterschiede zwischen der Kariesprävalenz bei } \\
\text { Schweizern und der Kariesprävalenz bei der Gruppe u.a.A. gering }\end{array}$} \\
\hline \multicolumn{3}{|c|}{$\begin{array}{l}\text { c Anteil der Gruppe u.a.A. gross; sehr grosse Unterschiede zwischen der Kariesprävalenz bei Schweizern und der Kariesprä- } \\
\text { valenz bei der Gruppe u.a.A. (daher separat aufgeführt) }\end{array}$} \\
\hline \multicolumn{3}{|c|}{ d 20 Zähne gewährleisten eine genügende Kaufähigkeit (386). } \\
\hline \multicolumn{3}{|l|}{$-=$ keine Angabe } \\
\hline
\end{tabular}

substanz, der durch von Bakterien des Zahnbelages (Plaque) produzierte, organische Säuren verursacht wird. Voraussetzung für diese Säureproduktion sind eine ungenügende Mundhygiene und die häufige Verfügbarkeit von Kohlenhydraten (vor allem von Zuckern) in der Mundhöhle.
Der Verlauf des Kariesbefalls in der Schweiz über mehr als vier Jahrzehnte ist bei verschiedenen Altersgruppen gut dokumentiert, insbesondere bei Schülern und Rekruten. Im Milchgebiss hat sich der Kariesbefall bei Schulanfängern seit den 1960 er Jahren um $80 \%$ verringert, im bleibenden Gebiss um $90 \%$ bei 
Volksschülern und um $80 \%$ bei Rekruten. Eine Abnahme des durchschnittlichen Kariesbefalls wurde auch bei Erwachsenen bis zur Altersgruppe der 40- bis 49-Jährigen zwischen 1992 und 1999 beobachtet. Diese Entwicklung wurde hauptsächlich durch die schnelle und flächendeckende Verbreitung fluoridhaltiger Zahnpasten (ab den 1960er Jahren) und durch die Einführung des fluoridierten (und jodierten) Speisesalzes (1983) ermöglicht. Auch Kariesvorbeugungsmassnahmen in den Schulen haben v.a. in den 1960er bis 1980er Jahren eine wichtige Rolle gespielt. Die verfügbaren Daten sind in Tabelle 3.13 zusammengefasst (386).

\section{Erosionen}

Der häufige direkte Kontakt von Säuren, die in Lebensmitteln enthalten sind, mit nicht von Plaque bedeckten Zahnoberflächen kann zu oberflächlichem Mineralverlust und damit zur schichtweisen Anätzung und irreversiblen Abtragung von Zahnhartsubstanz führen. Dieser Prozess wird als Erosion bezeichnet. Er kann grosse Zahnflächen betreffen und vor allem bei den Milchzähnen schnell voranschreiten.

Es gibt nur wenige Studien zur Prävalenz von Zahnschmelzerosionen in der Schweiz (387; 388). Sie zeigen, dass erste Anzeichen von Erosionen bereits im Kindesalter auftreten können. Wahrscheinlich nimmt die Anzahl von Zahnschmelzerosionen bei Jüngeren zu (389).

\subsubsection{Risiko- und Schutzfaktoren}

Während der Entwicklung der Zähne beeinträchtigen höchstens extremste Ernährungsdefizite die Zahnhartsubstanz. Nach dem Zahndurchbruch hat die Ernährung keinen systemi- schen Einfluss mehr auf die Zähne, sondern nur noch einen Einfluss von aussen (390). Karies und Erosion sind Folgen von lokalen chemischen und mechanischen Einwirkungen von Lebensmitteln auf die Zahnoberflächen.

\section{Karies}

Schlechte Mundhygiene (Plaque auf den Zähnen ist ein Symptom dafür), Gebrauch von Nuckelflaschen während des Einschlafens oder in der Nacht (Kleinkinder) und häufige Zwischenmahlzeiten bestehend aus kariogenen (v.a. zuckerhaltigen) Lebensmitteln und Getränken fördern Karies $(315 ; 391)$. Tiefes Einkommen und geringe Schulbildung scheinen Kofaktoren für Karies und ein schlechtes Gebiss zu sein (391;392).

Den stärksten schützenden Einfluss auf die Kariesentstehung hat die Mundhygiene mit fluoridhaltiger Zahnpasta. Wichtig ist, dass und wie geputzt wird: zwei- bis dreimal am Tag, am besten nach den Hauptmahlzeiten mit der richtigen Putztechnik. Weniger oft kariogene und säurehaltige Lebensmittel als Zwischenmahlzeit zu essen und zu trinken, unterstützt diese hygienische Massnahme. Besonders im Kindesalter sollte auf den Gebrauch von zuckerhaltigen Getränken während des Einschlafens oder in der Nacht verzichtet werden.

\section{Erosionen}

Ob Erosionen an den Zähnen auftreten, hängt von verschiedenen, sich gegenseitig beeinflussenden Faktoren ab $(315 ; 393)$ : Essgewohnheiten, Menge und Zusammensetzung des Speichels, Mundhygienegewohnheiten sowie häufiges Erbrechen (Bulimie). Die Erosivität von Lebensmitteln hängt von deren $\mathrm{pH}$-Wert, Pufferkapazität und Säuretyp sowie von deren Cal- 
cium-, Phosphat- und Fluoridgehalt ab. So können Lebensmittel und Getränke trotz ähnlicher $\mathrm{pH}$-Werte unterschiedlich stark erosiv wirken (393). Der häufige Konsum von säurehaltigen Lebensmitteln (saure Gurken, Essig, saure Bonbons, Früchte, Salatsaucen usw.) und Getränken (kohlensäurehaltige Süssgetränke, Alcopops, Sportgetränke, Orangensaft) in grossen Mengen sowie der Konsum säurehaltiger Lebensmittel in der Nacht fördern Erosionen. Je länger Lebensmittel und Getränke an den Zähnen haften, desto höher ist ihre Erosivität (393;394). Werden saure Lebensmittel oder Getränke länger im Mund behalten oder der Mund damit sogar noch gespült, werden Zahnmineralien ausgewaschen (395).

Zähneknirschen und Abrasion z.B. durch Zahnpasten (hoher Anteil "Putzkörper» in der Paste) können den Erosionsprozess verstärken (315;390). Deshalb sollten die Zähne nicht unmittelbar nach dem Genuss saurer Lebensmittel geputzt werden (393).

Zähne mit Erosionen bedürfen spezifischer Hygienemassnahmen: Verwendung einer weichen Zahnbürste und einer wenig scheuernden Zahnpasta. Weiter schützen ein geringerer Konsum von säurehaltigen Lebensmitteln (insbesondere von Getränken) sowie ein Verzicht auf solche Produkte während der Nacht vor Erosionen. Ebenso nützlich ist es, die säurepuffernde Eigenschaft von Milchprodukten zu nutzen, indem z.B. der Verzehr einer Frucht mit dem Trinken von Milch verknüpft wird.

\subsubsection{Bedeutung}

\section{Karies}

Die grossen Anstrengungen der vergangenen 40-50 Jahre in Kindergärten und Schulen zur Reduktion der Kariesprävalenz haben sich gelohnt und müssen beibehalten werden. Die gelernten Mundhygienemassnahmen müssen ständiger Bestandteil des Lebens bleiben und von den älteren Generationen an die jüngeren weitergegeben werden. Wichtig sind auch die schulzahnärztlichen Untersuchungen und die Schulzahnpflege bzw. -erziehung, weil sie alle Kinder erreicht - unabhängig von ihrer Herkunft. Nachholbedarf besteht bei den Kleinsten: Der relativ häufige Kariesbefall der Frontzähne bei 2-Jährigen (391) macht Vorsorgemassnahmen im Rahmen der Mütter-Väterberatung und der Vorsorgeuntersuchungen beim Kinderarzt erforderlich. So können alle Eltern gleichermassen, ungeachtet ihres sozialen Hintergrundes, über den richtigen Umgang mit Süssgetränken und über die bei Kleinkindern notwendige Zahnhygiene aufgeklärt werden.

\section{Erosionen}

Erosionen haben nicht die gleiche volksgesundheitliche Bedeutung wie Karies und sind bei normaler Ernährung klinisch nicht relevant. Sie können aber bei extremen Konsumgewohnheiten (z.B. zwei Liter kohlensäurehaltige Süssgetränke pro Tag) und bei Bulimie zu grossen Zahnhartsubstanzverlusten führen und umfangreiche zahnaufbauende Behandlungen nach sich ziehen (315;393). In einem zunehmenden Konsum kohlensäurehaltiger Süssgetränke inkl. Energydrinks und isotonischer Sportgetränke steckt ein nicht zu unterschätzendes Risiko für Zahnerosionen. Der Informationsstand der Be- 
völkerung über die nachhaltig zerstörerische Wirkung saurer Lebensmittel auf die Zähne sowie über entsprechende Verhaltensregeln dürfte gering sein, was die Gefahr von Erosionen durch ungeeignete Getränke erhöht.

\section{Zahnverlust}

Werden kranke Zähne nicht behandelt, kann dies zu ihrem Verlust führen. Ein unvollständiges oder schlecht sitzendes künstliches Gebiss kann - muss jedoch nicht - eine Mangelernährung zusätzlich begünstigen (331; Kapitel 3.10.1.2).

\subsection{Schlussfolgerungen und Ausblick}

Der Einfluss der Ernährung auf die Gesundheit kann nur bruchstückhaft beschrieben werden, weil Schweizer Daten zu Lebensmittelverzehr und ernährungsabhängigen Krankheiten sowie Informationen über ursächliche Zusammenhänge zwischen Ernährung und Erkrankungsrisiko fehlen. Neben einer fundierteren Verzehrserhebung könnten eine national repräsentative Kohortenstudie und eine umfassende Erhebung von Krankheiten und Risikofaktoren die grössten Lücken schliessen (Kapitel 4.9.1). Eine wichtige Zielgruppe hinsichtlich Vorbeugung ernährungsabhängiger Krankheiten sind Familien mit bildungsfernem Hintergrund (Kapitel 4.9.2).

Ein entscheidender vermeidbarer Risikofaktor für Stoffwechsel- und Herz-Kreislauf-Krankheiten ist Übergewicht. Die nächsten Jahre werden zeigen, wie nachhaltig der Trend zur Stagnation oder Abnahme der Adipositasprävalenz ist. Unabhängig davon muss die Politik Rah- menbedingungen schaffen, welche die körperliche Aktivität fördern, und es gleichzeitig ermöglichen, sich ausgewogen zu ernähren (Kapitel 4.9.5). Die Prävention muss bereits bei Normalgewichtigen ansetzen und früh im Leben beginnen (Kapitel 4.9.3). Die schweizweite Erfassung von Essstörungen bietet eine weitere wichtige Grundlage für gezielte Präventionsmassnahmen.

Präventionspotenzial bieten auch eine schrittweise Anpassung der Zusammensetzung von Lebensmitteln, allen voran eine Senkung des Salz-, Zucker- und Fettgehaltes, Massnahmen zur Einschränkung eines übermässigen Konsums alkoholischer und / oder gezuckerter Getränke und die Förderung des Konsums von Früchten und Gemüse sowie einer ausgewogenen Zufuhr der verschiedenen Fettsäuren (Tabelle 4.7).

Während bei der Selenzufuhr vorläufig kein Handlungsbedarf besteht, müssten Teile der Bevölkerung besser mit Eisen, Folsäure, Vitamin D und möglicherweise Jod versorgt werden. Das Risiko für Mangelernährung könnte durch Früherkennung und Betreuung in und besonders ausserhalb von Spitälern und Pflegeheimen vermindert werden. Die erfolgreichen Massnahmen in der Kariesprävention müssen beibehalten und für spezifische Zielgruppen intensiviert werden. 


\section{Danksagung}

Wir danken all denjenigen ganz herzlich, die uns durch Zusendung von Artikeln oder Informationen bei der Erstellung des Kapitels unterstützt haben. Ganz besonders danken wir denjenigen, die dies in Form von inhaltlichen Diskussionen und Rückmeldungen zum Manuskript getan haben: Isabelle Aeberli, Barbara Ballmer-Weber, Heike A. Bischoff-Ferrari, Matthias Bopp, Peter Burkhardt, Vincent Dudler, Monika Eichholzer, Sophia Fischer, Idris Guessous, Carola Imfeld, Thomas Imfeld, Reinhard Imoberdorf, Bettina Isenschmid, Judith Jenny-Burri, Marius Kraenzlin, Pedro Marques-Vidal, Rémy Meier, Giorgio Menghini, Simone Munsch, Katharina Quack Lötscher, Sabine Rohrmann, Susi Saxer, Karin Schindler, Bernd Schultes, Hanspeter Stamm, Susanne Stronski Huwiler.

\section{Literaturverzeichnis}

1. World Cancer Research Fund / American Institute for Cancer Research. Food, nutrition, physical activity and the prevention of cancer: a global perspective. Washington, DC: AICR, 2007.

2. JointWHO/FAO Expert Consultation. Diet, nutrition and the prevention of chronic diseases. Technical Report Series 916. Geneva:WHO, 2003.

3. Wietlisbach V, Paccaud F, Rickenbach M, Gutzwiller F. Trends in cardiovascular risk factors (19841993) in a Swiss region: results of three population surveys. Prev Med 1997;26(4):523-33.

4. Vollenweider P, Hayoz D, Preisig M, et al. Health examination survey of the Lausanne population: first results of the CoLaus study. Rev Med Suisse 2006;2(86):2528-33.

5. Galobardes B, Costanza MC, Bernstein MS, Delhumeau CH, Morabia A. Trends in risk factors for the major «lifestyle-related diseases» in Geneva, Switzerland, 1993-2000. Ann Epidemiol 2003;13(7):537-40.

6. Zimmermann-Sloutskis D, Morabia A, Beer-Borst S, Costanza MC. Swiss national health data collections. A proposition for a systematic comparison and evaluation. Berne: Office fédéral de la santé publique, 2006.

7. Bundesamt für Statistik. Gesundheit und Gesundheitsverhalten in der Schweiz 2007. Schweizerische Gesundheitsbefragung. Neuchâtel: Bundesamt für Statistik, 2010.

Internet: http://www.bfs.admin.ch/bfs/portal/de/ index/infothek/publ.html?publication ID =4092 (letzter Zugriff 30. September 2011).

8. Sucht Info Schweiz. Health Behaviour in Schoolaged Children (HBSC).

Internet: http://www.hbsc.ch (letzter Zugriff 30. September 2011).

9. Bopp M, Braun J, Faeh D, Gutzwiller F. Establishing a follow-up of the Swiss MONICA participants (1984-1993): record linkage with census and mortality data. BMC Public Health 2010;10:562. 
10. Gutzwiller F, Nater B, Martin J. Community-based primary prevention of cardiovascular disease in Switzerland: methods and results of the National Research Program (NRP 1A). Prev Med 1985;14(4):482-91.

11. Household survey on health in the NFP 8 (Somipops) II. Content and course of the main survey. Soz Praventivmed 1982;27(6):324-5.

12. Noack $H$, Weiss W. Un système intercantonal d'information sanitaire en Suisse. Rapport du responsable scientifique et du coordinateur, Projet intercantonal sur les indicateurs de santé (IGIP/ PROMES). Aarau/Lausanne, 1990.

13. Schweizer Haushalt-Panel (SHP). Internet: http://www.swisspanel.ch/?lang=de (letzter Zugriff 30. September 2011).

14. Gmel G, Gaume J, Faouzi M, Kulling JP, Daeppen JB. Who drinks most of the total alcohol in young men - risky single occasion drinking as normative behaviour. Alcohol 2008;43(6):692-7.

15. Bundesamt für Statistik. Schweizerische Gesundheitsbefragung 2007. Standardtabellen. Neuchâtel: Bundesamt für Statistik. Internet: http://www.bfs.admin.ch/bfs/portal/de/ index/infothek/publ.html?publicationID $=3502$ (letzter Zugriff 30. September 2011).

16. AMIS-PLUS. National Registry of Acute Myocardial Infarction in Switzerland. Internet: http://www.amis-plus.ch (letzter Zugriff 30. September 2011).

17. Schnyder U, Milos G, Mohler-Kuo M, Dermota P. Prävalenz von Essstörungen in der Schweiz. Schlussbericht. Bern: Bundesamt für Gesundheit, 2012.

Internet: http://www.bag.admin.ch/themen/ ernaehrung_bewegung/05207/13287/index. html?lang=de (letzter Zugriff 30. Juni 2012).

18. IMS Health $\mathrm{GmbH}$. Schweizerischer Diagnosen Index (SDI).

Internet: http://www.imshealth.ch/Basis/Start.asp? Ber $=$ PortPharm\&Men2=Integrated $\%$ 20Data $\% 20$ and\%20Applications\&Start=../Prod-DI/Produktpraesentation. htm\&sitetype $=$ c (letzter Zugriff 30. September 2011).
19. Bundesamt für Statistik. SchweizerischeTodesursachenstatistik: Richtlinien für die ärztliche Bescheinigung derTodesursachen. Statistik der Schweiz. Bern: Bundesamt für Statistik, 1996. Internet: http://www.bfs.admin.ch/bfs/portal/de/ index/infothek/publ.html?publicationID=332 (letzter Zugriff 30. September 2011).

20. Bundesamt für Statistik. Medizinische Statistik der Krankenhäuser.

Internet: http://www.bfs.admin.ch/bfs/portal/de/ index/infothek/erhebungen__quellen/blank/blank/ mkh/01.html (letzter Zugriff 30. September 2011).

21. Bopp M, Zellweger $U$, Faeh D. Routine data sources challenge international diabetes Federation extrapolations of national diabetes prevalence in Switzerland. Diabetes Care 2011;34(11):2387-9.

22. Schweizerisches Gesundheitsobservatorium (OBSAN). Gesundheitszustand. Spezifische Diagnosen: Myokardinfarkt, Herzinsuffizienz, Hirnschlag. Neuchâtel: OBSAN, 2008.

Internet: http://www.obsan.admin.ch/bfs/obsan/de/ index/04/01/ind26.indicator.149011.260103.html? open $=149001,149004 \# 149004$ (letzter Zugriff 30. September 2011).

23. Bundesamt für Statistik. Von Generation zu Generation. Entwicklung derTodesursachen 1970 bis 2004. Neuchâtel: Bundesamt für Statistik, 2008. Internet: http://www.bfs.admin.ch/bfs/portal/de/ index/infothek/publ.html?publicationID=3251 (letzter Zugriff 30. September 2011).

24. Bundesamt für Statistik. Todesursachenstatistik. Ursachen der Sterblichkeit 2005 und 2006. Neuchâtel: Bundesamt für Statistik, 2008. Internet: http://www.bfs.admin.ch/bfs/portal/de/ index/infothek/publ.html?publicationID =3308 (letzter Zugriff 30. September 2011).

25. Bopp M, Spoerri A, Zwahlen M, et al. Cohort Profile: the Swiss National Cohort - a longitudinal study of 6.8 million people. Int J Epidemiol 2009;38(2):379-84.

26. Faeh D, Bopp M. Excess weight in the canton of Zurich, 1992-2009: harbinger of a trend reversal in Switzerland? Swiss MedWkly 2010;140:w13090. 
27. Krebs $\mathrm{H}$, Keller R, Hornung R. Werbe- und Verkaufseinschränkungen fürTabakwaren, höhere Zigarettenpreise und Rauchverbote: Einstellungen der Schweizer Bevölkerung 2003-2006. In: Hans Krebs Kommunikation und Publikumsforschung, ed. Tabakmonitoring - Schweizerische Umfrage zumTabakkonsum. Zurich: University of Zurich, 2006.

28. Nationales Institut für Krebsepidemiologie und -registrierung (nicer).

Internet: http://www.nicer.org (letzter Zugriff 6. Juni 2012)

29. Meyer K, Simmet A, Arnold M, Mattle H, Nedeltchev K. Stroke events, and case fatalities in Switzerland based on hospital statistics and cause of death statistics. Swiss MedWkly 2009;139(56):65-9.

30. Bundesamt für Statistik. Krankenhausbehandlung - Detaillierte Daten, 1998-2009. Internet: http://www.bfs.admin.ch/bfs/portal/de/ index/themen/14/04/01/data/01/01.html (letzter Zugriff 30. September 2011).

31. Faeh D, Minder C, Gutzwiller F, Bopp M. Culture, risk factors and mortality: can Switzerland add missing pieces to the European puzzle? J Epidemiol Community Health 2009;63(8):639-45.

32. Boffetta P, Couto E, Wichmann J, et al. Fruit and vegetable intake and overall cancer risk in the European Prospective Investigation into Cancer and Nutrition (EPIC). J Natl Cancer Inst 2010;102(8):529-37.

33. Gonzalez CA, Riboli E. Diet and cancer prevention: Contributions from the European Prospective Investigation into Cancer and Nutrition (EPIC) study. Eur J Cancer 2010;46(14):2555-62.

34. Frisch AL, Schulz PJ. Ernährung, Bewegung und Körpergewicht:Wissen, Einstellung undWahrnehmung der Schweizer Bevölkerung. Ergebnisse der Bevölkerungsbefragungen von 2006 und 2009. Bern: Gesundheitsförderung Schweiz, 2010.
35. Schneider H, VenetzW, Gallani Berardo C. Overweight and obesity in Switzerland. Part 1: Cost burden of adult obesity in 2007. Bern: Bundesamt für Gesundheit, 2009.

Internet: http://www.bag.admin.ch/themen/ ernaehrung_bewegung/05207/05218/05232/ index.html?lang=de\&download=NHzLpZeg7t, Inp 6I0NTU042I2Z6In1acy4Zn4Z2qZpnO2Yuq2Z6gpJCldHt7fWym162epYbg2c_JjKbNoKSn6A (letzter Zugriff 30. September 2011).

36. World Health Organization. Body Mass Index classification. WHO, 1995.

Internet: http://apps.who.int/bmi/index.jsp? introPage=intro_3.html (letzter Zugriff 30. September 2011).

37. Marques-Vidal P, Bochud M, MooserV, Paccaud F, Waeber G, Vollenweider P. Obesity markers and estimated 10-year fatal cardiovascular risk in Switzerland. Nutr Metab Cardiovasc Dis 2009;19(7):462-8.

38. Marques-Vidal P, Bochud M, Mooser V, Paccaud F, Waeber G, Vollenweider P. Prevalence of obesity and abdominal obesity in the Lausanne population. BMC Public Health 2008;8:330.

39. Velho S, Paccaud F, Waeber G, Vollenweider P, Marques-Vidal P. Metabolically healthy obesity: different prevalences using different criteria. Eur J Clin Nutr 2010;64(10):1043-51.

40. Sebo P, Beer-Borst S, Haller DM, Bovier PA. Reliability of doctors' anthropometric measurements to detect obesity. Prev Med 2008;47(4):389-93.

41. Faeh D, Braun J, Tarnutzer S, Bopp M. Obesity but not overweight is associated with increased mortality risk. Eur J Epidemiol 2011;26(8):647-55.

42. ColeTJ, Bellizzi MC, Flegal KM, DietzWH. Establishing a standard definition for child overweight and obesity worldwide: international survey. BMJ 2000;320(7244):1240-3.

43. Kuczmarski RJ, Ogden CL, Grummer-Strawn LM, et al. CDC growth charts: United States. Adv Data 2000(314):1-27. 
44. Gutzwiller F, Leu R, Schulz HR. Household survey on health in the NFP 8 (Somipops) II. Content and course of the main survey. Soz Praventivmed $1982 ; 27(6): 324-5$.

45. Faeh D, Marques-Vidal P, Chiolero A, Bopp M. Obesity in Switzerland: do estimates depend on how body mass index has been assessed? Swiss Med Wkly 2008;138(13-14):204-10.

46. Saely $\mathrm{CH}$, Risch L, Frey F, et al. Body mass index, blood pressure, and serum cholesterol in young Swiss men: an analysis on 56784 army conscripts. Swiss MedWkly 2009;139(35-36):518-24.

47. Bopp M, Faeh D. Where have all the conscripts gone? Swiss MedWkly 2010;140(23-24):354.

48. Bundesamt für Gesundheit. Sammlung Monitoring-Projekte. BMI, Körpergewicht, Fettmessung. Bern: Bundesamt für Gesundheit. Internet: http://www.bag.admin.ch/themen/ ernaehrung_bewegung/05190/05294/index. html? lang=de\%23index.html?id1=0-2 (letzter Zugriff 30. September 2011).

49. Stamm H, Ackermann U, Frey D, et al. Monitoring der Gewichtsdaten der schulärztlichen Dienste der Städte Basel, Bern und Zürich. Auswertung der Daten des Schuljahres 2009/2010. Bern: Gesundheitsförderung Schweiz, 2011.

50. Stamm H, Wiegand D, Lamprecht M. Monitoring der Gewichtsdaten von Kindern und Jugendlichen in den Kantonen Graubünden, Wallis, Jura, Genf und Basel-Stadt sowie den Städten Freiburg, Bern und Zürich. Auswertung der Daten des Schuljahres 2008/2009. Bern: Gesundheitsförderung Schweiz, 2010.

51. Delgrande Jordan M, Annaheim B. Habitudes alimentaires, activité physique et statut pondéral chez les élèves de 11 à 15 ans en Suisse. Situation en 2006 et évolution récente. Résultats de l'Enquête internationale Health Behaviour in School-aged Children (HBSC). Rapport de recherche No 45. Lausanne: Institut suisse de prévention de l'alcoolisme et autres toxicomanies, 2009. Internet: http://www.sfa-ispa.ch/DocUpload/ rr_no45_HBSC_2006.pdf (letzter Zugriff 30. September 2011).
52. Stronski Huwiler S, Stamm H, Frey D, et al. Übergewicht und Adipositas bei Kindern und Jugendlichen im Kanton Zürich. Gesundheitsbericht des Kantons Zürich. Zürich: Institut für Sozial- und Präventivmedizin der Universität Zürich, 2012.

53. Connor Gorber S, Tremblay M, Moher D, Gorber B. A comparison of direct vs. self-report measures for assessing height, weight and body mass index: a systematic review. Obes Rev 2007;8(4):307-26.

54. Faeh D, Braun J, Bopp M. Underestimation of obesity prevalence in Switzerland: comparison of two methods for correction of self-report. Swiss Med Wkly 2009;139(51-52):752-6.

55. Bopp M, Faeh D. Who gives me five? Rounding preference for self reported height depends on language. BMJ 2008;337(a2950):1463.

56. Bopp M, Faeh D. End-digits preference for selfreported height depends on language. BMC Public Health 2008;8:342.

57. Gaspoz JM, Beer-Borst S, Costanza MC, Morabia A. Suivi des facteurs de risque cardiovasculaire dans la population genevoise de 1993 à 2007. Berne: Office fédérale de la santé publique, 2009. Internet: http://www.bag.admin.ch/themen/ ernaehrung_bewegung/05207/05213/index.html? lang=fr\&download=NHzLpZig7t, Inp6IONTU042I2 Z6In1ae2IZn4Z2qZpnO2Yuq2Z6gpJCJfH1_gWym 162dpYbUzd,Gpd6emK2Oz9aGodetmqaN19XI2Id voaCVZ,s- (letzter Zugriff 30. September 2011).

58. Clair C, Chiolero A, Faeh D, et al. Dose-dependent positive association between cigarette smoking, abdominal obesity and body fat: cross-sectional data from a population-based survey. BMC Public Health $2011 ; 11(1): 23$.

59. Schneider H, VenetzW, Gallani Berardo C. Overweight and obesity in Switzerland. Part 2: Overweight and obesity trends in children. Bern: Bundesamt für Gesundheit, 2009. Internet: http://www.bag.admin.ch/themen/ ernaehrung_bewegung/05207/05218/05232/ index.html? lang=de\&download=NHzLpZeg7t, Inp 6IONTU042I2Z6In1acy4Zn4Z2qZpnO2Yuq2Z6gpJCldHt7f2ym162epYbg2c_JjKbNoKSn6A-(letzter Zugriff 30. September 2011). 
60. Lasserre AM, Chiolero A, Paccaud F, Bovet P. Worldwide trends in childhood obesity. Swiss Med Wkly 2007;137(9-10):157-8.

61. Jeannot E, Mahler P, Duperrex O, Chastonay P. Evolution of overweight and obesity among elementary school children in Geneva. Swiss Med Wkly 2010;140:w13040.

62. Aeberli I, Henschen I, Molinari L, Zimmermann MB. Stabilisation of the prevalence of childhood obesity in Switzerland. Swiss MedWkly 2010;140:w13046.

63. Aeberli I, Ammann RS, Knabenhans M, Molinari L, Zimmermann MB. Decrease in the prevalence of paediatric adiposity in Switzerland from 2002 to 2007. Public Health Nutr 2010;13(6):806-11.

64. Peytremann-Bridevaux I, Faeh D, Santos-Eggimann B. Prevalence of overweight and obesity in rural and urban settings of 10 European countries. Prev Med 2007;44(5):442-6.

65. Lasserre AM, Chiolero A, Cachat F, Paccaud F, Bovet P. Overweight in Swiss children and associations with children's and parents' characteristics. Obesity 2007;15(12):2912-9.

66. Burgi F, Meyer U, Niederer I, et al. Socio-cultural determinants of adiposity and physical activity in preschool children: a cross-sectional study. BMC Public Health 2010;10:733.

67. Finucane MM, Stevens GA, Cowan MJ, et al. National, regional, and global trends in body-mass index since 1980: systematic analysis of health examination surveys and epidemiological studies with 960 country-years and 9.1 million participants. Lancet 2011;377(9765):557-67.

68. Organisation for Economic Co-operation and Development (OECD). Health at a Glance: Europe 2010. Geneva OECD, 2010.

Internet: http://www.oecd-ilibrary.org/socialissues-migration-health/health-at-a-glance-europe-2010_health_glance-2010-en (letzter Zugriff 30. September 2011).

69. Andreyeva T, Michaud PC, van Soest A. Obesity and health in Europeans aged 50 years and older. Public Health 2007;121(7):497-509.
70. Schopper D. Gesundes Körpergewicht bei Kindern und Jugendlichen. Was haben wir seit 2005 dazugelernt? Bern: Gesundheitsförderung Schweiz, 2010.

71. Beer-Borst S. Bus Santé: 14 years of risk factor surveillance in Geneva 2006.

Internet: http://www.bag.admin.ch/themen/ ernaehrung_bewegung/05190/05297/05301/ index.html? lang=de\&download =NHzLpZeg7t, In p6I0NTU042I2Z6In1acy4Zn4Z2qZpnO2Yuq2Z6gp JCFfH97f2ym162epYbg2c_JjKbNoKSn6A (letzter Zugriff 30. September 2011).

72. Martin B, Mäder U, Stamm H, Braun-Fahrländer C. Physical activity and health - what are the recommendations and where do we find the Swiss population. Schweizer Zeitschr "Sportmedizin und Sporttraumatologie" 2009;27(2):37-43.

73. Eichholzer M, Bovey F, Jordan P, Schmid M, Stoffel-Kurt N. Body weight related data: results of the 2007 Swiss Health Survey. PRAXIS (Bern 1994) 2010;99(15):895-906.

74. Stamm H, Fischer A, Wiegand D, Lamprecht M. Monitoring-System Ernährung und Bewegung (MOSEB). Indikatorensammlung zum Nationalen Programm Ernährung und Bewegung 2008-2012 (NPEB). Bern: Bundesamt für Gesundheit, 2009. Internet: http://www.bag.admin.ch/themen/ ernaehrung_bewegung/05190/07835/index.html? lang=de\&download=NHzLpZeg7t,Inp6I0NTU042 I2Z6In1acy4Zn4Z2qZpnO2Yuq2Z6gpJCldIJ_fWym162epYbg2c_JjKbNoKSn6A-- (letzter Zugriff 30. September 2011).

75. Lamprecht \& Stamm Sozialforschung und Beratung AG. Monitoring der langfristigen Strategie von Gesundheitsförderung Schweiz. Indikatoren zum Bereich "Gesundes Körpergewicht». Bern: Gesundheitsförderung Schweiz, 2011.

76. Aeberli I, Kaspar M, Zimmermann MB. Dietary intake and physical activity of normal weight and overweight 6 to 14 year old Swiss children. Swiss MedWkly 2007;137(29-30):424-30. 
77. Stettler N, SignerTM, Suter PM. Electronic games and environmental factors associated with childhood obesity in Switzerland. Obes Res 2004;12(6):896-903.

78. Ebenegger V, Marques-Vidal PM, Nydegger A, et al. Independent contribution of parental migrant status and educational level to adiposity and eating habits in preschool children. Eur J Clin Nutr 2011;65(2):210-8.

79. Ebenegger V, Marques-Vidal P, Barral J, Kriemler S, Puder JJ, Nydegger A. Eating habits of preschool children with high migrant status in Switzerland according to a new food frequency questionnaire. Nutr Res 2010;30(2):104-9.

80. Arbeitsgemeinschaft BASS, ZHAW, ISPM, M.I.S TREND. Gesundheitsmonitoring der Migrationsbevölkerung (GMM) in der Schweiz. Schlussbericht. Bern: Bundesamt für Gesundheit, Bundesamt für Migration, August 2011.

Internet: http://www.bag.admin.ch/themen/ gesundheitspolitik/07685/12533/12535/index. html? lang=de (letzter Zugriff 6. Juni 2012).

81. Keller SK, Schulz PJ. Distorted food pyramid in kids programmes: A content analysis of television advertising watched in Switzerland. Eur J Public Health 2011;21(3):300-5.

82. Meyer K, ed. Gesundheit in der Schweiz. Nationaler Gesundheitsbericht 2008. Schweizerisches Gesundheitsobservatorium (OBSAN). Bern: Verlag Hans Huber, 2009.

83. Faeh D, Bopp M. Educational inequalities in mortality and associated risk factors: German- versus French-speaking Switzerland. BMC Public Health 2010;10:567.

84. Marques-Vidal P, Bovet P, Paccaud F, Chiolero A. Changes of overweight and obesity in the adult Swiss population according to educational level, from 1992 to 2007. BMC Public Health 2010;10(1):87.

85. Faeh D, Braun J, Bopp M. Prevalence of obesity in Switzerland 1992-2007: the impact of education, income and occupational class. Obes Rev 2011;12(3):151-66.
86. Loetscher KC, Selvin S, Zimmermann R, Abrams B. Ethnic-cultural background, maternal body size and pregnancy outcomes in a diverse Swiss cohort. Women Health 2007;45(2):25-40.

87. Institut für Sozial- und Präventivmedizin der Universität Zürich, ed. Gesundheit im Kanton Zürich 2000-2006. Zürich: Institut für Sozial- und Präventivmedizin der Universität Zürich, 2007.

88. Marques-Vidal P, Vollenweider P,Waeber G, Paccaud F. Prevalence of overweight and obesity among migrants in Switzerland: association with country of origin. Public Health Nutr 2011:1-9.

89. Faeh D, Bopp M. Increase in the Prevalence of Obesity in Switzerland 1982-2007: Birth Cohort Analysis Puts Recent Slowdown into Perspective. Obesity 2010;18(3):644-6.

90. Schneider H, Dietrich ES, VenetzWP. Trends and stabilization up to 2022 in overweight and obesity in Switzerland, Comparison to France, UK, US and Australia. Int J Environ Res Public Health 2010;7:460-72.

91. Faeh D. Die vom Menschen gestaltete Umwelt als Gesundheitsdeterminante. Die Struktur der Übergewichtsepidemie. Schweizerische Ärztezeitung 2006;87(34):1453-8.

92. Galani $\mathrm{C}$, Schneider H. Health economic model in overweight and obese people in Switzerland. Bern: Bundesamt für Gesundheit, 2006.

93. Galani $\mathrm{C}, \mathrm{Schneider} \mathrm{H}$. Prevention and treatment of obesity with lifestyle interventions: review and meta-analysis. Int J Public Health 2007;52(6):34859.

94. Kriemler S, Zahner L, Schindler C, et al. Effect of school based physical activity programme (KISS) on fitness and adiposity in primary schoolchildren: cluster randomised controlled trial. BMJ 2010;340:c785.

95. Estoppey D, Paccaud F, Vollenweider P, MarquesVidal P. Trends in self-reported prevalence and management of hypertension, hypercholesterolemia and diabetes in Swiss adults, 1997-2007. BMC Public Health 2011;11:114. 
96. Carrera E, Maeder-Ingvar M, Rossetti AO, Devuyst G, Bogousslavsky J. Trends in risk factors, patterns and causes in hospitalized strokes over 25 years: The Lausanne Stroke Registry. Cerebrovasc Dis 2007;24(1):97-103.

97. Gostynski M, Engelter S, Papa S, Ajdacic-Gross V, Gutzwiller F, Lyrer P. Incidence of first-ever ischemic stroke in the Canton Basel-City, Switzerland: a population-based study 2002/2003. J Neurol 2006;253(1):86-91.

98. Levi F, Chatenoud L, Bertuccio P, Lucchini F, Negri E, La Vecchia C. Mortality from cardiovascular and cerebrovascular diseases in Europe and other areas of the world: an update. Eur $\mathrm{J}$ Cardiovasc Prev Rehabil 2009;16(3):333-50.

99. Stolt Steiger V, Goy JJ, Stauffer JC, et al. Significant decrease in in-hospital mortality and major adverse cardiac events in Swiss STEMI patients between 2000 and December 2007. Swiss Med Wkly 2009;139(31-32):453-7.

100. Schweizerische Herzstiftung. Zahlen und Daten über Herz-Kreislauf-Krankheiten in der Schweiz. Bern: Schweizerische Herzstiftung, 2008.

101. Bundesamt für Statistik. Spitalaufenthalte 2008. Ausgewählte Ergebnisse. Neuchâtel: Bundesamt für Statistik, 2010. Internet: http://www.bfs.admin.ch/bfs/portal/de/ index/infothek/publ.html?publicationID=3951 (letzter Zugriff 30. September 2011).

102. Galobardes B, Costanza MC, Bernstein MS, Delhumeau C, Morabia A. Trends in risk factors for lifestyle-related diseases by socioeconomic position in Geneva, Switzerland, 1993-2000: health inequalities persist. Am J Public Health 2003;93(8):1302-9.

103. Avendano M, Kunst AE, Huisman M, et al. Socioeconomic status and ischaemic heart disease mortality in 10 western European populations during the 1990s. Heart 2006;92(4):461-7.

104. Morabia A, Costanza MC. The obesity epidemic as harbinger of a metabolic disorder epidemic: trends in overweight, hypercholesterolemia, and diabetes treatment in Geneva, Switzerland, 1993-2003. Am J Public Health 2005;95(4):632-5.
105. Schleiffenbaum BE, Schaer DJ, Burki D, et al. Unexpected high prevalence of metabolic disorders and chronic disease among young male draftees the Swiss Army XXI experience. Swiss MedWkly 2006;136(11-12):175-84.

106. Chiolero A, Cachat F, Burnier M, Paccaud F, Bovet P. Prevalence of hypertension in schoolchildren based on repeated measurements and association with overweight. J Hypertens 2007;25(11):2209-17.

107. L'Allemand D, Wiegand S, ReinehrT, et al. Cardiovascular risk in 26'008 European overweight children as established by a multicenter database. Obesity 2008;16(7):1672-9.

108. Faeh D, Gutzwiller F, Bopp M. Lower mortality from coronary heart disease and stroke at higher altitudes in Switzerland. Circulation 2009;120(6):495-501.

109. Chiolero A, Peytremann-Bridevaux I, Paccaud F. Associations between obesity and health conditions may be overestimated if self-reported body mass index is used. Obes Rev 2007;8(4):373-4.

110. Peytremann-Bridevaux I, Santos-Eggimann B. Health correlates of overweight and obesity in adults aged 50 years and over: results from the Survey of Health, Ageing and Retirement in Europe (SHARE). Obesity and health in Europeans aged $\geq 50$ years. Swiss MedWkly 2008;138(17-18):261-6.

111. Firmann M, Mayor V, Vidal PM, et al. The CoLaus study: a population-based study to investigate the epidemiology and genetic determinants of cardiovascular risk factors and metabolic syndrome. BMC Cardiovasc Disord 2008;8:6.

112. Aeberli I, Spinas GA, Lehmann R, I'Allemand D, Molinari L, Zimmermann MB. Diet determines features of the metabolic syndrome in 6- to 14-year-old children. Int J Vitam Nutr Res 2009;79(1):14-23.

113. Marques-Vidal P, Pecoud A, Hayoz D, et al. Normal weight obesity: Relationship with lipids, glycaemic status, liver enzymes and inflammation. Nutr Metab Cardiovasc Dis 2010;20(9):669-75. 
114. Prior JO, Allenbach G, Darioli R, Masson J. Apports de la médecine nucléaire en cardiologie préventive: I'exemple du syndrome métabolique. Rev Med Suisse 2008;4:644-9.

115. Clerc O, Nanchen D, Cornuz J, et al. Alcohol drinking, the metabolic syndrome and diabetes in a population with high mean alcohol consumption. Diabet Med 2010;27(11):1241-9.

116. Simonetti GD, Raio L, Surbek D, Nelle M, Frey FJ, Mohaupt MG. Salt sensitivity of children with low birth weight. Hypertension 2008;52(4):625-30.

117. Bundesamt für Gesundheit. Salz Strategie 20082012. Strategiepapier zur Reduktion des Kochsalzkonsums. Bern: Bundesamt für Gesundheit, 2009. Internet: http://www.bag.admin.ch/themen/ ernaehrung_bewegung/05207/05216/index.html? lang=de\&download=NHzLpZeg7t, Inp6IONTU042| 2Z6In1acy4Zn4Z2qZpnO2Yuq2Z6gpJCHfYJ7f2ym 162epYbg2c_JjKbNoKSn6A-- (letzter Zugriff 30. September 2011).

118. Beer-Borst S, Costanza MC, Pechere-Bertschi A, Morabia A. Twelve-year trends and correlates of dietary salt intakes for the general adult population of Geneva, Switzerland. Eur J Clin Nutr 2009;63(2):155-64.

119. Bachmann M, Keller U. Salzkonsum und Bluthochdruck. Schweiz Med Forum 2008;8(50):968-72.

120. Sun B, Williams JS, Svetkey LP, Kolatkar NS, Conlin PR. Beta2-adrenergic receptor genotype affects the renin-angiotensin-aldosterone system response to the Dietary Approaches to Stop Hypertension (DASH) dietary pattern. Am J Clin Nutr 2010;92(2):444-9.

121. Bundesamt für Statistik. GesundheitszustandDaten, Indikatoren. Übersicht chronische Krankheiten. Neuchâtel: Bundesamt für Statistik, 2007. Internet: http://www.bfs.admin.ch/bfs/portal/ de/index/themen/14/02/01/key/02.html (letzter Zugriff 30. September 2011).
122. Eidgenössische Ernährungskommission. Fette in der Ernährung. Empfehlungen. Expertenbericht der EEK. Bern: Bundesamt für Gesundheit, 2010. Internet: http://www.bag.admin.ch/themen/ ernaehrung_bewegung/05207/05211/index. html? lang=de\&download=NHzLpZeg7t, Inp6I0N TU042I2Z6In1acy4Zn4Z2qZpnO2Yuq2Z6gpJCFf YR_gWym162epYbg2c_JjKbNoKSn6A-- (letzter Zugriff 30. September 2011).

123. Aeberli I, Molinari L, Spinas G, Lehmann R, I'Allemand D, Zimmermann MB. Dietary intakes of fat and antioxidant vitamins are predictors of subclinical inflammation in overweight Swiss children. Am J Clin Nutr 2006;84(4):748-55.

124. Nishida C, Uauy R. WHO Scientific Update on health consequences of trans fatty acids: introduction. Eur J Clin Nutr 2009;63 Suppl 2:S1-4.

125. Siri-Tarino PW, Sun Q, Hu FB, Krauss RM. Metaanalysis of prospective cohort studies evaluating the association of saturated fat with cardiovascular disease. Am J Clin Nutr 2010;91(3):535-46.

126. World Health Organization. Interim summary of conclusion and dietary recommendations on total fat \& fatty acids. From the joint FAO / WHO expert consultation on fats and fatty acids in human nutrition, 10-14 November 2008, Geneva. Internet: http://www.who.int/nutrition/topics/ FFA_summary_rec_conclusion.pdf (letzter Zugriff 30. September 2011).

127. Leiber F, Kreuzer M, Nigg D, Wettstein HR, Scheeder MR. A study on the causes for the elevated $\mathrm{n}-3$ fatty acids in cows' milk of alpine origin. Lipids 2005;40(2):191-202.

128. Bertschi I, Collomb M, Rist L, et al. Maternal dietary Alpine butter intake affects human milk: fatty acids and conjugated linoleic acid isomers. Lipids 2005;40(6):581-7.

129. Hauswirth CB, Scheeder MR, Beer JH. High omega-3 fatty acid content in alpine cheese: the basis for an alpine paradox. Circulation 2004;109(1):103-7. 
130. Eidgenössische Ernährungskommission. Kohlenhydrate in der Ernährung. Stellungnahme und Empfehlungen der EEK. Bern: Bundesamt für Gesundheit, 2010.

Internet: http://www.bag.admin.ch/themen/ ernaehrung_bewegung/05207/07326/index. html? lang=de\&download=NHzLpZeg7t, Inp6ION TU042I2Z6In 1acy4Zn4Z2qZpnO2Yuq2Z6gpJCHfIR5fWym162epYbg2c_JjKbNoKSn6A-- (letzter Zugriff 30. September 2011).

131. Arriola L, Martinez-Camblor P, Larranaga N, et al. Alcohol intake and the risk of coronary heart disease in the Spanish EPIC cohort study. Heart 2010;96(2):124-30.

132. Mukamal KJ, Chiuve SE, Rimm EB. Alcohol consumption and risk for coronary heart disease in men with healthy lifestyles. Arch Intern Med 2006;166(19):2145-50.

133. Foerster M, Marques-Vidal P, Gmel G, et al. Alcohol drinking and cardiovascular risk in a population with high mean alcohol consumption. Am J Cardiol 2009;103(3):361-8.

134. Marques-Vidal P, Bochud M, Paccaud F, et al. No interaction between alcohol consumption and HDL-related genes on HDL cholesterol levels. Atherosclerosis 2010;211(2):551-7.

135. O'Donnell MJ, Xavier D, Liu L, et al. Risk factors for ischaemic and intracerebral haemorrhagic stroke in 22 countries (the INTERSTROKE study): a casecontrol study. Lancet 2010;376(9735):112-23.

136. Sugiyama K, Kuriyama S, Akhter M, et al. Coffee consumption and mortality due to all causes, cardiovascular disease, and cancer in Japanese women. J Nutr 2010;140(5):1007-13.

137. Flammer AJ, Hermann F, Sudano I, et al. Dark chocolate improves coronary vasomotion and reduces platelet reactivity. Circulation 2007;116(21):237682.

138. Danon-Hersch N, Marques-Vidal P, Bovet P, et al. Prevalence, awareness, treatment and control of high blood pressure in a Swiss city general population: the CoLaus study. Eur J Cardiovasc Prev Rehabil 2009;16(1):66-72.
139. Addor V, Wietlisbach V, Narring F, Michaud PA. Cardiovascular risk factor profiles and their social gradient from adolescence to age 74 in a Swiss region. Prev Med 2003;36(2):217-28.

140. Bolland MJ, Avenell A, Baron JA, et al. Effect of calcium supplements on risk of myocardial infarction and cardiovascular events: meta-analysis. BMJ 2010;341:c3691.

141. Marques-Vidal P, Pecoud A, Hayoz D, et al. Prevalence and characteristics of vitamin or dietary supplement users in Lausanne, Switzerland: the CoLaus study. Eur J Clin Nutr 2009;63(2):273-81.

142. House AA, Eliasziw M, Cattran DC, et al. Effect of B-vitamin therapy on progression of diabetic nephropathy: a randomized controlled trial. JAMA 2010;303(16):1603-9.

143. Bundesamt für Statistik. Gesundheitsstatistik 2009. Neuchâtel: Bundesamt für Statistik, 2009. Internet: http://www.bfs.admin.ch/bfs/portal/de/ index/infothek/publ.html?publicationID=3499 (letzter Zugriff 30. September 2011).

144. Interpharma. Gesundheitswesen Schweiz. 2011.

145. Schweizerische Herzstiftung. Zahlen und Daten über Herz-Kreislauf-Krankheiten in der Schweiz. Bern: Schweizerische Herzstiftung, 2004.

146. Morabia A, Costanza MC. Determinants of hypercholesterolemia in Geneva: should the focus be on genes or environment? Rev Med Suisse 2005;1(34):2198-204.

147. Bundesamt für Gesundheit. Salz Strategie 2008 2012. Konsultation der interessierten Kreise. Bern: Bundesamt für Gesundheit, 2009.

Internet: http://www.bag.admin.ch/themen/ ernaehrung_bewegung/05207/05216/index. html?lang=de\&download=NHzLpZeg7t, Inp6ION TU042I2Z6In1acy4Zn4Z2qZpnO2Yuq2Z6gpJCHfYJ7gWym162epYbg2c_JjKbNoKSn6A-- (letzter Zugriff 30. September 2011).

148. MarthalerTM, Petersen PE. Salt fluoridationan alternative in automatic prevention of dental caries. Int Dent J 2005;55(6):351-8. 
149. Richter EK, Albash Shawish K, Scheeder MRL, Colombani PC. Trans fatty acid content of selected Swiss foods: The TransSwissPilot study. J Food Comp Anal 2009;22:479-84.

150. Ngo Sock ET, Le KA, Ith M, Kreis R, Boesch C, Tappy L. Effects of a short-term overfeeding with fructose or glucose in healthy young males. $\mathrm{Br} \mathrm{J}$ Nutr 2010;103(7):939-43.

151. Krebsliga Schweiz. Krebs in der Schweiz: wichtige Zahlen. Bern: Krebsliga Schweiz, 2011.

152. Nationales Institut für Krebsepidemiologie und Registrierung (nicer). Krebs in der Schweiz. Stand und Entwicklung von 1983 bis 2007. Neuchâtel, 2011.

153. Sonnenberg A. Differences in the birth-cohort patterns of gastric cancer and peptic ulcer. Gut 2010;59(6):736-43.

154. Schmassmann A, Oldendorf MG, Gebbers JO. Changing incidence of gastric and oesophageal cancer subtypes in central Switzerland between 1982 and 2007. Eur J Epidemiol 2009;24(10):603-9.

155. The HAPO Study Cooperative Research Group. Hyperglycemia and Adverse Pregnancy Outcomes. N Engl J Med 2008;358(19):1991-2002.

156. Ceschi M, Gutzwiller F, Moch H, Eichholzer M, Probst-Hensch NM. Epidemiology and pathophysiology of obesity as cause of cancer. Swiss Med Wkly 2007;137(3-4):50-6.

157. Kesteloot $\mathrm{H}$. Regional differences in mortality: a comparison between Austria, Hungary and Switzerland. Acta Cardiol 1999;54(6):299-309.

158. Cullati S, Charvet-Berard AI, PernegerTV. Cancer screening in a middle-aged general population: factors associated with practices and attitudes. BMC Public Health 2009;9:118.

159. Rapiti E, Fioretta G, Schaffar R, et al. Impact of socioeconomic status on prostate cancer diagnosis, treatment, and prognosis. Cancer 2009;115(23):5556-65.
160. RodriguezT, Altieri A, Chatenoud L, et al. Risk factors for oral and pharyngeal cancer in young adults. Oral Oncol 2004;40(2):207-13.

161. Gallus S, Bosetti C, Franceschi S, Levi F, Negri E, La Vecchia C. Laryngeal cancer in women: tobac$\mathrm{co}$, alcohol, nutritional, and hormonal factors. Cancer Epidemiol Biomarkers Prev 2003;12(6):514-7.

162. Randi G, Scotti L, Bosetti C, et al. Pipe smoking and cancers of the upper digestive tract. Int J Cancer 2007;121(9):2049-51.

163. Institut für Sozial- und Präventivmedizin der Universität Zürich, ed. Krebs im Kanton Zürich. Kurzbericht des Krebsregisters. Zürich: Institut für Sozial- und Präventivmedizin der Universität Zürich, 2009.

Internet: http://www.gesundheitsfoerderungzh.ch/fileadmin/user_upload/publikationen/ Berichte/Brosch_Krebs_Kanton_Z\%C3\%BCrich. pdf (letzter Zugriff 30. September 2011).

164. Eichholzer M, Bernasconi F, Jordan P, Stähelin HB. Body mass index and the risk of male cancer mortality of various sites: 17-year follow-up of the Basel cohort study. Swiss MedWkly 2005;135(12):27-33.

165. Bosetti C, La Vecchia C, Talamini R, et al. Energy, macronutrients and laryngeal cancer risk. Ann Oncol 2003;14(6):907-12.

166. Augustin LS, Gallus S, Bosetti C, et al. Glycemic index and glycemic load in endometrial cancer. Int J Cancer 2003;105(3):404-7.

167. Lucenteforte E, Bosetti C, Talamini R, et al. Diabetes and endometrial cancer: effect modification by body weight, physical activity and hypertension. Br J Cancer 2007;97(7):995-8.

168. Taylor B, Rehm J, Gmel G. Moderate alcohol consumption and the gastrointestinal tract. Dig Dis 2005;23(3-4):170-6.

169. Altieri A, Bosetti C, Gallus S, et al. Wine, beer and spirits and risk of oral and pharyngeal cancer: a case-control study from Italy and Switzerland. Oral Oncol 2004;40(9):904-9. 
170. Bosetti C, Levi F, Boffetta P, Lucchini F, Negri E, La Vecchia C. Trends in mortality from hepatocellular carcinoma in Europe, 1980-2004. Hepatology 2008;48(1):137-45.

171. Levi F, Pasche C, Lucchini F, Bosetti C, La Vecchia C. Processed meat and the risk of selected digestive tract and laryngeal neoplasms in Switzerland. Ann Oncol 2004;15(2):346-9.

172. Bundesamt für Gesundheit. Acrylamid. Internet: http://www.bag.admin.ch/themen/ lebensmittel/04861/04907/index.html? lang=de (letzter Zugriff 30. September 2011).

173. Pelucchi C, Galeone C, Levi F, et al. Dietary acrylamide and human cancer. Int J Cancer 2006;118(2):467-71.

174. Galeone C, Pelucchi C, Talamini R, et al. Role of fried foods and oral/pharyngeal and oesophageal cancers. Br J Cancer 2005;92(11):2065-9.

175. Pelucchi C, Franceschi S, Levi F, et al. Fried potatoes and human cancer. Int J Cancer 2003;105(4):558-60.

176. Galeone C, Talamini R, Levi F, et al. Fried foods, olive oil and colorectal cancer. Ann Oncol 2007;18(1):36-9.

177. Test: Acrylamid in Pommes Chips. K-Tipp 11/2006. Internet: http://www.ktipp.ch/tests/1024942/ Acrylamid_in_Pommes_Chips (letzter Zugriff 30. September 2011).

178. Figueiredo JC, Grau MV, Haile RW, et al. Folic acid and risk of prostate cancer: results from a randomized clinical trial. J Natl Cancer Inst 2009;101 (6):432-5.

179. Ericson U, Borgquist S, Ivarsson MI, et al. Plasma folate concentrations are positively associated with risk of estrogen receptor beta negative breast cancer in a Swedish nested case control study. J Nutr 2010;140(9):1661-8.

180. Pelucchi C, Talamini R, Negri E, et al. Folate intake and risk of oral and pharyngeal cancer. Ann Oncol 2003;14(11):1677-81.

181. Levi F, Pasche $C$, Lucchini F, Ghidoni R, Ferraroni M, La Vecchia C. Resveratrol and breast cancer risk. Eur J Cancer Prev 2005;14(2):139-42.
182. Bidoli E, Bosetti C, La Vecchia C, et al. Micronutrients and laryngeal cancer risk in Italy and Switzerland: a case-control study. Cancer Causes Control 2003;14(5):477-84.

183. GaravelloW, Lucenteforte E, Bosetti C, et al. Diet diversity and the risk of laryngeal cancer: a casecontrol study from Italy and Switzerland. Oral Oncol 2009;45(1):85-9.

184. Galeone C, Pelucchi C, Levi F, et al. Onion and garlic use and human cancer. Am J Clin Nutr 2006;84(5):1027-32.

185. Tavani A, Bertuzzi M, Talamini R, et al. Coffee and tea intake and risk of oral, pharyngeal and esophageal cancer. Oral Oncol 2003;39(7):695-700.

186. Tavani A, Pelucchi C, Parpinel M, et al. n-3 polyunsaturated fatty acid intake and cancer risk in Italy and Switzerland. Int J Cancer 2003;105(1):113-6.

187. Kuendig $\mathrm{H}$. Estimation du nombre de personnes alcoolo-dépendantes dans la population helvétique (Rapport de recherche No 56). Lausanne: Addiction Info Suisse, 2010.

Internet: http://www.sucht-info.ch/fileadmin/ user_upload/estimation_alcoolodependants_ RR56.pdf (letzter Zugriff 30. September 2011).

188. Wicki M, Gmel G. Alkohol-Intoxikationen Jugendlicher und junger Erwachsener. Ein Update der Sekundäranalyse der Daten Schweizer Spitäler bis 2007. Lausanne: Schweizerische Fachstelle für Alkohol- und andere Drogenprobleme, 2009.

189. Annaheim B, Gmel G. Alkoholkonsum in der Schweiz. Ein Synthesebericht zu Alkoholkonsum und dessen Entwicklung auf der Basis der Schweizerischen Gesundheitsbefragungen 1997 und 2002. Lausanne: Schweizerische Fachstelle für Alkohol- und andere Drogenprobleme, 2004.

190. Shaw JE, Sicree RA, Zimmet PZ. Global estimates of the prevalence of diabetes for 2010 and 2030. Diabetes Res Clin Pract 2010;87(1):4-14.

191. King H, Rewers M. Global estimates for prevalence of diabetes mellitus and impaired glucose tolerance in adults. WHO Ad Hoc Diabetes Reporting Group. Diabetes Care 1993;16(1):157-77. 
192. Amos AF, McCarty DJ, Zimmet P. The rising global burden of diabetes and its complications: estimates and projections to the year 2010. Diabet Med 1997;14 Suppl 5:S1-85.

193. Allemann S, Saner C, Zwahlen M, Christ ER, Diem P, Stettler C. Long-term cardiovascular and non-cardiovascular mortality in women and men with type 1 and type 2 diabetes mellitus: a 30-year follow-up in Switzerland. Swiss MedWkly 2009;139(39-40):576-83.

194. International Diabetes Federation (IDF). Diabetes Atlas. 2011.

195. Lin X, Song K, Lim N, et al. Risk prediction of prevalent diabetes in a Swiss population using a weighted genetic score - the CoLaus Study. Diabetologia 2009;52(4):600-8.

196. Neilson A, Schneider H. Obesity and its comorbidities: present and future importance on health status in Switzerland. Soz Präventivmed 2005;50(2):78-86.

197. Steffen R, Potoczna N, Bieri N, Horber FF. Successful multi-intervention treatment of severe obesity: a 7-year prospective study with $96 \%$ follow-up. Obes Surg 2009;19(1):3-12.

198. Le KA, Ith M, Kreis R, et al. Fructose overconsumption causes dyslipidemia and ectopic lipid deposition in healthy subjects with and without a family history of type 2 diabetes. Am J Clin Nutr 2009;89(6):1760-5.

199. Joosten MM, Chiuve SE, Mukamal KJ, Hu FB, Hendriks HF, Rimm EB. Changes in alcohol consumption and subsequent risk of type 2 diabetes in men. Diabetes 2011;60(1):74-9.

200. Thomann R, Lenherr C, Keller U. Glycaemic control in hospitalised diabetic patients at the University Hospital Basel in 2002 and in 2005. Swiss Med Wkly 2009;139(37-38):547-52.

201. Weber C, Schneider B, Lodwig V, Holm MV, Neeser K. Cost impact of blood glucose self-monitoring on complications of type 2 diabetes: a Swiss perspective (ROSSO study No. 11). Swiss Med Wkly 2007;137(39-40):545-50.
202. Schmitt-Koopmann I, Schwenkglenks M, Spinas GA, Szucs TD. Direct medical costs of type 2 diabetes and its complications in Switzerland. Eur J Public Health 2004;14(1):3-9.

203. Sandoz MS, Ess SM, Keusch GW, Schwenkglenks $M$, Szucs TD. Prevalence and direct medical costs of end-stage renal disease in patients with type 2 diabetes mellitus in Switzerland for 2001. Swiss MedWkly 2004;134(31-32):448-58.

204. Noussitou P, Monbaron D, VialY, Gaillard RC, Ruiz J. Gestational diabetes mellitus and the risk of metabolic syndrome: a population-based study in Lausanne, Switzerland. Diabetes Metab 2005;31(4):361-9.

205. Schwenkglenks M, Szucs, Th.D. Epidemiologie der Osteoporose und der Frakturen im Alter. In: Bundesamt für Gesundheit, ed. Osteoporose und Stürze im Alter. Ein Public-Health-Ansatz. Bern: Bundesamt für Gesundheit, 2004:27-34.

206. Seeman E. Estrogen, androgen, and the pathogenesis of bone fragility in women and men. Curr Osteoporos Rep 2004;2(3):90-6.

207. Lippuner K. Osteoporose: Definition, Diagnostik und Therapie. In: Bundesamt für Gesundheit, ed. Osteoporose und Stürze im Alter. Ein PublicHealth-Ansatz. Bern: Bundesamt für Gesundheit, 2004:7-15.

208. Rizzoli R, Burckhardt P. Alimentation et ostéoporose. In: Eichholzer M, Camenzind-Frey E, Matzke M, Amadò R, Ballmer PE, et al., eds. Fünfter Schweizerischer Ernährungsbericht. Bern: Bundesamt für Gesundheit, 2005:553-72.

209. Bonjour JP, ChevalleyT, Rizzoli R, Ferrari S. Geneenvironment interactions in the skeletal response to nutrition and exercise during growth. Med Sport Sci 2007;51:64-80.

210. Bonjour JP, ChevalleyT, Ferrari S, Rizzoli R. The importance and relevance of peak bone mass in the prevalence of osteoporosis. Salud Publica Mex 2009;51 Suppl 1:S5-17. 
211. Rizzoli R, Bianchi ML, Garabedian M, McKay HA, Moreno LA. Maximizing bone mineral mass gain during growth for the prevention of fractures in the adolescents and the elderly. Bone 2010;46(2):294305.

212. ChevalleyT, Bonjour J-P, Ferrari S, Rizzoli R. High-Protein Intake Enhances the Positive Impact of Physical Activity on BMC in Prepubertal Boys. Journal of Bone and Mineral Research 2008;23(1):131-42.

213. Ginty F, Cavadini C, Michaud PA, et al. Effects of usual nutrient intake and vitamin $\mathrm{D}$ status on markers of bone turnover in Swiss adolescents. Eur J Clin Nutr 2004;58(9):1257-65.

214. Heaney RP, Layman DK. Amount and type of protein influences bone health. Am J Clin Nutr 2008;87(5):1567S-70S.

215. OrchardTS, Cauley JA, Frank GC, et al. Fatty acid consumption and risk of fracture in the Women's Health Initiative. Am J Clin Nutr 2010;92(6):145260.

216. Dratva J, Merten S, Ackermann-Liebrich U. Vitamin D supplementation in Swiss infants. Swiss MedWkly 2006;136(29-30):473-81.

217. Burckhardt P. Vitamin D, Calcium-Versorgung und Osteoporose in der Schweiz. In: Keller U, Lüthy J, Amadò R, Battaglia-Richi E, Battaglia R, Casabianca A, et al., eds. Vierter Schweizerischer Ernährungsbericht. Bern: Bundesamt für Gesundheit, 1998:250-60.

218. Michel C, Burckhardt, P. Aspects nutritionnels de I'ostéoporose. In: Stähelin HB, Lüthy J, Casabianca A, Monnier N, Müller H-R, SchutzY, et al., eds. Dritter Schweizerischer Ernährungsbericht. Bern: Bundesamt für Gesundheit, 1991:449-58.

219. Bischoff-Ferrari HA, Can U, Staehelin HB, et al., Severe vitamin $D$ deficiency in Swiss hip fracture patients. Bone 2008;42(3):597-602.

220. Dumartheray EW, Krieg MA, Cornuz J, Whittamore DR, Lanham-New SA, Burckhardt P. Energy and nutrient intake of Swiss women aged 75-87 years. J Hum Nutr Diet 2006 19(6):431-5.
221. Bundesamt für Gesundheit. Vitamin-D-Gehalte im Serum von gesunden Erwachsenen. Bisher unveröffentlichte Daten, 2011.

222. Marques-Vidal P, Pecoud A, Hayoz D, et al. Prevalence and characteristics of vitamin or dietary supplement users in Lausanne, Switzerland: the CoLaus study. Bisher unveröffentlichte Daten, 2011.

223. Bischoff-Ferrari HA, Shao A, Dawson-Hughes B, Hathcock J, Giovannucci E, WillettWC. Benefitrisk assessment of vitamin D supplementation. Osteoporos Int 2010;21(7):1121-32.

224. Bischoff-Ferrari HA, WillettWC, Wong JB, et al. Prevention of nonvertebral fractures with oral vitamin D and dose dependency: a meta-analysis of randomized controlled trials. Arch Intern Med 2009;169(6):551-61.

225. Kriemler S, Zahner L, Puder JJ, et al. Weightbearing bones are more sensitive to physical exercise in boys than in girls during pre-and early puberty: a cross-sectional study. Osteoporos Int 2008; 19(12):1749-58.

226. KemmlerW, von Stengel S. Exercise and osteoporosis-related fractures: perspectives and recommendations of the sports and exercise scientist. Physicians Sportsmed 2011;39(1):1-17.

227. Bocalini DS, Serra AJ, dos Santos L, Murad N, Levy RF. Strength training preserves the bone mineral density of postmenopausal women without hormone replacement therapy. J Aging Health 2009;21(3):519-27.

228. Engelke K, KemmlerW, Lauber D, Beeskow C, Pintag R, KalenderW. Exercise maintains bone density at spine and hip EFOPS: a 3-year longitudinal study in early postmenopausal women. Osteoporosis International 2006;17(1):133-42.

229. Wallace BA, Cumming RG. Systematic review of randomized trials of the effect of exercise on bone mass in pre-and postmenopausal women. Calcif Tissue Int 2000;67(1):10-8.

230. Pye SR, DevakumarV, Boonen S, et al. Influence of lifestyle factors on quantitative heel ultrasound measurements in middle-aged and elderly men. CalcifTissue Int 2010;86(3):211-9. 
231. Lamprecht M, Fischer A, Stamm H. Das Sportverhalten der Schweizer Bevölkerung. Magglingen: Bundesamt für Sport, 2008.

232. Lamprecht M, Fischer A, Stamm H. Sport Schweiz 2008. Kinder- und Jugendbericht. Magglingen: Bundesamt für Sport, 2008.

233. Schweizerische Vereinigung gegen die Osteoporose. Osteoporose. Prävention - Diagnostik Behandlung. 2010.

Internet: http://www.svgo.ch/content/documents/ SVGO_Empfehlungen2010_V19April2010.pdf (letzter Zugriff 30. September 2011).

234. Mehler PS, Cleary BS, Gaudiani JL. Osteoporosis in anorexia nervosa. Eat Disord 2011;19(2):194202.

235. Bonjour J-P, Guéguen L, Palacios C, Shearer MJ, Weaver CM. Minerals and vitamins in bone health: the potential value of dietary enhancement. $\mathrm{Br} \mathrm{J}$ Nutr 2009;101(11):1581-96.

236. SheuY, Cauley J. The Role of Bone Marrow and Visceral Fat on Bone Metabolism. Current Osteoporosis Reports 2011;9(2):67-75.

237. Ronis M, Mercer K, Chen J-R. Effects of nutrition and alcohol consumption on bone loss. Current Osteoporosis Reports 2011;9(2):53-9.

238. FentonTR, Eliasziw M, Lyon AW, Tough SC, Hanley DA. Meta-analysis of the quantity of calcium excretion associated with the net acid excretion of the modern diet under the acid-ash diet hypothesis. Am J Clin Nutr 2008;88(4):1159-66.

239. FentonTR, Lyon AW, Eliasziw M, Tough SC, Hanley DA. Meta-analysis of the effect of the acid-ash hypothesis of osteoporosis on calcium balance. J Bone Miner Res 2009;24(11):1835-40.

240. Wynn E, Lanham-New SA, Krieg MA, Whittamore DR, Burckhardt P. Low estimates of dietary acid load are positively associated with bone ultrasound in women older than 75 years of age with a lifetime fracture. J Nutr 2008;138(7):1349-54.

241. FentonTR, Eliasziw M, Tough SC, Lyon AW, Brown JP, Hanley DA. Low urine $\mathrm{pH}$ and acid excretion do not predict bone fractures or the loss of bone mineral density: a prospective cohort study. BMC Musculoskelet Disord 2010;11:88.
242. Wynn E, Krieg MA, Lanham-New SA, Burckhardt P. Postgraduate Symposium Positive influence of nutritional alkalinity on bone health. Proceedings of the Nutrition Society 2010;69(01):166-73.

243. Frings-Meuthen P. Einfluss einer hohen Kochsalzzufuhr auf die Natriumspeicherung, den Knochenstoffwechsel und den Säure-Basen-Haushalt bei ambulanten sowie immobilisierten Probanden. Bonn: Rheinische Friedrich-Wilhelms-Universität, 2008.

244. Schwenkglenks M, Szucs TD. Krankheitslast und Kosten der Osteoporose bis 2020. In: Bundesamt für Gesundheit, ed. Osteoporose und Stürze im Alter. Ein Public-Health-Ansatz. Bern: Bundesamt für Gesundheit, 2004:39-44.

245. Lippuner K, Johansson H, Kanis JA, Rizzoli R. Remaining lifetime and absolute 10 -year probabilities of osteoporotic fracture in Swiss men and women. Osteoporos Int 2009;20(7):1131-40.

246. Rizzoli R, Birkhaeuser MH, Burckhadt $P$, Lippuner K, Kraenzlin ME. Osteoporose in der Schweiz im Jahr 2008: eine Aufforderung zurTat. Schweiz Med Forum 2008;8(Suppl.45):1-11.

247. WHO Scientific Group on the Assessment of Osteoporosis at Primary Health Care Level. Summary Meeting Report. Brussels, Belgium, 5-7 May 2004. Geneva:WHO, 2007.

248. Prince R, Devine A, Dick I, et al. The effects of calcium supplementation (milk powder or tablets) and exercise on bone density in postmenopausal women. Journal of Bone and Mineral Research 1995;10(7):1068-75.

249. Bonjour JP, Brandolini-Bunlon M, BoirieY, et al. Inhibition of bone turnover by milk intake in postmenopausal women. Br J Nutr 2008;100(4): 866-74.

250. Zamora SA, Rizzoli R, Belli DC, Slosman DO, Bonjour JP. Vitamin D supplementation during infancy is associated with higher bone mineral mass in prepubertal girls. J Clin Endocrinol Metab 1999;84(12):4541-4.

251. Norman K, Pichard C, Lochs H, Pirlich M. Prognostic impact of disease-related malnutrition. Clin Nutr 2008;27(1):5-15 
252. Rizzoli R, Ammann P, Birkhäuser M, et al. Osteoporose: von der osteodensitometrischen Diagnose zur Evaluation des absoluten Frakturrisikos.

Schweiz Med Forum 2010;10(6):111-3.

253. Meier R. Der überempfindliche MagendarmtraktNahrungsmittel mit negativen Effekten im Darm. Praxis 2009;98:395-401.

254. Terjung B, Lammert, F. Laktoseintoleranz: Neue Aspekte eines alten Problems. Dtsch med Wochenschr 2007;132:271-5.

255. Heyman MB. Lactose intolerance in infants, children, and adolescents. Pediatrics 2006;118(3):1279-86.

256. Eidgenössische Ernährungskommission. Zöliakie. Expertenbericht der EEK. Bern: Bundesamt für Gesundheit, 2010. Internet: http://www.bag.admin.ch/themen/ ernaehrung_bewegung/05207/10411/index.html? lang=de\&download=NHzLpZeg7t, Inp6IONTU042 I2Z6In1acy4Zn4Z2qZnnO2Yuq2Z6gpJCJeHx4g2ym162epYbg2c_JjKbNoKSn6A-- (letzter Zugriff 30. September 2011).

257. Wüthrich $B$. Nahrungsmittelallergien und -intoleranzen. In: Eichholzer M, Camenzind-Frey E, Matzke A, Amadò R, Ballmer PE, et al., eds. Fünfter Schweizerischer Ernährungsbericht. Bern: Bundesamt für Gesundheit, 2005:623-46.

258. Santer R, Rischewski J, von Weihe M, et al. The spectrum of aldolase $B(A L D O B$ ) mutations and the prevalence of hereditary fructose intolerance in Central Europe. Hum Mutat 2005;25(6):594.

259. Mishkin D, Sablauskas L, Yalovsky M, Mishkin S. Fructose and sorbitol malabsorption in ambulatory patients with functional dyspepsia: comparison with lactose maldigestion/malabsorption. Dig Dis Sci 1997;42(12):2591-8.

260. Beyerlein L, Pohl D, Delco F, Stutz B, Fried M, Tutuian R. Correlation between symptoms developed after the oral ingestion of $50 \mathrm{~g}$ lactose and results of hydrogen breath testing for lactose intolerance. Aliment PharmacolTher 2008;27(8): 659-65.
261. Ingram CJ, Mulcare CA, Itan Y, Thomas MG, Swallow DM. Lactose digestion and the evolutionary genetics of lactase persistence. Hum Genet 2009;124(6):579-91.

262. Scrimshaw NS, Murray EB. The acceptability of milk and milk products in populations with a high prevalence of lactose intolerance. Am J Clin Nutr 1988;48(4 Suppl):1079-159.

263. Tatar G, Elsurer R, Simsek H, et al. Screening of tissue transglutaminase antibody in healthy blood donors for celiac disease screening in the Turkish population. Dig Dis Sci 2004;49(9):1479-84.

264. Matek Z, Jungvirth-Hegedus M, Kolacek S. Epidemiology of coeliac disease in children in one Croatian county: possible factors that could affect the incidence of coeliac disease and adherence to a gluten-free diet (Part II). Coll Antropol 2000;24(2):397-404.

265. Federal Commission for Nutrition. Proteins in Human Nutrition. Expert report of the FCN. Zurich: Federal Office of Public Health, 2011. Internet: http://www.bag.admin.ch/themen/ ernaehrung_bewegung/05207/11924/index.html? lang=de\&download=NHzLpZeg7t, Inp6IONTU042 | 2Z6In1acy4Zn4Z2qZpnO2Yuq2Z6gpJCKdH13gWym162epYbg2c_JjKbNoKSn6A-- (letzter Zugriff 30. September 2011).

266. Theler B, Brockow K, Ballmer-Weber BK. Clinical presentation and diagnosis of meat allergy in Switzerland and Southern Germany. Swiss Med Wkly 2009;139(17-18):264-70.

267. EuroPrevall. Internet: www.europrevall.org (letzter Zugriff 30. September 2011).

268. Institute of Food Research. Food Allergy Information. Internet: http://www.foodallergens.info/ (letzter Zugriff 30. September 2011).

269. Vieths S, Scheurer S, Ballmer-Weber B. Current understanding of cross-reactivity of food allergens and pollen. Ann NY Acad Sci 2002;964:47-68. 
270. Mittag D, Vieths S, Vogel L, et al. Soybean allergy in patients allergic to birch pollen: clinical investigation and molecular characterization of allergens. J Allergy Clin Immunol 2004;113(1):148-54.

271. Steinke M, Fiocchi A, KirchlechnerV, et al. Perceived food allergy in children in 10 European nations. A randomised telephone survey. Int Arch Allergy Immunol 2007;143(4):290-5.

272. SchäferT. Epidemiologie der Nahrungsmittelallergie in Europa. Ernährung 2008;2:4-9.

273. Ballmer-Weber B, Wüthrich B. Die Nahrungsmittelallergie und ihre diätetische Behandlung. Aktuel Ernährungsmed 2001;26:196-201.

274. Miles S, Fordham R, Mills C, Valovirta E, Mugford M. A framework for measuring costs to society of IgE-mediated food allergy. Allergy 2005;60(8):9961003.

275. Bähler P, Bänziger O, Belli D, et al. Empfehlungen für die Säuglingsernährung 2008. Ernährungskommission der Schweizerischen Gesellschaft für Pädiatrie. Schweiz Med Forum 2008;8(20):366-9.

276. Koletzko B, Brönstrup A, Cremer M, Flothkötter $M$, et a. Säuglingsernährung und Ernährung der stillenden Mutter. Handlungsempfehlungen - Ein Konsensuspapier im Auftrag des bundesweiten Netzwerk Junge Familie. Monatsschr Kinderheilkd 2010;158:679-89.

277. Eidgenössisches Departement des Innern. Verordnung des EDI vom 23. November 2005 über Speziallebensmittel. Stand 1. November 2010. SR 817.022.104. Internet: http://www.admin.ch/ch/d/sr/8/ 817.022.104.de.pdf (letzter Zugriff 30. September 2011).

278. Fox M, Voordouw J, Mugford M, Cornelisse J, Antonides G, Frewer L. Social and economic costs of food allergies in Europe: development of a questionnaire to measure costs and health utility. Health Serv Res 2009;44(5):1662-78.

279. Mathieu J. What is orthorexia? J Am Diet Assoc 2005;105(10):1510-2.

280. Fischer S, Meyer AH, Hermann E, Tuch A, Munsch S. Night Eating Syndrome in young adults: Delineation from other eating disorders and clinical significance. Psychiatry Research (under revision).
281. Fischer S, Munsch S. Institut de Psychologie, Psychologie clinique de l'enfant et de l'adolescent, Université de Lausanne. Bisher unveröffentlichte Daten, 2010.

282. Drobnjak S, Ehlert U. Gewichts- und figurbezogene Sorgen bei Frauen im mittleren Lebensalter und ihr Zusammenhang mit negativer Affektivität und Bildung. Verhaltenstherapie (in press).

283. Narring F, Tschumper A, Inderwildi Bonivento I, et al. Gesundheit und Lebensstil 16- bis 20-Jähriger in der Schweiz (2002) SMASH 2002. Swiss Multicenter Adolescent Study on Health 2002. Lausanne: Institut universitaire de médecine social et préventive Lausanne, 2004 (Raisons de santé, 95b).

284. Buddeberg-Fischer B, Gnam G, Klaghofer R, Buddeberg C. Störungen des Essverhaltens bei Jugendlichen als Risiko für die Entwicklung einer Anorexie oder Bulimie. In: Keller U, Lüthy J, Amadò R, Battaglia-Richi E, Battaglia R, Casabianca $A$, et al., eds. Vierter Schweizerischer Ernährungsbericht. Bern: Bundesamt für Gesundheit, 1998:392-411.

285. Devaud C, Michaud PA, Narring F. Perceptions corporelles, comportements et dysfonctions alimentaires parmi les adolescents suisse: une enquête nationale. Rev Med Suisse Romande 1994;114(11):1009-15.

286. Sucht Info Schweiz. Health Behaviour in Schoolaged Children 2010 (HBSC). Bisher unveröffentlichte Daten, 2011.

287. Mathys N, Meyer Egli C, Matter S, Biedert R, Birkhäuser M. Retrospektive Befragung bei Schweizer Athletinnen zur Female AthleteTriad. Schweizer Zeitschr Sportmed Sporttraumat 2005;53(4):167-71.

288. Laederach-Hofmann K, Isenschmid, B. Übergewicht und Essstörungen - eine Einführung. Schweizer Zeitschr für Ernährungsmedizin 2007;5:4-12.

289. Milos G, Spindler A, Schnyder U, Martz J, Hoek HW, Willi J. Incidence of severe anorexia nervosa in Switzerland: 40 years of development. Int J Eat Dis 2004;35(3):250-8.

290. de Zwaan M, Friederich HC. Binge Eating Störung. Ther Umsch 2006;63(8):529-33. 
291. Giusti V, Heraief E, Gaillard RC, Burckhardt P. Predictive factors of binge eating disorder in women searching to lose weight. EatWeight Disord 2004;9(1):44-9.

292. Brownley KA, Berkman ND, Sedway JA, Lohr $\mathrm{KN}$, Bulik CM. Binge eating disorder treatment: a systematic review of randomized controlled trials. Int J Eat Disord 2007;40(4):337-48.

293. Hepp U, Milos G. Essstörungen. Eine Einführung. Schweiz Med Forum 2010;10(48):834-40.

294. Isenschmid B. Essstörungen - Basiswissen, neue Erkenntnisse, Trends und Komplikationen. Schweizer Zeitschr für Ernährungsmedizin 2007:5:13-21.

295. Schulze U, Holl, R.W., Goldbeck, L. Essstörungen und Typ-1-Diabetes im Kindes- und Jugendalter. Monatsschr Kinderheilkd 2008;156:887-92.

296. Steinhausen HC, Weber S. The outcome of bulimia nervosa: findings from one-quarter century of research. Am J Psychiatry 2009;166(12):1331-41.

297. Steinhausen HC. Outcome of eating disorders. Child Adolesc Psychiatr Clin N Am 2009;18(1): 225-42.

298. Hillebrand JJG, Geary N. Neuroendocrine aspects of the control of eating in eating disorders. Schweizer Zeitschr Ernährungsmed 2007;5:25-7.

299. Steinhausen HC, Gavez S, Winkler Metzke C. Psychosocial correlates, outcome, and stability of abnormal adolescent eating behavior in community samples of young people. Int J Eat Disord 2005;37(2):119-26.

300. Spindler A, Milos G. Links between eating disorder symptom severity and psychiatric comorbidity. Eat Behav 2007;8(3):364-73.

301. Domine F, Berchtold A, Akre C, Michaud PA, Suris JC. Disordered eating behaviors: what about boys? J Adolesc Health 2009;44(2):111-7.

302. Roth D, Meyer-Egli C, Kriemler S, et al. Female AthleteTriad. Schweizer Zeitschr «Sportmedizin und Sporttraumatologie» 2000;48:119-32.

303. Matter S, Marti B. Prävalenz von Risikofaktoren einer "Female Athlete Triad" bei Schweizer Spitzensportlerinnen. Schweizer Zeitschr "Sportmedizin und Sporttraumatologie» 2001;49(3):112-6.
304. Bonci CM, Bonci LJ, Granger LR, et al. National athletic trainers' association position statement: preventing, detecting, and managing disordered eating in athletes. J AthITrain 2008;43(1):80-108.

305. Rosendahl J, Bormann B, Aschenbrenner K, Aschenbrenner F, Strauss B. Dieting and disordered eating in German high school athletes and nonathletes. Scand J Med Sci Sports 2009;19(5): 731-9.

306. Meltzer LJ, Johnson SB, Prine JM, Banks RA, Desrosiers PM, Silverstein JH. Disordered eating, body mass, and glycemic control in adolescents with type 1 diabetes. Diabetes Care 2001;24(4):678-82.

307. Hepp U, Spindler A, Schnyder U, Kraemer B, Milos G. Post-traumatic stress disorder in women with eating disorders. EatWeight Disord 2007;12(1):e24-7.

308. Zens M. Essstörungen / Eating Disorders. Fachinformationen zu aktuellenThemen 2. Recherche spezial. Leibnitz-Institut für Sozialwissenschaften, ed. Bonn: Leibnitz-Institut für Sozialwissenschaften, 2010.

309. Steinhausen HC. The outcome of anorexia nervosa in the 20th century. Am J Psychiatry 2002;159(8):1284-93.

310. Fichter MM, Quadflieg N, Hedlund S. Long-term course of binge eating disorder and bulimia nervosa: relevance for nosology and diagnostic criteria. Int J Eat Disord 2008;41(7):577-86.

311. Westenhoefer J. Prevalence of eating disorders and weight control practices in Germany in 1990 and 1997. International Journal of Eating Disorders 2001;29(4):477-81.

312. Hudson JI, Lalonde JK, Coit CE, et al. Longitudinal study of the diagnosis of components of the metabolic syndrome in individuals with binge-eating disorder. Am J Clin Nutr 2010;91 (6):1568-73.

313. Jacobs MJ, Roesch S, Wonderlich SA, et al. Anorexia nervosa trios: behavioral profiles of individuals with anorexia nervosa and their parents. Psychol Med 2009;39(3):451-61. 
314. Field AE, Javaras KM, Aneja P, et al. Family, peer, and media predictors of becoming eating disordered. Arch Pediatr Adolesc Med 2008;162(6):574-9.

315. Imfeld C, ImfeldT. Reflux und Essstörungen - «Ein Fall für Zwei». Ther Umsch 2008;65:97-102.

316. Federal Commission for Nutrition. Vitamin D deficiency: Evidence, safety, and recommendations for the Swiss population. Expert report of the FCN. Zurich: Federal Office of Public Health, 2012. Internet: http://www.bag.admin.ch/themen/ ernaehrung_bewegung/05207/13246/index. html?lang=de (letzter Zugriff 30. Juni 2012).

317. Imoberdorf R, Meier R, Krebs P, et al. Prevalence of undernutrition on admission to Swiss hospitals. Clin Nutr 2010;29(1):38-41.

318. Venzin RM, Kamber N, KellerWC, Suter PM, ReinhartWH. How important is malnutrition? A prospective study in internal medicine. Eur J Clin Nutr 2009;63(3):430-6.

319. Nutrition DayTeam. Nutrition Day 2006 - Swiss Results. Vienna: Department of Internal Medicine III, Division of Endocrinology and Metabolism, Medical University Vienna, 2006.

320. Nutrition DayTeam. Nutrition Day 2010 - Swiss Results. Vienna: Department of Internal Medicine III, Division of Endocrinology and Metabolism, Medical University Vienna, 2010.

321. Saxer S, Hessler I, Ivanovic N. Prävalenzerhebung Schweiz 2010 (Alters- und Pflegeheim). St. Gallen: Institut für angewandte Pflegewissenschaft (IPW). Unveröffentlichte Daten, 2010.

322. Bourquin L, Senes HR, Germanier A, Kehtari R. Screening of community malnutrition by homecare staff in Neuchâtel-Switzerland. Rev Med Suisse 2008;4(179):2458-62.

323. GerberV, Krieg MA, Cornuz J, GuigozY, Burckhardt P. Nutritional status using the Mini Nutritional Assessment questionnaire and its relationship with bone quality in a population of institutionalized elderly women. J Nutr Health Aging 2003;7(3):140-5.
324. Expertengruppe des Europarates und Eidgenössische Ernährungskommission. Mangelernährung im Spital. Stellungnahme einer Expertengruppe des Europarates und Empfehlungen der EKK. Bern: Bundesamt für Gesundheit, 2005. Internet: http://www.bag.admin.ch/themen/ ernaehrung_bewegung/05207/05226/index.html? lang=de\&download =NHzLpZeg7t, Inp6IONTU042 I2Z6In1acy4Zn4Z2qZpnO2Yuq2Z6gpJCFe4R6g2ym162epYbg2c_JjKbNoKSn6A-- (letzter Zugriff 30. September 2011).

325. Ernst B, Thurnheer M, Schmid SM, Schultes B. Evidence for the necessity to systematically assess micronutrient status prior to bariatric surgery. Obes Surg 2009;19(1):66-73.

326. Gasteyger C, Suter M, Calmes JM, Gaillard RC, GiustiV. Changes in body composition, metabolic profile and nutritional status 24 months after gastric banding. Obes Surg 2006;16(3):243-50.

327. Adam O, Tesche A, Wolfram G. Impact of linoleic acid intake on arachidonic acid formation and eicosanoid biosynthesis in humans. Prostaglandins Leukot Essent Fatty Acids 2008;79(3-5):177-81.

328. Council of Europe. Food and nutritional care in hospitals: how to prevent undernutrition. Report and recommendations of the Committee of Experts on Nutrition, Food Safety and Consumer Protection. Council of Europe Publishing. Strasbourg, November 2002.

329. Schneider A. Mangelernährung bei Adipositas. Epidemiologie, Diagnostik und Therapie. Akt Ernähr Med 2008;33:280-3.

330. Rufenacht $U$, Ruhlin $M$, Wegmann M, Imoberdorf R, Ballmer PE. Nutritional counseling improves quality of life and nutrient intake in hospitalized undernourished patients. Nutrition 2010;26(1): 53-60.

331. Hamada MO, Garrett NR, Roumanas ED, et al. A randomized clinical trial comparing the efficacy of mandibular implant-supported overdentures and conventional dentures in diabetic patients. Part IV: Comparisons of dietary intake. J Prosthet Dent 2001;85(1):53-60. 
332. Volkert D. Die Rolle der Ernährung zur Prävention von Sarkopenie und Frailty. Schweiz Z Ernährungsmed 2009;7:25-31.

333. Santos-Eggimann B, Karmaniola A, SeematterBagnoud L, et al. The Lausanne cohort Lc65+: a population-based prospective study of the manifestations, determinants and outcomes of frailty. BMC Geriatr 2008;8:20.

334. Frei A. Mangelernährung im Spital-medizinische Kosten und Kosteneffektivität beiVerhinderung. Bern: Bundesamt für Gesundheit, 2006. Internet: http://www.bag.admin.ch/themen/ ernaehrung_bewegung/05207/05226/index.html? lang=de\&download=NHzLpZeg7t, Inp6IONTU042I 2Z6In1acy4Zn4Z2qZpnO2Yuq2Z6gpJCGd3t7f2ym 162epYbg2c_JjKbNoKSn6A-- (letzter Zugriff 30. September 2011).

335. Imhof L, Naef R, Mahrer-Imhof R. Forschungsprioritäten der gerontologischen Pflege. Pflege 2008;21:435-51.

336. Berthod G, Roduit J, Roulet M, Coti Bertrand P. Hospital undernutrition: how not to ignore it any more? Rev Med Suisse 2007;3(131):2466-71.

337. Schindler K, Pernicka E, Laviano A, et al. How nutritional risk is assessed and managed in European hospitals: a survey of 21'007 patients findings from the 2007-2008 cross-sectional nutritionDay survey. Clin Nutr 2010;29(5):552-9.

338. Salva A, Coll-Planas L, Bruce S, et al. Nutritional assessment of residents in long-term care facilities (LTCFs): recommendations of the task force on nutrition and ageing of the IAGG European region and the IANA. J Nutr Health Aging 2009;13(6):475-83.

339. Rüfenacht U, Rühlin M, Imoberdorf R, Ballmer PE. Das Tellerdiagramm: Ein sinnvolles Erfassungsinstrument für ungenügende Nahrungszufuhr bei Patienten im Krankenhaus. Aktuel Ernaehr Med 2006;31:66-72.

340. Eichholzer M, Bovey F, Jordan P, Probst-Hensch N, Stoffel-Kurt N. Daten zum Übergewicht und zu Ernährungsgewohnheiten aus der Schweizerischen Gesundheitsbefragung 2007. PRAXIS 2010;99:17-25.
341. Jans-Ruggli S, Baerlocher, K. Kenntnisse über Folsäure und Folsäurestatus bei Müttern: Häufige Einnahme von Folsäuresupplementen, aber ungenügende Prävention von Neuralrohrdefekten. In: Eichholzer M, Camenzind-Frey E, Matzke A, Amadò R, Ballmer PE et al., ed. Fünfter Schweizerischer Ernährungsbericht. Bern: Bundesamt für Gesundheit, 2005:71-86.

342. Eigenössische Ernährungskommission. Folsäure. Expertenbericht der EEK zur Prophylaxe von Neuralrohrdefekten. Bern: Bundesamt für Gesundheit, 2002.

Internet: http://www.bag.admin.ch/themen/ernaehrung_bewegung/05207/05212/index.html?lan$\mathrm{g}=$ de\&download=NHzLpZeg7t, Inp6IONTU042I2 Z6In1acy4Zn4Z2qZpnO2Yuq2Z6gpJCFeX1_fWym162epYbg2c_JjKbNoKSn6A-- (letzter Zugriff 30. September 2011).

343. Swiss Paediatric Surveillance Unit. SPSU-Jahresbericht 2007. BAG-Bulletin 2008;39:672-80.

344. Registry of Vaud - Switzerland EUROCAT. Prevalence of neural tube defects (NTD) in Canton Vaud 1992-2009. Lausanne: Service de Génétique Médicale, CHUV.

345. Eichholzer M, Tönz O, Zimmermann R. Folic acid: a public-health challenge. Lancet 2006;367(9519): 1352-61.

346. European Food Safety Authority. Folic acid: an update on scientific developments. EFSA meeting summary report. 21-22 January 2009, Uppsala/ Sweden. Parma: EFSA, 2010.

347. Rizzoli R, Eisman JA, Norquist J, et al. Risk factors for vitamin $D$ inadequacy among women with osteoporosis: an international epidemiological study. International Journal of Clinical Practice 2006;60(8):1013-9.

348. Bischoff-Ferrari HA, Dawson-Hughes B, Platz A, et al. Effect of high-dosage cholecalciferol and extended physiotherapy on complications after hip fracture: a randomized controlled trial. Arch Intern Med 2010;170(9):813-20. 
349. Burnand B, Sloutskis D, Gianoli F, et al. Serum 25-hydroxyvitamin D: distribution and determinants in the Swiss population. Am J Clin Nutr 1992;56(3):537-42.

350. Holick MF. Environmental factors that influence the cutaneous production of vitamin D. Am J Clin Nutr 1995;61 (3 Suppl):638S-45S.

351. Friedrich W. Handbuch der Vitamine. München: Urban\&Schwarzenberg, 1987.

352. Holick MF, Matsuoka LY, Wortsman J. Age, vitamin D, and solar ultraviolet. Lancet 1989;2(8671): 1104-5.

353. Lau KH, Baylink DJ. Vitamin D therapy of osteoporosis: plain vitamin $D$ therapy versus active vitamin $D$ analog (D-hormone) therapy. CalcifTissue Int 1999;65(4):295-306.

354. Dawson-Hughes B, Mithal A, Bonjour JP, et al. IOF position statement: vitamin $D$ recommendations for older adults. Osteoporos Int 2010;21(7): 1151-4.

355. Ross AC, Manson JE, Abrams SA, et al. The 2011 report on dietary reference intakes for calcium and vitamin D from the Institute of Medicine: what clinicians need to know. J Clin Endocrinol Metab 2011;96(1):53-8.

356. Joint World Health Organization / Centers for Disease Control and Prevention Technical Consultation on the Assessment of Iron Status at the Population Level (2004: Geneva / Switzerland). Assessing the iron status of populations: including literature reviews: report of a Joint World Health Organization / Centers for Disease Control and PreventionTechnical Consultation on the Assessment of Iron Status at the Population Level, Geneva, Switzerland, 6-8 April 2004. - 2nd ed. Geneva:WHO, 2007.

357. Andersson M, Egli IM, Zimmermann MB. Eisenmangel. Schweizer Zeitschr Ernährungsmed 2010;1:13-8.

358. Merlo CM, Wuillemin WA. Prävalenz und Ursachen von Anämien in einer städtischen Hausarztpraxis. Praxis 2008;97(13):713-8.
359. Mettler S, Zimmermann MB. Iron excess in recreational marathon runners. Eur J Clin Nutr 2010;64(5):490-4.

360. Hess SY, Zimmermann MB, Brogli S, Hurrell RF. A national survey of iron and folate status in pregnant women in Switzerland. Int J Vitam Nutr Res 2001;71(5):268-73.

361. Waldvogel Abramowski S, Canellini G, Vedy D, Tissot JD. Iron deficiency without anemia in young female blood donors: a reality that needs management. Poster. Forum Med Suisse 2008;8:(Suppl 40) $67 \mathrm{~S}$.

362. O'Meara A, Infanti L, Stebler C, et al. The value of routine ferritin measurement in blood donors. Transfusion 2011;51(10):2183-8.

363. Fehr J, Favrat B, Schleiffenbaum B, Krayenbühl PA, Kapanci C, von Orelli F. Diagnostic et traitement de la carence en fer sans anémie. Rev Med Suisse 2009;5:2229-34.

364. Schiesser D, Binet I, Tsinalis D, et al. Weekly lowdose treatment with intravenous iron sucrose maintains iron status and decreases epoetin requirement in iron-replete haemodialysis patients. Nephrol DialTransplant 2006;21(10):2841-5.

365. Schulzke JD, Tröger H, Amasheh M. Disorders of intestinal secretion and absorption. Best Pract Res Clin Gastroenterol 2009;23(3):395-406.

366. Biecker E, Stieger M, Zimmermann A, Reichen J. Autoimmune hepatitis, cryoglobulinaemia and untreated coeliac disease: a case report. Eur J Gastroenterol Hepatol 2003;15(4):423-7.

367. Aeberli I, Hurrell RF, Zimmermann MB. Overweight children have higher circulating hepcidin concentrations and lower iron status but have dietary iron intakes and bioavailability comparable with normal weight children. Int J Obes 2009;33(10):1111-7.

368. Hurrell R, Egli I. Iron bioavailability and dietary reference values. Am J Clin Nutr 2010;91(5):1461S-7S.

369. WHO / UNICEF / UNU. Iron Deficiency Anemia Assessment, Prevention, and Control. Geneva: World Health Organization, 2001. 
370. Kraemer K, Zimmermann MB, eds. Nutritional Anemia. Basel: Sight and Life Press, 2007.

371. Bundesamt für Statistik. Statistik der natürlichen Bevölkerungsbewegung (BEVNAT) 2009. Neuchâtel: Bundesamt für Statistik, 2010. Internet: http://www.bfs.admin.ch/bfs/portal/de/ index/infothek/publ.html?publicationID=3982 (letzter Zugriff 30. September 2011).

372. Beard J, Tobin B. Iron status and exercise. Am J Clin Nutr 2000;72(2 Suppl):594S-7S.

373. Andersson M, Aeberli I, Wust N, et al. The Swiss iodized salt program provides adequate iodine for school children and pregnant women, but weaning infants not receiving iodine-containing complementary foods as well as their mothers are iodine deficient. J Clin Endocrinol Metab 2010;95(12):5217-24.

374. Zimmermann MB, Jooste PL, Pandav CS. lodine-deficiency disorders. Lancet 2008;372(9645):1251-62.

375. World Health Organization, United Nations Children's Fund, International Council for the Control of lodine Deficiency Disorders. Assessment of iodine deficiency disorders and monitoring their elimination. 3rd edition. Geneva:WHO, 2007.

376. Zimmermann MB. Iodine deficiency. Endocr Rev 2009;30(4):376-408.

377. Zimmermann MB, Burgi $H$, Hurrell RF. Iron deficiency predicts poor maternal thyroid status during pregnancy. J Clin Endocrinol Metab 2007;92(9):3436-40.

378. Zimmermann MB. The influence of iron status on iodine utilization and thyroid function. Annu Rev Nutr 2006;26:367-89.

379. Burri J, Haldimann M, DudlerV. Selenium status of the Swiss population: assessment and change over a decade. JTrace Elem Med Biol 2008;22(2):112-9.

380. Haldimann M, VennerTY, Zimmerli B. Determination of selenium in the serum of healthy Swiss adults and correlation to dietary intake. JTrace Elem Med Biol 1996;10:31-45.
381. Jenny-Burri J, Haldimann M, DudlerV. Estimation of selenium intake in Switzerland in relation to selected food groups. Food Addit Contam Part A Chem Anal Control Expo Risk Assess 2010;27(11):1516-31.

382. Hahn A, Schuchardt JP. Selen - die Frage nach der optimalen Dosis. Schweizer Zeitschr Ernährungsmed 2011;9(1):15-24.

383. Neve J. Methods in determination of selenium states. JTrace Elem Electrolytes Health Dis 1991;5(1):1-17.

384. Thomson CD. Assessment of requirements for selenium and adequacy of selenium status: a review. Eur J Clin Nutr 2004;58(3):391-402.

385. Combs GF, Jr., Clark LC, Turnbull BW. An analysis of cancer prevention by selenium. Biofactors 2001;14(1-4):153-9.

386. Menghini G, Steiner M. Orale Gesundheit in der Schweiz-Stand 2006. Monitoring. OBSAN Arbeitsdokument 26. Neuchâtel: Bundesamt für Statistik, 2007.

Internet: http://www.bfs.admin.ch/bfs/portal/de/ index/infothek/publ.html?publication ID =2849 (letzter Zugriff 30. September 2011).

387. JaeggiT, Lussi A. Erosionen bei Kindern im frühen Schulalter. Schweiz Monatsschr Zahnmed 2004;114:876-81.

388. JaeggiT, Schaffner M, Burgin W, Lussi A. Erosions and wedge-shaped defects in Swiss Army recruits. Schweiz Monatsschr Zahnmed 1999;109(11):1170-82.

389. JaeggiT, Lussi A. Prevalence, incidence and distribution of erosion. In: Lussi A, ed. Dental Erosion Monogr Oral Sci. Basel: Karger, 2006:44-65.

390. ImfeldT. Ernährung, Nahrungsmittel und Zahngesundheit-De- und Remineralisation der Zähne. Ther Umsch 2008;65:69-73.

391. Menghini G, Steiner M, Thomet $E$, Roos $M$, ImfeldT. Caries prevalence in 2-year-old children in the city of Zurich. Community Dent Health 2008;25(3):154-60. 
392. Zitzmann NU, Staehelin K, Walls AW, Menghini G, Weiger R, Zemp Stutz E. Changes in oral health over a 10-yr period in Switzerland. Eur J Oral Sci 2008;116(1):52-9.

393. Lussi A, Schaffner M, JaeggiT, Grüninger A. Erosionen. Schweiz Monatszeitschr Zahnmed 2005;115(10):917-35.

394. Al-Dlaigan YH, Shaw L, Smith A. Dental erosion in a group of British 14-year-old school children. Part II: Influence of dietary intake. Br Dent J 2001;190(5):258-61.

395. Johansson AK, Lingstrom P, ImfeldT, Birkhed D. Influence of drinking method on tooth-surface $\mathrm{pH}$ in relation to dental erosion. Eur J Oral Sci 2004;112(6):484-9.

396. Janner M, Ballinari P, Mullis PE, Fluck CE. High prevalence of vitamin $D$ deficiency in children and adolescents with type 1 diabetes. Swiss MedWkly 2010;140:w13091.

397. Bundesamt für Gesundheit. Jodversorgung der Schweizer Bevölkerung. Ergebnisse aus einer nationalen Multizenterstudie. Bisher unveröffentlichte Daten, 2011. 


\section{Ernährungsmassnahmen zur Förderung der Gesundheit}

\section{Christian Ryser}

Alexia Fournier Fall

Sophie Frei

Andrea May 


\subsection{Zusammenfassung}

Ernährungsabhängige Krankheiten wie HerzKreislauf-Krankheiten, Diabetes mellitus und verschiedene Krebsformen tragen wesentlich zu den Gesundheitskosten in der Schweiz bei. Bestrebungen zur Förderung einer ausgewogenen Ernährung zwecks Verminderung des Risikos dieser Krankheiten sind daher auch aus ökonomischen Gründen sinnvoll. In der Schweiz orientieren sich Bund und Fachwelt dabei im Wesentlichen am übergeordneten Rahmen der Weltgesundheitsorganisation und der Europäischen Union. Den strategischen Rahmen auf Schweizer Ebene legt das Nationale Programm Ernährung und Bewegung des Bundes.

Massnahmen zur Förderung einer ausgewogenen Ernährung sollen evidenzbasiert sein. Um Wirkungen zu erzielen, wird empfohlen, Massnahmen sowohl auf der Verhaltens- als auch auf der Verhältnisebene zu implementieren. Die Evidenz, welche Massnahme oder welche Kombination von Massnahmen wirksam ist, ist aber nach wie vor relativ schwach: Oft fehlen Evaluationsergebnisse, wirksame Einzelmassnahmen sind nicht eruierbar, Wirksamkeitsüberprüfungen von Ernährungsinterventionen sind komplex. Aus nationalen und europäischen Interventionen können immerhin einzelne kontextbezogene Erfolgsfaktoren abgeleitet werden.

$\mathrm{Zu}$ den prioritären Handlungsempfehlungen für die Schweiz zählen die Verbesserung der aktuell ungenügenden Datenlage (v.a. in Bezug auf Lebensmittelverzehr, Ernährungsverhalten und ernährungsabhängige Krankheiten und Risikofaktoren), die Stärkung der Gesundheitsund Ernährungskompetenzen und die Förderung des Früchte- und Gemüsekonsums bei Kindern. Grosses Potenzial besteht weiter bei der Verbesserung der Konsumenteninformation (z.B. Nährwert- und Front-of-Pack-Kennzeichnung von Lebensmitteln) sowie bei der Optimierung der Zusammensetzung von Angeboten der Ausser-Haus-Verpflegung und im Bereich der Convenience-Produkte und Fertigmahlzeiten (Salz-, Fett- und Zuckergehalt, Fettqualität). Massnahmen zur Beschränkung der Vermarktung von Lebensmitteln und nichtalkoholischen Getränken mit einem hohen Gehalt an Fett, Zucker oder Salz, die sich an Kinder richten, sind ein weiterer Handlungsbereich mit Potenzial.

Bei der Umsetzung von Massnahmen sollten die föderalistische Struktur der Schweiz berücksichtigt und der multisektorale Ansatz (Einbezug aller Politikbereiche) verfolgt werden. Dies bedingt eine entsprechende Koordination zwischen nationalen, kantonalen und kommunalen Akteuren. Als Zielgruppen zukünftiger Ernährungsinterventionen sollten besonders Männer, junge Menschen und sozio-ökonomisch benachteiligte Bevölkerungsgruppen angesprochen werden, da bei diesen der grösste Handlungsbedarf besteht.

\subsection{Einleitung}

\section{Bedeutung und Potenzial von Ernährungs-} interventionen

Wie in vielen OECD-Staaten nehmen auch in der Schweiz die Gesundheitskosten schneller zu als das Brutto-Inlandprodukt $(1 ; 2)$. Ein wesentlicher Kostenfaktor ist die grosse Anzahl an Krankenhäusern und die zunehmende Zahl von Menschen mit nichtübertragbaren Krankheiten (Adipositas, Diabetes mellitus, Herz-KreislaufKrankheiten, Krebs usw.), die weniger intensiv, dafür aber regelmässig versorgt werden müssen. Übergewicht und Adipositas verursachten 
in der Schweiz im Jahr 2006 direkte und indirekte Kosten von CHF 5.8 Mia., was im Vergleich zu den Kosten im Jahr 2004 mehr als einer Verdoppelung gleichkommt (3). Die direkten Kosten übergewichtsbedingter Erkrankungen beliefen sich auf CHF 3.8 Mia. und entsprachen im Jahr 2006 7.3\% der gesamten Gesundheitskosten von CHF 52.7 Mia.

Der OECD-Bericht empfiehlt vor diesem Hintergrund, das Gesundheitssystem stärker auf Grundversorgung und Vorsorge auszurichten. Zurzeit fliessen in der Schweiz nur gerade 2.3\% aller Ausgaben im Gesundheitssektor in die Gesundheitsförderung und Prävention - der OECDDurchschnitt liegt bei $3.1 \%$ (1). Dies ist umso bedeutungsvoller, als der Anteil älterer Menschen an der Gesamtbevölkerung immer grösser wird und diese Menschen möglichst lange von einer hohen Lebensqualität profitieren möchten (4). Die Gesundheitskosten, die im Jahr 2008 von Menschen im Alter zwischen 56-90 Jahren verursacht wurden, betrugen CHF $31.5 \mathrm{Mia}$., was einem Anteil von nahezu 54\% der gesamten Gesundheitskosten entsprach (5). Die Alterung der Bevölkerung ist besonders im Bereich der Langzeitpflege für die über 65-Jährigen ein wichtiger Kostentreiber (6).

Das Mass an staatlichen Interventionen, das Recht auf Selbstbestimmung und die Übernahme von Selbstverantwortung sind immer wieder Themen öffentlicher Diskussionen in der Schweiz, nicht zuletzt auch im Ernährungs- und Gesundheitsbereich (7). In den vergangenen Jahren wurde vermehrt ein Erfolgsnachweis insbesondere von staatlichen Interventionen gefordert; die Wirksamkeitsüberprüfung ist auch im Art. 170 der Bundesverfassung festgehalten (8). Für die Beurteilung des Erfolgs von Interventionen ist die Gegenüberstellung der Ausgangs- grösse "Aufwand" und des erzielten "Nutzens» von entscheidender Bedeutung. Weil sich Interventionen im Ernährungsbereich aber nicht in geschlossenen Forschungssystemen abspielen, sondern in komplexen, vielschichtigen, dynamischen und unberechenbaren sozialen Systemen, dabei dem Einfluss von zahlreichen, nicht kontrollierbaren "Störgrössen» unterworfen sind sowie in der Regel erst langfristig Wirkung entfalten können, ist dieses Ursache-WirkungsPrinzip im Präventions- und Gesundheitsförderungsbereich nur in angepasster Form anzuwenden (9). Im Bereich der Übergewichtsprävention ist der Wirkungsnachweis von Interventionen zunehmend, wenn auch immer noch beschränkt (1;10-15). Klar ist, dass zur Umkehr der weltweiten Adipositasepidemie einzelne Interventionen nicht ausreichen, sondern ein breites Spektrum an Interventionen über alle Sektoren hinweg nötig ist. Diese Ausgangslage erschwert die Rechtfertigung von Interventionen insbesondere auf politischer Ebene.

In jüngsten Jahren wird allerdings auch in der Schweiz der politische Wille signalisiert, Gesundheit mehr und mehr als öffentliche Aufgabe anzuerkennen - wie z.B. durch die Verankerung des Themas Gesundheit in den sprachregionalen Lehrplänen (Kapitel 4.7.3).

\section{Inhalt dieses Kapitels}

Das vorliegende Kapitel 4 stützt sich auf die aktuellen Ernährungsempfehlungen (Kapitel 1), die Ernährungssituation in der Schweiz (Kapitel 2) und deren Bedeutung für die Gesundheit (Kapitel 3). Daraus leiten sich die wichtigsten Schlussfolgerungen, die dringlichsten Handlungsempfehlungen für die Schweiz zur Förderung einer ausgewogenen Ernährung sowie die dafür notwendigen Rahmenbedingungen ab. 


\subsection{Politische und gesetzliche Rahmenbedingungen in der Schweiz}

\section{Föderalismus}

Eines der wichtigen Merkmale des schweizerischen Gesundheitssystems stellt der Föderalismus mit der Kompetenzaufteilung zwischen Bund, Kantonen und Gemeinden dar $(1 ; 16 ; 17)$. Der Bund kann Prävention und Gesundheitsförderung nur so weit regeln, als ihm in der Bundesverfassung der Schweizerischen Eidgenossenschaft entsprechende Rechtsetzungskompetenzen zugewiesen wurden. Die Hauptverantwortung der öffentlichen Gesundheit liegt bei den Kantonen; dem Bund kommt eine subsidiäre Rolle zu, so dass er nur dort tätig wird, wo Kantone und Gemeinden über keine entsprechenden Zuständigkeiten verfügen.

Heute bestehen neben der Bundespolitik 26 kantonale Gesundheitspolitiken. Zunehmend setzen Bund und Kantone die Rahmenbedingungen für Gesundheitsförderung und Prävention nicht mehr alleine, sondern orientieren sich am vorgegebenen Rahmen der Europäischen Union (EU) und der Weltgesundheitsorganisation (WHO).

\section{Multisektorale Gesundheitspolitik}

Entsprechend dem modernen Gesundheitsverständnis mit einem umfassenden und multisektoralen Ansatz ist es erforderlich, Gesundheitsförderung und Prävention in allen Politikbereichen zu berücksichtigen $(18 ; 19)$. Für das Gesundheitsthema relevante Politikbereiche sind Landwirtschaft, Sport, Bildung, Verkehrs- und Raumordnung, Umwelt, Soziales, Arbeitsmarkt und Handel sowie Forschung. In all diesen Gebieten wurden von Experten zahlreiche und vielfältige politische Massnahmen erarbeitet, deren Wirksamkeit im Ausland bereits erprobt sind (2022). Der Weg zu einer multisektoralen Gesundheitspolitik wird im Nationalen Gesundheitsbericht 2008 dargelegt (23).

\section{Ernährungspolitik heute}

Wurde Ernährungspolitik früher mit der primären Aufgabe der Ernährungssicherung gleichgesetzt, so haben sich die Akzente in unserer Wohlstandsgesellschaft heute hin zu Gesundheit (Gesundheitsförderung und Prävention), Lebensmittelsicherheit und Nachhaltigkeit verschoben (Kapitel 1.2).

\subsubsection{Bundesebene}

\section{Gesundheitsschutz als Gesetzesauftrag}

Die krankheitsunspezifische Gesundheitsförderung ist heute erst im Rahmen der Förderung von Turnen und Sport gesetzlich verankert (24). Charakteristisch für die Schweiz ist nach wie vor die hohe Bedeutung der kurativen Medizin, welche politisch und rechtlich umfassend festgelegt ist (17). Demgegenüber ist die Gesetzgebung in den Bereichen Gesundheitsförderung und Prävention fragmentiert, uneinheitlich und meist sehr allgemein gehalten. Dies spiegelt den geringeren Stellenwert von Prävention und Gesundheitsförderung gegenüber Behandlung, Pflege und Rehabilitation wider.

In der Bundesverfassung ist heute lediglich festgehalten, dass der Bund Massnahmen zum Schutz der Gesundheit zu ergreifen hat (25). Damit hat er die Kompetenz, in den beiden Bereichen "Lebensmittel" (inkl. Alkohol und Ta-

\footnotetext{
${ }^{1}$ Der Begriff Lebensmittel umfasst auch Getränke.
} 
bak)» und "übertragbare, stark verbreitete und bösartige Krankheiten» Massnahmen zur Prävention und Gesundheitsförderung umzusetzen. Als Beispiel hat der Bund im Bereich Lebensmittel den maximalen Gehalt an Transfettsäuren in pflanzlichen Ölen und Fetten auf $2 \mathrm{~g}$ pro $100 \mathrm{~g}$ oder $100 \mathrm{ml}$ festgelegt (26).

Auf Bundesebene sind im Hinblick auf Massnahmen zur Prävention und Gesundheitsförderung neben dem Bundesverfassungsartikel zum Gesundheitsschutz (25) folgende weitere Verfassungsartikel von Bedeutung: Sportförderungsgesetz (27), Alkoholgesetz (28), Artikel zur Erhebung von Verbrauchssteuern auf Tabak und Tabakwaren sowie gebrannte Wasser (29) sowie der Artikel zum Schutz der Konsumentinnen und Konsumenten (30).

Zusätzlich wird in Artikel 19 des Bundesgesetzes über die Krankenversicherung (KVG) festgehalten, dass die Versicherer gemeinsam mit den Kantonen eine Institution betreiben, die Massnahmen zur Förderung der Gesundheit und zur Verhütung von Krankheiten anregt, koordiniert und evaluiert (31). Diese Aufgabe wird heute durch Gesundheitsförderung Schweiz wahrgenommen (Kapitel 4.6.1.2).
Information der Öffentlichkeit

Entsprechend dem Lebensmittelgesetz (LMG) vom Oktober 1992 «kann der Bund die Öffentlichkeit über ernährungswissenschaftliche Erkenntnisse von allgemeinem Interesse, welche namentlich für die Gesundheitsvorsorge und den Gesundheitsschutz von Bedeutung sind, informieren» (32).

\subsubsection{Kantonale Ebene}

Wichtige Rechtsgrundlagen für die Bereiche Gesundheitsförderung und Prävention findet man vor allem in den kantonalen Gesundheitsgesetzen, weshalb die Kantone als politische Akteure von zentraler Bedeutung sind. Regelungen, die konkrete Teilbereiche der Gesundheitsförderung und Prävention betreffen, finden sich in einigen Kantonen auch in den Schul-, Bildungs- und Jugendgesetzen (Tabelle 4.1).

Die Kantone bzw. die 26 kantonalen Bildungsdepartemente haben die Hauptverantwortung über den Bereich der obligatorischen Schulzeit. Dies ist insofern von Bedeutung, als Bildungsinstitutionen in vielerlei Hinsicht einen zentralen Beitrag zur Gesundheitsförderung leisten können (Kapitel 4.7.3). 


\section{> Ernährungsmassnahmen zur Förderung der Gesundheit}

Tabelle 4.1: Gesetzliche Verankerung von Gesundheitsförderung (GF) und Prävention (P) in den Kantonen, Stand 2010 (33)

\begin{tabular}{|c|c|c|c|c|c|}
\hline Kanton & $\begin{array}{l}\text { GF/P in der } \\
\text { Verfassung } \\
\text { verankert }\end{array}$ & $\begin{array}{c}\text { GF/P in einem } \\
\text { Gesundheits- } \\
\text { gesetz } \\
\text { verankert }\end{array}$ & $\begin{array}{c}\text { GF / P im } \\
\text { Schulgesetz / } \\
\text { schulärztlichen } \\
\text { Dienst verankert }\end{array}$ & $\begin{array}{c}\text { GF / P im } \\
\text { Bildungsgesetz } \\
\text { verankert }\end{array}$ & $\begin{array}{c}\text { GF / P im } \\
\text { Jugendgesetz } \\
\text { verankert }\end{array}$ \\
\hline Jura (JU) & $x$ & $x$ & $x$ & & $x$ \\
\hline $\begin{array}{l}\text { Freiburg (FR) } \\
\text { Glarus (GL) } \\
\text { Nidwalden (NW) } \\
\text { Uri (UR) }\end{array}$ & $x$ & $x$ & $x$ & & \\
\hline $\begin{array}{l}\text { Appenzell Ausser- } \\
\text { rhoden (AR) } \\
\text { Schaffhausen (SH) } \\
\text { St. Gallen (SG) } \\
\text { Zürich (ZH) }\end{array}$ & $x$ & $x$ & x (Verordnung) & & \\
\hline Baselland (BL) & & $x$ & $x$ (Verordnung) & $x$ & \\
\hline $\begin{array}{l}\text { Aargau (AG) } \\
\text { Bern (BE) } \\
\text { Graubünden (GR) } \\
\text { Neuenburg (NE) } \\
\text { Obwalden (OW) } \\
\text { Solothurn (SO) } \\
\text { Thurgau (TG) } \\
\text { Waadt (VD) }\end{array}$ & $x$ & $x$ & & & \\
\hline Basel-Stadt (BS) & $x$ & x (Entwurf) & & & \\
\hline $\begin{array}{l}\text { Appenzell Inner- } \\
\text { rhoden (Al) } \\
\text { Zug (ZG) }\end{array}$ & & $x$ & $x$ & & \\
\hline Luzern (LU) & & $x$ & x (Verordnung) & & \\
\hline Wallis (VS) & & $x$ & & & $x$ \\
\hline $\begin{array}{l}\text { Genf (GE) } \\
\text { Tessin (TI) }\end{array}$ & & $x$ & & & \\
\hline Schwyz (SZ) & & $x$ (Verordnung) & & & \\
\hline
\end{tabular}




\subsection{Strategische Grundlagen im Ernährungsbereich}

\subsubsection{Strategische Grundlagen der WHO}

Auf globaler Ebene wird die Strategie zur Förderung einer ausgewogenen Ernährung von der WHO vorgegeben. Auch der Bund und die im Ernährungsbereich tätigen Schweizer Fachgesellschaften orientieren sich an diesem übergeordneten Rahmen (Kapitel 4.4.3). Die Kernelemente von zwei zentralen WHO-Strategiepapieren werden nachfolgend dargelegt.

\section{Globale Strategie für Ernährung, Bewegung} und Gesundheit

Der Schweizer Bundesrat hat die "Globale Strategie für Ernährung, Bewegung und Gesundheit» der WHO ("Global strategy on diet, physical activity and health»; 20), die entsprechende WHA-Resolution (WHA 57.17) vom Mai 2004 (34) und damit den Auftrag, in diesen Bereichen aktiv zu werden, angenommen. Die Anpassung der WHO-Strategie auf die Verhältnisse in der Schweiz resultierte im Nationalen Programm Ernährung und Bewegung (NPEB) (Kapitel 4.6.1.1).

\section{Aktionsplan für die globale Strategie zur}

Prävention und Kontrolle von nichtübertragbaren Krankheiten 2008-2013

Der «Aktionsplan für die globale Strategie zur Prävention und Kontrolle von nichtübertragbaren Krankheiten 2008-2013" ("Action Plan for the Global Strategy for the Prevention and Control of Noncommunicable Diseases 2008-2013») fordert die Mitgliedstaaten der WHO auf, die wich- tigsten modifizierbaren Risikofaktoren für nichtübertragbare Krankheiten zu reduzieren (35). Dazu zählen der Tabakkonsum, unausgewogene Ernährung, körperliche Inaktivität und missbräuchlicher Alkoholkonsum. Im Hinblick auf eine ausgewogene Ernährung ruft die WHO dazu auf, dass einerseits lebensmittelbasierte Ernährungsempfehlungen etabliert und implementiert werden und dass andererseits über vier Massnahmen eine gesündere Lebensmittelzusammensetzung angestrebt wird: durch die Reduktion des Salzgehalts, die Vermeidung industriell hergestellter Transfettsäuren, die Reduktion des Gehalts an gesättigten Fettsäuren und durch die Beschränkung des Gehalts an freien Zuckern.

\subsubsection{Strategische Grundlagen in Europa}

Auf europäischer Ebene finden sich ebenfalls umfangreiche strategische Vorgaben zur Förderung einer ausgewogenen Ernährung, wovon nachfolgend eine für die Schweiz relevante Auswahl diskutiert wird.

Die Europäische Charta zur Bekämpfung der Adipositas

An der Ministerkonferenz der WHO vom November 2006 unterzeichneten die Mitgliedstaaten die "Europäische Charta zur Bekämpfung der Adipositas» ( European Charter on counteracting obesity») und bekräftigten damit ihren Willen, sie in ihren Staaten umzusetzen (36). Die Charta wurde auch von der Schweiz mitunterzeichnet. In ihren Grundsätzen fordert die Charta wirksamere und innovativere sektorenübergreifende Massnahmen, deren Anpassung 
an örtliche Gegebenheiten und neue Forschungsansätze auf allen Ebenen mit besonderer Berücksichtigung von Kindern, Jugendlichen und sozial benachteiligten Bevölkerungsgruppen. Spezielle Erwähnung finden die Optimierung der Energiebilanz durch ausgewogene Ernährung und körperliche Betätigung sowie die Erfassung der diesbezüglichen Fortschritte.

\section{Erster und Zweiter Europäischer Aktionsplan}

\section{Nahrung und Ernährung}

Der "Erste Aktionsplan Nahrung und Ernährung 2000-2005» ( Action Plan for Food and Nutrition Policy 2000-2005») der WHO Europa definierte im Jahr 2000 drei Strategien in den Bereichen Lebensmittelsicherheit, nachhaltige Ernährung und gesundes Ernährungsverhalten mit dem Ziel, die Prävalenz ernährungsabhängiger Krankheiten und deren Folgekosten zu reduzieren (37). Die von den Mitgliedstaaten aufgestellten Ziele wurden jedoch nicht bzw. nur teilweise erreicht, was auf den mangelnden Einsatz finanzieller und personeller Ressourcen, fehlendes politisches Engagement und ressortübergreifende Abstimmungsprobleme zurückgeführt wurde. Zu diesem Schluss kamen die Autoren des "Zweiten Europäischen Aktionsplans Nahrung und Ernährung 2007-2012» («European Action Plan for Food and Nutrition Policy 2007-2012»), der darauf abzielt, die Ernährungs- und Nahrungspolitik der europäischen Staaten besser zu koordinieren (38). Dieser Zweite Aktionsplan basiert auf dem "Aktionsplan für die globale Strategie zur Prävention und Kontrolle von nichtübertragbaren Krankheiten 2008-2013» (35; Kapitel 4.4.1) und auf der «Europäischen Charta zur Bekämpfung der Adipositas» (36).
Weissbuch "Ernährung, Übergewicht, Adipositas: Eine Strategie für Europa» Im Jahr 2007 nahm die EU im Rahmen des Weissbuches "Ernährung, Übergewicht, Adipositas: Eine Strategie für Europa» («White Paper on a Strategy for Europe on Nutrition, Overweight and Obesity related health issues») die Ziele des "Zweiten Europäischen Aktionsplans Nahrung und Ernährung 2007-2012» der WHO Europa auf und definierte Massnahmen zu deren Erreichung (39). Das Weissbuch legt einen Schwerpunkt auf handlungsorientierte Partnerschaften. Zu den wichtigsten Zielen gehören u.a. die Stärkung der Selbstverantwortung, die Verbesserung der Konsumenteninformation, die Überprüfung der Lebensmittelzusammensetzung, die Verbesserung der Datengrundlagen und die Förderung der Forschung.

Seit 2005 sind über die «EU-Aktionsplattform Ernährung, Bewegung und Gesundheit» ( «EU Platform for Action on Diet, Physical Activity and Health») knapp 300 freiwillige Aktionsversprechen zu Massnahmen in den Bereichen der Konsumenteninformation, Bildung und Erziehung, Werbung und Marketing, Zusammensetzung von Lebensmitteln sowie Bewegungsförderung formuliert worden (40). Die Mitglieder der Plattform stammen aus allen Wirtschaftssektoren, aus dem Konsumentenbereich und aus dem Bereich der Nichtregierungsorganisationen (NGO). Ein jährliches Monitoring überprüft den aktuellen Fortschritt und bewertet die Qualität der Massnahmen.

Eine Bestandesaufnahme aus dem Jahr 2010 zeigt, dass Aktivitäten in EU-Ländern vor allem im Bereich von Informationskampagnen sowie im Setting Schule verankert wurden, dass Aktivitäten im Bereich von Lebensmittelpreisen und im Setting Arbeitsplatz jedoch noch gänzlich 
fehlten (41). Zudem wird festgehalten, dass bisher noch keine Good-Practice-Projekte definiert werden konnten, obschon dies eine der Zielsetzungen der Plattform ist (40).

\subsubsection{Strategische Grundlagen in der Schweiz}

Die Schweiz hat sich an den internationalen Strategieentwicklungen der WHO und der WHO Europa aktiv beteiligt. Die "Europäische Charta zur Bekämpfung der Adipositas» der WHO Europa (36; Kapitel 4.4.2), die "Globale Strategie für Ernährung, Bewegung und Gesundheit» der WHO (20; Kapitel 4.4.1) und das Weissbuch "Ernährung, Übergewicht, Adipositas: Eine Strategie für Europa» der EU (39; Kapitel 4.4.2) stellten bei der Erarbeitung des NPEB wesentliche Grundlagen dar (Kapitel 4.6.1.1) und sind auch in der Umsetzungsphase wegweisend. Die Schweizer Salzstrategie, die von der EURahmenstrategie zur Salzreduktion abgeleitet wurde, ist ein gutes Beispiel für die konkrete Anpassung einer WHO-Empfehlung auf nationale Verhältnisse (42).

\section{Bundesebene}

Auf nationaler Ebene sind die strategischen Grundlagen zur Gesundheitsförderung und Prävention vor allem in den nationalen Schwerpunktprogrammen verankert, wie sie für die Bereiche Ernährung und Bewegung (NPEB, Kapitel 4.6.1.1), Alkohol, Tabak sowie Drogen existieren.

Eine Schlüsselrolle bei der Erarbeitung von Ernährungsempfehlungen für die Schweiz kommt der Eidgenössischen Ernährungskommission (EEK) zu, die dem Bundesrat als ausserparlamentarische Kommission beratend zur
Seite steht (Kapitel 1.2). Eine Arbeitsgruppe der EEK hat für die Jahre 2001-2010 zudem eine "Erste Nationale Ernährungspolicy» erarbeitet; darin ist als Hauptziel das gesunde Körpergewicht genannt (43). Auf dieser Grundlage wurden in den vergangenen Jahren verschiedene Programme und Projekte des Bundes ins Leben gerufen (Kapitel 4.6).

Strategisch tätig ist zudem die von den Kantonen und Krankenversicherern gemeinsam getragene Stiftung Gesundheitsförderung Schweiz, welche der Aufsicht des Bundes untersteht und "Gesundes Körpergewicht» als Kernthema definiert hat (44; Kapitel 4.6.1.2).

\section{Kantonale Ebene}

In den meisten Kantonen wurden in den letzten Jahren grundlegende Strategiepapiere (z.B. Leitbilder) sowie Konzepte zu spezifischen Themen wie z.B. Ernährung und Bewegung entwickelt (45). Darin wurden neben den Zielen einerseits Planungs- und Umsetzungsbedingungen und andererseits meist auch Schwerpunktthemen und Handlungsschwerpunkte formuliert. Letztere mündeten wiederum in Aktionspläne, z.B. das Luzerner Aktionsprogramm «Gesundes Körpergewicht» 2008-2011 (46).

Die Kantone arbeiten im Gesundheitsbereich vor allem bilateral und auf gesamtschweizerischer Ebene in der Schweizerischen Konferenz der kantonalen Gesundheitsdirektorinnen und -direktoren (GDK) zusammen. Dieses Koordinationsorgan vereint die für das Gesundheitswesen zuständigen Regierungsmitglieder der Kantone und zielt auf die Zusammenarbeit zwischen den 26 Kantonen und dem Bund ab.

Positionsbezüge und Koordinationsanstrengungen im Bereich der Gesundheitsförderung und Prävention gehören heute zu den Tätig- 
keitsschwerpunkten der GDK. In der Vereinigung der kantonalen Beauftragten für Gesundheitsförderung (VBGF), die der GDKangegliedert ist, werden gesundheitsfördernde Massnahmen der Kantone koordiniert und nationale Aktivitäten zur Gesundheitsförderung mit jenen der Kantone abgestimmt (47).

\subsection{Das Individuum im Spannungsfeld verschiedener Einflüsse}

\subsubsection{Von der Gesundheits- kompetenz zur Ernährungskompetenz}

Ausgehend vom übergeordneten Begriff der Gesundheitskompetenz $(48 ; 49)$ kann auch die Ernährungskompetenz ("Food Literacy») als ein umfassendes, wissenschaftlich jedoch wenig dokumentiertes Konzept für das individuelle Verhalten betrachtet werden. Ernährungskompetenz beinhaltet die Fähigkeit, den Ernährungsalltag selbstbestimmt, verantwortungsbewusst und genussvoll zu gestalten (50). Voraussetzung dafür ist, dass Ernährungsinformationen zugänglich sind, richtig verstanden und schliesslich im Gesamtzusammenhang beurteilt werden. Ein breites Wissen in den verschiedensten Themenbereichen ist dafür unerlässlich.

Es ist davon auszugehen, dass wie bei der Gesundheitskompetenz auch bei der Ernährungskompetenz das Lebensalter, das Einkommensniveau, der Gesundheitszustand und vor allem auch das Bildungsniveau entscheidende Einflussgrössen darstellen (51). Eine zielgruppengerechte Ernährungsbildung und -aufklä- rung ist deshalb von entscheidender Bedeutung. Bildungsinstitutionen kommt in diesem Zusammenhang eine besondere Rolle zu, da sie die individuellen Chancen erhöhen können, sich Ernährungskompetenzen anzueignen.

Die Bedeutung der Gesundheitskompetenz wurde von der Politik im Jahr 2007 erkannt. Damals wurde eine von 60 Parlamentariern unterzeichnete Motion zum Thema "Massnahmen zur Förderung der Gesundheitskompetenz in der Bevölkerung" zuhanden des Bundesrates eingereicht (52). Die Parlamentarier forderten u.a., das Thema Gesundheitskompetenz bei allen gesundheitlichen Gesetzgebungsvorhaben zu berücksichtigen (Kapitel 4.7.1).

\subsubsection{Lebenswelten}

Unser Lebensstil und damit unser Ernährungsund Bewegungsverhalten werden wesentlich von unserem Umfeld bzw. von unseren Lebenswelten geprägt, in denen wir uns bewegen. Über Veränderungen unseres Umfeldes bzw. unserer Lebenswelten kann insofern Einfluss darauf genommen werden, wie und wie oft wir uns ernähren und bewegen. Darin gründet der Gedanke der Verhältnisprävention, auch «strukturelle Prävention» genannt (53). Die Verhältnisprävention grenzt sich von der Verhaltensprävention insofern ab, als Letztere auf die direkte Veränderung des Verhaltens des Individuums abzielt.

\section{Gesamtgesellschaftliche Verantwortung}

Gesundheit ist im Hinblick auf eine höhere Lebensqualität erstrebenswert. Eine gesunde Gesellschaft ist auch aus wirtschaftlicher Sicht von grosser Bedeutung, ist sie doch gleichbedeutend mit leistungsfähigen Arbeitskräften. Ge- 
sundheitsfördernde Lebenswelten sind für den privaten Sektor deshalb unerlässlich.

Der Bund ist verantwortlich dafür, die Lebensmittelsicherheit zu gewährleisten und die Öffentlichkeit über besondere Ereignisse zu informieren, die für den Gesundheitsschutz von Bedeutung sind (32; Kapitel 4.3.1). Dahinter steht nicht zuletzt ein volkswirtschaftliches Interesse, verursachen die nichtübertragbaren Krankheiten doch den Hauptanteil der Gesundheitskosten (3; Kapitel 4.2).

Die Verbesserung der Ernährungssituation in der Schweiz und damit die Erhaltung der Gesundheit der Schweizer Bevölkerung liegen also im gemeinsamen Interesse des privaten und öffentlichen Sektors. Es sind innovative Ansätze gefragt, die sowohl den Gesundheitsschutz der Bevölkerung gewährleisten als auch den wirtschaftlichen Interessen des privaten Sektors gerecht werden. Die Ansätze können dabei sowohl im Bereich der Informationsvermittlung als auch in Lebensmitteln selbst (gesundheitsfördernde Zusammensetzung unter Berücksichtigung des Genusswertes) oder in finanziellen Anreizsystemen zur Förderung eines gesunden Ernährungsverhaltens liegen (z.B. Vergünstigung von Früchten und Gemüse).

Auf internationaler Ebene sind Steuern auf Lebensmittel und Getränke zunehmend ein Thema, obschon die wissenschaftliche Evidenz für deren Wirksamkeit verhältnismässig tief ist $(11 ; 54)$. So erhebt der dänische Staat seit Oktober 2011 eine Steuer auf fettreiche Lebensmittel (bzw. auf die darin enthaltenen gesättigten Fettsäuren), wobei einige Produkte wie z.B. Milch, Fisch und Eier davon befreit sind (55). Ob sich solche fiskalischen Massnahmen gesundheitsfördernd auswirken, wird sich erst in einigen Jahren zeigen.
Lebensmittelangebot: Verfügbarkeit, Preis, Werbung

Die Verfügbarkeit und der Preis von Lebensmitteln bestimmen zu einem wesentlichen Teil, was auf den Tisch kommt - dies trifft v.a. auf Familien mit tieferem Einkommensniveau zu. Die Bewerbung von Lebensmitteln hat ebenfalls einen Einfluss auf das Konsumverhalten von Familien. Gerade Familien mit Migrationshintergrund, die sich aufgrund von oftmals schwach ausgebildeten Gesundheits- und Ernährungskompetenzen selten an gesundheitsfördernden Verhaltensstrategien orientieren, sind solchen Einflüssen in verstärktem Mass ausgesetzt. Hinzu kommt, dass die Möglichkeit, energiedichte Lebensmittel konsumieren zu können, oft mit einem erstrebenswerten sozialen Status einhergeht (56).

\section{An Kinder gerichtete Werbung für Lebensmittel}

Der Anteil Werbung für Lebensmittel, der während Kinderfernsehprogrammen ausgestrahlt wird, liegt in der Schweiz bei über 25\% - die Hälfte davon betrifft Produkte mit hohem Zucker- und Fettgehalt (57). Nur wenige Fernsehspots bewerben Grundnahrungsmittel wie Früchte und Gemüse (58). Auch internationale Untersuchungen weisen einen hohen Anteil Werbung für zucker- und fettreiche Lebensmittel in Fernsehprogrammen nach (59). Sie wird zudem besonders zu Sendezeiten ausgestrahlt, die vor allem von Kindern bevorzugt werden. Nationale Regulierungen in diesem Bereich machen zunehmend weniger Sinn, weil der Zugang zu Fernsehprogrammen aus der ganzen Welt immer einfacher wird.

Das Marketing und die Bewerbung von Lebensmitteln und nichtalkoholischen Getränken 
mit einem hohen Gehalt an gesättigten und Transfettsäuren, Zucker oder Salz, die sich an Kinder richten, haben einen ungünstigen Einfluss auf ihr Ernährungsverhalten $(60 ; 61)$. Hinzu kommt der in der Werbebranche zunehmende Einsatz elektronischer Medien wie Internet, Applikationen für Mobiltelefone oder Computerspiele, der auf die klassische Fernseh- und Printwerbung abgestimmt wird. Diese Faktoren könnten einen zusätzlichen Einfluss auf das Kauf- und Konsumverhalten ausüben (62).

Die Foederation der Schweizerischen Nahrungsmittel-Industrien (fial) hat im Jahr 2008 einen Ideenkatalog zur Förderung einer ausgewogenen Ernährung erarbeitet; dessen Umsetzung durch die fial-Mitglieder hinkt diesem Erlass allerdings noch nach (63). Die fial empfiehlt in diesem Katalog u.a. den "Verzicht auf Werbung, die sich an noch nicht schulpflichtige Kinder richtet», und "Werbung mit schulpflichtigen Kindern als Zielgruppe verantwortungsvoll zu gestalten». Diese Vorschläge decken sich inhaltlich mit einem Positionspapier der fial zur Lebensmittelwerbung aus dem Jahr 2010 (64). Hier setzt auch die freiwillige Initiative "Swiss Pledge» der Schweizer Lebensmittel- und Getränkeindustrie an, die sich dazu bekennt, keine Werbung an Kinder unter 12 Jahren zu richten (65). Davon ausgenommen sind allerdings Produkte, die spezifische Kriterien auf der Grundlage von international anerkannten Ernährungsempfehlungen und / oder nationalen und internationalen Ernährungsrichtlinien erfüllen. Das Festlegen von international anerkannten Kriterien, welche von unabhängigen Stellen erarbeitet wurden (z.B. von der WHO in Zusammenarbeit mit den Mitgliedstaaten), steht zudem noch aus. "Swiss Pledge" wurde von der Expertengruppe "actionsanté» positiv bewertet und als Aktionsversprechen genehmigt (Kapitel 4.6.1.1).

\section{Ausser-Haus-Verpflegung}

Die Ausser-Haus-Verpflegung hat in den letzten Jahren an Bedeutung gewonnen (Kapitel 2.6.5). Die diesbezüglichen jährlichen Ausgaben sind in der Schweiz im 2010 gegenüber 2009 um CHF 3.3 Mia. auf CHF 26 Mia. gestiegen (66). Die Schnellverpflegung (Fast Food) wird dabei immer häufiger in Anspruch genommen; in der Altersgruppe der 15- bis 29-Jährigen steht sie mit 29.5\% aller Bezugsquellen bereits an zweiter Stelle (nach herkömmlicher Gastronomie). Ebenfalls von zunehmender Bedeutung ist die Gemeinschaftsverpflegung $(66 ; 67)$, die in der Schweiz täglich rund 1 Mio. Menschen verpflegt (68). Dies hat zur Folge, dass die quantitativen und qualitativen Anforderungen an die Angebote der AusserHaus-Verpflegung aus Präventionssicht steigen.

\section{Ausgaben für Lebensmittel und alkoholfreie Getränke}

In der langfristigen Perspektive sind die Ausgaben der privaten Haushalte für Lebensmittel und alkoholfreie Getränke rückläufig. Machten sie im Jahr 1945 noch über 35\% aller Ausgaben aus (69), belief sich deren Anteil am Haushaltsbudget im Jahr 2009 noch auf 7.0\% (70). Unterschiede können auch zwischen den Sprachregionen festgestellt werden: Der entsprechende Anteil machte während den Jahren 2006-2008 in der Deutschschweiz 6.9\% aus, in der Westschweiz $8.0 \%$ und im Tessin $8.7 \%$ (71). Der verhältnismässig tiefe Stellenwert von Lebensmitteln zeigt sich auch daran, dass Lebensmittelpreise höher eingeschätzt werden, als sie effektiv sind, und dass das Wissen über die Lebensmittelpreise kontinuierlich abnimmt (72). 
Betrachtet man nur die Konsumausgaben, machten Lebensmittel und alkoholfreie Getränke im Jahr 2009 in der Schweiz 12.3\% aus (70). Der Vergleich zu unseren Nachbarländern zeigt, dass sich die anteilsmässigen Ausgaben für Lebensmittel und alkoholfreie Getränke in einem ähnlichen Bereich bewegen: Sie liegen in Österreich bei $12.1 \%$ (Daten aus 2009; 73), in Deutschland bei $14.0 \%$ (Daten aus 2009; 74), in Frankreich bei 15.0\% (Daten aus 2006; 75) und in Italien bei 18.9\% (Daten aus 2009, allerdings inkl. alkoholische Getränke; 76).

\subsubsection{Von der Bildung über das Wissen zum Verhalten}

\section{Ernährungsbildung von Kindern}

Die Gewohnheitsbildung und die Prägung des Ernährungsverhaltens beginnen bereits in den ersten Lebensjahren. Werdende und neugewordene Eltern sind zudem besonders empfänglich für Empfehlungen zu Gesundheitsthemen, gerade auch im Ernährungsbereich (77). Den Elternberatungsstellen sowie den medizinischen Bezugspersonen kommt bei der Vermittlung von Ernährungsempfehlungen in dieser Lebensphase deshalb eine entscheidende Rolle zu. Verschiedene Projekte in der Schweiz (Kapitel 4.6.2) richten sich gezielt an werdende und neugewordene Eltern und verfolgen insofern die Strategie des frühen Einbezugs und der verstärkten Zusammenarbeit zwischen den relevanten Fachpersonen (Hebammen, Mütter-Väterberaterinnen, interkulturelle Vermittler, dipl. Ernährungsberaterinnen, Kinder- und Frauenärzte usw.).

In der vorschulischen Phase, in welcher die Inanspruchnahme des Gesundheitsversorgungs- systems gegenüber der Schwangerschaftsund Nachgeburtsphase in der Regel wieder abnimmt und die Einführung der Kleinkinder in die häusliche Esskultur stattfindet, sind Ernährungsbildung bzw. Ernährungskompetenzen der Eltern besonders wichtig. Studien bestätigen die wichtige Vorbildfunktion von Eltern; indem Eltern sicherstellen, dass Früchte und Gemüse zu Hause verfügbar sind, tragen sie zudem nachweislich zu einem erhöhten Früchte- und Gemüsekonsum ihrer Kinder bei (78). Veränderungen im Lebensstil (z.B. Wiederaufnahme einer Erwerbstätigkeit verbunden mit Fremdbetreuung der Kinder) haben einen starken Einfluss auf den Ernährungsalltag von Eltern und ihren Kindern. Konsum- und Dienstleistungsentscheidungen im Ernährungsbereich unterliegen in dieser Lebensphase oftmals verstärkt äusseren Einwirkungen, welche von den Eltern nicht direkt beeinflussbar sind (z.B. Verpflegungssituation in Kinderbetreuungsstätten).

Im Schulalter wird die Ernährungsbildung zunehmend von strukturellen Aspekten beeinflusst, u.a. von der "Interkantonalen Vereinbarung über die Harmonisierung der obligatorischen Schule vom 14. Juni 2007» (79). Dieses so genannte HarmoS-Konkordat ist am 1. August 2009 in Kraft getreten und gilt für diejenigen Kantone, welche dem Konkordat beigetreten sind. Es harmonisiert sowohl die übergeordneten Ziele der obligatorischen Schule als auch die wichtigsten strukturellen Eckwerte wie das Einschulungsalter, die Dauer der Schulstufen und deren Übergänge und benennt die Instrumente für die Systementwicklung und Qualitätssicherung auf gesamtschweizerischer Ebene (Kapitel 4.7.3).

Sechster Schweizerischer Ernährungsbericht | 221 
Im HarmoS-Konkordat wird weiter festgehalten, dass für die Betreuung der Schüler ausserhalb der Unterrichtszeit ein bedarfsgerechtes Angebot bestehen soll, wobei die Nutzung dieses Angebotes freiwillig und in der Regel kostenpflichtig ist. Dabei wird nicht ein «nationales Modell» vorgegeben; die so genannten Tagesstrukturen können Mittagstische, Tagesschulen oder andere Tagesstruktur-Angebote umfassen. Tagesschulen mit ganztägigen Betreuungsangeboten inklusive Mittagsverpflegung sind in den Kantonen nicht flächendeckend verbreitet. Einzig im Kanton Tessin und - weniger ausgeprägt - in den Kantonen Appenzell Ausserrhoden, Bern, Basel-Stadt, Nidwalden und Zürich besteht ein grösseres Angebot an Tagesschulen (80). Tagesstrukturen bieten ein grosses Potenzial für systematische Präventionsbemühungen. Die Vereinbarkeit von Familie und Berufstätigkeit wird erleichtert, Kinder mit Sozialisierungsschwächen können Defizite in diesem Bereich kompensieren und ihre Eltern bei der sozialen Integration unterstützt werden. Zahlreiche Gemeinden und Städte haben bereits mit Regelungen zum Verpflegungsangebot in Schulen, Kindergärten und Kindertagesstätten reagiert. So wurden beispielsweise in verschiedenen Kantonen Süssgetränkeautomaten aus den Schulen verbannt und in der Stadt Zürich Ernährungsrichtlinien zur Verbesserung des Angebotes erlassen (81; Kapitel 4.6.2).

\section{Ernährungswissen}

Die Thematik rund um das Ernährungswissen und Ernährungsverhalten in der Schweiz wird in den Kapiteln 2.6.2 und 2.6.3 ausführlich behandelt. Das Ernährungswissen bildet die Voraussetzung zur Steuerung eines nachhaltig gesunden Ernährungsverhaltens. Die Steigerung des
Wissens muss also ein vordringliches Ziel aller Präventionsbemühungen auf der Verhaltensebene sein, um eine Verbesserung oder Beibehaltung des Gesundheitszustandes erreichen zu können - ungeachtet der ebenso wichtigen Massnahmen auf der Verhältnisebene (Kapitel 4.5.2). Das Wissen umfasst in diesem Zusammenhang Kenntnisse über grundlegende Ernährungsempfehlungen bis hin zum Verständnis komplexer Zusammenhänge (Kapitel 4.5.1).

\section{Umstellung des Ernährungsverhaltens}

Es reicht nicht aus, das Ernährungsverhalten mittels blosser Wissenssteigerung beeinflussen zu wollen. Insbesondere das soziale Gefälle kann nicht alleine durch Ernährungsaufklärung auf kognitiver Ebene kompensiert werden.

Von zentraler Bedeutung zur Beeinflussung des Ernährungsverhaltens sind psychische Steuergrössen. Die schon seit einigen Jahren geführte Diskussion über die Unterscheidung der Begrifflichkeiten "Ernährung» und "Essen» gibt dabei entscheidende Aufschlüsse über die wirksame Ausrichtung von Ernährungsempfehlungen (82). Das stark emotional geprägte Essverhalten hat mit dem bewusst wahrgenommenen Ernährungsverhalten nicht viel gemeinsam. Die Menschen ernähren sich, um gesund zu bleiben oder es zu werden; sie essen und trinken aber - zumindest meist - aus Genuss und Freude, umgeben von Familienmitgliedern oder Freunden und einem gemütlichen Ambiente. Auf Menschen mit Essstörungen oder gestörtem Essverhalten treffen diese positiven Assoziationen allerdings nicht zu; im Gegenteil, bei ihnen dominieren beim Essen negative Emotionen wie Wut, Einsamkeit, Trauer usw. (Kapitel 3.9). Es ist generell aussichtslos, diesem auf Emotionen (seien diese nun positiv oder nega- 
tiv) aufgebauten Essverhalten mit rationalen, kognitiv ausgerichteten Empfehlungen zu begegnen. Vielmehr müssen emotionsregulierende Inhalte zur Kompetenz- und Gewohnheitsbildung einfliessen, wenn eine Ernährungsumstellung angestrebt wird. Diesen Ansatz verfolgen einerseits die individuellen Ernährungsberatungen durch dipl. Ernährungsberaterinnen HF / FH und andererseits auch verschiedene Programme und Projekte in der Schweiz (Kapitel 4.6), u.a. die Projekte der Fachstelle Prävention Essstörungen Praxisnah (PEP), die sich zielgruppenspezifisch für die Förderung emotionaler Kompetenzen, Körperzufriedenheit und psychischer Gesundheit engagieren $(83 ; 84)$.

\subsection{Laufende Programme und Projekte in der Praxis}

In der Schweiz gibt es verschiedene Programme und Projekte zur Förderung einer ausgewogenen Ernährung und ausreichend körperlicher Aktivität auf der Wissens- und Verhaltensebene (Verhaltensprävention) sowie zur Verbesserung der diesbezüglichen Rahmenbedingungen (Verhältnisprävention). Das vorliegende Kapitel stellt in einem ersten Teil die übergeordneten nationalen Programme (Kapitel 4.6.1) und in einem zweiten Teil eine Auswahl von laufenden nationalen und kantonalen Projekten vor (Kapitel 4.6.2).

\subsubsection{Nationale Programme}

\subsubsection{Nationales Programm Ernährung und Bewegung}

Das Nationale Programm Ernährung und Bewegung (NPEB) legt die nationale Strategie für die Förderung einer ausgewogenen Ernährung und ausreichend Bewegung fest (85). Das NPEB basiert auf der WHA-Resolution vom Mai 2004 (Kapitel 4.4.1) und dem Weissbuch «Ernährung, Übergewicht, Adipositas: Eine Strategie für Europa» der EU (Kapitel 4.4.2). Am 18. Juni 2008 hat der Bundesrat das Programm verabschiedet und das Bundesamt für Gesundheit (BAG) mit dessen Umsetzung beauftragt. Das NPEB definiert die langfristigen Ziele und prioritären Handlungsfelder für das Vorgehen auf nationaler Ebene und bildet die Grundlage für die Zusammenarbeit der verschiedenen Akteure. Die Hauptpartner sind das BAG, das Bundesamt für Sport (BASPO), Gesundheitsförderung Schweiz und die Kantone. Die Vision des NPEB ist es, die Bevölkerung und insbesondere die Jugendlichen zu motivieren, sich ausgewogen zu ernähren und genügend zu bewegen. Damit sollen Übergewicht, Adipositas, Essstörungen und weitere damit verbundene Krankheiten wirksam bekämpft werden. Im Rahmen des NPEB führt das BAG gemeinsam mit anderen Bundesämtern und Partnerinstitutionen verschiedene Projekte zur Förderung einer ausgewogenen Ernährung und ausreichend Bewegung auf der Verhaltens- und Verhältnisebene durch. Unter Berücksichtigung der soziokulturellen und sozioökonomischen Voraussetzungen will das NPEB die Eigenverantwortung der Konsumenten fördern, freiwillige Massnahmen der Wirtschaft unterstützen und die Lebensmittelsicherheit 
gewährleisten. Das Problem ernährungsabhängiger Krankheiten betrifft nicht nur die Gesundheitspolitik; auch andere Sektorpolitiken sind gefordert, angefangen bei der Landwirtschaft über die Bildung und den Langsamverkehr bis hin zur Agglomerationspolitik (Kapitel 4.3).

Nachfolgend werden drei ausgewählte Projekte des BAG vorgestellt, die im Rahmen des NPEB lanciert wurden.

\section{Salzstrategie 2008-2012}

Die Schweizer Salzstrategie basiert auf der EURahmenstrategie zur Salzreduktion $(42 ; 86)$. Sie zielt darauf ab, den Salzkonsum bis zum Jahr 2012 auf unter $8 \mathrm{~g}$ pro Tag und Person zu senken, und strebt längerfristig die von der WHO empfohlenen maximalen $5 \mathrm{~g}$ pro Tag und Person an. Die verfügbaren Daten zeigen, dass dieses Ziel noch nicht erreicht wurde (Kapitel 2.4.6). Die Umsetzung der Salzstrategie erfolgt über "actionsanté» und über die "Schweizer Qualitätsstandards für eine gesundheitsfördernde Gemeinschaftsgastronomie» (Tabelle 4.2), deren Ziel es ebenfalls ist, den Salzkonsum zu reduzieren, ohne dabei den Geschmack der Gerichte zu tangieren. In einem ersten Schritt setzte das BAG auf freiwillige Aktionsversprechen, bei denen die Partner von "actionsanté» (Lebensmittelindustrie und -handel) die SalzGrenzwerte selbst festlegten. In einem zweiten Schritt gibt das BAG Standards und Zielwerte an und versucht, Partner von "actionsanté" wiederum freiwillig - zur Einhaltung dieser Werte zu verpflichten. "actionsanté" - "besser essen, mehr bewegen"

Mit der Initiative "actionsanté" hat das BAG unter Einbezug der Wirtschaft neue Wege beschritten (87). Neu an "actionsanté» ist die Zusammenarbeit mit Unternehmen und Institutionen. Letztere können seit 2009 freiwillige Aktionsversprechen in den Bereichen Lebensmittelzusammensetzung und -angebot, Konsumenteninformation, Marketing und Werbung sowie Förderung eines bewegungsfreundlichen Umfelds abgeben. Mit der Initiative "actionsanté» möchte das BAG den Menschen ermöglichen, in einem Umfeld zu leben, das eine gesunde Lebensweise - d.h. einen aktiven Lebensstil und eine ausgewogene Ernährung - fördert. Eine breit abgestützte Jury mit Vertretern der Wissenschaft, der Konsumentenorganisationen, der Kantone und der Wirtschaft begleitet und bewertet die Aktivitäten und gibt Empfehlungen zuhanden des BAG ab. Bisher wurden insgesamt 21 Aktionsversprechen von 15 verschiedenen Akteuren abgegeben (Stand November 2011). "actionsanté» wurde im Rahmen der Evaluation von NPEB im Jahr 2011 positiv beurteilt, wobei bemerkt wurde, dass sich die bisherigen Aktionsversprechen fast vollumfänglich auf den Bereich der Lebensmittelzusammensetzung und dabei nur auf einzelne Produkte bzw. Produktgruppen konzentrieren (89).

\section{Monitoring-System Ernährung und Bewegung} Ein wichtiges Begleitinstrument des NPEB ist das Monitoring-System Ernährung und Bewegung (MOSEB), welches zum Ziel hat, die Datenlage zur Ernährungs- und Bewegungssituation in der Schweiz qualitativ und quantitativ zu verbessern (88). Qualitativ hochwertige Daten sind die Voraussetzung dafür, dass Entwicklun- 
gen verfolgt und Massnahmen überprüft, angepasst, initiiert oder auch sistiert werden können. MOSEB wurde im Rahmen der Evaluation von NPEB im Jahr 2011 sehr positiv beurteilt, wobei bemängelt wurde, dass die Nachhaltigkeit (regelmässige und schweizweite Erhebung der Kernindikatoren) noch zu wenig gewährleistet ist (89).

\section{Evaluation des NPEB}

Das NPEB wurde im Jahr 2011 evaluiert (89), wobei das Programm zu diesem Zeitpunkt erst seit rund 3 Jahren lief und ein Wirkungsnachweis insofern noch nicht möglich war. Zusammenfassend lässt sich sagen, dass die meisten NPEB-Projekte betreffend ihrer Umsetzung auf gutem Weg sind. Eine Ausnahme ist die Nationale Ernährungserhebung, die nicht im vorgesehenen Umfang umgesetzt werden kann. Der hauptsächliche Mehrwert von NPEB liegt darin, dass das Programm den Aktivitäten im Bereich Ernährung und Bewegung in der Schweiz einen konzeptionellen Rahmen gibt und dass die Legitimation für diese Aktivitäten verstärkt wird. Als positiv werden der multisektorale Ansatz, der Fokus auf der Verhältnisprävention (z.B. Lebensmittelzusammensetzung) und die Verbesserung von Informationsaustausch und Abstimmung zwischen den Hauptpartnern von NPEB beurteilt. Als Erfolgsfaktor wird u.a. die mit den KAP und Suisse Balance angestrebte Multiplikation von erprobten Projekten genannt. Die grössten Umsetzungsdefizite weist das NPEB im Bereich der Kommunikation gegen aussen und Koordination auf; weitere Schwachstellen sind, dass das NPEB den Bildungsbereich nicht einbezieht und keine messbaren Ziele aufweist.

\subsubsection{Strategie 2007-2018 "Gesundes Körpergewicht"}

Gesundheitsförderung Schweiz ist eine Stiftung, die von Kantonen und Krankenversicherern getragen wird und der Aufsicht des Bundes untersteht. Mit gesetzlichem Auftrag initiiert, koordiniert und evaluiert sie Massnahmen zur Förderung der Gesundheit (31). In ihrer langfristigen Strategie 2007-2018 hat die Stiftung "Gesundes Körpergewicht" als eines von drei Kernthemen definiert (neben "Gesundheitsförderung und Prävention stärken» und "Psychische Gesundheit - Stress») und sich dabei zum Ziel gesetzt, den langjährigen Trend zur zunehmenden Prävalenz von Übergewicht in der Bevölkerung bis zum Jahr 2018 nachhaltig zu stoppen (44).

\section{Kantonale Aktionsprogramme}

Im Jahr 2007 hat Gesundheitsförderung Schweiz als Teil ihrer Strategie 2007-2018 «Gesundes Körpergewicht" die Kantonalen Aktionsprogramme (KAP) lanciert; die Stiftung leistet als Programmpartner einen wichtigen Beitrag zur Zielerreichung des NPEB. Im Rahmen der KAP werden die Kantone dabei unterstützt, Massnahmen im Bereich Ernährung und Bewegung auf Projekt- und Policy-Ebene (Verankerung von Massnahmen durch Regulierungen, Gesetze, Verordnungen, Reglemente, Leitbilder oder Empfehlungen) zu ergreifen und sich sowohl intra- als auch interkantonal zu vernetzen (90). Die Kantone verpflichten sich dabei meistens für vier Jahre und für die Übernahme von mindestens der Hälfte der Programmkosten; unterstützt werden sie u.a. auch durch eine breit angelegte Öffentlichkeitsarbeit von Gesundheitsförderung Schweiz. Bis zum Jahr 2010 wurden in 17 Kantonen insgesamt 108 verschiedene Interventionsprojekte umgesetzt. Da zahlreiche 
Projekte in mehreren KAP realisiert werden, entspricht diese Zahl 177 Projektumsetzungen in 16 Programmen (Stand November 2010). Seit Januar 2011 führen 22 Kantone ein KAP durch (91).

Die KAP werden von Gesundheitsförderung Schweiz laufend evaluiert. Eine Zwischenevaluation über die Erhebungsjahre 2008/09 kommt zum Schluss, dass die KAP zwar zum Teil von finanziellen und personellen Engpässen bedroht sind, auf kantonaler Ebene aber von einem unterstützenden politischen Willen profitieren (92). Letzteres zeigt sich u.a. darin, dass $40 \%$ der KAP-Projekte in bestehende Strukturen integriert werden konnten (Eingliederung in die zuständigen Departemente).

Die Koordinationsbemühungen von Gesundheitsförderung Schweiz zwischen den Kantonen zeigen insofern Wirkung, als trotz sehr heterogener Massnahmen eine relativ hohe Standardisierung erreicht werden konnte (92). Die Zwischenevaluation zeigte ferner, dass die Westschweizer Kantone und das Tessin stärker untereinander vernetzt sind als die Deutschschweizer Kantone.

Für das Setting Kindergarten und Schule zeigte die Zwischenevaluation, dass der Erfolg von Massnahmen stark vom persönlichen Engagement der Multiplikatoren (Lehrpersonen) abhängt und dass die Multiplikatoren im Kleinkinder- und ausserschulischen Bereich im Rahmen der KAP vor allem dann gut erreicht werden, wenn einerseits der Policy-Bereich unterstützend angegangen wird oder wenn andererseits Partnerorganisationen miteinbezogen werden. Massnahmen auf der Policy-Ebene haben in den KAP allerdings eine noch zu geringe Bedeutung (92). Dies, obwohl in diesem Bereich mit vergleichsweise wenig Ressourcen eine hohe Wirkung erzielt werden könnte (11).
Im Bereich der Öffentlichkeitsarbeit und Information der Bevölkerung wird im Rahmen der KAP viel unternommen. Aufgrund der Komplexität der Wirkungszusammenhänge (Kapitel 4.2) ist es aber nicht möglich, eine Aussage über den qualitativen oder quantitativen Nutzen dieser Aktivitäten zu machen. Es scheint jedoch, dass die Ressourcen in diesem Bereich weniger effizient genutzt werden als in anderen Bereichen. Dies zeigt sich beispielsweise daran, dass die Kantone oftmals eigenes Kommunikationsmaterial (Broschüren, Darstellungen von Empfehlungen usw.) erstellen, statt auf Bestehendes zurückzugreifen (92).

\subsubsection{Weitere nationale Programme, Akteure und Netzwerke}

Die in den Kapiteln 4.6.1.1 und 4.6.1.2 genannten übergeordneten nationalen Programme des BAG und von Gesundheitsförderung Schweiz dienen zahlreichen Akteuren in der Schweiz als Basis für weitere Programme oder sind diesen angegliedert: z.B. action d (93), die Kampagne "5 am Tag» (94), die Schweizerische Stiftung zur Förderung des Stillens (95), die nationale Projektförderstelle Suisse Balance (96).

Neben dem BAG und Gesundheitsförderung Schweiz engagieren sich verschiedene weitere Akteure für eine ausgewogene Ernährung. Viele sind dem Netzwerk Nutrinet angeschlossen, welches auf ernährungsbezogene Gesundheitsförderung und Prävention abzielt (97). Nutrinet wird von der Schweizerischen Gesellschaft für Ernährung (SGE) koordiniert, die im Bereich der Ernährungsinformation und -aufklärung tätig ist (98).

Eines der zentralen nationalen Programme im Bildungsbereich ist "bildung + gesundheit Netzwerk Schweiz", das vom BAG getragen wird (99). Ziel des Netzwerkes ist die Veranke- 
rung von Gesundheitsförderung und Prävention in der Schule.

Ein weiteres nationales Netzwerk im Bildungsbereich ist das Schweizerische Netzwerk Gesundheitsfördernder Schulen (SNGS; 100). Es unterstützt Schulen dabei, sich unter Mitwirkung aller Beteiligten zu einer Organisation zu entwickeln, die gesundes Lehren und Lernen ermöglicht. Die regelmässigen Projektauswertungen zeigen, dass Themen rund um ein gesundes Ernährungs- und Bewegungsverhalten zwar Eingang in die Schulen finden, dies aber öfter auf die Primar- und Sekundarstufe I (also auf Schüler im Alter von 6-15 Jahren) zutrifft als auf die Sekundarstufe II (also auf Schüler im Jugendalter). Die Erfahrungen aus diversen Projekten zeigen, dass es schwierig ist, das Ernährungs- und Bewegungsverhalten von Jugendlichen nachhaltig zu beeinflussen, weil sich diese als selbstkompetent wahrnehmen. Zudem nehmen in diesem Alter die globale Gerechtigkeit und die Umwelt einen grösseren Stellenwert ein als Gesundheitsthemen.

Schliesslich sind verschiedene nationale Netzwerke mit Bezug zu Ernährung und Gesundheit auf politischer Ebene aktiv (einige Beispiele in alphabetischer Reihenfolge): Allianz Ernährung (101), Allianz Gesunde Schweiz (102), Allianz Gesundheitskompetenz (103), NGO-Allianz Ernährung, Bewegung, Körpergewicht (104).

\subsubsection{Nationale und kantonale Ernährungsprojekte}

Die nachfolgenden Tabellen stellen eine Auswahl von laufenden nationalen und überkantonalen (Tabelle 4.2), von in der Westschweiz und / oder im Tessin laufenden (Tabelle 4.3) und von in der Deutschschweiz laufenden (Tabelle 4.4) Projekten zur Förderung einer ausgewogenen Ernährung und ausreichend körperlicher Aktivität sowie zur Verbesserung der diesbezüglichen Rahmenbedingungen vor. Die Vielfalt an Projekten ist erfreulich, zeigt sie doch, dass das Thema Ernährung in der Schweiz zunehmend an Bedeutung gewinnt. Die grosse Projektvielfalt erfordert eine gute Koordination zwischen allen Beteiligten.

Die aufgeführten Projekte sind Beispiele aus der Praxis, welche die Qualitätskriterien für Projekte in der Gesundheitsförderung und Prävention erfüllen (z.B. diejenigen von Quintessenz; 105) und entweder bereits in Strukturen verankert sind, in mehreren Kantonen umgesetzt werden oder das Potenzial dazu haben, verbreitet zu werden. Die Tabellen erheben keinen Anspruch auf Vollständigkeit. Die Projekte sind jeweils in alphabetischer Reihenfolge aufgeführt. 
Tabelle 4.2: Übersicht über laufende nationale und überkantonale Ernährungsprojekte in der Schweiz, Stand November 2011

\begin{tabular}{|c|c|c|c|}
\hline $\begin{array}{l}\text { Projekt (Referenz) } \\
\text { Projektleitung }\end{array}$ & Kurzbeschrieb, Ziele & $\begin{array}{l}\text { Geographische } \\
\text { Reichweite }\end{array}$ & Settings \\
\hline $\begin{array}{l}\text { Bien manger à petit prix } \\
\text { (Gut, gesund und } \\
\text { günstig essen) } \\
\text { (106) } \\
\text { Fédération Romande des } \\
\text { Consommateurs FRC } \\
\text { (Konsumentenschutzver- } \\
\text { band der Romandie) }\end{array}$ & $\begin{array}{l}\text { Projekt, das Kurse, einen prak- } \\
\text { tischen Ratgeber (107), Umfragen } \\
\text { und Informationen auf Papier und } \\
\text { im Internet umfasst, mit denen } \\
\text { die Konsumenten für eine gute, } \\
\text { gesunde, qualitativ hochwertige } \\
\text { und preisgünstige Ernährung } \\
\text { sensibilisiert werden. }\end{array}$ & $\begin{array}{l}\text { Kantone Bern, Freiburg, } \\
\text { Genf, Jura, Neuenburg, } \\
\text { Tessin, Waadt, Wallis }\end{array}$ & Familien, Konsumenten \\
\hline $\begin{array}{l}\text { Caritas-Markt - gesund! } \\
\text { (109) } \\
\text { Caritas Schweiz }\end{array}$ & $\begin{array}{l}\text { Armutsbetroffene können in den } \\
\text { Caritas-Märkten frisches Gemüse } \\
\text { und Früchte besonders günstig } \\
\text { kaufen (finanzieller Anreiz zum } \\
\text { Konsum) und erhalten Informa- } \\
\text { tionen und konkreteTipps zur } \\
\text { Förderung der Ernährungs- und } \\
\text { Bewegungskompetenz. }\end{array}$ & National & $\begin{array}{l}\text { Caritas-Märkte in der } \\
\text { Schweiz }\end{array}$ \\
\hline $\begin{array}{l}\text { fit-4-future } \\
(110) \\
\text { Cleven-Stiftung }\end{array}$ & $\begin{array}{l}\text { Gesundheitsförderungsinitiative, } \\
\text { die Schulen und Eltern kostenlose } \\
\text { Events, Weiterbildungen und } \\
\text { Materialien zu den Themen Bewe- } \\
\text { gung, Ernährung und Brainfitness } \\
\text { anbietet. }\end{array}$ & National & Primarschulen \\
\hline $\begin{array}{l}\text { Fourchette verte } \\
\text { (112) } \\
\text { Fourchette verte Schweiz }\end{array}$ & $\begin{array}{l}\text { Gesundheitslabel für Restau- } \\
\text { rationsbetriebe und öffentliche } \\
\text { Einrichtungen, die ein ausgewo- } \\
\text { genes Tagesmenü anbieten, das } \\
\text { gewisse Kriterien erfüllt. }\end{array}$ & $\begin{array}{l}\text { Verschiedene Kantone in } \\
\text { der ganzen Schweiz } \\
\text { (Aargau, Bern, Freiburg, } \\
\text { Genf, Jura, Neuenburg, } \\
\text { Solothurn, Tessin, } \\
\text { Waadt, Wallis) }\end{array}$ & Restaurationsbetriebe \\
\hline $\begin{array}{l}\text { Gesundheitsfördernde } \\
\text { Gemeinschaftsgastro- } \\
\text { nomie } \\
(114) \\
\text { Berner Fachhochschule, } \\
\text { Fachbereich Gesundheit } \\
\text { (BFH) }\end{array}$ & $\begin{array}{l}\text { Systemische, kontinuierliche } \\
\text { und nachhaltige Umsetzung der } \\
\text { "Schweizer Qualitätsstandards } \\
\text { für eine gesundheitsfördernde } \\
\text { Gemeinschaftsgastronomie». In } \\
\text { Zusammenarbeit mit Betrieben } \\
\text { Ernährungsverhältnisse schaffen, } \\
\text { welche die Konsumenten bei der } \\
\text { Optimierung ihres Lebensstils } \\
\text { unterstützen. }\end{array}$ & $\begin{array}{l}\text { Deutsch- undWest- } \\
\text { schweiz, teilweise Tessin }\end{array}$ & $\begin{array}{l}\text { Private und öffentliche } \\
\text { Unternehmen mit einem } \\
\text { Verpflegungsangebot, } \\
\text { d.h. mit einem Schul- oder } \\
\text { Personalrestaurant, einer } \\
\text { Cafeteria, einer Spital- oder } \\
\text { Heimküche, einem Ver- } \\
\text { pflegungsautomaten usw. } \\
\text { Produzenten / Liefe- } \\
\text { ranten zuhanden der GG }{ }^{1}\end{array}$ \\
\hline
\end{tabular}

$1 \mathrm{GG}=$ Gemeinschaftsgastronomie 


\begin{tabular}{|c|c|c|c|c|}
\hline Zielgruppen & Multiplikatoren & Projektstart & $\begin{array}{l}\text { Multiplikation, } \\
\text { Vernetzung }\end{array}$ & Evaluationsergebnisse \\
\hline $\begin{array}{l}\text { Kinder, Jugend- } \\
\text { liche und Erwach- } \\
\text { sene, ältere Men- } \\
\text { schen, Migrations- } \\
\text { bevölkerung }\end{array}$ & $\begin{array}{l}\text { Eltern, } \\
\text { Grosseltern }\end{array}$ & 2009 & $\begin{array}{l}\text { Aufgrund des grossen Erfolgs } \\
\text { des Projekts in derWest- } \\
\text { schweiz und im Tessin wurde } \\
\text { eine deutsche Version der } \\
\text { Kurse und des Ratgebers in } \\
\text { Zusammenarbeit mit der Stif- } \\
\text { tung für Konsumentenschutz } \\
\text { erstellt (Ratgeber "Gut, gesund } \\
\text { und günstig essen»; 108). }\end{array}$ & Keine Evaluation vorhanden. \\
\hline $\begin{array}{l}\text { Armutsbetroffene } \\
\text { Erwachsene und } \\
\text { Familien }\end{array}$ & $\begin{array}{l}\text { Caritas-Markt } \\
\text { Mitarbeitende, } \\
\text { vereinzelt } \\
\text { Fachleute }\end{array}$ & 2010 & $\begin{array}{l}\text { Das Projekt wird in allen } \\
\text { Caritas-Märkten umgesetzt. }\end{array}$ & $\begin{array}{l}\text { Evaluationsergebnisse liegen } \\
\text { Ende } 2012 \text { vor. }\end{array}$ \\
\hline $\begin{array}{l}\text { Kinder im Alter } \\
\text { von 6-12 Jahren }\end{array}$ & $\begin{array}{l}\text { Schulleitungen, } \\
\text { Lehrpersonen, } \\
\text { Eltern }\end{array}$ & 2005 & $\begin{array}{l}\text { "fit-4-future» wird in } 483 \\
\text { Primarschulen der ganzen } \\
\text { Schweiz kostenlos durch- } \\
\text { geführt (Stand November } \\
\text { 2011). }\end{array}$ & $\begin{array}{l}\text { Ergebnisse im Bereich Bewe- } \\
\text { gung: signifikante Steigerung } \\
\text { der Bewegungsaktivität in } \\
\text { den Schulpausen (111). } \\
\text { Ergebnisse im Bereich Ernäh- } \\
\text { rung folgen } 2012 \text {. }\end{array}$ \\
\hline $\begin{array}{l}\text { Verschiedene } \\
\text { Labels für Klein- } \\
\text { kinder unter } \\
\text { 4 Jahren, Kinder } \\
\text { im Alter von } \\
\text { 4-15 Jahren, } \\
\text { Erwachsene, } \\
\text { ältere Menschen } \\
\text { über } 65 \text { Jahre }\end{array}$ & $\begin{array}{l}\text { Köche, Kleinkinder- } \\
\text { erzieherinnen, } \\
\text { Schul-und } \\
\text { Krippenleitungen, } \\
\text { Gemeindepoliti- } \\
\text { ker, Ernährungs- } \\
\text { beraterinnen }\end{array}$ & $\begin{array}{l}1993 \\
\text { (seit } 2009 \text { in } \\
\text { der Deutsch- } \\
\text { schweiz) }\end{array}$ & $\begin{array}{l}\text { Das Konzept «Fourchette } \\
\text { verte» ist in der ganzen } \\
\text { Schweiz verbreitet. } \\
\text { Seit } 2010 \text { besteht eine } \\
\text { Zusammenarbeit mit der } \\
\text { Lebensmittelindustrie zur } \\
\text { Beeinflussung der Zusam- } \\
\text { mensetzung von Produkten } \\
\text { für Restaurationsbetriebe. }\end{array}$ & $\begin{array}{l}\text { Das Label "Fourchette verte» } \\
\text { ist sehr bekannt, vor allem in } \\
\text { derWestschweiz und im Tes- } \\
\text { sin. } 75 \% \text { der befragten Per- } \\
\text { sonen im Kanton Genf geben } \\
\text { an, das Label zu kennen (113). }\end{array}$ \\
\hline $\begin{array}{l}\text { Gäste der } \mathrm{GG}^{1} \text {, } \\
\text { d.h. alle Alters- } \\
\text { gruppen }\end{array}$ & $\begin{array}{l}\text { Verantwortliche } \\
\text { (Unternehmen } \\
\text { und GG-Betrieb }{ }^{1} \text { ) } \\
\text { für das Verpfle- } \\
\text { gungsangebot; } \\
\text { Produzenten/ } \\
\text { Lieferanten; Fach- } \\
\text { personen der GG }{ }^{1} \\
\text { und Gesundheits- } \\
\text { förderung; Gäste }\end{array}$ & 2007 & $\begin{array}{l}\text { Das Projekt wird im Rahmen } \\
\text { des NPEB gemeinsam von } \\
\text { der Berner Fachhochschule } \\
\text { und der Schweizerischen } \\
\text { Gesellschaft für Ernährung } \\
\text { durchgeführt. }\end{array}$ & $\begin{array}{l}\text { Die Schweizer Qualitätsstan- } \\
\text { dards weisen der Praxis den } \\
\text { Weg zu gesunden Verhältnis- } \\
\text { sen und zeigen den in der GG }{ }^{1} \\
\text { anzutreffenden Personen- } \\
\text { gruppen die Anforderungen } \\
\text { an ihrVerhalten auf (115). }\end{array}$ \\
\hline
\end{tabular}




\begin{tabular}{|c|c|c|c|}
\hline $\begin{array}{l}\text { Projekt (Referenz) } \\
\text { Projektleitung }\end{array}$ & Kurzbeschrieb, Ziele & $\begin{array}{l}\text { Geographische } \\
\text { Reichweite }\end{array}$ & Settings \\
\hline $\begin{array}{l}\text { GORILLA } \\
\text { (116) } \\
\text { Schtifti Foundation }\end{array}$ & $\begin{array}{l}\text { Motiviert Kinder und Jugendliche, } \\
\text { sich mit denThemen Ernährung } \\
\text { und Bewegung für ein gesundes } \\
\text { Körpergewicht auseinanderzuset- } \\
\text { zen. In einem eLearning eignen } \\
\text { sie sich Wissen an, mit dem sie in } \\
\text { Tests Punkte für Individual- und } \\
\text { Schulhauspreise (u.a. Workshops) } \\
\text { sammeln können. }\end{array}$ & National & Internet, Schulen \\
\hline $\begin{array}{l}\text { Kebab+ } \\
\text { (118) } \\
\text { Migros Kulturprozent }\end{array}$ & $\begin{array}{l}\text { Unterstützung von Projekten, die } \\
\text { Jugendliche zur Reflexion der } \\
\text { gegenwärtigen Esskultur animie- } \\
\text { ren, die zu einer Auseinanderset- } \\
\text { zung mit gesunder Ernährung und } \\
\text { Bewegung sowie Begegnungen } \\
\text { motivieren und die gemeinsam } \\
\text { mit Jugendlichen entwickelt und } \\
\text { durchgeführt werden. }\end{array}$ & $\begin{array}{l}\text { Deutsch- und West- } \\
\text { schweiz }\end{array}$ & $\begin{array}{l}\text { Jugendarbeitsstellen, } \\
\text { lokale Jugendförde- } \\
\text { rungsstellen }\end{array}$ \\
\hline $\begin{array}{l}\text { KidBalù } \\
\text { (120) } \\
\text { Schweizerischer } \\
\text { Turnverband (STV) }\end{array}$ & $\begin{array}{l}\text { Das vielseitige, lebhafte Bewe- } \\
\text { gungsangebot Muki-Turnen (Mut- } \\
\text { ter-Kind-Turnen) wird über die } \\
\text { Aus- undWeiterbildung der Leite- } \\
\text { rinnen mit Bausteinen zum Thema } \\
\text { Essen undTrinken ergänzt. } \\
\text { Ziel ist eine Sensibilisierung für } \\
\text { ein gesundes Bewegungs- und } \\
\text { Ernährungsverhalten und dessen } \\
\text { Optimierung. }\end{array}$ & National & $\begin{array}{l}\text { Muki-Turnen (Elki-oder } \\
\text { Vaki-Turnen) }\end{array}$ \\
\hline $\begin{array}{l}\text { meingleichgewicht } \\
\text { (121) } \\
\text { Migros Kulturprozent }\end{array}$ & $\begin{array}{l}\text { Sensibilisierung von Behinder- } \\
\text { teninstitutionen für Gesundheits- } \\
\text { förderung und Ermunterung zur } \\
\text { Entwicklung von Projekten für } \\
\text { ein gesundes Körpergewicht in } \\
\text { diesen Institutionen. }\end{array}$ & $\begin{array}{l}\text { Deutsch- und West- } \\
\text { schweiz }\end{array}$ & $\begin{array}{l}\text { Institutionen für } \\
\text { Menschen mit einer } \\
\text { Behinderung }\end{array}$ \\
\hline $\begin{array}{l}\text { NUTRIKID } \\
\text { (122) } \\
\text { Schweizerische Gesell- } \\
\text { schaft für Ernährung (SGE) }\end{array}$ & $\begin{array}{l}\text { Ernährungserziehung und -ausbil- } \\
\text { dung von Kindern im Hinblick auf } \\
\text { eine ausgewogene Ernährung, } \\
\text { verbunden mit ausreichender } \\
\text { körperlicher Bewegung und Ent- } \\
\text { spannung. }\end{array}$ & National & $\begin{array}{l}\text { Kindergärten und } \\
\text { Primarschulen }\end{array}$ \\
\hline
\end{tabular}




\begin{tabular}{|c|c|c|c|c|}
\hline Zielgruppen & Multiplikatoren & Projektstart & $\begin{array}{l}\text { Multiplikation, } \\
\text { Vernetzung }\end{array}$ & Evaluationsergebnisse \\
\hline $\begin{array}{l}\text { Jugendliche } \\
\text { im Alter von } \\
\text { 10-18 Jahren }\end{array}$ & $\begin{array}{l}\text { Schulleitungen, } \\
\text { Lehrpersonen, } \\
\text { Schüler, Eltern, } \\
\text { breite Bevölke- } \\
\text { rung }\end{array}$ & $\begin{array}{l}2003 \text { (Vor- } \\
\text { läuferprojekt } \\
\text { "Schtifti } \\
\text { Freestyle } \\
\text { Tour») } \\
2010 \\
\text { («GORILLA») }\end{array}$ & $\begin{array}{l}\text { Die GORILLAWorkshops } \\
\text { können im Rahmen der KAP } \\
\text { eingesetzt werden. }\end{array}$ & $\begin{array}{l}\text { Eine Zwischenbilanz vom } \\
\text { Oktober } 2011 \text { zeigt, dass die } \\
\text { GORILLAWorkshops sowohl } \\
\text { bei den Jugendlichen als auch } \\
\text { bei den Lehrpersonen auf } \\
\text { grosse Zustimmung stossen } \\
\text { (117). } \\
\text { Die umfassenden Evaluations- } \\
\text { ergebnisse vom Frühling } 2012 \\
\text { lagen zum Zeitpunkt derText- } \\
\text { erstellung noch nicht vor. }\end{array}$ \\
\hline $\begin{array}{l}\text { Jugendliche } \\
\text { im Alter von } \\
\text { 13-20 Jahren }\end{array}$ & $\begin{array}{l}\text { Sozial-und } \\
\text { Jugendarbeiter }\end{array}$ & 2009 & $\begin{array}{l}\text { Multiplikation geschieht fort- } \\
\text { laufend über die Projektein- } \\
\text { gaben der Jugendlichen. }\end{array}$ & $\begin{array}{l}\text { Ergebnisse in der Vermittlung } \\
\text { von Kochkompetenzen bei } \\
\text { Jugendlichen (119). }\end{array}$ \\
\hline $\begin{array}{l}\text { Kinder im Alter } \\
\text { von 3-5 Jahren } \\
\text { und deren Eltern }\end{array}$ & $\begin{array}{l}\text { Muki-Leiterinnen } \\
\text { und STV-Ausbild- } \\
\text { nerinnen }\end{array}$ & $\begin{array}{l}2004 \\
\text { (Neulancie- } \\
\text { rung 2012) }\end{array}$ & $\begin{array}{l}\text { Das Projekt ist in die Ausbil- } \\
\text { dungsstrukturen des STV } \\
\text { integriert und erreicht somit } \\
\text { alle in der Schweiz aktiven } \\
\text { Muki-Leiterinnen. }\end{array}$ & $\begin{array}{l}\text { Das Projekt konnte sich nach } \\
\text { drei Jahren Laufzeit einer } \\
\text { hohen Beteiligung der Lei- } \\
\text { terinnen erfreuen. Dank der } \\
\text { sehr weiten Streuung konnte } \\
\text { mit dem Projekt ein sehr } \\
\text { vorteilhaftes Nutzen-Kosten- } \\
\text { Verhältnis erlangt werden. }\end{array}$ \\
\hline $\begin{array}{l}\text { Menschen mit } \\
\text { einer Behinde- } \\
\text { rung, die in } \\
\text { Institutionen } \\
\text { leben }\end{array}$ & $\begin{array}{l}\text { Institutions- und } \\
\text { Heimleiter, } \\
\text { Heimpersonal, } \\
\text { Ernährungs- und } \\
\text { Bewegungsfach- } \\
\text { personen, Ange- } \\
\text { hörige }\end{array}$ & 2011 & $\begin{array}{l}\text { Multiplikation geschieht fort- } \\
\text { laufend über die Projektein- } \\
\text { gaben der Institutionen. }\end{array}$ & Keine Evaluation vorhanden. \\
\hline $\begin{array}{l}\text { Kinder im Alter } \\
\text { von } 5-7,7-10 \text { und } \\
\text { 10-12 Jahren }\end{array}$ & $\begin{array}{l}\text { Lehrpersonen, } \\
\text { Eltern }\end{array}$ & $\begin{array}{l}2001 \text { (Modul } \\
\text { 10-12 Jahre) } \\
2006 \text { (Modul } \\
\text { 5-7 Jahre) } \\
\text { 2010 (Modul } \\
\text { 7-10 Jahre) }\end{array}$ & $\begin{array}{l}\text { NUTRIKID }{ }^{\circledR} \text { kommt neben } \\
\text { der Schweiz auch in Deutsch- } \\
\text { land, Italien und Ungarn zum } \\
\text { Einsatz. }\end{array}$ & Keine Evaluation vorhanden. \\
\hline
\end{tabular}




\section{$>$ Ernährungsmassnahmen zur Förderung der Gesundheit}

\begin{tabular}{|c|c|c|c|}
\hline $\begin{array}{l}\text { Projekt (Referenz) } \\
\text { Projektleitung }\end{array}$ & Kurzbeschrieb, Ziele & $\begin{array}{l}\text { Geographische } \\
\text { Reichweite }\end{array}$ & Settings \\
\hline $\begin{array}{l}\text { Procap bewegt } \\
\text { (123) } \\
\text { Procap Sport }\end{array}$ & $\begin{array}{l}\text { Sensibilisierung für und Motivie- } \\
\text { rung zu Gesundheitsförderung } \\
\text { (Schwerpunkte Ernährung und } \\
\text { Bewegung) für Menschen mit } \\
\text { einer kognitiven und / oder körper- } \\
\text { lichen Behinderung; Entwicklung } \\
\text { eines strukturierten Vorgehens in } \\
\text { Institutionen. }\end{array}$ & $\begin{array}{l}\text { Deutsch- undWest- } \\
\text { schweiz }\end{array}$ & $\begin{array}{l}\text { Institutionen für } \\
\text { Menschen mit einer } \\
\text { Behinderung (z.B. Ausbil- } \\
\text { dungs- undWohnheime); } \\
\text { weitere Settings sind } \\
\text { vorgesehen }\end{array}$ \\
\hline $\begin{array}{l}\text { Purzelbaum } \\
\text { (125) } \\
\text { Radix }\end{array}$ & $\begin{array}{l}\text { Konzept für mehr Bewegung und } \\
\text { gesunde Ernährung in Kinder- } \\
\text { gärten und Kindertagesstätten } \\
\text { (Weiterbildung und praxisnahe } \\
\text { Unterstützung von Lehrpersonen } \\
\text { und Leitenden von Kindertages- } \\
\text { stätten (Kitas); Elternabende } \\
\text { und -aktivitäten; Räumlichkeiten } \\
\text { werden bewegungsfreundlich } \\
\text { umgestaltet). }\end{array}$ & National & $\begin{array}{l}\text { Familienergänzende } \\
\text { Betreuungseinrich- } \\
\text { tungen (Kindergarten, } \\
\text { Kindertagesstätten / } \\
\text { Krippen) }\end{array}$ \\
\hline
\end{tabular}


Zielgruppen

Kinder, Jugendliche und Erwachsene mit einer Behinderung, die in Institutionen leben; Institutionsleitung; betreuendes Umfeld; Angehörige

\section{Multiplikatoren}

Institutions- und

Heimleiter,

Betreuer, Fachpersonal, Ernährungsund Bewegungsfachpersonen, Angehörige, Procap sport mit einem Coachingund Fachteam, Organisationen und Netzwerke im Ernährungs-und Bewegungsbereich

\section{Kinder im Alter} von 0-6 Jahren sowie ihre Eltern

Kindergartenlehr-
personen, Betreuungspersonen Kleinkindbereich

\section{Projektstart} 2009

Vernetzung

Produkte: Grundlagen und

Empfehlungen zu Ernährung / Bewegung / Gesundheit für Menschen mit Behinderung in Institutionen; Umsetzungsleitfaden (123).

Projekte: diverse Projekte für Schulen (GORILLA, Bodytalk PEP usw.), KAP Aargau, «meingleichgewicht» (121) usw.
Evaluationsergebnisse

Grössere Sensibilisierung und Motivation der Zielgruppen;

Erweiterung der fachlichen

Kenntnisse des betreuenden

Umfelds; Entwicklung eines

strukturierten Vorgehens zur Implementierung; Schaffung von fachlichen Grundlagen zu Ernährung / Bewegung / Gesundheit für Menschen mit Behinderung in Institutionen (124).

Schulleitungen,
Lehrpersonen,
Eltern
Schulleitungen Jugendliche im Alter von 5-16 Jahren

\section{Eltern}

\section{4}

2005

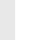

Das

Das Projekt ist Bestandteil
der Plattform "Purzelbaum
plus» und mit Projekten wie
"Papperla PEP» (83) und
"schnitz und drunder» (126)
vernetzt.
Aus Sicht vieler beteiligter Lehrpersonen und Eltern hat sich das Bewegungsverhalten der Kinder durch das Projekt «Purzelbaum» eindeutig verändert: Die Kinder sind sicherer und mutiger gewor- den und haben Freude, sich zu bewegen (127).

\section{Sehr hohe Zufriedenheit}

Ca. 6\% aller Schweizer Schulklassen bzw. 10\% aller Primarschulklassen setzen "schule bewegt» um. bei den teilnehmenden Lehrpersonen. 90\% der Lehrpersonen wollen die tägliche Bewegung weiterführen (129). 
Tabelle 4.3: Übersicht über laufende Ernährungsprojekte in der Westschweiz und / oder im Tessin, Stand November 2011

\begin{tabular}{|c|c|c|c|}
\hline $\begin{array}{l}\text { Projekt (Referenz) } \\
\text { Projektleitung }\end{array}$ & Kurzbeschrieb, Ziele & $\begin{array}{l}\text { Geographische } \\
\text { Reichweite }\end{array}$ & Settings \\
\hline $\begin{array}{l}\text { Guide école } \\
\text { (Ratgeber Schule) } \\
\text { (130) } \\
\text { Kantone Freiburg, Jura, } \\
\text { Neuenburg, Waadt und } \\
\text { Wallis }\end{array}$ & $\begin{array}{l}\text { Internetplattform für Lehrper- } \\
\text { sonen, Schulleitungen und Schul- } \\
\text { gesundheitspersonal, die ihnen } \\
\text { helfen soll, eine umfassende und } \\
\text { kohärente Politik zur Förderung } \\
\text { einer gesundheitsfördernden } \\
\text { Ernährung und Bewegung in den } \\
\text { Schulen umzusetzen. }\end{array}$ & $\begin{array}{l}\text { Kantone Freiburg, Jura, } \\
\text { Neuenburg, Waadt, } \\
\text { Wallis }\end{array}$ & Schulen, Internet \\
\hline $\begin{array}{l}\text { Les petits gourmets } \\
\text { à table } \\
\text { (Kleine Feinschmecker } \\
\text { zuTisch!) } \\
\text { (131) } \\
\text { Kanton Neuenburg }\end{array}$ & $\begin{array}{l}\text { Sensibilisierung von Fachper- } \\
\text { sonen in Betreuungseinrich- } \\
\text { tungen für Kleinkinder und von } \\
\text { Eltern für die Entwicklung von } \\
\text { Essgewohnheiten und des } \\
\text { Geschmacks bei Kindern. }\end{array}$ & Kanton Neuenburg & $\begin{array}{l}\text { Betreuungseinrich- } \\
\text { tungen für Kleinkinder }\end{array}$ \\
\hline $\begin{array}{l}\text { Movimento e gusto } \\
\text { con l'equilibrio giusto! } \\
\text { (Bewegung und genuss- } \\
\text { volle Ernährung im } \\
\text { Gleichgewicht) } \\
\text { (133) } \\
\text { Kantonsärztlicher Dienst } \\
\text { des KantonsTessin }\end{array}$ & $\begin{array}{l}\text { Projekt zur Förderung einer } \\
\text { ausgewogenen Ernährung und } \\
\text { gesundheitsfördernden Bewe- } \\
\text { gung in Kindergärten und Primar- } \\
\text { schulen durch Ausbildung der } \\
\text { Lehrpersonen und Information } \\
\text { der Eltern. Jede Schule, die teil- } \\
\text { nimmt, verpflichtet sich für eine } \\
\text { Teilnahme von zwei Jahren. }\end{array}$ & Tessin & $\begin{array}{l}\text { Kindergärten und Primar- } \\
\text { schulen }\end{array}$ \\
\hline $\begin{array}{l}\text { Pharmaciens malins } \\
\text { (Kluge Apotheker) } \\
\text { (134) } \\
\text { Association pharma Genève } \\
\text { (Genfer Apothekerverband) }\end{array}$ & $\begin{array}{l}\text { Sensibilisierung der Apotheker für } \\
\text { die aktuellen Empfehlungen im } \\
\text { Bereich Stillen, gesunde Ernäh- } \\
\text { rung und gesundheitsfördernde } \\
\text { Bewegung, damit sie diese Infor- } \\
\text { mationen und Ratschläge an ihre } \\
\text { Kundschaft weitergeben können. }\end{array}$ & Kanton Genf & Apotheken \\
\hline
\end{tabular}




\begin{tabular}{|c|c|c|c|c|}
\hline Zielgruppen & Multiplikatoren & Projektstart & $\begin{array}{l}\text { Multiplikation, } \\
\text { Vernetzung }\end{array}$ & Evaluationsergebnisse \\
\hline $\begin{array}{l}\text { Kinder und } \\
\text { Jugendliche im } \\
\text { Alter von } \\
\text { 0-20 Jahren }\end{array}$ & $\begin{array}{l}\text { Lehrpersonen, } \\
\text { Schulgesund- } \\
\text { heitspersonal, } \\
\text { Schulleitungen }\end{array}$ & 2009 & $\begin{array}{l}\text { Das Projekt ist Teil der Kan- } \\
\text { tonalen Aktionsprogramme } \\
\text { "Gesundes Körpergewicht" } \\
\text { in den Kantonen Freiburg, } \\
\text { Jura, Neuenburg, Waadt und } \\
\text { Wallis. }\end{array}$ & Keine Evaluation vorhanden. \\
\hline $\begin{array}{l}\text { Säuglinge und } \\
\text { Kleinkinder im } \\
\text { Alter von } \\
\text { 0-3 Jahren sowie } \\
\text { ihre Eltern }\end{array}$ & $\begin{array}{l}\text { Kleinkindererzie- } \\
\text { herinnen, Eltern, } \\
\text { Ernährungsbera- } \\
\text { terinnen }\end{array}$ & 2008 & $\begin{array}{l}\text { Das Projekt ist Teil des Kan- } \\
\text { tonalen Aktionsprogramms } \\
\text { "Ernährung und Bewegung" } \\
\text { des Kantons Neuenburg. }\end{array}$ & $\begin{array}{l}\text { Evaluationsbericht des } \\
\text { Projekts «Enfants, fruits et } \\
\text { légumes» des kantonalen } \\
\text { Gesundheitsdienstes des } \\
\text { Kantons Neuenburg (132). }\end{array}$ \\
\hline $\begin{array}{l}\text { Lehrpersonen und } \\
\text { Eltern von Kinder- } \\
\text { garten- und Pri- } \\
\text { marschulkindern } \\
\text { (3-11 Jahre) }\end{array}$ & $\begin{array}{l}\text { Lehrpersonen und } \\
\text { Eltern }\end{array}$ & 2005 & $\begin{array}{l}\text { Das Projekt ist Teil des Kan- } \\
\text { tonalen Aktionsprogramms } \\
\text { "Gesundes Körpergewicht" } \\
\text { des Kantons Tessin. }\end{array}$ & $\begin{array}{l}\text { Dokumente mit den Evalua- } \\
\text { tionsergebnissen zu den ver- } \\
\text { schiedenen Aktivitäten (133). }\end{array}$ \\
\hline $\begin{array}{l}\text { Schwangere und } \\
\text { stillende Frauen, } \\
\text { Kinder und } \\
\text { Jugendliche im } \\
\text { Alter von } \\
\text { 0-20 Jahren sowie } \\
\text { ihre Eltern }\end{array}$ & $\begin{array}{l}\text { Apotheker, Pharma- } \\
\text { assistentinnen, } \\
\text { Eltern }\end{array}$ & 2008 & $\begin{array}{l}\text { Das Projekt ist Teil des } \\
\text { Programms "Marchez et } \\
\text { mangez malin" (Gehen Sie } \\
\text { und essen Sie klug) des } \\
\text { kantonalen Gesundheitsför- } \\
\text { derungsplans des Kantons } \\
\text { Genf. } \\
\text { Die Erfahrungen des Kantons } \\
\text { Genf lassen sich in andere } \\
\text { Kantone übertragen. Mehre- } \\
\text { reWestschweizer Kantone } \\
\text { haben daher beschlossen, } \\
\text { das Konzept zu übernehmen } \\
\text { und es an ihre Rahmenbedin- } \\
\text { gungen anzupassen. }\end{array}$ & $\begin{array}{l}\text { Die Bedeutung und die Wir- } \\
\text { kungen des Projekts konnten } \\
\text { nachgewiesen werden. } \\
\text { Es wurden verschiedene } \\
\text { Empfehlungen und Verbes- } \\
\text { serungsvorschläge für die } \\
\text { Weiterführung des Projekts } \\
\text { formuliert (135). }\end{array}$ \\
\hline
\end{tabular}




\begin{tabular}{|c|c|c|c|}
\hline $\begin{array}{l}\text { Projekt (Referenz) } \\
\text { Projektleitung }\end{array}$ & Kurzbeschrieb, Ziele & $\begin{array}{l}\text { Geographische } \\
\text { Reichweite }\end{array}$ & Settings \\
\hline $\begin{array}{l}\text { Planificateur de menus } \\
\text { pour camps } \\
\text { (Menüplanung für Lager) } \\
\text { (136) } \\
\text { Kantonales Programm } \\
\text { "CCa marche!» (Es/man geht!) } \\
\text { des Kantons Waadt }\end{array}$ & $\begin{array}{l}\text { Internetplattform, um saisonale } \\
\text { und ausgewogene Menüs für } \\
\text { Gruppen von Kindern und Jugend- } \\
\text { lichen zusammenzustellen, die } \\
\text { Mengen zu berechnen und Ein- } \\
\text { kaufslisten zu erstellen. }\end{array}$ & Kanton Waadt & $\begin{array}{l}\text { Schulen, Jugendorgani- } \\
\text { sationen, Sportclubs, } \\
\text { Ferienlagerveranstalter }\end{array}$ \\
\hline $\begin{array}{l}\text { Programme d'éducation } \\
\text { nutritionnelle du canton } \\
\text { du Jura } \\
\text { (Ernährungserziehungs- } \\
\text { programm des Kantons } \\
\text { Jura) } \\
\text { (137) } \\
\text { Bildungsdepartement und } \\
\text { Gesundheitsdepartement } \\
\text { des Kantons Jura }\end{array}$ & $\begin{array}{l}\text { Sensibilisierungsprogramm für } \\
\text { die Ernährungserziehung in der } \\
\text { Schule zwecks Vorbeugung von } \\
\text { Übergewicht und Adipositas bei } \\
\text { Kindern. Die Ernährungslehre ist } \\
\text { im Lehrplan der Primarschulen } \\
\text { des Kantons Jura verankert. }\end{array}$ & Kanton Jura & $\begin{array}{l}\text { Kindergärten und Primar- } \\
\text { schulen }\end{array}$ \\
\hline $\begin{array}{l}\text { Senso5 } \\
\text { (138) } \\
\text { Fondation Senso5 }\end{array}$ & $\begin{array}{l}\text { Pädagogisches und Ernährungs- } \\
\text { erziehungsprojekt in der Schule, } \\
\text { das auf einem multidisziplinären, } \\
\text { sensoriellen Ansatz basiert. }\end{array}$ & Kanton Wallis & Schulen \\
\hline $\begin{array}{l}\text { Tutti Frutti } \\
\text { (141) } \\
\text { Kantonales Programm } \\
\text { "Ça marche!» (Es / man geht!) } \\
\text { des Kantons Waadt }\end{array}$ & $\begin{array}{l}\text { Ateliers für Familien mit Fragen im } \\
\text { Zusammenhang mit Ernährung, } \\
\text { Bewegung und Schlaf. }\end{array}$ & Kanton Waadt & $\begin{array}{l}\text { Präventionsstellen des } \\
\text { Kantons Waadt, Familien }\end{array}$ \\
\hline
\end{tabular}




\begin{tabular}{|c|c|c|c|c|}
\hline Zielgruppen & Multiplikatoren & Projektstart & $\begin{array}{l}\text { Multiplikation, } \\
\text { Vernetzung }\end{array}$ & Evaluationsergebnisse \\
\hline $\begin{array}{l}\text { Kinder und } \\
\text { Jugendliche im } \\
\text { Alter von } \\
\text { 7-20 Jahren }\end{array}$ & $\begin{array}{l}\text { Lehrpersonen, } \\
\text { Köche von Ferien- } \\
\text { lagern, Sportleiter, } \\
\text { Jugendleiter }\end{array}$ & 2010 & $\begin{array}{l}\text { Das Projekt ist Teil des kanto- } \\
\text { nalen Aktionsprogramms } \\
\text { "Ça marche!» (Es / man geht!) } \\
\text { des Kantons Waadt. }\end{array}$ & $\begin{array}{l}\text { Per Ende } 2011 \text { fast } \\
4200 \text { Planungen erstellt. }\end{array}$ \\
\hline $\begin{array}{l}\text { Kinder im Alter } \\
\text { von 5-13 Jahren }\end{array}$ & $\begin{array}{l}\text { Lehrpersonen, } \\
\text { Schulgesund- } \\
\text { heitspersonal, } \\
\text { Ernährungsbera- } \\
\text { terinnen, Schullei- } \\
\text { tungen / Lehrplan- } \\
\text { verantwortliche }\end{array}$ & $\begin{array}{l}2006 \text { (Primar- } \\
\text { schulen) } \\
2008 \text { (Kinder- } \\
\text { gärten) }\end{array}$ & $\begin{array}{l}\text { Das Projekt ist Teil des Kan- } \\
\text { tonalen Aktionsprogramms } \\
\text { «Ernährung und Bewegung» } \\
\text { des Kantons Jura. }\end{array}$ & Keine Evaluation vorhanden. \\
\hline $\begin{array}{l}\text { Kinder und } \\
\text { Jugendliche }\end{array}$ & $\begin{array}{l}\text { Lehrpersonen, } \\
\text { Schulleitungen }\end{array}$ & 2003 & $\begin{array}{l}\text { Das Projekt ist Teil des Kan- } \\
\text { tonalen Aktionsprogramms } \\
\text { "Gesundes Körpergewicht" } \\
\text { des Kantons Wallis. }\end{array}$ & $\begin{array}{l}\text { Eine ganze Reihe von Indika- } \\
\text { toren wurde erarbeitet, um } \\
\text { dieWirkung des Projekts } \\
\text { bezüglich Gesundheitsförde- } \\
\text { rung und Nachhaltigkeit zu } \\
\text { evaluieren }(139 ; 140) \text {. Evalua- } \\
\text { tionsergebnisse liegen im } \\
\text { Jahr } 2012 \text { vor (138). }\end{array}$ \\
\hline $\begin{array}{l}\text { Kinder im Alter } \\
\text { von 0-6 Jahren } \\
\text { sowie ihre Eltern }\end{array}$ & $\begin{array}{l}\text { Kinderärzte, Pfle- } \\
\text { gefachpersonal } \\
\text { für Kleinkinder, } \\
\text { Kleinkindererzie- } \\
\text { herinnen }\end{array}$ & 2008 & $\begin{array}{l}\text { Das Projekt ist Teil des kanto- } \\
\text { nalen Aktionsprogramms } \\
\text { "Ça marche!» (Es / man geht!) } \\
\text { des Kantons Waadt. }\end{array}$ & $\begin{array}{l}\text { Evaluationsbericht zu den } \\
\text { Ateliers Tutti Frutti (142). }\end{array}$ \\
\hline
\end{tabular}


Tabelle 4.4: Übersicht über laufende Ernährungsprojekte in der Deutschschweiz, Stand November 2011

\begin{tabular}{|c|c|c|c|}
\hline $\begin{array}{l}\text { Projekt (Referenz) } \\
\text { Projektleitung }\end{array}$ & Kurzbeschrieb, Ziele & $\begin{array}{l}\text { Geographische } \\
\text { Reichweite }\end{array}$ & Settings \\
\hline $\begin{array}{l}\text { Bewegter Lebensstart } \\
\text { (143) } \\
\text { Stiftung Pro UKBB } \\
\text { (Universitätskinderspital } \\
\text { beider Basel) }\end{array}$ & $\begin{array}{l}\text { Umfassendes Angebot zu Ernäh- } \\
\text { rung und Bewegung bestehend } \\
\text { ausWeiterbildungsveranstal- } \\
\text { tungen für Fachpersonen im Früh- } \\
\text { bereich, einer Informationsplatt- } \\
\text { form im Internet sowie Bewe- } \\
\text { gungs- und Ernährungskursen für } \\
\text { Eltern und ihre Säuglinge. }\end{array}$ & $\begin{array}{l}\text { Kantone Basel-Stadt, } \\
\text { Baselland und Solothurn }\end{array}$ & $\begin{array}{l}\text { Spital, Arztpraxen, } \\
\text { Mütter-Väterberatungs- } \\
\text { stellen, Quartierzentren }\end{array}$ \\
\hline $\begin{array}{l}\text { Bodytalk PEP } \\
\text { (84) } \\
\text { Fachstelle Prävention } \\
\text { Essstörungen Praxisnah } \\
\text { (PEP) }\end{array}$ & $\begin{array}{l}\text { Projekt für Kinder und Jugend- } \\
\text { liche zum selbstbewussten } \\
\text { Umgang mit sich, dem eigenen } \\
\text { Wohlbefinden und der eigenen } \\
\text { Attraktivität (kostenlose Work- } \\
\text { shops für Schulen). }\end{array}$ & Deutschschweiz & $\begin{array}{l}\text { Primar- und Sekundar- } \\
\text { schulen, Organisationen } \\
\text { und Vereine }\end{array}$ \\
\hline $\begin{array}{l}\text { feel-ok.ch } \\
(144) \\
\text { Radix }\end{array}$ & $\begin{array}{l}\text { Internetplattform zu } 11 \text { Gesund- } \\
\text { heitsthemen (z.B. Ernährung, } \\
\text { Über- und Untergewicht, Ess- } \\
\text { störungen, Bewegung, Alkohol, } \\
\text { Tabak usw.). }\end{array}$ & $\begin{array}{l}\text { Deutschschweiz, } \\
\text { Österreich (feelok.at), } \\
\text { Deutschland (feelok.de) }\end{array}$ & $\begin{array}{l}\text { Internet, Schulen, } \\
\text { Freizeit }\end{array}$ \\
\hline $\begin{array}{l}\text { klemon } \\
\text { (146) } \\
\text { Schweizerischer Fach- } \\
\text { verband Adipositas im } \\
\text { Kindes- und Jugendalter (akj) }\end{array}$ & $\begin{array}{l}\text { Elternzentrierte Frühinterventions- } \\
\text { massnahme für Kleinkinder } \\
\text { mit Essproblemen oder Über- } \\
\text { gewichtsrisiko (mehrmonatige } \\
\text { Beratungen). }\end{array}$ & $\begin{array}{l}\text { Deutschschweiz } \\
\text { (Kantone Aargau, Bern, } \\
\text { Nidwalden, Obwalden, } \\
\text { Solothurn, Thurgau, Uri, } \\
\text { Zürich) }\end{array}$ & $\begin{array}{l}\text { Mütter-Väterberatungs- } \\
\text { stellen in enger Zusam- } \\
\text { menarbeit mit Pädiatrie- } \\
\text { und Hausarztpraxen }\end{array}$ \\
\hline
\end{tabular}




\begin{tabular}{|c|c|c|c|c|}
\hline Zielgruppen & Multiplikatoren & Projektstart & $\begin{array}{l}\text { Multiplikation, } \\
\text { Vernetzung }\end{array}$ & Evaluationsergebnisse \\
\hline $\begin{array}{l}\text { Säuglinge und } \\
\text { Kleinkinder im } \\
\text { Alter von } \\
\text { 0-3 Jahren sowie } \\
\text { ihre Eltern }\end{array}$ & $\begin{array}{l}\text { Schwangere und } \\
\text { Eltern mit Neuge- } \\
\text { borenen / Klein- } \\
\text { kindern sowie } \\
\text { Akteure und Insti- } \\
\text { tutionen, die zur } \\
\text { Beratungskette } \\
\text { dieser Zielgruppe } \\
\text { gehören }\end{array}$ & 2008 & $\begin{array}{l}\text { Die Weiterbildungsveran- } \\
\text { staltungen und die Internet- } \\
\text { plattform stehen weiteren } \\
\text { interessierten Kantonen zur } \\
\text { Verfügung. }\end{array}$ & Keine Evaluation vorhanden. \\
\hline $\begin{array}{l}\text { Kinder und } \\
\text { Jugendliche im } \\
\text { Alter von } \\
\text { 9-20 Jahren }\end{array}$ & $\begin{array}{l}\text { Lehrpersonen } \\
\text { und andere Mul- } \\
\text { tiplikatoren, die } \\
\text { mit Jugendlichen } \\
\text { arbeiten }\end{array}$ & 2005 & $\begin{array}{l}\text { Das Projekt steht weiteren } \\
\text { Kantonen und Projekten als } \\
\text { Dienstleistungsangebot } \\
\text { der Fachstelle PEP zurVer- } \\
\text { fügung. }\end{array}$ & $\begin{array}{l}\text { Evaluationsergebnisse liegen } \\
\text { Ende } 2012 \text { vor. }\end{array}$ \\
\hline $\begin{array}{l}\text { Jugendliche im } \\
\text { Alter von } \\
\text { 12-17 Jahren }\end{array}$ & $\begin{array}{l}\text { Lehrpersonen, } \\
\text { Jugendarbeiten- } \\
\text { de, Leiter von } \\
\text { Sportvereinen und } \\
\text { weitere Multipli- } \\
\text { katoren }\end{array}$ & 1999 & $\begin{array}{l}\text { Die Internetplattform bündelt } \\
\text { das Fachwissen eines inter- } \\
\text { nationalen Netzwerkes, das } \\
\text { aus über hundert Institutio- } \\
\text { nen besteht. }\end{array}$ & $\begin{array}{l}\text { Die Evaluation des Pro- } \\
\text { gramms «Mein Gewicht» } \\
\text { weist in Bezug auf Verständ- } \\
\text { lichkeit, Interessantheit und } \\
\text { Nützlichkeit der Inhalte für } \\
\text { Jugendliche positive } \\
\text { Ergebnisse auf (145). }\end{array}$ \\
\hline $\begin{array}{l}\text { Kleinkinder } \\
\text { im Alter von } \\
\text { 2-5 Jahren mit } \\
\text { Essproblemen oder } \\
\text { Übergewichts- } \\
\text { risiko sowie ihre } \\
\text { Eltern }\end{array}$ & $\begin{array}{l}\text { Mütter-Väterbera- } \\
\text { terinnen, Kinder- } \\
\text { ärzte, Hausärzte }\end{array}$ & 2006 & $\begin{array}{l}\text { Das Projekt wird in verschie- } \\
\text { denen Kantonen im Rahmen } \\
\text { der KAP umgesetzt und } \\
\text { steht weiteren Kantonen zur } \\
\text { Verfügung. }\end{array}$ & $\begin{array}{l}\text { Ungünstige Verhaltensmus- } \\
\text { ter und Gewohnheiten von } \\
\text { Kindern und ihren Familien } \\
\text { können durch die Intervention } \\
\text { adäquat angegangen und } \\
\text { positiv beeinflusst werden } \\
\text { (147). }\end{array}$ \\
\hline $\begin{array}{l}\text { Säuglinge und } \\
\text { Kleinkinder im } \\
\text { Alter von } \\
\text { 0-4 Jahren sowie } \\
\text { ihre Mütter }\end{array}$ & $\begin{array}{l}\text { Mütter-Väterbera- } \\
\text { terinnen }\end{array}$ & 2005 & $\begin{array}{l}\text { Das Projekt wurde in der Ost- } \\
\text { schweiz lanciert, wird seit } \\
2009 \text { über das Netzwerk der } \\
\text { regionalen Caritas-Stellen } \\
\text { auf weitere Regionen über- } \\
\text { tragen und ist Teil der KAP } \\
\text { Bern, Luzern, St. Gallen und } \\
\text { Thurgau. } \\
\text { «Miges Balù» ist u.a. mit } \\
\text { dem Projekt «klemon» ver- } \\
\text { netzt (146). }\end{array}$ & $\begin{array}{l}\text { Die Nutzung des Beratungs- } \\
\text { angebotes durch Familien mit } \\
\text { Migrationshintergrund aus } \\
\text { dem ehemaligen Jugosla- } \\
\text { wien, Sri Lanka und der Türkei } \\
\text { ist aufgrund der spezifischen } \\
\text { Ausrichtung der Beratungs- } \\
\text { stellen angestiegen. Die } \\
\text { Partizipation von Schlüssel- } \\
\text { personen aus der Zielgruppe } \\
\text { ist unerlässlich (149). }\end{array}$ \\
\hline
\end{tabular}




\begin{tabular}{|c|c|c|c|}
\hline $\begin{array}{l}\text { Projekt (Referenz) } \\
\text { Projektleitung }\end{array}$ & Kurzbeschrieb, Ziele & $\begin{array}{l}\text { Geographische } \\
\text { Reichweite }\end{array}$ & Settings \\
\hline $\begin{array}{l}\text { Papperla PEP } \\
\text { (83) } \\
\text { Fachstelle Prävention } \\
\text { Essstörungen Praxisnah } \\
\text { (PEP) }\end{array}$ & $\begin{array}{l}\text { Modellprojekt zur Integration von } \\
\text { Emotionsregulation und Körperei- } \\
\text { genwahrnehmung in bestehende } \\
\text { Ernährungs-und Bewegungspro- } \\
\text { jekte sowie Bildungs- und Betreu- } \\
\text { ungseinrichtungen für Kinder. }\end{array}$ & Deutschschweiz & $\begin{array}{l}\text { Bestehende Ernährungs- } \\
\text { und Bewegungsprojekte } \\
\text { sowie Bildungs- und } \\
\text { Betreuungseinrich- } \\
\text { tungen }\end{array}$ \\
\hline $\begin{array}{l}\text { Präventive Ernährungs- } \\
\text { und Bewegungsberatung } \\
\text { in der Schwangerschaft } \\
\text { PEBS } \\
\text { (150) } \\
\text { Geburtshilfliche Poliklinik } \\
\text { des Universitätsspitals } \\
\text { Zürich }\end{array}$ & $\begin{array}{l}\text { Ernährungs- und Bewegungs- } \\
\text { beratung mit niederschwelligen } \\
\text { Fitnesskursen während und nach } \\
\text { der Schwangerschaft (kosten- } \\
\text { loses Angebot für Schwangere), } \\
\text { insbesondere für Frauen mit } \\
\text { Migrationshintergrund. }\end{array}$ & $\begin{array}{l}\text { Stadt Zürich, Gemeinden } \\
\text { im Kanton Zürich, seit } \\
2012 \text { im Kanton Grau- } \\
\text { bünden }\end{array}$ & Spital und Arztpraxen \\
\hline $\begin{array}{l}\text { Primano - Fördermodul } \\
\text { Ernährung } \\
\text { (152) } \\
\text { Direktion für Bildung, } \\
\text { Soziales und Sport der Stadt } \\
\text { Bern }\end{array}$ & $\begin{array}{l}\text { Initiative der Stadt Bern zur Förde- } \\
\text { rung von Kindern im Vorschulalter } \\
\text { mit Schwerpunkt auf sozio-öko- } \\
\text { nomisch benachteiligte Familien. } \\
\text { Neben Hausbesuchsprogrammen } \\
\text { und Quartierarbeit werden Spiel- } \\
\text { gruppen und Kindertagesstätten } \\
\text { in so genannten Fördermodulen } \\
\text { in ihrer Förder- und Elternarbeit } \\
\text { u.a. zumThema Ernährung unter- } \\
\text { stützt. }\end{array}$ & Stadt Bern & $\begin{array}{l}\text { Familienergänzende } \\
\text { Betreuungseinrich- } \\
\text { tungen, Quartierzentren, } \\
\text { bei Familien zu Hause }\end{array}$ \\
\hline $\begin{array}{l}\text { Projekte rund um die } \\
\text { Pausenverpflegung } \\
\text { (154) } \\
\text { Verschiedene } \\
\text { Projektleitungen }\end{array}$ & $\begin{array}{l}\text { Projekte zu Znünibox, Pausen- } \\
\text { kiosk, Pausenapfel, Pausenmilch } \\
\text { und Ernährungsrichtlinien für } \\
\text { Schulen (Zürich; 81) usw. }\end{array}$ & $\begin{array}{l}\text { Verschiedene Städte und } \\
\text { Kantone in der Deutsch- } \\
\text { schweiz }\end{array}$ & $\begin{array}{l}\text { Kindergärten, Primar- } \\
\text { und Sekundarschulen }\end{array}$ \\
\hline
\end{tabular}




\begin{tabular}{|c|c|c|c|c|}
\hline Zielgruppen & Multiplikatoren & Projektstart & $\begin{array}{l}\text { Multiplikation, } \\
\text { Vernetzung }\end{array}$ & Evaluationsergebnisse \\
\hline $\begin{array}{l}\text { Kinder im Alter } \\
\text { von 0-8 Jahren } \\
\text { sowie ihre Eltern }\end{array}$ & $\begin{array}{l}\text { Fachpersonen } \\
\text { aus Pädagogik, } \\
\text { Betreuung und } \\
\text { Therapie, die mit } \\
\text { Kindern im Alter } \\
\text { von 0-8 Jahren } \\
\text { und deren Eltern } \\
\text { arbeiten }\end{array}$ & 2008 & $\begin{array}{l}\text { Das Projekt steht weiteren } \\
\text { Kantonen und Projekten als } \\
\text { Dienstleistungsangebot } \\
\text { der Fachstelle PEP zurVer- } \\
\text { fügung. }\end{array}$ & $\begin{array}{l}\text { Evaluationsergebnisse liegen } \\
\text { Ende } 2012 \text { vor. }\end{array}$ \\
\hline $\begin{array}{l}\text { Säuglinge und } \\
\text { Kleinkinder im } \\
\text { Alter von } \\
\text { 0-3 Jahren sowie } \\
\text { ihre Mütter }\end{array}$ & $\begin{array}{l}\text { Gynäkologen, } \\
\text { Pädiater, } \\
\text { Hausärzte, } \\
\text { Hebammen, } \\
\text { Ernährungsbera- } \\
\text { terinnen, Bewe- } \\
\text { gungsfachleute, } \\
\text { medizinisches } \\
\text { Praxispersonal }\end{array}$ & 2009 & $\begin{array}{l}\text { Das Projekt wurde im } \\
\text { Universitätsspital Zürich } \\
\text { lanciert und wird seit } 2011 \\
\text { auf Gemeindeebene (Kanton } \\
\text { Zürich) multipliziert. Es ist } \\
\text { Teil des KAP Zürich. Transfer } \\
\text { und Multiplikation in den } \\
\text { Kanton Graubünden (KAP) } \\
\text { sind in Planung. }\end{array}$ & $\begin{array}{l}\text { Mütter mit Migrationshin- } \\
\text { tergrund werden über PEBS } \\
\text { erreicht; berufstätige, eher } \\
\text { bildungsferne Schwangere } \\
\text { sowie Schwangere, die } \\
\text { bereits Kinder haben, sind } \\
\text { dagegen schwieriger zu moti- } \\
\text { vieren (151). }\end{array}$ \\
\hline $\begin{array}{l}\text { Säuglinge und } \\
\text { Kleinkinder im } \\
\text { Alter von } \\
\text { 0-4 Jahren sowie } \\
\text { ihre Eltern }\end{array}$ & $\begin{array}{l}\text { Betreuungsper- } \\
\text { sonen im Klein- } \\
\text { kindbereich, Haus- } \\
\text { besucherinnen }\end{array}$ & 2007 & $\begin{array}{l}\text { Primano und das Fördermo- } \\
\text { dul Ernährung wurden in vier } \\
\text { Pilotquartieren in der Stadt } \\
\text { Bern erprobt und sollen ab } \\
2013 \text { in das geplante Frühför- } \\
\text { derungs-Grundangebot der } \\
\text { Stadt Bern integriert werden. }\end{array}$ & $\begin{array}{l}\text { Familienergänzende Betreu- } \\
\text { ungseinrichtungen sind } \\
\text { ein geeignetes Setting zur } \\
\text { Förderung einer gesunden } \\
\text { Ernährung, u.a. weil ein guter } \\
\text { Zugang zu den Eltern besteht } \\
\text { und das Gelernte in den Ver- } \\
\text { pflegungssituationen sofort } \\
\text { und immer wieder angewandt } \\
\text { werden kann (153). }\end{array}$ \\
\hline $\begin{array}{l}\text { Kinder und } \\
\text { Jugendliche }\end{array}$ & $\begin{array}{l}\text { Schüler, Lehrper- } \\
\text { sonen, Eltern, } \\
\text { Schulleitungen, } \\
\text { Schulhausabwarte }\end{array}$ & $\begin{array}{l}\text { Unterschied- } \\
\text { lich }\end{array}$ & $\begin{array}{l}\text { Oftmals werden Projekte } \\
\text { zur Bewegungsförderung } \\
\text { wie z.B. das Projekt «Purzel- } \\
\text { baum» mit demThema Zwi- } \\
\text { schenverpflegung ergänzt; } \\
\text { so ist das Projekt "Znünibox» } \\
\text { integrierter Bestandteil von } \\
\text { "Purzelbaum» (125). } \\
\text { Im Kanton Zürich ist die } \\
\text { Anleitung zu gesunden Zwi- } \\
\text { schenmahlzeiten Bestandteil } \\
\text { der Besuche durch Schul- } \\
\text { zahnpflege-Instruktoren in } \\
\text { fast allen Kindergärten und } \\
\text { Primarschulen. }\end{array}$ & $\begin{array}{l}\text { Die Ernährungsrichtlinien } \\
\text { für Stadtzürcher Horte und } \\
\text { Schulen werden sowohl von } \\
\text { den Betreuern als auch von } \\
\text { den Kindern gut akzeptiert; } \\
\text { die gesetzten Ziele wurden } \\
\text { erreicht. Als problematisch } \\
\text { erwiesen sich allerdings die } \\
\text { Richtlinien betreffend Süs- } \\
\text { sigkeiten und Süssgetränke } \\
\text { (155). }\end{array}$ \\
\hline
\end{tabular}




\begin{tabular}{|c|c|c|c|}
\hline $\begin{array}{l}\text { Projekt (Referenz) } \\
\text { Projektleitung }\end{array}$ & Kurzbeschrieb, Ziele & $\begin{array}{l}\text { Geographische } \\
\text { Reichweite }\end{array}$ & Settings \\
\hline $\begin{array}{l}\text { schnitz und drunder } \\
\text { (126) } \\
\text { Radix }\end{array}$ & $\begin{array}{l}\text { Beratungsangebot für Betriebe } \\
\text { zur Förderung eines gesundheits- } \\
\text { förderlichen, kindergerechten } \\
\text { und regionalen Verpflegungsan- } \\
\text { gebotes. }\end{array}$ & Deutschschweiz & $\begin{array}{l}\text { Betriebe mit einem Ver- } \\
\text { pflegungsangebot ( } z \text {.B. } \\
\text { familien- und schulergän- } \\
\text { zende Betreuungsein- } \\
\text { richtungen, Schulen) }\end{array}$ \\
\hline $\begin{array}{l}\text { Schnitz\&Schwatz } \\
\text { (156) } \\
\text { Katakult - Fabrik für } \\
\text { Projektkultur }\end{array}$ & $\begin{array}{l}\text { Bildende Kinderfernsehsendung } \\
\text { im Bereich Ernährung und Bewe- } \\
\text { gung. Das edukative Format ist als } \\
\text { lustvollerWettbewerb zwischen } \\
\text { Primarschulklassen-Teams kon- } \\
\text { zipiert. }\end{array}$ & Deutschschweiz & $\begin{array}{l}\text { TV, Website, Primar- } \\
\text { schulen }\end{array}$ \\
\hline $\begin{array}{l}\text { Schulstufenkonzept } \\
\text { Rüebli, Zimt \& Co. } \\
\text { (158) } \\
\text { Gesundheitsdepartement } \\
\text { Kanton Basel-Stadt, } \\
\text { Gesundheitsförderung und } \\
\text { Prävention }\end{array}$ & $\begin{array}{l}\text { Angebote für die Primarschulen in } \\
\text { Basel-Stadt: } \\
\text { 1. Klasse: Ernährungskiste; } \\
\text { 2. Klasse: Interaktive Ernährungs- } \\
\quad \text { ausstellung; } \\
\text { 3. Klasse: Lebensmittelpyramide; } \\
\text { 4. Klasse: Flyer «Frühstück aus } \\
\text { allerWelt». } \\
\text { In der 1. Klasse erhalten alle Pri- } \\
\text { marschulen in Basel-Stadt eine } \\
\text { Ernährungskiste mit Materialien } \\
\text { für die 1.-4. Primarstufe. }\end{array}$ & Kanton Basel-Stadt & Primarschulen \\
\hline $\begin{array}{l}\text { Tacco \& Flip } \\
\text { (159) } \\
\text { Gesundheitsförderung } \\
\text { Baselland }\end{array}$ & $\begin{array}{l}\text { Sensibilisierung von Kindergar- } \\
\text { ten- und Primarschulkindern, } \\
\text { Lehrpersonen und Eltern im Kan- } \\
\text { ton Baselland für ein gesundes } \\
\text { Ernährungs- und Bewegungsver- } \\
\text { halten. }\end{array}$ & Kanton Baselland & $\begin{array}{l}\text { Kindergärten und Primar- } \\
\text { schulen }\end{array}$ \\
\hline
\end{tabular}




\begin{tabular}{|c|c|c|c|c|}
\hline Zielgruppen & Multiplikatoren & Projektstart & $\begin{array}{l}\text { Multiplikation, } \\
\text { Vernetzung }\end{array}$ & Evaluationsergebnisse \\
\hline $\begin{array}{l}\text { Kinder und } \\
\text { Jugendliche }\end{array}$ & $\begin{array}{l}\text { Verpflegungsver- } \\
\text { antwortliche und } \\
\text { Mitarbeitende in } \\
\text { den Betrieben, } \\
\text { Köche, Liefe- } \\
\text { ranten }\end{array}$ & 2006 & $\begin{array}{l}\text { Das Projekt wurde im Kanton } \\
\text { Baselland lanciert und wird } \\
\text { seit } 2011 \text { über Radix in der } \\
\text { Deutschschweiz multipli- } \\
\text { ziert. Das Angebot ist in der } \\
\text { Plattform "Purzelbaum plus» } \\
\text { eingebettet und mit dem } \\
\text { Projekt «Purzelbaum» (125), } \\
\text { "Fourchette verte» (112) } \\
\text { und "Gesundheitsfördernde } \\
\text { Gemeinschaftsgastrono- } \\
\text { mie» (114) vernetzt. }\end{array}$ & Keine Evaluation vorhanden. \\
\hline $\begin{array}{l}\text { Kinder im Alter } \\
\text { von 7-12 Jahren }\end{array}$ & $\begin{array}{l}\text { Schulleitungen, } \\
\text { Primarschullehr- } \\
\text { personen, Eltern, } \\
\text { Grosseltern, brei- } \\
\text { te Bevölkerung }\end{array}$ & 2010 & $\begin{array}{l}\text { Vernetzung mit dem Projekt } \\
\text { "fit-4-future» (110). }\end{array}$ & $\begin{array}{l}\text { Das Projekt ist kindergerecht, } \\
\text { originell und innovativ und } \\
\text { trägt dank Fachkompetenz } \\
\text { und Professionalität zur Stär- } \\
\text { kung der Gesundheitskompe- } \\
\text { tenzen von Kindern bei (157). }\end{array}$ \\
\hline $\begin{array}{l}\text { Kinder im Alter } \\
\text { von 7-12 Jahren }\end{array}$ & $\begin{array}{l}\text { Primarschullehr- } \\
\text { personen, Schul- } \\
\text { leitungen }\end{array}$ & 2007 & $\begin{array}{l}\text { Lehrpersonen werden im } \\
\text { Rahmen der Aktion «Znüni- } \\
\text { box» über das Projekt infor- } \\
\text { miert. Die Ausstellung steht } \\
\text { den Primarschulklassen im } \\
\text { Rahmen des Schwerpunkt- } \\
\text { programmes "Gesundes } \\
\text { Körpergewicht» des Kantons } \\
\text { Basel-Stadt kontinuierlich zur } \\
\text { Verfügung. }\end{array}$ & $\begin{array}{l}\text { Entsprechend einer Lehrerbe- } \\
\text { fragung im 2007/08 halten die } \\
\text { Lehrpersonen das Angebot } \\
\text { für sinnvoll und attraktiv. } \\
\text { Im Rahmen der kommenden } \\
\text { Schulreform wird das Kon- } \\
\text { zept } 2012 \text { überarbeitet und } \\
\text { erweitert. }\end{array}$ \\
\hline $\begin{array}{l}\text { Kinder im Alter } \\
\text { von 4-12 Jahren, } \\
\text { Kindergarten- und } \\
\text { Primarschullehr- } \\
\text { personen, Eltern }\end{array}$ & $\begin{array}{l}\text { Eltern, Kinder- } \\
\text { garten- und } \\
\text { Primarschul- } \\
\text { lehrpersonen, } \\
\text { Schulleitungen, } \\
\text { Gesundheitsför- } \\
\text { derung Baselland } \\
\text { mit Coaching- und } \\
\text { Fachteam, Orga- } \\
\text { nisationen in den } \\
\text { Bereichen Ernäh- } \\
\text { rung, Bewegung } \\
\text { und Gesundheits- } \\
\text { förderung }\end{array}$ & $\begin{array}{l}2003 \\
\text { (Projekt } \\
\text { im Kanton } \\
\text { Baselland } \\
\text { seit Ende } \\
2011 \text { abge- } \\
\text { schlossen, } \\
\text { Materialien } \\
\text { aber noch } \\
\text { verfügbar) }\end{array}$ & $\begin{array}{l}\text { Das im Projekt entwickelte } \\
\text { "Znüni-Zvieri-Blatt» kommt } \\
\text { in acht weiteren Kantonen } \\
\text { zum Einsatz. } \\
\text { Leitfaden zur Umsetzung von } \\
\text { Elternabenden, Leitfaden zur } \\
\text { Umsetzung des Projektes } \\
\text { sowie weitere Materialien } \\
\text { (159). } \\
\text { Vernetzung: KAP Kanton } \\
\text { Baselland. }\end{array}$ & $\begin{array}{l}\text { Bei den Kindern wurde eine } \\
\text { Verbesserung des gesund- } \\
\text { heitsrelevanten Wissens und } \\
\text { Verhaltens festgestellt. In } \\
\text { einigen beteiligten Schulen } \\
\text { wurden das zuckerfreie und } \\
\text { gemeinsame Znüni verankert } \\
\text { und zusätzliche Bewegungs- } \\
\text { angebote in den Schulalltag } \\
\text { und Unterricht integriert } \\
\text { (160). }\end{array}$ \\
\hline
\end{tabular}




\section{$>$ Ernährungsmassnahmen zur Förderung der Gesundheit}

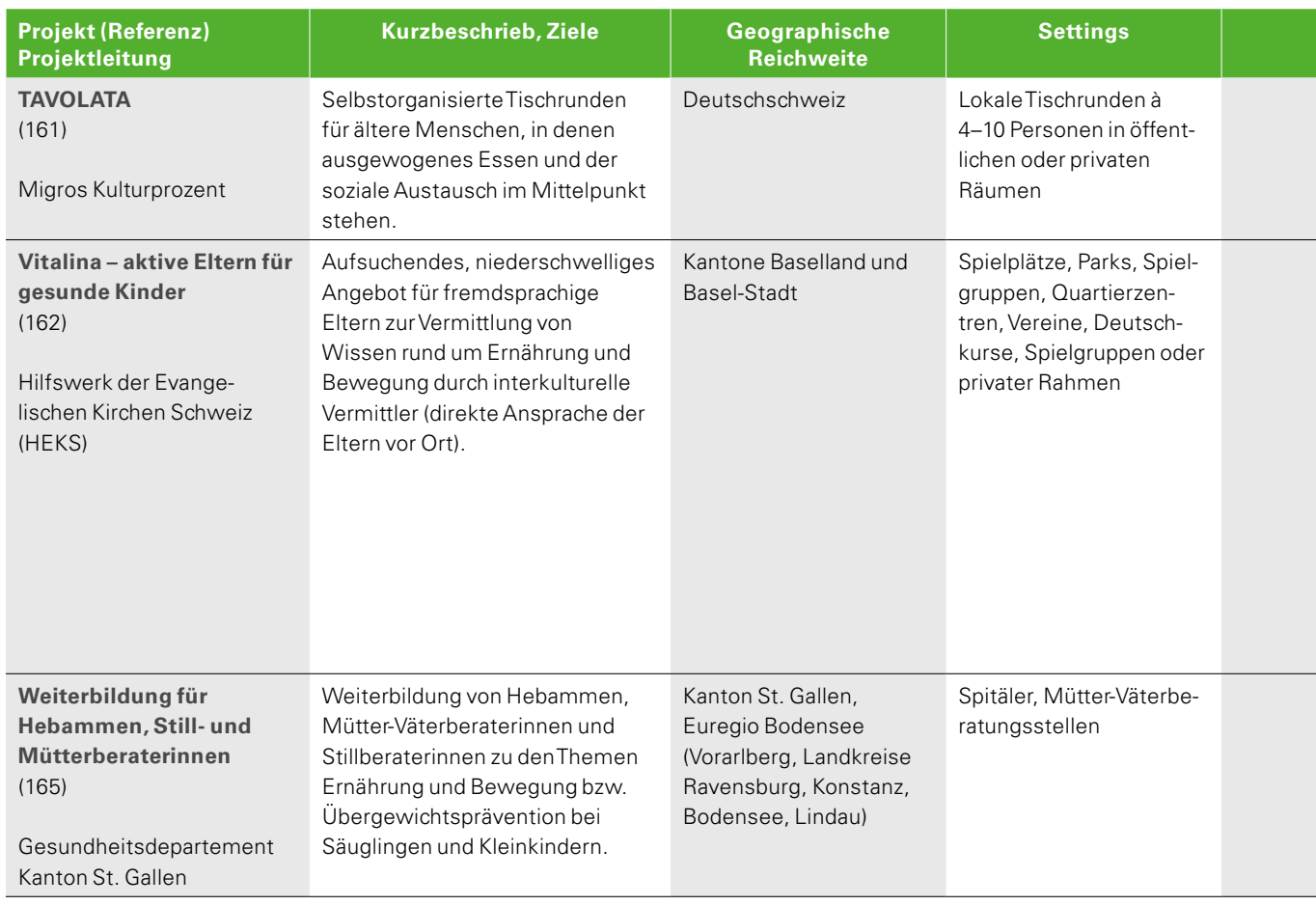




\begin{tabular}{|c|c|c|c|c|}
\hline Zielgruppen & Multiplikatoren & Projektstart & $\begin{array}{l}\text { Multiplikation, } \\
\text { Vernetzung }\end{array}$ & Evaluationsergebnisse \\
\hline $\begin{array}{l}\text { Ältere Menschen } \\
\text { ab } 55 \text { Jahren }\end{array}$ & $\begin{array}{l}\text { Impulsveranstal- } \\
\text { tungen für Kon- } \\
\text { taktpersonen }\end{array}$ & 2010 & $\begin{array}{l}\text { Kooperationen mit } \\
\text { Pro Senectute, Spitex, } \\
\text { Kirchgemeinden und } \\
\text { Gesundheitsverantwort- } \\
\text { lichen in Gemeinden. }\end{array}$ & Keine Evaluation vorhanden. \\
\hline $\begin{array}{l}\text { Kinder im Alter } \\
\text { von 0-6 Jahren } \\
\text { sowie ihre fremd- } \\
\text { sprachigen Eltern }\end{array}$ & $\begin{array}{l}\text { Interkulturelle } \\
\text { Vermittler, Mütter- } \\
\text { Väterberaterinnen }\end{array}$ & 2008 & $\begin{array}{l}\text { Das Projekt wird in den } \\
\text { Kantonen Baselland und } \\
\text { Basel-Stadt im Rahmen des } \\
\text { KAP umgesetzt und steht } \\
\text { weiteren Kantonen zurVer- } \\
\text { fügung. }\end{array}$ & $\begin{array}{l}\text { Interkulturelle Vermittler kön- } \\
\text { nen das Vertrauen der Eltern } \\
\text { gewinnen. Es besteht zusätz- } \\
\text { lich zum Austausch mit den } \\
\text { interkulturellen Vermittlern } \\
\text { auch derWunsch, sich mit } \\
\text { anderen Eltern über Gesund- } \\
\text { heits-, Ernährungs- und Erzie- } \\
\text { hungsfragen auszutauschen } \\
\text { (163). } \\
\text { Eine externe Evaluation } \\
\text { wurde im Oktober } 2011 \text { abge- } \\
\text { schlossen (164). }\end{array}$ \\
\hline $\begin{array}{l}\text { Säuglinge und } \\
\text { Kleinkinder im } \\
\text { Alter von } \\
\text { 0-3 Jahren sowie } \\
\text { ihre Eltern }\end{array}$ & $\begin{array}{l}\text { Hebammen, } \\
\text { Mütter-Väterbera- } \\
\text { terinnen, Stillbera- } \\
\text { terinnen }\end{array}$ & 2004 & $\begin{array}{l}\text { Das Weiterbildungskonzept } \\
\text { steht anderen Kantonen als } \\
\text { Grundlage zur Planung von } \\
\text { eigenen Weiterbildungsver- } \\
\text { anstaltungen zur Verfügung. }\end{array}$ & $\begin{array}{l}\text { DieWeiterbildung sollte } \\
\text { für einen längerfristigen } \\
\text { Lösungsansatz bereits in die } \\
\text { Grundausbildung der unter- } \\
\text { schiedlichen Berufsgruppen } \\
\text { integriert werden (166). }\end{array}$ \\
\hline
\end{tabular}




\subsection{Laufende Aktivitäten auf politischer und gesetzlicher Ebene}

\subsubsection{Aktivitäten auf Bundesebene}

\section{Präventionsgesetz}

Gesundheitsförderung und Prävention sind heute auf Bundesebene noch zu wenig gesetzlich verankert und die Zuständigkeiten zersplittert: Es fehlt an Koordination und Transparenz bezüglich Angebot und Leistung (Kapitel 4.3.1). Viele Akteure arbeiten aufgrund der zahlreichen Spezialgesetze nebeneinander, ohne ihr Wirken aufeinander abzustimmen. Deshalb wurden im Jahr 2007 vom Bundesrat Vorarbeiten für ein neues Bundesgesetz gestartet, welches Prävention, Gesundheitsförderung und Früherkennung als vierte Säule des Schweizer Gesundheitssystems (neben Behandlung, Pflege und Rehabilitation) stärken soll. Das Gesetz soll zudem die heute fehlende spezialgesetzliche Grundlage für Massnahmen des Bundes im Bereich der Prävention und Früherkennung von nichtübertragbaren und psychischen Krankheiten darstellen. Der Entwurf des «Bundesgesetzes über Prävention und Gesundheitsförderung» oder kurz "Präventionsgesetz» wurde am 30. September 2009 vom Bundesrat an die Eidgenössischen Räte überwiesen (167).

Das neue Bundesgesetz zielt darauf ab, eine Gesamtstrategie mit übergeordneten Zielen festzulegen, um bestehende Doppelspurigkeiten zu vermeiden und Lücken zu beheben. Es ist ein Aufgaben- und Organisationsgesetz und enthält somit keine spezifische Präventionsmassnahmen. Das Präventionsgesetz regelt insbesondere die Steuerung und Koordination (z.B. über von Bund und Kantonen gemeinsam definierte nationale Ziele), die Aufgabenteilung zwischen Bund und Kantonen, Massnahmen zur Sicherstellung der Qualität und zur Förderung der Wirksamkeit von Prävention und Gesundheitsförderung sowie die Rahmenbedingungen für die Verwendung des KVG-Prämienzuschlags und der Tabakpräventionsabgabe und für die Gewährung von Finanzhilfen an NGOs. Zudem enthält es Bestimmungen zur Gesundheitsberichterstattung sowie die Weiterentwicklung und Harmonisierung der Datenerhebung zu nichtübertragbaren Krankheiten (168).

\section{Integrierte Versorgung}

Der Bundesrat hat im Jahr 2011 seine gesundheitspolitische Strategie verabschiedet (169). Diese zielt u.a. auf eine Stärkung der integrierten Versorgung ab, womit in erster Linie die eng aufeinander abgestimmte Zusammenarbeit der Leistungserbringer über die gesamte Behandlungskette hinweg angestrebt wird. ManagedCare-Modelle bieten aber auch Möglichkeiten, ergänzende Leistungen anzubieten wie z.B. im Bereich der Früherkennung und -intervention von ernährungsabhängigen Krankheiten. Die in diesem Zusammenhang formulierte ManagedCare-Vorlage wurde im Herbst 2011 vom Parlament angenommen; zum Zeitpunkt der Texterstellung war die Referendumsfrist noch nicht abgelaufen.

\section{Cassis-de-Dijon-Prinzip}

Da der Handel von Lebensmitteln und $\mathrm{Ge}$ brauchsgegenständen heute grenzüberschreitend erfolgt, ist auch die rechtliche Situation im Lebensmittelbereich seit Anfang der 1990er Jahre den internationalen Regelungen angepasst worden. 
Um die Behinderungen des grenzüberschreitenden Handelsverkehrs abzubauen, wurde das Bundesgesetz über die technischen Handelshemmnisse (THG) revidiert (170). Kern der Vorlage bildet die autonome Einführung des so genannten "Cassis-de-Dijon-Prinzips" durch die Schweiz, d.h. dessen Anwendung durch die Schweiz auf bestimmte Einfuhren aus der Europäischen Gemeinschaft (EG) und dem Europäischen Wirtschaftsraum (EWR). Das bedeutet, dass seit dem Jahr 2010 in der Schweiz auch solche Produkte in Verkehr gebracht werden dürfen, welche nach den Vorschriften der EG oder eines EG-/ EWR-Mitgliedstaates hergestellt und dort rechtmässig in Verkehr sind. Um dem Gesundheitsschutz gerecht zu werden, gilt im Lebensmittelbereich eine zusätzliche Sonderregelung: Lebensmittel, die durch Anwendung des Cassis-de-Dijon-Prinzips in die Schweiz kommen, müssen zuerst vom BAG bewilligt werden, bevor sie in der Schweiz vertrieben werden dürfen. Die Bewilligung wird erteilt, sofern das betreffende Lebensmittel die Sicherheit und Gesundheit von Menschen nicht gefährdet und die Anforderungen an die Produktinformation des Ursprungslandes erfüllt sind.

\section{Lebensmittelgesetz}

Im Mittelpunkt der aktuellen Totalrevision des Lebensmittelgesetzes (LMG) steht die Harmonisierung mit dem europäischen Recht, um den Schutz der Konsumenten zu erhöhen und den Handel zu erleichtern (171). Seit Januar 2012 wird im Parlament darüber beraten; das revidierte Gesetz wird frühestens Ende 2013 in Kraft treten.

Parallel zur laufenden Totalrevision des LMG wurden und werden zahlreiche Verordnungen des Lebensmittelrechts zwecks Harmonisie- rung mit dem europäischen Recht vom Eidgenössischen Departement des Innern (EDI) und dem BAG revidiert (172). Diese neuen Regelungen bauen Handelshemmnisse mit der EU ab und erleichtern den freien Warenverkehr. Einige dieser Anpassungen sind in Bezug auf ein gesundes Körpergewicht relevant (z.B. Lebensmittelkennzeichnung).

Die Verordnung der Schweiz über Kennzeichnung und Anpreisung von Lebensmitteln enthält bereits Bestimmungen über die Zulässigkeit von nährwert- und gesundheitsbezogenen Angaben wie "fettarm», "reich an Vitamin C" oder "Calcium ist ein wichtiger Bestandteil von Knochen und Zähnen» (173). Die EU wird in nächster Zukunft für alle diese so genannten "Nutrition Claims» und "Health Claims» zulässige Angaben festlegen (174). Die gegenwärtig gültige Schweizer Verordnung wird infolgedessen voraussichtlich durch Regelungen aus der EU ergänzt werden.

Der Ministerrat der EU hat im September 2011 einer neuen Verordnung betreffend Information der Verbraucher über Lebensmittel zugestimmt, welche im Dezember 2011 in Kraft getreten ist (175). Sie enthält neue grundsätzliche Vorgaben über die Kennzeichnung von Lebensmitteln; u.a. ist die Angabe des Energiegehaltes und von sechs Nährstoffen (Fett, gesättigte Fettsäuren, Kohlenhydrate, Zucker, Proteine und Salz) verpflichtend.

\section{Gesundheitsabkommen mit der EU}

Zwischen der Schweiz und der EU laufen gegenwärtig Verhandlungen über ein Abkommen zwecks einer vertieften Zusammenarbeit im Agrar-, Lebensmittel- und Gesundheitsbereich (Gesundheitsabkommen; 176). Gegenstand und Ziel der Verhandlungen ist neben dem An- 
schluss an Früh- und Schnellwarnsysteme auch die Teilnahme am EU-Gesundheitsprogramm 2008-2013. Bedingung ist jedoch die Übernahme der vorhandenen EU-Gesetzgebung im Bereich der öffentlichen Gesundheit. Während dieser Verhandlungsteil unproblematisch ist, sind die Agrarverhandlungen stark umstritten, so dass mittelfristig mit keinem erfolgreichen Verhandlungsabschluss gerechnet wird.

Beim EU-Gesundheitsprogramm 2008-2013 handelt es sich um ein zentrales Instrument zur Förderung der öffentlichen Gesundheit (177). Es bildet den Rahmen für die Finanzierung von Gesundheitsprojekten und umfasst insbesondere Bereiche wie die Gesundheitsförderung, die Gesundheitsbildung und -information oder die Prävention, inkl. Massnahmen zur Förderung einer ausgewogenen Ernährung. Eine Teilnahme am EU-Gesundheitsprogramm 2008-2013 würde es allen im Bereich der Gesundheitsförderung und Prävention tätigen Schweizer Akteuren erlauben, EU-weite Projekte einzugeben, zu leiten oder sich solchen anzuschliessen. Ein solches EU-weites Projekt ist der "European Nutrition and Health Report" (ENHR; 178; Kapitel 2.5.1).

\section{Agrarpolitik 2014-2017}

Mit der Agrarpolitik 2014-2017 will der Bundesrat die landwirtschaftliche Produktion stärken, die Umweltleistungen steigern und die bäuerlichen Einkommen verbessern (179). Kernelement ist die Weiterentwicklung des Direktzahlungssystems, dessen Wirksamkeit und Effizienz verbessert werden sollen. Die Direktzahlungen an die Landwirtschaft sollen künftig konsequent auf die von der Bevölkerung gewünschten gemeinwirtschaftlichen Leistungen ausgerichtet werden (z.B. Förderung vielfältiger
Kulturlandschaften, Förderung der Biodiversität usw.). Auf Gesetzesstufe fand die Vernehmlassung im Jahr 2011 statt; die Vernehmlassung des Verordnungspakets ist für Sommer 2013 geplant und die Inkraftsetzung für 2014.

Die Landwirtschaft bzw. die sie steuernde Agrarpolitik hat einen bedeutenden Einfluss auf Lebensmittelangebot und -preise und damit auf die Ernährung und die öffentliche Gesundheit. Umso wichtiger wäre es im Hinblick auf eine multisektorale Gesundheitspolitik, dass das Thema Gesundheit in die Agrarpolitik 20142017 einfliesst. Die Resultate der Vernehmlassung zur Agrarpolitik 2014-2017 lagen zum Zeitpunkt der Texterstellung noch nicht vor.

\section{Ernährungsforschung}

Der Forschungsbedarf im Bereich Ernährung ist gross. Der Nutzen nationaler Forschungsergebnisse für die Praxis zeigt sich auf verschiedenen Ebenen: z.B. bei der Entwicklung nationaler Ernährungsempfehlungen oder bei der Planung von Ernährungsinterventionen, die auf Gesundheitsförderung und Prävention abzielen. Internationale Forschungsdaten können aus verschiedenen Gründen nicht uneingeschränkt auf die Schweiz übertragen werden; einen Einfluss haben u.a. Unterschiede im Lebensstil (Traditionen, Religion usw.), in den Ernährungsgewohnheiten, in den verfügbaren Lebensmitteln und in den gesetzlichen und politischen Rahmenbedingungen.

Der Bundesrat hat im Frühling 2011 beschlossen, das Nationale Forschungsprogramm "Gesunde Ernährung und nachhaltige Lebensmittelproduktion» (NFP 69) zu lancieren (180). Das NFP 69 zielt u.a. darauf ab, praxisorientierte Wissensgrundlagen bereitzustellen, wie in der Schweiz eine ausgewogene Ernährung ge- 
fördert werden kann. Es ist das erste vom Schweizerischen Nationalfonds finanzierte Ernährungsforschungsprogramm und wird als entsprechend grosse Chance für den Forschungsstandort Schweiz gewertet.

\section{Politische Vorstösse auf Bundesebene}

Tabelle 4.5 dokumentiert die Vorstösse im Bundesparlament (National- und Ständerat) zwischen 1990 und Ende 2010 in den Bereichen
"Gesundheitsförderung und Prävention» (als übergreifender Bereich) und "Gesundes Körpergewicht" und stellt sie zum Vergleich den Vorstössen in den Bereichen "Alkohol» und «Tabak» gegenüber $(181 ; 182)$.

Während die traditionellen Präventionsthemen "Alkohol» und "Tabak» schon während der 1990er Jahre im Rahmen der Gesundheitspolitik diskutiert wurden, sind die Themen «Ernährung und Bewegung" sowie "Gesundheitsför-

Tabelle 4.5: Auswertung von parlamentarischen Vorstössen zwischen 1990 und 2010 (183)

\begin{tabular}{|c|c|c|c|c|c|}
\hline Jahr / Legislatur & $\begin{array}{l}\text { Gesundheits- } \\
\text { förderung / } \\
\text { Prävention }^{\text {a }}\end{array}$ & $\begin{array}{l}\text { Gesundes } \\
\text { Körpergewicht }\end{array}$ & Alkohol & Tabak & Total \\
\hline 1990-1999 & 8 & 13 & - & - & 21 \\
\hline 2000 & 0 & 0 & 2 & 3 & 5 \\
\hline 2001 & 0 & 0 & 4 & 2 & 6 \\
\hline 2002 & 0 & 2 & 4 & 5 & 11 \\
\hline 2003 & 0 & 2 & 2 & 4 & 8 \\
\hline $\begin{array}{l}\text { 46. Legislatur } \\
\text { (2000-2003) }\end{array}$ & 0 & 4 & 12 & 14 & 30 \\
\hline 2004 & 4 & 6 & 5 & 4 & 19 \\
\hline 2005 & 6 & 6 & 7 & 5 & 24 \\
\hline 2006 & 4 & 6 & 5 & 5 & 20 \\
\hline 2007 & 5 & 4 & 4 & 1 & 14 \\
\hline $\begin{array}{l}\text { 47. Legislatur } \\
\text { (2004-2007) }\end{array}$ & 19 & 22 & 21 & 15 & 77 \\
\hline 2008 & 8 & 3 & 4 & 1 & 16 \\
\hline 2009 & 6 & 1 & 0 & 0 & 7 \\
\hline 2010 & 3 & 0 & 3 & 0 & 6 \\
\hline Total 2000-2010 & 36 & 30 & 40 & 30 & 136 \\
\hline
\end{tabular}

- = keine Daten vorhanden

a In der Spalte "Gesundheitsförderung / Prävention» sind alle Vorstösse aufgeführt, die Gesundheitsförderung und Prävention allgemein thematisieren. Vorstösse zu den Themen "Gesundes Körpergewicht", "Alkohol» oder "Tabak» sind darin nicht eingeschlossen. 
derung" relativ jung und weniger etabliert. Die Themen "Gesundes Körpergewicht" und "Gesundheitsförderung und Prävention» haben auf der politischen Agenda erst in den vergangenen Jahren allmählich an Bedeutung und Aufmerksamkeit gewonnen (Tabelle 4.5). Im Übergang von der 46. zur 47. Legislatur versechsfachten sich diesbezügliche Vorstösse. Die Abnahme der letzten Jahre hängt sicherlich mit dem Engagement für ein künftiges Präventionsgesetz zusammen, gilt es doch, die daraus resultierenden Massnahmen abzuwarten.

Die Vorstösse seitens der Parlamentarier zu "Ernährung und Bewegung» sowie "Gesundheitsförderung" hatten in der Vergangenheit meist Kontroll- und Auskunftscharakter (Anfragen, Interpellationen) und wurden nicht in ihrer Funktion als Gesetzgeber (Motionen, Postulate und Initiativen) eingereicht. Regulierungen im Bereich von Werbung und Marketing oder fiskalische Massnahmen wie die Einführung einer Lenkungssteuer waren in der Bundespolitik nicht mehrheitsfähig; entsprechende Vorstösse wurden abgelehnt oder abgeschrieben. Diese Zurückhaltung gegenüber Massnahmen, welche eng mit dem individuellen Verhalten und ökonomischen Interessen verbunden sind, ist charakteristisch für das grundsätzlich liberal geprägte Schweizer Gesundheitssystem; Eingriffe in die alltäglichen Gewohnheiten des Einzelnen und in die Freiheiten der Wirtschaft sind nur schwer durchsetzbar.

\subsubsection{Aktivitäten auf kantonaler Ebene}

Zurzeit gibt es keine aktuelle, vollständige Übersicht darüber, in welchen Kantonen welche Projekte zu Gesundheitsförderung und Prävention laufen und welche finanziellen Ressourcen hierfür aufgewendet werden. Bekannt ist jedoch, dass sich die Kantone im Rahmen der KAP auf Projekte zu den Themen Ernährung und Bewegung konzentrieren (variierende Zielgruppen je nach Kanton). Dieser Erfolg lässt sich auch auf das Engagement von Gesundheitsförderung Schweiz zurückführen (Kapitel 4.6.1.2).

Die kantonalen Gesundheitsgesetze sind besonders interessant, weil sie in beinahe allen Kantonen in jüngster Zeit überarbeitet wurden oder eine Überarbeitung läuft bzw. zumindest geplant ist (Tabelle 4.6). In den älteren Gesetzen wurden der Gesundheitsförderung und Prävention wenig oder gar keine Bedeutung geschenkt. Die Rede war - falls überhaupt - meist von Vorsorge; die Gesetzgebung beschränkte sich auf Massnahmen zur Verhinderung übertragbarer Krankheiten.

Heute kommt der Gesundheitsförderung und Prävention wesentlich höhere Bedeutung zu: Die beiden Begriffe werden breiter definiert, in den neueren Gesundheitsgesetzen ist ihnen meistens ein ganzer, eigener Abschnitt gewidmet, und teilweise nennen einzelne Gesetzesartikel gar spezifische Themenfelder der Gesundheitsförderung und Prävention (182).

Tabelle 4.6 gibt einen zusammenfassenden Überblick über den Stand und die Dynamik der Gesundheitsgesetze bzw. der Revisionsprozesse in den Kantonen. 
Tabelle 4.6: Gesundheitsgesetze in den Kantonen, Stand Oktober 2011 (182)

\begin{tabular}{|c|c|c|c|}
\hline Kanton & Inkraftsetzung & $\begin{array}{c}\text { Regelungen zu Gesundheitsförderung } \\
\text { und Prävention }\end{array}$ & Revision \\
\hline Aargau (AG) & 2010 & enthalten & - \\
\hline Appenzell Ausserrhoden (AR) & 2008 & enthalten & - \\
\hline Appenzell Innerrhoden (Al) & 1998 & enthalten & - \\
\hline Baselland (BL) & 2009 & enthalten in eigenem Abschnitt & - \\
\hline Basel-Stadt (BS) & 2012 & enthalten in eigenem Abschnitt & - \\
\hline Bern (BE) & 2011 & enthalten in eigenem Bereich & - \\
\hline Freiburg (FR) & 1999 & enthalten & - \\
\hline Genf (GE) & 2006 & enthalten in eigenem Abschnitt & - \\
\hline Glarus (GL) & 2007 & enthalten in eigenem Abschnitt & - \\
\hline Graubünden (GR) & 2005 & enthalten in eigenem Abschnitt & - \\
\hline Jura (JU) & 1990 & enthalten & - \\
\hline Luzern (LU) & 2005 & enthalten in eigenem Abschnitt & - \\
\hline Neuenburg (NE) & 1995 & enthalten in eigenem Kapitel & - \\
\hline Nidwalden (NW) & 2008 & enthalten in eigenem Abschnitt & - \\
\hline Obwalden (OW) & 1991 & in allgemeinen Zielsetzungen geregelt & geplant \\
\hline Schaffhausen (SH) & $\begin{array}{l}\text { voraussichtlich } \\
\qquad 2013\end{array}$ & im Entwurf als eignes Kapitel vorgesehen & läuft \\
\hline Schwyz (SZ) & 2004 & enthalten & - \\
\hline Solothurn (SO) & 1999 & enthalten & - \\
\hline St. Gallen (SG) & 1980 & enthalten & $\begin{array}{l}\text { in Aussicht } \\
\text { gestellt }\end{array}$ \\
\hline Tessin (TI) & 1989 & enthalten & - \\
\hline Thurgau (TG) & - & $\begin{array}{l}\text { nur in allgemeinen Gesundheitszielen betref- } \\
\text { fend öffentliche Gesundheit enthalten }\end{array}$ & geplant \\
\hline Uri (UR) & 2008 & enthalten in eigenem Abschnitt & - \\
\hline Waadt (VD) & 2009 & enthalten in eigenem Abschnitt & - \\
\hline Wallis (VS) & 2008 & enthalten in eigenem Abschnitt & - \\
\hline Zug (ZG) & 2008 & enthalten in eigenem Abschnitt & - \\
\hline Zürich (ZH) & 2008 & enthalten in eigenem Abschnitt & - \\
\hline
\end{tabular}




\subsubsection{Aktivitäten im Bildungsbereich}

Die Schulen als Bildungsinstitutionen und insofern die dafür hauptverantwortlichen 26 kantonalen Bildungsdepartemente können in vielerlei Hinsicht einen Beitrag zu Gesundheitsförderung und Prävention leisten (Kapitel 4.5.3).

Zurzeit existieren in der Schweizer Volksschule 26 Lehrpläne. Die Ernährungs- und Bewegungserziehung ist in den Lehrplänen je nach Kanton an unterschiedlichen Stellen verankert: im Sachunterricht (je nach Kanton: Natur Mensch Mitwelt NMM, Mensch und Umwelt, Realien), im Hauswirtschaftsunterricht oder als fächerübergreifende Thematik (184). Diese Ausgangslage ist aber im Wandel begriffen.

\section{HarmoS-Konkordat}

Am 1. August 2009 ist die «Interkantonale Vereinbarung über die Harmonisierung der obligatorischen Schule vom 14. Juni 2007» (HarmoSKonkordat) in Kraft getreten. Sie harmonisiert die wichtigsten strukturellen Eckwerte (Schuleintrittsalter, Schulpflicht, Dauer der Bildungsstufen) und die Bildungsziele der obligatorischen Schule (79; Kapitel 4.5.3). Die übergeordneten Ziele der obligatorischen Schule werden im HarmoS-Konkordat als Bereiche der Grundbildung aufgeführt. Das Thema Bewegung und Gesundheit stellt einen der fünf Bildungsbereiche dar. Ein aus Präventionssicht weiterer wichtiger Aspekt ist schliesslich, dass das HarmoSKonkordat die Schaffung eines bedarfsgerechten Angebotes für die Betreuung der Schüler ausserhalb der Unterrichtszeit verlangt (so genannte Tagesstrukturen), wobei die Nutzung dieses Angebotes freiwillig und in der Regel kostenpflichtig ist (Kapitel 4.5.3).
Über den Beitritt zum HarmoS-Konkordat entscheidet jeder Kanton einzeln. Bisher sind dem HarmoS-Konkordat 15 Kantone beigetreten (Stand Oktober 2011); sie repräsentieren zusammen 76.3\% der Wohnbevölkerung. Bei 4 Kantonen ist der Entscheid noch ausstehend (79).

\section{Lehrpläne}

Mit den beiden sprachregionalen Lehrplänen für die Deutsch- und Westschweiz wird durch die kantonalen Erziehungsdirektoren eine neue Lehrplangeneration für die obligatorische Schulzeit erarbeitet (185). Diese Lehrpläne orientieren sich an den Bildungsbereichen des HarmoSKonkordates und verfolgen nebst einer Harmonisierung der Lehrpläne in der Schweiz u.a. auch die Verankerung von Gesundheitsthemen im Unterricht auf allen Stufen. Beide Lehrplan-Projekte bieten das Potenzial, Ernährungsbildung harmonisiert, effizient und nachhaltig im Unterricht zu verankern und einen wichtigen Beitrag zu mehr Chancengerechtigkeit zu leisten.

Der Lehrplan 21 mit Einführungsbeginn im Jahr 2014 setzt das HarmoS-Konkordat in den Deutschschweizer Kantonen in Form von Fachbereichen sowie überfachlichen Themen um (186). Da viele Bildungsanliegen nicht einem einzelnen Fachbereich entsprechen und der Fächerkanon nicht beliebig ausgeweitet werden kann, hat der Lehrplan 21 die drei überfachlichen Themen Berufsvorbereitung, Umgang mit Informationstechnik und Medien sowie Bildung für Nachhaltige Entwicklung (BNE) definiert (187). In Letzterem ist auch der Bereich Ernährung angesiedelt (neben weiteren Themen wie Gesundheit, Umwelt usw.). Wissen und Kompetenzen sollen die Schüler befähigen, komplexe Zusammenhänge und Sachverhalte zu reflektieren; die Ernährung eignet sich durch ihre vielfältigen globalen, saisonalen 
und sozialen Aspekte sowie ihre persönlichen Bezüge bestens dazu. Ab Anfang 2013 ist eine Konsultation der Lehrplanvorlage geplant. In dieser wird die Zuordnung von BNE zu den einzelnen Fachbereichen ersichtlich sein (188).

In der Westschweiz und im Tessin ist der gemeinsame Lehrplan bereits einen Schritt weiter; der Plan d'études romand (PER) wurde im August 2009 von allen Kantonen der Westschweiz und dem Kanton Tessin verabschiedet (189). Im PER gehören Gesundheit und Wohlbefinden als eines von fünf Elementen zum Bildungsinhalt des neuen Fachs "Formation Générale».

\subsection{Erfolgsfaktoren}

\subsubsection{Theorie und Praxis}

Erfolgsfaktoren sind Eigenschaften einer Intervention, die dazu beitragen, dass eine Intervention eine beabsichtigte Wirkung zeigt, indem sie eine hohe Akzeptanz geniesst, die Adressaten sensibilisiert und bestenfalls eine nachhaltige Veränderung des Verhaltens und / oder der Verhältnisse zur Folge hat. Die Wirkung ernährungsbezogener Massnahmen zu beurteilen ist aufgrund der Komplexität des Ursache-Wirkungs-Prinzips allerdings ein schwieriges Unterfangen (Kapitel 4.2). Oftmals sind nur Aussagen über Resultate in Teilbereichen zulässig, z.B. über die Zielgruppenerreichung, die Integration in den sozialen und strukturellen Kontext oder die langfristige Verankerung der Massnahme.

Hinzu kommt, dass sich die wissenschaftliche Literatur bisher grösstenteils darauf konzentriert hat zu beschreiben, was gemacht werden soll, statt sich auch damit zu beschäftigen, wie etwas gemacht werden soll, um erfolgreich zu sein. Im Bereich der Adipositasprävention ist die Forschung verhältnismässig am weitesten fortgeschritten; der Rahmen für Best-PracticePrinzipien umfasst in diesem Bereich: Engagement der Gemeinde, Programmdesign und -planung, Evaluation, Implementierung und Nachhaltigkeit sowie verantwortungsvolle Führung und Transparenz (13).

Modelle können helfen, Zusammenhänge und Wirkungsweisen von Interventionen in der Gesundheitsförderung und Prävention nachvollziehbar zu machen. Ein solches Modell ist das Ergebnismodell von Gesundheitsförderung Schweiz (190). Es zeigt u.a., dass sich die Gesundheit der Bevölkerung nur mit Interventionen beeinflussen lässt, welche auf allen Ebenen (Verhaltens- und Verhältnisebene; nationale, kantonale, kommunale Ebene) und in allen Sektoren (Politik, Wirtschaft, Bildung usw.) angesiedelt sind. In der Regel lassen sich Massnahmen nicht nur auf der einen oder anderen Ebene umsetzen, da sie sich gegenseitig beeinflussen.

Interventionen - ob auf der Verhaltens- oder Verhältnisebene - müssen ferner verschiedenste Kontexte berücksichtigen: den institutionellen, lokalen, regionalen, nationalen und internationalen Kontext auf der Policy-Ebene, der gesetzlichen Ebene sowie der sozio-ökonomischen und soziokulturellen Ebene (191). Die Praxis zeigt, dass einzelne isolierte Massnahmen diesen komplexen Anforderungen nicht gerecht werden können. In der Schweiz werden aufgrund der ausgeprägt föderalistischen Struktur und der grossen regionalen Heterogenität deshalb zahlreiche kleinere, aber wenig flächendeckende und umfassende Massnahmen umgesetzt. Bei der Beurteilung der Wirksamkeit von Massnahmen ist der Kontext immer zu berücksichtigen. 


\subsubsection{Ernährungsinterventionen in der Schweiz: Erfolgsfaktoren}

Kapitel 4.6 beschreibt die in der Schweiz laufenden Programme und Projekte zur Förderung einer ausgewogenen Ernährung und ausreichend körperlicher Aktivität auf der Wissensund Verhaltensebene sowie zur Verbesserung der diesbezüglichen Rahmenbedingungen. Angesichts ihrer bisher relativ kurzen Interventionsdauer, der grossen Heterogenität und der im Kapitel 4.8.1 erwähnten methodischen Einschränkungen ist es (noch) nicht möglich nachzuweisen, dass die beschriebenen, individuellen Massnahmen eine nachhaltige gesundheitsfördernde Verhaltensänderung zur Folge haben und / oder sich direkt auf die Inzidenz und Prävalenz von Krankheiten und ihre Risikofaktoren auswirken.

Als Beispiel sei in diesem Zusammenhang auf die mögliche Stabilisierung der Prävalenz von Übergewicht und Adipositas bei Kindern in der Schweiz hingewiesen (Kapitel 3.3.1). Diese kann als Teilerfolg gewertet werden - auch wenn noch nicht feststeht, ob es sich nur um einen vorübergehenden oder um einen nachhaltigen Trend handelt. Ob dieser Teilerfolg allerdings auf einzelne oder die Summe aller Präventionsmassnahmen oder auch auf völlig andere Einflussfaktoren zurückzuführen ist, kann nicht abschliessend beurteilt werden. Eine weitere Hypothese besteht darin, dass eine natürliche Sättigung in der Steigerung der Prävalenzrate von Übergewicht und Adipositas bei Kindern eingetreten ist.

Aus diesen Gründen lassen sich aus den bisher in der Schweiz durchgeführten Interventionen keine allgemein gültigen Erfolgsfaktoren ableiten. Aus den verschiedenen Programmen und Projekten können aber einzelne Erfolgsfaktoren abgeleitet werden, die allerdings immer kontextbezogen sind und somit von der Zielsetzung der Intervention, von den Zielgruppen, vom Umfeld usw. abhängen. Nachfolgend werden eine Auswahl von Erfolgsfaktoren dargelegt.

Generell ist der Erfolg von Interventionen abhängig von der Art und vom Grad der Zielgruppenerreichung. Der Einbezug von Schlüsselpersonen bzw. -institutionen in die Planung, Umsetzung und Evaluation von Interventionen (Partizipation) ist deshalb ein wichtiger Erfolgsfaktor (192). Schlüsselpersonen bzw. -institutionen variieren je nach Zielgruppe der Intervention:

- Bei Interventionen, die auf Personen mit Migrationshintergrund abzielen, haben sich interkulturelle Vermittler als Schlüsselpersonen (163) und Ausländervereine als Schlüsselinstitutionen (192) erwiesen.

- Bei Interventionen, die auf Kleinkinder abzielen, gilt der institutionelle Zugang, z.B. über Mütter-Väterberatungsstellen oder familienergänzende Betreuungseinrichtungen, als Erfolgsfaktor $(83 ; 125 ; 126 ; 131 ; 143 ; 146 ; 148 ; 152$; 162;165). Die Ballabeina-Studie, eine wissenschaftlich begleitete Intervention zur Verhaltensänderung bei Kindern mit Migrationshintergrund im Vorschulalter, fand im Setting von Betreuungseinrichtungen für Kleinkinder statt und wählte damit einen solchen institutionellen Ansatz (193). Bei Kleinkindern gelten zudem deren Eltern als Schlüsselpersonen (194), u.a. weil sie für Empfehlungen zu Gesundheitsthemen besonders empfänglich sind (Kapitel 4.5.3). Multisektorale Interventionen mit Erziehungs- und Umfeldorientierung, die im 
frühesten Kindesalter ansetzen und von langer Laufdauer sind, scheinen generell beachtlichen Erfolg zu haben $(131 ; 141 ; 146)$.

- Bei Interventionen, die auf Kinder der Primarschulstufe abzielen, hat sich die Schule als Absender von Ernährungsempfehlungen bewährt (81).

Das Setting Schule, insbesondere die Schule mit Ganztagesstrukturen, scheint ein idealer Ort für Interventionen zu sein (Kapitel 4.8.3). Über die Schule können einerseits Kinder aller sozialen Schichten und ihre Bezugspersonen (Lehrpersonen, Eltern) erreicht werden. Andererseits kann im Setting Schule auch auf und über das Angebot (Schulkantinen, Schulfrühstück, Pausenverpflegung, Automaten, Verpflegungsanbieter im schulnahen Umfeld) Einfluss genommen werden. Im besten Fall werden Kinder und Jugendliche während der ganzen obligatorischen Schulzeit von geeigneten Interventionen begleitet, damit die Prinzipien einer ausgewogenen Ernährung nachhaltig auf der Wissens- und Verhaltensebene verankert werden können (195). Allerdings ist darauf zu achten, das System Schule und seine Multiplikatoren (Lehrpersonen) nicht mit komplexen Präventionsmassnahmen in verschiedenen Gesundheitsbereichen gleichzeitig zu belasten. Hier setzt «bildung + gesundheit Netzwerk Schweiz" an, welches sich für die Gesundheitsförderung und Prävention im schulischen Kontext einsetzt und das Thema Gesundheit über seine Mitgliederorganisationen ganzheitlich in die Schule trägt (Kapitel 4.6.1.3).

Ein weiterer Erfolgsfaktor ernährungsbezogener Interventionen dürfte die Berücksichtigung und der Einbezug des Umfeldes bzw. der Lebenswelten der Zielgruppe sein (Kapitel 4.5.2 und 4.5.3). Als Beispiel sei hier die Zielgruppe der Jugendlichen der Sekundarstufe genannt. Bei dieser Zielgruppe hat sich gezeigt, dass Ernährungsbotschaften besonders dann auf Interesse stossen, wenn sie auf ihre geschlechtsspezifischen Lebenswelten hin angepasst und in Verbindung mit anderen Themen wie Freizeitverhalten, Konsum, Nachhaltigkeit usw. vermittelt werden (196). Die Erfahrungen des Schweizerischen Netzwerkes Gesundheitsfördernder Schulen (SNGS) bestätigen dies (Kapitel 4.6.1.3).

Konkrete Erfolgsfaktoren konnten u.a. auch aus dem Projekt Miges Balù (z.B. breit abgestützte Trägerschaft mit verschiedenen Organisationen, Vernetzung mit anderen Projekten; 192), aus dem Projekt Senso5 (z.B. Integration schlüsselfertiger Aktivitäten in den laufenden Lehrplan, Motivation der Lehrpersonen, Interesse und Unterstützung der Eltern, Freude der Kinder; 197) und aus den Ernährungsrichtlinien der Stadt Zürich (z.B. Vorbildfunktion von Erwachsenen, Unterstützung der Eltern, genügend finanzielle Ressourcen; 155) abgeleitet werden.

\subsubsection{Ernährungsinterventionen in Europa: Evidenz und Erfolgsfaktoren}

\section{Setting Schule}

Obschon das Setting Schule als idealer Ort für Interventionen gilt, ist die Evidenz zur Wirksamkeit von Interventionen zwecks Förderung einer ausgewogenen Ernährung und / oder Prävention von Übergewicht in diesem Setting relativ schwach und variiert je nach Interventionsmethode stark $(91 ; 194 ; 198-202)$. Überzeugend ist 
die Evidenz bei Multikomponenten-Interventionen (203) und insbesondere bei solchen zur Förderung des Früchte- und Gemüsekonsums, die auf Kinder abzielen und Ernährungsbildung im Unterricht mit einer erhöhten Verfügbarkeit von Früchten und Gemüse vor Ort kombinieren (194). Als vielversprechend im Jugendalter erweisen sich computerbasierte und personalisierte Ansätze der Ernährungsbildung (198).

Zur Prävention von Übergewicht sind Interventionen mit Erziehungs- und Umfeldorientierung, die sowohl das Thema Ernährung als auch das Thema Bewegung aufgreifen (198), sowie Interventionen mit längerer Laufdauer (204) erfolgversprechend.

Abschliessend muss festgehalten werden, dass Interventionen im Setting Schule nur dann wirksam sein können, wenn sie in einem übergeordneten Programm verankert sind (Einbezug der Familie, Gemeinde usw.; 91).

\section{Schulfruchtprogramme in Schulen}

Die EU stellt seit Herbst 2009 Mittel für Schulfruchtprogramme zur Förderung des Früchteund Gemüsekonsums in Schulen zur Verfügung. Die Finanzierung erfolgt über die Gemeinsame Agrarpolitik; die Zusammenarbeit mit dem Gesundheitssektor ist ausgezeichnet etabliert und dokumentiert (205-207). Schulfruchtprogramme führen nachweislich zu einer Erhöhung des Ernährungswissens und zu einer nachhaltigen Steigerung des Früchte- und Gemüsekonsums (194;208;209).

Deutschland hat im Jahr 2009 erstmals Erfolgsfaktoren benannt und Standards gesetzt, an denen sich zukünftige Schulfruchtprogramme orientieren können (208): u.a. kostenlose Abgabe der Früchte an alle, vielfältiges und täglich wechselndes Früchteangebot hoher Quali- tät (Genussreife), verlässliche Logistik, tägliche Belieferung der Schulen.

\section{Medienkompetenz}

Erfolgversprechend sind weiter auch Interventionen, die Kompetenzen im Umgang mit Medien fördern, zum Verständnis von Informationen beitragen und das Erkennen von Werbebotschaften unterstützen $(91 ; 210)$. Dies nicht zuletzt deshalb, weil energiedichte Lebensmittel und Süssgetränke in der Werbung omnipräsent sind (Kapitel 4.5.2).

\section{Europäische Programme zur Förderung einer} ausgewogenen Ernährung

Aus den nationalen Programmen zur Förderung einer ausgewogenen Ernährung, die gegenwärtig in Europa laufen, können weitere wertvolle Erkenntnisse gewonnen werden, die mit gewissen Einschränkungen auch auf die Schweiz übertragen werden können (Einschränkungen z.B. aufgrund des föderalistischen Systems der Schweiz, Kapitel 4.3).

In Frankreich läuft bereits die dritte Phase 2011-2015 des "Programme National Nutrition Santé» (PNNS; 211), die auf den Evaluationsergebnissen der ersten beiden Programmphasen 2001-2005 und 2006-2010 basiert (212;213). Die Hauptziele von PNNS 2006-2010 wurden im Jahr 2004 gesetzlich verankert (214). Die Komplementarität und die Synergien der Kommunikationsaktionen (z.B. Broschüren, Logo, Auszeichnungen, Medienkampagnen, Ernährungsprinzipien; Abbildung 1.5) werden als Erfolgsfaktoren des PNNS erachtet. Sie ermöglichten einen Referenzrahmen festzulegen, der u.a. mit Aktionen im Bereich Lebensmittelangebot (Charta für das Engagement der Unternehmen zur Verbesserung der ernährungsphysiologischen Qualität 
von Lebensmitteln usw.) und mit lokalen Aktionen (Charta für lokale und regionale Betriebe) ergänzt wurde (215).

Die nationale Kampagne "Change4Life" in England zur Förderung einer ausgewogenen Ernährung und genügend Bewegung wurde Anfang 2009 in der Öffentlichkeit lanciert (216). Zur Kampagne existiert eine umfassende Evaluation, in der konkrete Erfolgsfaktoren genannt sind (217): u.a. die Einbettung der Kampagne in eine übergeordnete nationale Strategie oder die Nutzung bestehender Netzwerke.

Der nationale Aktionsplan "IN FORM Deutschlands Initiative für gesunde Ernährung und mehr Bewegung" zielt darauf ab, das Ernährungs- und Bewegungsverhalten in Deutschland nachhaltig zu verbessern, und wirkt sowohl auf der Verhaltens- als auch auf der Verhältnisebene (218). Der Aktionsplan ist u.a. aufgrund seiner langfristigen Perspektive von besonderem Interesse: Er setzt sich zum Ziel, bis 2020 sichtbare Ergebnisse zu erreichen. Als besonders erfolgreich zur Erreichung der Zielgruppen gelten u.a. die Qualitätsstandards, die von der Wissenschaft und Praxis gemeinsam für alle Bereiche der institutionellen Ausser-Haus-Verpflegung erarbeitet wurden, und die unterstützenden und begleitenden Massnahmen zur Verbreitung dieser Standards (wie z.B. die Einrichtung von so genannten "Schulvernetzungsstellen»; 219). Den gleichen Ansatz verfolgen die "Schweizer Qualitätsstandards für eine gesundheitsfördernde Gemeinschaftsgastronomie» (Tabelle 4.2). Der Erfolg von «IN FORM» liegt darin, dass es sehr viele Initiativen auf verschiedenen Ebenen mit unterschiedlichen Akteuren gibt und dass "IN FORM» ein Dach und Strukturen für die verschiedenen Akteure und Initiativen schafft (220).

\subsection{Handlungsempfehlungen für die Schweiz}

\subsubsection{Datenlücken schliessen}

Sowohl Kapitel 2 als auch Kapitel 3 weisen mehrfach auf die unzureichende und unsichere Datenlage im Bereich der Ernährung und $\mathrm{Ge}$ sundheit in der Schweiz hin. Mit dem Projekt MOSEB wurde zwar der Grundstein gelegt, um die Datenlage systematisch zu verbessern (Kapitel 4.6.1.1). Es fehlen aber nach wie vor qualitativ hochwertige und repräsentative Daten zum Lebensmittelverzehr und Ernährungsverhalten in der Schweiz sowie Schweizer Daten zum Einfluss der Ernährung auf Krankheiten und ihre Risikofaktoren.

Diese Daten sind nötig, um Massnahmen zur Förderung einer ausgewogenen Ernährung planen, umsetzen und schliesslich überprüfen zu können. Bei qualitativ und quantitativ unzureichender Datenlage kann die Wirksamkeit von Massnahmen nicht gemessen werden, was wiederum die Weiterentwicklung der Massnahmen erschwert.

Die Verbesserung der Datenlage liegt im Interesse und in der Verantwortung vieler Akteure und ist deshalb eine gesamtgesellschaftliche Aufgabe, die entsprechende finanzielle und personelle Ressourcen, die Berücksichtigung internationaler Erkenntnisse und geeignete Forschungs- und Messmethoden voraussetzt. 


\subsubsection{Ernährungskommunikation auf vulnerable Zielgruppen ausrichten}

Kapitel 1 zeigt auf, dass in der Schweiz offizielle Ernährungsempfehlungen für nahezu alle Altersgruppen bzw. Lebensphasen vorhanden sind. Lücken bestehen hingegen im Bereich der Ernährungskommunikation, die sich an spezifische Bevölkerungsgruppen mit unterdurchschnittlichem Ernährungsbewusstsein und -wissen und damit mit geringen Ernährungskompetenzen richten. Dazu zählen besonders Männer, junge Menschen und sozio-ökonomisch benachteiligte Menschen (z.B. mit Migrationshintergrund, geringem Einkommen, tiefem Bildungsniveau usw.; Kapitel 2.6.6). Diese Zielgruppen sind von besonderer Bedeutung, weil sie gleichzeitig die Bevölkerungsgruppen darstellen, die ernährungsabhängigen Krankheitsrisiken am stärksten ausgesetzt sind. Das Körpergewicht von Kindern steht beispielsweise in einem direkten Zusammenhang zum Bildungsniveau ihrer Eltern (Kapitel 3.3.2), und die Prävalenz von Übergewicht und Adipositas bei Kindern ist mit ihrer Nationalität assoziiert (Abbildung 3.2). Diese vulnerablen Bevölkerungsgruppen (Männer, junge Menschen, sozio-ökonomisch benachteiligte Menschen) sind nicht nur im Rahmen zukünftiger Kommunikationsbestrebungen von Bedeutung, sondern generell bei der Entwicklung und Ausrichtung künftiger Interventionen.

Bei der zielgruppenspezifischen Ernährungskommunikation ist nicht nur der Inhalt der vermittelten Ernährungsbotschaften relevant, sondern vor allem auch die Art der Vermittlung. Ein gutes Verständnis der Adressaten, ihres Lebensstils und Ernährungsverhaltens sowie ihres
Umfeldes und ihrer Lebenswelten ist deshalb die wichtigste Voraussetzung.

Den Nährwert- und Front-of-Pack-Kennzeichnungen von Lebensmitteln sind in diesem Zusammenhang besondere Beachtung zu schenken. Die heutigen Kennzeichnungssysteme in der Schweiz sind schwierig zu verstehen und werden im Prozess der Kaufentscheidung verhältnismässig wenig genutzt (Kapitel 2.6.4). Eine einheitliche und verständliche Nährwertund/oder Front-of-Pack-Kennzeichnung kann zur Orientierung im Lebensmittelangebot und zur gesunden Wahl beim Kaufentscheid beitragen. Gerade Menschen mit geringen Ernährungskompetenzen könnten wesentlich profitieren.

\subsubsection{Familienergänzende Betreuungseinrichtungen und Schulen stärken}

Die frühe Bildung und Prägung durch Elternhaus, Schule und Gemeinschaft sind für die Entwicklung von Gesundheits- und Ernährungskompetenzen und für die Förderung eines gesunden Ernährungsverhaltens wesentlich (Kapitel 4.5.1 und 4.5.3).

Familienergänzende Betreuungseinrichtungen (z.B. Spielgruppen, Kinderkrippen), Kindergärten und Schulen sind deshalb von besonderer Bedeutung. Sie können und sollen einerseits den Grundstein für eine altersgerechte und nachhaltige Ernährungsbildung legen und andererseits gesundheitsfördernde Rahmenbedingungen schaffen. Dies bedingt allerdings eine Stärkung ihrer Stellung und Vermittlerfunktion mit finanziellen und personellen Ressourcen. Bildungsinvestitionen sind immer auch Investitionen in die Gesundheit. 


\subsubsection{Potenzial föderalistischer Strukturen nutzen}

Top-Down-Strategien laufen in der Schweiz Gefahr, am Widerstand der Kantone oder der Interessenverbände zu scheitern. Die Zusammenarbeit nationaler, kantonaler und kommunaler Behörden sowie weiterer Akteure ist unabdingbar, setzt jedoch eine nicht zu unterschätzende Koordination und Rollenklärung voraus. Gesetzliche Grundlagen wie das Präventionsgesetz (Kapitel 4.7.1) könnten einen wichtigen Beitrag leisten, indem darin die Zuständigkeiten und Verantwortlichkeiten von Bund und Kantonen geregelt werden.

Eine besondere Rolle kommt den fast 2600 Gemeinden in der Schweiz zu. Sie sind zuständig für zahlreiche budgetrelevante Entscheidungen, welche einen unmittelbaren Einfluss auf die Verhältnisebene haben: z.B. betreffend Kindergärten und Schulen, Rahmenbedingungen für Verpflegungsangebote, raumplanerische Massnahmen usw. Die lokalen Strukturen in Gemeinden bieten den verschiedenen Stakeholdern zudem gute Vernetzungsmöglichkeiten und können einen wichtigen Beitrag zum Gelingen von Interventionen leisten. Internationale Studien zeigen, dass Programme mit langer Laufdauer, die Interventionen in Schule und Gemeinde kombinieren, vielversprechende Erfolge im Bereich der Prävention von Übergewicht erzielen (91;204).

\subsubsection{Politik mobilisieren}

Die multisektorale Gesundheitspolitik verlangt, dass Gesundheitsförderung und Prävention in allen Politikbereichen berücksichtigt werden (Kapitel 4.3). Im Hinblick auf eine ausgewogene Ernährung stehen neben der Gesundheitspolitik v.a. die Agrar- und die Bildungspolitik in der Pflicht.

Die Agrarpolitik hat einen bedeutenden Einfluss auf die verfügbaren Lebensmittel und insofern auf die Preisbildung. Konkrete Massnahmen erfordern allerdings nicht nur politischen Willen, sondern auch die Zusammenarbeit zwischen Produzenten, Industrie und Handel.

Die Bildungspolitik kann auf verschiedenen Ebenen einen Beitrag zu Gesundheitsförderung und Prävention leisten (Kapitel 4.5.3 und 4.7.3). Die Entwicklungen der letzten Jahre sind positiv zu werten (HarmoS-Konkordat, sprachregionale Lehrpläne) und sollten aus gesamtgesellschaftlicher Sicht weitergeführt werden.

Gesetzgebungsverfahren in der Schweiz stehen in einer engen Abhängigkeit von der EUGesetzgebung. Die Kohärenz innerhalb der Schweizer Gesetzgebung (z.B. im Hinblick auf den Gesundheitsschutz der Bevölkerung, Kapitel 4.3.1) sollte dabei allerdings nicht untergraben werden. Bei zukünftigen Gesetzesrevisionen sollte die Gelegenheit, Voraussetzungen für ein Umfeld zu schaffen, welches eine ausgewogene Ernährung fördert, wahrgenommen werden (Kapitel 4.7.1).

Im Hinblick auf die Prävention von Übergewicht und Adipositas gelten Massnahmen auf der Policy-Ebene generell als kosteneffizient (11). Zur Umkehr der globalen Adipositasepidemie gilt der Staat als wichtigster Akteur - dies nicht zuletzt aufgrund seiner gesetzlich veran- 
kerten Aufgabe, Massnahmen zum Schutz der Gesundheit zu ergreifen (25; Kapitel 4.3.1). Allerdings trägt der Staat nicht die alleinige Verantwortung; die Umkehr der Adipositasepidemie wird nur im Verbund mit allen beteiligten Akteuren gelingen (Wirtschaft, NGOs usw.). Nicht zuletzt ist auch Eigenverantwortung jedes Einzelnen gefragt.

\subsubsection{Privatwirtschaft mobilisieren}

Die Ausser-Haus-Verpflegung, die Schnellverpflegung und die Verwendung von ConvenienceProdukten und Fertigmahlzeiten zu Hause haben in der Schweiz an Bedeutung gewonnen (Kapitel 2.6.5 und 4.5.2) und bieten somit grosses Potenzial für eine gesundheitsfördernde Ernährung. Dass dieses Potenzial noch nicht ausgeschöpft ist, belegt nicht zuletzt eine Schweizer Studie, nach der fehlendes GastroAngebot der am dritthäufigsten genannte Hinderungsgrund für eine ausgewogene Ernährung ist (Kapitel 2.6.2). Angesprochen sind einerseits Lebensmittelindustrie und -handel, Gastronomie und ihre Lieferanten, andererseits aber auch alle Entscheidungsträger, die dazu beitragen können, dass die Verpflegung in öffentlichen und privaten Institutionen gesundheitlichen Kriterien genügt. Dabei gilt, die lebensmitteltechnologische Machbarkeit und die sensorischen Eigenschaften der Angebote zu berücksichtigen.

Im Rahmen von "actionsanté» sollen die Akteure der Privatwirtschaft weiterhin zu freiwilligen Massnahmen zur Förderung einer ausgewogenen Ernährung motiviert werden, sei dies z.B. bei der Anpassung von Portionengrössen oder bei der Überprüfung der Lebensmittelrezepturen. Darüber hinaus sollten sich Akteure der Privatwirtschaft verstärkt in den Bereichen der Konsumenteninformation sowie des Marketings und der Werbung verpflichten, die bei den Aktionsversprechen im Rahmen von "actionsanté" bisher untervertreten sind (Kapitel 4.6.1.1).

In diesem Zusammenhang sei auf die Selbstkontrolle durch die Lebensmittelindustrie zur Beschränkung der Vermarktung von Lebensmitteln und nichtalkoholischen Getränken mit einem hohen Gehalt an Fett, Zucker oder Salz, die sich an Kinder richten, hingewiesen. Diese hat sich auf internationaler Ebene als wenig wirksam erwiesen (221;222). Die WHO hat deshalb im Anschluss an die WHA-Konferenz vom Mai 2010 Empfehlungen formuliert, die über die Selbstkontrolle hinausgehen (223). Ein gangbarer Weg wäre beispielsweise, dass die Vermarktung von Lebensmitteln an die Bedingung geknüpft wäre, dass diese einem vorgegebenen Nährwertprofil («nutrient profile») entsprechen. Eine andere Möglichkeit wäre z.B., eine generelle Einigung mit der Lebensmittelindustrie zu finden betreffend Lebensmittel, die beworben bzw. nicht mehr beworben werden dürfen. Dabei müssten insbesondere auch die elektronischen Medien miteinbezogen werden, nehmen sie im heutigen Freizeitverhalten doch eine zunehmende Rolle ein. Greifen die von der WHO formulierten Massnahmen nicht, sind auch gesetzlich festgelegte Richtlinien für Werbung im Fernsehen und in anderen Medien in Betracht zu ziehen. 


\subsubsection{Dringliche Massnahmen für die Schweiz}

Tabelle 4.7 stellt einige dringliche Massnahmen für die Schweiz aus Sicht der Autoren dar; sie stellt keinen Anspruch auf Vollständigkeit. Sie stützt sich u.a. auf die von der WHO genannten Elemente einer umfassenden Interventionsstrategie (54).

Bei der Umsetzung ist zu beachten, dass erst das Zusammenspiel von Interventionen auf allen Ebenen (Verhältnisebene mit Politik, Wirtschaft, Infrastruktur, Organisation, Institution usw. und Verhaltensebene mit sozialen Gruppen, Gemeinschaften, Individuen) und die Berücksichtigung aller Sektoren (insbesondere des Bildungssektors) Wirkung zeigen können. Nur eine Vielfalt von Massnahmen, die sowohl inhaltlich als auch zeitlich gut aufeinander abgestimmt sind, auf den lokalen Kontext hin angepasst wurden und sich gegenseitig begünstigen, kann eine messbare und nachhaltige Wirkung entfalten $(54 ; 229)$.

\subsection{Schlussfolgerungen und Ausblick}

Die Förderung einer ausgewogenen Ernährung bedingt die Kombination von Massnahmen auf der Verhaltens- und Verhältnisebene, die auf ihre Wirksamkeit hin überprüft wurden. Informationen zu Erfolgsfaktoren liegen jedoch nur wenige vor. Aus diesem Grund müssen Bestrebungen zur Förderung einer ausgewogenen Ernährung von einer zeitgleich umgesetzten Evaluationsstrategie begleitet werden, welche idealerweise mit der Bereitstellung von Ausgangsdaten (z.B. Daten zum Lebensmittelver- zehr, zum Ernährungsverhalten und zur Prävalenz von Krankheiten und Risikofaktoren) sowie mit der Analyse der Verhältnisebene beginnt (1). Nur so ist es möglich, die Wirkung von Ernährungsinterventionen zu messen und im positiven Fall den Nutzen aufzuzeigen bzw. im negativen Fall eine Anpassung der Intervention vorzunehmen oder sie ganz zu sistieren. Nicht zuletzt dienen Evaluationsergebnisse auch dazu, Präventionsbemühungen zu verankern und zu legitimieren. Letzteres ist insofern von Bedeutung, als davon auszugehen ist, dass die öffentlichen Diskussionen um die KostenNutzen-Frage sowie um das richtige Mass an staatlichen Interventionen im Ernährungs- und Gesundheitsbereich, das Recht auf Selbstbestimmung und die Übernahme von Selbstverantwortung in nächster Zukunft weitergeführt werden.

Die Planung, Umsetzung und Evaluation von Interventionen zur Förderung einer ausgewogenen Ernährung auf der Verhaltens- und Verhältnisebene sollten zudem vermehrt an Erfahrungen aus dem europäischen Ausland anknüpfen, was die Projektebene betrifft (nach Vorbild der Programmebene, Kapitel 4.4.3). Nicht zuletzt aufgrund der föderalistischen Struktur der Schweiz können internationale Erkenntnisse allerdings selten 1:1 auf die Schweiz übertragen werden.

Besondere Bedeutung hat in der Schweiz die Forderung, Gesundheitsförderung und Prävention in allen Politikbereichen zu berücksichtigen (multisektoraler Ansatz). Dies setzt allerdings die Koordination und Rollenklärung von nationalen, kantonalen und kommunalen Akteuren voraus. Dem Bund kommt hier sicherlich die Führungsrolle zu, da nur er die Strategie aller relevanten Bereiche (z.B. Finanzen, Land- 
Tabelle 4.7: Dringliche Massnahmen für die Schweiz und Verantwortlichkeiten

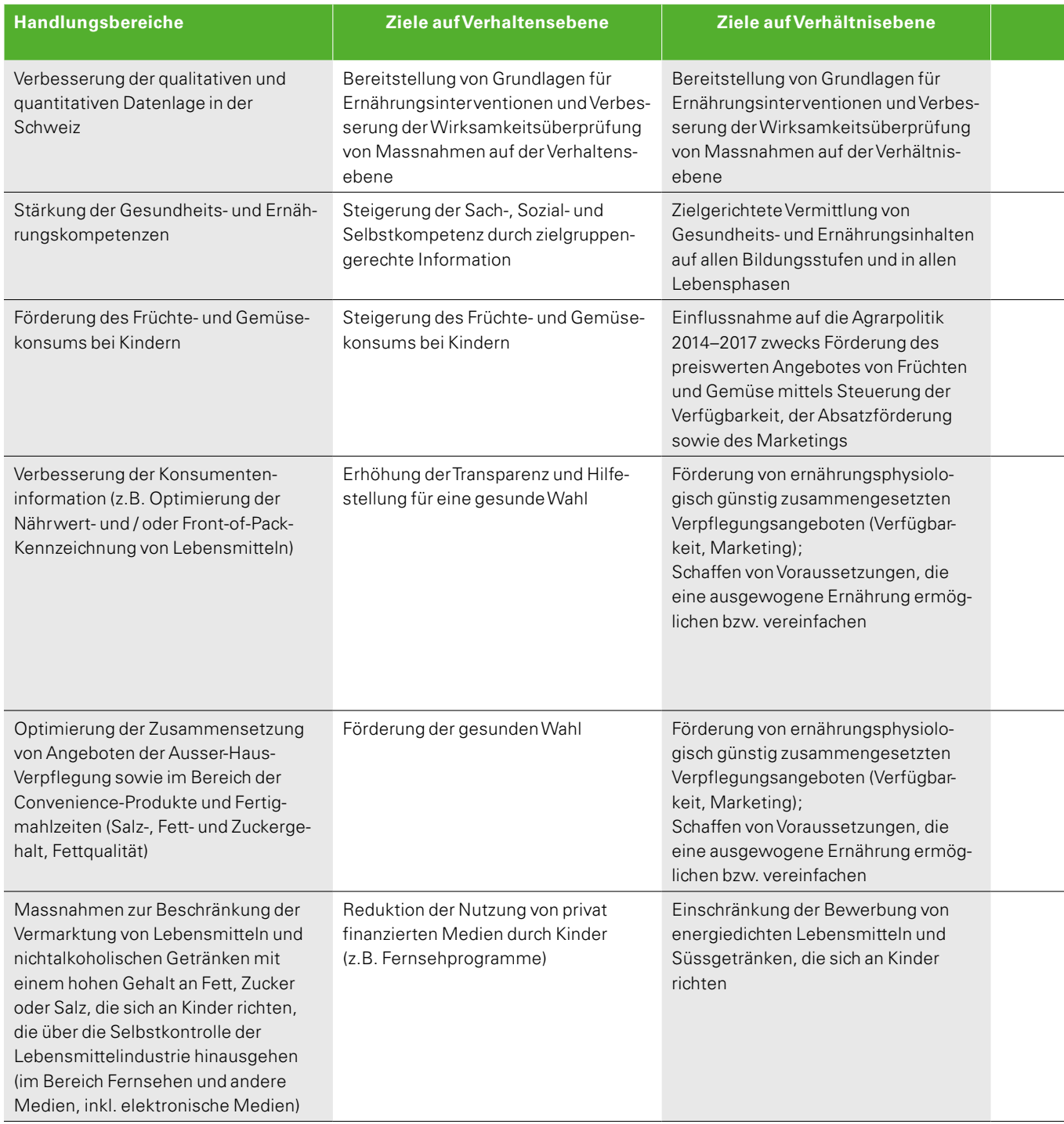


Verantwortliche Akteure auf Verhältnisebene

Bund, Kantone, Gemeinden, Forschungsinstitutionen, Fachorganisationen und -personen

Kantone, Gemeinden, Schulen, Fachorganisationen und-personen
Machbarkeit, Ansatzmöglichkeit

Mit MOSEB wurde ein erster Grund-

stein gelegt (Kapitel 4.6.1.1).
Weiterführende Informationen, Evidenz

Kapitel 2, 3, 4.9.1
Diverse Massnahmen bieten Ansatzmöglichkeiten (Tabellen 4.2-4.4, Kapitel 4.7.3).
Kapitel 4.5.1, 4.5.3

Kapitel 4.7.1, 4.8.3, 4.9.5

$(194 ; 208 ; 209)$

Umsetzung eines gramms in der Schweiz unter den heutigen Rahmenbedingungen nicht machbar ist.

Die Bestrebungen des BAG zur Einführung eines "Healthy Choice Labels" scheiterten am Widerstand der Privatwirtschaft (225). Gemeinsam mit Konsumentenorganisationen und der Lebensmittelwirtschaft wird die SGE Massnahmen testen und Vorschläge erarbeiten, welche die Konsumenten in der Schweiz zukünftig bei der gesunden Wahl unterstützen.

Mit den «Schweizer Qualitätsstandards für eine gesundheitsfördernde Gemeinschaftsgastronomie" wurde eine wichtige Grundlage geschaffen (Tabelle 4.2).
Kapitel 2.6.4, 4.9.2

$(10 ; 11 ; 54)$

Kapitel 2.6.5, 4.5.2, 4.9.6

$(11 ; 54 ; 114)$
Bund, Kantone, Gemeinden, Individualund Gemeinschaftsgastronomie, Lebensmittelindustrie und -handel, Lieferanten, Betriebe
Die Initiative "actionsanté» bietet den Rahmen für freiwillige Aktionsversprechen der Privatwirtschaft im Bereich Marketing und Werbung (Kapitel 4.6.1.1).
Kapitel 4.5.2, 4.9.6

$(10 ; 11 ; 223 ; 226-228)$

Bund, Lebensmittelind
-handel, Gastronomie 
wirtschaft, Bildung usw.) koordinieren kann ein für die Eindämmung der Gesundheitskosten entscheidender Faktor (10). Hier setzt das NPEB an, das inhaltlich weitergeführt und mit entsprechenden Ressourcen ausgerüstet werden muss, damit die Aspekte der Gesundheit und Lebensqualität in andere nationale Sektorpolitiken aufgenommen werden. Die Verknüpfung der Themen Ernährung und Bewegung im Rahmen des NPEB sollte im Hinblick auf das übergeordnete Ziel der Prävention und Gesundheitsförderung beibehalten werden.

In Abstimmung dazu muss die erfolgreiche Zusammenarbeit von Gesundheitsförderung Schweiz mit den Kantonen im Rahmen der KAP die ebenfalls den kombinierten Ansatz von Ernährung und Bewegung verfolgen - weitergeführt und auf eine solide Basis gestellt werden.

Der Bund setzt zur Förderung einer ausgewogenen Ernährung und ausreichend Bewegung vor allem auf freiwillige Massnahmen der Privatwirtschaft. Ein wichtiges Ziel ist dabei die Optimierung der Zusammensetzung von Angeboten der Ausser-Haus-Verpflegung sowie im Bereich der Convenience-Produkte und Fertigmahlzeiten (Salz-, Fett- und Zuckergehalt, Fettqualität). Auch bei der Konsumenteninformation (z.B. Nährwert- und Front-of-Pack-Kennzeichnung von Lebensmitteln) besteht grosses Optimierungspotenzial. Hierzu und bei der Bewerbung von Lebensmitteln und nichtalkoholischen Getränken mit einem hohen Gehalt an Fett, Zucker oder Salz bei Kindern und deren direkten Bezugspersonen sind regulierende Massnahmen in Betracht zu ziehen. Solche Regelungen im Bereich von Werbung und Marketing wären unter der Bedingung, dass das politische Feld dafür entsprechend vorbereitet werden könnte, ein sinnvoller und gangbarer Weg. Weitere prio- ritäre Handlungsfelder sind schliesslich die Stärkung der Gesundheits- und Ernährungskompetenzen sowie die Förderung des Früchte- und Gemüsekonsums bei Kindern. Allen diesen Handlungsempfehlungen gemeinsam ist, dass sie eine multisektorale Gesundheitspolitik voraussetzen und die Zusammenarbeit von Akteuren aus den verschiedensten Sektoren erfordern (Wirtschaft, Bildung, Landwirtschaft usw.). 


\section{Literaturverzeichnis}

1. Organisation for Economic Co-operation and Development, World Health Organisation.

OECD reviews of health systems: Switzerland 2011. OECD Publishing, 2011.

2. Organisation for Economic Co-operation and Development. Health at a Glance: Europe 2010. OECD Publishing, 2010.

3. Bundesamt für Statistik. Statistisches Lexikon der Schweiz. Kosten des Gesundheitswesens seit 1960. Neuchâtel: Bundesamt für Statistik, 2011. Internet: http://www.bfs.admin.ch/bfs/portal/de/ index/infothek/lexikon/lex/0.html (letzter Zugriff 28. November 2011).

4. Bundesamt für Statistik. Szenarien der Bevölkerungsentwicklung der Kantone 2010-2035. Internet: http://www.bfs.admin.ch/bfs/portal/de/ index/news/01/nip_detail.html?gnplD=2011-574 (letzter Zugriff 28. November 2011).

5. Bundesamt für Statistik. Statistisches Lexikon der Schweiz. Kosten, Finanzierung - Daten, Indikatoren. Kosten nach Alter und Geschlecht. Gesundheitskosten in 2009 nach Alter und Geschlecht. Neuchâtel: Bundesamt für Statistik, 2011. Internet: http://www.bfs.admin.ch/bfs/portal/de/ index/themen/14/05/blank/key/05.html (letzter Zugriff 28. November 2011).

6. Colombier C, WeberW. Ausgabenprojektionen für das Gesundheitswesen bis 2050. Working Paper des Ökonomenteams EFV, Nr. 10. Eidgenössische Finanzverwaltung, 2008.

7. Flückiger-Bäni S. Rigide Ernährungsvorschriften zur Erziehung der Bürger? Interpellation 09.4320, eingereicht am 11.12.2009.

Internet: http://www.parlament.ch/d/suche/ seiten/geschaefte.aspx?gesch_id=20094320 (letzter Zugriff 28. November 2011).

8. Bundesverfassung der Schweizerischen Eidgenossenschaft vom 18. April 1999 (Stand am 1. Januar 2011); SR 101, Art. 170 Überprüfung der Wirksamkeit.

Internet: http://www.admin.ch/ch/d/sr/101/ a170.html (letzter Zugriff 28. November 2011).
9. Kemm J. The limitations of "evidence-based" public health. J Eval Clin Pract 2006;12(3):319-24.

10. Organisation for Economic Co-operation and Development. Obesity and the economics of prevention: fit not fat. OECD Publishing, 2010.

11. Gortmaker S, Swinburn BA, Levy D et al. Changing the future of obesity: science, policy, and action. Lancet 2011;378:838-47.

12. Galani C, Schneider H. Effectiveness of preventive and therapeutic interventions in overweight and obesity. A Health Technology Assessment. Basel: HealthEcon AG, 2006.

Internet: http://www.bag.admin.ch/themen/ ernaehrung_bewegung/05207/05218/05231/ index.html? lang=de\&download $=\mathrm{NHzLpZeg7t}$, Inp 6IONTU042I2Z6In1acy4Zn4Z2qZpnO2Yuq2Z6gpJ CGe4N2gWym162epYbg2c_JjKbNoKSn6A-(letzter Zugriff 28. November 2011).

13. King L, GillT, Allender S, Swinburn B. Best practice principles for community-based obesity prevention: development, content and application. Obes Rev 2011;12(5):329-38.

14. Birch LL, Ventura AK. Preventing childhood obesity: what works? Int J Obes 2009;33(1):74-81.

15. Lemmens VE, Oenema A, Klepp KI, Henriksen HB, Brug J. A systematic review of the evidence regarding efficacy of obesity prevention interventions among adults. Obes Rev 2008;9(5):446-55.

16. Bundesamt für Gesundheit. Dialog Nationale Gesundheitspolitik.

Internet: http://www.nationalegesundheit.ch/de/ index.html (letzter Zugriff 28. November 2011).

17. Achtermann W, Berset C. Gesundheitspolitiken in der Schweiz. Potential für eine nationale Gesundheitspolitik. Band 1: Analyse und Perspektiven. Bern: Bundesamt für Gesundheit, 2006.

18. The Ottawa Charter for Health Promotion. First International Conference on Health Promotion, Ottawa, 21 November 1986.

Internet: http://www.who.int/healthpromotion/ conferences/previous/ottawa/en/index.html (letzter Zugriff 28. November 2011). 
19. Konferenz der Vereinten Nationen für Umwelt und Entwicklung. AGENDA 21. Rio de Janeiro, 1992. Internet: http://www.un.org/depts/german/conf/ agenda21/agenda_21.pdf (letzter Zugriff 28. November 2011).

20. World Health Organization. Global strategy on diet, physical activity and health. WHO, 2004. Internet: http://www.who.int/dietphysicalactivity/ strategy/eb11344/strategy_english_web.pdf (letzter Zugriff 28. November 2011).

21. World Health Organization. The challenge of obesity in the WHO - European Region and the strategies for response. Copenhagen: World Health Organization, 2007. Internet: http://www.euro.who.int/_data/assets/ pdf_file/0010/74746/E90711.pdf (letzter Zugriff 28. November 2011).

22. Bundesministerium für Gesundheit. Nationaler Aktionsplan Ernährung inkl. Massnahmenübersicht und Planung 2011. Wien: Bundesministerium für Gesundheit, 2011.

Internet: http://www.bmg.gv.at/cms/home/ attachments/1/3/0/CH1046/CMS1264514565545/ nape_210111.pdf (letzter Zugriff 28. November 2011).

23. Meyer K, ed. Gesundheit in der Schweiz. Nationaler Gesundheitsbericht 2008. Schweizerisches Gesundheitsobservatorium (Obsan). Bern: Verlag Hans Huber, 2009.

24. Bundesgesetz über die Förderung von Sport und Bewegung (Sportförderungsgesetz SpoFöG). Internet: http://www.admin.ch/ch/d/ff/2011/4893. pdf (letzter Zugriff 28. November 2011).

25. Bundesverfassung der Schweizerischen Eidgenossenschaft vom 18. April 1999 (Stand am 1. Januar 2011); SR 101, Art. 118 Schutz der Gesundheit.

Internet: http://www.admin.ch/ch/d/sr/101/ a118.html (letzter Zugriff 28. November 2011).

26. Eidgenössisches Departement des Innern. Verordnung des EDI über Speiseöl, Speisefett und daraus hergestellte Erzeugnisse. Stand 22. März 2009. Internet: http://www.admin.ch/ch/d/ sr/8/817.022.105.de.pdf (letzter Zugriff 28. November 2011).
27. Bundesverfassung der Schweizerischen Eidgenossenschaft vom 18. April 1999 (Stand am 1. Januar 2011); SR 101, Art. 68 Sport. Internet: http://www.admin.ch/ch/d/sr/101/ a68.html (letzter Zugriff 28. November 2011).

28. Bundesverfassung der Schweizerischen Eidgenossenschaft vom 18. April 1999 (Stand am 1. Januar 2011); SR 101, Art. 105 Alkohol. Internet: http://www.admin.ch/ch/d/sr/101/ a105.html (letzter Zugriff 28. November 2011).

29. Bundesverfassung der Schweizerischen Eidgenossenschaft vom 18. April 1999 (Stand am 1. Januar 2011); SR 101, Art. 131 Besondere Verbrauchssteuern. Internet: http://www.admin.ch/ch/d/sr/101/ a131.html (letzter Zugriff 28. November 2011).

30. Bundesverfassung der Schweizerischen Eidgenossenschaft vom 18. April 1999 (Stand am 1. Januar 2011); SR 101, Art. 97 Schutz der Konsumentinnen und Konsumenten. Internet: http://www.admin.ch/ch/d/sr/101/ a97.html (letzter Zugriff 28. November 2011).

31. Bundesgesetz über die Krankenversicherung vom 18. März 1994 (Stand am 1. Januar 2011); SR 832.10, Art. 19 Förderung der Verhütung von Krankheiten. Internet: http://www.admin.ch/ch/d/sr/832_10/ a19.html (letzter Zugriff 28. November 2011).

32. Bundesgesetz über Lebensmittel und Gebrauchsgegenstände (Lebensmittelgesetz LMG) vom 9. Oktober 1992 (Stand am 1. April 2008); SR 817.0, Art. 1 Zweck, Art. 2 Geltungsbereich. Internet: http://www.admin.ch/ch/d/sr/817_0/ index.html (letzter Zugriff 28. November 2011).

33. Fachstelle für Gesundheitspolitik. Gesundheitsförderung junger Menschen im Kanton Baselland. Gesetzliche und politische Rahmenbedingungen 2010. Daten zur Verfügung gestellt von Hanimann A und Wiesli R, Fachstelle für Gesundheitspolitik, 2010. 
34. World Health Organization. Fifty-seventh World Health Assembly. WHA 57.17 Global strategy on diet, physical activity and health. 2004. Internet: http://apps.who.int/gb/ebwha/pdf_files/ WHA57/A57_R17-en.pdf (letzter Zugriff 28. November 2011).

35. World Health Organization. 2008-2013 Action plan for the global strategy for the prevention and control of noncommunicable diseases. Geneva: WHO, 2008. Internet: http://whqlibdoc.who.int/publications/ 2009/9789241597418_eng.pdf (letzter Zugriff 28. November 2011).

36. World Health Organization Europe. European charter on counteracting obesity. WHO European Ministerial Conference on Counteracting Obesity, 2006.

Internet: http://www.euro.who.int/__data/assets/ pdf_file/0009/87462/E89567.pdf (letzter Zugriff 28. November 2011).

37. World Health Organization Europe. The first action plan for food and nutrition policy 2000-2005. WHO European Region. WHO, 2001. Internet: http://www.euro.who.int/__data/assets/ pdf_file/0013/120244/E72199.pdf (letzter Zugriff 28. November 2011).

38. World Health Organization Europe. WHO European Action plan for food and nutrition policy 2007-2012. WHO, 2008.

Internet: http://www.euro.who.int/__data/assets/ pdf_file/0017/74402/E91153.pdf (letzter Zugriff 28. November 2011).

39. Commission of the European Communities. White Paper on A strategy for Europe on nutrition, overweight and obesity related health issues. Brussels, 2007.

Internet: http://ec.europa.eu/health/ph_determinants/ life_style/nutrition/documents/nutrition_wp_en.pdf (letzter Zugriff 28. November 2011).
40. European Commission. Evaluation of the european platform for action on diet, physical activity and health. Final report July 2010. Brussels: European Commission, 2010.

Internet: http://ec.europa.eu/health/nutrition_physical_activity/docs/evaluation_frep_en.pdf (letzter Zugriff 28. November 2011).

41. European Commission. Directorate General for Health and Consumers. Strategy for Europe on nutrition, overweight and obesity related health issues. Implementation progress report. December 2010.

Internet: http://ec.europa.eu/health/nutrition_physical_activity/docs/implementation_report_en.pdf (letzter Zugriff 28. November 2011).

42. High Level Group on nutrition and physical activity. EU Framework for National Salt Initiatives, verabschiedet am 1. Juli 2008. Internet: http://ec.europa.eu/health/archive/ ph_determinants/life_style/nutrition/documents/ salt_initiative.pdf (letzter Zugriff 28. November 2011).

43. Bundesamt für Gesundheit. Aktionsplan Ernährung und Gesundheit: Eine Ernährungspolicy für die Schweiz. Bern: Bundesamt für Gesundheit, 2001. Internet: http://www.bag.admin.ch/themen/ ernaehrung_bewegung/05207/05215/index.html? lang=de\&download=NHzLpZeg7t, Inp6IONTU04 2I2Z6In1acy4Zn4Z2qZpnO2Yuq2Z6gpJCFeXx7fWym162epYbg2c_JjKbNoKSn6A (letzter Zugriff 28. November 2011).

44. Gesundheitsförderung Schweiz. Auftrag und Ziele. Internet: http://www.gesundheitsfoerderung.ch/ pages/uebersicht/Ueber_uns/m_auftrag_und_ ziele.php (letzter Zugriff 28. November 2011).

45. May A, Castella F, Wiesli R. Die Kantonalen Beauftragten für Gesundheitsförderung. Situationsanalyse und Entwicklungspotential. Bern: Vereinigung der Kantonalen Beauftragten für Gesundheitsförderung in der Schweiz, ed., 2010. Internet: http://www.vbgf-arps.ch/logicio/client/ vbgf/file/BerichtVBGF/Bericht_010210.pdf (letzter Zugriff 28. November 2011). 
46. Kantonsärztliche Dienste Luzern. Luzerner Aktionsprogramm "Gesundes Körpergewicht" 2008-2011.

47. Vereinigung der Kantonalen Beauftragten für Gesundheitsförderung in der Schweiz. Internet: http://www.vbgf-arps.ch/logicio/ pmws/indexDOM.php?client_id=vbgf\&page_ id=home\&lang_iso639=de (letzter Zugriff 28. November 2011).

48. Sommerhalder K, AbelT. Gesundheitskompetenz: Eine konzeptionelle Einordnung. Bern: Institut für Sozial- und Präventivmedizin, 2007.

49. Kickbusch I. Die Gesundheitsgesellschaft: Megatrends der Gesundheit und deren Konsequenzen für Politik und Gesellschaft. Werbach-Gamburg: Verlag für Gesundheitsförderung, 2006.

50. BEST Institut für berufsbezogene Weiterbildung und Personaltraining. Food Literacy. Schmackhafte Angebote für die Erwachsenenbildung und Beratung. Internet: http://www.gutessen.at/uploads/ FL_guidelines_de.pdf (letzter Zugriff 28. November 2011)

51. Darmon N, Drewnowski A. Does social class predict diet quality? Am J Clin Nutr 2008;87(5):1107-17.

52. Heim B. Gesundheitskompetenz: Zentraler Faktor im Gesundheitswesen. Motion 07.3525, eingereicht am 22.06.2007. Internet: http://www.parlament.ch/d/suche/ seiten/geschaefte.aspx?gesch_id=20073525 (letzter Zugriff 28. November 2011).

53. Gutzwiller F, Paccaud F, eds. Sozial- und Präventivmedizin. Public Health. 3. vollständig überarbeitete Auflage. Bern: Verlag Hans Huber, 2007.

54. World Health Organization. Interventions on diet and physical activity: what works. Summary report. Geneva:WHO, 2009. Internet: http://www.who.int/dietphysicalactivity/ summary-report-09.pdf (letzter Zugriff 28. November 2011).
55. Retsinformation Danmark. Lov nr. 247 af 30/03/2011. Lov om afgift af mættet fedt i visse fødevarer (fedtafgiftsloven). Danmark: Skatteministeriet, 2011.

Internet: https://www.retsinformation.dk/Forms/ R0710.aspx?id=136314 (letzter Zugriff

28. November 2011).

56. Arbeitsgemeinschaft BASS, ZHAW, ISPM, M.I.S TREND. Gesundheitsmonitoring der Migrationsbevölkerung (GMM) in der Schweiz. Schlussbericht. Bern: Bundesamt für Gesundheit, Bundesamt für Migration, August 2011.

Internet: http://www.bag.admin.ch/themen/ gesundheitspolitik/07685/12533/12535/index. html?lang=de (letzter Zugriff 6. Juni 2012).

57. Keller SK, Schulz PJ. Distorted food pyramid in kids programmes: a content analysis of television advertising watched in Switzerland. Eur J Public Health 2011;21(3):300-5.

58. Schulz PJ, Keller S, Hartung U. Bericht zur KIWI Studie. Lebensmittelwerbung für Kinder: eine Inhaltsanalyse des Schweizer Fernsehens März-August 2006. Lugano: Universität Lugano, Health Care Communication Laboratory, 2006. Internet: http://www.bag.admin.ch/themen/ ernaehrung_bewegung/05207/05218/05234/ index.html?lang=de\&download=NHzLpZeg7t, Inp 6IONTU042I2Z6In1acy4Zn4Z2qZpnO2Yuq2Z6gpJ CGdn9_f2ym162epYbg2c_JjKbNoKSn6A-(letzter Zugriff 28. November 2011).

59. Kelly B, Halford JC, Boyland EJ et al. Television food advertising to children: a global perspective. Am J Public Health 2010;100(9):1730-6.

60. Hastings G, Stead M, McDermott L. Review of research on the effects of food promotion to children. Final Report. Glasgow: The University of Strathclyde, 2003. Internet: http://www.food.gov.uk/multimedia/pdfs/ foodpromotiontochildren1.pdf (letzter Zugriff 28. November 2011). 
61. McGinnis JM, Appleton Gootman J, Kraak VI. Food marketing to children and youth. Threat or opportunity? Washington, D.C.: National Academies Press, 2006.

62. Hebden L, King L, Kelly B. Art of persuasion: an analysis of techniques used to market foods to children. J Paediatr Child Health 2011;47(11): 776-82.

63. Foederation der Schweizerischen NahrungmittelIndustrien. Ernährung und Übergewicht: Massnahmen, mit welchen Unternehmungen der Schweizer Nahrungsmittel-Industrie positive Beiträge für eine ausgewogenere Ernährung leisten können. 2008.

Internet: http://www.fial.ch/files/documents/de/ Ideenkatalog_Ernaehrung.pdf (letzter Zugriff 28. November 2011).

64. Foederation der Schweizerischen NahrungmittelIndustrien. Positionspapier der NahrungsmittelIndustrie zum Thema Übergewicht und Ernährung. 2010.

Internet: http://www.fial.ch/files/documents/de/ Positionspapier_Ernaehrung.pdf (letzter Zugriff 28. November 2011).

65. Swiss Pledge. Internet: http://www.swiss-pledge.ch (letzter Zugriff 28. November 2011).

66. GastroSuisse. Branchenspiegel 2011. Zürich: GastroSuisse, 2011.

67. Forschungsgruppe «Good Practice-Gemeinschaftsgastronomie», eds. CATI-Befragung Gesundheitsfördernde Gemeinschaftsgastronomie. Executive Summary. Bern: Berner Fachhochschule, Fachbereich Gesundheit, 2011. Internet: http://www.goodpractice-gemeinschaftsgastronomie.ch/fileadmin/user_upload/ downloads_de/D_CATI11_Excecutive_summary_ 110929.pdf (letzter Zugriff 28. November 2011).

68. Schweizer Verband für Spital-, Heim- und Gemeinschaftsgastronomie.

Internet: http://www.svg.ch/ (letzter Zugriff

28. November 2011).
69. Bundesamt für Statistik. Taschenstatistik der Schweiz 2011. Neuchâtel: Bundesamt für Statistik, 2011. Internet: http://www.bfs.admin.ch/bfs/portal/de/ index/infothek/publ.Document.140347.pdf (letzter Zugriff 28. November 2011).

70. Bundesamt für Statistik. Haushaltsbudgeterhebung 2009. Medienmitteilung vom 15. November 2011.

Internet: http://www.bfs.admin.ch/bfs/portal/de/ index/news/medienmitteilungen.Document. 150098.pdf (letzter Zugriff 28. November 2011).

71. Bundesamt für Statistik. Haushaltsbudgeterhebung 2006-2008. Haushaltseinkommen und-ausgaben nach Sprachregion. Internet: http://www.bfs.admin.ch/bfs/portal/de/ index/themen/20/02/blank/key/einkommen0/ niveau. html (letzter Zugriff 28. November 2011).

72. GfK Switzerland AG. Spezialstudie "Der Preis ist heiss" oder wie gut kennen die Schweizer die Preise? Hergiswil: GfK Switzerland AG, 2011.

73. Statistik Austria, Bundesanstalt Statistik Österreich. Konsumerhebung 2009/10. Internet: http://www.statistik.at/web_de/statistiken/ soziales/verbrauchsausgaben/konsumerhebung_ 2009_2010/index.html (letzter Zugriff 28. November 2011).

74. Statistisches Bundesamt Deutschland. Laufende Wirtschaftsrechnungen 2009.

75. Institut national de la statistique et des études économiques. Enquête Budget de famille 2006. Internet: http://www.insee.fr/fr/themes/ document.asp?ref_id=BDF06 (letzter Zugriff 28. November 2011).

76. Istituto nazionale di statistica. I consumi delle famiglie. Anno 2009. Roma: Istituto nazionale di statistica, 2011.

Internet: http://www3.istat.it/dati/catalogo/ 20110405_00/ann_11_16_I_consumi_delle_ famiglie_2009.pdf (letzter Zugriff 28. November 2011). 
77. MerkleT, Wippermann C. Eltern unter Druck. Selbstverständnisse, Befindlichkeiten und Bedürfnisse von Eltern in verschiedenen Lebenswelten. Stuttgart: Lucius \& Lucius Verlagsgesellschaft, 2008.

78. Pearson N, Biddle SJ, GorelyT. Family correlates of fruit and vegetable consumption in children and adolescents: a systematic review. Public Health Nutr 2009;12(2):267-83.

79. Schweizerische Konferenz der kantonalen Erziehungsdirektoren. HarmoS-Konkordat. Interkantonale Vereinbarung über die Harmonisierung der obligatorischen Schule.

Internet: http://edudoc.ch/record/24711/ files/HarmoS_d.pdf (letzter Zugriff 28. November 2011).

80. Schweizerische Konferenz der kantonalen Erziehungsdirektoren. Kantonsumfrage 2010/2011. Internet: http://www.edk.ch/dyn/13341.php (letzter Zugriff: 28. November 2011).

81. Schulgesundheitsdienste Stadt Zürich. Ernährungsrichtlinien für die Schulen der Stadt Zürich. Internet: http://www.stadt-zuerich.ch/content/dam/ stzh/ssd/Deutsch/Gesundheit\%20Praevention/ Schularzt/Formulare\%20und\%20Merkblaetter/ Praevention_Beratung/Ernaehrung/Ernaehrungsrichtlinien.pdf (letzter Zugriff 28. November 2011).

82. EllrottT.Wie kann man "gesunde Ernährung" schmackhaft machen? Welche Barrieren gibt es auf diesem Weg? Nationale Fachtagung der Schweizerischen Gesellschaft für Ernährung. Bern, 2011. Internet: http://www.sge-ssn.ch/de/ich-und-du/ die-sge/events/archiv-2011/ (letzter Zugriff 6. Juni 2012).

83. Fachstelle Prävention Essstörungen Praxisnah (PEP). Papperla PEP. Internet: http://www.pepinfo.ch/index.php?id=34 (letzter Zugriff 28. November 2011).

84. Fachstelle Prävention Essstörungen Praxisnah (PEP). Bodytalk PEP. Internet: http://www.pepinfo.ch/index.php?id=33 (letzter Zugriff 28. November 2011).
85. Bundesamt für Gesundheit. Nationales Programm Ernährung und Bewegung (NPEB) 2008-2012. Bern: Bundesamt für Gesundheit, 2008. Internet: http://www.bag.admin.ch/themen/ernaehrung_bewegung/13227/index.html?lang=de (letzter Zugriff 6. Juni 2012).

86. Bundesamt für Gesundheit. Salz Strategie 2008-2012.

Internet: http://www.bag.admin.ch/themen/ ernaehrung_bewegung/05207/05216/index. html? lang=de (letzter Zugriff 28. November 2011).

87. Bundesamt für Gesundheit. actionsanté - besser essen, mehr bewegen.

Internet: www.actionsante.ch (letzter Zugriff 28. November 2011).

88. Bundesamt für Gesundheit. Monitoring-System Ernährung und Bewegung. Internet: http://www.bag.admin.ch/themen/ernaehrung_bewegung/05190/index.html?lang=de (letzter Zugriff 6. Juni 2012).

89. INFRAS / IUMSP / Maud Krafft Consulting. Evaluation NPEB 2008-2012. Zürich, 2011.

90. Gesundheitsförderung Schweiz. Kantonale Aktionsprogramme für mehr Bewegung und gesunde Ernährung im Alltag.

Internet: http://www.gesundheitsfoerderung.ch/ pages/Gesundes_Koerpergewicht/Programme_ Projekte/kantone.php (letzter Zugriff 28. November 2011).

91. Schopper D. Gesundes Körpergewicht bei Kindern und Jugendlichen. Was haben wir seit 2005 dazugelernt? Bern und Lausanne: Gesundheitsförderung Schweiz, 2010.

92. Furrer C, Fässler S, Lauberau B, Wight N, Balthasar A. Evaluation Kantonale Aktionsprogramme Gesundes Körpergewicht. Zwischensynthese 2010 der Umsetzung und derWirkungen. Luzern: Interface, 2010. Internet: http://www.gesundheitsfoerderung. ch/pdf_doc_xls/d/gesundes_koerpergewicht/ grundlagen_wissen/Evaluation/Evaluation-KAPZwischensynthese-2010-de.pdf (letzter Zugriff 28. November 2011). 
93. Gesundheitsförderung Schweiz, Helsana, Visana. action d-diabetes aktiv vorbeugen.

Internet: http://www.actiond.ch/logicio/pmws/ actiond__home__de.html (letzter Zugriff 28. November 2011).

94. Krebsliga Schweiz, Bundesamt für Gesundheit. Nationale Kampagne "5 am Tag". Internet: http://www.5amtag.ch/ (letzter Zugriff 28. November 2011).

95. Schweizerische Stiftung zur Förderung des Stillens. Internet: http://www.allaiter.ch/logicio/pmws/ indexDOM.php?client_id=stillen\&page id=aktuell\&lang_iso639=de (letzter Zugriff 28. November 2011).

96. Bundesamt für Gesundheit, Gesundheitsförderung Schweiz. Suisse Balance: Die nationale Projektförderstelle Ernährung und Bewegung. Internet: www.suissebalance.ch (letzter Zugriff 28. November 2011)

97. Nutrinet. Netzwerk Ernährung und Gesundheit. Internet: http://www.nutrinet.ch (letzter Zugriff 28. November 2011).

98. Schweizerische Gesellschaft für Ernährung. Internet: http://www.sge-ssn.ch (letzter Zugriff 28. November 2011).

99. bildung + gesundheit Netzwerk Schweiz. Internet: http://www.bildungundgesundheit.ch (letzter Zugriff 28. November 2011).

100. Radix. Schweizerisches Netzwerk Gesundheitsfördernder Schulen.

Internet: http://www.radix.ch/index.cfm/ AD965B64-A6A5-9FE2-88B2B40A6956040A/ (letzter Zugriff 28. November 2011).

101. Allianz Ernährung. Internet: http://www.sge-ssn.ch/de/wissenschaftund-forschung/experten/allianz-ernaehrung/ (letzter Zugriff 6. Juni 2012).

102. Allianz "Gesunde Schweiz». Internet: http://www.allianzgesundeschweiz.ch/ (letzter Zugriff 28. November 2011).
103. Allianz Gesundheitskompetenz. Internet: http://www.gesundheitsfoerderung.ch/ pages/Gesundheitsfoerderung_und_Praevention/ Programme_Projekte/allianz_gesundheitskompetenz.php?lang $=d$ (letzter Zugriff 28. November 2011).

104. NGO-Allianz Ernährung, Bewegung, Körpergewicht. Internet: http://cardiovascsuisse.ch/index.php? id=1791 (letzter Zugriff 28. November 2011).

105. Gesundheitsförderung Schweiz. quint-essenz. Qualitätsentwicklung in Gesundheitsförderung und Prävention.

Internet: http://www.quint-essenz.ch/de/ (letzter Zugriff 28. November 2011).

106. Fédération Romande des Consommateurs. Cours "Bien manger à petit prix ". Internet: http://frc.ch/categorie/alimentationdistribution/manger-sainement/ (letzter Zugriff 6. Juni 2012).

107. Clerc A, Pfenniger B, DelacrétazT. Guide «Bien manger à petit prix». Equilibre alimentaire, acheter malin pour bien manger, recettes avec propositions d'Annick Jeanmairet. Lausanne: Fédération Romande des Consommateurs, 2010.

108. Stiftung für Konsumentenschutz. Ratgeber "Gut, gesund und günstig essen». Bern: Stiftung für Konsumentenschutz, 2011.

109. Caritas Schweiz. Caritas-Markt-gesund! Internet: http://www.caritas-markt.ch/de/ p103001366.html (letzter Zugriff 28. November 2011).

110. Cleven-Stiftung. fit-4-future. Internet: http://www.fit-4-future.ch (letzter Zugriff 28. November 2011).

111. Universität Basel, Institut für Sport und Sportwissenschaften. Evaluation der fit-4-future Bewegungsförderungsmittel: Zusammenfassung. November 2010. Internet: http://www.fit-4-future.ch/Uploads/ Documents/divers/zfs_f4f_bewegungsfoerderungsmittel.pdf (letzter Zugriff 28. November 2011). 
112. Fourchette verte suisse. Fourchette verte. Internet: http://www.fourchetteverte.ch/de/ (letzter Zugriff 28 November 2011).

113. Démoscope. Fourchette verte, section genevoise. Démoscope, 2010.

114. Berner Fachhochschule, Fachbereich Gesundheit. Good Practice-Gemeinschaftsgastronomie. Internet: http://www.goodpractice-gemeinschaftsgastronomie.ch (letzter Zugriff 28. November 2011).

115. Forschungsgruppe «Good Practice-Gemeinschaftsgastronomie", eds. Projektzusammenfassung. Bern: Berner Fachhochschule, Fachbereich Gesundheit, 2008.

Internet: http://www.goodpractice-gemeinschaftsgastronomie.ch/fileadmin/user_upload/ downloads_de/D_Projektzusammenfassung_ V2.2_100806.pdf (letzter Zugriff 28. November 2011).

116. Schtifti Foundation. GORILLA. Internet: http://www.gorilla.ch (letzter Zugriff 28. November 2011).

117. Stamm H, Lamprecht M. Evaluation des GORILLAProjekts. Standortbestimmung und Zwischenbilanz per Ende Oktober 2011. Zürich: Lamprecht und Stamm Sozialforschung und Beratung AG, 2011.

118. Migros Kulturprozent. Kebab+. Internet: http://www.kebabplus.ch (letzter Zugriff 28. November 2011).

119. Kratzer J, Loos V. Kebab+. Projektevaluation Kochen mit Jugendlichen (MigrantInnen). 2009. Internet: http://www.kebabplus.ch/data/ OA393936-CEA5-3CB2EDB56137751BFD46/Evaluation_Kochen1_09.pdf (letzter Zugriff 28. November 2011).

120. SchweizerischerTurnverband. KidBalù. Internet: http://www.stv-fsg.ch/newsmedia/news/ detail/article/2004/06/24/archivierte-news-3/ (letzter Zugriff 28. November 2011).
121. Migros Kulturprozent. meingleichgewicht. Menschen mit Behinderung und gesundes Körpergewicht. Internet: http://www.meingleichgewicht.ch (letzter Zugriff 28. November 2011).

122. Schweizerische Gesellschaft für Ernährung. NUTRIKID. Internet: http://www.nutrikid.ch (letzter Zugriff 28. November 2011).

123. Procap. Procap bewegt. Internet: http://www.procap-bewegt.ch (letzter Zugriff 28. November 2011).

124. Interface. Pilotphase Procap bewegt. Bericht der externen Evaluation zuhanden der Projektleitung. Luzern: Interface, 2011. Internet: http://www.interface-politikstudien.ch/ downloads/deutsch/Be_Procap_bewegt.pdf (letzter Zugriff 28. November 2011).

125. Radix. Purzelbaum. Internet: http://www.radix.ch/index.cfm/ 0C0464D2-0BE8-08F2-816BAAB45F8F6A43/ (letzter Zugriff 28. November 2011).

126. Radix. schnitz und drunder. Internet: http://www.gesundheit.lu.ch/index/ gesundheitsfoerderung/gewicht/schnitz_und_ drunder.htm (letzter Zugriff 6. Juni 2012).

127. Kirchhofer J, Steffen T, Müller B, Jauch A. Schlussbericht zur Evaluation des Projekts Burzelbaum Bewegter Kindergarten. Basel: Gesundheitsförderung und Prävention Basel-Stadt, 2007. Internet: http://upload.sitesystem.ch/B2DBB48B7E/ 5B4613A676/0497BFF887.pdf (letzter Zugriff 28. November 2011).

128. Bundesamt für Sport. Schule bewegt. Internet: http://www.schulebewegt.ch/ (letzter Zugriff 28. November 2011).

129. Bundesamt für Sport. schule bewegt: Jahresberichte. Internet: http://www.schulebewegt.ch/internet/ Schulebewegt/de/home/auswertung/zahlen_ fakten.html (letzter Zugriff 28. November 2011). 
130. Cantons du Jura, Fribourg, Neuchâtel, Valais et Vaud. Guide "Bouger, manger à l'école». Internet: http://www.guide-ecole.ch (letzter Zugriff 28. November 2011).

131. Canton de Neuchâtel. Les petits gourmets à table.

132. Ehmig SC, Schneider M. Evaluation du projet "Enfants, fruits et légumes» du Service cantonal de la santé publique du canton de Neuchâtel. Lugano: Institut de Communication de la Santé, 2009.

133. Repubblica e Cantone Ticino, Ufficio del medico cantonale. Movimento e gusto con l'equilibrio giusto.

134. Association pharmaGenève. Pharmaciens malins. Internet: http://www.pharmageneve.ch/ (letzter Zugriff 28. November 2011).

135. Association pharmaGenève. Engagement der Apotheker für das Programm «Marchez et mangez malin!». Internet: http://www.gesundheitsfoerderung.ch/ pdf_doc_xls/d/gesundes_koerpergewicht/ allgemeines/Netzwerktagung_11/ParallelSessions/ PS-1_Pharmaciens-malins_Forni_Haas_de.pdf (letzter Zugriff 28. November 2011).

136. Programme cantonal «Ça marche! » du canton de Vaud. Planificateur de menus pour camps. Internet: http://www.ca-marche.ch/ PlanificateurMenus/ (letzter Zugriff 28. November 2011).

137. Département de la Formation et Département de la Santé du canton du Jura. Programme d'éducation nutritionnelle du canton du Jura. Internet: http://www.fondationo2.ch/fr/Programme/ Alimentation-et-activite-physique/ Alimentation-activite-physique.html (letzter Zugriff 28. November 2011).

138. Fondation Senso5. Projet Senso5. Internet: http://www.senso5.ch (letzter Zugriff 28. November 2011).

139. Fondation Senso5. Newsletter 2010;3.

140. Fondation Senso5. Senso 5 en chiffres. Année scolaire 2011-2012.
141. Programme cantonal "Ça marche! » du canton de Vaud. Tutti Frutti.

Internet: http://www.ca-marche.ch/index.php? rubrique $=217 \&$ langue $=F$ (letzter Zugriff 28. November 2011).

142. Besson G. Rapport d'évaluation des ateliers Tutti Frutti. Lausanne, 2010.

143. Stiftung Pro UKBB (Universitätskinderspital beider Basel). Bewegter Lebensstart. Internet: http://www.bewegter-lebensstart.ch (letzter Zugriff 28. November 2011).

144. Radix. feel-ok. Internet: http://www.feelok.ch/de_CH/ jugendliche/jugendliche.cfm (letzter Zugriff 28. November 2011).

145. Radix. feel ok. Evaluation der Inhalte der neuen Sektion "Ich und mein Gewicht». Zürich: Radix, 2010. Internet: http://www.feelok.ch/files/ wissenschaftlicheBerichte/ 2010_ichMein Gewicht.pdf (letzter Zugriff 28. November 2011).

146. Schweizerischer Fachverband Adipositas im Kindes- und Jugendalter. klemon: Ein Angebot für Kleinkinder mit Essproblemen oder Übergewichtsrisiko. Internet: http://www.klemon.ch (letzter Zugriff 28. November 2011).

147. Neuenschwander-Simoni M, Quinteros-Hungerbühler I, Schnoz D. Prävention und Gesundheitsförderung bei Kleinkindern mit Risiko zu Übergewicht. Projekt klemon. Evaluationsbericht 2007 (Pilotphase). Zürich: Institut für Sucht- und Gesundheitsforschung, 2007. Internet: http://v2.klemon.ch/fileadmin/ Downloads/Dossier_MVB/_KLEMON.Eval-Bericht. 2007.V8. light.pdf (letzter Zugriff 28. November 2011).

148. Caritas Schweiz. Miges Balù.

149. Miges Balù. Externe Projektevaluation im Auftrag von Suisse Balance. Genf: Evaluanda, 2009. 
150. Geburtshilfliche Poliklinik des Universitätsspitals Zürich. PEBS: Präventive Ernährungs- und Bewegungsberatung in der Schwangerschaft bis ein Jahr nach Geburt.

Internet: http://www.leichter-leben-zh.ch/ projekte-nach-zielpersonen/projekte-saeuglinge/ (letzter Zugriff 28. November 2011).

151. Quack Lötscher K. Präventive Ernährungs- und Bewegungsberatung in der Schwangerschaft bis ein Jahr nach der Geburt. Zwischenevaluation: Erstes Projektjahr. Zürich: Universitätsspital Zürich, 2010.

152. Direktion für Bildung, Soziales und Sport der Stadt Bern. Primano, Fördermodul Ernährung. Internet: http://www.primano.ch (letzter Zugriff 28. November 2011).

153. Alsaker FD, Gantenbein-Schaffner B. Evaluation of Primano: Final Report Nutrition. Bern: Universität Bern, Institut für Psychologie, 2009.

154. Suisse Balance. Gesunde Pausen- und Mittagsverpflegung. Eine Übersicht an Projekten, Angeboten und Unterlagen.

Internet: http://www.suissebalance.ch/logicio/ client/suissebalance/file/SB_Materialien/ Uebersichtsliste_Pausenkiosk_Mittagstisch_ PDF_Aktuell_2011.pdf (letzter Zugriff 28. November 2011).

155. Papandreou A. Evaluationsbericht zur Umsetzung der Ernährungsrichtlinien an Stadtzürcher Horten. Zürich: Schulgesundheitsdienste der Stadt Zürich, 2010.

Internet: http://www.stadt-zuerich.ch/content/dam/ stzh/ssd/Deutsch/Gesundheit\%20Praevention/ Schulgesundheitsdienste/Publikationen \%20und\% 20Broschueren/V12\%20Evaluationsbericht $\% 20$ Ernrichtl_def.pdf (letzter Zugriff 28. November 2011).

156. Katakult-Fabrik für Projektkultur.

Schnitz\&Schwatz - das gesunde Fernsehen für Kinder. Internet: http://www.schnitzundschwatz.ch (letzter Zugriff 28. November 2011).
157. Bolliger-Salzmann H. Evaluatorische Reflexionen über das Projekt Schnitz\&Schwatz (S\&S). Bern: Universität Bern, Institut für Sozial- und Präventivmedizin, 2010.

158. Gesundheitsdepartement Kanton Basel-Stadt, Gesundheitsförderung und Prävention. Schulstufenkonzept Rüebli, Zimt \& Co. Internet: http://www.gesundheitsdienste. bs.ch/projekte/ruebli-zimt.htm (letzter Zugriff 28. November 2011).

159. Gesundheitsförderung Baselland. Tacco \& Flip. Internet: http://www.baselland.ch/main_ ernaehrung-htm.280406.0.html (letzter Zugriff 28. November 2011).

160. Tacco \& Flip. Externe Projektevaluation im Auftrag von Suisse Balance. Genf: Evaluanda, 2009.

161. Migros Kulturprozent, Pädagogische Hochschule Zürich. TAVOLATA. Internet: http://www.tavolata.net (letzter Zugriff 28. November 2011).

162. Hilfswerk der Evangelischen Kirchen Schweiz (HEKS). Vitalina - aktive Eltern für gesunde Kinder. Internet: http://www.baselland.ch/0-5_vitalina-htm. 311511.0.html (letzter Zugriff 28. November 2011).

163. Ruder A. Vitalina - Aktive Eltern für gesunde Kinder. Evaluationsbericht. Basel: Hilfswerk der Evangelischen Kirchen Schweiz, 2010.

164. Gloor D, Meier H. Evaluation «Vitalina - aktive Eltern für gesunde Kinder». Schinznach-Dorf: Gesundheitsförderung Baselland, 2011.

165. Gesundheitsdepartement des Kantons St. Gallen. Kinder im Gleichgewicht.

Internet: http://www.kinder-im-gleichgewicht.ch/ (letzter Zugriff 28. November 2011).

166. Ehmig SC, Furrer C, Lenares J. Evaluation des Modulprojekts Weiterbildung für Hebammen, Stillund Mütterberaterinnen in St. Gallen. Ergebnisse von Befragungen und Beobachtungen. Lugano: Institut für Gesundheitskommunikation, 2009.

167. Botschaft zum Bundesgesetz über Prävention und Gesundheitsförderung (Präventionsgesetz, PrävG) vom 30. September 2009. Internet: http://www.admin.ch/ch/d/ff/2009/ 7071.pdf (letzter Zugriff 28. November 2011). 
168. Die Bundesversammlung - das Schweizer Parlament. Botschaft vom 30. September 2009 zum Bundesgesetz über Prävention und Gesundheitsförderung (Präventionsgesetz, PrävG). Zusammenfassung. Internet: http://www.parlament.ch/d/suche/seiten/ legislaturrueckblick.aspx?rb_id=20090076 (letzter Zugriff 28. November 2011).

169. Generalsekretariat des Eidgenössischen Departements des Innern. Die Strategie des Bundesrats in der Gesundheitspolitik. Bern, Juni 2011. Internet: http://www.edi.admin.ch/themen/00391/05211/index.html?lang=de (letzter Zugriff 28. November 2011).

170. Botschaft zurTeilrevision des Bundesgesetzes über die technischen Handelshemmnisse vom 25. Juni 2008.

Internet: http://www.admin.ch/ch/d/ff/2008/7275.pdf (letzter Zugriff 28. November 2011).

171. Botschaft zum Bundesgesetz über Lebensmittel und Gebrauchsgegenstände. Entwurf 2011. Internet: http://www.bag.admin.ch/themen/ lebensmittel/04865/05022/07826/index.html? lang=de\&download=NHzLpZeg7t, Inp6IONTU042। 2Z6In1acy4Zn4Z2qZpnO2Yuq2Z6gpJCKdHx5f2ym 162epYbg2c_JjKbNoKSn6A-- (letzter Zugriff 28. November 2011).

172. Bundesamt für Gesundheit. Revisionen der Verordnungen des Lebensmittelrechts. Bern, 2010. Internet: http://www.bag.admin.ch/themen/ lebensmittel/04865/05022/07910/index.html? lang=de (letzter Zugriff 28. November 2011).

173. Eidgenössisches Departement des Innern. Verordnung des EDI über die Kennzeichnung und Anpreisung von Lebensmitteln. Stand 28. November 2010. Internet: http://www.admin.ch/ch/d/sr/8/ 817.022.21.de.pdf (letzter Zugriff 28. November 2011).
174. European Food Safety Authority. Health claim applications.

Internet: http://www.efsa.europa.eu/en/nda/ ndaclaims.htm (letzter Zugriff 28. November 2011).

175. Europäisches Parlament und Rat der Europäischen Union. Verordnung (EU) Nr. 1169/2011 vom

\section{Oktober 2011.}

Internet: http://eur-lex.europa.eu/LexUriServ/ LexUriServ.do?uri=OJ:L:2011:304:0018:0063:DE: PDF (letzter Zugriff 28. November 2011).

176. Bundesamt für Gesundheit. Verhandlungen Schweiz - EU in den Bereichen Lebensmittelsicherheit und öffentliche Gesundheit. Internet: http://www.bag.admin.ch/themen/ internationales/07419/07460/index.html?lang=de (letzter Zugriff 28. November 2011).

177. Europäische Kommission. Generaldirektion Gesundheit und Verbraucher: Gesundheitsprogramm 2008-2013.

Internet: http://ec.europa.eu/health/archive/ ph_programme/documents/prog_booklet_de.pdf (letzter Zugriff 28. November 2011).

178. European Nutrition and Health Report 2009. Elmadfa I (ed). Forum of nutrition, Vol. 62. Internet: http://www.univie.ac.at/enhr/index.htm (letzter Zugriff 28. November 2011).

179. Eidgenössisches Volkswirtschaftsdepartement. Agrarpolitik 2014-2017.

Internet: http://www.evd.admin.ch/themen/ 00192/00195/index.html?lang=de (letzter Zugriff 28. November 2011).

180. Schweizerischer Nationalfonds zur Förderung der wissenschaftlichen Forschung. NFP 69 "Gesunde Ernährung und nachhaltige Lebensmittelproduktion". Internet: http://www.snf.ch/D/forschung/ Forschungsprogramme/neue/Seiten/ _xc_nfp69.aspx (letzter Zugriff 28. November 2011).

181. RielleY, Wirz R, Wiesli R. Gesundheitspolitisches Monitoring in Bund, Kantonen und Städten. Bericht 2/2009 zuhanden der Stiftung Gesundheitsförderung Schweiz. Bern: Fachstelle für Gesundheitspolitik, 2009. 
182. RielleY, Hanimann A, Wiesli R. Gesundheitspolitisches Monitoring in Bund, Kantonen und Städten. Bericht 2011 zuhanden der Stiftung Gesundheitsförderung Schweiz. Bern: Fachstelle für Gesundheitspolitik, 2011.

183. Die Bundesversammlung - das Schweizer Parlament. Curia Vista Geschäftsdatenbank. Internet: http://www.parlament.ch/d/suche/ seiten/curia-vista.aspx (letzter Zugriff 28. November 2011).

184. Schweizerische Konferenz der kantonalen Erziehungsdirektoren. Lehrpläne in der Schweiz. Internet: http://www.edk.ch/dyn/18094.php (letzter Zugriff 28. November 2011).

185. Schweizerische Konferenz der kantonalen Erziehungsdirektoren. Lehrpläne und Lehrmittel. Internet: http://www.edk.ch/dyn/12927.php (letzter Zugriff 28. November 2011).

186. Schweizerische Konferenz der kantonalen Erziehungsdirektoren. Lehrplan 21. Internet: http://www.lehrplan.ch (letzter Zugriff: 28. November 2011).

187. Schweizerische Konferenz der kantonalen Erziehungsdirektoren. Bildung für Nachhaltige Entwicklung. Internet: http://www.edk.ch/dyn/12048.php (letzter Zugriff 28. November 2011)

188. Geschäftsstelle der deutschsprachigen EDKRegionen, ed. Grundlagen für den Lehrplan 21, verabschiedet von der Plenarversammlung der deutschsprachigen EDK-Regionen am 19.3.2010. Luzern: Geschäftsstelle der deutschsprachigen EDK-Regionen, 2010. Internet: http://www.lehrplan.ch/sites/default/ files/Grundlagenbericht.pdf (letzter Zugriff 28. November 2011).

189. Conférence Intercantonale de l'Instruction Publique de la Suisse romande et duTessin. Plan d'études romand (PER). Internet: http://www.plandetudes.ch/ (letzter Zugriff 28. November 2011).
190. Cloetta B, Spörri-Fahrni A, Spencer B, Ackermann G, Broesskamp-Stone U, Ruckstuhl B. Anleitung zum Ergebnismodell von Gesundheitsförderung Schweiz. Modell zurTypisierung von Ergebnissen der Gesundheitsförderung und Prävention. Zweite überarbeitete Version. Bern: Gesundheitsförderung Schweiz, 2005.

Internet: http://www.gesundheitsfoerderung.ch/ pdf_doc_xls/d/gesundheitsfoerderung_und_ praeventition/tipps_tools/N107713_

Ergebnismodell_Anleitung_de.pdf (letzter Zugriff 28. November 2011).

191. Gesundheitsförderung Schweiz. Best Practice Kriterien (Best Practice-Konzept Gesundheitsförderung Schweiz). Bern: Gesundheitsförderung Schweiz, 2007.

Internet: http://www.gesundheitsfoerderung.ch/ pdf_doc_xls/d/gesundheitsfoerderung_und_ praeventition/tipps_tools/20100623_BestPractice-Kriterien-d_Version-1.1.pdf (letzter Zugriff 28. November 2011).

192. Ostschweizer Verein für das Kind. Miges Balù 1 und II, Projektabschlussbericht. St. Gallen: Ostschweizer Verein für das Kind, 2008.

193. Puder JJ, Marques-Vidal P, Schindler C et al. Effect of multidimensional lifestyle intervention on fitness and adiposity in predominantly migrant preschool children (Ballabeina): cluster randomised controlled trial. BMJ 2011;343:d6195.

194. Van Cauwenberghe E, Maes L, Spittaels $\mathrm{H}$ et al. Effectiveness of school-based interventions in Europe to promote healthy nutrition in children and adolescents: systematic review of published and "grey» literature. Br J Nutr 2010;103(6):781-97.

195. Mooney E, Kelly-Blakeney E, Mc Cloat A, Black D. Primary SchoolTeachers' experiences of teaching healthy eating within the curriculum. A report for the Standing Conference on Teacher Education North and South (SCOTENS). Northern Ireland: The Centre for Cross Border Studies, 2011. 
196. Robert Koch Institut. Erkennen - Bewerten Handeln: Zur Gesundheit von Kindern und Jugendlichen in Deutschland. Berlin und Köln: Bundeszentrale für gesundheitliche Aufklärung, 2008.

197. Haefliger-Rossier F. Projet Senso5. Cinq sens pour apprendre...; Cinq sens pour manger...; Cinq sens pour le plaisir. 2010.

198. De Bourdeaudhuij I, van Cauwenberghe E, Spittaels $\mathrm{H}$ et al. School-based interventions promoting both physical activity and healthy eating in Europe: a systematic review within the HOPE project. Obes Rev 2011;12(3):205-16.

199. Jaime PC, Lock K. Do school based food and nutrition policies improve diet and reduce obesity? Prev Med 2009;48(1):45-53.

200. Doak CM, VisscherTL, Renders CM, Seidell JC. The prevention of overweight and obesity in children and adolescents: a review of interventions and programmes. Obes Rev 2006;7(1):111-36.

201. BrownT, Summerbell C. Systematic review of school-based interventions that focus on changing dietary intake and physical activity levels to prevent childhood obesity: an update to the obesity guidance produced by the National Institute for Health and Clinical Excellence. Obes Rev 2009;10(1):110-41.

202. Kropski JA, Keckley PH, Jensen GL. School-based obesity prevention programs: an evidence-based review. Obesity 2008;16(5):1009-18.

203. Luckner H, Moss JR, Gericke CA. Effectiveness of interventions to promote healthy weight in general populations of children and adults: a meta-analysis. Eur J Public Health (in press).

204. Gonzalez-Suarez C, Worley A, Grimmer-Somers K, Dones V. School-based interventions on childhood obesity: a meta-analysis. Am J Prev Med 2009;37(5):418-27.

205. European Commission. Towards a possible European School Fruit Scheme. Brussels: European Commission, 2008. Internet: http://ec.europa.eu/agriculture/fruitand-vegetables/school-fruit-scheme/documents/ public/consultation_en.pdf (letzter Zugriff 28. November 2011).
206. Rat der Europäischen Union. Verordnung (EG) Nr. 13/2009 vom 18. Dezember 2008. Amtsblatt der Europäischen Union, 2009. Internet: http://eur-lex.europa.eu/LexUriServ/ LexUriServ.do?uri=OJ:L:2009:005:0001:0004: DE:PDF (letzter Zugriff 28. November 2011).

207. Bundesministerium für Ernährung, Landwirtschaft und Verbraucherschutz. Gesetz zur Durchführung gemeinschaftsrechtlicherVorschriften über das Schulobstprogramm (Schulobstgesetz SchulObG) vom 29. September 2009. Bonn: Bundesministerium für Ernährung, Landwirtschaft und Verbraucherschutz, 2009.

Internet: http://npl.ly.gov.tw/pdf/7052.pdf (letzter Zugriff 28. November).

208. 5 am Tag e.V. Abschlussbericht zum Pilotprojekt Schulfrucht im Rahmen des Nationalen Aktionsplans. Band I. Mainz, 2009. Internet: http://www.5amtag-schulfrucht.de/ fileadmin/user_upload/Presse/Pressemeldungen/ Abschlussbericht_Schulfrucht_Band1.pdf (letzter Zugriff 28. November 2011).

209. De Sa J, Lock K. Will European agricultural policy for school fruit and vegetables improve public health? A review of school fruit and vegetable programmes. Eur J Public Health 2008;18(6):558-68.

210. Task Force on Community Preventive Services. Guide to Community Preventive Services. Obesity prevention and control: interventions in community settings.

Internet: http://www.thecommunityguide.org/ obesity/communitysettings.html (letzter Zugriff 28. November 2011).

211. Ministère duTravail, de l'Emploi et de la Santé. Programme national nutrition santé 2011-2015. Paris: Ministère duTravail, de l'Emploi et de la Santé, 2011. Internet: http://www.sante.gouv.fr/IMG/pdf/ PNNS_2011-2015.pdf (letzter Zugriff 28. November 2011). 
212. Hercberg S. Eléments de bilan du PNNS (2001-2005) et propositions de nouvelles stratégies pour le PNNS2 (2006-2008). Pour une grande mobilisation nationale de tous les acteurs pour la promotion de la nutrition en France. Paris: Centre de Recherche en Nutrition Humaine d'lle-de-France, Unité de Surveillance et d'Epidémiologie Nutritionnelle, 2006. Internet: http://lesrapports.ladocumentationfrancaise.fr/BRP/064000322/0000.pdf (letzter Zugriff 28. November 2011)

213. Jourdain Menninger D, Lecoq G, Guedj J, Boutet P, Danel JB, Mathieu G. Evaluation du programme national nutrition santé PNNS2 2006-2010. Inspection générale des affaires sociales, Conseil général de l'alimentation, de l'agriculture et des espaces ruraux. Avril 2010.

Internet: http://lesrapports.ladocumentationfrancaise.fr/BRP/104000214/0000.pdf (letzter Zugriff 28. November 2011).

214. Legifrance. Loi n $2004-806$ du 9 août 2004 relative à la politique de santé publique. Article annexe. Internet: http://www.legifrance.gouv.fr/ affichTexteArticle.do;jsessionid=11BE590B269D C9B4736D5E1D77D9323F.tpdjo15v_3?idArticle= LEGIARTI000006697614\&cidTexte=LEGITEXT00 $0005823063 \&$ dateTexte $=20111123$ (letzter Zugriff 28. November 2011).

215. Daten zur Verfügung gestellt von der Programmleitung des Programme National Nutrition Santé (Frankreich). November 2011.

216. Department of Health. Change4life - Eat well, move more, live longer. Internet: http://www.nhs.uk/change4life/Pages/ change-for-life.aspx (letzter Zugriff 28. November 2011).

217. Department of Health. Change4life. Change4life one year on. In support of healthy weight, healthy lives. London: Department of Health, 2010. Internet: http://www.dh.gov.uk/prod_consum_dh/ groups/dh_digitalassets/@dh/@en/documents/ digitalasset/dh_115511.pdf (letzter Zugriff 28. November 2011).
218. Bundesministerium für Ernährung, Landwirtschaft und Verbraucherschutz, Bundesministerium für Gesundheit. IN FORM - Deutschlands Initiative für gesunde Ernährung und mehr Bewegung. Nationaler Aktionsplan zur Prävention von Fehlernährung, Bewegungsmangel, Übergewicht und damit zusammenhängenden Krankheiten. Internet: http://www.in-form.de/nc/profiportal/ in-form-presse/medien.html?tx_drblob_pi1 \% 5 BdownloadUid \%5D=4 (letzter Zugriff 28. November 2011).

219. Bundesministerium für Ernährung, Landwirtschaft und Verbraucherschutz, Bundesministerium für Gesundheit. IN FORM - Deutschlands Initiative für gesunde Ernährung und mehr Bewegung. Qualitätsstandards.

Internet: http://www.in-form.de/buergerportal/ in-form-sein/bewusste-ernaehrung/auswaertsessen/qualitaetsstandards-fuer-die-ausser-hausverpflegung.html (letzter Zugriff 28. November 2011).

220. Daten zur Verfügung gestellt von der Geschäftsstelle des nationalen Aktionsplans IN FORM (Deutschland). November 2011.

221. Harris JL, Schwartz MB, Brownell KD. Evaluating the nutrition quality and marketing of children's cereals. Yale University, Rudd Center for Food Policy and Obesity, 2009.

222. Romero-Fernández M, Royo-Bordonada MA, Rodríguez-Artalejo F. Compliance with self-regulation of television food and beverage advertising aimed at children in Spain. Public Health Nutr 2009;13(7):1013-21.

223. World Health Organization. Set of recommendations on the marketing of foods and non-alcoholic beverages to children. Geneva: World Health Organization, 2010.

224. Gesundheitsförderung Schweiz, Merck Sharp \& Dohme-Chibret AG. Die volkswirtschaftliche Bedeutung von Gesundheitskompetenz. Eine Studie, ein Gespräch am runden Tisch und drei Interviews. Zürich und Bern: Merck Sharp \& Dohme-Chibret AG, Gesundheitsförderung Schweiz, 2008. 
225. Schweizerische Gesellschaft für Ernährung .

Projekt «Healthy Choice Label».

Internet: http://www.sge-ssn.ch/de/wissenschaftund-forschung/sge-projekte/healthy-choice-label/ (letzter Zugriff6. Juni 2012).

226. Ferguson CJ, Muñoz ME, Medrano MR. Advertising influences on young children's food choices and parental influence. Journal Pediatr 2012;160(3):452-5.

227. Council on Communications and Media, StrasburgerVC. Children, adolescents, obesity, and the media. Pediatrics 2011;128(1):201-8.

228. Zimmerman FJ, Bell JF. Associations of television content type and obesity in children. Am J Public Health 2010;100(2):334-40.

229. University of Bologna. Review of policy actions, data available for their analysis and existing evaluations throughout Europe. Work Package 1: Benchmarking nutrition policies in Europe, their evaluation and identification of success and failures. Eat well, 2010. 


\section{Kapitel 1}

Tabelle 1.1:

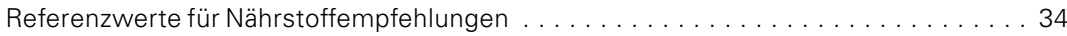

Tabelle 1.2: $\quad$ Ernährungsempfehlungen in der Schweiz, Deutschland, Frankreich und Italien:

Botschaften an die Bevölkerung und Mengenempfehlungen . . . . . . . . . . . . . 36

Abbildung 1.1: SGE-Ernährungsscheibe für Kinder. . . . . . . . . . . . . . . . . . . . 19

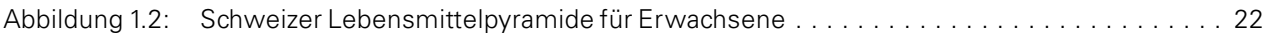

Abbildung 1.3: Ernährungskreis und dreidimensionale Lebensmittelpyramide der DGE . . . . . . . . 38

Abbildung 1.4: Die neue Lebensmittelpyramide der mediterranen Ernährung . . . . . . . . . . . . . 39

Abbildung 1.5: Plakat derTreppe, welche die 9 Ernährungsprinzipien gemäss PNNS

(Programme National Nutrition Santé) für Erwachsene darstellt . . . . . . . . . . . . . . . 40

\section{Kapitel 2}

Tabelle 2.1: $\quad$ Durchschnittlicher Verbrauch an Gemüse pro Kopf und Jahr in $\mathrm{kg} \ldots \ldots \ldots$. . . . . 58

Tabelle 2.2: $\quad$ Durchschnittlicher Verbrauch an Früchten pro Kopf und Jahr in $\mathrm{kg} \ldots \ldots \ldots \ldots$

Tabelle 2.3: Durchschnittlicher Verbrauch an Getreide, Kartoffeln und Hülsenfrüchten

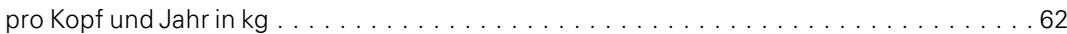

Tabelle 2.4: Durchschnittlicher Verbrauch an Milch und Milchprodukten pro Kopf und Jahr in kg . . . . 64

Tabelle 2.5: $\quad$ Durchschnittlicher Verbrauch an Fleisch, Fisch und Eiern pro Kopf und Jahr in kg . . . . . . 66

Tabelle 2.6: $\quad$ Durchschnittlicher Verbrauch an Nüssen und Samen pro Kopf und Jahr in $\mathrm{kg}$. . . . . . 67

Tabelle 2.7: $\quad$ Durchschnittlicher Verbrauch an Ölen und Fetten pro Kopf und Jahr in kg . . . . . . . 67

Tabelle 2.8: Durchschnittlicher Verbrauch an Zucker und Honig pro Kopf und Jahr in kg . . . . . . . . 68

Tabelle 2.9: Durchschnittlicher Verbrauch an nichtalkoholischen Getränken pro Kopf

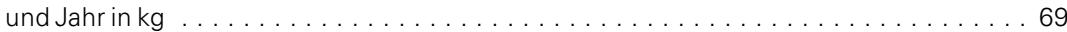

Tabelle 2.10: Durchschnittlicher Verbrauch an alkoholischen Getränken pro Kopf und Jahr in Liter . . . 69

Tabelle 2.11: Zuordnung von Lebensmitteln in der Schweizer Nährwertdatenbank zu den verbrauchten Lebensmitteln . . . . . . . . . . . . . . . . . . . . . . . . 76

Tabelle 2.12: Durchschnittlicher Verbrauch und angenäherter Verzehr an Energie, energieliefernden Nährstoffen und Wasser pro Person undTag (Durchschnitt 2007/08) . . . . . . . . . . . 82

Tabelle 2.13: Durchschnittlicher Verbrauch und angenäherterVerzehr an Fettsäuren, Cholesterin, Alkohol und Nahrungsfasern pro Person und Tag (Durchschnitt 2007/08) . . 86

Tabelle 2.14: DurchschnittlicherVerbrauch und angenäherterVerzehr an fettlöslichen Vitaminen pro Person undTag (Durchschnitt 2007/08) . . . . . . . . . . . . . . . . 88

Tabelle 2.15: DurchschnittlicherVerbrauch und angenäherter Verzehr an wasserlöslichen Vitaminen pro Person undTag (Durchschnitt 2007/08) . . . . . . . . . 90

Tabelle 2.16: Durchschnittlicher Verbrauch und angenäherter Verzehr an Mineralstoffen pro Person und Tag (Durchschnitt 2007/08) . 
Tabelle 2.17: Täglicher Verbrauch an Energie, energieliefernden Nährstoffen, Nahrungsfasern und Cholesterin im Vergleich zu den Angaben im 5., 4., 3. und 2. Schweizerischen Ernährungsbericht und zur gewichteten empfohlenen Zufuhr . . . . . . . . . . . . . 94

Tabelle 2.18: Täglicher angenäherter Verzehr an Energie, energieliefernden Nährstoffen, Nahrungsfasern und Cholesterin im Vergleich zu den Angaben im 5. und 3. Schweizerischen Ernährungsbericht und zur gewichteten empfohlenen Zufuhr. . . . . 95

Tabelle 2.19: Täglicher Verbrauch an Vitaminen im Vergleich zu den Angaben im 5. und 4. Schweizerischen Ernährungsbericht und zur gewichteten empfohlenen Zufuhr . . . . 96

Tabelle 2.20: Täglicher angenäherter Verzehr an Vitaminen im Vergleich zu den Angaben im 5. und 3. Schweizerischen Ernährungsbericht und zur gewichteten empfohlenen Zufuhr

Tabelle 2.21: Täglicher Verbrauch an Mineralstoffen im Vergleich zu den Angaben im 5., 4. und 2. Schweizerischen Ernährungsbericht und zur gewichteten empfohlenen Zufuhr 98

Tabelle 2.22: Täglicher angenäherter Verzehr an Mineralstoffen im Vergleich zu den Angaben im 5. und 3. Schweizerischen Ernährungsbericht und zur gewichteten empfohlenen Zufuhr . . . . . . . . . . . . . . . . . . . . . . . . . . . . . . . . . . . . . 99

Tabelle 2.23: Mittels Food Frequency Questionnaire erhobene durchschnittliche Nährstoffeinnahme im Jahr 2003 bei 401 Frauen im Alter von 75-87 Jahren . . . . . . . . . . . . . . . 100

Tabelle 2.24: Durchschnittliche Nährstoffeinnahme von normalgewichtigen Kindern in der Deutschschweiz im Alter von 6-14 Jahren . . . . . . . . . . . . . . . . . . . . . . . . 101

Tabelle 2.25: Verbrauch von Lebensmitteln durch die Schweizer Bevölkerung im Vergleich zum Verzehr von Lebensmitteln durch die europäische Bevölkerung (Angaben in kg pro Kopf und Jahr) . . . . . . . . . . . . . . . . . . . . . . . . . . 104

Tabelle 2.26: Täglicher Verbrauch und angenäherter Verzehr an Energie und energieliefernden Nährstoffen durch die Schweizer Bevölkerung im Vergleich zu den Angaben im Europäischen Ernährungs- und Gesundheitsbericht 2009 . . . 104

Tabelle 2.27: Durchschnittlicher Verbrauch und angenäherter Verzehr an Vitaminen durch die Schweizer Bevölkerung im Vergleich zu den Angaben im Europäischen Ernährungs- und Gesundheitsbericht 2009 . . . . . . . . . . . . . . . . . . . . . . 105

Tabelle 2.28: Durchschnittlicher Verbrauch und angenäherter Verzehr an Mineralstoffen durch die Schweizer Bevölkerung im Vergleich zu den Angaben im Europäischen Ernährungs- und Gesundheitsbericht $2009 \ldots \ldots \ldots \ldots \ldots \ldots$

Abbildung 2.1: Verschiedene Methoden zur Erhebung von Ernährungsdaten . . . . . . . . . . 52

Abbildung 2.2: Prozentuale Veränderungen im Pro-Kopf-Verbrauch einzelner Lebensmittelgruppen 2007/08 im Vergleich zu 2001/02

Abbildung 2.3: Verbrauchsmengen der verschiedenen Lebensmittelgruppen von 1979/80 bis $2007 / 08$ (in kg pro Kopf und Jahr).

Abbildung 2.4: Jährlicher Pro-Kopf-Verbrauch alkoholischer Getränke in der Schweiz zwischen 1990 und 2010 
Abbildung 2.5: Häufigkeit des Alkoholkonsums nach Geschlecht (Gesamtbevölkerung ab 15 Jahren) zwischen 1992 und 2007 . . . . . . . . . . . . . . . . . . . . . . . . . . . . . . . 72

Abbildung 2.6: Prozentuale Anteile der 11-bis 15-jährigen Knaben und Mädchen mit wöchentlichem Alkoholkonsum zwischen 1986 und 2010 . . . . . . . . . . . . . . . . . . . . . . . . . . . 73

Abbildung 2.7: Prozentualer Beitrag der einzelnen Lebensmittelgruppen zum Verbrauch von Energie, energieliefernden Nährstoffen, Nahrungsfasern und Cholesterin . . . . . . . 78

Abbildung 2.8: Prozentualer Beitrag der einzelnen Lebensmittelgruppen zum Verbrauch von Vitaminen . . . . . . . . . . . . . . . . . . . . . . . . 79

Abbildung 2.9: Prozentualer Beitrag der einzelnen Lebensmittelgruppen zum Verbrauch von

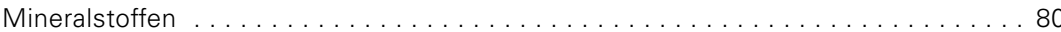

Abbildung 2.10: AngenäherterVerzehr an Energie und prozentualer Anteil der energieliefernden Nährstoffe pro Person undTag in den Jahren 1987, 2001/02 und 2007/08

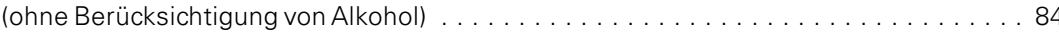

Abbildung 2.11: Anteil Personen, die nicht auf bestimmte Sachen in ihrer Ernährung achten, aufgeschlüsselt nach Sprachregion und Erhebungsjahr . . . . . . . . . . . . . . . . . . 109

Abbildung 2.12: Abfrage des Ernährungswissens der Bevölkerung in der Deutschschweiz . . . . . . . . . 110

Abbildung 2.13: Nutzung von Nährwertinformationen . . . . . . . . . . . . . . . . . . . 112

Abbildung 2.14: Konsumhäufigkeit von Haupt- und Zwischenmahlzeiten bei Frauen und Männern. . . . . . 115

Abbildung 2.15: Verpflegung in Restaurant, Kantine / Mensa und Bistro . . . . . . . . . . . . . . . 116

Abbildung 2.16: Konsum von Gemüse und Gemüsesäften, Früchten und Fruchtsäften, Fisch sowie Fleisch und Wurstwaren in den drei Sprachregionen . . . . . . . . . . . . . . . 117

Abbildung 2.17: Konsumhäufigkeit von Guetzli, Keksen, süssen Backwaren sowie Schokolade, Pralinen und Schokoriegeln . . . . . . . . . . . . . . . . . . . . . . . . . . . . 118

\section{Kapitel 3}

Tabelle 3.1: $\quad$ Prävalenz von erfragten Erkrankungen und Störungen bei Personen ab 15 Jahren in Privathaushalten in der Schweiz, nach Geschlecht, 1992-2007. . . . . . . . . . . . . . . 132

Tabelle 3.2: Definition von BMI-Klassen bei Erwachsenen nach WHO . . . . . . . . . . . . . . . . 134

Tabelle 3.3: $\quad$ Prävalenz von Übergewicht und Adipositas bei Erwachsenen, Kindern und Jugendlichen in der Schweiz: Neuere Datenquellen (Auswahl, seit 2003).

Tabelle 3.4: Altersstandardisierte Mortalitätsraten ernährungsabhängiger Krankheiten pro 100'000 Männer oder Frauen in der Schweiz, 1980-2008

Tabelle 3.5: Prävalenz von Risikofaktoren für Herz-Kreislauf-Krankheiten nach Geschlecht in zwei Studienpopulationen, 1993-2007

Tabelle 3.6: Häufigste Krebsformen in der Schweiz (Inzidenz und Mortalität pro 100'000 Männern oder Frauen), 5-Jahres-Perioden zwischen 1983-2007 . . . . . . . . . . . . 152

Tabelle 3.7: Häufigkeit von Lebensmittelunverträglichkeiten in der Schweiz . . . . . . . . . 163

Tabelle 3.8: $\quad$ Auswahl von häufigen kreuzreagierenden Pollen- und Lebensmittelallergien . . . . . . 164 
Tabelle 3.9: Häufigkeit von gestörtem Essverhalten in der Schweiz, Daten aus den Jahren 2002-2010 . . . . . . . . . . . . . . . . . . . . . . . . . . . . 167

Tabelle 3.10: Häufigkeit von Mangelernährung in der Schweiz, Daten aus den Jahren 2003-2010 _ . 172

Tabelle 3.11: Vitamin-D-Status verschiedener Bevölkerungsgruppen der Schweiz, Daten aus den Jahren $2004-2010$. . . . . . . . . . . . . . . . . . . . . . . . . . . . . . . . . 176

Tabelle 3.12: Prävalenz von Eisenmangel, Anämie und Eisenmangelanämie in der Schweizer Bevölkerung: Resultate einzelner Studien (Daten aus den Jahren 1996-2009) . . . . . . 180

Tabelle 3.13: Kariesprävalenz in der Schweiz nach Bevölkerungsgruppe, Daten aus den Jahren 1999-2006 . . . . . . . . . . . . . . . . . . . . . . . . . . . . . . . . . . . . . . 183

Abbildung 3.1: Prävalenz von Übergewicht und Adipositas kombiniert (BMI $\left.\geq 25 \mathrm{~kg} / \mathrm{m}^{2}\right)$ im Kanton Zürich und in der übrigen Deutschschweiz, nach Geschlecht, 1992-2009 . . . . . . . . . 138

Abbildung 3.2: Prävalenz von Übergewicht und Adipositas kombiniert (BMI $\left.\geq 25 \mathrm{~kg} / \mathrm{m}^{2}\right)$ bei Schülern der 4. und 8. Klasse nach Nationalität (Schweizer oder Ausländer) in der Stadt Zürich, Schuljahre 1994/95 bis 2009/10 . . . . . . . . . . . . . . . . . . . . . . 139

Abbildung 3.3: Relatives Sterberisiko nach BMI-Klasse bei MONICA-Teilnehmern nach Raucherstatus . . . . . . . . . . . . . . . . . . . . . . . . . . . . . . . . . 142

Abbildung 3.4: Prävalenz von Diabetes mellitus in der Schweiz aufgrund unterschiedlicher Quellen, nach Alter, Geschlecht und Jahr, Daten aus den Jahren 1995-2008 . . .

Abbildung 3.5: 25-Hydroxy-Vitamin-D-Konzentrationen im Serum von gesunden, erwachsenen Blutspendern, Dezember 2009 bis November 2010

\section{Kapitel 4}

Tabelle 4.1: Gesetzliche Verankerung von Gesundheitsförderung und Prävention in den Kantonen, Stand 2010 . . . . . . . . . . . . . . . . . . . . . . . . . 214

Tabelle 4.2: $\quad$ Übersicht über laufende nationale und überkantonale Ernährungsprojekte in der Schweiz, Stand November 2011 . . . . . . . . . . . . . . . . . . . . . 228

Tabelle 4.3: Übersicht über laufende Ernährungsprojekte in derWestschweiz und / oder im Tessin, Stand November 2011 . . . . . . . . . . . . . . . . . . . . . . . . . 234

Tabelle 4.4: $\quad$ Übersicht über laufende Ernährungsprojekte in der Deutschschweiz, Stand November 2011 . . . . . . . . . . . . . . . . . . . . . . . . . . . . . . . . . 238

Tabelle 4.5: $\quad$ Auswertung von parlamentarischen Vorstössen zwischen 1990 und 2010 . . . . . . . 249

Tabelle 4.6: Gesundheitsgesetze in den Kantonen, Stand Oktober $2011 \ldots \ldots \ldots \ldots$. . . . . . . 251

Tabelle 4.7: Dringliche Massnahmen für die Schweiz und Verantwortlichkeiten . . . . . . . . . . 262 


\begin{tabular}{|c|c|}
\hline $25(\mathrm{OH}) \mathrm{D}$ & 25-Hydroxy-Vitamin-D \\
\hline AN & Anorexia nervosa \\
\hline BAG & Bundesamt für Gesundheit \\
\hline BASPO & Bundesamt für Sport \\
\hline BED & Binge-Eating-Störung (Binge Eating Disorder) \\
\hline BMI & Body-Mass-Index \\
\hline BN & Bulimia nervosa \\
\hline $\mathrm{Cl}$ & Confidence Interval (Vertrauensintervall) \\
\hline Colaus & Cohorte Lausannoise \\
\hline $\mathrm{DACH}$ & Deutschsprachige Gesellschaften für Ernährung (Deutschland, Österreich, Schweiz) \\
\hline DACH-Referenzwerte & $\begin{array}{l}\text { Referenzwerte für die Nährstoffzufuhr der deutschsprachigen Gesellschaften für } \\
\text { Ernährung }\end{array}$ \\
\hline DGE & Deutsche Gesellschaft für Ernährung \\
\hline DHA & Docosahexaensäure (docosahexaenoic acid) \\
\hline EDI & Eidgenössisches Departement des Innern \\
\hline EEK & Eidgenössische Ernährungskommission \\
\hline EFSA & Europäische Lebensmittelsicherheitsbehörde (European Food Safety Authority) \\
\hline EK SGP & Ernährungskommission der Schweizerischen Gesellschaft für Pädiatrie \\
\hline ENHR II & $\begin{array}{l}\text { Europäischer Ernährungs- und Gesundheitsbericht } 2009 \text { (European Nutrition and } \\
\text { Health Report 2009) }\end{array}$ \\
\hline EPA & Eicosapentaensäure (eicosapentaenoic acid) \\
\hline ETH & Eidgenössische Technische Hochschule \\
\hline EU & Europäische Union \\
\hline FAO & Food and Agriculture Organization \\
\hline FFQ & Food Frequency Questionnaire \\
\hline fial & Foederation der Schweizerischen Nahrungsmittel-Industrien \\
\hline GDA & Guideline Daily Amount \\
\hline GDK & $\begin{array}{l}\text { Schweizerische Konferenz der kantonalen Gesundheitsdirektorinnen und } \\
\text {-direktoren }\end{array}$ \\
\hline HarmoS-Konkordat & Interkantonale Vereinbarung über die Harmonisierung der obligatorischen Schule \\
\hline HBSC & Health Behaviour in School-aged Children \\
\hline HDL & High Density Lipoprotein \\
\hline ICD & International Classification of Diseases \\
\hline IE & Internationale Einheiten \\
\hline ISSFAL & International Society for the Study of Fatty Acids and Lipids \\
\hline KAP & Kantonale Aktionsprogramme \\
\hline KVG & Bundesgesetz über die Krankenversicherung \\
\hline LDL & Low Density Lipoprotein \\
\hline LMG & Lebensmittelgesetz \\
\hline
\end{tabular}




$\begin{array}{ll}\text { MONICA } & \text { Monitoring of trends and determinants in cardiovascular disease } \\ \text { MOSEB } & \text { Monitoring-System Ernährung und Bewegung } \\ \text { MUFA } & \text { Einfach ungesättigte Fettsäuren (mono-unsaturated fatty acids) } \\ \text { NGO } & \text { Nichtregierungsorganisation (Non-Governmental Organization) } \\ \text { NPEB } & \text { Nationales Programm Ernährung und Bewegung (NPEB) } \\ \text { OECD } & \text { Organisation for Economic Co-operation and Development } \\ \text { ON } & \text { Orthorexia nervosa } \\ \text { PAL } & \text { Physical Activity Level } \\ \text { PEP } & \text { Fachstelle Prävention Essstörungen Praxisnah } \\ \text { PNNS } & \text { Programme National Nutrition Santé } \\ \text { PUFA } & \text { Mehrfach ungesättigte Fettsäuren (poly-unsaturated fatty acids) } \\ \text { RE } & \text { Retinoläquivalente } \\ \text { SEB } & \text { Schweizerischer Ernährungsbericht } \\ \text { SFA } & \text { Gesättigte Fettsäuren (saturated fatty acids) } \\ \text { SGB } & \text { Schweizerische Gesundheitsbefragung } \\ \text { SGE } & \text { Schweizerische Gesellschaft für Ernährung } \\ \text { SGP } & \text { Schweizerische Gesellschaft für Pädiatrie } \\ \text { SNC } & \text { Swiss National Cohort } \\ \text { SMASH } & \text { Swiss Multicenter Adolescent Survey on Health } \\ \text { TFA } & \text { Transfettsäuren (trans-fatty acids) } \\ \text { UL } & \text { Tolerable Upper Intake Level } \\ \text { WHA } & \text { World Health Assembly } \\ \text { WHO } & \text { Weltgesundheitsorganisation (World Health Organization) } \\ \text { ZÜGS } & \text { Zürcher Übergewichtsstudie } \\ & \end{array}$


24-h-Recall

52, 93, 101, 103, 107, 108, 119

25-Hydroxy-Vitamin-D

$3,175,176,177,178$

A

Abendessen

109, 114

Acrylamid

154

actionsanté

220, 224, 260, 263, 270

Adipositas

$\mathrm{VI}, 6,7,8,43,51,109,128,129,132-156,159$, $170,171,173,179,190,204,210,211,215,216$, $217,223,236,238,254,258,259,260$

Adipositasprävalenz $6,9,128,131,135,137,138,139,143,145,150$, 186

Adipositasprävention

$7,143,159,253$

Agrarpolitik

102,104-106, 248, 256, 259, 262

Agrarstatistik

4, 50, 52-54, 59, 61, 62, 65-69, 102,104-106, 119

Aktionsplan Ernährung und Gesundheit 41

Aktionsplan für die globale Strategie zur Prävention und Kontrolle von nichtübertragbaren Krankheiten

215,216

Aktionsplattform Ernährung, Bewegung und Gesundheit 216

Aktionsversprechen

$216,220,224,260,262$

Alkohol

$\mathrm{VI}, 7,21,25-27,30,31,70-72,82,84-86,88,90$, $94,95,105,116,128,131,140,148,151-156$, $158,160,212,213,215,217,238,249$ alkoholische Getränke

$4,5,21,37,50,54-56,69-71,74,77,81-86,88$, $90,92,83,84,94-99,104,116,140,148,154$, 186,221

Allergie

$17,34,128,163,164,165$

Allergieprävention

18

Allianz Ernährung

227

Allianz Gesunde Schweiz 227

Allianz Gesundheitskompetenz 227

ältere Menschen $2,4,5,7,8,23,30,31,100,103,128,138,141$, $155,156,160,161,173,176,177,211,229,244$

Anämie

93, 179, 180

Anorexia nervosa (AN) 166-171

atherosklerotische Krankheiten 28

Ausgaben für Lebensmittel 220,221

Ausser-Haus-Konsum 70

Ausser-Haus-Verpflegung $12,50,114,118,210,220,257,260,262,264$

\section{B}

Beikost

$14,17,18,98,165$

Beschränkung derVermarktung von Lebensmitteln und nichtalkoholischen Getränken 210, 260, 262

Bewegter Lebensstart 238 
Bewegung

$8,12,15,20,23,24,35,37,41,51,140,143,156$, $160,210,215-218,223-225,227-244,249,250$, $252,256,257,264$

Bien manger à petit prix 228

Bildung $3,8,11,12,41,144,149,151,155,184,186$, $212,213,216,218,221,223-228,230,232,234$, 238, 240, 244, 248, 252, 253, 256, 261, 262, 264

Bildung für Nachhaltige Entwicklung (BNE) 252

bildung + gesundheit Netzwerk Schweiz 226, 255

Bildungsniveau $3,4,6,9,10,11,51,74,108,118,119,128,141$, $143,151,168,218,258$

Bildungspolitik 12,259

\section{Binge-Eating-Störung (BED)} 168-171

Blutdruck 29, 30, 31, 128, 131, 144, 145, 146, 148, 150

Bluthochdruck $\mathrm{VI}, 6,30,31,132,144-146,149,161,181$

Blutzuckerspiegel 156

Body-Mass-Index (BMI) 77, 133-136, 138-142, 145, 146, 153, 158, 169, 176

Bodytalk PEP 233, 238

Brot $20,31,36,60,91,112,166$

Bulimia nervosa (BN) 168-171

Bundesamt für Gesundheit (BAG) $\mathrm{VI}, 2,15,223$

Bundesgesetz über die technischen Handelshemmnisse (THG) 247
Bundesverfassung

$12,27,211,212$

Bus Santé

$57,71,91,96,136,140,144,145,146,158$

\section{C}

Calcium

$5,7,14,20,24,27,75,79,80,91,92,98,99,100$, $101,106,128,149,152,159,160,161,165,247$

Caritas-Markt-gesund! 228

Carotinoide

$5,79,85,88,96,97,105$

Cassis-de-Dijon-Prinzip 246

Change4Life 257

Charta $215,216,217,256,257$

Chlorid $5,31,79,80,90,92,98,99$

Cholesterin $\mathrm{VI}, 6,28,29,78,80,83,84,86,94,95,131,132$, $144-148,154$

Codex Alimentarius 16

CoLaus $33,71,75,94,136,144,145,146,149,154,160$ Convenience-Produkte $12,50,116,118,210,260,262,264$

D

Datenquellen $108,130,132,134,136,144,156$

Deutsche Gesellschaft für Ernährung (DGE) 36

Diabetes

VI, 6-8, 21, 28, 30, 128, 131, 132, 144-146, 149, $153,156,161,170,210$ 
Diabetes mellitus Typ 1 156, 170, 176

Diabetes mellitus Typ 2 $\mathrm{VI}, 7,21,128,144,153,156,158,159$

Dioxine 3,32

Docosahexaensäure (DHA) 29

\section{$\bar{E}$}

\section{Eicosapentaensäure (EPA)} 29

Eidgenössische Ernährungskommission (EEK) $2,15,36,85$

Eier $4,21,25,26,35,37,55,58,63,66,76,78-80,82$, $83,84,86,88,90,91,92,99,103,104,163,164$, 182,219

einfach ungesättigte Fettsäuren (MUFA) $28,29,35,78-80,83,86,94,95,150$

Eisen

$5,8,14,20,24,26,50,75,78-80,92-94,95$, $98-102,106,119,171,179,186$

Eisenmangel $93,94,107,128,179,180,181$

Energie $2-6,11,14,17,18,20-25,27-30,34,35,38,50$, $51,53,74-85,94,95,100-102,104,107,112$, $114,119,143,150,156,158,172,247$

Energiebedarf $17,20,22,23,30,94,95,166$

Energiebilanz $15,35,140,216$

Energiedichte $30,141,219,256,262$

Energieeinnahme 140,153

energieliefernde Nährstoffe 50, 74, 75, 78, 82, 84, 94, 95, 100, 102, 104, 107

Energieverbrauch $30,50,81,94,105,140$
Energy Drinks 70,185

Erfolgsfaktor $10,210,225,253-257,261$

Ergebnismodell 253

ernährungsabhängige Krankheiten VI, 2, 5, 14-16, 21, 31, 40, 41, 130, 131, 133, 142, $144,186,210,216,224,246,258$

Ernährungsbewusstsein $3,9,11,108,111,113,118,119,258$

Ernährungsbildung $11,218,221,252,256,258$

Ernährungsempfehlungen VI, 2, 3, 11, 13-17, 20, 24, 32, 34, 35-38, 40, 41, $53,55,63,84,113,119,211,215,217,220-222$, $248,255,258$

Ernährungserhebung $4,51,107,108,112,119,120,225$

Ernährungserhebungsmethoden $51,52,105,107$

Ernährungsforschung 15,248

Ernährungskommunikation 11,258

Ernährungskompetenz $10,11,210,218,219,221,262,264$

Ernährungskreis 38

Ernährungspolicy 41,217

Ernährungspolitik 41,212

Ernährungsscheibe 14,19

Ernährungsstrategie 12

Ernährungsverhalten VI, 9, 11, 14, 16, 27, 41, 51-53, 107-111, 114, $119,166,210,216,219-222,230,257,258,261$ 
Ernährungswissen

$3,4,9,27,41,50,108-113,118,119,222,256$

\section{Erosion}

$17,182,184-186$

essentielle Fettsäuren

28, 29

\section{Essstörung}

$128,130,131,141,166-171$

Ess- und Trinkverhalten

20, 130, 131

Essverhalten

$128,141,166-170,222,223$

\section{Europa}

$\mathrm{VI}, 4,8,10,11,14,16,31,35,40,50,53,86$, $102-107,130,139,151,161-164,173,202,210$, 212, 215-217, 223, 247, 255, 256, 261

Europäische Charta zur Bekämpfung der Adipositas 215, 217

Europäische Lebensmittelsicherheitsbehörde (EFSA)

34, 102

Europäischer Aktionsplan Nahrung und Ernährung 35,216

European Food Information Resource (EuroFIR) 107

European Nutrition and Health Report II (ENHR II) 103,248

Evaluation

$51,100,224-245,253,254,256,257$

\section{F}

Fachgesellschaft

$2,14,165,215$

Fachstelle Prävention Essstörungen Praxisnah (PEP)

$223,238,240$

familienergänzende Betreuungseinrichtungen $10,11,232,240,254,258,263$

Fast Food $20,21,116,141,220$ feel-ok.ch

238

Fehlernährung

129

Fertigmahlzeiten

$12,31,116,117,210,260,262,264$

Fett

$2,27,28,35,37,50,80,82-84,94,95,100-102$, $104,110-113,117,128,147,158,160,186,210$, $219,247,260,262,264$

Fette

$2,4-6,24,28,30,34,37,50,54,56,65,67,74$, 77-83, 85-88, 90, 92, 103, 104, 140, 147, 150, 156,213

Fettsäuren

$24,26,28,29,50,65,75,80,83,86,113,147$, $150,155,158,160,186,215,219,247$

Fettstoffwechselstörung $128,145-146,150,158$

Fisch

$3,4,21,24,26,29,32,33,35,37,50,54-56,63$, $66,80,82,86,88-90,92,99,103,104,112,114$, $116,117,128,140,147,163,164,182,219$

fiskalische Massnahmen

219,250

fit-4-future

228,243

Fleisch

$4,18,21,24-27,35,37,50,54-56,63,66,74,76$, 78-80, 82-92, 94, 95, 103, 104, 110, 116, 117, $128,140,151,152,154,163,164,179,182$

Fleischprodukte $4,31,50,55,56,63,74,75,78-80,82-92,94,95$, $104,128,152,154$

Föderalismus

$11,210,212,253,256,259,261$

Foederation der Schweizerischen NahrungsmittelIndustrien (fial) 220

Folgenahrung $14,16,17$ 


\section{Folsäure}

$5,8,25,33,34,50,79,88,90,96,97,102,105$, $106,119,128,154,165,171,174,186$

Folsäuremangel 174

Folsäuresupplement $88,174,175$

Food Frequency Questionnaire (FFQ) 52, 100, 108, 111, 119

Food Literacy 218

Formation Générale 253

Forschung $8,28,51,166,212,216,248,253$

Fourchette verte 228,242

Front-of-Pack-Kennzeichnung $4,112,113,117,118,128,113,118,140,141$, $148,150,154,155,186,210,258,262,264$

\section{Früchte}

$\mathrm{VI}, 3,10,15,18,24,30,33,35,36,41,54,57,60$, $61,76,79,81,82,85,86,88-90,92,104,111$, $114,119,152,163-165,174,185,210,219,221$, $228,256,262,264$

Fruchtsaft $17,30,57,61,68,69,77,82,114,117,148$

Fructose 30, 68, 147, 148, 150, 158, 162, 198, 201

Fructoseintoleranz $162,163,165,166$

Fructosemalabsorption $162,163,165$

Fructoseunverträglichkeit 162

Früherkennung $128,143,149,156,159,161,165,186,246$

Frühstück $109,114,115,242,255$
Functional Food

33

\section{G}

Gastronomie

$12,111,119,220,260,263$

Gemüse

$\mathrm{VI}, 3,4,6,15,18,21,24,30,32,33,35,36,38$, $41,50,54-59,70,74,76,78-82,85-92,94$, $103-105,111,114,117,119,128,140,141,148$, $150,152,154,155,163-165,174,186,210,219$, $221,228,256,262,264$

gesättigte Fettsäuren (SFA)

$28,35,78,80,83,86,94,95,113,147,158,215$, 219,247

gestörtes Essverhalten 166, 167, 168, 169, 170, 222, 283

Gesundheitsabkommen 247

Gesundheitsfördernde Gemeinschaftsgastronomie $224,228,243,257,263$

Gesundheitsförderung $8,12,20,27,41,211-214,217,218,223,225$, 226-242, 246, 248-253, 255, 259, 261, 264

Gesundheitsförderung Schweiz $213,217,223,225,226,250,253,264$

Gesundheitsgesetz $213,214,250,251$

Gesundheitskompetenz $218,227,243$

Gesundheitskosten $6,8,133,142,210,211,219,265$

Gesundheitspolitik $12,41,212,224,248,249,259,264$

Gesundheitsschutz $16,212,213,219,247,259$

Getränk $4,5,19,21,37,50,51,54-56,68-71,74,75,77$, $80-86,88,90,92,94-99,104,112,116,141,148$, $154,185,186,221,222,240,256,262$ 
Getreide

$4,5,18,21,30,33,36,50,55-57,60,62,76$,

$78-82,85-88,90,92,95,103,104,112,113$, 174,182

Getreideprodukte

$19,21,30,36,165$

Gewicht

$37,129,132,134,135,158,168-170,239$,

gewichtete empfohlene Zufuhr

$50,77,81,82,87-99,102$

Globale Strategie für Ernährung, Bewegung und Gesundheit

215,217

\section{Glucose}

$30,68,147,150$

glykämischer Index

$29,85,153,158$

\section{GORILLA \\ 230, 233 \\ Guide école \\ 234 \\ H}

Handlungsempfehlungen

$10,210,211,257,264$

Handlungsfelder

223, 264

Harmos

221, 222, 252, 259

Health Behaviour in School-aged Children (HBSC)

$72,122,131,136,137,140,168,284$

Health Claim

247

Herz-Kreislauf-Krankheit

$\mathrm{VI}, 6-8,28,31,33,83,84,128,141,143-151$, $155,156,186,210$

Hirnschlag

$6,132,143,144,146,148,149$
Honig

$4,5,26,30,55,68,78-92$

Hülsenfrüchte

$4,18,21,30,36-38,50,55-57,59,60,62,76$, $78-80,82,86,88,90,92,103,150,164$

Hypertonie

VI, 6, 30, 31, 132, 144-146, 149, 161, 181

Hyperurikämie

145

I

Industrie

$112,118,119,220,224,229,259,260,262,263$

IN FORM

257

Insulinempfindlichkeit

$28,29,30,147$

Insulinresistenz

153, 158

Integrierte Versorgung

246

Interkulturelle Vermittler

$10,221,244,245,254$

Intervention

$8-11,210,211,225,238,239,246,248,253$, $254,261,262$

Inzidenz

$128,131,133,149,151,152,158,161,164,169$, $171,174,254$

Istituto Nazionale di Ricerca per gli Alimenti e la Nutrizione (INRAN)

36,39

$\mathbf{J}$

Jod

$5,8,14,37,50,80,93,95-99,102,106,107,119$, $128,150,171,181,186$

Jodmangel

181

Joghurt

$18,37,55,62,64$ 
Jugendliche

$2,14,20,31,103,133-136,138,140,147,160$, $166,175,176,216,223,227-242,255$

\section{$\mathbf{K}$}

Kaffee

$25,68,69,75,77,93,148,155$

Kalium

5, 79, 80, 90, 92, 98-100, 106

Kantonale Aktionsprogramme (KAP)

$9,225,226,231-245,235,245,250,264$

Karies

$17,128,182-185$

Kartoffeln

$4,19,20,21,30,36,55-60,62,76,78-80,82$, $86-88,90,92,103,117,154,164$

Käse

$25,31,37,54,63-65,76,91$

Kebab+

230

Kennzeichnung

$3,4,11,16,17,53,112,113,118,165,210,247$,

258, 262, 264

KidBalù

230

Kinder

3-12, 14, 18-20, 22, 31-33, 96, 97, 101, 103, $107,128,133-142,145,147,158,160,163-165$, $176,179,181,184,185,210,216,219-222,226$, 228-245, 254-256, 258-260, 264

Kinder und Jugendliche

$31,134-136,160,176,230-242,255$

klemon

238

Kohlenhydrate

2, 5, 27-30, 34, 50, 78-80, 83-85, 94, 95,

100-102, 104, 147, 148, 150, 154, 158, 183, 247

Konferenz der kantonalen

Gesundheitsdirektorinnen und -direktoren (GDK) 217,218
Konsumenten

$3,16,21,32,41,70,108,111,113,118,119,182$, 213, 223, 228, 247, 263

Konsumenteninformation

$210,216,224,260,262,264$

Konsumentenorganisationen 224, 263

Koronare Herzkrankheit $6,33,143,144,146,148$

Körpergewicht

$11,17,21,24-26,30,33,35,109,135,143,153$, $166,168,171,217,225,227,230,234-237,243$, $247,249,250,258$

körperliche Aktivität

$5,7,20,22,24,26,30,77,81,101,128,129,140$, $143,145,150,155,159-161,186,223,227,254$

Körperwahrnehmung 21, 128, 166

Kosten

$6,8,133,142,158,161,166,171,173,210,211$, $216,219,231,261,264$

Krankenversicherer

217,225

Krebs

$6-8,21,31,33,34,128-131,133,141,148,149$, $151-156,174,178,210$

L

Label

$4,112,113,228,263$

Lactose

162

Lactoseintoleranz 162-165

Landwirtschaft

$8,41,60,212,224,248,264$

Lebensmittelallergie 162-164, 166

lebensmittelbasierte Ernährungsempfehlungen $14,16,40,215$ 
Lebensmittelgesetz

$8,16,32,213,247$

Lebensmittelpyramide

$2,14,20-26,28,36,38,39,111,119,242$

Lebensmittelsicherheit

$32,34,51,212,216,219,224$

Lebensmittelunverträglichkeit

$128,130,162-166$

Lebensmittelverbrauch

$4,50,53,54,75,81,98,102,103,107,119,130$, 140

Lebensmittelverzehr

$4,11,51,52,101-103,107,108,175,186,210$, 257,261

Lebensphase

$11,26,27,29,221,258,262$

Lebenswelten

$218,219,255,258$

Lehrplan

$211,236,252,253,255,259$

Lehrplan 21
252

Lenkungssteuer

250

Les petits gourmets à table

234

$\alpha$-Linolensäure

29

Linolsäure

28,29

\section{M}

Magnesium

5, 80, 92, 98-102, 106, 171

Mahlzeitenverteilung 21

Makronährstoff $2,3,8,24,27,29$

Managed Care 246
Mangelernährung

$5,8,15,23,24,128,130,131,141,143,171-173$, 186

maximale Knochenmasse (peak bone mass) 20, 159

mehrfach ungesättigte Fettsäuren (PUFA)

$28,29,33,35,50,65,78,80,83,86,94,95,102$

meingleichgewicht

230, 232

metabolisches Syndrom

145, 148, 156

Methylquecksilber

33

Miges Balù

239, 255

Migrationsbevölkerung

162, 163, 228

Mikronährstoff

$5,11,50,51,74,75,78,100,102,107,119,154$

Milch

$3-5,17-19,24-27,37,50,55,56,62-64,74,76$, 78-99, 103, 104, 114, 119, 160, 163, 165, 182, $185,219,240$

Milchprodukt $3-5,19,21,24,26,27,35,37,50,55,56,62-64$, $74,76,78-95,103,104,110,111,114,119,147$, $165,182,185$

Mineralstoff $23,25,34,90,92,98,99,103,106$

Mineralstoffsupplement

$5,75,149$

Mineralwasser

$68-70,75,81,82,86,88,90,92$

Mittagessen 109, 114, 115

Monitoring-System Ernährung und Bewegung (MOSEB)

51,224

Morbidität

$5,131,143,147,161$ 


\section{Mortalität}

5, 128, 131-133, 142-145, 147-149, 151-153, 155,173

Movimento e gusto con l'equilibrio giusto! 234

multisektorale Gesundheitspolitik

$12,212,248,259,264$

Muttermilch

$3,17,25,32,181$

Muttermilchersatzpräparate 17

Mütter-Väterberaterinnen

$221,239,244,245$

Mütter-Väterberatungsstellen

$10,238,244,254$

\section{N}

n-3-Fettsäuren

$24,26,29,75,147,155$

n-6-Fettsäuren

$24,29,75,147$

Nachhaltigkeit

$27,212,225,237,253,255$

Nährstoffbasierte Ernährungsempfehlungen 16,40

Nährstoffzufuhr

$2,15,21,24,34,77,101$

Nahrungsfasern

$30,34,50,74,75,78-80,82,85,86,94,95$,

$100-102,128,147,150,152,165$

Nährwertdatenbank

$74-77,83,98,107$

Nährwertkennzeichnung

$3,11,16,53,112,113,118,210,258,262,264$

Nationale Ernährungserhebung

4, 107, 119, 225

Nationales Programm Ernährung und Bewegung (NPEB)

$8,12,35,41,223$
Natrium

$2,3,5,31,79,80,90-92,98,99,106,107$

Neuralrohrdefekt

$33,128,174$

NGO

$216,227,246,260$

NGO-Allianz Ernährung, Bewegung, Körpergewicht 227

Niacin

$5,79,87,90,96,97,105$

nichtalkoholische Getränke

$9,55,56,61,68,69,75,77-82,85,86,90,92,94$, $116,210,219,220,260,262,264$

Nitrat

32,33

Nitrosamine

33

Nüsse

$4,18,21,23,29,35,37,54-56,65,67,76,78-80$, $82,86,88,90,92,147,163,164$

NUTRIKID ${ }^{\circledR}$

230

Nutrinet

226

Nutrition Claim

247

o

Öle

$4-6,21,28,29,37,50,54-56,65,67,74,77-83$, $85,86,88,90,92,104,150$

Organochlorpestizide 32

Orthorexia nervosa (ON)

166, 169

Osteopenie

159

Osteoporose

7, 20, 128, 132, 159-162, 176, 177 
Ovo-Lacto-Vegetarier

$26,27,89$

ovo-lacto-vegetarische Ernährung

26

$\mathbf{P}$

Pantothensäure

$5,79,80,89,90,96,97$

Papperla PEP

232,240

Pausenverpflegung

240, 255

peak bone mass (maximale Knochenmasse) 20, 159, 198

pflanzliche Öle

$4,6,21,29,37,65,67,80,87,103,147,213$

Pharmaciens malins

234

Phosphor

$5,14,20,78,80,92,98-100,106$

physical activity level (PAL)

$22,77,81,94,95,101$

Plan d'études romand (PER) 253

Planificateur de menus pour camps 236

Policy

216, 225, 226, 253, 259

Portionengrösse

$9,12,26,52,53,260$

Prävalenz

5, 6, 128-149, 156-159, 162-164, 168, 171, 177,

$180,184,216,225,254,258,261$

\section{Prävention}

$8,12,16,41,51,130,150,155,159,165,174$, $182,186,210-218,223,225-227,238,240,242$, 246, 248-253, 255, 256, 259, 261, 264

Präventionsgesetz

$8,41,246,250,259$
Präventive Ernährungs- und Bewegungsberatung in der Schwangerschaft (PEBS)

240

Primano - Fördermodul Ernährung 240

Procap bewegt 232

Programme d'éducation nutritionnelle du canton du Jura

236

Programme National Nutrition Santé (PNNS) $36,40,48,256$

Protein

$2,5,7,14,20,23,24,26,27,30,34,50,78-80$, $82,84,94,95,100-102,104,128,159-161,172$

Purzelbaum

$233,240,243$

\section{$\overline{\mathbf{Q}}$}

Quecksilberrückstände 33

$\mathbf{R}$

Radix

$232,238,242,243$

Referenzwerte für die Nährstoffzufuhr der deutschsprachigen Gesellschaften für Ernährung (DACHReferenzwerte)

$2,3,15,24,27,28,31,32,34,40,77,81,94-101$, 105, 106

Regulierung

$219,225,250$

Reis

$36,60,62,76$

Retinoläquivalent (RE)

$85,88,96,97$

Risikofaktor

VI, 2, 5-7, 11, 14, 31, 128, 129, 131, 133, 134, $140,141,143-145,148,149,152,154,156,158$, $159,170,174,179,186$ 
Risikowahrnehmung

32

Rückstände

$17,32,33$

\section{$\mathbf{s}$}

Saccharose

$30,68,85,147,148$

Salz

$2,3,6,9,18,21,31,32,35,37,91,95-97,102$,

$107,113,115,117,128,129,147,150,152,161$, $181,186,210,215,217,220,224,247,260,262$, 264

\section{Salzstrategie}

$3,31,91,150,217,224$

Säugling

$2,3,5,8,14,16,17,18,25,29,32,181$

Säuglingsanfangsnahrung

$14,16,17$

Säuglingsfolgenahrung

16,17

Schnellverpflegung

$12,220,260$

Schnitz\&Schwatz

242

schnitz und drunder

232, 242

Schule

9-12, 20, 143, 184, 185, 216, 221, 222, 226-242, $252,255,256,258,259,263$

schule bewegt

232

Schulfruchtprogramm

$10,256,263$

Schulstufenkonzept Rüebli, Zimt \& Co.

242

\section{Schutzfaktor}

$129,130,140,141,144,148,151,152,154,156$, $158,159,160,169,172,174,175,179,181,182$, 184
Schweizer Haushalt-Panel (SHP)

131,136

Schweizerische Gesellschaft für Ernährung (SGE)

$2,14,15,19-23,36,120,230$

Schweizerische Gesundheitsbefragung (SGB)

$57,63,70,75,101,108,114,116,119,131-136$, $138,170,174$

Schweizerisches Netzwerk Gesundheitsfördernder Schulen (SNGS)

227

Schwermetall

3,32

sekundäre Pflanzeninhaltsstoffe

155

Selen

$5,8,26,75,98,99,128,152,171,181,182$

Selenmangel

181,182

Senso5

236, 255

Setting

$9,10,216,226,228,234,238,240,254-256$

Sirup

68

Snack

115,141

Snacking

20,21

\section{Sport}

$8,23,25,26,41,140,160,168,169,170,198$, $200,212,223,232,240$

Spurenelement

24

Steuern

213,219

Stillen

$2,3,14,17,18,24,25,27,30-33,81,97,101$, $109,128,129,160,165,175,181,226,234$

Stoffwechselstörung $145,170,171$ 
Suisse Balance

225,226

Supplement

$3,5,7,20,25,27,32,33,75,86,88,94,128,149$, $154,160,161,165,174-176,178,182$

Süssgetränk

$19,21,68,69,80,81,112,141,148,185,222$, $240,256,262$

\section{Süssigkeit}

$21,37,241$

Süssstoff 30

Swiss Multicenter Adolescent Survey on Health (SMASH) 166-169

\section{Swiss Pledge}

220

$\mathbf{T}$

Tacco \& Flip 242

Tagesstrukturen

$10,222,232,252,255$

TAVOLATA

244

Tee

$17,68,69,70,75,77,148$

Teigwaren $20,36,60,99,112,166,182$

tierische Fette

$4,55,65,67,79,103,128,147,150$

Tocopheroläquivalente

96,97

Tolerable Upper Intake Level (UL)

$25,30,33,34$

Transfettsäuren (TFA)

$9,28,75,147,150,213,215,220$

Trinkverhalten

$20,70,130,131$
Tutti Frutti

236

$\mathbf{U}$

Übergewicht

VI, 6-8, 21, 30, 31, 41, 51, 109, 117, 128, 129,

$131-159,179,186,210,216,217,223,225,236$,

254-256, 258, 259

Umfeld

$9,12,51,129,155,218,224,233,254,255,256$, 258,259

Umweltkontaminanten

3,32

Unterernährung

$5,8,14,15,23,24,128,130,131,141,143$,

$171-173,186$

Untergewicht

$31,109,132,133,134,135,136,137,141,143$,

168,238

Unterversorgung

$8,32,50,85,86,89,102,106,107,119,171,181$

V

Veganer

$26,27,89$

Vegetarier

$2,14,26,27,89$

Vegetarische Ernährung

$21,26,27$

Vereinigung der kantonalen Beauftragten für

Gesundheitsförderung (VBGF)

218

Verhaltensprävention

218, 223

Verhältnisprävention

$218,223,225$

Verzehrsverhalten

$4,50,53,108,111,112,118$

Vitalina - aktive Eltern für gesunde Kinder 244 
Vitamin A

$5,79,80,85,88,96,97,105$

Vitamin B1

5, 14, 20, 78, 79, 87, 90, 96, 97, 102, 105

Vitamin B2

$5,80,79,87,90,96,97,105$

Vitamin B6

5, 78-80, 87, 90, 96, 97, 100, 105

Vitamin B12

$5,24,27,33,79,89,91,96,97,171$

Vitamin C

$5,79,89,91,96,97,100,101,105,179,247$

Vitamin D

$2,3,5,7,8,18,20,32,79,80,86,89,96,97$,

$100-102,105,106,119,128,159,160,161,171$,

175-178, 186

Vitamin-D-Mangel

175-177

Vitamin $\mathrm{E}$

$5,87,89,96,97,100,105$

Vitaminsupplement

$25,33,154$

Vollkorn

$30,35,36,76,112,150$

Vorsorge

$16,185,211,213,250$

Vorstösse

$8,249,250$

$\mathbf{W}$

Wasser

$17,19,27,34-36,68,70,75,77,78,81,82,92$, 112,213

Weissbuch «Ernährung, Übergewicht, Adipositas:

Eine Strategie für Europa»

216, 217, 223

Weiterbildung für Hebammen, Still- und Mütterberaterinnen

244
Weltgesundheitsorganisation (WHO)

$2,3,14,16,17,28,29,31,32,35,41,85,91,134$, $161,179,210,212,215-217,224,260,261$

Werbung

$4,9,10,51,141,216,219,220,224,250,256$, $260,262,264$

Wirksamkeit

$10,12,143,181,210-212,219,222,223,246$, $248,253,255-257,260-262$

Wirkung

$9,10,28,30,31,85,86,178,186,210,211,226$, $235,253,261$

\section{Z}

Zahngesundheit

18, 171, 182-186, 207

Zink

$5,24,26,78,80,93,95,98,99,106,165,171$

Zöliakie

$162,163,165,166,170$

Zucker

$4,5,7,9,18,30,35,37,50,55,56,68,78-82,85$, $86,88,90,92,110-113,117,128,140,145,150$, $183,186,210,215,219,220,247,260,262,264$

Zuckeraustauschstoff 30

Zuckerstoffwechselstörung 158

Zusatzstoff 32,117

Zwischenverpflegung $18,114,115,184,241$ 



\section{Autor Zusammenfassung}

Dr. Michael Beer

Bundesamt für Gesundheit

Abteilung Lebensmittelsicherheit

3003 Bern

michael.beer(at)bag.admin.ch

\section{Autoren Kapitel 1}

Kapitelverantwortung

PD Dr. Jürg Lüthy

Salvias $\mathrm{GmbH}$

Tobelackerstrasse 14

8620Wetzikon

info(at)salvias.ch

Co-Autoren

Angelika Hayer

(Kapitel 1.3.2, 1.3.3, 1.3.4)

Schweizerische Gesellschaft für Ernährung SGE

Schwarztorstrasse 87

Postfach 8333

3001 Bern

a.hayer(at)sge-ssn.ch

Muriel Jaquet

(Kapitel 1.7)

Schweizerische Gesellschaft für Ernährung SGE

Schwarztorstrasse 87

Postfach 8333

3001 Bern

m.jaquet(at)sge-ssn.ch

\section{Autoren Kapitel 2}

Kapitelverantwortung

Alexandra Schmid

Agroscope Liebefeld-Posieux ALP

Schwarzenburgstrasse 161

3003 Bern

alexandra.schmid(at)alp.admin.ch

Co-Autoren

Prof. Dr. Christine Brombach

(Kapitel 2.5)

Zürcher Hochschule für AngewandteWissenschaften

Life Sciences und Facility Management

Campus Grüental

8820Wädenswil

broc(at)zhaw.ch

Dr. Sabine Jacob

(Berechnungen Verbrauchs- und Verzehrsdaten)

Steinbrüchelstrasse 69

8053 Zürich

sabine.jacob(at)hispeed.ch

Iso Schmid

(Aufbereitung und Zusammenstellung der

Verbrauchsdaten)

Schweizerischer Bauernverband

SBV Statistik

Lauerstrasse 10

5201 Brugg

iso.schmid(at)sbv-usp.ch

Dr. Robert Sieber

(Berechnungen Verbrauchs- und Verzehrsdaten)

Agroscope Liebefeld-Posieux ALP

Schwarzenburgstrasse 161

3003 Bern

robsieber(at)hotmail.com 
Prof. Dr. Michael Siegrist

(Kapitel 2.6)

ETH Zürich

Consumer Behavior

Universitätsstrasse 22

8092 Zürich

msiegrist(at)ethz.ch

\section{Autoren Kapitel 3}

Kapitelverantwortung

PD Dr. med. David Fäh

Universität Zürich

Institut für Sozial- und Präventivmedizin

Hirschengraben 84

8001 Zürich

david.faeh(at)uzh.ch

Co-Autor

Dr. Annette Matzke

(Kapitel 3.7-3.11)

Mattenstrasse 18F

3600Thun

a.matzke(at)hispeed.ch

\section{Autoren Kapitel 4}

\section{Kapitelverantwortung}

Christian Ryser

Schweizerische Gesellschaft für Ernährung SGE

Schwarztorstrasse 87

Postfach 8333

3001 Bern

ch.ryser(at)sge-ssn.ch
Co-Autoren

Dr. Alexia Fournier Fall

(Tabellen 4.2, 4.3)

Commission de prévention et de promotion

de la santé du GRSP

c/o Ligues de la Santé

Avenue de Provence 12

1007 Lausanne

cpps(at)fvls.vd.ch

Sophie Frei

(Tabellen 4.2, 4.4)

Suisse Balance

Die nationale Projektförderstelle Ernährung und

Bewegung

Effingerstrasse 40

Postfach 8172

3001 Bern

frei(at)suissebalance.ch

Andrea May

(Kapitel 4.3, 4.7)

polsan $\mathrm{GmbH}$

Fachstelle für Gesundheitspolitik

Effingerstrasse 54

3001 Bern

andrea.may(at)polsan.ch 


\section{Redaktionskommission}

Vorsitzender

Prof. Dr. med. Ulrich Keller

Endokrinologische Praxis

Missionsstrasse 24

4055 Basel

ulrich.keller(at)unibas.ch

\section{Mitglieder}

Evelyne Battaglia Richi

Studio di consulenza dietetica

Via Motta 15

6900 Lugano

evelyne.battaglia(at)mangiasano.ch

Dr. Michael Beer

Bundesamt für Gesundheit

Abteilung Lebensmittelsicherheit

3003 Bern

michael.beer(at)bag.admin.ch

Prof. Dr. med. Roger Darioli

5, ch. des Fleurs

1007 Lausanne

roger.darioli(at)hospvd.ch

Prof. Dr. Katharina Meyer, MPH

Inselspital, Universitätsspital Bern

Universitätspoliklinik für Endokrinologie,

Diabetologie \& Klinische Ernährung

3010 Bern

katharina.meyer(at)insel.ch
Dr. Andrea Renggli

Bundesamt für Gesundheit

Abteilung Lebensmittelsicherheit

Sektion Ernährungs- undToxikologische Risiken

Stauffacherstrasse 101

8004 Zürich

andrea.renggli(at)bag.admin.ch

Dr. Christine Römer-Lüthi

Berner Fachhochschule Gesundheit

Murtenstrasse 10

3008 Bern

christine.roemer-luethi(at)bfh.ch

Nadine Stoffel-Kurt

Bundesamt für Gesundheit

Abteilung Nationale Präventionsprogramme

Sektion Ernährung und Bewegung

3003 Bern

nadine.stoffel-kurt(at)bag.admin.ch 


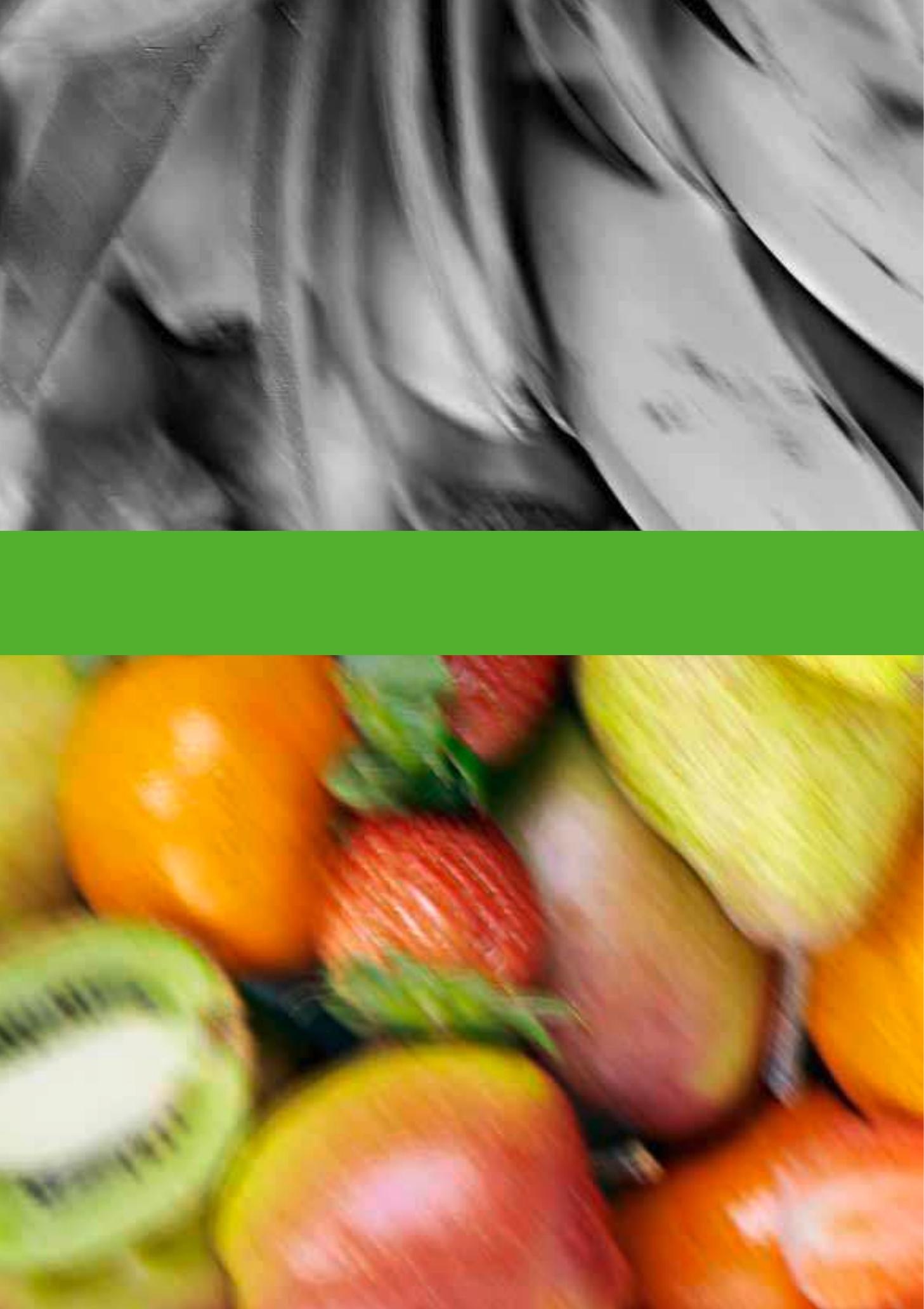